\title{
Longitudinal radiomics for prognosis in non-small cell lung cancer
}

Citation for published version (APA):

van Timmeren, J. (2019). Longitudinal radiomics for prognosis in non-small cell lung cancer. [Doctoral Thesis, Maastricht University]. Ipskamp Printing BV. https://doi.org/10.26481/dis.20190705jt

Document status and date:

Published: 01/01/2019

DOI:

10.26481/dis.20190705jt

Document Version:

Publisher's PDF, also known as Version of record

\section{Please check the document version of this publication:}

- A submitted manuscript is the version of the article upon submission and before peer-review. There can be important differences between the submitted version and the official published version of record.

People interested in the research are advised to contact the author for the final version of the publication, or visit the DOI to the publisher's website.

- The final author version and the galley proof are versions of the publication after peer review.

- The final published version features the final layout of the paper including the volume, issue and page numbers.

Link to publication

\footnotetext{
General rights rights.

- You may freely distribute the URL identifying the publication in the public portal. please follow below link for the End User Agreement:

www.umlib.nl/taverne-license

Take down policy

If you believe that this document breaches copyright please contact us at:

repository@maastrichtuniversity.nl

providing details and we will investigate your claim.
}

Copyright and moral rights for the publications made accessible in the public portal are retained by the authors and/or other copyright owners and it is a condition of accessing publications that users recognise and abide by the legal requirements associated with these

- Users may download and print one copy of any publication from the public portal for the purpose of private study or research.

- You may not further distribute the material or use it for any profit-making activity or commercial gain

If the publication is distributed under the terms of Article $25 \mathrm{fa}$ of the Dutch Copyright Act, indicated by the "Taverne" license above, 


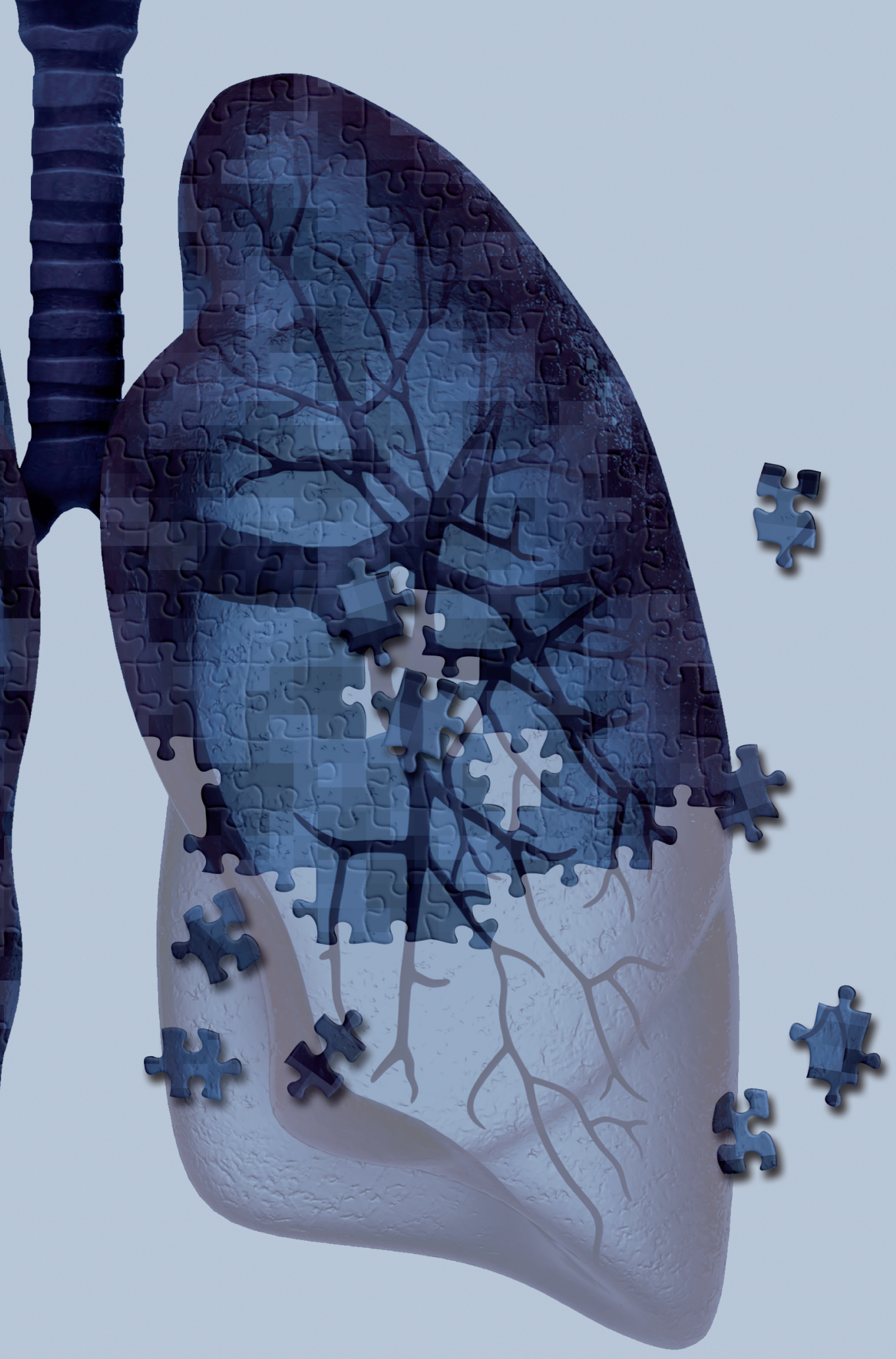

\section{LONGITUDINAL RADIOMICS}

for prognosis in non-small cell lung cancer Janjita van Tinsmsneren. 



\section{Longitudinal radiomics for prognosis in non-small cell lung cancer}

Janita van Timmeren 


\section{Production}

Printing: Ipskamp Printing BV, Enschede

ISBN: 978-94-028-1444-6

(c) Copyright J.E. van Timmeren, Maastricht 2019 


\title{
Longitudinal radiomics for prognosis in non-small cell lung cancer
}

\author{
Proefschrift \\ ter verkrijging van de graad van doctor aan de Universiteit Maastricht, \\ op gezag van de Rector Magnificus, Prof. dr. Rianne M. Letschert, \\ volgens het besluit van het College van Decanen, \\ in het openbaar te verdedigen \\ op vrijdag 5 juli 2019 om 12.00 uur \\ door
}

Janna Elizabeth van Timmeren 


\section{Promotor}

Prof. dr. P. Lambin

\section{Copromotoren}

Dr. ir. W. van Elmpt

Dr. ir. R.T.H. Leijenaar

\section{Beoordelingscommissie}

Prof. dr. ir. F.J.W. Verhaegen (voorzitter)

Prof. dr. R. Boellaard (Universitair Medisch Centrum Groningen)

Prof. dr. A.-M.C. Dingemans

Prof. dr. M.E. Kooi

Prof. dr. J.J. Sonke (Nederlands Kanker Instituut) 


\section{CONTENTS}

Page

Introduction

Chapter 1 Introduction and outline of the thesis

Longitudinal radiomics

Chapter 2 Survival prediction of non-small cell lung cancer patients using radiomics analyses of cone-beam CT images

Chapter 3 Feature selection methodology for longitudinal cone-

Chapter 4 Longitudinal radiomics of cone-beam CT images from non-small cell lung cancer patients: evaluation of the added prognostic value for overall survival and locoregional recurrence

Chapter 5 Tumor regression during radiotherapy for non-small cell lung cancer patients using cone-beam computed

\section{Challenges} tomography

Chapter 6 Test-retest data for radiomics feature stability analysis, generalizable or study specific?

Chapter 7 Influence of gray-level discretization on radiomic feature stability for different CT scanners, tube currents

Chapter 8 Challenges and caveats of a multi-center retrospective radiomics study: an example of early treatment response assessment for NSCLC patients using FDG$\mathrm{PET} / \mathrm{CT}$ radiomics

\section{Future and discussion}

Chapter 9 Complementary value of deep learning and radiomics for prognosis and histology classification in non-small cell lung cancer

Chapter 10 General discussion and future perspectives

\section{Appendices}

Summary

Samenvatting

Valorization addendum

Curriculum Vitae

Publication list

Dankwoord 



\section{Chapter \\ Introduction and outline of the thesis}

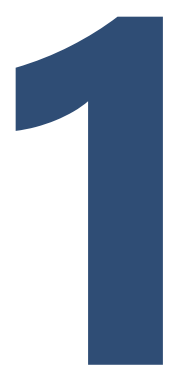





\section{Cancer}

Genetic changes in the body can cause uncontrollable grow of cells that result in a malignant tumor, which is able to invade into different structures and spread through the body (metastasize). The generic term for this phenomenon is cancer, which is the Latin translation of 'crab'. This word is associated to the appearance of tumors, which usually have enlarged veins and irregular shapes. Cancer accounted for 8.2 million deaths worldwide in 2012 [1] and 9.6 million estimated deaths in 2018 [2]. Incidence and mortality worldwide are presented in Figure 1.1.

There exist over 100 different types of cancer. In this thesis, the focus will be on lung cancer, which is the most common cause of death from cancer worldwide in both men and women, accounting for $18.4 \%$ of all cancer deaths [1, 2]. As the known risk factors include smoking and air pollution, most incidences of lung cancer are found in more developed regions, with an incidence rate of 40.4 per 10,000 men and 19.4 per 10,000 women in Western Europe [2]. In Europe, on average $12.6 \%$ of patients diagnosed with lung cancer were still alive after 5 years [3].

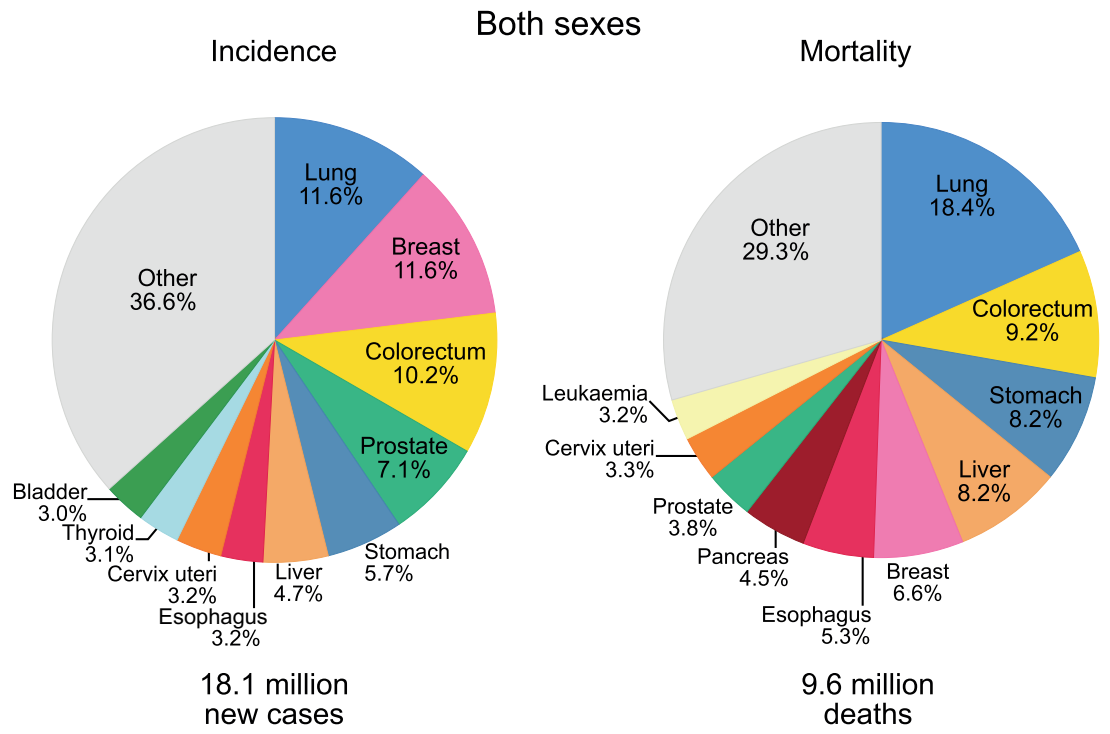

Figure 1.1. Distribution of incidence and mortality of the 10 most common cancers worldwide in 2018 [2].

From Global cancer statistics 2018: GLOBOCAN estimates of incidence and mortality worldwide for 36 cancers in 185 countries by Freddie Bray, Jacques Ferlay, Isabelle Soerjomataram, et al.

Copyright (C) 2018 by John Wiley and Sons, Inc. Reprinted by permission of John Wiley \& Sons, Inc.

\section{Lung cancer}

Two main types of lung cancer can be distinguished: small-cell lung cancer and nonsmall cell lung cancer (NSCLC). The latter accounts for more than $80 \%$ of all lung cancers $[4,5]$. Among the cancers classified as NSCLC, several histologic subtypes can be described, including adenocarcinoma, squamous cell carcinoma and large cell 
carcinoma [6]. Generally, a biopsy (i.e. a sample of tissue) will be taken prior to treatment to decide on the pathologic classification [7]. In some cases, not enough tissue can be obtained for classification, and the terminology 'not-otherwisespecified' (NOS) is used [8]. About $10 \%$ of NSCLC patients are classified with NOS in Europe [3].

Besides the histology, the stage of tumor will be determined prior to a treatment decision. A TNM Classification of Malignant Tumors (TNM) was developed to describe the stage of a tumor by classifying the location and size of the (primary) tumor $(\mathrm{T})$, the extent of spread in regional lymph nodes $(\mathrm{N})$ and the existence of distant metastases (M) $[9,10]$. The combination of $T, N$ and $M$ summarizes the overall stage (e.g. I, IIla). Patients with stage I are usually described as early-staged, whereas patients with stage III are considered to have locally-advanced disease and patients with stage IV suffer from metastatic disease.

For NSCLC, generally also mutation status is assessed. The presence of mutations in, for instance, epidermal growth factor receptor (EGFR) or Kirsten rat sarcoma viral oncogene homolog (KRAS), or gene rearrangements in anaplastic lymphoma kinase (ALK) or c-ros oncogene 1 (ROS1), will influence the decision of first-line treatment.

\section{Patient}

Besides the stage of the cancer, mutation status and the (histological) type of cancer, a patient's condition is examined upon initial treatment decision [8]. The pretreatment evaluation includes assessment of treatment risk, comorbidities and expected quality of life after treatment [8]. Patient's general health is typically assessed using the World Health Organization (WHO) performance status [11], which is related to the ability of the patient to perform activities in daily life on a scale from 0 (perfect health) to 5 (death). Furthermore, for lung cancer patients, pulmonary function tests are performed to acquire the Forced Expiration Volume in 1 second $\left(\mathrm{FEV}_{1}\right)$ and/or maximal oxygen consumption ( $\left.\mathrm{VO}_{2} \max \right)$ [8]. Moreover, additional information from the patient is collected, including smoking status, medical history and demographic information like age and gender.

\section{Treatment}

Cancer treatment is with either palliative or curative intent [12]. In the first case, the disease is considered incurable and the treatment is intended to reduce pain and to improve or prolong quality of life. On the other hand, curative treatment is intended to eradicate the disease using a more radical treatment. In this thesis, only patients receiving curative treatment intention are considered.

Here, several types of treatment are possible, including surgery, radiation treatment (radiotherapy) or systemictreatment (e.g.chemotherapy or immunotherapy) [8]. Patients with early-stage disease will usually be treated with surgical resection or stereotactic body radiation therapy (SBRT). Patients with stage II or III will usually receive a combined treatment, e.g. surgery followed by chemotherapy or radiotherapy combined with chemotherapy $[8,13]$. Concurrent chemoradiotherapy is the preferred treatment for stage IIIA and IIIB patients, as this was shown to improve 5 -year survival 
rates [14]. The alternative for patients with relevant comorbidities is induction chemotherapy followed by radiotherapy (known as sequential chemoradiotherapy).

\section{Radiotherapy}

Radiation therapy (RT) is an important treatment option for lung cancer patients, more than $50 \%$ receive radiotherapy at least once. For NSCLC patients with unresectable locally-advanced disease, radiotherapy is the preferred choice of treatment [8]. For lung cancer, external beam radiotherapy (EBRT) is most often used, which means that the source of radiation is located outside the body. The radiation, e.g. particles or photons with high energy originating from a linear accelerator (linac), will be administered to the body to damage cancer cells. Radiation dose is measured in Gray (Gy). Higher doses will result in a larger kill of cancer cells, but will also damage normal, healthy tissue to a large extent, which can lead to toxicity. Therefore, the radiation dose is administered in small fractions to give the healthy tissue the opportunity to recover. The rationale of fractionation is that the complex cell killing and repair mechanism is different between cancer tissue and healthy tissue [15]. Fractionation schedules are varying between institutes, but a commonly used scheme is 30-33 daily fractions of 2 Gy over 6-7 weeks of treatment [8].

\section{Medical imaging}

Medical imaging plays a fundamental role during the period from diagnosis to follow-up after treatment. During the radiotherapy process many image modalities are involved, including computed tomography (CT), positron emission tomography (PET) and cone-beam CT (CBCT).

\section{Computed tomography}

A CT scan is a three-dimensional (3D) image consisting of stacked 2D cross-sectional images of the body, acquired using a source of X-rays and a detector (i.e. CT scanner). The appearance of tissue on the image depends on the amount of attenuation of the $\mathrm{X}$-rays. At image reconstruction, attenuation coefficients are linearly transformed to obtain CT numbers. Therefore, the pixels in a CT image have quantitative values measured in Hounsfield Units $(\mathrm{HU})$. Water is represented by $0 \mathrm{HU}$, whereas air has $-1000 \mathrm{HU}$.

\section{Positron emission tomography}

For tumor visualization, 18 -fluor-fluorodeoxyglucose $\left({ }^{18} \mathrm{~F}-\mathrm{FDG}\right)$-PET is most commonly used in clinical practice [16]. A radionuclide is labeled to a glucose molecule (tracer) and administered to the body. The radionuclide is an unstable isotope that emits positrons, which will hit an electron and transform into two photons (annihilation process). These photons will be detected by a ring of detectors around the patient. Due to varying tracer uptake in tissues, high or low intensity areas are visualized on a PET image. Tumor cells have a higher metabolic activity and will thus have a higher uptake of these radiolabeled glucose molecules. This makes it possible to distinguish the tumor from healthy cells on a PET image. 


\section{PET/CT}

Combining PET and CT implies that the biological processes (PET) and the anatomy (CT) can be imaged simultaneously on a single device (i.e. PET/CT scanner). A PET/CT image is generally acquired to perform radiation treatment planning [17]. Using this treatment planning scan, the tumor and organs at risk (OAR) can be accurately located and delineated by the radiation oncologist by drawing contours around the regions of interest (ROI). For lung cancer, this includes amongst others segmentation of the tumor, lungs, heart, esophagus and spinal cord. The segmented primary tumor is called the gross tumor volume (GTV). The CT image and the contours will be used to make a radiation treatment plan, which contains the direction, size and energy of all radiation beams and accurately calculates how much dose will be delivered to the tumor and the surrounding tissues.

\section{Cone-beam CT}

Because of the high precision of a radiation treatment, it is of major importance that the position of the patient matches the position at the moment the treatment planning PET/CT scan was made to ensure the dose is delivered to the correct location. To verify the position of the patient and to capture interfractional changes, usually a cone-beam $\mathrm{CT}$ (CBCT) image is made prior to the delivery of a radiotherapy fraction [18]. Moreover, the CBCT images can guide decisions about adaptations to the treatment plan in case significant changes in the patient anatomy occur: adaptive radiotherapy (ART).

A CBCT system is mounted to the linac and uses kilovoltage $(\mathrm{kV})$ energy $\mathrm{X}$-rays in a cone-shaped beam [19]. The $\mathrm{X}$-rays are detected behind the patient using a flat panel detector. CBCT images have inferior image quality compared to conventional CT images, caused by the different technique and lower irradiation dose, which causes more scatter, artifacts and lower contrast. Moreover, CBCT images are more susceptible to motion artifacts (e.g. blurring) as the acquisition of one scan takes about 40 seconds up to two minutes [18].

The frequency of $C B C T$ imaging during radiotherapy differs per institution. In clinical practice, for lung cancer patients, a CBCT scan is generally made prior to the first three radiotherapy fractions and then weekly. In other institutions, CBCT scans are made prior to each radiotherapy fraction, which is usually daily or sometimes even twice-daily. In case of daily imaging, this means that usually large numbers of CBCT images are stored per patient, up to 30 or 40 . Figure 1.2 shows an example of a treatment planning $\mathrm{CT}$ image and weekly $\mathrm{CBCT}$ images of the same patient.

\section{Personalized treatment}

Although information about tumor stage, histology, general patient health and patient choice are taken into account upon initial treatment decision, fully individualized radiation treatment does currently not exist. Prediction models could help to adjust a treatment to patient-specific demands. These models are developed and validated to predict patient outcome, e.g. overall survival, disease recurrence or toxicity. Prediction models can aid in clinical decision making and are generally part of decision support systems, which are essential in the individualized treatment 

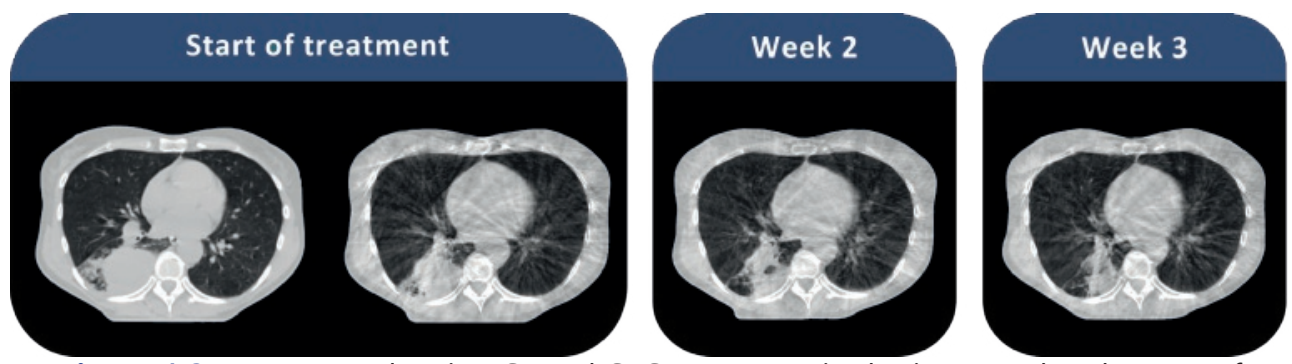

Figure 1.2. Treatment planning $C T$ and $C B C T$ scan acquired prior to and the start of treatment (left), CBCT acquired at the second week of treatment (middle) and CBCT acquired at the third week of treatment (right).

approach [20]. To build these models, factors that have either prognostic or predictive value, are required. A predictive factor estimates the potential benefit of a specific therapy, whereas a prognostic factor is related to a certain patient outcome regardless of the therapy $[21,22]$. In this thesis, only prognostic factors are considered to either predict overall survival or locoregional recurrence for NSCLC patients.

Many factors derived from different information sources could potentially be incorporated in a prediction model. Prognostic factors for NSCLC include biological factors (e.g. circulating immune cells, protein expressions, gene mutations), clinical parameters (e.g. age, weight loss), treatment factors (e.g. dose) or tumor related factors (e.g. tumor size, histological classification) [10]. Moreover, qualitative and quantitative information can be derived from the medical images that are acquired during the treatment process. The qualitative information can be summarized in semantic features, which describe the appearance of the tumor and/or the lungs, for example the presence of calcification or tumor cavitation. On the other hand, quantitative features can be derived which are defined by a mathematical formula and provide a measure for the phenotype of the tumor, e.g. shape and texture [23, 24]. The principle of extracting these large amounts of quantitative features is called radiomics. Image acquisition is performed in clinical practice, which means that radiomic features can be extracted without the need for extra patient imaging burden. Moreover, they are able to capture 3D-information, fast and userindependent.

\section{Radiomics workflow}

The workflow of radiomics consists of image acquisition, segmentation of the ROI, feature extraction, feature selection and analysis of these features (e.g. modeling). Figure 1.3 summarizes the workflow of radiomics.

\section{Image acquisition}

Acquiring medical images is the crucial step for initiating the radiomics workflow. Any type of modality can be used here, but CT, CBCT and PET are the modalities of interest in this thesis. Often, the images included in a radiomics study are collected a few years after the patient was treated (retrospective data). This means that the images are usually acquired in a non-controlled setting, using a variation of different 
imaging systems, acquisition parameters and reconstruction settings. Therefore, imaging heterogeneities are often present within and between datasets.

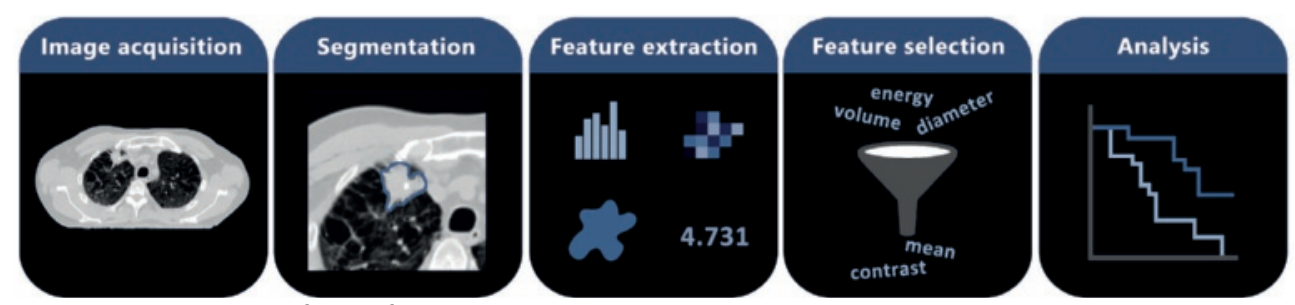

Figure 1.3. Workflow of radiomics.

\section{Segmentation}

Segmentation is an important step in the radiomics workflow, because this step defines the ROI for radiomic feature extraction. The GTV, which was delineated for treatment planning purposes, is the considered ROI in this thesis.

\section{Feature extraction}

Over thousands of radiomic features can be extracted from images. These can roughly be divided into five categories: first order statistics, shape, texture, fractal and filtered features. The first order statistics features describe the distribution of intensity levels within the ROI, including minimum, mean, skewness and kurtosis. The texture features describe the relationship between one voxel and its neighbor and describe amongst others the coarseness, contrast and energy. Filtered features are calculated after a certain type of filter (e.g. wavelet or Laplacian of Gaussian (LoG)) is applied to the image.

\section{Feature selection}

Prior to modeling, a feature reduction step is essential, as the number of variables is generally much larger than the number of patients, resulting in high risks for overfitting the model. First of all, redundant features should be removed, as highly correlated features represent the same phenotype. Moreover, features that are susceptible to variations (e.g. multi-observer segmentation variations) should be removed, as these are not reproducible. Moreover, features that change much in a test-retest situation, e.g. a situation in which two images are acquired in a short time span without changing the settings, can be considered unstable. Due to natural biological processes in the body, two subsequent images will never be exactly the same, but the radiomic features should not be too sensitive for these small variations.

\section{Modeling}

After feature reduction, a modeling technique can be chosen to build a prognostic model. Several studies have evaluated the performance of different machine learning techniques for prediction modeling. One of the methods that is used throughout this thesis is least absolute shrinkage and selection operator (LASSO), which is a form of penalized regression that minimizes regression coefficients to zero [25]. It is a 
powerful method for large numbers of variables as it performs a feature selection and regularization simultaneously.

The performance of a prediction model can be summarized using several measures. This includes Kaplan-Meier curves, which are survival curves of groups of patients that are commonly used to visualize the ability of the model to distinguish between high and low risk groups. Moreover, Harrell's concordance index (c-index) is a measure that indicates a model's discriminative power and ranges from 0.5 (random) to 1.0 (perfect discrimination).

\section{This thesis: radiomics for prognosis in NSCLC}

The prognostic value of radiomic features extracted from CT for NSCLC has been studied before. In 2012, a four-feature radiomic signature was developed to predict overall survival for NSCLC patients [26], which was further validated in external cohorts [27]. Subsequently, multiple other models were developed for prognosis in NSCLC using baseline imaging acquired prior to treatment. Additionally, some studies have investigated the prognostic value of radiomic features changing during the course of treatment (i.e. longitudinal radiomics), for example using weekly PET or CT imaging [28].

Longitudinal information allows for monitoring changes over the course of treatment, which could contribute to individualized decisions about (early) treatment adaptations. CBCT imaging is a potential powerful candidate for longitudinal radiomics, as large numbers of these images are acquired in clinical practice. In case the radiomic features contain prognostic value, this could improve prediction models to assess (early) response to treatment on an individual basis. Personalized treatment based on patient's characteristics is thought to achieve better patient outcomes because of better informed treatment decisions. These ideas have resulted in the following hypothesis which is investigated in this thesis:

Quantitative information derived from medical images acquired prior to and during treatment describing the (changing) phenotype of the tumor, provides prognostic information for non-small cell lung cancer patients.

\section{Feasibility of $C B C T$ radiomics}

Although $C B C T$ images could be an ideal modality for longitudinal radiomics for prognosis in NSCLC, the lower image quality and susceptibility for artifacts raises questions about the feasibility of extracting radiomic features from $C B C T$ images. Therefore, the first aim of this thesis was to investigate the feasibility of longitudinal radiomics using $C B C T$ imaging and subsequently to investigate the prognostic value of these features for NSCLC patients.

In Chapter 2, the feasibility of extracting radiomic features from CBCT images was evaluated by investigating the interchangeability between CT- and CBCTextracted radiomic features. In Chapter 3, a feature selection methodology was developed to select radiomic features that are relevant in a longitudinal setting. Features that are too stable and do not change over the course of treatment will not contain additional information over features acquired prior to treatment (at baseline). This chapter evaluates which features do change over the course of treatment. The 
prognostic value of these radiomic features extracted from CBCT images during radiotherapy, is evaluated in Chapter 4 . Whereas Chapter 4 evaluates all radiomic features, Chapter 5 focusses on the change of tumor volume during treatment and the relationship between this change and patient outcome.

\section{Challenges of radiomics}

The entire radiomics process faces multiple challenges and the reproducibility is prone to variations in image acquisition and reconstruction settings, segmentation of the ROI, pre-processing of the images and implementation of the mathematical calculations. Several studies performed in this thesis were influenced by these factors, therefore the challenges are an important theme throughout the thesis. The aim of the second part of the thesis was to evaluate some (technical) challenges in the field of radiomics. Chapter 6 compares two test-retest datasets to investigate feature stability. In Chapter 7 the results of a phantom study are shown, in which the influence of gray-level discretization on radiomic feature stability was investigated. Chapter 8 presents a multicenter retrospective radiomics study that faces challenges in terms of cohort size and heterogeneities within and between the datasets.

\section{Future}

A recent development is deep learning. Although the principle is old, the use of deep networks for image classification recently achieved high performances. In Chapter 9, the potential of deep learning was investigated. The complementary value of radiomics and deep learning was evaluated for NSCLC patients. Chapter 10 completes the thesis with an extensive discussion about the barriers and future prospects of (longitudinal) radiomics for prognosis in NSCLC.

Figure 1.4 shows a summary of the outline in this thesis. 


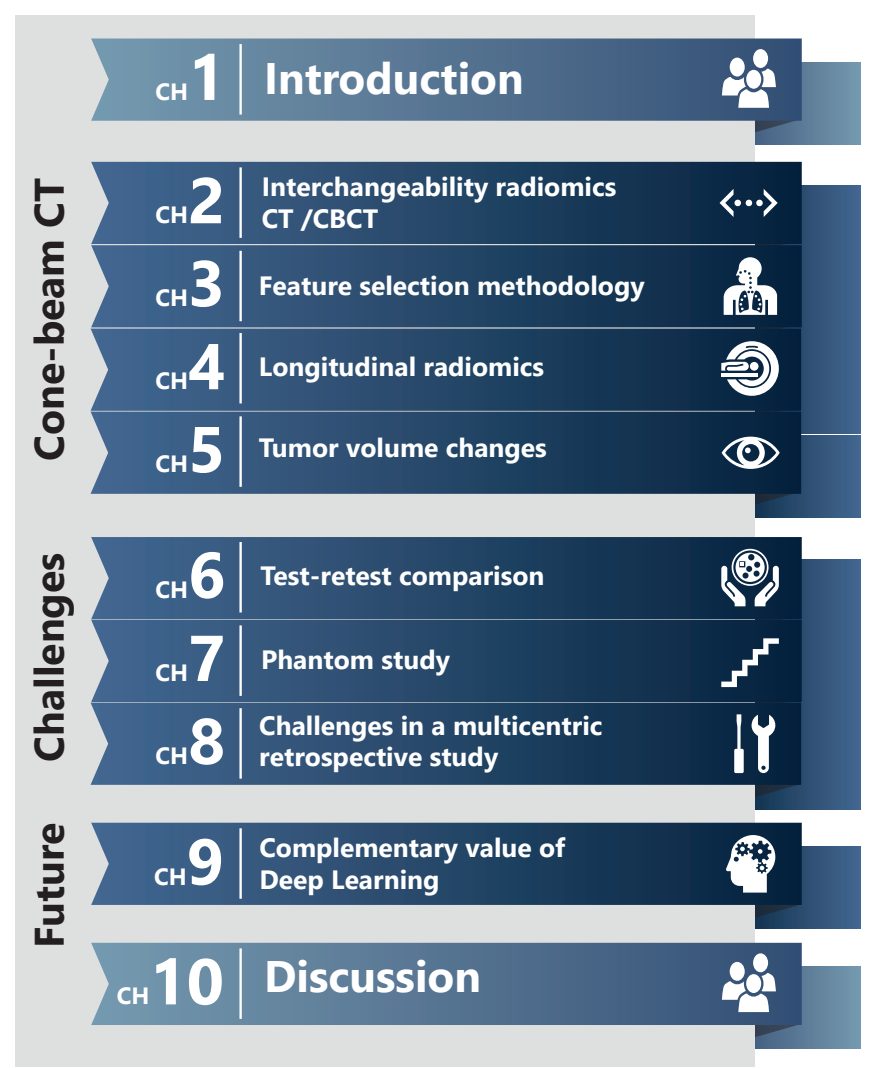

Figure 1.4. Outline of the thesis. 


\section{References}

[1] Torre LA, Bray F, Siegel RL, Ferlay J, Lortet-Tieulent J, Jemal A. Global cancer statistics, 2012. CA Cancer J Clin. 2015;65:87-108.

[2] Bray F, Ferlay J, Soerjomataram I, Siegel RL, Torre LA, Jemal A. Global cancer statistics 2018: GLOBOCAN estimates of incidence and mortality worldwide for 36 cancers in 185 countries. CA Cancer J Clin. 2018.

[3] Francisci S, Minicozzi P, Pierannunzio D, Ardanaz E, Eberle A, Grimsrud TK, et al. Survival patterns in lung and pleural cancer in Europe 1999-2007: Results from the EUROCARE-5 study. Eur J Cancer. 2015;51:2242-53.

[4] Jemal A, Bray F, Center MM, Ferlay J, Ward E, Forman D. Global cancer statistics. CA Cancer J Clin. 2011;61:69-90.

[5] Molina JR, Yang P, Cassivi SD, Schild SE, Adjei AA. Non-small cell lung cancer: epidemiology, risk factors, treatment, and survivorship. Mayo Clin Proc. 2008;83:584-94.

[6] Goldstraw P, Ball D, Jett JR, Le Chevalier T, Lim E, Nicholson AG, et al. Non-small-cell lung cancer. Lancet. 2011;378:1727-40.

[7] Travis WD, Brambilla E, Nicholson AG, Yatabe Y, Austin JHM, Beasley MB, et al. The 2015 World Health Organization Classification of Lung Tumors: Impact of Genetic, Clinical and Radiologic Advances Since the 2004 Classification. J Thorac Oncol. 2015; 10:1243-60.

[8] Postmus PE, Kerr KM, Oudkerk M, Senan S, Waller DA, Vansteenkiste J, et al. Early and locally advanced non-small-cell lung cancer (NSCLC): ESMO Clinical Practice Guidelines for diagnosis, treatment and follow-up. Ann Oncol. 2017;28:iv1-iv21.

[9] Goldstraw P, Chansky K, Crowley J, Rami-Porta R, Asamura H, Eberhardt WE, et al. The IASLC Lung Cancer Staging Project: Proposals for Revision of the TNM Stage Groupings in the Forthcoming (Eighth) Edition of the TNM Classification for Lung Cancer. J Thorac Oncol. 2016;11:39-51.

[10] Brierley JD, Gospodarowicz MK, C. W. TNM Classification of Malignant Tumours. 8th ed: Oxford: John Wiley \& Sons, Inc.; 2016.

[11] Oken MM, Creech RH, Tormey DC, Horton J, Davis TE, McFadden ET, et al. Toxicity and response criteria of the Eastern Cooperative Oncology Group. Am J Clin Oncol. 1982;5:64955.

[12] Numico G, Russi E, Merlano M. Best supportive care in non-small cell lung cancer: is there a role for radiotherapy and chemotherapy? Lung Cancer. 2001;32:213-26.

[13] Tsao MS, Marguet S, Le Teuff G, Lantuejoul S, Shepherd FA, Seymour L, et al. Subtype Classification of Lung Adenocarcinoma Predicts Benefit From Adjuvant Chemotherapy in Patients Undergoing Complete Resection. J Clin Oncol. 2015;33:3439-46.

[14] Auperin A, Le Pechoux C, Rolland E, Curran WJ, Furuse K, Fournel P, et al. Meta-analysis of concomitant versus sequential radiochemotherapy in locally advanced non-small-cell lung cancer. J Clin Oncol. 2010;28:2181-90.

[15] Mitchell G. The rationale for fractionation in radiotherapy. Clin J Oncol Nurs. 2013:17:412-7.

[16] Bunyaviroch T, Coleman RE. PET evaluation of lung cancer. J Nucl Med. 2006;47:451-69.

[17] Gregoire V, Haustermans K, Geets X, Roels S, Lonneux M. PET-based treatment planning in radiotherapy: a new standard? J Nucl Med. 2007;48 Suppl 1:68s-77s.

[18] Boda-Heggemann J, Lohr F, Wenz F, Flentje M, Guckenberger M. kV cone-beam CTbased IGRT: a clinical review. Strahlenther Onkol. 2011;187:284-91.

[19] Oelfke U, Tucking T, Nill S, Seeber A, Hesse B, Huber P, et al. Linac-integrated kV-cone beam CT: technical features and first applications. Med Dosim. 2006;31:62-70.

[20] Lambin P, van Stiphout RG, Starmans MH, Rios-Velazquez E, Nalbantov G, Aerts HJ, et al. Predicting outcomes in radiation oncology--multifactorial decision support systems. Nat Rev Clin Oncol. 2013;10:27-40.

[21] Ballman KV. Biomarker: Predictive or Prognostic? J Clin Oncol. 2015;33:3968-71.

[22] Ettinger DS, Aisner DL, Wood DE, Akerley W, Bauman J, Chang JY, et al. NCCN Guidelines Insights: Non-Small Cell Lung Cancer, Version 5.2018. J Natl Compr Canc Netw. 2018;16:80721. 
[23] Gillies RJ, Kinahan PE, Hricak H. Radiomics: Images Are More than Pictures, They Are Data. Radiology. 2016;278:563-77.

[24] Lambin P, Rios-Velazquez E, Leijenaar R, Carvalho S, van Stiphout RG, Granton P, et al. Radiomics: extracting more information from medical images using advanced feature analysis. Eur J Cancer. 2012;48:441-6.

[25] Tibshirani R. Regression shrinkage and selection via the lasso. J Royal Stat Soc. 1996:26788.

[26] Aerts HJ, Velazquez ER, Leijenaar RT, Parmar C, Grossmann P, Carvalho S, et al. Decoding tumour phenotype by noninvasive imaging using a quantitative radiomics approach. Nat Commun. 2014;5:4006.

[27] Leijenaar RT, Carvalho S, Hoebers FJ, Aerts HJ, van Elmpt WJ, Huang SH, et al. External validation of a prognostic CT-based radiomic signature in oropharyngeal squamous cell carcinoma. Acta Oncol. 2015;54:1423-9.

[28] Fave X, Zhang L, Yang J, Mackin D, Balter P, Gomez D, et al. Delta-radiomics features for the prediction of patient outcomes in non-small cell lung cancer. Sci Rep. 2017;7:588. 

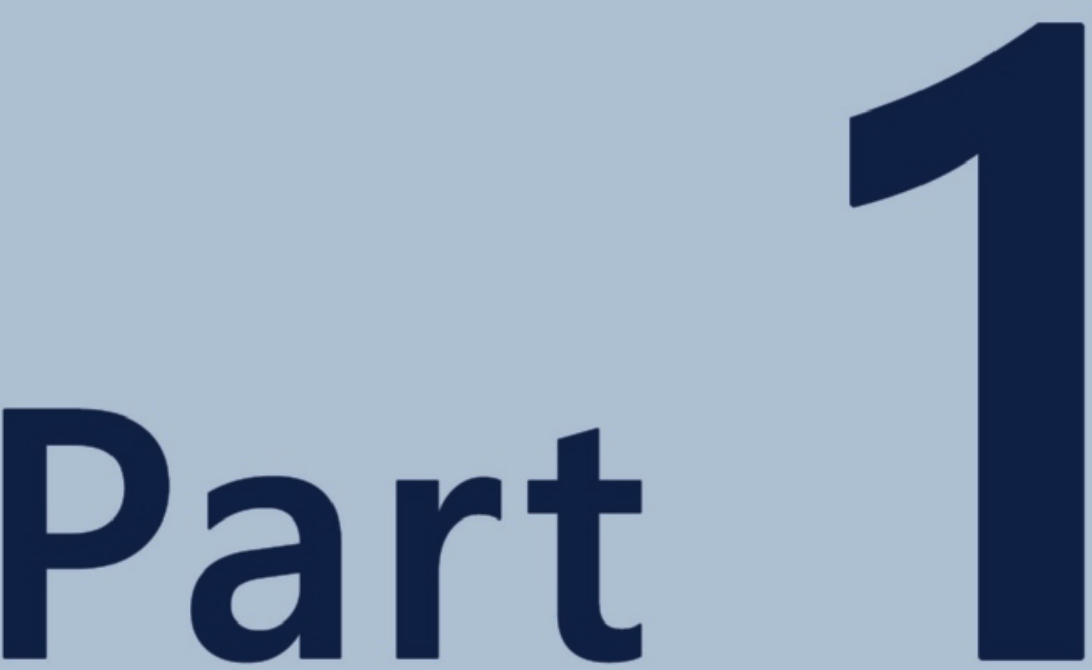



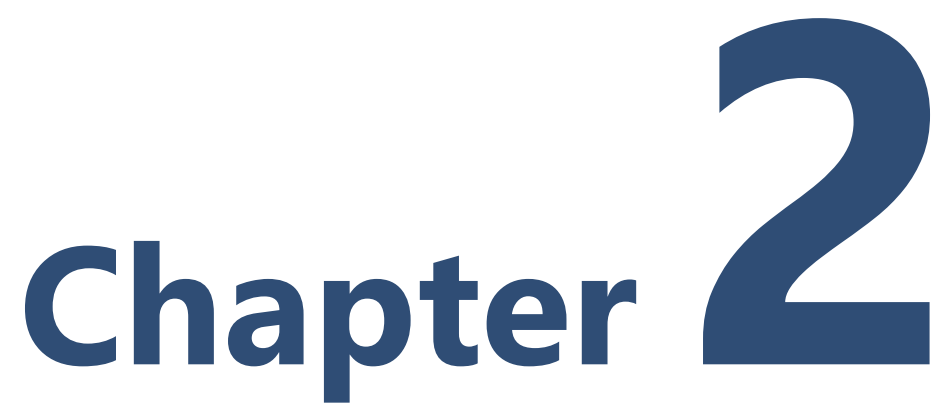

\section{Survival prediction of non-small cell lung cancer patients using radiomics analyses of cone-beam CT images}

Authors

Janna E. van Timmeren, Ralph T.H. Leijenaar, Wouter van Elmpt, Bart Reymen, Cary Oberije, René Monshouwer, Johan Bussink, Carsten Brink, Olfred Hansen,

Philippe Lambin

Adapted from

Radiotherapy \& Oncology. 2017 April; 123(3):363-9.

DOI: 10.1016/j.radonc.2017.04.016 


\section{ABSTRACT}

\section{Background and Purpose}

In this study we investigated the interchangeability of planning CT and cone-beam CT (CBCT) extracted radiomic features. Furthermore, a previously described CT based prognostic radiomic signature for non-small cell lung cancer (NSCLC) patients using CBCT based features was validated.

\section{Material and Methods}

One training dataset of 132 and two validation datasets of 62 and 94 stage I-IV NSCLC patients were included. Interchangeability was assessed by performing a linear regression on $C T$ and $C B C T$ extracted features. A two-step correction was applied prior to model validation of a previously published radiomic signature.

\section{Results}

$13.3 \%$ (149 out of 1119) of the radiomic features, including all features of the previously published radiomic signature, showed an $R^{2}$ above 0.85 between intermodal imaging techniques. For the radiomic signature, Kaplan-Meier curves were significantly different between groups with high and low prognostic value for both modalities. Harrell's concordance index was 0.69 for CT and 0.66 for CBCT models for dataset 1 .

\section{Conclusions}

The results show that a subset of radiomic features extracted from CT and CBCT images are interchangeable using simple linear regression. Moreover, a previously developed radiomics signature has prognostic value for overall survival in three $\mathrm{CBCT}$ cohorts, showing the potential of $\mathrm{CBCT}$ radiomics to be used as prognostic imaging biomarker. 


\section{Introduction}

With 1.6 million deaths in 2012, lung cancer is the most common cause of death from cancer worldwide $[1,2]$. Lung cancer is also the most frequently diagnosed cancer with 1.82 million new cases in 2012, comprising $12.9 \%$ of the worldwide incidence. Improved disease outcome and a subsequent increase in a patient's chance of survival can be achieved by individualized treatment [3-5]. To this end, biomarkers are needed $[6,7]$.

Medical imaging has become a cornerstone of personalized cancer treatment over the past decades. Novel advanced imaging analysis techniques such as radiomics - extracting quantitative features from medical images such as computed tomography $(\mathrm{CT})$, positron emission tomography (PET), or magnetic resonance imaging (MRI) can identify a patient's response to treatment or the probability of developing side effects [3, 8-14].

Furthermore, a longitudinal approach where the change of quantitative radiomic features (i.e. delta radiomics) is examined, may also aid in early response assessment compared to the use of only baseline (imaging) characteristics [15-17]. However, in most studies, PET-, CT- or MRI-scans are only performed at baseline or at very limited number of points in time, hampering the possibility for timely treatment adaptation. However, during radiotherapy for NSCLC patients, threedimensional (3D) cone-beam CT (CBCT) images are routinely obtained for patient set-up and positioning verification [18]. These images could provide valuable information about day-to-day changes of the tumor during the course of treatment [19].

Radiomics based on CBCT imaging therefore offers a possibility for (early) treatment adaptation using the changes of imaging biomarkers over time. Where the prognostic value of conventional CT images is already known $[9,11,20]$, the potential of $\mathrm{CBCT}$ radiomics still needs to be investigated especially because image quality of CBCT is generally worse compared to conventional CT images. Therefore, in this study we aimed to compare radiomics for $\mathrm{CT}$ and $\mathrm{CBCT}$ by investigating the interchangeability of radiomic features extracted from both modalities. Furthermore, we validated a previously published CT-based radiomics signature (a Cox regression model based on imaging only, without clinical parameters) [20, 21], using three independent $C B C T$ datasets to validate the model and to evaluate the prognostic potential of $C B C T$ imaging compared to $C T$ imaging. 


\section{Methods and Materials}

\section{Patients}

Three NSCLC cohorts from three different institutes were included in this study. All patients received radiation therapy with curative intent. Patients that received less than 40 Gy were excluded from the analysis. Moreover, patients referred to postoperative radiotherapy or simultaneous treatment of brain metastases were excluded, as well as patients with prior history of lung cancer.

The first dataset consists of 132 stage I-IV patients treated between January 2012 and January 2014 at Maastro Clinic, Maastricht, the Netherlands. Data are provided online on www.cancerdata.org [22]. The second dataset consists of 62 stage I-IIIB patients receiving treatment between January 2009 and January 2011 at Radboud University Medical Center, Nijmegen, the Netherlands. The third dataset consists of 94 stage I-IIIB patients, a subset of the cohort used in a previous study on CBCT imaging [19], treated between November 2007 and December 2011 at Odense University Hospital, Odense, Denmark. This retrospective study was approved by each respective institutional review board.

\section{Image acquisition}

The images of the treatment planning CT (pCT) scan and the images of the conebeam CT (CBCT) scan prior to the first radiotherapy fraction were used for all analyses in this study. Details of all image acquisitions can be found in Supplementary Material $2 \mathrm{~A}$.

\section{Feature extraction}

The gross tumor volume (GTV) of the primary tumor was manually delineated on the CT scan by experienced radiation oncologists and used for treatment planning. For each patient, the GTV was registered to the CBCT image using a deformable transformation field obtained by performing non-rigid registration of the $\mathrm{PCT}$ image and the CBCT image $[23,24]$. Afterwards, all contours were visually checked and manually adjusted when necessary by an experienced radiation oncologist.

Radiomic features were extracted from the delineated tumor regions of the PCT and CBCT images. A total of 1119 radiomic features were calculated, divided into five groups: tumor intensity $(n=19)$, texture $(n=95)$, wavelet $(n=912)$, Laplacian of Gaussian $(n=74)$, and shape $(n=19)$. Emphasis was placed on the features of the previously published prognostic radiomic signature: I) tumor intensity: 'Energy', II) texture: 'Gray-level Nonuniformity, III) wavelet: 'Gray-level Nonuniformity HLH', and IV) shape: 'Compactness' [20]. All features were automatically extracted using inhouse developed software, using Matlab 2014a (MathWorks, Natick, Massachusetts, U.S.A.). A mathematical description of all features can be found in Supplementary Material 2K.

\section{Correction}

A two-step correction procedure was performed on CBCT prior to model validation, which will be explained in further detail below. The first step comprises an intensity 
value correction and the second step is a radiomic feature normalization. The workflow of correcting CBCT is shown in Figure 2.1.

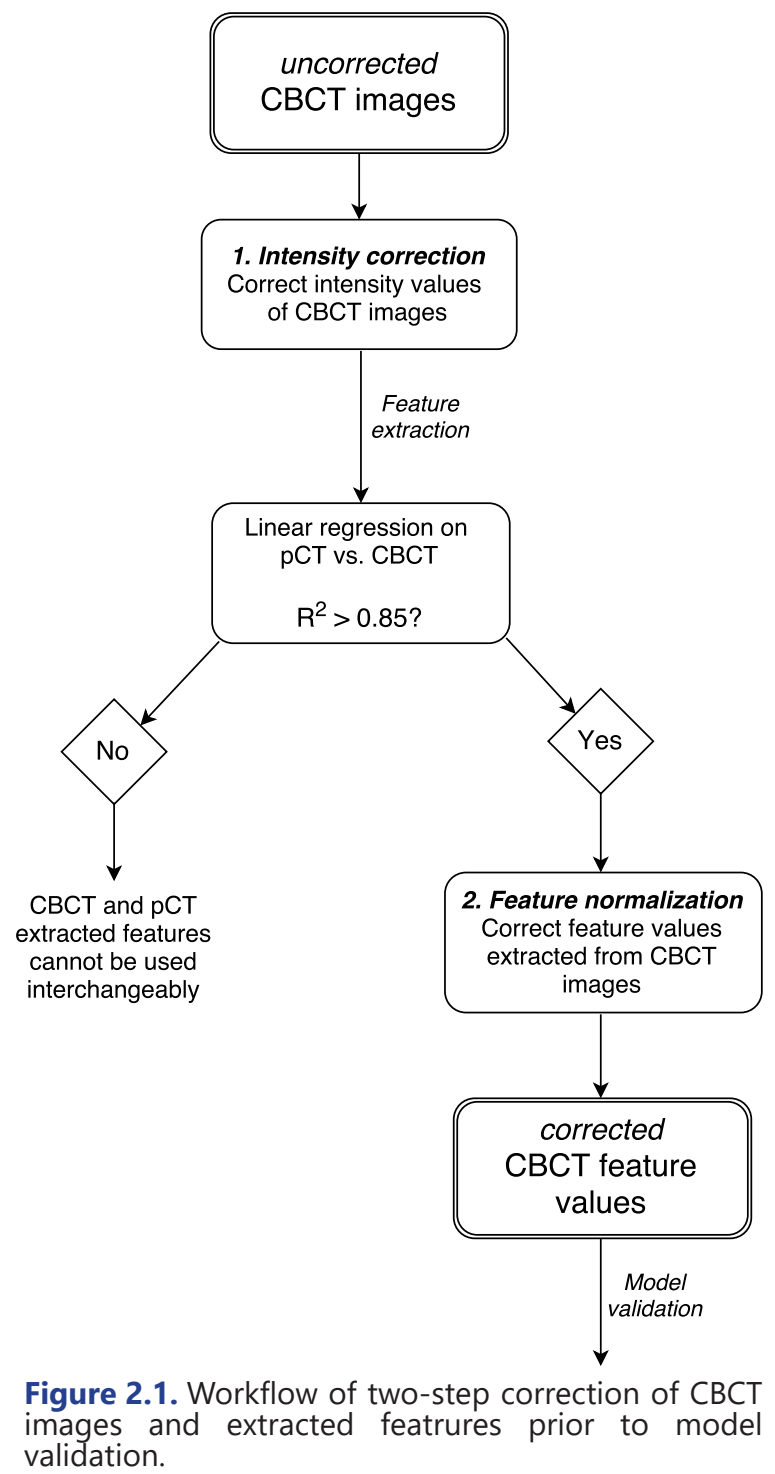

Step 1, the intensity correction, was performed to equalize the distribution of the intensity levels between $C B C T$ images. To find the correction factor, the mean intensity level in a region of interest (ROI) of approximately $5 \mathrm{~cm}^{2}$ in the heart was derived for each patient in the CBCT image. This ROI was chosen because typical Hounsfield units were known and because an area of this size at that location could be drawn for all images. The reference value was set to $50 \mathrm{HU}$, since according to 
literature typical Hounsfield units in myocardium and blood are between 40 and 60 HU [25]. A scaling factor was calculated using (mean intensity level + 1000) / (reference value +1000 ). Correction factors, derived for individual patients, were multiplied with intensity levels of $\mathrm{CBCT}$ images prior to feature extraction. In this study we decided to apply the intensity correction for all images, instead of defining a certain range around the reference value of $50 \mathrm{HU}$ within which some intensity values could be accepted.

Step 2 is the radiomic feature normalization, this was performed to correct CBCT extracted features with a fixed calibration factor in case of a linear relationship between $\mathrm{CT}$ and $\mathrm{CBCT}$ radiomic feature values. The correction factor was derived by performing a linear regression on the $\mathrm{CBCT}$ and $\mathrm{PCT}$ extracted features. A coefficient of determination $\left(R^{2}\right)$ above 0.85 was considered to indicate a strong linear relationship between $\mathrm{PCT}$ and $\mathrm{CBCT}$ feature values, meaning that the linear regression parameters (slope and intercept) could be used to correct radiomic feature values derived from CBCT. This procedure was performed for all datasets for the features of the radiomic signature prior to model validation.

The linear regression parameters were used to derive correction factors for feature normalization for the radiomic signature, but at the same time they provide a measure of interchangeability between conventional $\mathrm{PCT}$ and $\mathrm{CBCT}$ imaging for all radiomic features. Only dataset 1 was used to perform a linear regression on all 1119 radiomic features.

For dataset 1 , the slice thickness of all CBCT images matches with the $\mathrm{pCT}$ images (3 $\mathrm{mm}$ ), whereas in dataset 2 and 3 there is discrepancy in slice thicknesses $(1 \mathrm{~mm}$ for $\mathrm{CBCT}$ and $3 \mathrm{~mm}$ for $\mathrm{pCT}$ ). Therefore, analysis of linear regression parameters of the features of the radiomic signature was performed before and after resampling into equal voxel sizes of $1 \times 1 \times 3 \mathrm{~mm}^{3}$ using linear interpolation.

\section{Validation of previously published radiomics signature}

Model validation was performed on $\mathrm{PCT}$ and $\mathrm{CBCT}$ images of all datasets to validate its prognostic value and to compare prognostic values of the two modalities. The previously published Cox regression model consisted of 4 radiomic features [20]. Overall survival, measured from the start of radiotherapy, was used as endpoint. Patients alive at the end of follow-up were considered right-censored.

The linear predictors (LP), defined as the sum of each coefficient multiplied by the respective feature value with adjustment for average feature values [20], $\Sigma_{i} \beta_{i}\left(x_{i}-\overline{x_{i}}\right)$, were calculated for $\mathrm{PCT}$ and CBCT before and after correction to provide another measure for the interchangeability and to visualize the effect of the two-step correction procedure. The median value of the LP derived from the original dataset was -0.11916 [20], and used to separate patients with high prognostic value (i.e. linear predictor below the median) from patients with low prognostic value.

Model validation was performed on all datasets using multiple methods [26], similar as described in Leijenaar et al. [21]. For the first measure of validation, Cox regression was performed on the prognostic index $\left(\mathrm{Pl}\right.$, defined as $\Sigma_{i} \beta_{i} x_{i}$, equal to LP without 
adjustment for average feature values) to determine the calibration slope, where a slope equal to 1 means that the relative risk model is still valid and there is no need for recalibration. A log-rank (LR) test was used to test whether the slope was different from 1 . Secondly, model fit was evaluated by performing the Cox regression on the individual features of the signature with variable coefficients plus the PI with a fixed coefficient of 1 (i.e. offsetting the PI), and performing a joint test that all feature coefficients are 0 . Thirdly, discrimination of the model was assessed by calculating Harrell's concordance index (c-index), ranging from 0.5 (no discrimination) to 1 (perfect discrimination). Finally, Kaplan-Meier curves were made to visualize the potential discrimination between survival curves of groups with high or low prognostic value based on a median prediction threshold of the original model data of Aerts et al. [20] and a LR test was performed to test the significance of the curve split.

To compare the performance of the original model on corrected $C B C T$ to a new model on original $C B C T$ images, the coefficients of the same four variables of the radiomic signature were refitted on the $\mathrm{CBCT}$ images of dataset 1 without applying correction step 1 or 2 . The performance of this recalibrated model was evaluated for all three datasets.

To investigate the relevance of using this radiomics model for comparing prognostic performance of $\mathrm{pCT}$ and $\mathrm{CBCT}$, we compared the model's performance to the recognized prognostic factor 'TNM-stage' for dataset 1 . Four dummy variables with $\mathrm{T} 2, \mathrm{~T} 3 / 4, \mathrm{~N} 1 / 2$ and N3 were created, using $\mathrm{T} 1$ and N0 as reference categories. We compared the performance of a model with TNM-stage to the original radiomic signature model and also calculated the performance of a model with both the radiomic signature and TNM-stage. Even though we performed this small comparison with clinical data, we want to emphasize that it was not the purpose of this study to develop a new highly prognostic model incorporating different types of information.

All statistical analyses were performed using R (version 3.2.2 and 3.2.3), using the packages stats, survcomp and $r m s$.

\section{Results}

For 17 CBCT images of dataset 1, the field of view (FOV) did not cover the entire tumor volume, so these patients were excluded from further analyses. Moreover, 13 patients had not enough margin around the tumor volume to be able to calculate any filtered radiomic features without needing to perform padding which required imputing values and were therefore also excluded from the analysis. This resulted in a total of 102 CBCT images available for analysis. In Figure 2.2 an example of a treatment planning $\mathrm{CT}$ and corresponding $\mathrm{kV}$ cone-beam $\mathrm{CT}$ image from dataset 1 is shown.

For dataset 2, 6 patients had to be excluded because the registration of the images to transfer the delineated GTV did not work or the tumor was not visible on the CBCT due to blurring or decreased image quality. This resulted in a total of 56 patients available for analysis in dataset 2 .

For dataset 3, no patients were excluded. 
A

B
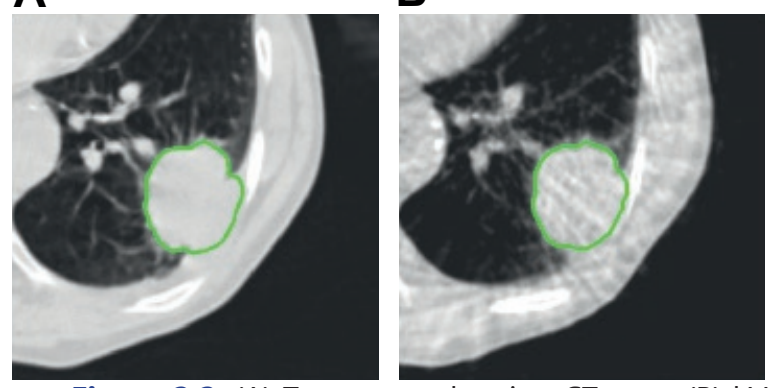

Figure 2.2. (A) Treatment planning CT scan, (B) kV cone-beam CT scan of the same patient prior to the first fraction of treatment. The GTV is indicated in green.

Clinical variables of all patients included in the analysis are shown in Table S2.1 in Supplementary Material 2B. All patients, including the stage IV patients who had 1 or 2 metastases, were treated with curative intent.

Prior to model validation, correction was applied on $\mathrm{CBCT}$ images and features. The mean intensity level in a ROI in the heart ranged from -65 to 117 (median 17.5) for CBCT images acquired in dataset 1, from -364 to 225 (median -242) for dataset 2 and from -372 to 226 (median -113) for dataset 3 (Figure S2.1). This shows large variability between patients and explains why correction step 1 , intensity correction, was applied. The distribution of intensity levels in the GTV before and after intensity correction was summarized in histograms for all datasets, shown in Figure S2.2.

After intensity value corrections, linear regression was performed on the PCT and $C B C T$ extracted radiomic features. The coefficient of determination $\left(R^{2}\right)$ was calculated for all 1119 radiomic features acquired from dataset 1 . A total of 149 out of $1119(13.3 \%)$ features had an $R^{2}$ above 0.85 . This included 2 out of $19(10.5 \%)$ features from the 'tumor intensity' group, 14 out of 95 (14.7\%) features from the 'texture' group, 5 out of 74 (6.8\%) from the 'Laplacian of Gaussian' group, 118 out of 912 (12.9\%) features from the 'wavelet' group and 10 out of 19 (52.6\%) features from the 'shape' group. Scatterplots and linear regression parameters of the 100 features with highest $\mathrm{R}^{2}$ are shown in Figure S2.3 and Table S2.2, respectively.

The results of linear regression for the four features of the previously published radiomic signature are shown in Table S2.3 for all datasets. Scatterplots for CBCT versus $\mathrm{pCT}$ for the four radiomic signature features are shown in Figure 2.3 for all datasets (using original voxel sizes). The parameters of the linear regression, i.e. the slopes and intercepts, were used to correct $C B C T$ feature values of all CBCT images of that respective dataset (correction step 2).

Linear predictor values of the radiomics signature were derived from $C B C T$ imaging before and after performing the two-step correction method. Linear predictors of individual patients of all datasets are shown in Supplementary Material $2 \mathrm{H}$. 
After the two-step correction procedure, model validation was performed. All validation statistics are shown in Table 2.1 for both $\mathrm{pCT}$ and CBCT. For dataset 1 , the proportion of Stage I patients was relatively large, therefore model validation was performed separately for the patients with Stage I $(n=42)$ and for the patients with Stage II or higher $(n=60)$. These results are shown in Table S2.4.

Table 2.1. Model validation results for $\mathrm{PCT}$ and $\mathrm{CBCT}$ for all datasets.

\begin{tabular}{lllll} 
& & $\begin{array}{l}\text { Calibration slope on the PI } \\
\text { (SE, } p \text {-value LR test) }\end{array}$ & $\begin{array}{l}\text { Joint test on } \\
\text { all coefficients, } \\
p \text {-value }\end{array}$ & $\begin{array}{l}\text { Harrell's c-index }(95 \% \mathrm{Cl}, \\
p \text {-value })\end{array}$ \\
\hline Dataset 1 & $\mathrm{pCT}$ & $1.77(\mathrm{SE}=0.42, p=0.09)$ & $p=0.013$ & $0.69\left(0.63-0.75, p=9.9^{*} 10^{-10}\right)$ \\
& $\mathrm{CBCT}$ & $1.90(\mathrm{SE}=0.44, p=0.06)$ & $p=0.027$ & $0.66\left(0.59-0.73, p=4.8^{\star} 10^{-6}\right)$ \\
Dataset 2 & $\mathrm{pCT}$ & $0.90(\mathrm{SE}=0.28, p=0.72)$ & $p=0.84$ & $0.61(0.52-0.70, p=0.016)$ \\
& $\mathrm{CBCT}$ & $1.17(\mathrm{SE}=0.33, p=0.62)$ & $p=0.53$ & $0.63(0.54-0.72, p=0.0052)$ \\
Dataset 3 & $\mathrm{pCT}$ & $0.73(\mathrm{SE}=0.30, p=0.35)$ & $p=0.28$ & $0.59(0.53-0.65, p=0.0036)$ \\
& $\mathrm{CBCT}$ & $0.36(\mathrm{SE}=0.23, p=0.0017)$ & $p=0.0024$ & $0.59(0.53-0.66, p=0.0063)$ \\
\hline
\end{tabular}

Significant differences between Kaplan-Meier curves of groups with high and low prognostic value were found for both the $\mathrm{pCT}$ cohort and the CBCT cohort ( $p=0.00064$ and $p=0.00020$, respectively) in dataset 1 . For dataset 2 , the Kaplan-Meier curves did not split significantly for $\mathrm{pCT}(p=0.43)$ and for $\mathrm{CBCT}(p=0.12)$. For dataset 3 , differences between Kaplan-Meier curves were not significant for both $\mathrm{pCT}(p=0.05)$ and CBCT $(p=0.19)$. Survival curves are shown in Figure 2.4. Additional Kaplan-Meier curves with different forms and steps of the correction procedure are shown in Supplementary Material 2J.

The model consisting of the same four radiomic signature features, but recalibrated on original, uncorrected CBCT images of dataset 1, achieved a c-index of $0.69(95 \%$ $\left.\mathrm{Cl} 0.63-0.75, p=4.0^{*} 10^{-10}\right)$ for dataset 1 , a c-index of $0.43(95 \% \mathrm{Cl} 0.34-0.51, p=0.08)$ for dataset 2 and $0.45(95 \% \mathrm{Cl} 0.38-0.52, p=0.16)$ for dataset 3 (all uncorrected images). All other statistics also showed that this recalibrated model could not be validated on original CBCT images of dataset 2 and 3 (not shown).

For dataset 1, the c-index of TNM-stage was found to be $0.62(95 \% \mathrm{Cl} 0.54$ $-0.71, p=0.0044$ ), but model performance of the radiomic signature did not improve when including staging in the original model, with a c-index of $0.66(95 \% \mathrm{Cl} 0.58-$ $\left.0.73, p=2.0^{*} 10^{-5}\right)$ and $0.65\left(95 \% \mathrm{Cl} 0.58-0.72, p=5.1 * 10^{-5}\right)$ for $\mathrm{pCT}$ and $\mathrm{CBCT}$ imaging, respectively. 

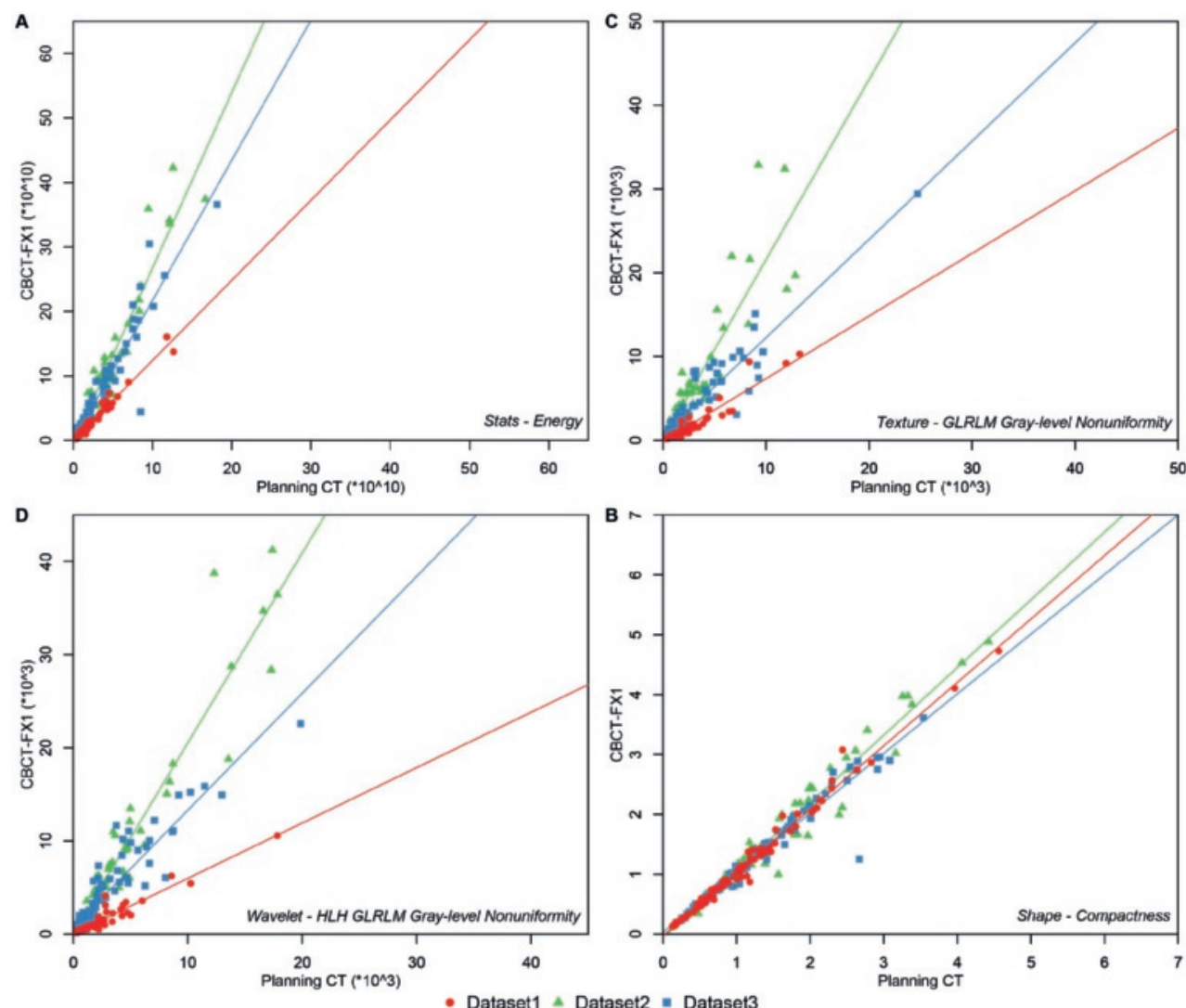

Figure 2.3. Scatterplots of CBCT extracted radiomic features versus $\mathrm{pCT}$ extracted radiomic features. Scatterplots derived for dataset 1 (dots), dataset 2 (triangles) and dataset 3 (squares). (A) tumor intensity-'Energy', (B) texture-'Gray-level Nonuniformity', (C) wavelet - 'HLH Gray-level Nonuniformity', (D) shape - 'Compactness'. GLRLM: Graylevel Runlength Matrix, H: High, L: Low.

\section{Discussion}

A small proportion (13.3\%) of radiomic features retrieved from planning CT are interchangeable with those retrieved from cone beam CTs. This group of features did include all features from the previously published prognostic radiomic signature [20]. Moreover, we tested its prognostic value in all three cohorts for $\mathrm{PCT}$ as well as CBCT. The similarity of linear predictors, after the two-step correction method, estimated by the Cox model of the radiomic signature shows that prognostic information can be obtained interchangeably from $\mathrm{PCT}$ or $\mathrm{CBCT}$ images. It has already been shown that image features derived from $\mathrm{CBCT}$ can potentially serve as an early biomarker for assessing response to treatment $[27,28]$. To the best of our knowledge, only one study investigated the potential of CBCT imaging in the field of radiomics [29]. However, these authors only performed test-retest analyses to assess the reproducibility of radiomic features obtained from $C B C T$ imaging. The current study shows that a subset of radiomic features and the prognostic value of the radiomic 
signature are interchangeable between $\mathrm{pCT}$ and $\mathrm{CBCT}$, showing the potential for using radiomics on $\mathrm{CBCT}$ imaging.

Kaplan-Meier curves of $\mathrm{pCT}$ and $\mathrm{CBCT}$ images are very similar for all datasets after correction, indicating that the same information can be derived from $\mathrm{kV}$ conebeam $\mathrm{CT}$ images as from conventional spiral planning $\mathrm{CT}$. Differences between the Kaplan-Meier curves between datasets could be explained by differences in cohorts, e.g. tumors were on average larger in dataset 2 and $3\left(109.2 \mathrm{~cm}^{3}\right.$ and $71.1 \mathrm{~cm}^{3}$ vs. $42.2 \mathrm{~cm}^{3}$ ) and also more stage III patients were included compared to dataset 1. More similar Kaplan-Meier curves are expected when cohorts are more comparable in both size and characteristics. For dataset 2, the non-significant split of the KaplanMeier curves could potentially be explained by the low number of patients (16 out of 56) in the high prognostic group.
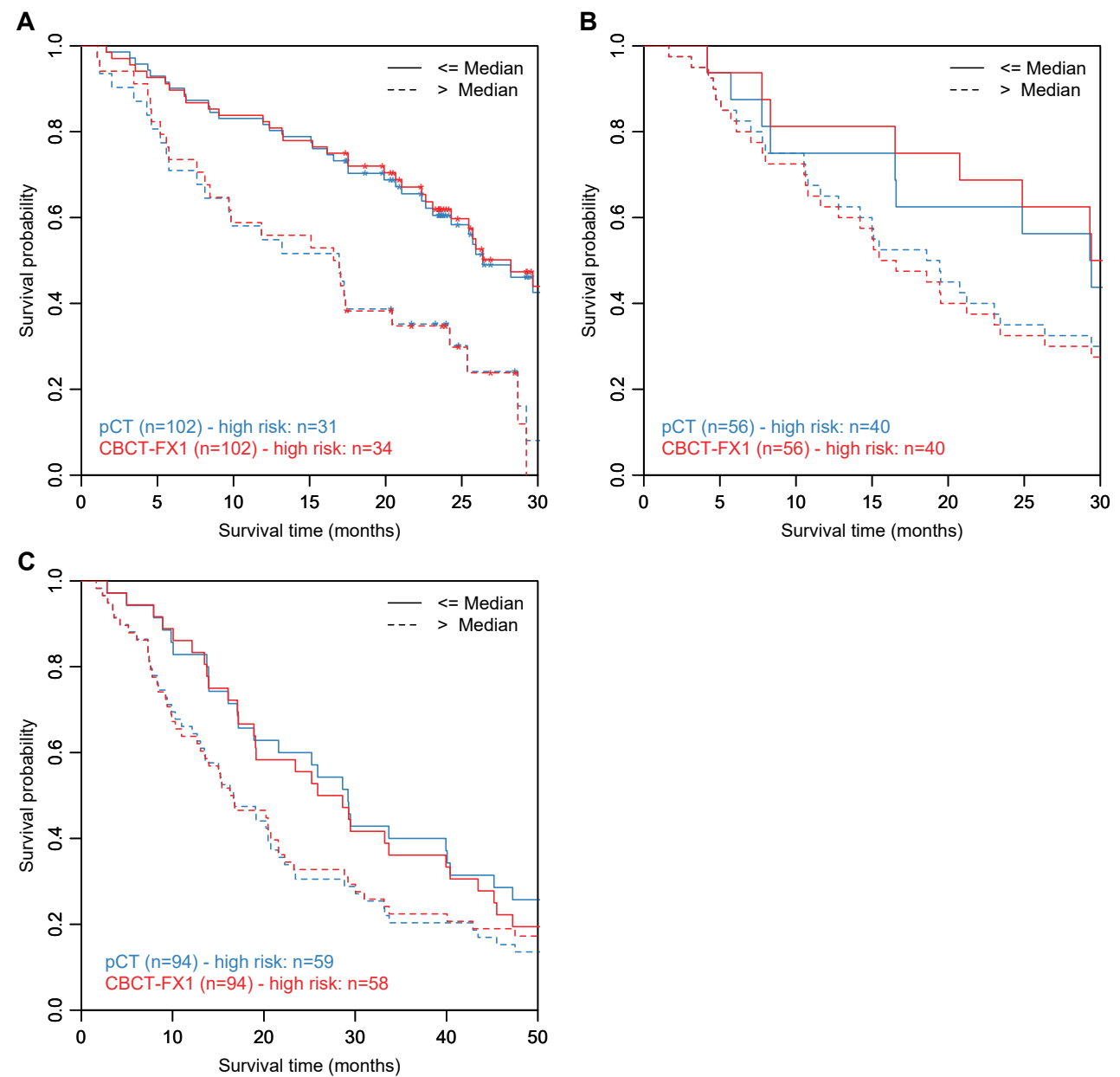

Figure 2.4. Kaplan-Meier curves for $\mathrm{pCT}$ and $\mathrm{CBCT}$. Kaplan-Meier curves are based on model predictions of the radiomic signature, split by a median prediction threshold derived by Aerts et al. [20]: (A) Dataset 1, (B) Dataset 2 and (C) Dataset 3. 
In this study, overall survival was analyzed as outcome. Although this outcome is highly relevant for patients, its association with imaging features is expected to be less strong than local tumor control or disease progression. Using this outcome, the prognostic performance of $\mathrm{PCT}$ as well as $\mathrm{CBCT}$ might increase.

There are several challenges in using $\mathrm{CBCT}$ imaging for radiomics. Extracted textural features typically depend on reconstruction and scanning parameters [2932]. Therefore, to be consistent throughout this study, only CBCT images with a $3 \mathrm{~mm}$ slice thickness were included in dataset 1 , which is identical to the slice thickness in the pCT images. Nonetheless, the CBCTs of dataset 2 and 3 all had a slice thickness of $1 \mathrm{~mm}$, which could partly explain the differences in linear regression parameters and model validation results between datasets. After resampling all slice thicknesses into an equal size of $3 \mathrm{~mm}$, energy features of CBCT images of dataset 2 and 3 decreased by a factor 3 , as expected since energy is directly related to the number of voxels. The resampling resulted in more similar results between datasets in terms of linear regression parameters. However, the influence of slice thickness on the radiomic feature values needs further investigation [33]. Furthermore, other unknown factors might influence the interchangeability between $\mathrm{PCT}$ and $\mathrm{CBCT}$ radiomics or the consistency of radiomics' performance on $\mathrm{CT}$ images. Potentially, a radiomics approved reconstruction or corrections afterwards as performed in this study, could in general improve the consistency and utility of radiomics in medical imaging.

Besides this, the detector size of the cone-beam CT has a limited field of view (FOV) that may not be large enough for extensive tumors and off-axis patient positions. Moreover, for calculating filtered radiomic features, a certain margin is necessary to avoid padding with artificial values. These issues could potentially be solved by stitching together two acquisitions, or extracting radiomic features using the image slice with the largest tumor cross section, which should still be investigated for CBCT imaging [10, 34].

Other challenges with $\mathrm{CBCT}$ images for radiomics include their increased noise and sensitivity to (strike) artefacts compared to conventional CT images. Due to the prolonged scan time of approximately one minute, CBCT images are more susceptible for motion artefacts. Respiratory correlated CT imaging minimizes the breathing artefacts, which is not the case for respiration-averaged CBCT images. Four-dimensional (4D) CBCT imaging might overcome this problem, however possible decreased image quality and increased noise compared to 3D CBCT imaging [35] may be observed and $4 D$ CBCT images are typically not acquired in routine clinical practice. More research is needed to investigate the influence of artefacts in CBCT radiomics.

This study shows that after applying correction, comparable prognostic information can be derived from CBCT images as from CT images for NSCLC patients. Apparently, the radiomic signature is a subset of features that is able to derive the important prognostic information which is not affected by differences in image acquisition and reconstruction between $\mathrm{CT}$ and $\mathrm{CBCT}$. When performing radiomics, one should perform extensive test-retest and stability analyses to select most reliable imaging features, which is especially important when using lower quality images like cone-beam CT. In the future, new models can be developed based on CBCT extracted 
radiomic features in combination with other (clinical) parameters that potentially have improved prognostic performance. Developing new models was outside the scope of the current study.

Since multiple scans are routinely made in clinical practice, $C B C T$ imaging could potentially be used in a longitudinal fashion to develop so-called "delta radiomics". Tumor changes occurring over the course of treatment, e.g. cell death, accelerated proliferation and reoxygenation, could then be monitored using radiomics metrics. Since this study only shows the interchangeability with planning $\mathrm{CT}$ radiomics, further research is needed to explore the performance of delta radiomic features extracted from $\mathrm{kV}$ cone-beam $\mathrm{CT}$ images for the (improved) prediction of survival and/or to quantify accelerated proliferation as a predictor for local progression.

\section{Conclusion}

This study shows that a selection of radiomic features are interchangeable when extracted from either planning CT or cone-beam CT images. The performance of the previously developed CT-based radiomic signature on CBCT images shows that prognostic information on overall survival can be derived from CBCT images of nonsmall cell lung cancer patients acquired prior to the first fraction of treatment, providing a two-step correction procedure was performed. These results show great potential for the use of radiomics on $\mathrm{CBCT}$ images. $\mathrm{CBCT}$ radiomics can now be further evaluated for its potential to monitor tumor changes over the course of treatment (the so-called "delta radiomics" approach).

\section{Acknowledgments}

This work was supported by the ERC advanced grant (ERC-ADG-2015, $\mathrm{n}^{\circ} 694812$ Hypoximmuno) and the QulC-ConCePT project, which is partly funded by EFPI A companies and the Innovative Medicine Initiative Joint Undertaking (IMI JU) under Grant Agreement No. 115151. This research is also supported by the Dutch technology Foundation STW (grant $n^{\circ}$ 10696 DuCAT \& n P14-19 Radiomics STRaTegy), which is the applied science division of NWO, and the Technology Programme of the Ministry of Economic Affairs. Authors also acknowledge financial support from the EU 7th framework program (ARTFORCE - $n^{\circ} 257144$, REQUITE - $n^{\circ}$ 601826), SME Phase 2 (EU proposal 673780 - RAIL), the European Program H2020-2015-17 (BD2Decide - PHC30-689715 and ImmunoSABR - $n^{\circ}$ 733008), Interreg V-A Euregio MeuseRhine ("Euradiomics"), Kankeronderzoekfonds Limburg from the Health Foundation Limburg, Alpe d'HuZes-KWF (DESIGN), EUROSTARS (DART) and the Dutch Cancer Society. Authors thank the contribution of Anisha Gogineni for editing. 


\section{References}

[1] Torre LA, Bray F, Siegel RL, Ferlay J, Lortet-Tieulent J, Jemal A. Global cancer statistics, 2012. CA Cancer J Clin. 2015;65:87-108.

[2] Ferlay J, Soerjomataram I, Dikshit R, Eser S, Mathers C, Rebelo M, et al. Cancer incidence and mortality worldwide: sources, methods and major patterns in GLOBOCAN 2012. Int J Cancer. 2015;136:E359-86.

[3] Lambin P, van Stiphout RG, Starmans MH, Rios-Velazquez E, Nalbantov G, Aerts HJ, et al. Predicting outcomes in radiation oncology--multifactorial decision support systems. Nat Rev Clin Oncol. 2013;10:27-40.

[4] Lambin P, Roelofs E, Reymen B, Velazquez ER, Buijsen J, Zegers CM, et al. 'Rapid Learning health care in oncology' - an approach towards decision support systems enabling customised radiotherapy'. Radiother Oncol. 2013;109:159-64.

[5] Lambin P, Zindler J, Vanneste BG, De Voorde LV, Eekers D, Compter I, et al. Decision support systems for personalized and participative radiation oncology. Adv Drug Deliv Rev. 2016.

[6] Oberije C, De Ruysscher D, Houben R, van de Heuvel M, Uyterlinde W, Deasy JO, et al. A Validated Prediction Model for Overall Survival From Stage III Non-Small Cell Lung Cancer: Toward Survival Prediction for Individual Patients. Int J Radiat Oncol Biol Phys. 2015;92:935-44. [7] Oberije C, Nalbantov G, Dekker A, Boersma L, Borger J, Reymen B, et al. A prospective study comparing the predictions of doctors versus models for treatment outcome of lung cancer patients: a step toward individualized care and shared decision making. Radiother Oncol. 2014;112:37-43.

[8] Carvalho S, Leijenaar RT, Velazquez ER, Oberije C, Parmar C, van Elmpt W, et al. Prognostic value of metabolic metrics extracted from baseline positron emission tomography images in non-small cell lung cancer. Acta Oncol. 2013;52:1398-404.

[9] Fried DV, Tucker SL, Zhou S, Liao Z, Mawlawi O, Ibbott G, et al. Prognostic value and reproducibility of pretreatment $C T$ texture features in stage III non-small cell lung cancer. Int J Radiat Oncol Biol Phys. 2014;90:834-42.

[10] Ganeshan B, Panayiotou E, Burnand K, Dizdarevic S, Miles K. Tumour heterogeneity in non-small cell lung carcinoma assessed by $\mathrm{CT}$ texture analysis: a potential marker of survival. Eur Radiol. 2012;22:796-802.

[11] Coroller TP, Grossmann P, Hou Y, Rios Velazquez E, Leijenaar RT, Hermann G, et al. CTbased radiomic signature predicts distant metastasis in lung adenocarcinoma. Radiother Oncol. 2015;114:345-50.

[12] Lambin P, Rios-Velazquez E, Leijenaar R, Carvalho S, van Stiphout RG, Granton P, et al. Radiomics: extracting more information from medical images using advanced feature analysis. Eur J Cancer. 2012;48:441-6.

[13] Kumar V, Gu Y, Basu S, Berglund A, Eschrich SA, Schabath MB, et al. Radiomics: the process and the challenges. Magn Reson Imaging. 2012;30:1234-48.

[14] Gillies RJ, Kinahan PE, Hricak H. Radiomics: Images Are More than Pictures, They Are Data. Radiology. 2016;278:563-77.

[15] Aerts HJ, Grossmann P, Tan Y, Oxnard GG, Rizvi N, Schwartz LH, et al. Defining a Radiomic Response Phenotype: A Pilot Study using targeted therapy in NSCLC. Sci Rep. 2016;6:33860.

[16] Cunliffe A, Armato SG, 3rd, Castillo R, Pham N, Guerrero T, Al-Hallaq HA. Lung texture in serial thoracic computed tomography scans: correlation of radiomics-based features with radiation therapy dose and radiation pneumonitis development. Int J Radiat Oncol Biol Phys. 2015;91:1048-56.

[17] Antunes J, Viswanath S, Rusu M, Valls L, Hoimes C, Avril N, et al. Radiomics Analysis on FLT-PET/MRI for Characterization of Early Treatment Response in Renal Cell Carcinoma: A Proof-of-Concept Study. Transl Oncol. 2016;9:155-62.

[18] Jaffray DA, Siewerdsen JH, Wong JW, Martinez AA. Flat-panel cone-beam CT for imageguided radiation therapy. Int J Radiation Oncology Biol Psych. 2002;53:1337-49.

[19] Brink C, Bernchou U, Bertelsen A, Hansen O, Schytte T, Bentzen SM. Locoregional control of non-small cell lung cancer in relation to automated early assessment of tumor regression on cone beam computed tomography. Int J Radiat Oncol Biol Phys. 2014;89:916-23. 
[20] Aerts HJ, Velazquez ER, Leijenaar RT, Parmar C, Grossmann P, Carvalho S, et al. Decoding tumour phenotype by noninvasive imaging using a quantitative radiomics approach. Nat Commun. 2014;5:4006.

[21] Leijenaar RT, Carvalho S, Hoebers FJ, Aerts HJ, van Elmpt WJ, Huang SH, et al. External validation of a prognostic CT-based radiomic signature in oropharyngeal squamous cell carcinoma. Acta Oncol. 2015;54:1423-9.

[22] van Timmeren JE, Leijenaar RTH, van Elmpt W, Reymen B, Oberije C, Monshouwer R, et al. Data from: Survival prediction of non-small cell lung cancer patients using radiomics analyses of cone-beam CT images. 2017.

[23] Moteabbed M, Sharp GC, Wang Y, Trofimov A, Efstathiou JA, Lu H-M. Validation of a deformable image registration technique for cone beam CT-based dose verification. Med Phys. 2015;42:196-205.

[24] Veiga C, McClelland J, Moinuddin S, Lourenço A, Ricketts K, Annkah J, et al. Toward adaptive radiotherapy for head and neck patients: Feasibility study on using CT-to-CBCT deformable registration for "dose of the day" calculations. Med Phys. 2014;41:031703.

[25] Cardiac CT imaging : diagnosis of cardiovascular disease. New York, NY: Springer Berlin Heidelberg; 2016.

[26] P. R, Altman DG. External validation of a Cox prognostic model, principles and methods. BMC Medical Research Methodology. 2013;13.

[27] Bertelsen A, Schytte T, Bentzen SM, Hansen O, Nielsen M, Brink C. Radiation dose response of normal lung assessed by Cone Beam CT - a potential tool for biologically adaptive radiation therapy. Radiother Oncol. 2011;100:351-5.

[28] Bernchou U, Hansen O, Schytte T, Bertelsen A, Hope A, Moseley D, et al. Prediction of lung density changes after radiotherapy by cone beam computed tomography response markers and pre-treatment factors for non-small cell lung cancer patients. Radiother Oncol. 2015;117:17-22.

[29] Fave X, Mackin D, Yang J, Zhang J, Fried D, Balter $P$, et al. Can radiomics features be reproducibly measured from $\mathrm{CBCT}$ images for patients with non-small cell lung cancer? Med Phys. 2015;42:6784.

[30] Mackin D, Fave X, Zhang L, Fried D, Yang J, Taylor B, et al. Measuring Computed Tomography Scanner Variability of Radiomics Features. Invest Radiol. 2015;50:757-65.

[31] Balagurunathan Y, Gu Y, Wang H, Kumar V, Grove O, Hawkins S, et al. Reproducibility and Prognosis of Quantitative Features Extracted from CT Images. Transl Oncol. 2014;7:72-87.

[32] Balagurunathan Y, Kumar V, Gu Y, Kim J, Wang H, Liu Y, et al. Test-retest reproducibility analysis of lung CT image features. J Digit Imaging. 2014;27:805-23.

[33] Zhao B, Tan Y, Tsai WY, Qi J, Xie C, Lu L, et al. Reproducibility of radiomics for deciphering tumor phenotype with imaging. Sci Rep. 2016;6:23428.

[34] Ganeshan B, Goh V, Mandeville HC, Ng QS, Hoskin PJ, Miles KA. Non-small cell lung cancer: histopathologic correlates for texture parameters at CT. Radiology. 2013;266:326-36.

[35] Lee S, Yan G, Lu B, Kahler D, Li JG, Sanjiv SS. Impact of scanning parameters and breathing patterns on image quality and accuracy of tumor motion reconstruction in 4D CBCT: a phantom study. Journal of Applied Clinical Medical Physics. 2015;16. 


\section{Supplementary Material}

\section{Supplementary Material 2A - Image acquisitions}

\section{Dataset 1}

For each patient a respiratory correlated $4 D$ CT scan in treatment position for radiotherapy planning purposes and multiple $C B C T$ s during radiation treatment were acquired. The $50 \%$ expiration phase of the $4 \mathrm{D} \mathrm{CT}$ was used for delineation and radiotherapy treatment planning. Voxels dimensions were $0.98 \times 0.98 \times 3 \mathrm{~mm}^{3}$.

The cone-beam CT scans were acquired from a TrueBeam Radiotherapy System (Varian Medical Systems, Palo Alto, CA, USA). All CBCT images were acquired according to the lung protocol, with a tube voltage of $125 \mathrm{kVp}$ reconstructed into a matrix of $512 \times 512 \times 54$ pixels. Images were reconstructed using filtered back projection [1] into images with an in-plane pixel size of $0.91 \times 0.91 \mathrm{~mm}^{2}$. CBCT images with $3 \mathrm{~mm}$ slice thickness were included in the study to match the slice thickness of the planning $\mathrm{CT}$ images.

\section{Dataset 2}

Planning CT scans in this dataset are acquired during free-breathing and with contrast for radiotherapy planning purposes. Voxel dimensions ranged from $0.98 \times 0.98 \times 3$ $\mathrm{mm}^{3}$ to $1.12 \times 1.12 \times 3 \mathrm{~mm}^{3}$.

The cone-beam CT scans were acquired from an Elekta XVI Systems (Elekta $A B$, Stockholm, Sweden). All CBCT images were acquired with a tube voltage of 120 $\mathrm{kVp}$ and reconstructed into a matrix of $410 \times 410 \times 264$ pixels. Images were reconstructed into images with an isotropic voxel size of $1 \mathrm{~mm}$ using filtered back projection.

\section{Dataset 3}

Planning CT scans in this dataset are acquired during free-breathing and with contrast for radiotherapy planning purposes. Voxel dimensions ranged from $0.98 \times 0.98 \times 2.5$ $\mathrm{mm}^{3}$ to $0.98 \times 0.98 \times 3 \mathrm{~mm}^{3}$.

The cone-beam CT scans were acquired from an Elekta XVI Systems (Elekta $A B$, Stockholm, Sweden). All CBCT images were acquired with a tube voltage of 120 $k V p$ and reconstructed into a matrix of $410 \times 410 \times 264$ pixels. Images were reconstructed into images with an isotropic voxel size of $1 \mathrm{~mm}$ using filtered back projection. 


\section{Supplementary Material 2B - Clinical variables}

Table S2.1. Demographic information of the entire population included in this study.

\begin{tabular}{|c|c|c|c|}
\hline & $\begin{array}{l}\text { Dataset } 1 \\
(n=102)\end{array}$ & $\begin{array}{l}\text { Dataset } 2 \\
(n=56)\end{array}$ & $\begin{array}{l}\text { Dataset } 3 \\
(\mathrm{n}=94)\end{array}$ \\
\hline \multicolumn{4}{|l|}{ Age } \\
\hline Range (median) & $42-87(71)$ & $41-85(64)$ & $42-83(68)$ \\
\hline Mean \pm SD & $69.8 \pm 9.9$ & $63.8 \pm 10.9$ & $66.9 \pm 8.5$ \\
\hline \multicolumn{4}{|l|}{ Gender } \\
\hline Male & 65 (63.7\%) & 39 (69.6\%) & 45 (47.9\%) \\
\hline Female & 37 (36.3\%) & 17 (30.4\%) & 49 (52.1\%) \\
\hline \multicolumn{4}{|l|}{ Stage } \\
\hline I & 42 (41.2\%) & $4(7.1 \%)$ & $6(6.4 \%)$ \\
\hline II & $8(7.8 \%)$ & $8(14.3 \%)$ & $4(4.3 \%)$ \\
\hline III & 45 (44.1\%) & $43(76.8 \%)$ & $84(89.4 \%)$ \\
\hline IV & 7 (6.9\%) & $0(0 \%)$ & $0(0 \%)$ \\
\hline Unknown & $0(0 \%)$ & $1(1.8 \%)$ & $0(0 \%)$ \\
\hline \multicolumn{4}{|l|}{ Histology } \\
\hline Adenocarcinoma & 20 (19.6\%) & $18(32.1 \%)$ & 34 (36.2\%) \\
\hline Squamous cell carcinoma & 46 (45.1\%) & $20(35.7 \%)$ & $42(44.7 \%)$ \\
\hline Large cell carcinoma & 7 (6.9\%) & $5(8.9 \%)$ & $5(5.3 \%)$ \\
\hline Undifferentiated & $0(0 \%)$ & $0(0 \%)$ & $6(6.4 \%)$ \\
\hline Neoplasm, malignant & $0(0 \%)$ & $2(3.6 \%)$ & $0(0 \%)$ \\
\hline Not specified & 29 (28.4\%) & $11(19.6 \%)$ & 7 (7.4\%) \\
\hline \multicolumn{4}{|l|}{$\mathrm{GTV}\left(\mathrm{cm}^{3}\right)$} \\
\hline Range (median) & $0.6-412(18.8)$ & $1.1-484(80.2)$ & $2.1-399(43.3)$ \\
\hline Mean \pm SD & $42.2 \pm 64.1$ & $109.2 \pm 109.5$ & $71.1 \pm 74.4$ \\
\hline \multicolumn{4}{|l|}{ Received dose (Gy) } \\
\hline Range (median) & $46.8-79.2(67.0)$ & $44.0-67.5(66.0)$ & $66.0-66.0(66.0)$ \\
\hline Mean \pm SD & $65.7 \pm 6.1$ & $64.0 \pm 5.3$ & $64.3 \pm 2.7$ \\
\hline \multicolumn{4}{|l|}{$\begin{array}{l}\text { Days between planning } \mathrm{CT} \text { and } \\
\text { first radiotherapy fraction }\end{array}$} \\
\hline Range (median) & $4-18(8)$ & $3-21(13)$ & $5-21(11)$ \\
\hline \multicolumn{4}{|l|}{ Median survival (months) } \\
\hline Median $[95 \% \mathrm{Cl}]$ & 24.3 [20.4 - 29.2] & $20.1[15.1-29.4]$ & $20.3[16.3-28.6]$ \\
\hline \multicolumn{4}{|l|}{ Events at time of analysis } \\
\hline Number & 60 & 44 & 82 \\
\hline
\end{tabular}




\section{Supplementary Material 2C - Intensity values histogram in the heart}

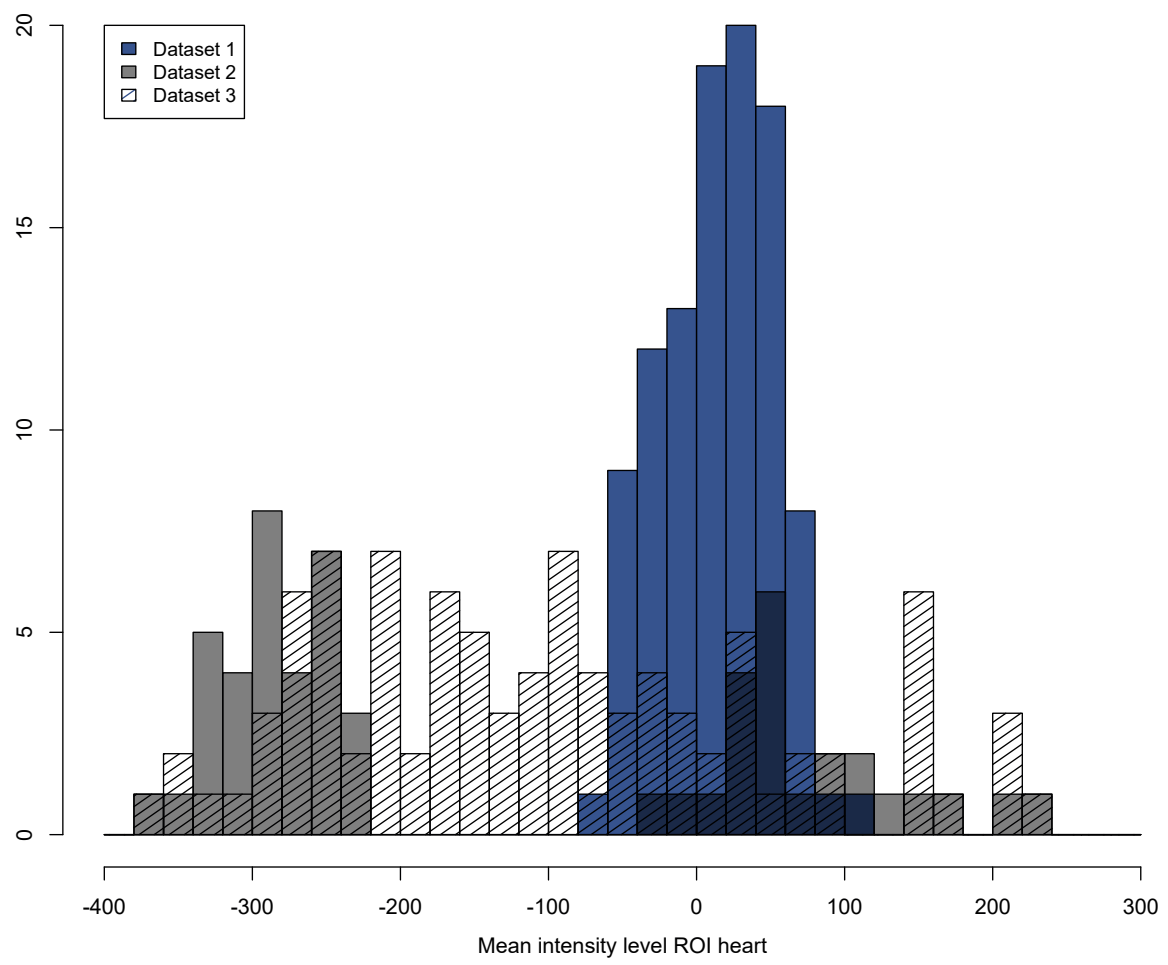

Figure S2.1. Histogram of mean intensity values in a ROI in the heart derived from the cone-beam CT images. 


\section{Supplementary Material 2D - Intensity values histogram GTV}

A

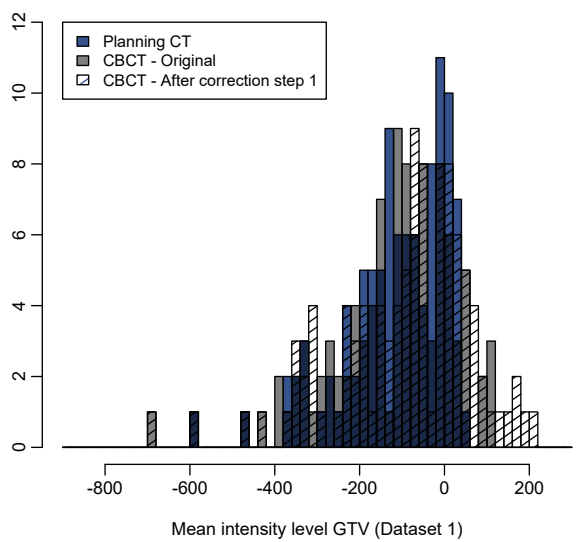

C

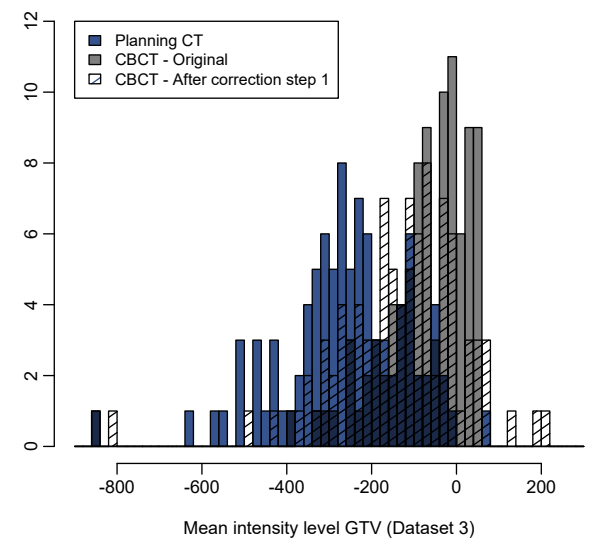

B

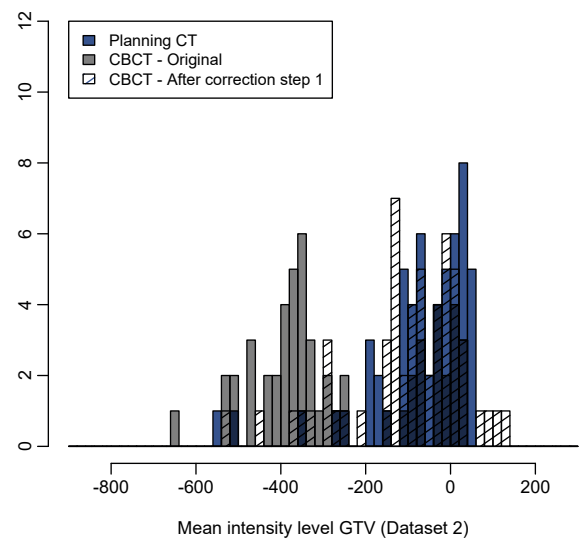

Figure S2.2. Histograms of mean intensity values of the GTV, for planning $C T$, original CBCT images and CBCT images after correction step 1. (A) Dataset 1, (B) Dataset 2, (C) Dataset 3. 


\section{Supplementary Material 2E - Scatterplots of interchangeable radiomic features}
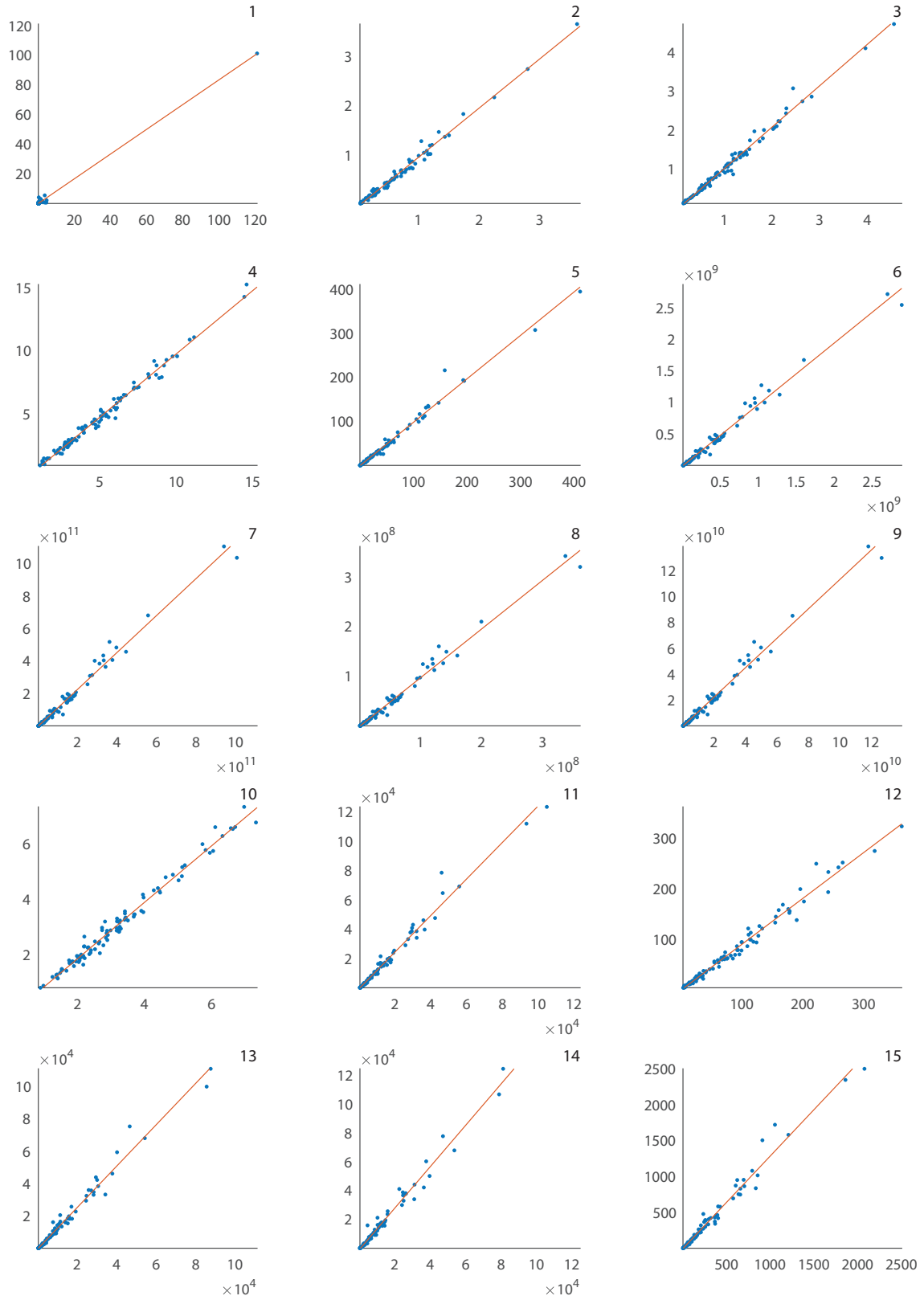

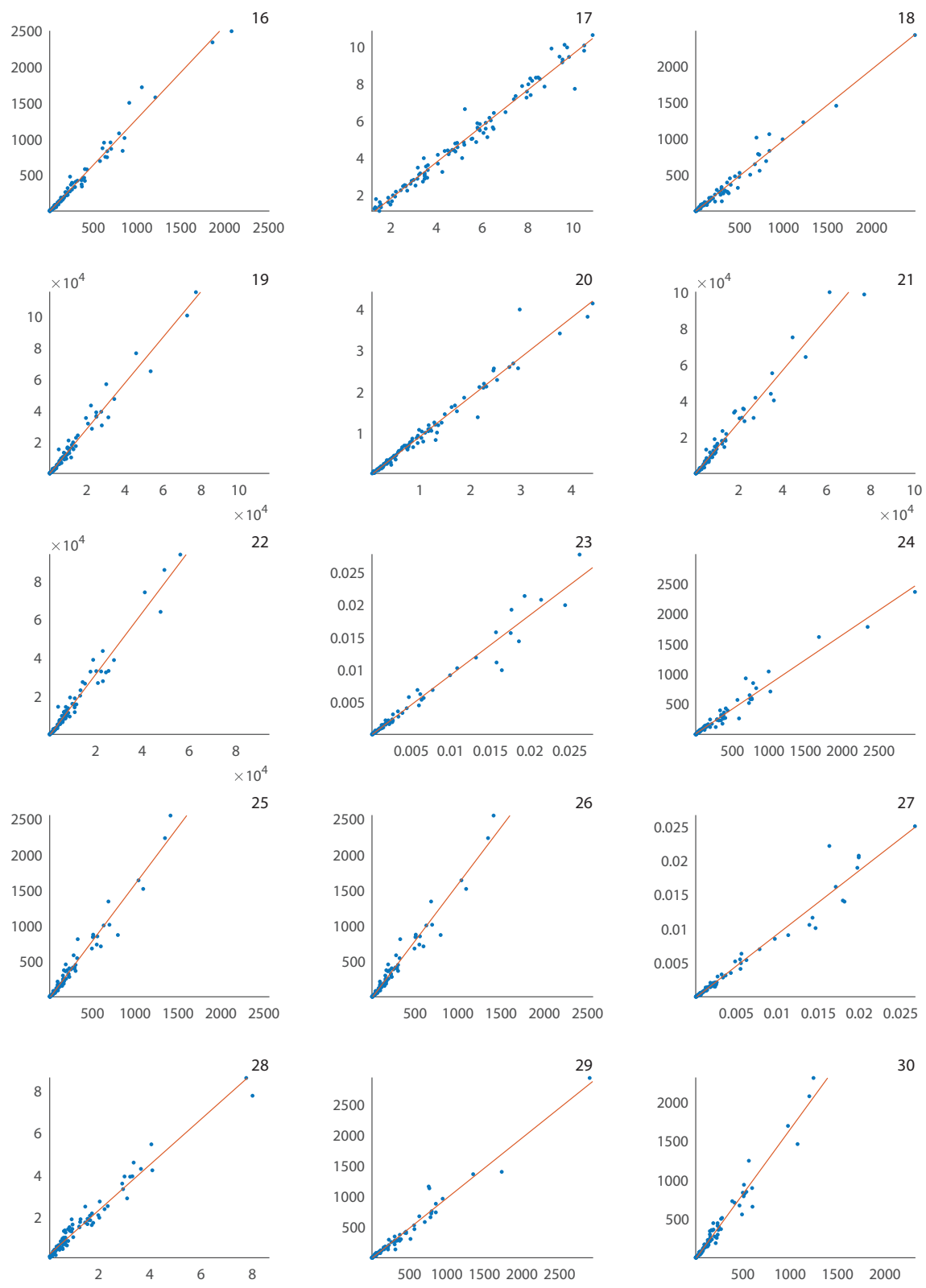

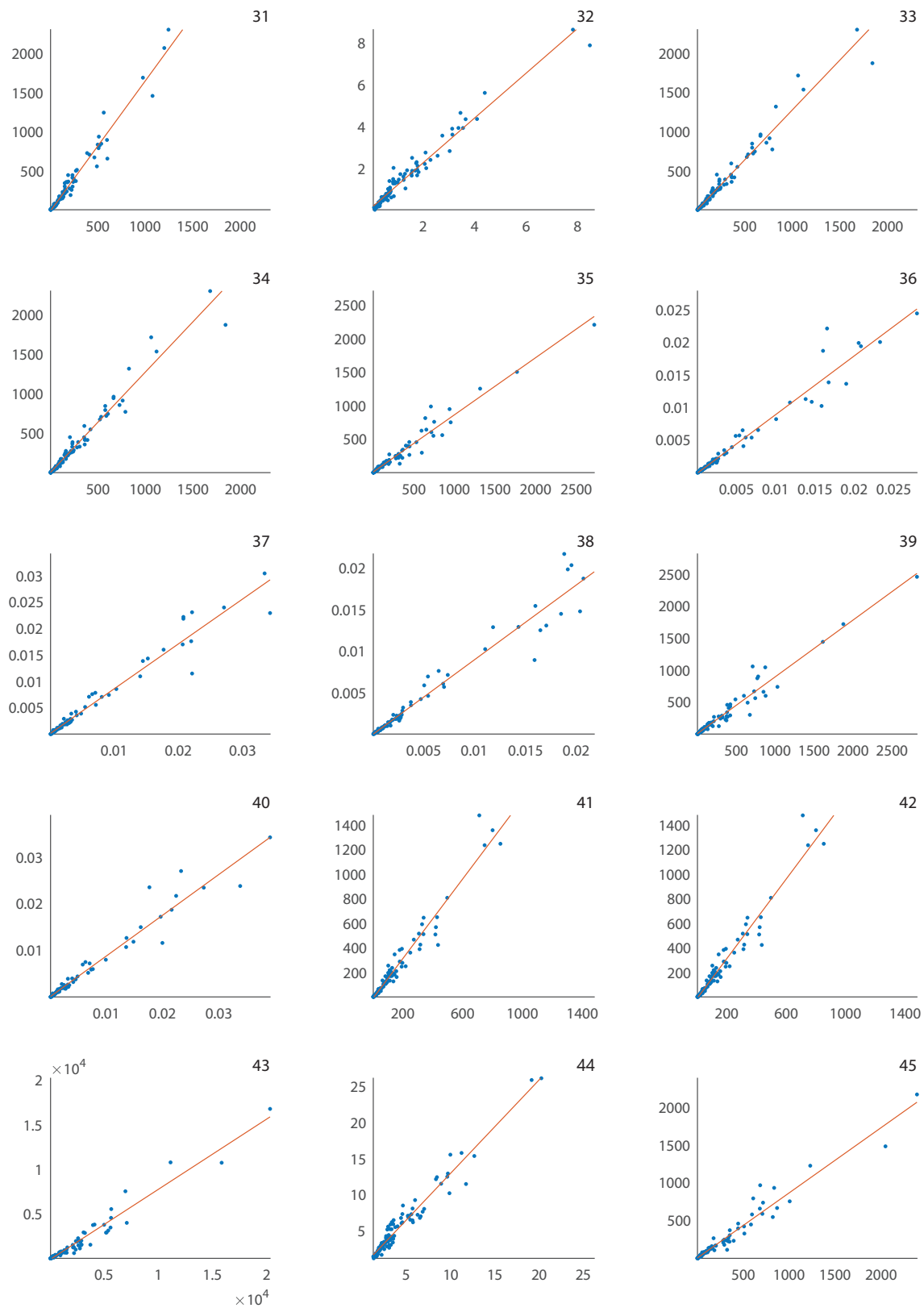

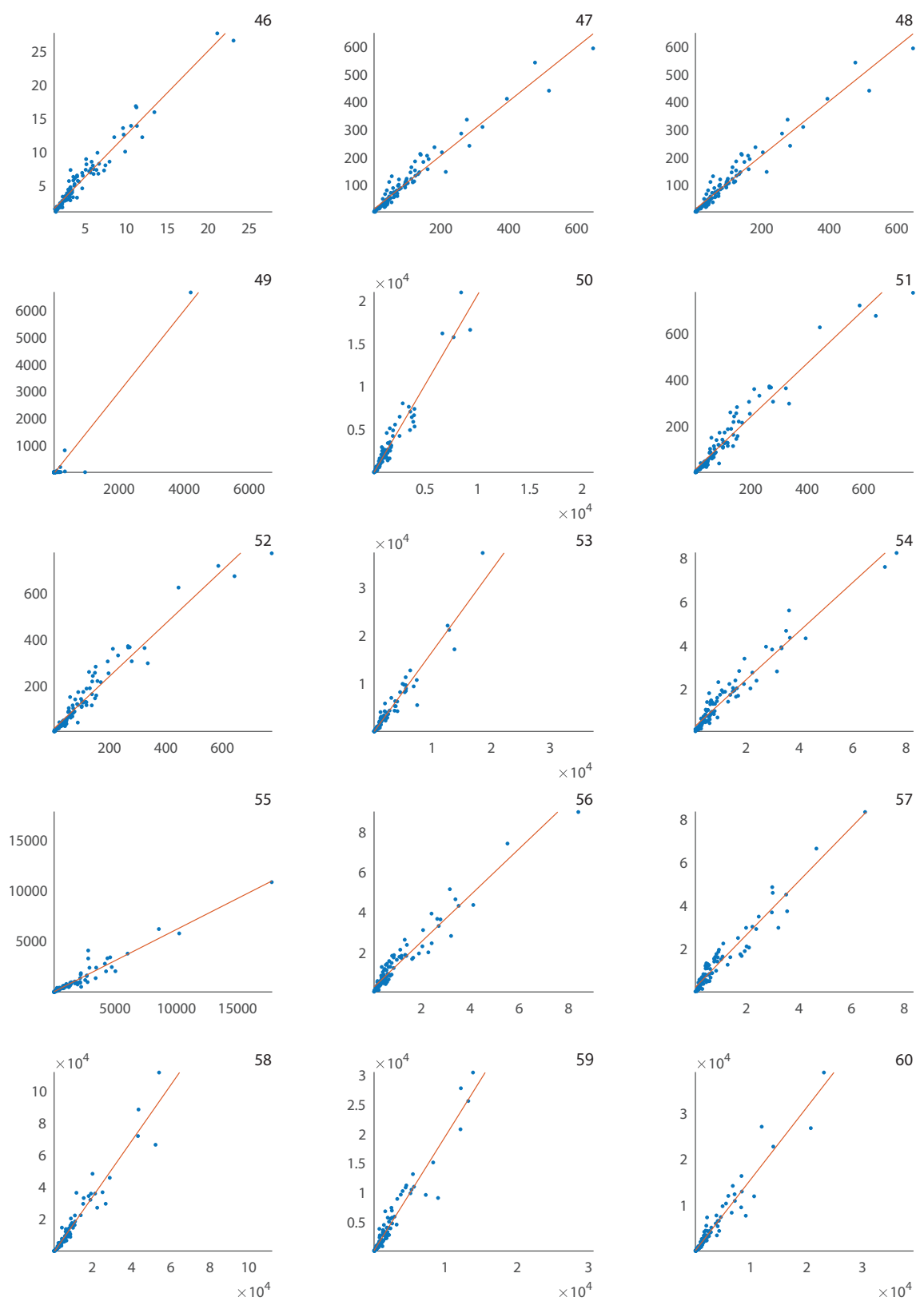

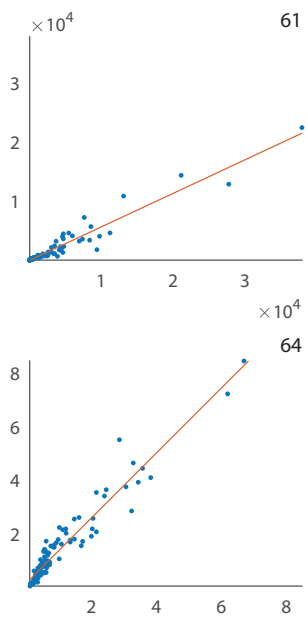

67
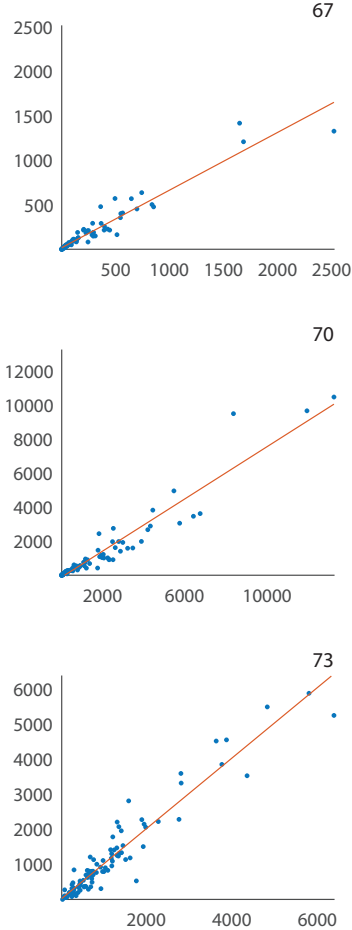

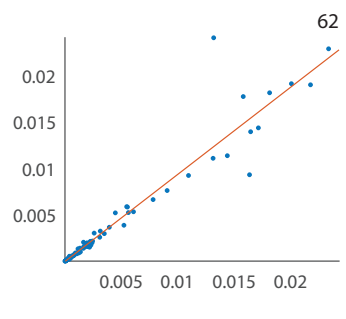

65

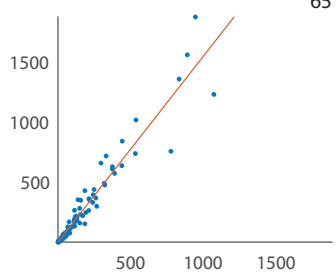

68
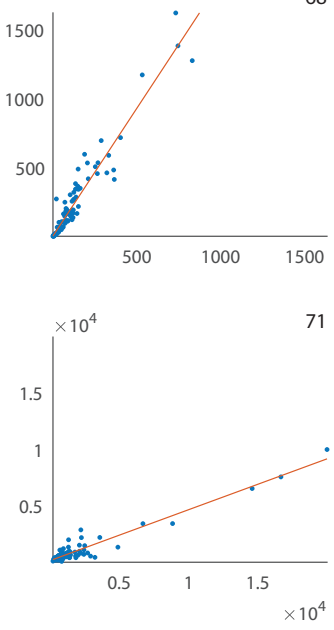

74

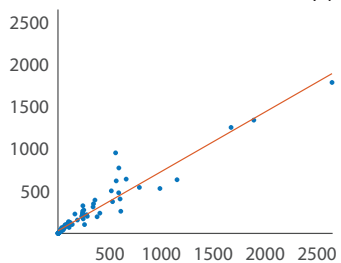

63

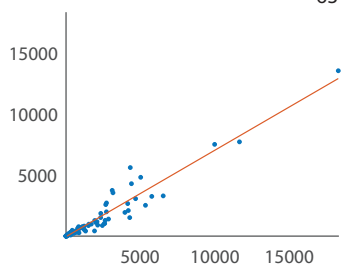

66

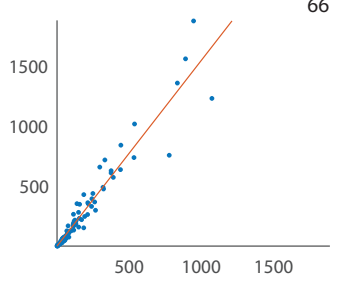

69
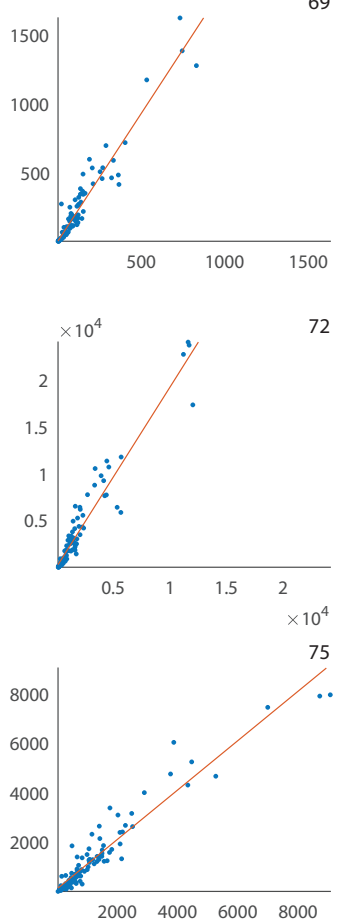

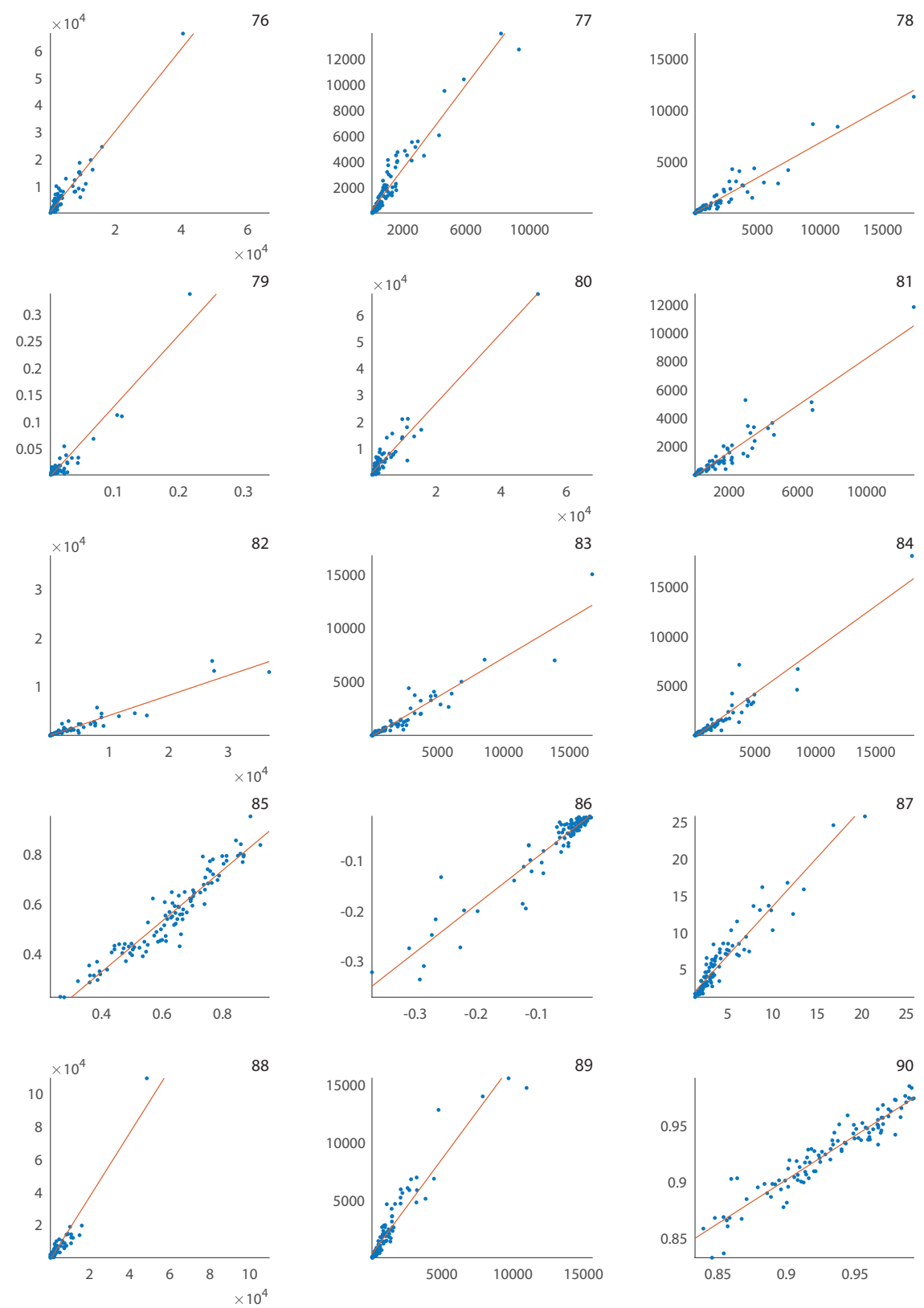

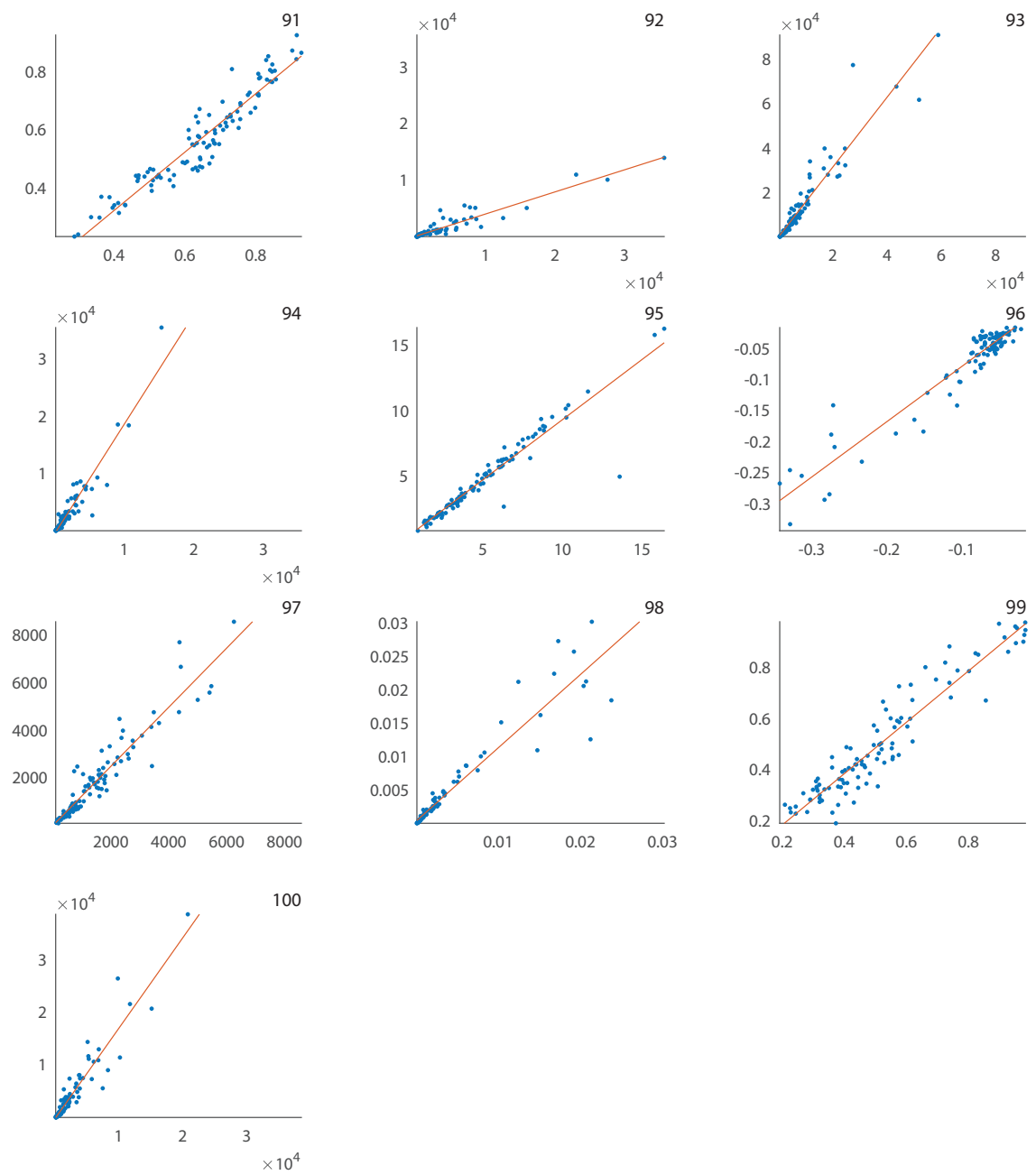

Figure S2.3. Scatterplots of 100 features with highest $R^{2}$, derived from $p C T$ and CBCT images of dataset 1 , without any corrections applied, ranking in descending $\mathrm{R}^{2}$ order. 


\section{Supplementary Material 2F - Linear regression parameters of interchangeable radiomic features}

Table S2.2. First 100 features with highest $\mathrm{R}^{2}$, derived from $\mathrm{pCT}$ and $C B C T$ images of dataset 1 , without any corrections applied. Plot numbers correspond to the scatterplots in Figure S2.3 of this Supplementary Material. Abbreviations of feature names are explained at the end of this Supplementary Material.

\begin{tabular}{|c|c|c|c|c|c|c|}
\hline Plot\# & Featurename & $\mathrm{R}^{2}$ & Slope & $p$-value & Intercept & $p$-value \\
\hline 1 & $\begin{array}{l}\text { LoG (sigma } 2.5 \mathrm{~mm}) \\
\text { Stats - minimum (positive) }\end{array}$ & 0.995 & 0.834 & $<0.0001$ & $2.7 \mathrm{E}-02$ & 0.70 \\
\hline 2 & Shape - Least axis length & 0.989 & 0.996 & $<0.0001$ & $-2.7 \mathrm{E}-02$ & 0.0019 \\
\hline 3 & Shape - Compactness 1 & 0.988 & 1.059 & $<0.0001$ & $-3.2 E-02$ & 0.032 \\
\hline 4 & Shape - Maximum diameter 2D3 & 0.988 & 1.001 & $<0.0001$ & $-2.1 \mathrm{E}-01$ & 0.00084 \\
\hline 5 & Volume & 0.986 & 0.988 & $<0.0001$ & $-7.8 \mathrm{E}-01$ & 0.38 \\
\hline 6 & Wavelet LLL Stats - Total energy & 0.984 & 0.980 & $<0.0001$ & $-3.4 \mathrm{E}+06$ & 0.64 \\
\hline 7 & Wavelet LLL Stats - Energy & 0.984 & 1.140 & $<0.0001$ & $-1.4 \mathrm{E}+09$ & 0.64 \\
\hline 8 & Stats - Total energy & 0.984 & 0.980 & $<0.0001$ & $-5.0 \mathrm{E}+05$ & 0.58 \\
\hline 9 & Stats - Energy & 0.984 & 1.140 & $<0.0001$ & $-2.0 \mathrm{E}+08$ & 0.58 \\
\hline 10 & Shape - Surface to volume ratio & 0.983 & 1.018 & $<0.0001$ & $-1.6 \mathrm{E}-01$ & 0.0012 \\
\hline 11 & Wavelet HHH - GLRLM RLN & 0.981 & 1.242 & $<0.0001$ & $-6.6 \mathrm{E}+01$ & 0.86 \\
\hline 12 & Shape - Surface & 0.981 & 0.911 & $<0.0001$ & $-1.4 \mathrm{E}+00$ & 0.30 \\
\hline 13 & Wavelet LHH - GLRLM RLN & 0.980 & 1.277 & $<0.0001$ & $-6.1 E+01$ & 0.86 \\
\hline 14 & Wavelet HLH - GLRLM RLN & 0.979 & 1.434 & $<0.0001$ & $-5.0 \mathrm{E}+02$ & 0.18 \\
\hline 15 & Wavelet HHH - GLDZM IN & 0.976 & 1.292 & $<0.0001$ & $-5.8 \mathrm{E}+00$ & 0.51 \\
\hline 16 & Wavelet HHH - GLSZM IN & 0.976 & 1.292 & $<0.0001$ & $-5.8 \mathrm{E}+00$ & 0.51 \\
\hline 17 & Shape - Maximum diameter 2D2 & 0.974 & 0.973 & $<0.0001$ & $-9.6 \mathrm{E}-02$ & 0.29 \\
\hline 18 & Wavelet HHH - NGLDM SM & 0.972 & 0.982 & $<0.0001$ & $-1.7 \mathrm{E}+00$ & 0.81 \\
\hline 19 & Wavelet HHL - GLRLM RLN & 0.971 & 1.455 & $<0.0001$ & $-1.7 \mathrm{E}+02$ & 0.68 \\
\hline 20 & Shape - Minor axis length & 0.970 & 0.955 & $<0.0001$ & $-1.7 \mathrm{E}-02$ & 0.45 \\
\hline 21 & Wavelet LHH - GLRLM RLN & 0.969 & 1.432 & $<0.0001$ & $3.0 \mathrm{E}+00$ & 0.99 \\
\hline 22 & Wavelet LHL - GLRLM RLN & 0.967 & 1.615 & $<0.0001$ & $-6.1 E+02$ & 0.12 \\
\hline 23 & Wavelet LHH - NGTDM coarseness & 0.967 & 0.929 & $<0.0001$ & $-2.6 \mathrm{E}-05$ & 0.82 \\
\hline 24 & Wavelet LHL - NGLDM SM & 0.966 & 0.822 & $<0.0001$ & $1.2 \mathrm{E}+01$ & 0.14 \\
\hline 25 & Wavelet HLH - GLDZM IN & 0.965 & 1.609 & $<0.0001$ & $-1.8 \mathrm{E}+01$ & 0.094 \\
\hline 26 & Wavelet HLH - GLSZM IN & 0.965 & 1.609 & $<0.0001$ & $-1.8 \mathrm{E}+01$ & 0.094 \\
\hline 27 & Wavelet HLH - NGTDM coarseness & 0.965 & 0.939 & $<0.0001$ & $-1.1 \mathrm{E}-04$ & 0.33 \\
\hline 28 & Wavelet LHH - GLDZM DZV & 0.965 & 1.086 & $<0.0001$ & $1.7 \mathrm{E}-01$ & $<0.0001$ \\
\hline 29 & Wavelet LHH - NGLDM SM & 0.964 & 0.982 & $<0.0001$ & $-5.9 \mathrm{E}+00$ & 0.51 \\
\hline 30 & Wavelet HHL - GLDZM IN & 0.964 & 1.671 & $<0.0001$ & $-1.7 \mathrm{E}+01$ & 0.094 \\
\hline 31 & Wavelet HHL - GLSZM IN & 0.964 & 1.671 & $<0.0001$ & $-1.7 \mathrm{E}+01$ & 0.094 \\
\hline 32 & Wavelet HHH - GLDZM DZV & 0.962 & 1.067 & $<0.0001$ & $1.7 \mathrm{E}-01$ & $<0.0001$ \\
\hline 33 & Wavelet LHH - GLDZM IN & 0.961 & 1.278 & $<0.0001$ & $2.8 \mathrm{E}+00$ & 0.78 \\
\hline 34 & Wavelet LHH - GLSZ IN & 0.961 & 1.278 & $<0.0001$ & $2.8 \mathrm{E}+00$ & 0.78 \\
\hline 35 & Wavelet HLH - NGLDM SM & 0.960 & 0.856 & $<0.0001$ & $1.2 \mathrm{E}+01$ & 0.15 \\
\hline 36 & Wavelet HHL - NGTDM coarseness & 0.959 & 0.904 & $<0.0001$ & $-6.5 \mathrm{E}-05$ & 0.61 \\
\hline 37 & Wavelet LHL - NGTDM coarseness & 0.957 & 0.852 & $<0.0001$ & $1.6 \mathrm{E}-04$ & 0.31 \\
\hline 38 & Wavelet HLL - NGTDM coarseness & 0.956 & 0.901 & $<0.0001$ & 2.5E-06 & 0.98 \\
\hline
\end{tabular}




\begin{tabular}{|c|c|c|c|c|c|c|}
\hline 39 & Wavelet HHL - NGLDM SM & 0.956 & 0.889 & $<0.0001$ & $8.0 E+00$ & 0.39 \\
\hline 40 & Wavelet LLH- NGTDM coarseness & 0.955 & 0.876 & $<0.0001$ & $1.1 \mathrm{E}-04$ & 0.50 \\
\hline 41 & Wavelet LHL - GLDZM IN & 0.954 & 1.620 & $<0.0001$ & $-1.2 E+01$ & 0.14 \\
\hline 42 & Wavelet LHL - GLSZM IN & 0.954 & 1.620 & $<0.0001$ & $-1.2 \mathrm{E}+01$ & 0.14 \\
\hline 43 & Wavelet LHL - GLRLM GLN & 0.953 & 0.790 & $<0.0001$ & $-9.8 \mathrm{E}+01$ & 0.11 \\
\hline 44 & Wavelet LHH - GLDZM LDE & 0.952 & 1.295 & $<0.0001$ & $1.1 \mathrm{E}-01$ & 0.46 \\
\hline 45 & Wavelet LLH - NGLDM SM & 0.951 & 0.865 & $<0.0001$ & $6.1 \mathrm{E}+00$ & 0.49 \\
\hline 46 & Wavelet HHH - GLDZM LDE & 0.950 & 1.248 & $<0.0001$ & $1.9 \mathrm{E}-01$ & 0.25 \\
\hline 47 & Wavelet LLL - GLDZM IN & 0.947 & 0.981 & $<0.0001$ & $1.1 \mathrm{E}+01$ & 0.0017 \\
\hline 48 & Wavelet LLL - GLSZM IN & 0.947 & 0.981 & $<0.0001$ & $1.1 \mathrm{E}+01$ & 0.0017 \\
\hline 49 & GLSZM LILAE & 0.940 & 1.514 & $<0.0001$ & $-3.3 E+01$ & 0.045 \\
\hline 50 & Wavelet LHL - NGLDM DN & 0.940 & 2.086 & $<0.0001$ & $-4.9 E+01$ & 0.66 \\
\hline 51 & GLDZM IN & 0.938 & 1.146 & $<0.0001$ & $1.2 \mathrm{E}+01$ & 0.013 \\
\hline 52 & GLSZM IN & 0.938 & 1.146 & $<0.0001$ & $1.2 \mathrm{E}+01$ & 0.013 \\
\hline 53 & Wavelet LHH - NGLDM DN & 0.937 & 1.687 & $<0.0001$ & $-8.8 \mathrm{E}+01$ & 0.60 \\
\hline 54 & Wavelet HLH - GLDZM DZV & 0.936 & 1.112 & $<0.0001$ & $2.4 \mathrm{E}-01$ & $<0.0001$ \\
\hline 55 & Wavelet HLH - GLRLM GLN & 0.934 & 0.614 & $<0.0001$ & $3.5 E+01$ & 0.45 \\
\hline 56 & Wavelet LLH - GLDZM DZV & 0.932 & 1.159 & $<0.0001$ & $2.4 \mathrm{E}-01$ & $<0.0001$ \\
\hline 57 & Wavelet LHL - GLDZM DZV & 0.932 & 1.240 & $<0.0001$ & 2.1E-01 & $<0.0001$ \\
\hline 58 & Wavelet HLL - GLRLM RLN & 0.931 & 1.744 & $<0.0001$ & $-6.8 \mathrm{E}+02$ & 0.27 \\
\hline 59 & Wavelet HLH - NGLDM DN & 0.931 & 1.966 & $<0.0001$ & $2.3 \mathrm{E}+01$ & 0.90 \\
\hline 60 & Wavelet HHH - NGLDM DN & 0.928 & 1.568 & $<0.0001$ & $7.1 \mathrm{E}+01$ & 0.73 \\
\hline 61 & Wavelet LHL - NGLDM GLN & 0.927 & 0.569 & $<0.0001$ & $-3.8 \mathrm{E}+01$ & 0.70 \\
\hline 62 & Wavelet HHH - NGTDM coarseness & 0.926 & 0.947 & $<0.0001$ & $-3.4 \mathrm{E}-05$ & 0.84 \\
\hline 63 & Wavelet HHL - GLRLM GLN & 0.925 & 0.708 & $<0.0001$ & $-4.2 E+01$ & 0.50 \\
\hline 64 & Wavelet HHL - GLDZM DZV & 0.924 & 1.209 & $<0.0001$ & $2.2 \mathrm{E}-01$ & $<0.0001$ \\
\hline 65 & Wavelet LLH - GLDZM IN & 0.923 & 1.556 & $<0.0001$ & $-2.1 \mathrm{E}+00$ & 0.86 \\
\hline 66 & Wavelet LLH - GLSZM IN & 0.923 & 1.556 & $<0.0001$ & $-2.1 \mathrm{E}+00$ & 0.86 \\
\hline 67 & NGLDM SM & 0.922 & 0.651 & $<0.0001$ & $1.8 \mathrm{E}+01$ & 0.024 \\
\hline 68 & Wavelet HLL - GLDZM IN & 0.922 & 1.868 & $<0.0001$ & $-1.1 \mathrm{E}+00$ & 0.91 \\
\hline 69 & Wavelet HLL - GLSZM IN & 0.922 & 1.868 & $<0.0001$ & $-1.1 \mathrm{E}+00$ & 0.91 \\
\hline 70 & GLRLM GLN & 0.921 & 0.768 & $<0.0001$ & $-1.1 \mathrm{E}+02$ & 0.080 \\
\hline 71 & Wavelet HLL - GLRLM LRHGE & 0.919 & 0.447 & $<0.0001$ & $3.3 \mathrm{E}+02$ & $<0.0001$ \\
\hline 72 & Wavelet HHL - NGLDM DN & 0.919 & 1.918 & $<0.0001$ & $2.3 \mathrm{E}+02$ & 0.17 \\
\hline 73 & Wavelet LLL - GLSZM SZN & 0.918 & 1.006 & $<0.0001$ & $2.5 \mathrm{E}+01$ & 0.60 \\
\hline 74 & Wavelet HLL - NGLDM SM & 0.917 & 0.704 & $<0.0001$ & $3.2 E+01$ & 0.0013 \\
\hline 75 & GLSZM SZN & 0.917 & 1.000 & $<0.0001$ & $1.4 \mathrm{E}+02$ & 0.028 \\
\hline 76 & Wavelet HLH - GLDZM HILDE & 0.916 & 1.519 & $<0.0001$ & $2.6 \mathrm{E}+02$ & 0.34 \\
\hline 77 & Wavelet LLL - NGLDM DN & 0.915 & 1.628 & $<0.0001$ & $2.2 \mathrm{E}+02$ & 0.016 \\
\hline 78 & Wavelet HLL - GLRLM GLN & 0.913 & 0.682 & $<0.0001$ & $4.0 \mathrm{E}+01$ & 0.52 \\
\hline 79 & GLDZM LILDE & 0.913 & 1.328 & $<0.0001$ & $-3.2 \mathrm{E}-03$ & 0.010 \\
\hline 80 & Wavelet HHL - GLDZM HILDE & 0.913 & 1.316 & $<0.0001$ & $7.7 \mathrm{E}+02$ & 0.0040 \\
\hline 81 & Wavelet HHH - GLRLM GLN & 0.912 & 0.828 & $<0.0001$ & $-5.5 E+01$ & 0.32 \\
\hline 82 & Wavelet LLL - NGLDM GLN & 0.910 & 0.411 & $<0.0001$ & $2.4 \mathrm{E}+01$ & 0.78 \\
\hline 83 & Wavelet LLH - GLRLM GLN & 0.908 & 0.729 & $<0.0001$ & $-6.0 \mathrm{E}+01$ & 0.40 \\
\hline 84 & Wavelet LHH - GLRLM GLN & 0.907 & 0.882 & $<0.0001$ & $-1.4 \mathrm{E}+02$ & 0.073 \\
\hline
\end{tabular}




$\begin{array}{llllllr}85 & \text { Wavelet HHH - GLDZM ZP } & 0.907 & 1.015 & <0.0001 & -7.4 \mathrm{E}-02 & 0.00060 \\ 86 & \text { Wavelet LHH - GLCM infoCorr1 } & 0.906 & 0.952 & <0.0001 & 4.2 \mathrm{E}-03 & 0.17 \\ 87 & \text { Wavelet HLH - GLDZM LDE } & 0.906 & 1.325 & <0.0001 & 3.9 \mathrm{E}-01 & 0.077 \\ 88 & \text { Wavelet LHH - GLDZM HILDE } & 0.905 & 1.941 & <0.0001 & -1.2 \mathrm{E}+03 & 0.0027 \\ 89 & \text { NGLDM DN } & 0.902 & 1.660 & <0.0001 & 3.2 \mathrm{E}+02 & 0.0073 \\ 90 & \text { Wavelet LLL - GLCM invDiffnorm } & 0.902 & 0.781 & <0.0001 & 2.0 \mathrm{E}-01 & <0.0001 \\ 91 & \text { Wavelet LHH - GLDZM ZP } & 0.902 & 1.005 & <0.0001 & -7.7 \mathrm{E}-02 & 0.00069 \\ 92 & \text { Wavelet HLL - NGLDM GLN } & 0.901 & 0.390 & <0.0001 & 1.3 \mathrm{E}+02 & 0.093 \\ 93 & \text { GLRLM RLN } & 0.900 & 1.568 & <0.0001 & 4.3 \mathrm{E}+02 & 0.50 \\ 94 & \text { Wavelet LHH - GLSZM SZN } & 0.900 & 1.905 & <0.0001 & -3.1 \mathrm{E}+02 & 0.092 \\ 95 & \text { Shape - Maximum Diameter 2D1 } & 0.900 & 0.920 & <0.0001 & 1.5 \mathrm{E}-01 & 0.40 \\ 96 & \text { Wavelet HHL - GLCM infoCorr1 } & 0.900 & 0.891 & <0.0001 & 1.1 \mathrm{E}-02 & 0.0016 \\ 97 & \text { Wavelet HHH - GLDZM DZN } & 0.899 & 1.245 & <0.0001 & 5.1 \mathrm{E}+00 & 0.95 \\ 98 & \text { NGTDM coarseness } & 0.896 & 1.095 & <0.0001 & 4.3 \mathrm{E}-04 & 0.088 \\ 99 & \text { Wavelet HHH - GLCM infoCorr2 } & 0.896 & 1.009 & <0.0001 & -1.7 \mathrm{E}-02 & 0.39 \\ 100 & \text { Wavelet HHH - GLSZM SZN } & 0.894 & 1.712 & <0.0001 & -7.6 \mathrm{E}+01 & 0.75\end{array}$




\section{Supplementary Material 2G - Linear regression parameters of the radiomic signature}

Table S2.3. Results of a linear regression performed on features derived from $\mathrm{pCT}$ and $\mathrm{CBCT}$. Feature 1: Stats - Energy, Feature 2: GLRLM - Gray-level Nonuniformity, Feature 3: Wavelet HLH GLRLM Gray-level Nonuniformity, Feature 4: Shape - Compactness. GLRLM: Gray-level Run-Length Matrix, H: High, L: Low.

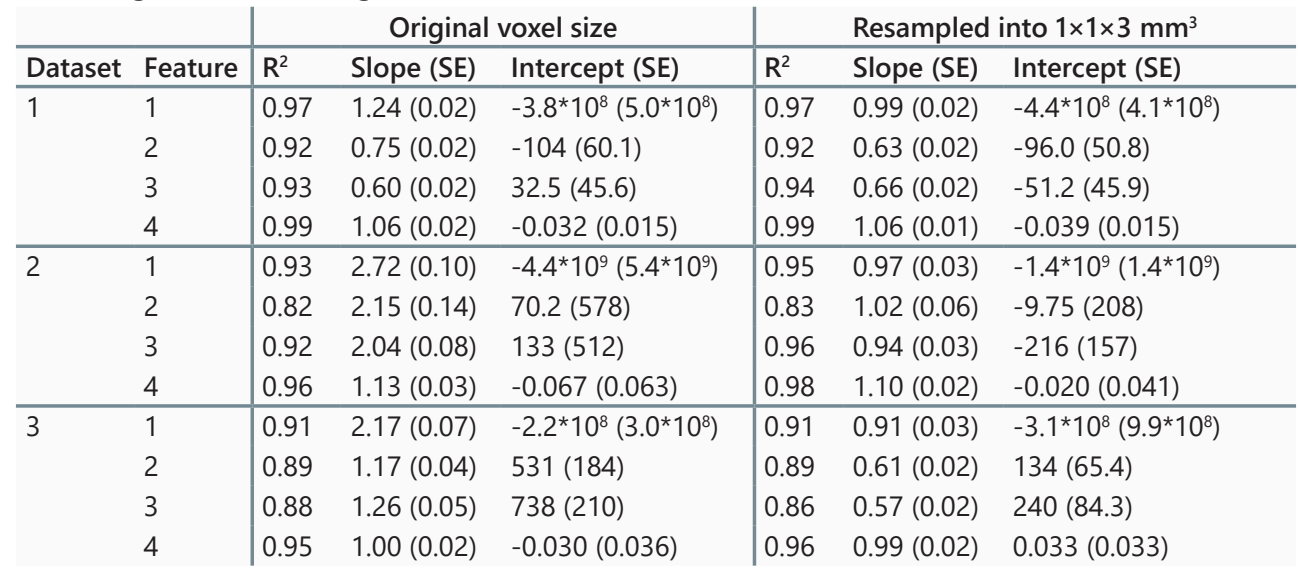




\section{Supplementary Material 2H - Linear predictors of individual patients}
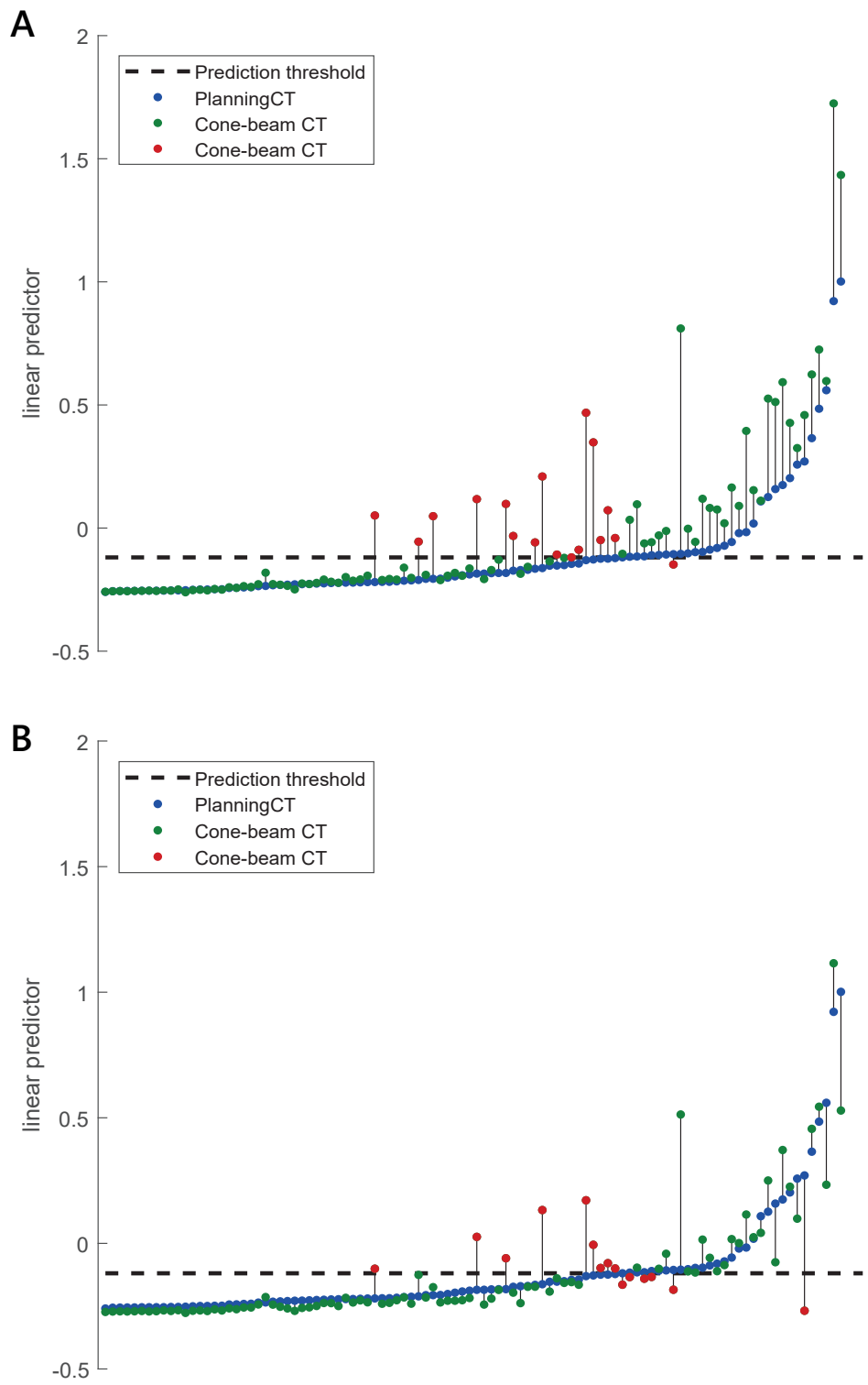

Figure S2.4. Linear predictors of individual patients of dataset 1. The horizontal black line represents the prediction threshold and the dots indicate the linear predictors derived from planning CT (blue) or cone-beam CT (green or red). Each pair of dots (either blue-green or bluered) corresponds to one patient. Data is ranked in ascending order based on linear predictors derived from planning $C T$. Dots for $C B C T$ were indicated in green for prediction correspondence with CT and red for non-prediction-correspondence. (A) Uncorrected images, (B) after the twostep correction procedure. 
A

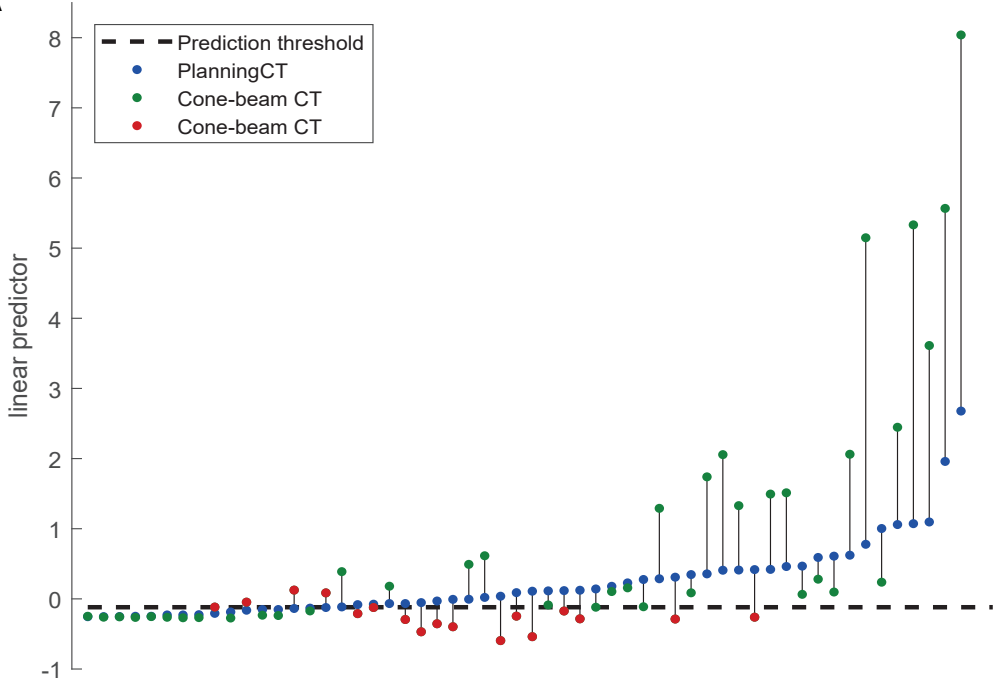

B

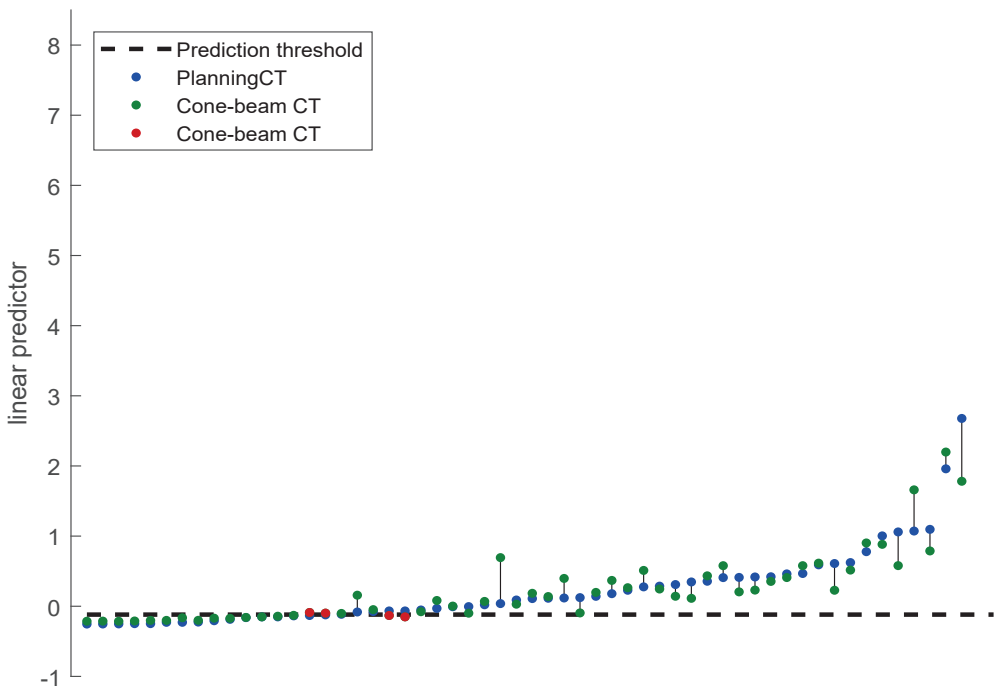

Figure S2.5. Linear predictors of individual patients of dataset 2. The horizontal black line represents the prediction threshold and the dots indicate the linear predictors derived from planning CT (blue) or cone-beam CT (green or red). Each pair of dots (either blue-green or bluered) corresponds to one patient. Data is ranked in ascending order based on linear predictors derived from planning $\mathrm{CT}$. Dots for $\mathrm{CBCT}$ were indicated in green for prediction correspondence with $\mathrm{CT}$ and red for non-prediction-correspondence. (A) Uncorrected images, (B) after the twostep correction procedure. 


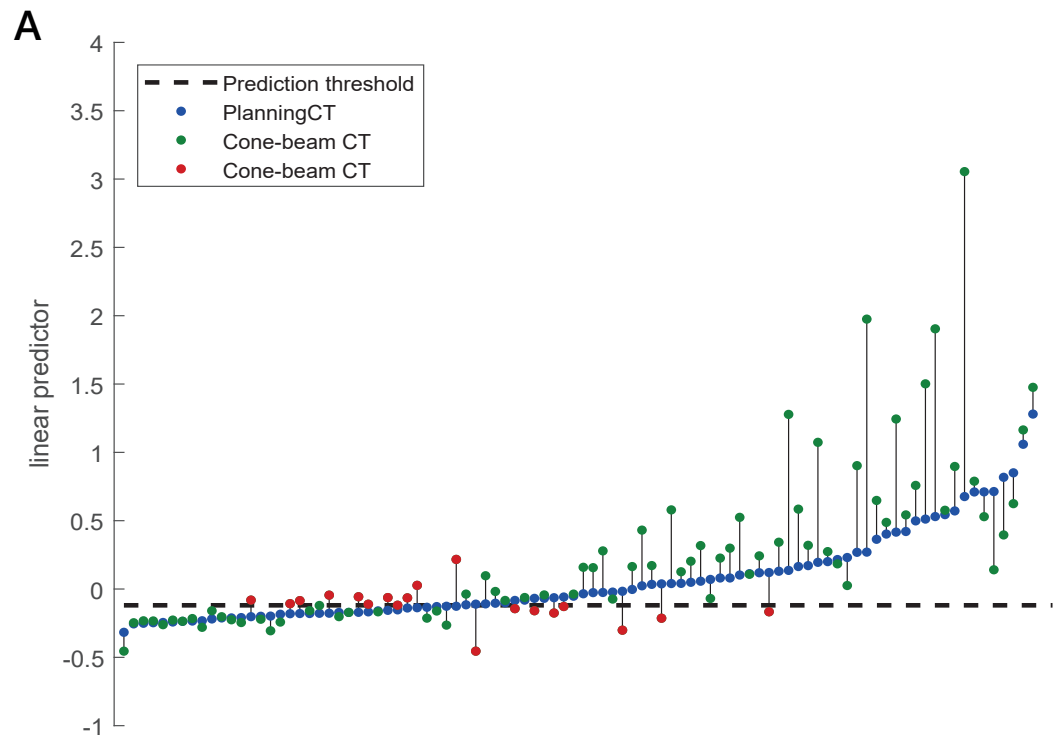

B

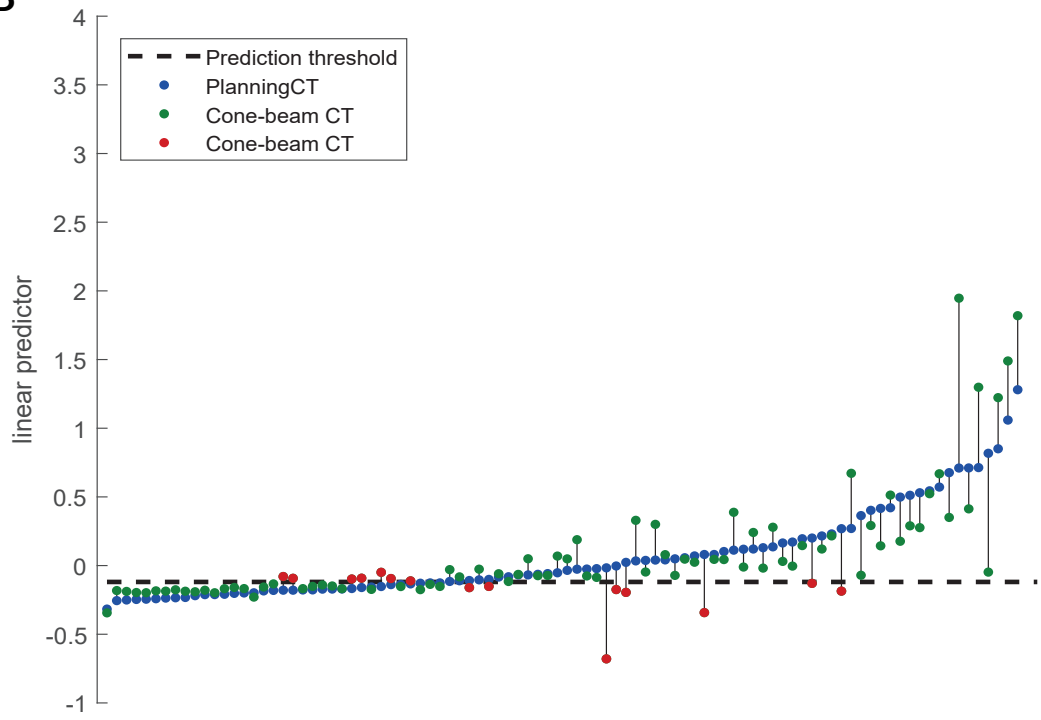

Figure S2.6. Linear predictors of individual patients of dataset 3. The horizontal black line represents the prediction threshold and the dots indicate the linear predictors derived from planning CT (blue) or cone-beam CT (green or red). Each pair of dots (either blue-green or bluered) corresponds to one patient. Data is ranked in ascending order based on linear predictors derived from planning CT. Dots for $C B C T$ were indicated in green for prediction correspondence with $\mathrm{CT}$ and red for non-prediction-correspondence. (A) Uncorrected images, (B) after the twostep correction procedure. 


\section{Supplementary Material 2I - Model validation of dataset 1 split by overall stage}

Table S2.4. Model validation results for $\mathrm{PCT}$ and $\mathrm{CBCT}$ for dataset 1, split by overall stage.

\begin{tabular}{|c|c|c|c|c|}
\hline & & $\begin{array}{l}\text { Calibration slope on the PI } \\
\text { (SE, } p \text {-value LR test) }\end{array}$ & $\begin{array}{l}\text { Joint test on all } \\
\text { coefficients, } p \text {-value }\end{array}$ & $\begin{array}{l}\text { Harrell's c-index } \\
(95 \% \mathrm{Cl}, p \text {-value })\end{array}$ \\
\hline \multirow[t]{2}{*}{$\begin{array}{l}\text { All patients } \\
(\mathrm{n}=102)\end{array}$} & $\mathrm{pCT}$ & $1.77(\mathrm{SE}=0.42, p=0.09)$ & $p=0.013$ & $\begin{array}{l}0.69(0.63-0.75 \\
\left.p=9.9 * 10^{-10}\right)\end{array}$ \\
\hline & $\mathrm{CBCT}$ & $1.90(\mathrm{SE}=0.44, p=0.06)$ & $p=0.027$ & $\begin{array}{l}0.66(0.59-0.73 \\
\left.p=4.8^{\star} 10^{-6}\right)\end{array}$ \\
\hline \multirow[t]{2}{*}{$\begin{array}{l}\text { Stage I } \\
(n=42)\end{array}$} & pCT & $9.42(\mathrm{SE}=2.36, p=0.006)$ & $p=0.0033$ & $\begin{array}{l}0.77(0.70-0.84 \\
\left.p=3.6^{*} 10^{-14}\right)\end{array}$ \\
\hline & $\mathrm{CBCT}$ & $6.98(\mathrm{SE}=1.90, p=0.002)$ & $p=0.0055$ & $\begin{array}{l}0.73(0.65-0.81 \\
\left.p=3.2^{*} 10^{-8}\right)\end{array}$ \\
\hline \multirow[t]{2}{*}{$\begin{array}{l}\text { Stage II-IV } \\
(\mathrm{n}=60)\end{array}$} & $\mathrm{pCT}$ & $1.33(\mathrm{SE}=0.54, p=0.54)$ & $p=0.73$ & $\begin{array}{l}0.64(0.56-0.72 \\
\left.p=8.8^{*} 10^{-4}\right)\end{array}$ \\
\hline & $\mathrm{CBCT}$ & $1.32(\mathrm{SE}=0.50, p=0.56)$ & $p=0.54$ & $\begin{array}{l}0.61(0.51-0.70 \\
p=0.02)\end{array}$ \\
\hline
\end{tabular}




\section{Supplementary Material 2J - Kaplan-Meier curves of all datasets}
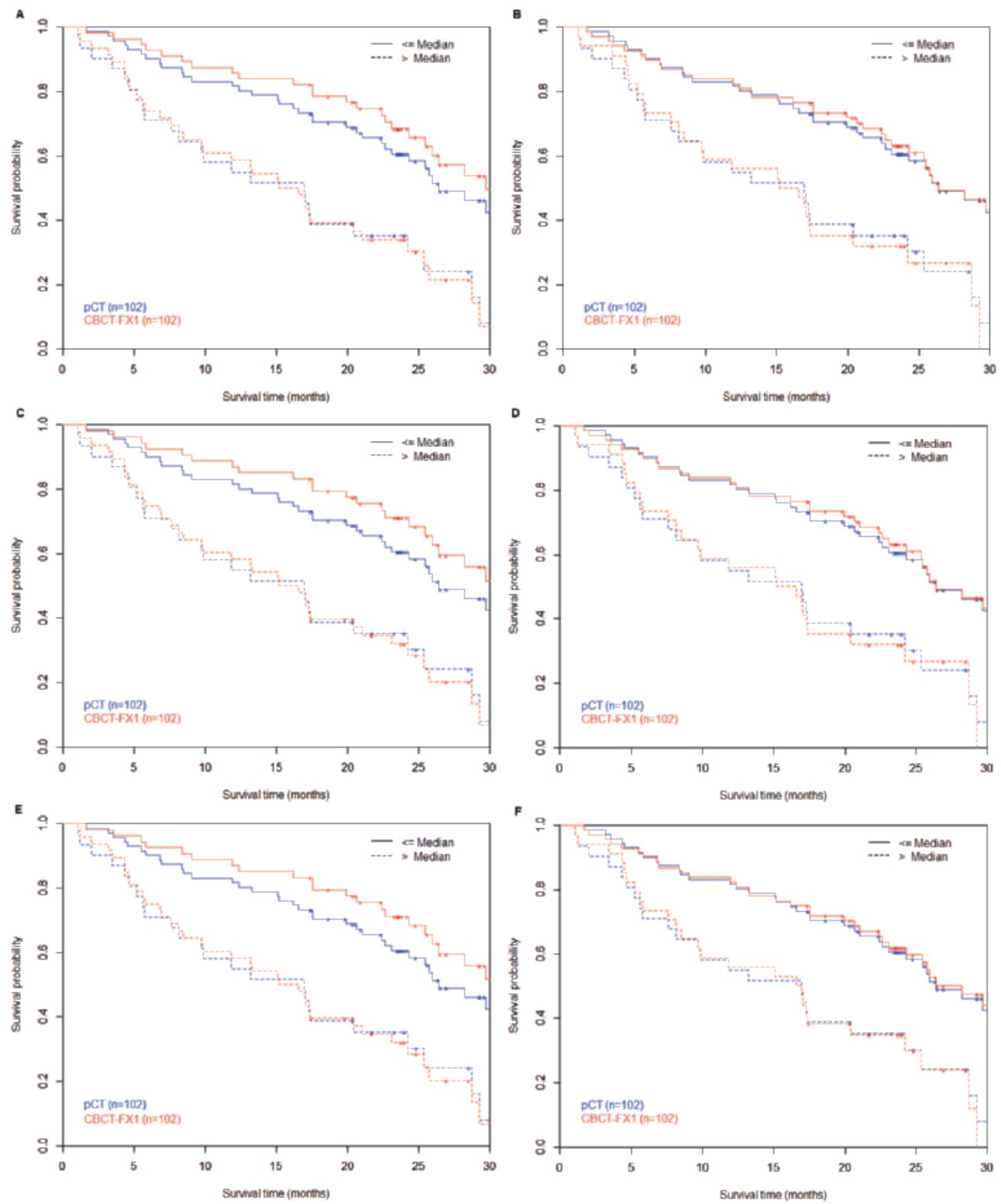

Figure S2.7. Kaplan-Meier curves of dataset 1. The The panels represent the split in groups with high and low prognostic value for $\mathrm{CT}$ (blue) and $\mathrm{CBCT}$ (red) after different steps of correction. In the first row of panels (A and B), correction step 1 (intensity correction) was not applied. In the middle row of panels ( $C$ and $D$ ), intensity correction was applied but using a pooled correction factor (one dataset instead of one per image). In the lower row of panels (E and F), correction step 1 was applied (using individual correction factors). Correction step 2 (feature normalization) was only applied int he right clumn of panels (B, D and F). 

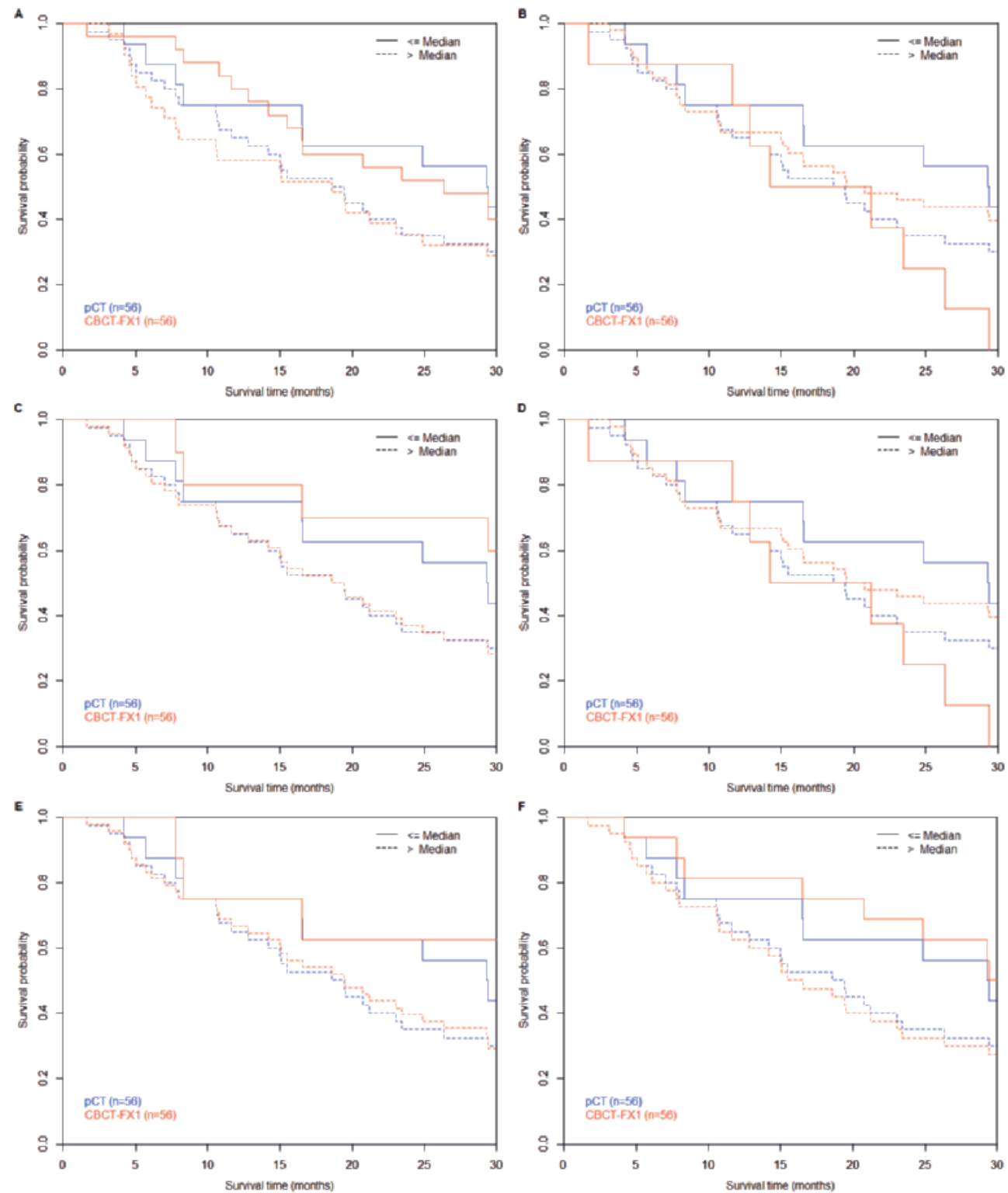

Figure S2.8. Kaplan-Meier curves of dataset 2. The panels represent the split in groups with high and low prognostic value for $\mathrm{CT}$ (blue) and $\mathrm{CBCT}$ (red) after different steps of correction. In the first row of panels (A and B), correction step 1 (intensity correction) was not applied. In the middle row of panels ( $C$ and $D$ ), intensity correction was applied but using a pooled correction factor (one per dataset instead of one per image). In the lower row of panels (E and F), correction step 1 was applied (using individual correction factors). Correction step 2 (feature normalization) was only applied in the right column of panels ( $B, D$ and $F)$. 

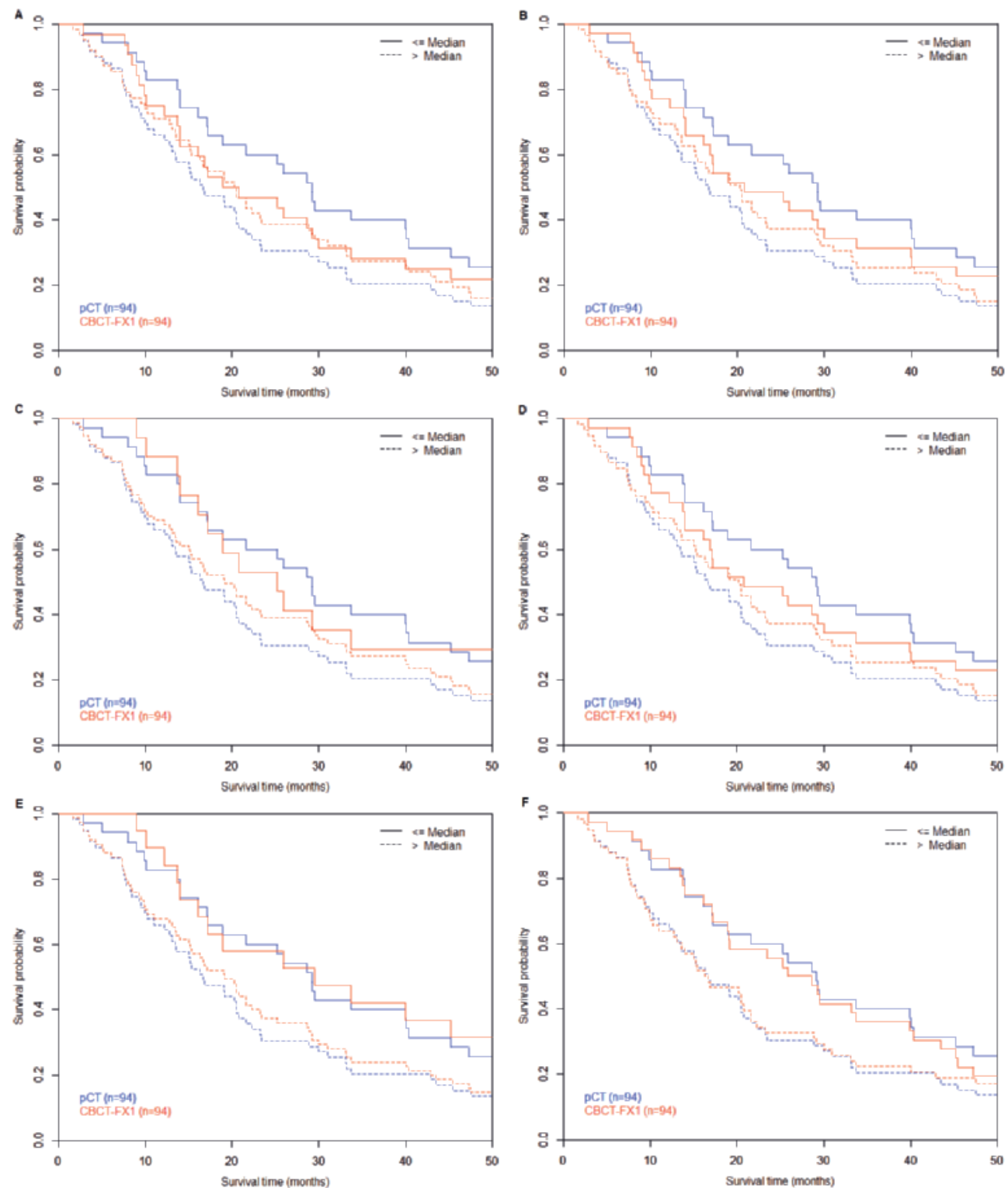

Figure S2.9. Kaplan-Meier curves of dataset 3. The panels represent the split in groups with high and low prognostic value for CT (blue) and CBCT (red) after different steps of correction. In the first row of panels (A and B), correction step 1 (intensity correction) was not applied. In the middle row of panels ( $C$ and $D$ ), intensity correction was applied but using a pooled correction factor (one per dataset instead of one per image). In the lower row of panels ( $E$ and $F$ ), correction step 1 was applied (using individual correction factors). Correction step 2 (feature normalization) was only applied in the right column of panels (B, D and F). 


\section{Supplementary Material 2K - Feature descriptions}

\section{First-order gray-level statistics}

First-order gray-level statistics describe the distribution of gray values within the volume. Let $X$ denote the three dimensional image matrix with $N$ voxels, $P$ the first order histogram, $P(i)$ the fraction of voxels with intensity level $i$ and $N_{l}$ the number of discrete intensity levels.

\section{Energy}

$$
\text { energy }=\sum_{i=1}^{N} X(i)^{2}
$$

Energy is also known as the sum of squares.

\section{Entropy}

$$
\text { entropy }=\sum_{\mathrm{i}=1}^{\mathrm{N}_{1}} \mathrm{P}(\mathrm{i}) \log _{2} \mathrm{P}(\mathrm{i})
$$

\section{Kurtosis}

$$
\text { kurtosis }=\frac{\frac{1}{\mathrm{~N}} \sum_{\mathrm{i}=1}^{\mathrm{N}}(\mathrm{X}(\mathrm{i})-\overline{\mathrm{X}})^{4}}{\left(\frac{1}{\mathrm{~N}} \sum_{\mathrm{i}=1}^{\mathrm{N}}(\mathrm{X}(\mathrm{i})-\overline{\mathrm{X}})^{2}\right)^{2}}
$$

where $\bar{X}$ is the mean of $X$.

\section{Maximum}

The maximum intensity value of $X$.

\section{Mean}

$$
\operatorname{maximum}=\max (\mathrm{X})
$$

The mean gray-value of $X$.

\section{Mean absolute deviation}

$$
\text { mean }=\frac{1}{N} \sum_{i=1}^{N} X(i)
$$

The mean of the absolute deviations of all voxel intensities around the mean intensity value.

where $\bar{X}$ is the mean of $X$.

$$
\text { mean absolute deviation }=\frac{1}{N} \sum_{i=1}^{N}|X(i)-\bar{X}|
$$

\section{Median}

The sample median of $X$, or the $50^{\text {th }}$ percentile of $X$.

\section{Minimum}

The minimum intensity value of $X$.

\section{Range}

$$
\operatorname{minimum}=\min (X)
$$

The range of intensity values of $X$.

\section{Root mean square (RMS)}

$$
\text { range }=\max (X)-\min (X)
$$

The quadratic mean, or the square root of the mean of squares of all voxel intensities.

$$
\text { RMS }=\sqrt{\frac{\sum_{\mathrm{i}}^{\mathrm{N}} \mathrm{X}(\mathrm{i})^{2}}{\mathrm{~N}}}
$$


11. Skewness

$$
\text { skewness }=\frac{\frac{1}{\mathrm{~N}} \sum_{\mathrm{i}=1}^{\mathrm{N}}(\mathrm{X}(\mathrm{i})-\overline{\mathrm{X}})^{3}}{\left(\sqrt{\frac{1}{\mathrm{~N}} \sum_{\mathrm{i}=1}^{\mathrm{N}}(\mathrm{X}(\mathrm{i})-\overline{\mathrm{X}})^{2}}\right)^{3}}
$$

where $\bar{X}$ is the mean of $X$.

12. Standard deviation

$$
\text { standard deviation }=\left(\frac{1}{\mathrm{~N}-1} \sum_{\mathrm{i}=1}^{\mathrm{N}}(\mathrm{X}(\mathrm{i})-\overline{\mathrm{X}})^{2}\right)^{1 / 2}
$$

where $\bar{X}$ is the mean of $X$.

\section{Robust mean absolute deviation}

The mean absolute deviation ( 0 ) of only those voxels in $X$ with a gray value between the $10^{\text {th }}$ and $90^{\text {th }}$ percentile.

\section{4. $10^{\text {th }}$ percentile}

The $10^{\text {th }}$ percentile of $X$, a robust alternative to the minimum gray value (8).

15. $90^{\text {th }}$ percentile

The $90^{\text {th }}$ percentile of $X$, a robust alternative to the maximum gray value (4).

16. Interquartile range

The interquartile range is defined as the $75^{\text {th }}$ minus the $25^{\text {th }}$ percentile of $X$.

17. Uniformity

$$
\text { uniformity }=\sum_{\mathrm{i}=1}^{\mathrm{N}_{\mathrm{l}}} \mathrm{P}(\mathrm{i})^{2}
$$

\section{Variance}

$$
\text { variance }=\frac{1}{\mathrm{~N}-1} \sum_{\mathrm{i}=1}^{\mathrm{N}}(\mathrm{X}(\mathrm{i})-\overline{\mathrm{X}})^{2}
$$

where $\bar{X}$ is the mean of $X$. Variance is the square of the standard deviation (12). 


\section{Geometric features}

Geometric features describe the shape and size of the volume of interest. Let $V$ be the volume and $A$ the surface area of the volume of interest. Let $N$ be the total number of voxels, $X=\left\{\vec{X}_{1}, \vec{X}_{2}, \ldots, \vec{X}_{N}\right\}$ the set of $\mathrm{N}$ Cartesian coordinate vectors and $I=\left\{I_{1}, I_{2}, \ldots, I_{N}\right\}$ the corresponding intensity values.

19. Asphericity

\section{Centroid distance}

$$
\text { asphericity }=\left(\frac{1}{36 \pi} \frac{A^{3}}{V^{2}}\right)^{\frac{1}{3}}-1
$$

The centroid distance is the Euclidean distance between the geometric centroid $\left(C_{g}\right)$ and the centroid weighing each voxel by its intensity value $\left(C_{i}\right)$. The centroid distance is a measure of how close the high intensity values are to the geometric center.

\section{Compactness 1}

$$
\begin{gathered}
\mathrm{C}_{\mathrm{g}}=\frac{1}{\mathrm{~N}} \sum_{\mathrm{i}=1}^{\mathrm{N}} \overrightarrow{\mathrm{X}}_{\mathrm{i}} \\
\mathrm{C}_{\mathrm{i}}=\frac{\sum_{\mathrm{i}=1}^{\mathrm{N}} \overrightarrow{\mathrm{X}}_{\mathrm{i}}}{\sum_{\mathrm{i}=1}^{\mathrm{N}} \mathrm{I}} \\
\text { centroid distance }=\left\|\mathrm{C}_{\mathrm{g}}-\mathrm{C}_{\mathrm{i}}\right\|
\end{gathered}
$$

Compactness is a measure of how much the volume resembles a sphere, as described by Aerts et al. [2].

\section{Compactness 2}

$$
\text { compactness } 1=\frac{\mathrm{V}}{\sqrt{\pi} \mathrm{A}^{\frac{2}{3}}}
$$

\section{Compactness 3}

$$
\text { compactness } 2=36 \pi \frac{\mathrm{V}^{2}}{\mathrm{~A}^{3}}
$$

$$
\text { compactness } 3=\frac{\mathrm{V}}{\sqrt{\pi} \mathrm{A}^{\frac{3}{2}}}
$$

A dimensionless alternative to Compactness (21), as described by Aerts et al. [2].

\section{Maximum diameter}

The maximum diameter is the largest pairwise difference between voxels on the surface of the volume, in $3 \mathrm{D}$ and for each plane separately. The following diameters are calculated:

24.1. The maximum three-dimensional tumor diameter.

24.2. The maximum two-dimensional diameter of all transversal planes.

24.3. The maximum two-dimensional diameter of all sagittal planes.

24.4. The maximum two-dimensional diameter of all coronal planes.

\section{Major axis length}

Axis lengths are measures of the extent of the volume along its three principle axis. Principle component analysis (PCA) on the $x, y$ and $z$ coordinates of all voxels within the volume is used to determine the three orthogonal eigenvectors and corresponding eigenvalues $\left(\lambda_{\max }, \lambda_{\text {minor }}, \lambda_{\text {min }}\right)$. The major axis length is the largest eigenvalue $\left(\lambda_{\max }\right)$ as determined by PCA.

\section{Minor axis length}

The largest eigenvalue $\left(\lambda_{\text {minor }}\right)$ as determined by PCA.

27. Least axis length

The smallest eigenvalue $\left(\lambda_{\min }\right)$ as determined by PCA.

28. Elongation

$$
\text { elongation }=\frac{\lambda_{\operatorname{minor}}}{\lambda_{\max }}
$$




\section{Flatness}

30. Spherical disproportion [3]

$$
\text { flatness }=\frac{\lambda_{\min }}{\lambda_{\max }}
$$

Spherical disproportion is a measure of how much the volume resembles a sphere.

$$
\text { spherical disproportion }=\frac{\mathrm{A}}{4 \pi \mathrm{R}^{2}}
$$

Where $A$ is the surface area and $R$ is the radius of a sphere with the same volume as the tumor, obtained through:

\section{Sphericity [3]}

$$
R=\sqrt[3]{\frac{3 V}{4 \pi}}
$$

Sphericity is a measure of how much the volume resembles a sphere.

\section{Surface area}

$$
\text { sphericity }=\frac{\pi^{\frac{1}{3}}(6 \mathrm{~V})^{\frac{2}{3}}}{\mathrm{~A}}=\frac{\left(36 \pi \mathrm{V}^{2}\right)^{\frac{1}{3}}}{\mathrm{~A}}
$$

The surface area is calculated by triangulation (i.e. dividing the surface into connected triangles, which define the isosurface enclosing the volume) and is defined as:

$$
\text { surface area }=\sum_{\mathrm{i}=1}^{\mathrm{N}} \frac{1}{2}\left|\mathrm{a}_{\mathrm{i}} \mathrm{b}_{\mathrm{i}} \times \mathrm{a}_{\mathrm{i}} \mathrm{c}_{\mathrm{i}}\right|
$$

Where $N$ is the total number of triangles covering the surface and $a, b$ and $c$ are edge vectors of the triangles.

\section{Surface to volume ratio}

\section{Volume}

$$
\text { surface to volume ratio }=\frac{\mathrm{A}}{\mathrm{V}}
$$

The volume is defined as the number of voxels within the volume multiplied by the voxel volume.

$$
\text { volume }=\mathrm{Nv}
$$

Where $v$ is the volume of a single voxel. 


\section{Gray-Level Co-occurrence Matrix based features}

Gray-level co-occurrence matrix (GLCM) based features, as originally described by Haralick et al. [4]. A normalized GLCM is defined as $P(i, j ; \delta, \alpha)$, a matrix with size $N_{g} \times N_{g}$ describing the second-order joint probability function of an image, where the $(i, j)$ th element represents the number of times the combination of intensity levels $i$ and $j$ occur in two pixels in the image, that are separated by a distance of $\delta$ pixels in direction $\alpha$, and $N_{g}$ is the maximum discrete intensity level in the image. Let:

$P(i, j)$ be the normalized (i.e. $\left.\sum P(i, j)=1\right)$ co-occurrence matrix, generalized for any $\delta$ and $\alpha$,

$p_{x}(i)=\sum_{j=1}^{N_{g}} P(i, j)$,

$p_{y}(j)=\sum_{i=1}^{N_{g}} P(i, j)$,

$\mu_{x}$ be the mean of $p_{x}$, where $\mu_{x}=\sum_{i=1}^{N_{g}} \sum_{j=1}^{N_{g}} i P(i, j)$

$\mu_{y}$ be the mean of $p_{y}$, where $\mu_{y}=\sum_{i=1}^{N_{g}} \sum_{j=1}^{N_{g}} j P(i, j)$

$\sigma_{x}$ be the standard deviation of $p_{x}$, where $\sigma_{x}^{2}=\sum_{i=1}^{N_{g}} \sum_{j=1}^{N_{g}} P(i, j)\left(i-\mu_{x}\right)^{2}$

$\sigma_{y}$ be the standard deviation of $p_{y}$, where $\sigma_{y}^{2}=\sum_{i=1}^{N_{g}} \sum_{j=1}^{N_{g}} P(i, j)\left(j-\mu_{y}\right)^{2}$

$p_{x+y}(k)=\sum_{i=1}^{N_{g}} \sum_{j=1}^{N_{g}} P(i, j), i+j=k, k=2,3, \ldots, 2 N_{g^{\prime}}$

$p_{x-y}(k)=\sum_{i=1}^{N_{g}} \sum_{j=1}^{N_{g}} P(i, j),|i-j|=k, k=0,1, \ldots, N_{g}-1$,

$H X Y 1=-\sum_{i=1}^{N_{g}} \sum_{j=1}^{N_{g}} P(i, j) \ln \left(p_{x}(i) p_{y}(j)\right)$,

$H X Y 2=-\sum_{i=1}^{N_{g}} \sum_{j=1}^{N_{g}} p_{x}(i) p_{y}(j) \ln \left(p_{x}(i) p_{y}(j)\right)$.

$H X=-\sum p_{x} \ln \left(p_{x}\right)$

$H Y=-\sum p_{y} \ln \left(p_{y}\right)$

35. Average $(\mu)$

$$
\text { average }(\mu)=\frac{\sum_{\mathrm{i}=1}^{\mathrm{Ng}} \sum_{\mathrm{j}=1}^{\mathrm{Ng}}(\mathrm{i}+\mathrm{j}) \mathrm{P}(\mathrm{i}, \mathrm{j})}{2}
$$

Note that for a symmetrical GLCM, $\mu=\mu_{x}=\mu_{y}$.

\section{Autocorrelation}

$$
\text { autocorrelation }=\sum_{\mathrm{i}=1}^{\mathrm{N}_{\mathrm{g}}} \sum_{\mathrm{j}=1}^{\mathrm{N}_{\mathrm{g}}} \mathrm{ijP}(\mathrm{i}, \mathrm{j})
$$

37. Cluster Prominence

$$
\text { cluster prominence }=\sum_{\mathrm{i}=1}^{\mathrm{N}_{\mathrm{g}}} \sum_{\mathrm{j}=1}^{\mathrm{N}_{\mathrm{g}}}\left[\mathrm{i}+\mathrm{j}-\mu_{\mathrm{x}}-\mu_{\mathrm{y}}\right]^{4} \mathrm{P}(\mathrm{i}, \mathrm{j})
$$

38. Cluster Shade

$$
\text { cluster shade }=\sum_{\mathrm{i}=1}^{\mathrm{N}_{\mathrm{g}}} \sum_{\mathrm{j}=1}^{\mathrm{N}_{\mathrm{g}}}\left[\mathrm{i}+\mathrm{j}-\mu_{\mathrm{x}}-\mu_{\mathrm{y}}\right]^{3} \mathrm{P}(\mathrm{i}, \mathrm{j})
$$

39. Cluster Tendency

$$
\text { cluster tendency }=\sum_{i=1}^{N_{g}} \sum_{j=1}^{N_{g}}\left[i+j-\mu_{x}-\mu_{y}\right]^{2} P(i, j)
$$

40. Contrast [5]

$$
\text { contrast }=\sum_{\mathrm{i}=1}^{\mathrm{N}_{\mathrm{g}}} \sum_{\mathrm{j}=1}^{\mathrm{N}_{\mathrm{g}}}|\mathrm{i}-\mathrm{j}|^{2} \mathrm{P}(\mathrm{i}, \mathrm{j})=\sum_{\mathrm{k}=0}^{\mathrm{N}_{\mathrm{g}}-1} \mathrm{k}^{2} \mathrm{p}_{\mathrm{x}-\mathrm{y}}(\mathrm{k})
$$


41. Correlation

$$
\text { correlation }=\frac{\sum_{\mathrm{i}=1}^{\mathrm{N}_{\mathrm{g}}} \sum_{\mathrm{j}=1}^{\mathrm{N}_{\mathrm{g}}} \mathrm{ijP}(\mathrm{i}, \mathrm{j})-\mu_{\mathrm{x}} \mu_{\mathrm{y}}}{\sigma_{\mathrm{x}} \sigma_{\mathrm{y}}}
$$

42. Difference Average $\left(\mu_{x-y}\right)$

$$
\text { difference average }\left(\mu_{\mathrm{x}-\mathrm{y}}\right)=\sum_{\mathrm{k}=0}^{\mathrm{N}_{\mathrm{g}}-1} \mathrm{kp}_{\mathrm{x}-\mathrm{y}}
$$

43. Difference Entropy

$$
\text { difference entropy }=-\sum_{i=0}^{N_{g}-1} P_{x-y}(i) \log _{2}\left[P_{x-y}(i)\right]
$$

44. Difference Variance

$$
\text { difference variance }=\sum_{i=0}^{N_{g}-1}\left(i-\mu_{x-y}\right)^{2} P_{x-y}(i)
$$

45. Dissimilarity

$$
\operatorname{dissimilarity}=\sum_{i=1}^{N_{g}} \sum_{j=1}^{N_{g}}|i-j| P(i, j)
$$

46. Energy [6]

$$
\text { energy }=\sum_{i=1}^{N_{g}} \sum_{j=1}^{N_{g}}[P(i, j)]^{2}
$$

This feature is also called Angular Second Moment (ASM) and Uniformity [5].

47. Entropy $(H)$

48. Homogeneity 1

$$
\operatorname{entropy}(H)=-\sum_{i=1}^{N_{g}} \sum_{j=1}^{N_{g}} P(i, j) \log _{2}[P(i, j)]
$$

This feature is also called Inverse Difference [5].

$$
\text { homogeneity } 1=\sum_{i=1}^{N_{g}} \sum_{j=1}^{N_{g}} \frac{P(i, j)}{1+|i-j|}
$$

49. Homogeneity 2 [6]

$$
\text { homogeneity } 2=\sum_{i=1}^{N_{g}} \sum_{j=1}^{N_{g}} \frac{P(i, j)}{1+|i-j|^{2}}
$$

This feature is also called Inverse Difference Moment [5].

50. Informational Measure of Correlation 1 (IMC1)

Where $\mathrm{H}$ is the entropy (47).

$$
\mathrm{IMC1}=\frac{\mathrm{H}-\mathrm{HXY} 1}{\max \{\mathrm{HX}, \mathrm{HY}\}}
$$

51. Informational Measure of Correlation 2 (IMC2)

$$
\mathrm{IMC} 2=\sqrt{1-\mathrm{e}^{-2(\mathrm{HXY} 2-\mathrm{H})}}
$$

Where $H$ is the entropy (47).

52. Inverse Difference Moment Normalized (IDMN)

$$
\operatorname{IDMN}=\sum_{i=1}^{N_{g}} \sum_{j=1}^{N_{g}} \frac{P(i, j)}{1+\left(\frac{|i-j|^{2}}{N_{g}^{2}}\right)}
$$


53. Inverse Difference Normalized (IDN)

$$
I D N=\sum_{i=1}^{N_{g}} \sum_{j=1}^{N_{g}} \frac{P(i, j)}{1+\left(\frac{|i-j|}{N_{g}}\right)}
$$

54. Inverse variance

$$
\text { inverse variance }=\sum_{i=1}^{N_{g}} \sum_{j=1}^{N_{g}} \frac{P(i, j)}{|i-j|^{2}} \quad, i \neq j
$$

55. Maximal Correlation Coefficient

maximal correlation coefficient $=\sqrt{\text { second largest eigenvalue of } Q}$

$$
\mathrm{Q}=\sum_{\mathrm{k}=1}^{\mathrm{N}_{\mathrm{g}}} \frac{\mathrm{P}(\mathrm{i}, \mathrm{k}) \mathrm{P}(\mathrm{j}, \mathrm{k})}{\mathrm{p}_{\mathrm{x}}(\mathrm{i}) \mathrm{p}_{\mathrm{y}}(\mathrm{k})}
$$

56. Maximum Probability

$$
\text { maximum probability }=\max \{P(i, j)\}
$$

57. Sum average (SA)

$$
\text { sum average }(S A)=\sum_{i=2}^{2 N_{g}}\left[i_{x+y}(i)\right]
$$

58. Sum entropy

$$
\text { sum entropy }=-\sum_{\mathrm{i}=2}^{2 \mathrm{~N}_{\mathrm{g}}} \mathrm{P}_{\mathrm{x}+\mathrm{y}}(\mathrm{i}) \log _{2}\left[\mathrm{P}_{\mathrm{x}+\mathrm{y}}(\mathrm{i})\right]
$$

59. Sum variance

$$
\text { sum variance }=\sum_{\mathrm{i}=2}^{2 \mathrm{~N}_{\mathrm{g}}}(\mathrm{i}-\mathrm{SA})^{2} \mathrm{P}_{\mathrm{x}+\mathrm{y}}(\mathrm{i})
$$

60. Variance (sum of squares)

$$
\text { variance }=\sum_{i=1}^{N_{g}} \sum_{j=1}^{N_{g}}(i-\mu)^{2} P(i, j)
$$




\section{Gray-Level Run-Length Matrix based features}

Gray-level run-length matrix (GLRLM) based features, as described by Galloway et al. [7]. Run length metrics quantify gray-level runs in an image. A gray-level run is defined as the length in number of pixels, of consecutive pixels that have the same gray-level value. In a gray-level run length matrix $p(i, j \mid \theta)$, the $(i, j)$ th element describes the number of times $j$ a gray-level $i$ appears consecutively in the direction specified by $\theta$. Let:

$p(i, j)$ be the $(i, j)$ th entry in the given run-length matrix $p$, generalized for any direction $\theta$, $N_{g}$ the number of discrete intensity values in the image,

$N_{r}$ the maximum run length,

$N_{s}$ the total number of runs, where $N_{s}=\sum_{i=1}^{N_{g}} \sum_{j=1}^{N_{r}} p(i, j)$,

$p_{r}$ the sum distribution of the number of runs with run length $j$, where $p_{r}(j)=\sum_{i=1}^{N_{g}} p(i, j)$,

$p_{g}$ the sum distribution of the number of runs with gray-level $i$, where $p_{g}(i)=\sum_{j=1}^{N_{r}} p(i, j)$,

$N_{p}$ the number of voxels in the image, where $N_{p}=\sum_{j=1}^{N_{r} j} p_{r}$,

$p_{n}(i, j)$ the normalized run-length matrix, where $p_{n}(i, j)=\frac{p(i, j)}{N_{s}}$,

$\mu_{r}$ the mean run length, where $\mu_{r}=\sum_{i=1}^{N_{g}} \sum_{j=1}^{N_{r}} j p_{n}(i, j)$,

$\mu_{g}$ the mean gray-level, where $\mu_{g}=\sum_{i=1}^{N_{g}} \sum_{j=1}^{N_{r}} i p_{n}(i, j)$.

61. Short Run Emphasis (SRE)

62. Long Run Emphasis (LRE)

$$
\mathrm{SRE}=\frac{1}{\mathrm{~N}_{\mathrm{s}}} \sum_{\mathrm{j}=1}^{\mathrm{N}_{\mathrm{r}}} \frac{\mathrm{p}_{\mathrm{r}}}{\mathrm{j}^{2}}
$$

63. Gray-Level Non-uniformity (GLN)

$$
\text { LRE }=\frac{1}{N_{s}} \sum_{j=1}^{N_{r}} j^{2} p_{r}
$$

64. Gray-Level Non-uniformity Normalized (GLNN)

$$
\mathrm{GLN}=\frac{1}{\mathrm{~N}_{\mathrm{s}}} \sum_{\mathrm{i}=1}^{\mathrm{N}_{\mathrm{g}}} \mathrm{p}_{\mathrm{g}}^{2}
$$

65. Run Length Non-uniformity (RLN)

$$
\text { GLNN }=\frac{1}{\mathrm{~N}_{\mathrm{s}}^{2}} \sum_{\mathrm{i}=1}^{\mathrm{N}_{\mathrm{g}}} \mathrm{p}_{\mathrm{g}}^{2}
$$

$$
\mathrm{RLN}=\frac{1}{\mathrm{~N}_{\mathrm{s}}} \sum_{\mathrm{j}=1}^{\mathrm{N}_{\mathrm{r}}} \mathrm{p}_{\mathrm{r}}^{2}
$$

66. Run Length Non-uniformity Normalized (RLNN)

67. Run Percentage (RP)

$$
\operatorname{RLNN}=\frac{1}{\mathrm{~N}_{\mathrm{s}}^{2}} \sum_{\mathrm{j}=1}^{\mathrm{N}_{\mathrm{r}}} \mathrm{p}_{\mathrm{r}}^{2}
$$

68. Low Gray-level Run Emphasis (LGRE)

$$
\mathrm{RP}=\frac{\mathrm{N}_{\mathrm{s}}}{\mathrm{N}_{\mathrm{p}}}
$$

$$
\text { LGRE }=\frac{1}{\mathrm{~N}_{\mathrm{s}}} \sum_{\mathrm{i}=1}^{\mathrm{N}_{\mathrm{g}}} \frac{\mathrm{p}_{\mathrm{g}}}{\mathrm{i}^{2}}
$$


69. High Gray-level Run Emphasis (HGRE)

$$
\text { HGRE }=\frac{1}{\mathrm{~N}_{\mathrm{s}}} \sum_{\mathrm{i}=1}^{\mathrm{N}_{\mathrm{g}}} \mathrm{i}^{2} \mathrm{p}_{\mathrm{g}}
$$

70. Short Run Low Gray-level Emphasis (SRLGE)

$$
\text { SRLGE }=\frac{1}{N_{\mathrm{s}}} \sum_{\mathrm{i}=1}^{\mathrm{N}_{\mathrm{g}}} \sum_{\mathrm{j}=1}^{\mathrm{N}_{\mathrm{r}}} \frac{\mathrm{p}(\mathrm{i}, \mathrm{j})}{\mathrm{i}^{2} \mathrm{j}^{2}}
$$

71. Short Run High Gray-level Emphasis (SRHGE)

$$
\text { SRHGE }=\frac{1}{N_{s}} \sum_{i=1}^{N_{g}} \sum_{j=1}^{N_{r}} \frac{p(i, j) i^{2}}{j^{2}}
$$

72. Long Run Low Gray-level Emphasis (LRLGE)

$$
\text { LRLGE }=\frac{1}{N_{\mathrm{s}}} \sum_{\mathrm{i}=1}^{\mathrm{N}_{\mathrm{g}}} \sum_{\mathrm{j}=1}^{\mathrm{N}_{\mathrm{r}}} \frac{\mathrm{p}(\mathrm{i}, \mathrm{j}) \mathrm{j}^{2}}{\mathrm{i}^{2}}
$$

73. Long Run High Gray-level Emphasis (LRHGE)

74. Gray-Level Variance (GLV)

$$
\text { LRHGE }=\frac{1}{N_{\mathrm{s}}} \sum_{\mathrm{i}=1}^{\mathrm{N}_{\mathrm{g}}} \sum_{\mathrm{j}=1}^{\mathrm{N}_{\mathrm{r}}} \mathrm{p}(\mathrm{i}, \mathrm{j}) \mathrm{i}^{2} \mathrm{j}^{2}
$$

75. Run Length Variance (RLV)

$$
G L V=\sum_{i=1}^{N_{g}} \sum_{j=1}^{N_{r}}\left(i-\mu_{g}\right)^{2} p_{n}(i, j)
$$

76. Run Entropy (RE) [8]

$$
R L V=\sum_{i=1}^{N_{g}} \sum_{j=1}^{N_{r}}\left(j-\mu_{r}\right)^{2} p_{n}(i, j)
$$

$$
R E=-\sum_{i=1}^{N_{g}} \sum_{j=1}^{N_{r}} p_{n}(i, j \mid \theta) \log _{2}\left[p_{n}(i, j)\right]
$$




\section{Gray-Level Size-Zone Matrix based features}

Gray-level size-zone matrix (GLSZM) based features, as described by Thibault et al. [9, 10]. A gray-level size-zone matrix describes the amount of homogeneous connected areas within the volume, of a certain size and intensity. The $(i, j)$ th entry of the $\operatorname{GLSZM} p(i, j)$ is the number of connected areas of gray-level (i.e. intensity value) $i$ and size $j$. GLSZM features therefore describe homogeneous areas within the tumor volume, describing tumor heterogeneity at a regional scale [11]. Let:

$p(i, j)$ be the $(i, j)$ th entry in the given GLSZM $p$,

$N_{g}$ the number of discrete intensity values in the image,

$N_{z}$ the size of the largest, homogeneous region in the volume of interest,

$N_{s}$ the total number of homogeneous regions (zones), where $N_{s}=\sum_{i=1}^{N_{g}} \sum_{j=1}^{N_{z}} p(i, j)$,

$p_{z}$ the sum distribution of the number of zones with size $j$, where $p_{z}(j)=\sum_{i=1}^{N_{g}} p(i, j)$,

$p_{g}$ the sum distribution of the number of zones with gray-level $i$, where $p_{g}(i)=\sum_{j=1}^{N_{z}} p(i, j)$,

$N_{p}$ the number of voxels in the image, where $N_{p}=\sum_{j=1}^{N_{z} j} p_{z}$,

$p_{n}(i, j)$ the normalized size-zone matrix, where $p_{n}(i, j)=\frac{p(i, j)}{N_{s}}$,

$\mu_{z}$ the mean zone size, where $\mu_{z}=\sum_{i=1}^{N_{g}} \sum_{j=1}^{N_{z}} j p_{n}(i, j \mid \theta)$,

$\mu_{g}$ the mean gray-level, where $\mu_{g}=\sum_{i=1}^{N_{g}} \sum_{j=1}^{N_{z}} i p_{n}(i, j \mid \theta)$.

77. Small Area Emphasis (SAE)

78. Large Area Emphasis (LAE)

$$
\mathrm{SAE}=\frac{1}{\mathrm{~N}_{\mathrm{s}}} \sum_{\mathrm{j}=1}^{\mathrm{N}_{\mathrm{z}}} \frac{\mathrm{p}_{\mathrm{z}}}{\mathrm{j}^{2}}
$$

79. Intensity Non-uniformity (IN)

$$
\text { LAE }=\frac{1}{N_{s}} \sum_{j=1}^{N_{z}} j^{2} p_{z}
$$

80. Intensity Non-uniformity Normalized (INN)

$$
\text { IN }=\frac{1}{\mathrm{~N}_{\mathrm{s}}} \sum_{\mathrm{i}=1}^{\mathrm{N}_{\mathrm{g}}} \mathrm{p}_{\mathrm{g}}^{2}
$$

81. Size-Zone Non-uniformity (SZN)

$$
\mathrm{INN}=\frac{1}{\mathrm{~N}_{\mathrm{s}}^{2}} \sum_{\mathrm{i}=1}^{\mathrm{N}_{\mathrm{g}}} \mathrm{p}_{\mathrm{g}}^{2}
$$

$$
\mathrm{SZN}=\frac{1}{\mathrm{~N}_{\mathrm{S}}} \sum_{\mathrm{j}=1}^{\mathrm{N}_{\mathrm{Z}}} \mathrm{p}_{\mathrm{Z}}^{2}
$$

82. Size-Zone Non-uniformity Normalized (SZNN)

83. Zone Percentage (ZP)

$$
\mathrm{SZNN}=\frac{1}{\mathrm{~N}_{\mathrm{S}}^{2}} \sum_{\mathrm{j}=1}^{\mathrm{N}_{\mathrm{Z}}} \mathrm{p}_{\mathrm{z}}^{2}
$$

84. Low Intensity Emphasis (LIE)

$$
\mathrm{ZP}=\frac{\mathrm{N}_{\mathrm{s}}}{\mathrm{N}_{\mathrm{p}}}
$$

$$
\text { LIE }=\frac{1}{\mathrm{~N}_{\mathrm{s}}} \sum_{\mathrm{i}=1}^{\mathrm{N}_{\mathrm{g}}} \frac{\mathrm{p}_{\mathrm{g}}}{\mathrm{i}^{2}}
$$


85. High Intensity Emphasis (HIE)

86. Low Intensity Small Area Emphasis (LISAE)

$$
\mathrm{HIE}=\frac{1}{\mathrm{~N}_{\mathrm{s}}} \sum_{\mathrm{i}=1}^{\mathrm{N}_{\mathrm{g}}} \mathrm{i}^{2} \mathrm{p}_{\mathrm{g}}
$$

87. High Intensity Small Area Emphasis (HISAE)

$$
\text { LISAE }=\frac{1}{\mathrm{~N}_{\mathrm{s}}} \sum_{\mathrm{i}=1}^{\mathrm{N}_{\mathrm{g}}} \sum_{\mathrm{j}=1}^{\mathrm{N}_{\mathrm{z}}} \frac{\mathrm{p}(\mathrm{i}, \mathrm{j})}{\mathrm{i}^{2} \mathrm{j}^{2}}
$$

$$
\text { HISAE }=\frac{1}{N_{s}} \sum_{i=1}^{N_{g}} \sum_{j=1}^{N_{z}} \frac{p(i, j) i^{2}}{j^{2}}
$$

88. Low Intensity Large Area Emphasis (LILAE)

$$
\text { LILAE }=\frac{1}{\mathrm{~N}_{\mathrm{s}}} \sum_{\mathrm{i}=1}^{\mathrm{N}_{\mathrm{g}}} \sum_{\mathrm{j}=1}^{\mathrm{N}_{\mathrm{z}}} \frac{\mathrm{p}(\mathrm{i}, \mathrm{j}) \mathrm{j}^{2}}{\mathrm{i}^{2}}
$$

89. High Intensity Large Area Emphasis (HILAE)

$$
\text { HILAE }=\frac{1}{N_{s}} \sum_{i=1}^{N_{g}} \sum_{j=1}^{N_{z}} p(i, j) i^{2} j^{2}
$$

90. Intensity Variance (IV)

$$
\mathrm{IV}=\sum_{\mathrm{i}=1}^{\mathrm{N}_{\mathrm{g}}} \sum_{\mathrm{j}=1}^{\mathrm{N}_{\mathrm{Z}}}\left(\mathrm{i}-\mu_{\mathrm{g}}\right)^{2} \mathrm{p}_{\mathrm{n}}(\mathrm{i}, \mathrm{j})
$$

91. Size-Zone Variance (SZV)

$$
S Z V=\sum_{i=1}^{N_{g}} \sum_{j=1}^{N_{z}}\left(j-\mu_{z}\right)^{2} p_{n}(i, j)
$$

92. Zone Entropy (ZE)

$$
Z E=\sum_{i=1}^{N_{g}} \sum_{j=1}^{N_{z}} p_{n}(i, j) \log _{2}\left[p_{n}(i, j)\right]
$$




\section{Gray-Level Distance-Zone Matrix based features}

Gray-level distance-zone matrix (GLDZM) based features, as described by Thibault et al. [12]. A graylevel distance-zone matrix describes the amount of homogeneous connected areas within the volume, of a certain intensity and distance to the shape border. The shape border is defined by 6 -connectedness in 3D (i.e. a voxel is on the border, if at least one face is exposed). In contrast to the original definition by Thibault et al. [12], the minimum distance to the border is 1 , instead of 0 (i.e. voxels on the border have a distance of 1$)$, to allow for correct feature calculations. The $(i, j)$ th entry of the $\operatorname{GLDZM} p(i, j)$ is the number of connected areas of gray-level (i.e. intensity value) $i$ and minimum distance $j$ to the shape border. GLSZM features therefore describe the radial distribution of homogeneous areas within the tumor volume. Let:

$p(i, j)$ be the $(i, j)$ th entry in the given GLDZM $p$,

$N_{g}$ the number of discrete intensity values in the image,

$N_{d}$ the largest distance of a homogeneous region in the volume of interest to the shape border,

$N_{s}$ the total number of homogeneous regions (zones), where $N_{s}=\sum_{i=1}^{N_{g}} \sum_{j=1}^{N_{d}} p(i, j)$,

$p_{d}$ the sum distribution of the number of zones with distance $j$, where $p_{z}(j)=\sum_{i=1}^{N_{g}} p(i, j)$,

$p_{g}$ the sum distribution of the number of zones with gray-level $i$, where $p_{g}(i)=\sum_{j=1}^{N_{z}} p(i, j)$,

$N_{p}$ the number of voxels in the image, where $N_{p}=\sum_{j=1}^{N_{d} j} j p_{d}$,

$p_{n}(i, j)$ the normalized size-zone matrix, where $p_{n}(i, j)=\frac{p(i, j)}{N_{s}}$,

$\mu_{d}$ the mean distance, where $\mu_{d}=\sum_{i=1}^{N_{g}} \sum_{j=1}^{N_{d} j} p_{n}(i, j \mid \theta)$,

$\mu_{g}$ the mean gray-level, where $\mu_{g}=\sum_{i=1}^{N_{g}} \sum_{j=1}^{N_{d}} i p_{n}(i, j \mid \theta)$.

\section{Small Distance Emphasis (SDE)}

$$
\operatorname{SDE}=\frac{1}{\mathrm{~N}_{\mathrm{s}}} \sum_{\mathrm{j}=1}^{\mathrm{N}_{\mathrm{d}}} \frac{\mathrm{p}_{\mathrm{d}}}{\mathrm{j}^{2}}
$$

94. Large Distance Emphasis (LDE)

$$
L D E=\frac{1}{N_{s}} \sum_{j=1}^{N_{d}} j^{2} p_{d}
$$

95. Intensity Non-uniformity (IN)

$$
\text { IN }=\frac{1}{\mathrm{~N}_{\mathrm{s}}} \sum_{\mathrm{i}=1}^{\mathrm{N}_{\mathrm{g}}} \mathrm{p}_{\mathrm{g}}^{2}
$$

96. Intensity Non-uniformity Normalized (INN)

97. Distance-Zone Non-uniformity (DZN)

$$
\mathrm{INN}=\frac{1}{\mathrm{~N}_{\mathrm{s}}^{2}} \sum_{\mathrm{i}=1}^{\mathrm{N}_{\mathrm{g}}} \mathrm{p}_{\mathrm{g}}^{2}
$$

$$
\text { DZN }=\frac{1}{\mathrm{~N}_{\mathrm{s}}} \sum_{\mathrm{j}=1}^{\mathrm{N}_{\mathrm{d}}} \mathrm{p}_{\mathrm{d}}^{2}
$$

98. Distance-Zone Non-uniformity Normalized (DZNN)

99. Zone Percentage (ZP)

$$
\text { DZNN }=\frac{1}{\mathrm{~N}_{\mathrm{s}}^{2}} \sum_{\mathrm{j}=1}^{\mathrm{N}_{\mathrm{d}}} \mathrm{p}_{\mathrm{d}}^{2}
$$

$$
\mathrm{ZP}=\frac{\mathrm{N}_{\mathrm{s}}}{\mathrm{N}_{\mathrm{p}}}
$$


100. Low Intensity Emphasis (LIE)

101. High Intensity Emphasis (HIE)

$$
\text { LIE }=\frac{1}{\mathrm{~N}_{\mathrm{s}}} \sum_{\mathrm{i}=1}^{\mathrm{N}_{\mathrm{g}}} \frac{\mathrm{p}_{\mathrm{g}}}{\mathrm{i}^{2}}
$$

102. Low Intensity Small Distance Emphasis (LISDE)

$$
\mathrm{HIE}=\frac{1}{\mathrm{~N}_{\mathrm{s}}} \sum_{\mathrm{i}=1}^{\mathrm{N}_{\mathrm{g}}} \mathrm{i}^{2} \mathrm{p}_{\mathrm{g}}
$$

$$
\operatorname{LISDE}=\frac{1}{N_{s}} \sum_{i=1}^{N_{g}} \sum_{j=1}^{N_{d}} \frac{p(i, j)}{i^{2} j^{2}}
$$

103. High Intensity Small Distance Emphasis (HISDE)

$$
\text { HISDE }=\frac{1}{N_{s}} \sum_{i=1}^{N_{g}} \sum_{j=1}^{N_{d}} \frac{p(i, j) i^{2}}{j^{2}}
$$

104. Low Intensity Large Distance Emphasis (LILDE)

$$
\text { LILDE }=\frac{1}{N_{s}} \sum_{i=1}^{N_{g}} \sum_{j=1}^{N_{d}} \frac{p(i, j) j^{2}}{i^{2}}
$$

105. High Intensity Large Distance Emphasis (HILDE)

106. Intensity Variance (IV)

$$
\text { HILDE }=\frac{1}{N_{s}} \sum_{i=1}^{N_{g}} \sum_{j=1}^{N_{d}} p(i, j) i^{2} j^{2}
$$

107. Distance-Zone Variance (DZV)

$$
I V=\sum_{i=1}^{N_{g}} \sum_{j=1}^{N_{d}}\left(i-\mu_{g}\right)^{2} p_{n}(i, j)
$$

108. Distance-Zone Entropy (DZE)

$$
S Z V=\sum_{i=1}^{N_{g}} \sum_{j=1}^{N_{d}}\left(j-\mu_{d}\right)^{2} p_{n}(i, j)
$$

$$
\text { DZE }=\sum_{i=1}^{N_{g}} \sum_{j=1}^{N_{d}} p_{n}(i, j) \log _{2}\left[p_{n}(i, j)\right]
$$




\section{Neighborhood Gray-Tone Difference Matrix based features}

Neighborhood gray-tone difference matrix (NGTDM) based features, as described by Amadasun and King [13]. The $i$ th entry of the NGTDM $s(i \mid d)$ is the sum of gray-level differences of voxels with intensity $i$ and the average intensity $A_{i}$ of their neighboring voxels within a distance $d$. In contrast to the original paper, a complete neighborhood is not required and $A_{i}$ is determined over the valid voxels. Let:

$n_{i}$ be the number of voxels with gray-level $i$,

$N_{v}=\sum n_{i}$, the total number of voxels (defined as $n^{2}$ by Amadasun and King [13]),

$s(i)=\left\{\begin{array}{cl}\sum_{n_{i}}\left|i-A_{i}\right| & \text { for } n_{i}>0 \\ 0 & \text { otherwise }\end{array}\right.$, generalized for any distance $d$,

$N_{g}$ be the maximum discrete intensity level in the image,

$p(i)=\frac{n_{i}}{N_{v}}$, the probability of gray-level $i$,

$N_{p}$, the total number of gray-levels present in the image.

109. Coarseness

$$
\text { coarseness }=\frac{1}{\varepsilon+\sum_{i=\mathrm{i}}^{N g} p(i) s(i)}
$$

Where $\varepsilon$ is a small number to prevent coarseness becoming infinite.

110. Contrast

111. Busyness

$$
\text { contrast }=\left(\frac{1}{N_{p}\left(1-N_{p}\right)} \sum_{i=1}^{N_{g}} \sum_{j=1}^{N_{g}} p(i) p(j)(i-j)^{2}\right)\left(\frac{1}{N_{v}} \sum_{i=i}^{N_{g}} s(i)\right)
$$

112. Complexity

$$
\text { busyness }=\frac{\sum_{\mathrm{i}=\mathrm{i}}^{\mathrm{Ng}} \mathrm{p}(\mathrm{i}) \mathrm{s}(\mathrm{i})}{\sum_{\mathrm{i}=\mathrm{i}}^{\mathrm{Ng}} \sum_{\mathrm{j}=\mathrm{i}}^{\mathrm{Ng}}|\mathrm{ip}(\mathrm{i})-\mathrm{jp}(\mathrm{j})|}, \quad \mathrm{p}(\mathrm{i}) \neq 0, \quad \mathrm{p}(\mathrm{j}) \neq 0
$$

113. Strength

$$
\text { complexity }=\frac{1}{N_{v}} \sum_{i=i}^{N_{g}} \sum_{j=i}^{N_{g}}|i-j| \frac{p(i) s(i)+p(j) s(j)}{p(i)+p(j)}, \quad p(i) \neq 0, p(j) \neq 0
$$

$$
\text { strength }=\frac{\sum_{\mathrm{i}=\mathrm{i}}^{\mathrm{Ng}_{\mathrm{g}}} \sum_{\mathrm{j}=\mathrm{i}}^{\mathrm{N}_{\mathrm{g}}}[\mathrm{p}(\mathrm{i})+\mathrm{p}(\mathrm{j})](\mathrm{i}-\mathrm{j})^{2}}{\varepsilon+\sum_{\mathrm{i}=\mathrm{i}}^{\mathrm{Ng}_{\mathrm{g}}} \mathrm{s}(\mathrm{i})}, \quad \mathrm{p}(\mathrm{i}) \neq 0, \quad \mathrm{p}(\mathrm{j}) \neq 0
$$




\section{Neighboring Gray-Level Dependence Matrix based features}

Neighboring gray-level dependence matrix (NGLDM) based features, as described by Sun and Wee [14]. NGLDM features are invariant under spatial rotation. The $(i, j)$ th entry of the $\operatorname{NGLDM~} p(i, j \mid d, a)$ describes the number of neighborhoods with center voxel gray-level (i.e. intensity value) $i$ and dependence (i.e. number of dependent voxels) $k=j-1$. A neighborhood are all voxels within a distance $d$ from the center voxel. The center voxel and a neighboring voxel are dependent if their absolute gray value difference $\leq a$, the dependency coarseness parameter. The features originally specified by Sun and Wee are analogous to the GLRLM and GLSZM features, and the feature set is extended accordingly. Let: $p(i, j)$ be the $(i, j)$ th entry in the given NGLDM $p$, generalized for any $d$ and $a$, $N_{g}$ the number of discrete intensity values in the image,

$N_{d}$ the maximum dependence value,

$N_{s}$ the total number of neighborhoods, where $N_{s}=\sum_{i=1}^{N_{g}} \sum_{j=1}^{N_{Z}} p(i, j)$,

$p_{d}$ the sum distribution of the number of neighborhoods with dependence $j=k+1$, where $p_{d}(j)=$ $\sum_{i=1}^{N_{g}} p(i, j)$,

$p_{g}$ the sum distribution of the number of neighborhoods with center voxel gray-level $i$, where $p_{g}(i)=$ $\sum_{j=1}^{N_{d}} p(i, j)$,

$p_{n}(i, j)$ the normalized NGLDM, where $p_{n}(i, j)=\frac{p(i, j)}{N_{s}}$,

$\mu_{d}$ the mean dependence, where $\mu_{r}=\sum_{i=1}^{N_{g}} \sum_{j=1}^{N_{z}} j p_{n}(i, j \mid \theta)$,

$\mu_{g}$ the mean gray-level, where $\mu_{g}=\sum_{i=1}^{N_{g}} \sum_{j=1}^{N_{Z}} i p_{n}(i, j \mid \theta)$.

Note: By definition, the number of voxels in the image $\left(N_{p}\right)$ equals the total number of neighborhoods $\left(N_{S}\right)$, since in our implementation every voxel is considered to have a neighborhood. Feature "dependence percentage" $\left(\frac{N_{S}}{N_{p}}\right)$, which is the equivalent to run-length feature "run percentage" (RP; 67), is therefore omitted, because it will always evaluate to 1 .

114. Small Dependence Emphasis (SDE)

$$
\operatorname{SDE}=\frac{1}{\mathrm{~N}_{\mathrm{s}}} \sum_{\mathrm{j}=1}^{\mathrm{N}_{\mathrm{d}}} \frac{\mathrm{p}_{\mathrm{d}}}{\mathrm{j}^{2}}
$$

This feature is also called Small Number Emphasis [14].

115. Large Dependence Emphasis (LDE)

$$
\mathrm{LDE}=\frac{1}{\mathrm{~N}_{\mathrm{s}}} \sum_{\mathrm{j}=1}^{\mathrm{N}_{\mathrm{d}}} \mathrm{j}^{2} \mathrm{p}_{\mathrm{d}}
$$

This feature is also called Large Number Emphasis [14].

116. Gray-Level Non-uniformity (GLN)

117. Gray-Level Non-uniformity Normalized (GLNN)

$$
\mathrm{GLN}=\frac{1}{\mathrm{~N}_{\mathrm{s}}} \sum_{\mathrm{i}=1}^{\mathrm{N}_{\mathrm{g}}} \mathrm{p}_{\mathrm{g}}^{2}
$$

$$
\text { GLNN }=\frac{1}{N_{\mathrm{s}}^{2}} \sum_{\mathrm{i}=1}^{\mathrm{N}_{\mathrm{g}}} \mathrm{p}_{\mathrm{g}}^{2}
$$


118. Dependence Non-uniformity (DN)

$$
\mathrm{DN}=\frac{1}{\mathrm{~N}_{\mathrm{s}}} \sum_{\mathrm{j}=1}^{\mathrm{N}_{\mathrm{d}}} \mathrm{p}_{\mathrm{z}}^{2}
$$

This feature is also called Number Non-uniformity [14].

119. Dependence Non-uniformity Normalized (DNN)

120. Low Gray-level Emphasis (LGE)

$$
\mathrm{DNN}=\frac{1}{\mathrm{~N}_{\mathrm{s}}^{2}} \sum_{\mathrm{j}=1}^{\mathrm{N}_{\mathrm{z}}} \mathrm{p}_{\mathrm{z}}^{2}
$$

121. High Gray-level Emphasis (HGE)

$$
\mathrm{LGE}=\frac{1}{\mathrm{~N}_{\mathrm{s}}} \sum_{\mathrm{i}=1}^{\mathrm{N}_{\mathrm{g}}} \frac{\mathrm{p}_{\mathrm{g}}}{\mathrm{i}^{2}}
$$

$$
\mathrm{HGE}=\frac{1}{\mathrm{~N}_{\mathrm{s}}} \sum_{\mathrm{i}=1}^{\mathrm{N}_{\mathrm{g}}} \mathrm{i}^{2} \mathrm{p}_{\mathrm{g}}
$$

122. Low Gray-level Small Dependence Emphasis (LGSDE)

$$
\text { LGSDE }=\frac{1}{\mathrm{~N}_{\mathrm{s}}} \sum_{\mathrm{i}=1}^{\mathrm{N}_{\mathrm{g}}} \sum_{\mathrm{j}=1}^{\mathrm{N}_{\mathrm{d}}} \frac{\mathrm{p}(\mathrm{i}, \mathrm{j})}{\mathrm{i}^{2} \mathrm{j}^{2}}
$$

123. High Gray-level Small Dependence Emphasis (HGSDE)

$$
\operatorname{HGSDE}=\frac{1}{\mathrm{~N}_{\mathrm{s}}} \sum_{\mathrm{i}=1}^{\mathrm{N}_{\mathrm{g}}} \sum_{\mathrm{j}=1}^{\mathrm{N}_{\mathrm{d}}} \frac{\mathrm{p}(\mathrm{i}, \mathrm{j}) \mathrm{i}^{2}}{\mathrm{j}^{2}}
$$

124. Low Gray-level Large Dependence Emphasis (LGLDE)

$$
\text { LGLDE }=\frac{1}{N_{s}} \sum_{i=1}^{N_{g}} \sum_{j=1}^{N_{d}} \frac{p(i, j) j^{2}}{i^{2}}
$$

125. High Gray-level Large Dependence Emphasis (HGLDE)

126. Gray-level Variance (GLV)

$$
\text { HGLDE }=\frac{1}{N_{\mathrm{s}}} \sum_{\mathrm{i}=1}^{\mathrm{N}_{\mathrm{g}}} \sum_{\mathrm{j}=1}^{\mathrm{N}_{\mathrm{d}}} \mathrm{p}(\mathrm{i}, \mathrm{j}) \mathrm{i}^{2} \mathrm{j}^{2}
$$

127. Dependence Variance (DV)

$$
G L V=\sum_{i=1}^{N_{g}} \sum_{j=1}^{N_{d}}\left(i-\mu_{g}\right)^{2} p_{n}(i, j)
$$

$$
D V=\sum_{i=1}^{N_{g}} \sum_{j=1}^{N_{d}}\left(j-\mu_{d}\right)^{2} p_{n}(i, j)
$$

128. Dependence Entropy (DE), also called Entropy [14]

$$
D E=\sum_{i=1}^{N_{g}} \sum_{j=1}^{N_{d}} p_{n}(i, j) \log _{2}\left[p_{n}(i, j)\right]
$$

Note: the definition of entropy by Sun and Wee [14] uses the dependence counts $(p)$ instead of the dependence probabilities $\left(p_{n}\right)$.

129. Second Moment (SM) [14]

$$
\mathrm{SM}=\frac{\sum_{\mathrm{i}=1}^{\mathrm{N}_{\mathrm{g}}} \sum_{\mathrm{j}=1}^{\mathrm{N}_{\mathrm{d}}} \mathrm{p}(\mathrm{i}, \mathrm{j})^{2}}{\mathrm{~N}_{\mathrm{s}}}
$$

Note: for this feature, defined by Sun and Wee [14], there is no gray-level run length equivalent. 


\section{Wavelet features}

Wavelet transform effectively decouples textural information by decomposing the original image, in a similar manner as Fourier analysis, in low- and high-frequencies. In this study a discrete, one-level and undecimated three dimensional wavelet transform was applied to each $C T$ and $C B C T$ image, which decomposes the original image $X$ into 8 decompositions. Consider $L$ and $H$ to be a low-pass (i.e. a scaling) and, respectively, a high-pass (i.e. a wavelet) function, and the wavelet decompositions of $X$ to be labeled as $X_{L L L}, X_{L L H}, X_{L H L}, X_{L H H}, X_{H L L}, X_{H L H}, X_{H H L}$ and $X_{H H H}$. For example, $X_{L L H}$ is then interpreted as the high-pass sub band, resulting from directional filtering of $X$ with a low-pass filter along $x$-direction, a low pass filter along $y$-direction and a high-pass filter along $z$-direction and is constructed as:

$$
X_{L L H}(i, j, k)=\sum_{p=1}^{N_{L}} \sum_{q=1}^{N_{L}} \sum_{r=1}^{N_{H}} L(p) L(q) H(r) X(i+p, j+q, k+r)
$$

Where $N_{L}$ is the length of filter $L$ and $N_{H}$ is the length of filter $H$. The other decompositions are constructed in a similar manner, applying their respective ordering of low or high-pass filtering in $x, y$ and z-direction. Wavelet decomposition of the image $X$ is schematically depicted in Figure S2.10. Since the applied wavelet decomposition is undecimated, the size of each decomposition is equal to the original image and each decomposition is shift invariant. Because of these properties, the original tumor delineation of the gross tumor volume (GTV) can be applied directly to the decompositions after wavelet transform. In this study "Coiflet 1" wavelet was applied. For each decomposition we computed the first order gray-level statistics and the textural features (GLCM, GLRLM, GLSZM).

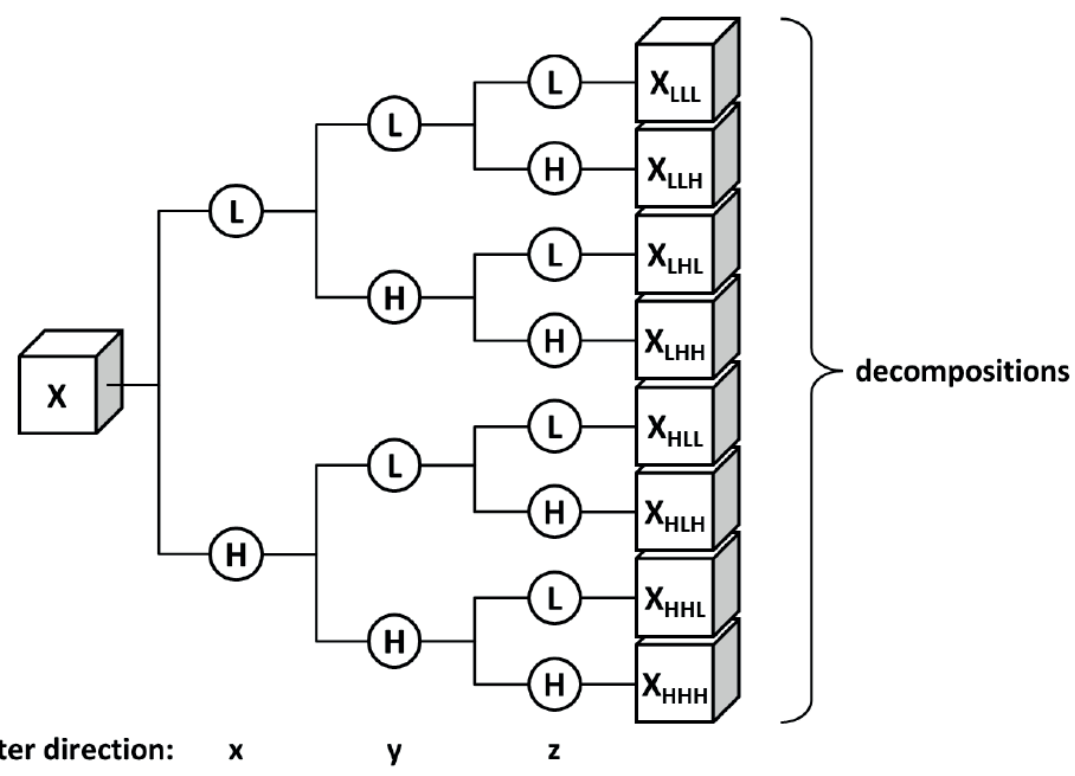

Figure S2.10. Schematic of the undecimated three dimensional wavelet transform applied to each $C T / C B C T$ image. The original $X$ is decomposed into 8 decompositions, by directional low-pass (i.e. a scaling) and high-pass (i.e. a wavelet) filtering: $\mathrm{X}_{\mathrm{LL}} \mathrm{X}_{\mathrm{LLH}}$ $\mathrm{X}_{\mathrm{LH} L^{\prime}} \mathrm{X}_{\mathrm{LHH}^{\prime}} \mathrm{X}_{\mathrm{HLL}} \mathrm{X}_{\mathrm{HLH}}, \mathrm{X}_{\mathrm{HHL}}$ and $\mathrm{X}_{\mathrm{HHH}}$ 


\section{Laplacian of Gaussian features}

The Laplacian of an image brings out areas of rapid intensity change and is usually used for edge detection. A Gaussian filter is applied prior to the Laplacian to smooth the image and reduce noise. Textural properties representing features of different degrees of coarseness can then be calculated. The equation of a LoG with a 2D kernel:

$$
\operatorname{LoG}(x, y)=-\frac{1}{\pi \sigma^{4}}\left[1-\frac{x^{2}+y^{2}}{2 \sigma^{2}}\right] e^{-\frac{x^{2}+y^{2}}{2 \sigma^{2}}}
$$

Texture size (fine to coarse) is highlighted by modifying the Gaussian radius parameter $\sigma$ (e.g. from 0.5 $\mathrm{mm}$ to $5 \mathrm{~mm}$, with $0.5 \mathrm{~mm}$ increments). Each value of $\sigma$ provides a filtered image. First-order gray-level statistics (described earlier) are determined for each filtered image, as well as for only the positive part of each filtered image. 


\section{References}

[1] Feldkamp LA, Davis LC, Kress JW. Practical cone-beam algorithm. J Opt Soc Am A. 1984;1:612-9.

[2] Aerts HJ, Velazquez ER, Leijenaar RT, Parmar C, Grossmann P, Carvalho S, et al. Decoding tumour phenotype by noninvasive imaging using a quantitative radiomics approach. Nat Commun. 2014;5:4006.

[3] Sousa JR, Silva AC, de Paiva AC, Nunes RA. Methodology for automatic detection of lung nodules in computerized tomography images. Comput Methods Programs Biomed. 2010;98:114.

[4] Haralick RM, Shanmugam K, Dinstein I. Textural features of image classification. IEEE Trans Syst, Man, Cybern Syst. 1973;3:610-21.

[5] Clausi DA. An analysis of co-occurrence texture statistics as a function of grey level quantization. Can J Remote Sens. 2002;28:45-62.

[6] Soh L, Tsatsoulis C. Texture analysis of SAR sea ice imagery using gray level co-occurrence matrices. IEEE Trans Geosci Remote Sens. 1999;37:780-95.

[7] Galloway M. Texture analysis using gray level run lengths. Computer Graphics and Image Processing. 1975;4:172-9.

[8] Albregtsen F, Nielsen B, Danielsen HE. Adaptive gray level run length features from class distance matrices. Proceedings 15th International Conference on Pattern Recognition ICPR2000 2000. p. 738-41 vol.3.

[9] Thibault G, Fertil B, Navarro C, L., Pereira S, Cau P, Lévy N, et al. Texture indexes and gray level size zone matrix. Application to cell nuclei classification. 10th International Conference on Pattern Recognition and Information Processing, PRIP 2009. Minsk, Belarus2009. p. 140--5. [10] Tixier F, Hatt M, Le Rest CC, Le Pogam A, Corcos L, Visvikis D. Reproducibility of tumor uptake heterogeneity characterization through textural feature analysis in 18F-FDG PET. J Nucl Med. 2012;53:693-700.

[11] Tixier F, Le Rest CC, Hatt M, Albarghach N, Pradier O, Metges JP, et al. Intratumor heterogeneity characterized by textural features on baseline 18F-FDG PET images predicts response to concomitant radiochemotherapy in esophageal cancer. J Nucl Med. 2011;52:36978.

[12] Thibault G, Angulo J, Meyer F. Advanced Statistical Matrices for Texture Characterization: Application to Cell Classification. IEEE Trans Biomed Eng. 2014;61:630-7.

[13] Amadasun $M$, King R. Textural features corresponding to textural properties. IEEE Trans Syst, Man, Cybern Syst. 1989;19:1264-74.

[14] Sun C, Wee WG. Neighboring gray level dependence matrix for texture classification. Comput Vis Graph Image Process. 1983;23:341-52. 




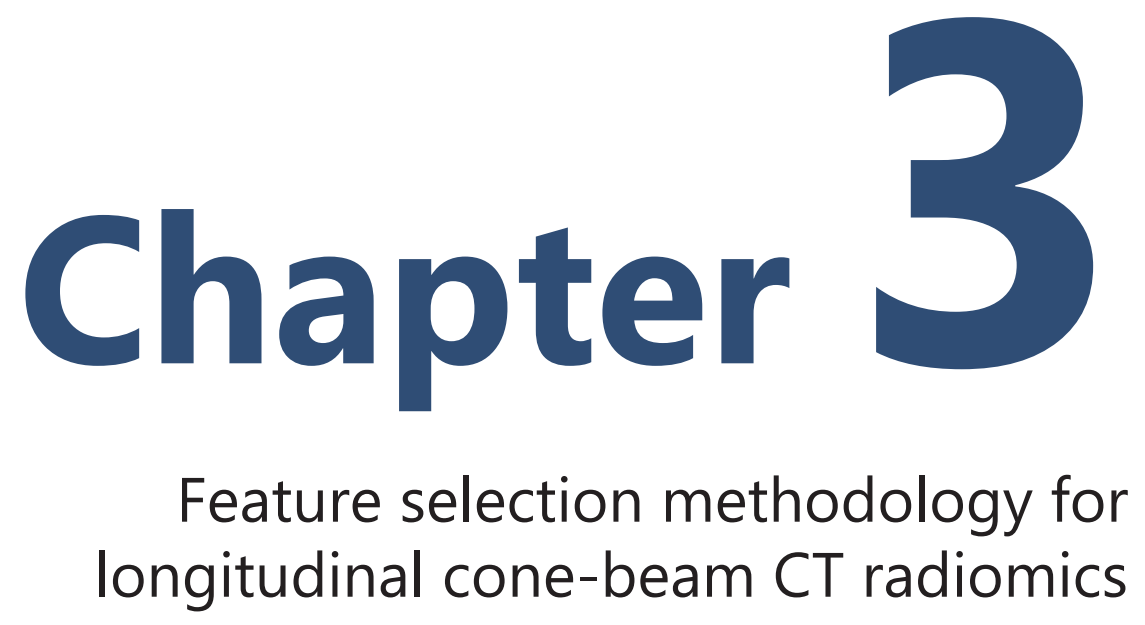

Authors

Janna E. van Timmeren, Ralph T.H. Leijenaar, Wouter van Elmpt, Bart Reymen, Philippe Lambin

Adapted from Acta Oncologica. 2017 August; 56(11):1537-1543. DOI: 10.1080/0284186X.2017.1350285 


\section{ABSTRACT}

\section{Background}

Cone-beam CT (CBCT) scans are typically acquired daily for positioning verification of non-small cell lung cancer (NSCLC) patients. Quantitative information, derived using radiomics, can potentially contribute to (early) treatment adaptation. The aims of this study were to 1) describe and investigate a methodology for feature selection of a longitudinal radiomics approach 2) investigate which time-point during treatment is potentially useful for early treatment response assessment.

\section{Materials and methods}

For 90 NSCLC patients CBCT scans of the first two fractions of treatment (considered as 'test-retest' scans) were analyzed, as well as weekly CBCT images. One hundred and sixteen radiomic features were extracted from the GTV of all scans and subsequently absolute and relative differences were calculated between weekly CBCT images and the CBCT of the first fraction. Test-retest scans were used to determine the smallest detectable change $(\mathrm{C}= \pm 1.96 * \mathrm{SD})$ allowing for feature selection by choosing a minimum number of patients for which a feature should change more than ' $C$ ' to be considered as relevant. Analysis of which features change at which moment during treatment was used to investigate which time-point is potentially relevant to extract longitudinal radiomics information for early treatment response assessment.

\section{Results}

A total of six absolute delta features changed for at least ten patients at week 2 of treatment and increased to 61 at week 3, 79 at week 4 and 85 at week 5. There was $93 \%$ overlap between features selected at week 3 and the other weeks.

\section{Conclusion}

This study describes a feature selection methodology for longitudinal radiomics that is able to select reproducible delta radiomics features that are informative due to their change during treatment, which can potentially be used for treatment decisions concerning adaptive radiotherapy. Nonetheless, the prognostic value of the selected delta radiomic features should be investigated in future studies. 


\section{Introduction}

Healthcare is moving towards a trend of personalized medicine replacing the 'onesize-fits-all' principle. In personalized medicine, early and accurate stratification of patients based on their prognosis, is essential to tailor treatment [1]. To this extent, radiomics could provide useful quantitative information [2, 3]. This is the principle of extracting large amounts of quantitative features from medical images acquired in clinical practice. Combining different types of information, i.e. radiomics, genomics, demographics, models can be developed to predict treatment outcome and improve treatment decisions [4-7].

Previously, the prognostic value of radiomic features extracted from Positron Emission Tomography (PET) and Computed Tomography (CT) images acquired prior to treatment has been investigated [8-11]. The potential of using cone-beam CT $(C B C T)$ for this purpose was also explored $[12,13]$. A recent study demonstrated the potential added prognostic value of delta radiomic features extracted from CT images [14], which indicates that monitoring the course of treatment in a longitudinal radiomics approach can potentially be useful for (early) treatment adaptation. For lung cancer patients, CBCT images are generally acquired daily for treatment positioning verification, which makes this ideal for longitudinal treatment response assessment with radiomics.

To assure robust and reproducible radiomics analyses, an essential step in the process is feature selection, in which features considered as non-informative are eliminated. For radiomics on pre-treatment images (baseline), test-retest methods are often used to eliminate unstable features [15-20]. However, stable features that do not change over the course of treatment are not informative in case of a longitudinal radiomics approach. Moreover, only relying on test-retest data could eliminate informative features for which the change over several weeks of treatment is much larger than the natural fluctuation in a test-retest setting. Therefore, the first aim of this study was to investigate a methodology for feature selection that takes into account not only single time-point robustness, but also potential longitudinal information of radiomic features derived from CBCT imaging. The second aim of this study was to investigate at which time-point during treatment radiomic features extracted from CBCT images change to explore the possibility of using longitudinal radiomics information for (early) treatment adaptation. 


\section{Materials and Methods}

\section{Data acquisition}

Stage II-IV NSCLC patients treated with curatively intended radiotherapy between March 2013 and February 2015 at Maastro Clinic, Maastricht, the Netherlands, were included in this study. Patients that received less than 40 Gy were excluded from the analysis, as well as patients with SBRT (Stereotactic Body Radiation Treatment), postoperative radiotherapy, simultaneous treatment of brain metastases or prior history of lung cancer. Retrospective analysis of weekly CBCT scans (CBCT1a, CBCT2, CBCT3, CBCT4 and CBCT5) was performed. Additionally, CBCT scans of the second treatment fraction (CBCT1b) were analyzed. Patients for which one of these scans were missing were also excluded from the analysis. This resulted in a cohort of 115 NSCLC patients. Patients for which the tumor was not distinguishable on one of the CBCT images due to atelectasis or blurring $(n=10)$ were excluded. Moreover, patients for which the field of view (FOV) was too small to cover the entire 3D tumor volume in one the CBCT images $(n=15)$ were also excluded from the analysis. Characteristics of the remaining 90 NSCLC patients are summarized in Table 3.1.

All CBCT images were acquired from a TrueBeam Radiotherapy System (Varian Medical Systems, Palo Alto, CA) according to the lung protocol with a tube voltage of $125 \mathrm{kVp}$. Images were reconstructed using filtered back projection [21] into images with an in-plane pixel size of $0.91 \times 0.91 \mathrm{~mm}^{2}$ and a slice thickness of either $2 \mathrm{~mm}$ or $3 \mathrm{~mm}$. To improve robustness of the analyses, all images were resampled [22] using cubic interpolation into a voxel size of $1 \times 1 \times 3 \mathrm{~mm}^{3}$ since this most closely resembles the voxel size of the majority of CBCT scans in the cohort.

\section{Feature extraction}

The gross tumor volume (GTV), defined as the primary tumor without lymph nodes, was copied to all CBCT images using a non-rigid registration of the delineation originating from treatment planning $\mathrm{CT}$ images using the Morphons algorithm in REGGUI (http://openreggui.org) [23]. All delineations were manually verified and adjusted when the algorithm failed to perform an acceptable registration of the images. Delineations of all 540 CBCT images were verified by an experienced radiation oncologist. Radiomic features were automatically extracted from the GTV of all CBCT scans using in-house developed software, using Matlab 2014a (MathWorks, Natick, Massachusetts, U.S.A.). Mathematical descriptions of the extracted 116 features are described elsewhere [12]. This included 95 'Texture' features, 2 'Shape' features and 19 'First order statistics (Stats)' features. For each feature, absolute differences compared to baseline were calculated: $C B C T n-C B C T 1 a$, as well as relative differences compared to baseline: (CBCTn - CBCT1a) / CBCT1a. This resulted in a total of 232 delta radiomic features.

\section{Feature selection}

The main aim of feature selection is to select radiomic features with single time-point robustness as well as longitudinal radiomics information. This implies that features having a change during treatment that is larger than the difference that would 
Table 3.1. Demographic information of the entire patient population included in this study.

$$
\mathrm{n}=90
$$

Age

Range (median)

Mean \pm SD

Gender

\section{Male}

Female

Stage

I

II

III

IV

Unknown

Histology

Adenocarcinoma

Squamous cell carcinoma

Large cell carcinoma

Not otherwise specified

GTV $\left(\mathrm{cm}^{3}\right)$

Range (median)

Mean \pm SD

Received dose (Gy)

Range (median)

Mean \pm SD

Radiotherapy schedule

30 x 1.5Gy (twice daily) + 10-12 x 2Gy (daily)

$24 \times 2.75 G y$ (daily)

$39-41 \times 1.8 \mathrm{~Gy}$ (daily)

Other

$$
\begin{aligned}
& 45.0-85.0(66.0) \\
& 66.5 \pm 10.0
\end{aligned}
$$

$52(59 \%)$

$38(42 \%)$

$3(3 \%)$

$6(7 \%)$

$61(68 \%)$

$13(17 \%)$

7 (8\%)

26 (29\%)

$34(38 \%)$

$3(3 \%)$

$27(30 \%)$

$0.7-285.1(34.9)$

$53.6 \pm 61.0$

$45.0-73.8(69.0)$

$66.3 \pm 5.5$

$59(66 \%)$

$14(16 \%)$

$8(9 \%)$

$9(10 \%)$

be expected by chance are interesting to select. We aimed to determine the $95 \%$ confidence interval of the 'smallest detectable change'. These boundaries could then be used in the longitudinal radiomics feature selection procedure by considering features as relevant when they have a detectable change during treatment for at least a minimum amount of patients.

We determined a threshold for the smallest detectable change (C), as proposed by Bland and Altman [24]:

$$
\mathrm{C}= \pm 1.96 * \mathrm{SD}
$$

where SD is the standard deviation of differences between feature values calculated on CBCT1a and CBCT1b. For 95\% of repeated measurements the difference is then expected to be less than $C$. For each feature, differences between CBCT1a and 
CBCT1 $\mathrm{b}$ were first plotted against their average. We then tested for constant variances by grouping the residuals into two groups based on the average feature value. Levene's test was used to test for equal variances of these groups. When we failed to reject the null hypothesis of equal variances (i.e. the SD of differences cannot be assumed to be equal for the entire range of feature values), a log-transformation was applied to the data. After back transforming from the log scale, $\mathrm{C}$ then represents a relative threshold (i.e. the absolute threshold then varies with feature value).

When all boundaries were calculated, for each of the features we could determine for how many patients there was a detectable change after a certain period of treatment. This could be used as a criterion to perform feature selection, e.g. to select delta radiomic features that exhibit a change for at least $10 \%$ of patients. This procedure of feature selection is summarized in a workflow diagram which can be found in Figure S3.1.

\section{Analyses}

The differences against averages plots, which were used to calculate $C$ for the feature selection procedure, were created for two features to be able to visualize the procedure described in 'Feature selection'. This was performed for 'Gray-level cooccurrence matrix - Average (GLCM - Average)', which is the average GLCM intensity value, and 'Gray-level distance-zone matrix - Intensity Non-uniformity (GLDZM $(N)$ ', which describes the distribution of the number of zones over the intensity values. The value of GLDZM - IN is low when the number of zones are distributed equally over all intensity levels. Different representations of the differences versus averages plot are shown for the absolute and relative delta radiomic features (but the results are the same).

To get some insight in the development of feature values over the course of treatment, we displayed the features' change over the course of treatment with respect to baseline for tumor volume, for a feature that does change for only a small amount of patients and a feature that changes for a large amount of patients.

The selection of features varies during treatment, meaning that we could explore which time-points are potentially relevant to be used to derive longitudinal radiomics information for early treatment adaptation. For instance as long as not a single feature changes, it is not helpful to derive longitudinal radiomics information for early response assessment. Therefore, we investigated the number of delta radiomic features that would have been selected at week 2 , week 3 , week 4 and week 5 of treatment when choosing a minimum of ' $x$ ' number of patients. Moreover, we investigated which features were selected at every timepoint in order to study the overlap between them.

\section{Correlation with volume}

To investigate whether selected delta radiomic features contain complementary information compared to change in tumor volume, we performed a correlation analysis in which we calculated Spearman's rank correlation coefficient for each feature with respect to volume. A rho value above 0.8 was considered as a significant correlation. The features calculated at all weeks of treatment were correlated to 
volume at that time-point. Moreover, correlation of the delta radiomic features with the change in volume was assessed for all selected features.

\section{Results}

In Figure 3.1, examples of the scatterplots for feature selection are shown. In this case, the p-value of Levene's test was significant for GLDZM - IN, indicating log transformation of the data was needed. For GLCM - Average, for ten patients the change at week 4 of treatment is larger than the smallest detectable change, whereas this is 15 for GLDZM - IN.
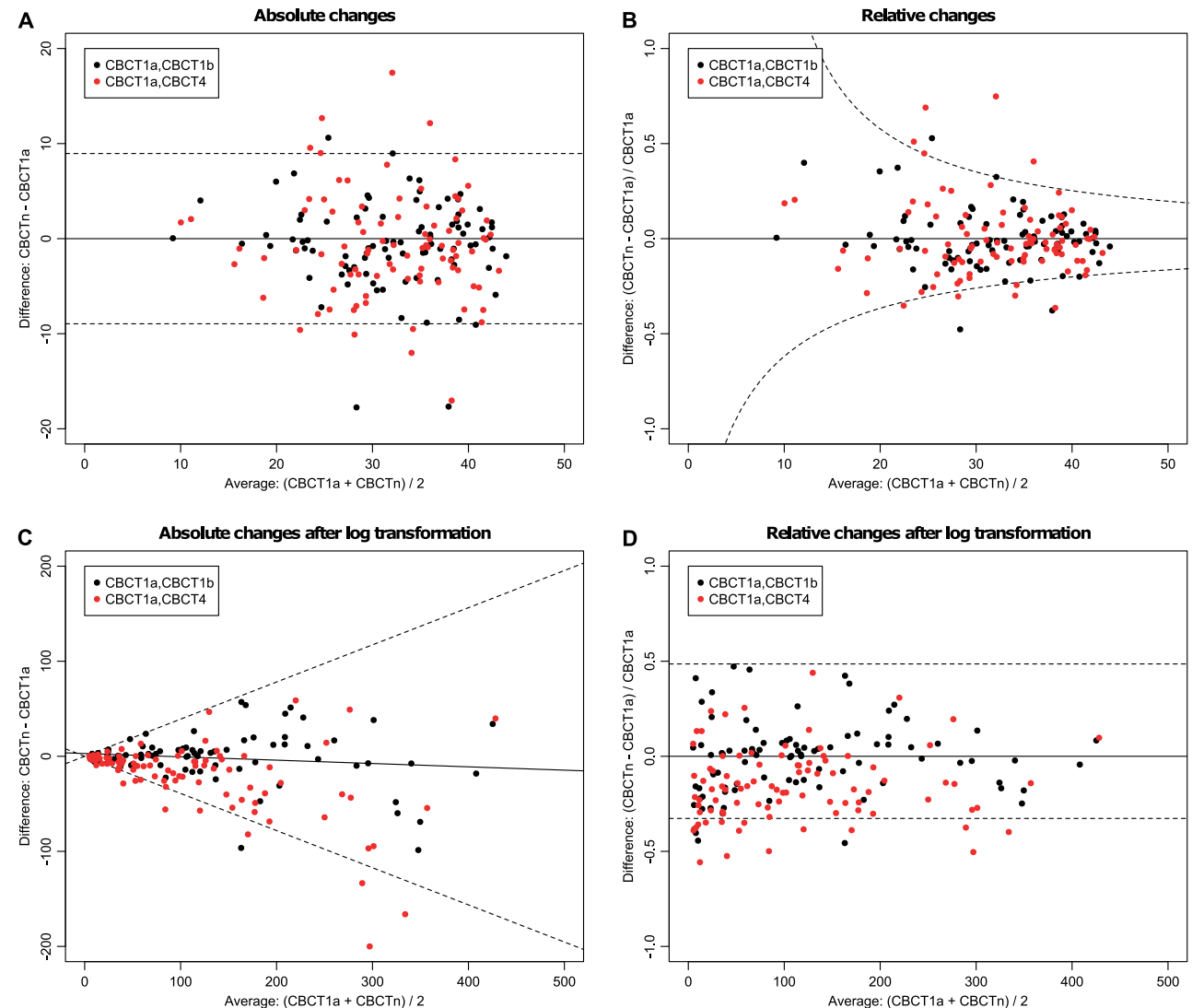

Figure 3.1. Example of scatterplots of differences versus averages for 'Gray-level cooccurrence matrix - Average (GLCM - Average)' (A and B) and 'Gray-level distance-zone matrix - Intensity Non-uniformity (GLDZM - IN)' (C and D) in which the $95 \%$ boundaries of smallest detectable change are indicated with a dashed line. Log transformation of the data was needed for the bottom feature. The left two plots indicate the absolute delta radiomic features and the right two plots the relative delta radiomic features.

In Figure 3.2, we have shown three examples of features changing over time with respect to the start of treatment. The absolute delta radiomic feature 'Gray-level Cooccurrence Matrix - Informational Measure of Correlation 1 (GLCM infoCorr1)', 
which is an information theoretic correlation, only changed for a few patients (seven at week 5 of treatment), shown in Figure 3.2B. The relative delta radiomic feature 'Gray-level Size Zone Matrix - High Intensity Large Area Emphasis (GLSZM - HILAE)', which emphasizes large zone sizes and high gray-levels thus having a high value for images with relatively large areas with high intensity, changes for a lot of patients (29 at week 5 of treatment), shown in Figure 3.2C.

A
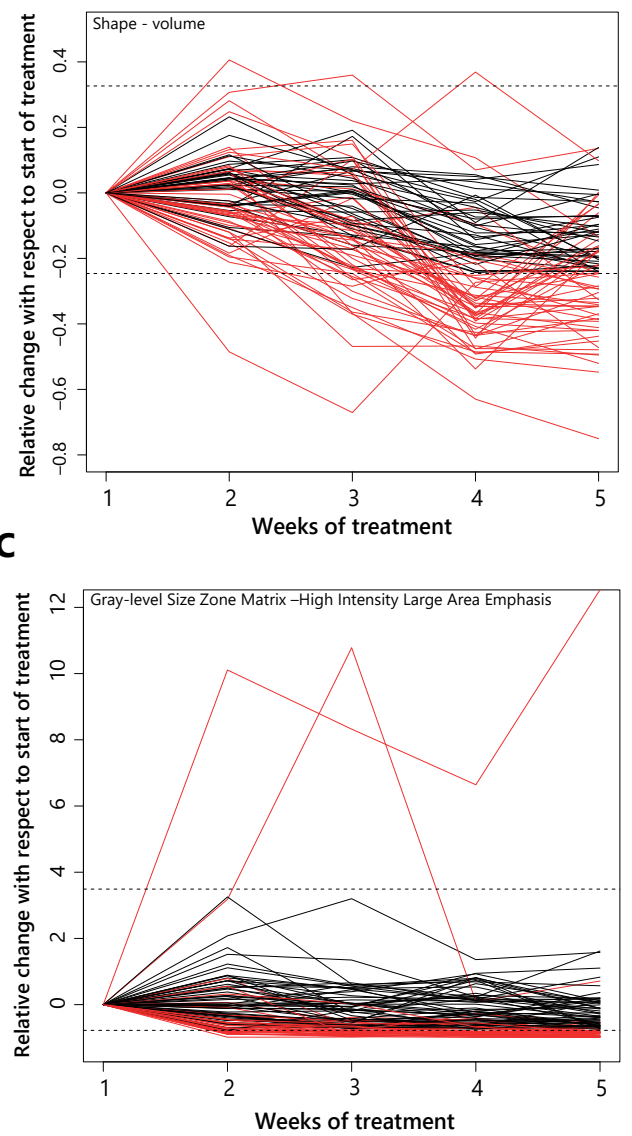

B

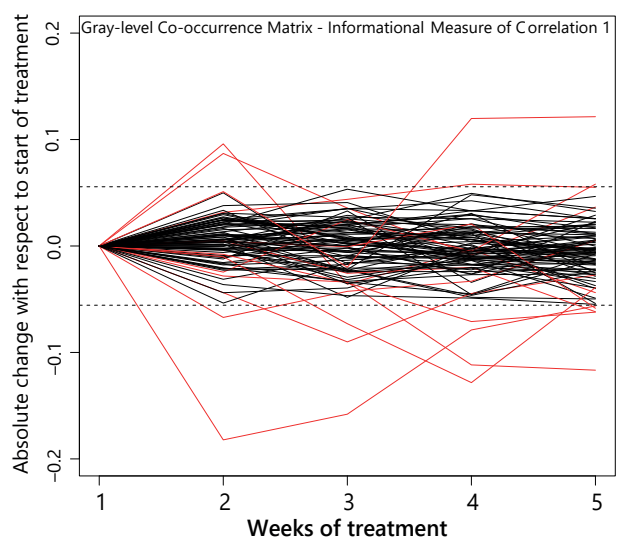

Figure 3.2. Example for the change of features over time for all patients the cohort. Dashed lines indicate the calculated thresholds of the smallest detectable change $(C)$ and each line represents a patient. For some patients, the line exceeds $C$ during treatment, meaning that there was a change larger than expected by chance.

Figure 3.3 shows a fast decrease in the number of features that are selected when increasing the criteria of number of patients. Moreover, the number of selected features increases during treatment. Note that only half of the total number of 232 delta radiomic features are shown, since the graphs are equal for absolute delta radiomic features and relative delta radiomic features. 


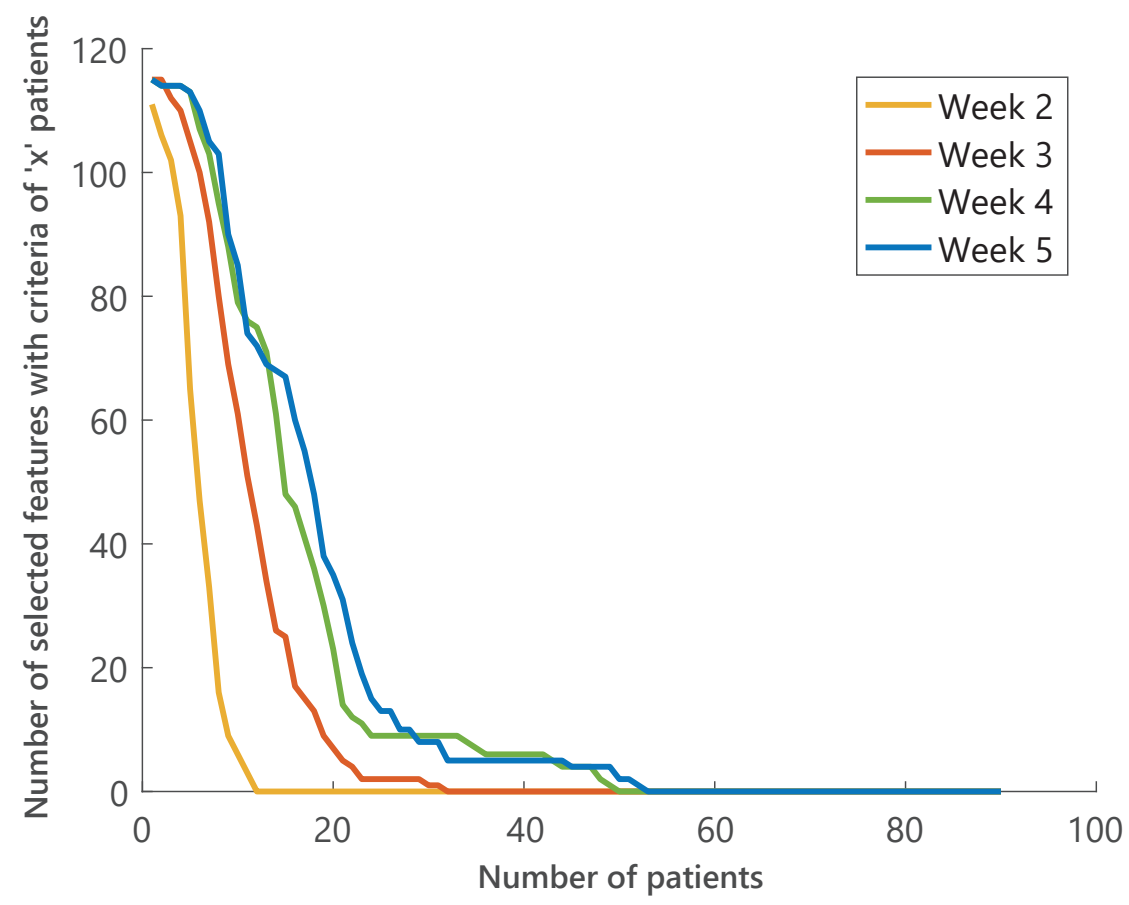

Figure 3.3. Number of features that change for an ' $x$ ' number of patients.

Based on the progress of the number of selected features shown in Figure 3.3, a (arbitrary chosen) number of ten patients was set as selection criteria. Using this setting, 85 out of 116 -absolute or relative- delta radiomic features were selected at week 5 of treatment, 79 at week 4, 61 at week 3 and six at week 2 .

Figure 3.4 shows the overlap between the selected delta radiomic features over treatment.

\section{Correlation with volume}

Twenty-five out of 116 features were correlated (rho > 0.8) to volume at baseline, 25 out of 116 at week 2, 29 at week 3, 26 at week 4 and 25 out of 116 (96\% overlap with week 1) at week 5 of treatment. For the delta radiomic features, two out of 116 were highly correlated to the change of volume at week 2 of treatment, three out of 116 at week 3 , eight out of 116 in week 4 and seven out of 116 in week 5 . This was $0 \%$, $5 \%, 10 \%$ and $8 \%$ of the selected delta radiomic features at week 2 , week 3 , week 4 and week 5 , respectively. The correlation between two delta radiomic features and the volume change is shown in Figure S3.2, as well as a scatterplot of Spearman's correlation coefficients of all delta radiomic features and volume change. 


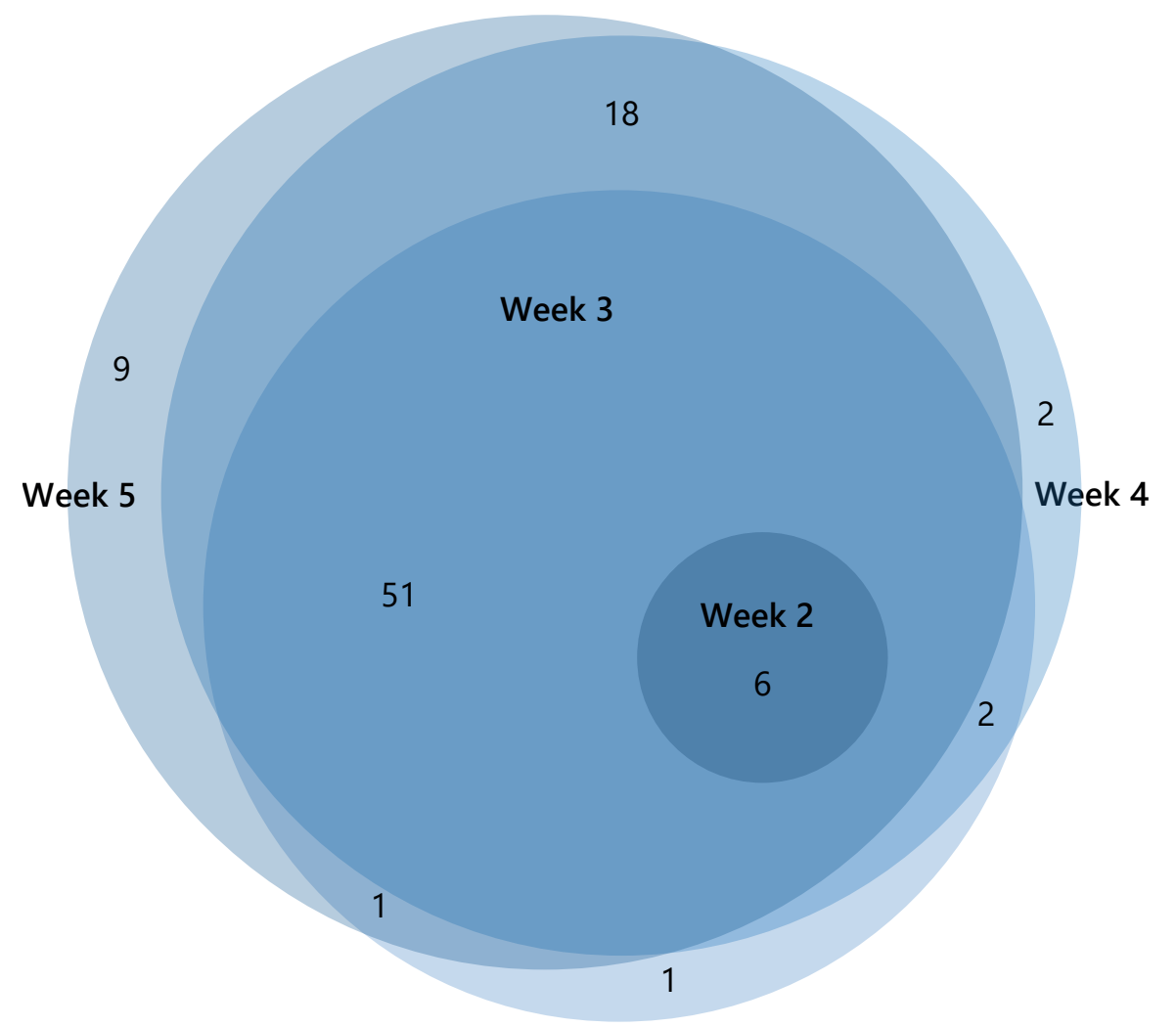

Figure 3.4. Overlap between delta radiomic features that change after 1 week, 2 weeks, 3 weeks and 4 weeks after the start of treatment.

\section{Discussion}

The first aim of this paper was to investigate and describe a feature selection methodology for the selection of both reproducible (single time-point robustness: test-retest) and informative (changing during time) features in a longitudinal radiomics approach. Combining baseline and longitudinal radiomics information could potentially contribute to a more informative and prognostic model than solely baseline information. However, it is important to perform a conceptually different feature selection procedure for baseline radiomic features and delta radiomic features. Whereas in baseline feature selection stable and robust features can be selected using a conventional threshold method based on an intra-class correlation coefficient, delta features should be reproducible but not stable over longer time periods of weeks into treatment and change during treatment for at least some patients to be informative. In this study, we proposed a workflow for the selection of delta radiomic features, which can be combined with baseline radiomic features in future model development. In this method, the criteria of the number of patients for which a feature should change is arbitrary, therefore allowing for a flexible feature 
selection procedure that can be dependent on the research question.

Besides the feature selection based on a change for a minimum amount of patients, the information from the Euler diagrams (Figure 3.4) that show the overlap in selected delta radiomic features after a certain period of treatment, can potentially be used as further feature selection: features that only change at a single time-point during treatment are most likely not very informative.

The second aim was to investigate which time-point is potentially useful to derive longitudinal radiomics information for early treatment response assessment, since personalized radiotherapy and decision making can be improved when we are able to predict the need for adaptive radiotherapy [25]. In the current analysis, a criterion of ten patients that needed changes was used for feature selection, showing that six delta radiomic features do only change at week 2 of treatment. This indicates that this time-point might be too early to detect enough change in tumor characteristics that can be used to decide for treatment adaptation. However, at week 3 of treatment, a large number of features show a change for at least ten patients, indicating that in the third week of treatment it would already be possible to extract useful radiomics information. The overlap in features show that all features that change at week 2 remain important during the rest of treatment. At week 3, 4 and 5, there is not much difference in which and how many features are selected, indicating that the majority of features already change at week 3 of treatment, which still allows for treatment adaptation if necessary.

A limitation of this study is the fact that much less radiomic features $(n=116)$ were calculated compared to the available number of features that were extracted in a previous study [12]. The reason for this was that no filtered features $(n=986)$ were calculated, as the margin around the tumor volume was often too small to be able to calculate any filtered radiomic features without needing to perform padding which required imputing values. Moreover, only the shape features 'Shape - volume' and 'Shape - surface' were calculated since the other shape features are not defined in case a GTV consists of multiple volumes. Because of the limited size of the dataset, we chose to not extract the filtered and shape features instead of excluding the patients with multiple volumes $(n=26)$ or a small margin $(n=10)$ in any of the six analyzed CBCT scans.

This study shows that delta radiomic features independent to volume change during treatment, showing that longitudinal radiomics contains complementary information. When longitudinal radiomics information is derived from CBCT images at week 3 or 4 of treatment and this information has prognostic value, it is possible to improve clinical decisions for early treatment adaptation. Nevertheless, the prognostic value of the features selected in the described method is currently unknown. To date, not many studies investigated the evaluation of metrics extracted from CBCT images for correlation with outcome, whereas this could provide important information for (early) treatment adaptations. However, a recent study investigated tumor regression acquired from $\mathrm{CBCT}$ images and related this to locoregional control and overall survival [26]. This study showed that CBCT imaging is feasible for detecting early changes and the authors were able to show that tumor regression was related to a worse prognosis. Moreover, another study used the early density changes of 
healthy lung tissue seen on $\mathrm{CBCT}$ images to predict the risk of developing normal tissue toxicity $[27,28]$. These studies show that $C B C T$ is a feasible image modality to detect early changes during treatment. Besides common metrics like tumor volume, more information can be extracted from medical images using radiomics, potentially able to more accurately monitor tumor changes over the course of treatment. Previously, radiomics has been shown to be able to predict outcome for NSCLC using $C T$ imaging $[6,19,29,30]$. Moreover, a recent study showed that similar prognostic information can be derived using $C B C T$ imaging [31]. These studies have led to the hypothesis that deriving quantitative features from $C B C T$ images acquired during treatment, using radiomics, could provide additional information to potentially improve personalized treatment adaptation. Therefore, the next step in the development of longitudinal radiomics is investigation of the prognostic value of the selected delta radiomic features extracted using $\mathrm{CBCT}$ imaging on clinical endpoints like overall survival or locoregional control. Moreover, the methodology for incorporating trends of feature values over time in prognostic models should be investigated.

To conclude, this study described a feature selection methodology for longitudinal CBCT radiomics for NSCLC patients that select reproducible delta radiomics features that are informative due to their change during the treatment. When these changes are linked to patient's outcome in the future, this can potentially contribute to (early) treatment adaptations.

\section{Acknowledgments}

Author PL acknowledges financial support from ERC advanced grant (ERC-ADG-2015, $\mathrm{n}^{\circ}$ 694812 - Hypoximmuno). This research is also supported by the Dutch Technology Foundation STW (grant $n^{\circ} 10696$ DuCAT \& $n^{\circ}$ P14-19 Radiomics STRaTegy), which is the applied science division of NWO, and the Technology Programme of the Ministry of Economic Affairs. Author $\mathrm{PL}$ also acknowledges financial support from the EU 7th framework program (ARTFORCE - $\mathrm{n}^{\circ}$ 257144, REQUITE - $n^{\circ}$ 601826), SME Phase 2 (EU proposal 673780 - RAIL), EUROSTARS (DART), the European Program H2020-2015-17 (BD2Decide - PHC30-689715 and ImmunoSABR - n ${ }^{\circ}$ 733008), Interreg V-A Euregio Meuse-Rhine ("Euradiomics"), Kankeronderzoekfonds Limburg from the Health Foundation Limburg and the Dutch Cancer Society. 


\section{References}

[1] Lambin P, Zindler J, Vanneste B, van de Voorde L, Jacobs M, Eekers D, et al. Modern clinical research: How rapid learning health care and cohort multiple randomised clinical trials complement traditional evidence based medicine. Acta Oncol. 2015;54:1289-300.

[2] Lambin P, Rios-Velazquez E, Leijenaar R, Carvalho S, van Stiphout RG, Granton P, et al. Radiomics: extracting more information from medical images using advanced feature analysis. Eur J Cancer. 2012;48:441-6.

[3] Kumar V, Gu Y, Basu S, Berglund A, Eschrich SA, Schabath MB, et al. Radiomics: the process and the challenges. Magn Reson Imaging. 2012;30:1234-48.

[4] Lambin P, van Stiphout RG, Starmans MH, Rios-Velazquez E, Nalbantov G, Aerts HJ, et al. Predicting outcomes in radiation oncology--multifactorial decision support systems. Nat Rev Clin Oncol. 2013;10:27-40.

[5] Lambin P, Roelofs E, Reymen B, Velazquez ER, Buijsen J, Zegers CM, et al. 'Rapid Learning health care in oncology' - an approach towards decision support systems enabling customised radiotherapy'. Radiother Oncol. 2013;109:159-64.

[6] Tang C, Liao Z, Hess K, Chance WW, Zhuang Y, Jensen G, et al. Prognosis and predictors of site of first metastasis after definitive radiation therapy for non-small cell lung cancer. Acta Oncol. 2016;55:1022-8.

[7] Aerts HJ. The Potential of Radiomic-Based Phenotyping in Precision Medicine: A Review. JAMA Oncol. 2016.

[8] Aerts HJ, Velazquez ER, Leijenaar RT, Parmar C, Grossmann P, Carvalho S, et al. Decoding tumour phenotype by noninvasive imaging using a quantitative radiomics approach. Nat Commun. 2014;5:4006.

[9] Leijenaar RT, Carvalho S, Hoebers FJ, Aerts HJ, van Elmpt WJ, Huang SH, et al. External validation of a prognostic CT-based radiomic signature in oropharyngeal squamous cell carcinoma. Acta Oncol. 2015;54:1423-9.

[10] Carvalho S, Leijenaar RT, Velazquez ER, Oberije C, Parmar C, van Elmpt W, et al. Prognostic value of metabolic metrics extracted from baseline positron emission tomography images in non-small cell lung cancer. Acta Oncol. 2013;52:1398-404.

[11] Parmar C, Leijenaar RT, Grossmann P, Rios Velazquez E, Bussink J, Rietveld D, et al. Radiomic feature clusters and prognostic signatures specific for Lung and Head \& Neck cancer. Sci Rep. 2015;5:11044.

[12] van Timmeren JE, Leijenaar RTH, van Elmpt W, Reymen B, Oberije C, Monshouwer R, et al. Survival prediction of non-small cell lung cancer patients using radiomics analyses of conebeam CT images. Radiotherapy and Oncology. 2017.

[13] Fave X, Mackin D, Yang J, Zhang J, Fried D, Balter $P$, et al. Can radiomics features be reproducibly measured from $\mathrm{CBCT}$ images for patients with non-small cell lung cancer? Med Phys. 2015;42:6784.

[14] Fave X, Zhang L, Yang J, Mackin D, Balter P, Gomez D, et al. Delta-radiomics features for the prediction of patient outcomes in non-small cell lung cancer. Sci Rep. 2017;7:588.

[15] Leijenaar RT, Carvalho S, Velazquez ER, van Elmpt WJ, Parmar C, Hoekstra OS, et al. Stability of FDG-PET Radiomics features: an integrated analysis of test-retest and inter-observer variability. Acta Oncol. 2013;52:1391-7.

[16] van Timmeren JE, Leijenaar RT, van Elmpt W, Wang J, Zhang Z, Dekker A, et al. Test-Retest Data for Radiomics Feature Stability Analysis: Generalizable or Study-Specific? Tomography. 2016;2.

[17] Balagurunathan Y, Gu Y, Wang H, Kumar V, Grove O, Hawkins S, et al. Reproducibility and Prognosis of Quantitative Features Extracted from CT Images. Transl Oncol. 2014;7:72-87.

[18] Balagurunathan Y, Kumar V, Gu Y, Kim J, Wang H, Liu Y, et al. Test-retest reproducibility analysis of lung CT image features. J Digit Imaging. 2014;27:805-23.

[19] Fried DV, Tucker SL, Zhou S, Liao Z, Mawlawi O, Ibbott G, et al. Prognostic value and reproducibility of pretreatment $\mathrm{CT}$ texture features in stage III non-small cell lung cancer. Int J Radiat Oncol Biol Phys. 2014;90:834-42.

[20] Zhao B, Tan Y, Tsai WY, Qi J, Xie C, Lu L, et al. Reproducibility of radiomics for deciphering 
tumor phenotype with imaging. Sci Rep. 2016;6:23428.

[21] Feldkamp LA, Davis LC, Kress JW. Practical cone-beam algorithm. J Opt Soc Am A. 1984;1:612-9.

[22] Shafiq-Ul-Hassan M, Zhang GG, Latifi K, Ullah G, Hunt DC, Balagurunathan Y, et al. Intrinsic dependencies of $\mathrm{CT}$ radiomic features on voxel size and number of gray levels. Med Phys. 2017;44:1050-62.

[23] Janssens G, Jacques L, Orban de Xivry J, Geets X, Macq B. Diffeomorphic registration of images with variable contrast enhancement. Int J Biomed Imaging. 2011;2011:891585.

[24] Bland JM, Altman DG. Statistical Methods for Assessing agreement between two methods of clinical measurement. Lancet. 1986;1:307-10.

[25] Berkovic P, Paelinck L, Lievens Y, Gulyban A, Goddeeris B, Derie C, et al. Adaptive radiotherapy for locally advanced non-small cell lung cancer, can we predict when and for whom? Acta Oncol. 2015;54:1438-44.

[26] Brink C, Bernchou U, Bertelsen A, Hansen O, Schytte T, Bentzen SM. Locoregional control of non-small cell lung cancer in relation to automated early assessment of tumor regression on cone beam computed tomography. Int J Radiat Oncol Biol Phys. 2014;89:916-23.

[27] Bertelsen A, Schytte T, Bentzen SM, Hansen O, Nielsen M, Brink C. Radiation dose response of normal lung assessed by Cone Beam CT - a potential tool for biologically adaptive radiation therapy. Radiother Oncol. 2011;100:351-5.

[28] Bernchou U, Hansen O, Schytte T, Bertelsen A, Hope A, Moseley D, et al. Prediction of lung density changes after radiotherapy by cone beam computed tomography response markers and pre-treatment factors for non-small cell lung cancer patients. Radiother Oncol. 2015;117:17-22.

[29] Ahn SY, Park CM, Park SJ, Kim HJ, Song C, Lee SM, et al. Prognostic value of computed tomography texture features in non-small cell lung cancers treated with definitive concomitant chemoradiotherapy. Invest Radiol. 2015;50:719-25.

[30] Aerts HJ, Grossmann P, Tan Y, Oxnard GG, Rizvi N, Schwartz LH, et al. Defining a Radiomic Response Phenotype: A Pilot Study using targeted therapy in NSCLC. Sci Rep. 2016;6:33860.

[31] van Timmeren JE, Leijenaar RTH, van Elmpt W, Reymen B, Oberije C, Monshouwer R, et al. Survival prediction of non-small cell lung cancer patients using radiomics analyses of conebeam CT images. Radiother Oncol. 2017;123:363-9. 


\section{Supplementary Material}

\section{Supplementary Material 3A - Workflow}

\section{STEP 1}

Plot of absolute differences against averages

STEP 2

Perform Levene's test and if $p<0.05$, perform log transformation of the data

STEP 3

Calculate 95\% confidence intervals for the 'smallest detectable change' for delta radiomic features

STEP 4

Select delta radiomic features that show a change for at least ' $x$ ' patients

Figure S3.1. Workflow of feature selection procedure. The need for a log-transformation is based on absolute differences using Levene's test. The smallest detectable change is represented by an absolute threshold for absolute differences and a relative threshold for relative differences. After log transformation, this is exactly the opposite. 
Supplementary Material 3B - Feature correlation with delta volume

A

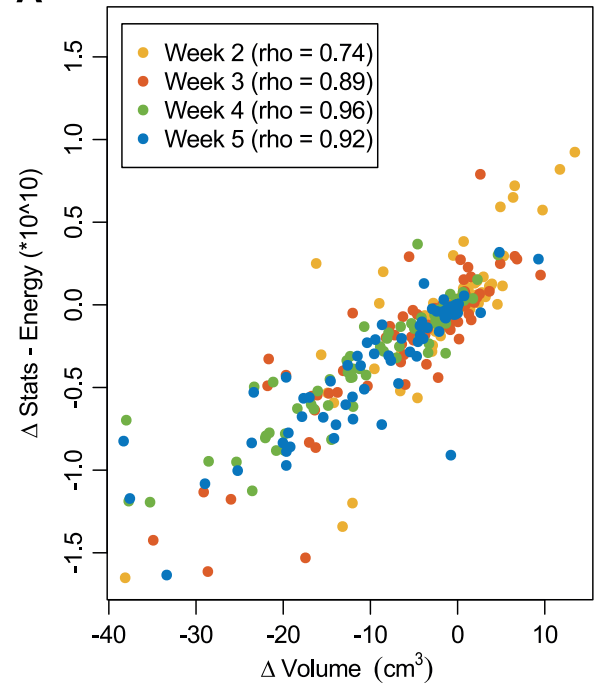

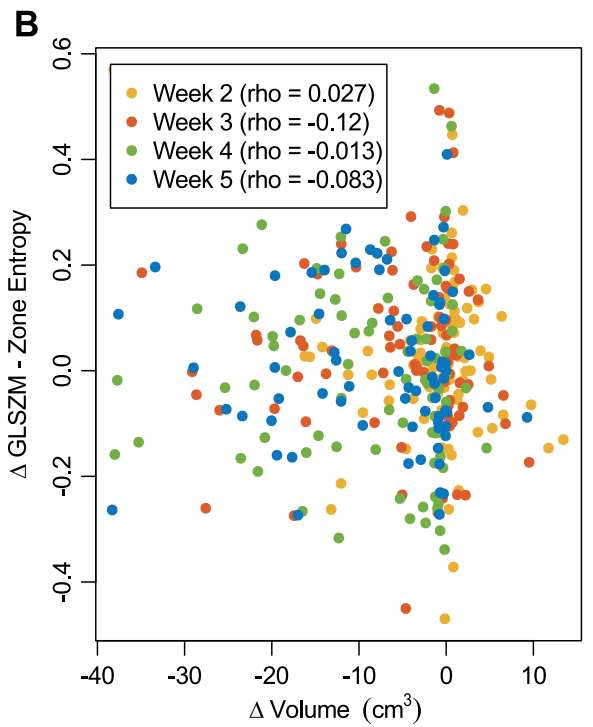

C

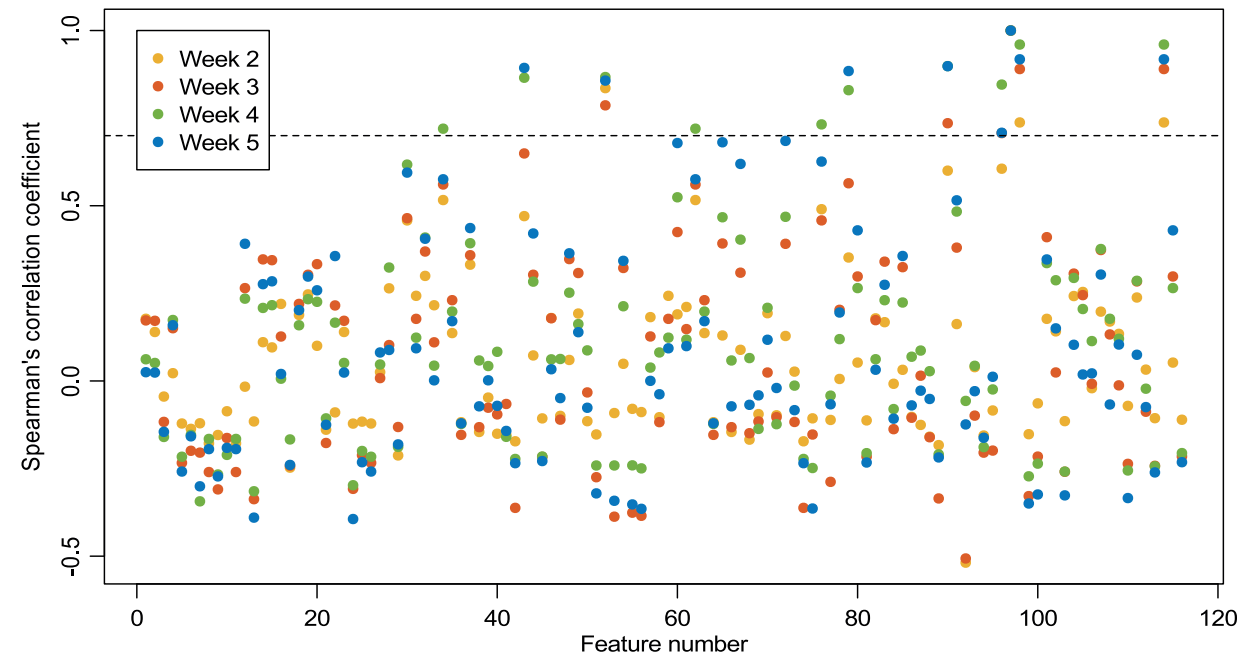

Figure S3.2. Spearman's correlation coefficient of delta radiomic features versus volume change. Correlation of delta 'Stats - Energy' (A) and 'GLSZM - Zone Entropy' (B) with delta volume (changes with respect to week1) and Spearman's correlation coefficient of all 116 absolute delta radiomic features with respect to volume (C). 




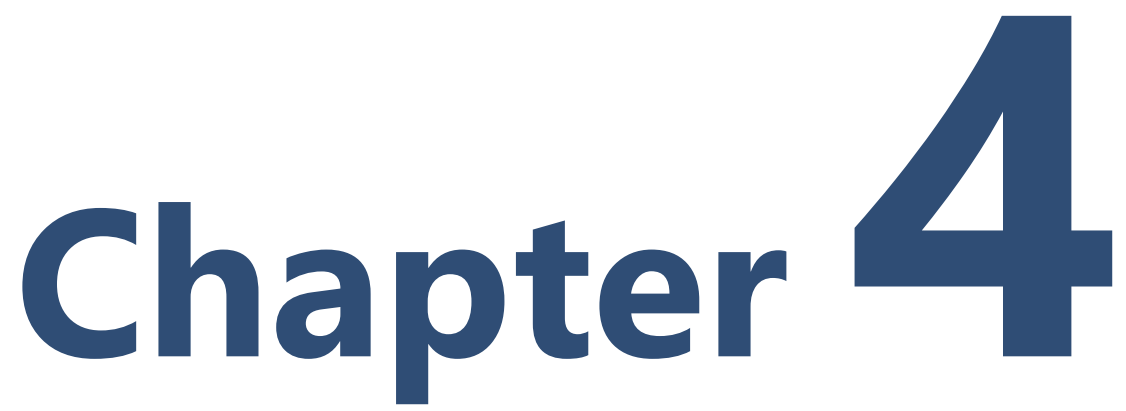

Longitudinal radiomics of cone-beam CT images from non-small cell lung cancer patients: evaluation of the added prognostic value for overall survival and locoregional recurrence

Authors

Janna E. van Timmeren, Wouter van Elmpt, Ralph T.H. Leijenaar, Bart Reymen, René Monshouwer, Johan Bussink, Leen Paelinck, Evelien Bogaert, Carlos De Wagter, Elamin Elhaseen, Yolande Lievens, Olfred Hansen, Carsten Brink,

Philippe Lambin

Adapted from Radiotherapy \& Oncology. 2019 July; 136:78-85. DOI: 10.1016/j.radonc.2019.03.032 


\begin{abstract}
Background and purpose

The prognostic value of radiomics for non-small cell lung cancer (NSCLC) patients has been investigated for images acquired prior to treatment, but no prognostic model has been developed that includes the change of radiomic features during treatment. Therefore, the aim of this study was to investigate the potential added prognostic value of a longitudinal radiomics approach using cone-beam computed tomography (CBCT) for NSCLC patients.
\end{abstract}

\title{
Materials and methods
}

This retrospective study includes a training dataset of 141 stage I-IV NSCLC patients and three external validation datasets of 94,61 and 41 patients, all treated with curative intended (chemo)radiotherapy. The change of radiomic features extracted from CBCT images was summarized as the slope of a linear regression. The CBCT slope-features and CT-extracted features were used as input for a Cox proportional hazards model. Moreover, prognostic performance of clinical parameters was investigated for overall survival and locoregional recurrence. Model performances were assessed using Kaplan-Meier curves and c-index.

\section{Results}

The radiomics model contained only CT-derived features and reached a c-index of 0.63 for overall survival and could be validated on the first validation dataset. No model for locoregional recurrence could be developed that validated on the validation datasets. The clinical parameters model could not be validated for either overall survival or locoregional recurrence.

\section{Conclusion}

In this study we could not confirm our hypothesis that longitudinal CBCT-extracted radiomic features contribute to improved prognostic information. Moreover, performance of baseline radiomic features or clinical parameters was poor, probably affected by heterogeneity within and between datasets. 


\section{Introduction}

Quantitative image analysis has been widely applied using treatment planning computed tomography (CT) images acquired at baseline prior to radiation treatment [1]. Investigation of the prognostic and predictive performance of large amounts of quantitative image features (i.e. radiomics) could eventually lead to the discovery of biomarkers that can contribute to the development of precision medicine [2-4]. The prognostic value of radiomic features extracted from $\mathrm{CT}$ images has already been shown for non-small cell lung cancer (NSCLC) [5-7]. Besides that, the potential added value of $\mathrm{CT}$ imaging during treatment has been explored for outcome prediction for both lung cancer and head-and-neck cancer $[8,9]$. These during-treatment images were acquired in a research setting and are generally not included in the clinical workflow. Nonetheless, a large number of cone-beam CT (CBCT) images are usually available, since these are acquired in clinical practice prior to radiation treatment fractions for positioning verification purposes. This means that additional potential prognostic information could be derived without extra patient burden. CBCT images have previously been proposed to monitor tumor response during treatment, either using radiomics [10-12] or simpler metrics like volume, density and mean Hounsfield units (HU) [13-16]. Early prognostic information could aid in clinical decision making regarding treatment adaption.

We hypothesize that the change of radiomic features might contain additional information over baseline radiomic information, since it would be possibly able to capture an early treatment response. A previous study showed that radiomic features extracted from cone-beam CT images acquired at the first radiotherapy fraction contains similar prognostic performance for overall survival as baseline CT measurements [10]. Moreover, a recent study showed that radiomic features acquired from $\mathrm{CBCT}$ images do change more than the day-to-day variability during the course of treatment [11]. Nevertheless, the prognostic value of these changes in feature values has not yet been explored. Therefore, in the current study we investigated the potential added prognostic value of a longitudinal radiomics approach using CBCT images, over the prognostic value of CT-extracted radiomic features, for non-small cell lung cancer (NSCLC) patients. 


\section{Methods}

\section{Patients}

Four patient cohorts from four different institutes were collected retrospectively, consisting of 141 (Dataset 1), 94 (Dataset 2), 61 (Dataset 3) and 41 patients (Dataset 4). Two clinical endpoints were considered in this study: overall survival and locoregional recurrence, both measured from the start of radiotherapy for all cohorts. Additionally, clinical parameters including age, gender, TNM-stage, overall stage and histology were collected. Detailed information about data collection, patients and exclusion criteria can be found in Supplementary Material 4A. The study was approved by Maastro Clinic's Institutional Review Board. Data collection was approved by each institutional ethics commitee.

\section{Imaging}

All patients in the cohort received a treatment planning $\mathrm{CT}(\mathrm{pCT})$ image and at least four $\mathrm{CBCT}$ images acquired during the course of radiotherapy. Moreover, the first available CBCT image was acquired prior to the first (FX1) or second (FX2) radiotherapy fraction. Patients were excluded from the study in case the field-of-view (FoV) of one or multiple CBCT image(s) did not comprise the entire primary tumor volume, which was the case for 19 patients in Dataset 1. Also if the required minimum number of 4 $\mathrm{CBCT}$ images was not available, patients were excluded. This was the case for 5 patients of Dataset 1 and 1 patient of Dataset 3. Technical details on the image acquisitions are presented in Supplementary Material $4 B$, as well as a histogram visualizing the timing of all 2154 analyzed CBCT images (Figure S4.1).

For all CBCT images, an intensity value correction was applied before feature extraction, using a region of interest $(\mathrm{ROI})$ in the heart and a reference value from literature of $50 \mathrm{HU}$ [17]. This is identical to the first step of the correction procedure described in more detail in [10].

\section{Tumor segmentation}

The treatment planning delineations of the primary gross tumor volume (GTV) on the $\mathrm{pCT}$ images were used to define corresponding tumor segmentations on all subsequent cone-beam CT images with the deformable registration algorithm 'Morphons' of the open source software REGGUI (http://openreggui.org). For Datasets 3 and 4, deformable registration in MIM (MIM Software Inc, Cleveland, $\mathrm{OH}$, USA) was used. The use of MIM reduced the workload by being able to register the images and adjust the contours in one-go. We experienced similar performance for the deformable registration of both software packages and believe this did not introduce a bias. Moreover, deformable registration was followed by manual adjustments to improve the delineations by removing spikes, loose pixels and irregularities or in case the registration procedure was not able to properly register the images. All tumor segmentations were checked and if needed manually adjusted by experienced radiation oncologists. In Dataset 1, for two patients the original GTV delineation contained two separate volumes and it was not possible to define which one was the primary tumor. Moreover, for 12 patients in Dataset 1, the tumor was 
indefinable on the CBCT images due to infiltration, atelectasis or image blurring. These 14 patients were therefore not included, resulting in the final cohort of 141 patients. For Dataset 4, the tumor was indefinable on the CBCT images for 4 patients.

\section{Feature extraction}

A total of 2317 radiomic features were extracted from all pCT and CBCT images included in the analysis, using in-house developed software programmed in Matlab 2014a (Mathworks, Natick, MA). Additional information about the features and image pre-processing can be found in Supplementary Material 4C.

\section{Cone-beam CT radiomics}

Features were extracted from weekly or bimonthly CBCT scans with at least four time points available for each patient throughout the entire treatment. Due to the limited number of data points, linear regression was performed to estimate the development of the radiomic features over time. Features values were first divided by the initial value to obtain relative numbers to also include baseline information. Then, a linear regression was performed and the slope parameter of the linear regression was then used as a new CBCT feature that captures the longitudinal information into one single feature. The coefficient of determination $\left(R^{2}\right)$ was evaluated to verify the goodness of fit.

\section{Feature selection}

Feature selection was performed on the training dataset: Dataset 1 . The process consisted of several steps: 1) Feature clean-up, 2) Selecting only changing CBCT features and calculate linear regression slope, 3) Combine CBCT-slope feature set with $\mathrm{PCT}$ feature set and 4) Remove correlated features OR calculate principal components. The steps are explained in further detail in Supplementary Material 4D and the resulting feature selection numbers are provided in the Results section.

\section{Model development and validation}

As agreed upon prior to the analysis, three models were developed using only Dataset 1 as training dataset. The first model was based on radiomics features itself (CBCT-slope and $\mathrm{pCT}$ ). The second model was based on the calculated principal components (PCs) from the radiomic features. To investigate the clinical relevance, the prognostic performance of radiomics was also compared to those of clinical parameters: the input of the third model included all available clinical parameters without radiomic features. Interaction terms were not addressed in this study.

Penalized Cox proportional hazard models were developed using a Least Absolute Shrinkage and Selection Operator (LASSO) procedure [18]. A 10-fold cross validation procedure was repeated 200 times to stabilize the method and to find the optimal lambda value with the smallest error. The performance of this Cox model was evaluated without retraining the coefficients. The prognostic index (PI), defined as $\Sigma_{i} \beta_{i} x_{i}$, was calculated for each patient. The distribution of the PIs on each dataset was evaluated to visualise as suggested by Royston and Altman [19]. Moreover, the model fit was evaluated by calculating estimating the regression coefficient 
('calibration slope') on the PI of the validation datasets, where a slope of 1 would indicate that the discrimination in the validation datasets is similar. Furthermore, the model misclassification was tested by offsetting the original PI and running a Cox regression on the covariates in the validation datasets. A significant $p$-value of the joint test on all regression coefficients would indicate a lack of fit of one or more variables.

The performance of Cox model was evaluated using the Kaplan-Meier curves, split between a low-risk and high-risk group using the median PI-value of all patients. A log-rank (LR) test was performed to evaluate the significance of the split. Moreover, Harrell's concordance index (c-index) was calculated to assess the discriminative power of the model, which is a measure that ranges from 0.5 (no discrimination) to 1.0 (perfect discrimination) [20].

The developed models on Dataset 1 were validated on Datasets 2, 3 and 4 or a combination of all validation datasets. The Kaplan-Meier curves were evaluated as well as the c-index.

All statistics and analyses were performed using R (version 3.4.3).

\section{Evaluation of the study design}

The study design was evaluated using two methods: the Radiomics Quality Score (available online on www.radiomics.world) and the TRIPOD classification [21, 22]. The TRIPOD checklist for "Prediction Model Development and Validation" was used to guide the study and the "TRIPOD Adherence data extraction checklist" was used to calculate a score $(0-100 \%)$ for the final study (both available online on www.tripodstatement.org).

\section{Results}

The patient characteristics of the final cohorts are shown in Table 4.1. Missing data in Dataset 1 was corrected using mean imputation: for 1 patient the WHO status was unknown, for 7 patients the smoking status was unknown and for 28 patients the $\mathrm{FEV}_{1}$ was unknown. A comparison of the overall survival and locoregional recurrence is shown in Figure 4.1.

A summary of the feature selection workflow is provided in Figure 4.2. Feature selection resulted in a final set of 283 radiomic features, consisting of 88 CBCT-slope features and 195 CT features. A total of 50 principal components described $95 \%$ of the variance in the data and were subsequently used as input for the modeling. The median of the mean $\mathrm{R}^{2}$ values calculated per feature by averaging over all patients, showing the goodness of fit of the linear regression for acquiring the CBCT-slope features, was $0.40 \pm 0.05$ (median $\pm \mathrm{SD}$ ).

\section{Model performance}

Three models were developed for both overall survival and locoregional recurrence, using different input variables: 1 ) radiomic features (models 1.1 and 1.2), 2) principal components (models 2.1 and 2.2) and 3) clinical parameters (models 3.1 and 3.2). Model 1.1 was developed to predict overall survival and resulted in a three-feature 
Table 4.1. Patient characteristics for all datasets. The italic, bold numbers indicate to which dataset the respective variable was significantly different $(p<0.05)$.

\begin{tabular}{|c|c|c|c|c|}
\hline & $\begin{array}{r}\text { Dataset } 1 \\
(\mathrm{n}=141)\end{array}$ & $\begin{array}{l}\text { Dataset } 2 \\
(\mathrm{n}=94)\end{array}$ & $\begin{array}{l}\text { Dataset } 3 \\
(\mathrm{n}=61)\end{array}$ & $\begin{array}{l}\text { Dataset } 4 \\
(\mathrm{n}=41)\end{array}$ \\
\hline Age & 3,4 & 3 & 1 & 1,2 \\
\hline Range (median) & $45-86(70)$ & $42-83(68)$ & $39-83(65)$ & $41-85(62)$ \\
\hline Mean \pm SD & $68.6 \pm 9.6$ & $67.0 \pm 8.5$ & $64.2 \pm 9.3$ & $62.3 \pm 10.7$ \\
\hline Gender & 4 & 4 & 1,2 & \\
\hline Male & $86(60.6 \%)$ & 45 (47.9\%) & $48(78.7 \%)$ & $26(63.4 \%)$ \\
\hline Female & 56 (39.4\%) & 49 (52.1\%) & 13 (21.3\%) & 15 (36.6\%) \\
\hline WHO performance status & 2,3 & 1,3 & 1,2 & \\
\hline 0 & $16(11.3 \%)$ & 27 (28.7\%) & 42 (68.8\%) & \\
\hline 1 & 96 (68.1\%) & 53 (56.4\%) & 15 (24.6\%) & \\
\hline 2 & 24 (17.0\%) & 14 (14.9\%) & $4(6.6 \%)$ & \\
\hline 3 & $4(2.8 \%)$ & $0(0 \%)$ & $0(0 \%)$ & \\
\hline \multicolumn{5}{|l|}{ Stage } \\
\hline 1 & $12(8.5 \%)$ & $4(4.3 \%)$ & $0(0 \%)$ & $2(4.9 \%)$ \\
\hline ॥ & 15 (10.6\%) & 7 (7.4\%) & $3(4.9 \%)$ & 4 (9.7\%) \\
\hline IIla & 44 (31.2\%) & $50(53.2 \%)$ & 37 (60.7\%) & 25 (61.0\%) \\
\hline $\mathrm{IIlb}$ & 55 (39.0\%) & 33 (35.1\%) & $21(34.4 \%)$ & 10 (24.4\%) \\
\hline IV & $15(10.6 \%)^{*}$ & $0(0 \%)$ & $0(0 \%)$ & $0(0 \%)$ \\
\hline Histology & + & & & \\
\hline Adenocarcinoma & 37 (26.2\%) & 34 (36.2\%) & $34(55.7 \%)$ & $14(34.1 \%)$ \\
\hline Squamous cell carcinoma & 60 (42.6\%) & 42 (44.7\%) & 22 (36.1\%) & 15 (36.6\%) \\
\hline Large cell carcinoma & $5(3.5 \%)$ & $5(5.3 \%)$ & $3(4.9 \%)$ & $3(7.3 \%)$ \\
\hline Undifferentiated & $0(0 \%)$ & $6(6.4 \%)$ & $0(0 \%)$ & $0(0 \%)$ \\
\hline Not otherwise specified & $39(27.7 \%)$ & $7(7.4 \%)$ & $2(3.3 \%)$ & $9(22.0 \%)$ \\
\hline \multicolumn{5}{|l|}{ GTV $\left(\mathrm{cm}^{3}\right)$} \\
\hline Range (median) & $0.61-341(38)$ & $2.1-397(38)$ & $1.5-425(36)$ & $1.7-415(55)$ \\
\hline Mean \pm SD & $62.3 \pm 70.6$ & $70.2 \pm 74.6$ & $72.3 \pm 93.0$ & $90.1 \pm 72.3$ \\
\hline \multicolumn{5}{|l|}{ Concurrent chemotherapy } \\
\hline Yes & 90 (63.8\%) & $63(67.0 \%)$ & 35 (57.4\%) & \\
\hline No & 51 (36.2\%) & 31 (33.0\%) & 26 (42.6\%) & \\
\hline Interval CT - RT (Days) & $2,3,4$ & 1,3 & 1,2 & 1 \\
\hline Range (median) & $3-16(7)$ & $5-21(11)$ & $6-39(13)$ & $6-21(13)$ \\
\hline Mean \pm SD & $7.2 \pm 1.6$ & $10.9 \pm 2.4$ & $15.6 \pm 7.7$ & $12.7 \pm 3.9$ \\
\hline Received RT dose (Gy) & $2,3,4$ & $1,3,4$ & $1,2,4$ & $1,2,3$ \\
\hline Range (median) & $45-76(69)$ & $60-66(66)$ & $60-70(70)$ & $52-67.5(66)$ \\
\hline Mean \pm SD & $66.4 \pm 5.6$ & $64.3 \pm 2.7$ & $68.5 \pm 3.1$ & $65.2 \pm 3.2$ \\
\hline Radiotherapy schedule & $2,3,4$ & 1 & 1 & 1 \\
\hline $30-35 * 2$ Gy (daily) & $0(0 \%)$ & $94(100 \%)$ & $61(100 \%)$ & 41 (100\%) \\
\hline $\begin{array}{l}30 \times 1.5 \mathrm{~Gy} \text { (twice daily) }+ \\
5-12 \times 2 \mathrm{~Gy} \text { (daily) }\end{array}$ & 78 (54.9\%) & $0(0 \%)$ & $0(0 \%)$ & $0(0 \%)$ \\
\hline $23-24$ x 2.75 Gy (daily) & 28 (19.7\%) & $0(0 \%)$ & $0(0 \%)$ & $0(0 \%)$ \\
\hline $38-42$ x 1.8Gy (daily) & 26 (18.3\%) & $0(0 \%)$ & $0(0 \%)$ & $0(0 \%)$ \\
\hline Other & $10(7.0 \%)$ & $0(0 \%)$ & $0(0 \%)$ & $0(0 \%)$ \\
\hline \multicolumn{5}{|l|}{ Overall survival (year) } \\
\hline Median [range] & $2.0[0.1-4.8]$ & $1.7[0.1-9.8]$ & $2.8[0.4-7.7]$ & $1.8[0.4-6.5]$ \\
\hline \multicolumn{5}{|l|}{ Events at time of analysis } \\
\hline Survival & 91 & 84 & 32 & 31 \\
\hline Follow-up (year) & 2,3 & $1,3,4$ & 2,4 & $1,2,3$ \\
\hline Median [range] & $3.0[0.2-4.8]$ & $8.6[6.6-9.8]$ & $3.4[1.1-7.7]$ & $5.7[5.3-6.5]$ \\
\hline
\end{tabular}


A

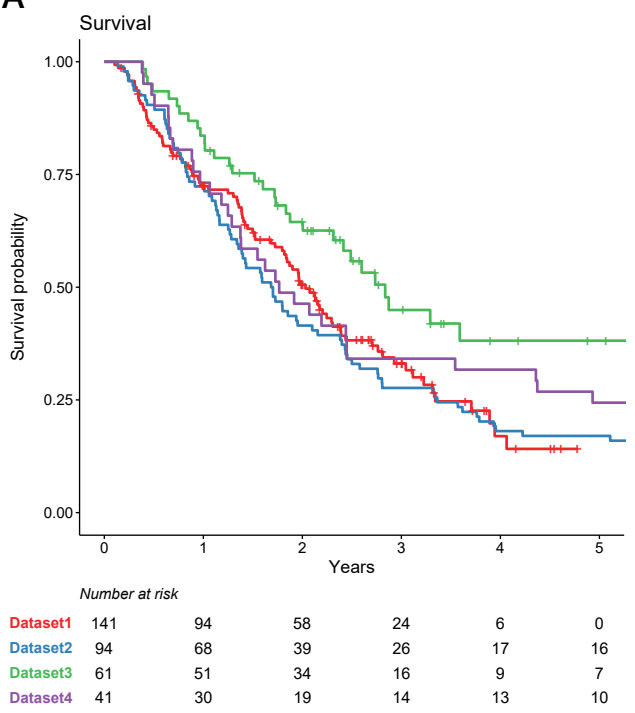

B

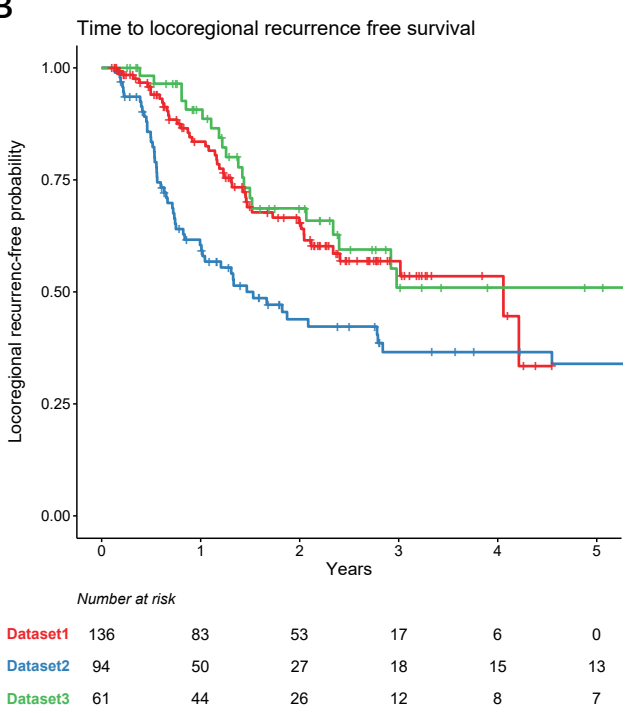

Figure 4.1. Kaplan-Meier curves showing the overall survival (A) and locoregional recurrence (B) of all datasets.

model containing only CT features. Model 1.2 for locoregional recurrence resulted in a six-feature model, consisting of three CBCT-slope features and three CT features. Models 2.1 and 2.2 could not be build, as no prognostic features could be identified for either overall survival or locoregional recurrence using the principal components as input. Model 3.1 included six clinical features to predict overall survival. Model 3.2 is a three-feature clinical model to predict locoregional recurrence.

All models with the feature's respective $\beta$-coefficients, plus the median and range of the feature values in Dataset 1 (which was used for training), are summarized in Table 4.2. Explanations of all features in these models can be found in Supplementary Material 4E. The distribution of the PIs, the calibration slope of the PI and the joint test on all regression coefficients are shown in Supplementary Material 4F. The C-indices of all identified prognostic models are shown in Figure 4.3. The clinical model contained parameters which were not available for Datasets 3 and 4 (WHO performance status), so this model was only validated in Dataset 2. The Kaplan-Meier curves for the radiomics model predicting overall survival (model 1.1) are shown in Figure 4.4. The Kaplan-Meier curves for model 1.2, for predicting locoregional recurrence, are shown in Figure S4.3.

\section{Evaluation of the study design}

The study design was evaluated using the Radiomics Quality Score (RQS) and the TRIPOD statement. We achieved an RQS of $53.8 \%$ as feature reduction was applied, three validation datasets were available and a clinical model was investigated. On the other hand, there was no phantom study involved, the correlation with underlying biology was not investigated, the data were not available from a prospective study registered in a trial database and no cost-effective analysis was performed. On the 


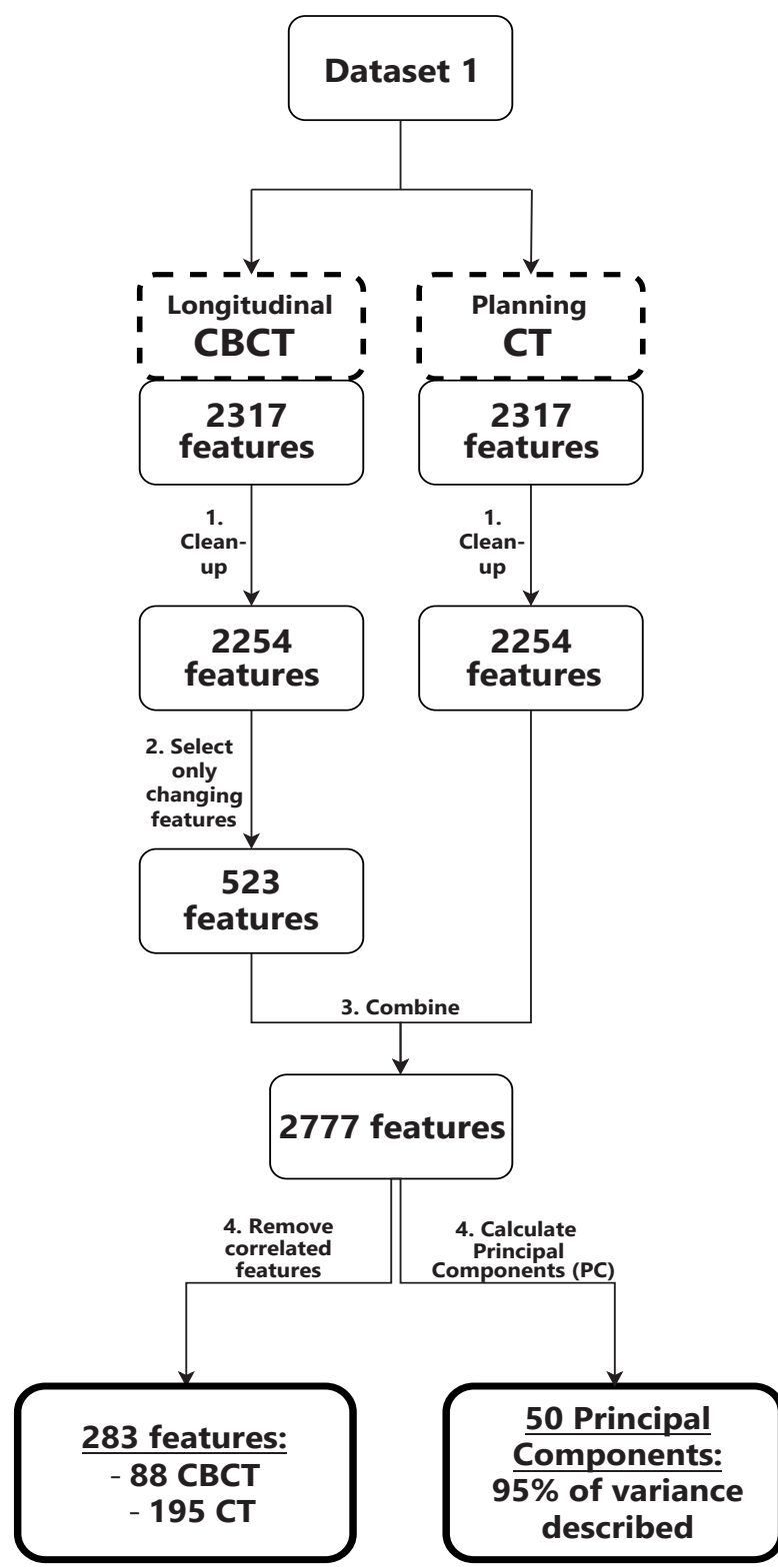

Figure 4.2. Schematic overview of the feature selection process applied to Dataset 1 . The initial 2317 features were initially reduced to 2254 features by a clean-up (see text) and the CBCT features were successively reduced based on time variance (see text). Finally the combined set of features (2777) are modelled either after removable based on correlations or by use of PCA in order to investigate model stabilities. 


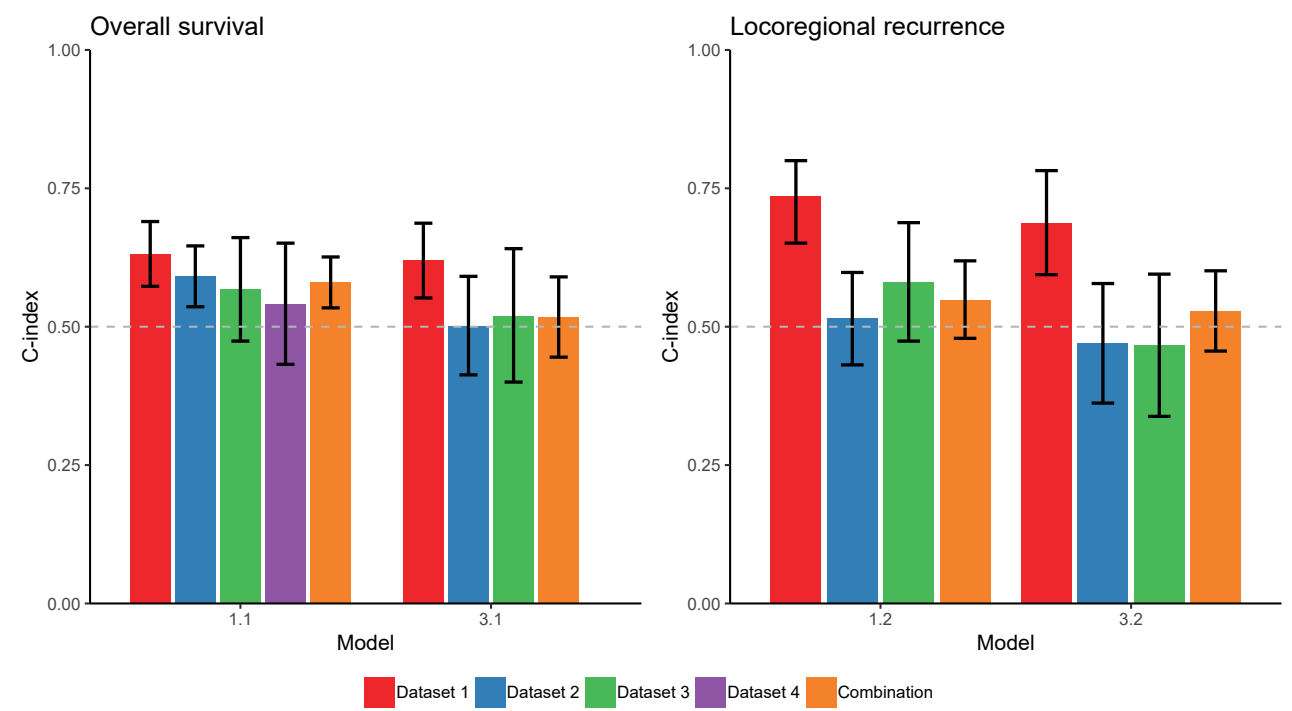

Figure 4.3. C-indices of the prognostic models identified for overall survival (left) and locoregional recurrence (right). The combination was either datasets 2, 3 and 4 (model 1.1) or datasets 2 and 3 (models 1.2, 3.1 and 3.3). Models 1.1 and 1.2 contain only radiomic features and models 3.1 and 3.2 contain only clinical parameters. For models 2.1 and 2.2 no prognostic features could not be identified.

other hand, feature reduction was performed and three validation datasets were available to validate the model without retraining and without adaption of the cutoff value. Moreover, the radiomics model performance was compared to that of clinical parameters, including TNM-stage and volume, which are commonly seen as the gold standard. The TRIPOD checklist for Prediction Model Development and Validation Scoring [20, 21], using the TRIPOD items for 'Development and external validation (of the same model)', reached a score of $81 \%$. The latter can be found in Supplementary Material $4 \mathrm{H}$.

\section{Discussion}

The aim of this study was to investigate the complementary prognostic value of CBCT radiomic features for overall survival and locoregional recurrence of NSCLC patients. We hypothesized that the change of radiomic features contains additional information over baseline radiomic information. To this extent, models were developed using CBCT-slope and planning CT-derived radiomic features. Model performances were compared to that of a model including solely clinical parameters.

For the first model, selected $C B C T$-slope and $\mathrm{pCT}$ features were used as input for LASSO. A prognostic model was identified for overall survival, which contains three $\mathrm{CT}$ based radiomic features. The performance of the model was average (c-index $0.63[95 \% \mathrm{Cl} 0.57-0.69]$ ), but it could be validated on Dataset 2 (c-index 0.59 [95\% $\mathrm{Cl} 0.54-0.65]$ ) and also on the combined set of Datasets 2, 3 and 4. A model developed by Fave et al. [9] containing clinical parameters, baseline CT-radiomic 
Table 4.2. Model features with corresponding model coefficients and indication the median and range of the feature values in training Dataset 1. Model 1.1 and model 3.1 were developed to predict overall survival and model 1.2 and model 3.2 were developed to predict locoregional recurrence. For models 2.1 and 2.2, no prognostic features were identified. Feature definitions are presented in Supplementary Material.

\section{Model $\quad \beta$-coefficients Feature names $\quad$ Median feature value [range]}

\begin{tabular}{clll}
\hline Models 1.1 and 1.2: radiomics features for overall survival and locoregional recurrence, respectively \\
Model 1.1 & $-4.25 \cdot 10^{-3}$ & CT_LoG_sigma_2_5_mm_3D_pos_IH_mode & $1[1-20]$ \\
& $9.66 \cdot 10^{-4}$ & CT_Loclnt_peakLocal & $882.4[414.5-1442.7]$ \\
& $1.96 \cdot 10^{-4}$ & CT_Wavelet_LLL_GLCM_inverseVar & $0.30[0.13-0.47]$ \\
Model 1.2 & $3.12 \cdot 10^{-3}$ & CT_LoG_sigma_2_5_mm_3D_pos_IH_maxGradl & $12[1-29]$ \\
& 0.0255 & CT_LoG_sigma_5_5_mm_3D_IH_p10 & $11[2.8-22]$ \\
-0.665 & CT_Wavelet_HHH_Stats_median & $0.011[-1.28-0.84]$ \\
-11.0 & CBCT-slope_Wavelet_HHL_GLCM_maxCorr & $0.0033[-0.0088-0.021]$ \\
-2.42 & CBCT-slope_Wavelet_HLH_GLCM_infoCorr2 & $0.0035[-0.0070-0.022]$ \\
3.46 & CBCT-slope_Wavelet_HLL_GLCM_inverseVar & $-0.0022[-0.020-0.016]$
\end{tabular}

Models 2.1 and 2.2: principal components for overall survival and locoregional recurrence, respectively No models could be identified

Models 3.1 and 3.2: clinical variables for overall survival and locoregional recurrence, respectively Model $3.10 .424 \quad$ WHO 2 Dummy variables

$0.0567 \quad$ Concomitant

$0.453 \quad$ Stage II

$0.165 \quad$ Stage IIla

$-0.121 \quad$ Histology (other)

$0.178 \quad$ Histology (squamous cell)

Model 3.20 .122 WHO 1

Dummy variables

0.223

Stage IIla

$0.558 \quad$ Histology (squamous cell)

features and delta-CT radiomic features found a c-index of 0.675 , but no external validation was present and weekly CT imaging acquired in a research setting was used for this study. For locoregional recurrence, six radiomic features were selected including three CBCT-slope features and three CT features. This model did not reach significance in any of the validation datasets. Also in the study of Fave et al. [9], no delta CT features were significant predictors for locoregional recurrence.

For the second model, principal components were used as input for LASSO. For both overall survival and locoregional recurrence, no prognostic models could be identified.

The third and last models, containing clinical parameters, achieved a c-index of 0.62 for overall survival and 0.69 for locoregional recurrence on Dataset 1 . Both models did not validate on either Dataset 2, Dataset 3 or a combination of both.

Previous studies have shown that a four-feature radiomics model derived from CT images has prognostic value for NSCLC patients. Also, a previous study showed that this model could also be validated using $\mathrm{CBCT}$ images acquired prior to the first radiotherapy fraction [10]. Therefore, we also investigated the performance of a previously developed radiomic signature [5] during treatment. The c-index was 0.59 for all datasets combined, which was not significantly different compared to the validation performance in other studies $[5,10,23]$. The performance of the signature 

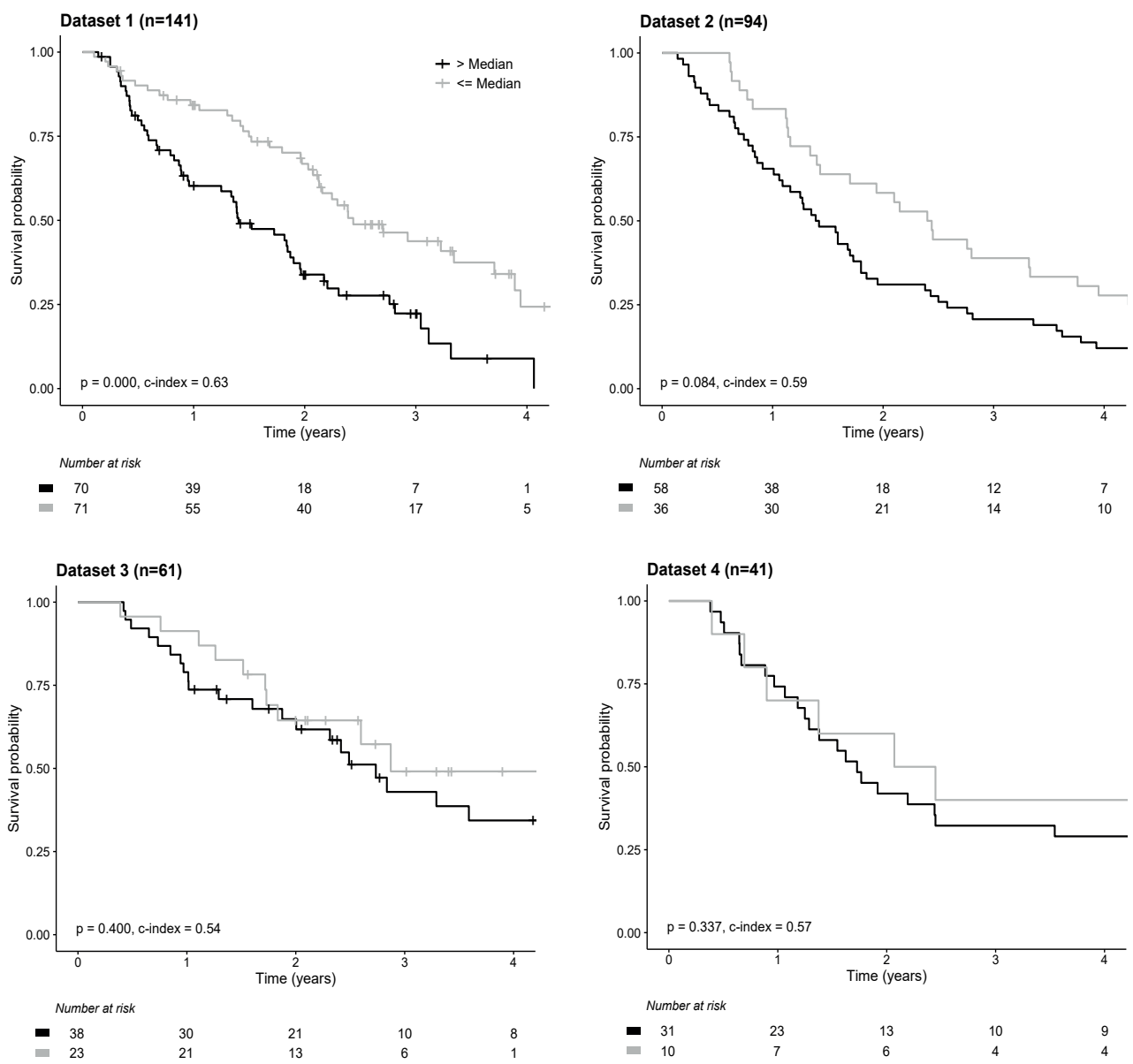

Figure 4.4. Kaplan-Meier curves of model 1.1 containing three $\mathrm{CT}$ features to predict overall survival. (A) Training: Dataset 1, (B) validation 1: Dataset 2, (C) validation 2: Dataset 3, (D) validation 3: Dataset 4. For Datasets 2 and 4, there were no censored data up to 4 years of follow-up.

on CBCT images acquired during radiation treatment shows that the signature is robust over time, but the prognostic value does not improve at later time points (data not shown).

Complementary prognostic value of longitudinal $C B C T$ radiomic features could not be shown in this study. No CBCT-slope features were selected by LASSO to predict overall survival. For locoregional recurrence, CBCT-slope features were selected, but the model did not validate on any of the validation datasets. The lack of prognostic value of CBCT-slope features can be caused by several factors. First of all, the slope surrogate might not be the best method to use for representing CBCT features changing over time. Only a few data points per patient were included to estimate the slope, since the GTV segmentations on the CBCT were time-consuming as they needed manual verification and adjustment. Due to this limited number of 
data points available, the slope is sensitive to outliers. Potentially, automatic segmentations, for instance atlas-based methods or deep learning, could improve this methodology $[24,25]$. Besides the uncertainty in the slope parameters due to the limited number of data points, it is possible that the development of CBCT extracted radiomic features over time is not linear. A more advanced model to represent the behavior of radiomic features over time might be more appropriate (e.g. a quadratic model or polynomial). However, the limited number of available data points in combination with a model with more degrees of freedom would have resulted in overfitting and most likely a false-positive result. Furthermore, it is also possible that the slope features contain no prognostic information, whereas the absolute difference between the feature values of two $C B C T$ scans does have prognostic value. In a recent study it was shown that a large number of features change more than expected by chance in week 3 and week 4 of treatment [11], but this was not yet related to outcome information. The lack of prognostic value of current CBCT features may also be influenced by the low image quality of cone-beam CT. Although a previous study showed that a batch of radiomic features are interchangeable between planning CT and cone-beam $\mathrm{CT}$, other features might be subject by high levels of noise. Several efforts are being made for improving the image quality of CBCT images, e.g. using artefact reduction techniques, hardware developments, improvement of 4D imaging or the implementation of iterative reconstruction methods [26-28]. In this study three different CBCT systems were involved: Varian Truebeam (Dataset 1), Elekta Synergy (Datasets 2 and 4) and Varian Clinac (Dataset 3). The image quality of these three systems differs substantially, which could have affected the lack of validation performance for the model containing CBCT slope features. Furthermore, the CBCT images analyzed in the current study are free-breathing, which for lung tumors causes a blurring of the region of interest. Considering all these factors, it is likely that (some of) these factors hamper the discovery of prognostic value in CBCT-extracted radiomic features. Once the image quality of CBCT is improved and automatic segmentation methods are developed, it would be interesting to redo the analysis.

A factor that could have influenced the validation performance of model 1.2 (containing CBCT-slope features to predict locoregional recurrence) is the software package used to perform the deformable registration to transfer the GTV contour of the $\mathrm{PCT}$ onto the CBCT images: a different software package was used in Datasets 1 and 2 than in Datasets 3 and 4. Although we have not experienced a difference between the two methods and the radiation oncologist manually verified all contours, this could potentially have resulted in a systematic bias in the data. Nevertheless, this model also did not validate on Dataset 2, for which the same software package was used as in training Dataset 1 . Moreover, this does not explain the lack of validation performance of the models containing only pCT-features or clinical parameters.

In general, models based on (pre)treatment imaging or parameters do most likely not contain enough information to more accurately predict a future event like death. Treatment changes, and in particular second-line or third-line therapies, have an enormous effect on overall survival. Another explanation for the poor validation performance could be the intra- and inter-dataset heterogeneity and the limited sample size of the validation datasets, which are common phenomena of retrospective 
(multicentric) studies. Comparing patient characteristics and scanner parameters show that the datasets are not much alike, which makes it difficult for a model to perform well on the external dataset. Locoregional recurrence free survival in Dataset 2 is worse compared to the other datasets, which might be caused by an earlier detection of tumor recurrence because of the use of PET in the follow-up program in this institution [29]. Overall survival is better in Dataset 3, in which a larger number of patients have WHO performance status 0 compared to the other datasets. Moreover, radiotherapy schedule, age and imaging protocols differed between datasets. Therefore, we recommend to perform future studies on larger, more homogeneous datasets.

Several validation measures were evaluated to investigate the potential cause for the fact that the developed models could not be validated on the validation datasets. The distribution of the prognostic indices for the radiomics model which was developed for overall survival is comparable for all datasets. Moreover, the calibration slope of the PI was not significantly different from 1 for any of the validation datasets. Furthermore, the joint test on all coefficients was not significant, showing that there is no evidence for a lack of fit. These results show that model covariates and coefficients are comparable in the validation datasets, which indicates that it is most likely that the lack of validation is a result of the small cohort sizes. We recommend to perform future studies on larger, more homogeneous datasets.

In this study we could not confirm our initial hypothesis that longitudinal radiomic features extracted from $C B C T$ images contribute to improved prognostic information. Nevertheless, the model developed for overall survival containing only $\mathrm{CT}$ features, could be validated in one external dataset and also on a combination of all three external validation datasets. On the other side, validation of the models containing only clinical parameters showed poor performance, which indicates that there is still a lack of reproducible prognostic models for outcome prediction in NSCLC.

\footnotetext{
Acknowledgments

Philippe Lambin acknowledges financial support from ERC advanced grant (ERC-ADG-2015, $n^{\circ}$ 694812 - Hypoximmuno), ERC-2018-PoC ( $\left.n^{\circ} 81320-\mathrm{CL}-\mathrm{IO}\right)$. This research is also supported by the Dutch technology Foundation STW (grant $n^{\circ} 10696$ DuCAT \& $n^{\circ}$ P14-19 Radiomics STRaTegy), which is the applied science division of NWO, and the Technology Programme of the Ministry of Economic Affairs. Authors also acknowledge financial support from SME Phase 2 (RAIL - n $\left.{ }^{\circ} 673780\right)$, EUROSTARS (DART, DECIDE, COMPACT), the European Program H20202015-17 (BD2Decide - PHC30-689715, ImmunoSABR - n 733008, PREDICT - ITN - n 766276), TRANSCAN Joint Transnational Call 2016 (JTC2016 "CLEARLY"- $\mathrm{n}^{\circ}$ UM 2017-8295), Interreg V-A Euregio Meuse-Rhine ("Euradiomics"), Kankeronderzoekfonds Limburg from the Health Foundation Limburg and the Dutch Cancer Society.

Olfred Hansen and Carsten Brink acknowledge support from AgeCare (Academy of Geriatric Research at Odense University Hospital).
} 


\section{References}

[1] Lambin P, Rios-Velazquez E, Leijenaar R, Carvalho S, van Stiphout RG, Granton P, et al. Radiomics: extracting more information from medical images using advanced feature analysis. Eur J Cancer. 2012;48:441-6.

[2] O'Connor JP, Aboagye EO, Adams JE, Aerts HJ, Barrington SF, Beer AJ, et al. Imaging biomarker roadmap for cancer studies. Nat Rev Clin Oncol. 2017;14:169-86.

[3] Lambin P, Leijenaar RTH, Deist TM, Peerlings J, de Jong EEC, van Timmeren J, et al. Radiomics: the bridge between medical imaging and personalized medicine. Nat Rev Clin Oncol. 2017; $14: 749-62$.

[4] Avanzo M, Stancanello J, El Naqa I. Beyond imaging: The promise of radiomics. Phys Med. 2017;38:122-39.

[5] Aerts HJ, Velazquez ER, Leijenaar RT, Parmar C, Grossmann P, Carvalho S, et al. Decoding tumour phenotype by noninvasive imaging using a quantitative radiomics approach. Nat Commun. 2014;5:4006.

[6] Ahn SY, Park CM, Park SJ, Kim HJ, Song C, Lee SM, et al. Prognostic value of computed tomography texture features in non-small cell lung cancers treated with definitive concomitant chemoradiotherapy. Invest Radiol. 2015;50:719-25.

[7] Coroller TP, Grossmann P, Hou Y, Rios Velazquez E, Leijenaar RT, Hermann G, et al. CTbased radiomic signature predicts distant metastasis in lung adenocarcinoma. Radiother Oncol. 2015;114:345-50.

[8] Leger S, Zwanenburg A, Pilz K, Zschaeck S, Zophel K, Kotzerke J, et al. CT imaging during treatment improves radiomic models for patients with locally advanced head and neck cancer. Radiother Oncol. 2018.

[9] Fave X, Zhang L, Yang J, Mackin D, Balter P, Gomez D, et al. Delta-radiomics features for the prediction of patient outcomes in non-small cell lung cancer. Sci Rep. 2017;7:588.

[10] van Timmeren JE, Leijenaar RTH, van Elmpt W, Reymen B, Oberije C, Monshouwer R, et al. Survival prediction of non-small cell lung cancer patients using radiomics analyses of conebeam CT images. Radiother Oncol. 2017;123:363-9.

[11] van Timmeren JE, Leijenaar RTH, van Elmpt W, Reymen B, Lambin P. Feature selection methodology for longitudinal cone-beam CT radiomics. Acta Oncol. 2017:1-7.

[12] Fave X, Mackin D, Yang J, Zhang J, Fried D, Balter P, et al. Can radiomics features be reproducibly measured from $\mathrm{CBCT}$ images for patients with non-small cell lung cancer? Med Phys. 2015;42:6784.

[13] Bernchou U, Hansen O, Schytte T, Bertelsen A, Hope A, Moseley D, et al. Prediction of lung density changes after radiotherapy by cone beam computed tomography response markers and pre-treatment factors for non-small cell lung cancer patients. Radiother Oncol. 2015;117:17-22.

[14] Bertelsen A, Schytte T, Bentzen SM, Hansen O, Nielsen M, Brink C. Radiation dose response of normal lung assessed by Cone Beam CT - a potential tool for biologically adaptive radiation therapy. Radiother Oncol. 2011;100:351-5.

[15] Rosen BS, Hawkins PG, Polan DF, Balter JM, Brock KK, Kamp JD, et al. Early changes in serial CBCT-measured parotid gland biomarkers predict chronic xerostomia after head and neck radiotherapy. Int J Radiat Oncol Biol Phys. 2018.

[16] Brink C, Bernchou U, Bertelsen A, Hansen O, Schytte T, Bentzen SM. Locoregional control of non-small cell lung cancer in relation to automated early assessment of tumor regression on cone beam computed tomography. Int J Radiat Oncol Biol Phys. 2014;89:916-23.

[17] Cardiac CT imaging : diagnosis of cardiovascular disease. New York, NY: Springer Berlin Heidelberg; 2016.

[18] Tibshirani R. Regression shrinkage and selection via the lasso. J Royal Stat Soc. 1996:26788.

[19] Royston P, Altman DG. External validation of a Cox prognostic model: principles and methods. BMC Med Res Methodol. 2013;13:33.

[20] Harrell FE, Jr., Califf RM, Pryor DB, Lee KL, Rosati RA. Evaluating the yield of medical tests. Jama. 1982;247:2543-6. 
[21] Collins GS, Reitsma JB, Altman DG, Moons KG. Transparent Reporting of a multivariable prediction model for Individual Prognosis or Diagnosis (TRIPOD): the TRIPOD statement. Ann Intern Med. 2015;162:55-63.

[22] Moons KG, Altman DG, Reitsma JB, loannidis JP, Macaskill P, Steyerberg EW, et al. Transparent Reporting of a multivariable prediction model for Individual Prognosis or Diagnosis (TRIPOD): explanation and elaboration. Ann Intern Med. 2015;162:W1-73.

[23] de Jong EEC, van Elmpt W, Rizzo S, Colarieti A, Spitaleri G, Leijenaar RTH, et al. Applicability of a prognostic CT-based radiomic signature model trained on stage I-III non-small cell lung cancer in stage IV non-small cell lung cancer. Lung Cancer. 2018;124:6-11.

[24] Schipaanboord B, Boukerroui D, Peressutti D, van Soest J, Lustberg T, Kadir T, et al. Can atlas-based auto-segmentation ever be perfect? Insights from Extreme Value Theory. IEEE Trans Med Imaging. 2018.

[25] Lustberg T, van Soest J, Gooding M, Peressutti D, Aljabar P, van der Stoep J, et al. Clinical evaluation of atlas and deep learning based automatic contouring for lung cancer. Radiother Oncol. 2018;126:312-7.

[26] Star-Lack J, Sun $M$, Oelhafen $M$, Berkus $T$, Pavkovich J, Brehm $M$, et al. A modified McKinnon-Bates (MKB) algorithm for improved 4D cone-beam computed tomography (CBCT) of the lung. Med Phys. 2018.

[27] Martin R, Ahmad M, Hugo G, Pan T. Iterative volume of interest based 4D cone-beam CT. Med Phys. 2017;44:6515-28.

[28] Zhu L, Xie Y, Wang J, Xing L. Scatter correction for cone-beam CT in radiation therapy. Med Phys. 2009;36:2258-68.

[29] Pan Y, Brink C, Schytte T, Petersen H, Wu YL, Hansen O. Planned FDG PET-CT Scan in Follow-Up Detects Disease Progression in Patients With Locally Advanced NSCLC Receiving Curative Chemoradiotherapy Earlier Than Standard CT. Medicine (Baltimore). 2015;94:e1863. 


\section{Supplementary Material}

\section{Supplementary Material 4A - Patients}

Dataset 1 contains 141 patients from Maastro Clinic, Maastricht, The Netherlands, treated between January 2012 and March 2015. Dataset 2 consists of 94 patients from Odense University Hospital, Odense, Denmark [1], treated between November 2007 and December 2011. Dataset 3 contains 61 patients from Ghent University Hospital, Ghent, Belgium, treated between April 2010 and March 2016. Dataset 4 contains 41 patients from Radboud University Medical Center, Nijmegen, The Netherlands, treated between January 2009 and January 2011. The study was approved by Maastro Clinic's Institutional Review Board. Data collection was approved by each institutional ethics committee. Inclusion criteria for patient characteristics and imaging were amongst others: patients diagnosed with stage I-IV non-small cell lung cancer (NSCLC) and received curatively intended (chemo)radiation therapy. Patients treated with stereotactic body radiation therapy (SBRT) were excluded from the cohort, as well as patients that received a total radiation dose less than $45 \mathrm{~Gy}$. Patients with prior history of lung cancer, postoperative radiation treatment or simultaneous treatment of brain metastasis were also excluded from the analysis.

Two clinical endpoints were considered in this study: overall survival and locoregional recurrence. The latter was not available for Dataset 4. Patient outcomes were collected in January 2018 for Datasets 1, 2 and 3 and in August 2016 for Dataset 4. Patients still alive at the end of follow-up or patients without recurrence at the end of follow-up were considered right censored. For all patients, clinical parameters including age, gender, TNM-stage, overall stage and histology were collected. Additionally, WHO performance status and information about chemotherapy (sequential versus concurrent) was available for Dataset 1, 2, and 3. Smoking status and forced expiration volume in 1 second $\left(\mathrm{FEV}_{1}\right)$ were available for Dataset 1 and 2. Mean imputation was used in case of missing values. For discrete variables, values were rounded to the nearest integer. Dunn's test was used to compare continuous variables between datasets, using the false discovery rate (fdr) p-value adjustment method. Fisher's exact test was used to compare categorical variables, followed by $\mathrm{fdr} p$-value adjustments. Kaplan-Meier curves were used to evaluate the differences in overall survival and locoregional recurrence between datasets and log-rank test was used to test for significance. 


\section{Supplementary Material 4B - Imaging}

\section{Dataset 1}

For each patient a respiratory correlated $4 D C T$ scan in treatment position for radiotherapy planning purposes and multiple $\mathrm{CBCTs}$ during radiation treatment were acquired. The $50 \%$ expiration phase of the $4 D$ CT was used for delineation and radiotherapy treatment planning. Voxels dimensions were $0.98 \times 0.98 \times 3 \mathrm{~mm}^{3}$.

The cone-beam CT scans were acquired from a TrueBeam Radiotherapy System (Varian Medical Systems, Palo Alto, CA, USA). All CBCT images were acquired according to the lung protocol, with a tube voltage of $125 \mathrm{kVp}$ reconstructed into a matrix of $512 \times 512 \times 54$ pixels or $512 \times 512 \times 81$ pixels. Images were reconstructed using filtered backprojection [2] into images with an in-plane pixel size of $0.91 \times 0.91$ $\mathrm{mm}$. Most CBCT images were reconstructed into a $2 \mathrm{~mm}$ or $3 \mathrm{~mm}$ slice thickness.

\section{Dataset 2}

Planning CT scans in this dataset are acquired during free-breathing and with contrast for radiotherapy planning purposes. Voxel dimensions ranged from $0.98 \times 0.98 \times 3$ $\mathrm{mm}^{3}$ to $1.12 \times 1.12 \times 3 \mathrm{~mm}^{3}$.

The cone-beam CT scans were acquired from an Elekta XVI Systems (Elekta $A B$, Stockholm, Sweden). All CBCT images were acquired with a tube voltage of 120 $\mathrm{kVp}$ and reconstructed into a matrix of $410 \times 410 \times 264$ pixels. Images were reconstructed into images with an isotropic voxel size of $1 \mathrm{~mm}$ using filtered backprojection.

\section{Dataset 3}

Planning CT scans in this dataset are acquired during free-beathing and either with or without contrsast for radiotherapy planning purposes. In-plane voxel size ranged from $0.85 \times 0.85 \mathrm{~mm}^{2}$ to $1.17 \times 1.17 \mathrm{~mm}^{2}$ with a slice thickness of either $2 \mathrm{~mm}$ or $5 \mathrm{~mm}$.

The cone-beam CT scans were acquired from the On-Board Imager (OBI) of the Varian Clinac iX System (Varian Medical Systems, Palo Alto, CA, USA). All CBCT images were acquired according to the lung protocol, with a tube voltage of $120 \mathrm{kVp}$ reconstructed into a matrix of $384 \times 384 \times 64$ pixels. Images were reconstructed using filtered backprojection [2] into images with an in-plane pixel size of $1.17 \times 1.17 \mathrm{~mm}^{2}$ and a $2.5 \mathrm{~mm}$ slice thickness.

\section{Dataset 4}

Planning CT scans in this dataset are acquired during free-breathing and with contrast for radiotherapy planning purposes. Voxel dimensions ranged from $0.98 \times 0.98 \times 2.5 \mathrm{~mm}^{3}$ to $0.98 \times 0.98 \times 3 \mathrm{~mm}^{3}$.

The cone-beam CT scans were acquired from an Elekta XVI Systems (Elekta $A B$, Stockholm, Sweden). All CBCT images were acquired with a tube voltage of 120 $\mathrm{kVp}$ and reconstructed into a matrix of $410 \times 410 \times 264$ pixels. Images were reconstructed into images with an isotropic voxel size of $1 \mathrm{~mm}$ using filtered backprojection. 


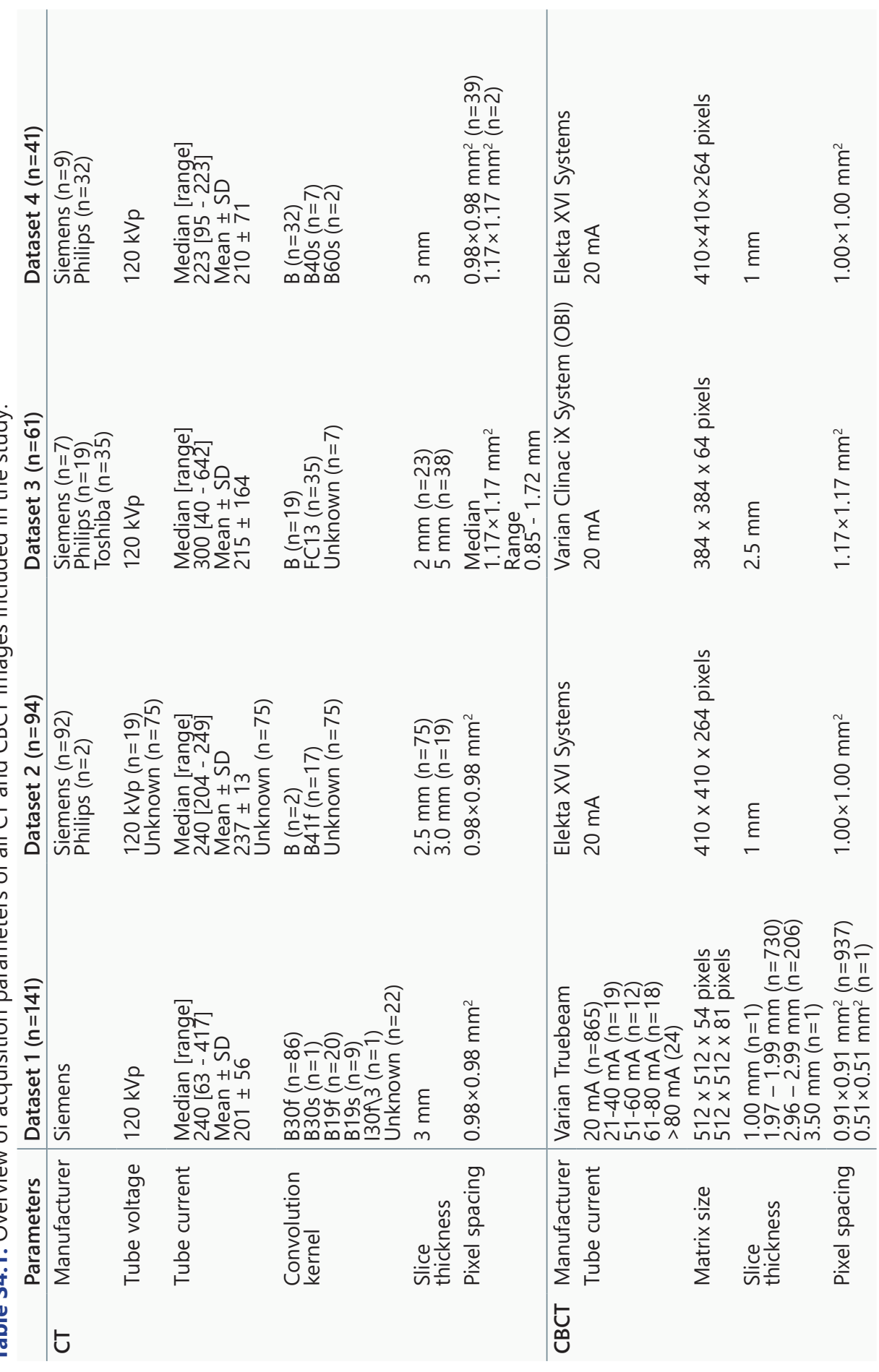


In total 2154 CBCT were analyzed: 938 scans for Dataset 1, 451 scans for Dataset 2, 366 scans for Dataset 3 and 263 scans for Dataset 4.

A

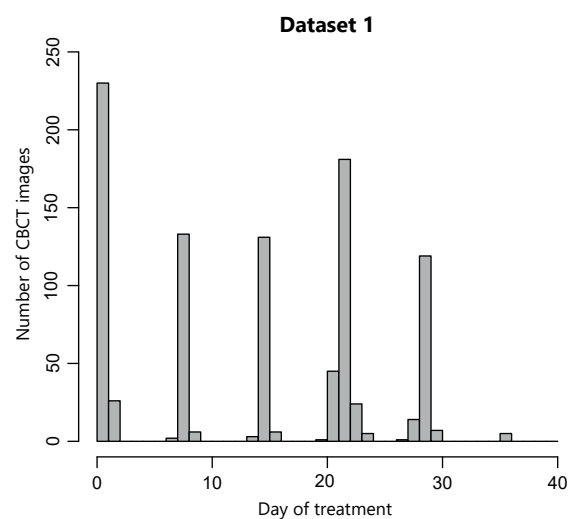

C

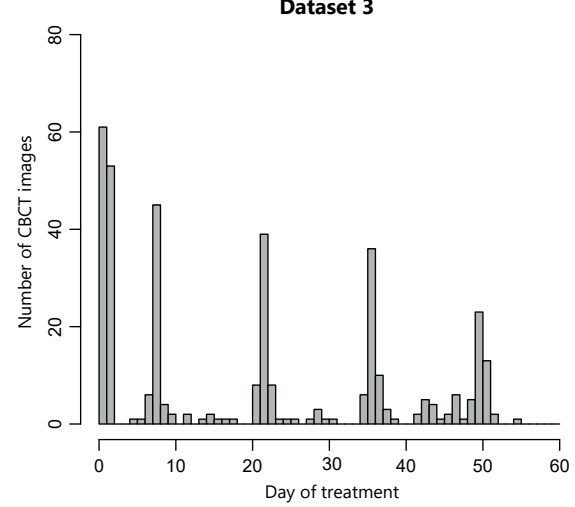

B

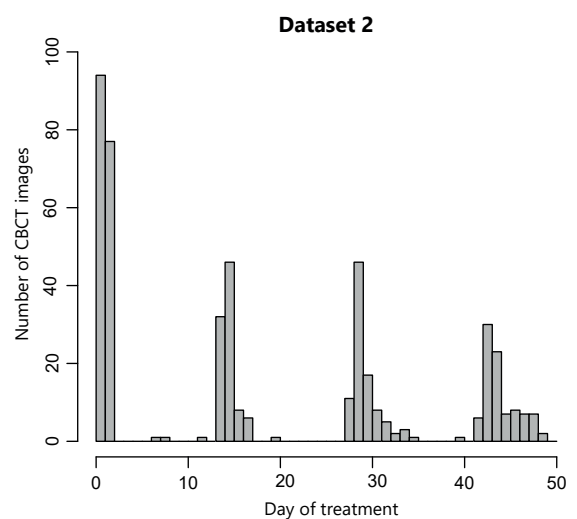

D

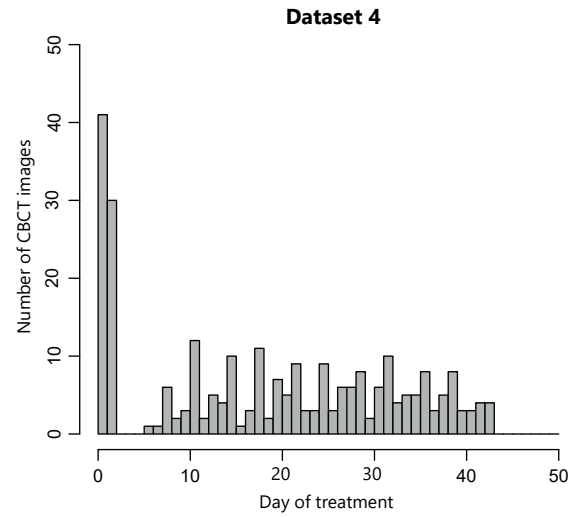

Figure S4.1. Histogram of all cone-beam CT images included per dataset and their timing during treatment. 


\section{Supplementary Material 4C - Feature extraction}

Features can be categorized in different groups: 1) Morphological 'Shape', 2) Fractal, 3) Local Intensity 'Loclnt', 4) Statistical features 'Stats', 5) Intensity-volume histogram 'IVH', 6) Textural features, including gray-level co-occurrence matrix ' $G L C M^{\prime}$ ', graylevel run length matrix 'GLRLM', gray-level size zone matrix 'GLSZM', neighborhood gray-tone difference matrix 'NGTDM', gray-level distance zone matrix 'GLDZM', neighborhood gray-level dependence matrix 'NGLDM', 7) Laplacian of Gaussian filter 'LoG' (prior to group 4), and 8) Wavelet filter (prior to group 6). Mathematical descriptions of all features are described in [3]. Prior to calculation of all features except those in group 4, a gray-level discretization was applied using a bin width of $25 \mathrm{HU}$ and an offset of $0 \mathrm{HU}$ such that the center position of one of the bins was 12.5 $\mathrm{HU}$. Moreover, all images were resampled to isotropic voxel size of $1 \times 1 \times 3 \mathrm{~mm}^{3}$ using cubic interpolation, as this improves the stability of radiomics and allows for more meaningful feature comparisons between scans with different voxel sizes [4]. 


\section{Supplementary Material 4D - Feature selection}

The feature selection process, which was performed on Dataset 1 , consists of four steps, which are explained in further detail below.

\section{Clean-up}

First of all, a clean-up of features that contain missing values or have a number of unique values that is lower than a threshold, which was arbitrarily set to $10 \%$ of the number of patients. The latter for example removes the feature 'Volume number', which is the number of volumes in the ROI. Since we only considered the primary tumor in this study, the feature value was always equal to 1 and thus uninformative.

\section{Select only changing features}

The features extracted from the cone-beam $\mathrm{CT}$ images were selected based on the approach described in detail in van Timmeren et al. [4]. This implies that only radiomic features that change more than expected by chance were considered relevant for a longitudinal radiomics approach. In this study, the features that change more than the variability in the first two radiotherapy fractions for at least $10 \%$ of patients in the two time points closest to $2 / 3$ of treatment, were selected. Due to different fractionation schemes within and between the datasets, the preferred time points at $2 / 3$ of treatment were chosen based on the number of prescribed fractions for each individual patient.

\section{Combine feature sets}

Next, the selected CBCT features were converted to the slope surrogates as explained before (CBCT-slope) and subsequently added to the features extracted from the treatment planning $\mathrm{CT}$ to acquire a complete set of features.

4. Remove correlated features OR calculate Principal Components (PC)

This complete set of features (CBCT-slope $+\mathrm{CT}$ ) was subjected to either a principal component analysis (PCA) or an additional feature selection procedure that removes highly correlated radiomic features, similar as described in Yip et al. [5]. In this procedure of removing correlated features, a correlation matrix was created using Spearman's rank correlation coefficient. From all feature pairs with a correlation above 0.85 , the features with the highest average correlation coefficient were removed. This procedure was performed to remove redundant information from the initial feature set. The CT-extracted feature "Shape - volume" was added to the final feature set in case it was removed during the feature selection process. 


\section{Supplementary Material 4E - Explanation of features in models}

\section{Model 1.1}

CT LoG sigma $25 \mathrm{~mm}$ 3D pos $\mathrm{IH}$ mode is the mode (most frequently occurring intensity level) of the Intensity Histogram (IH) calculated from the positive (pos) part of the filtered CT image. The image is filtered with a Laplacian of Gaussian (LoG) filter with a Gaussian standard deviation (radius) $\sigma$ of $2.5 \mathrm{~mm}$. The $\mathrm{IH}$ is the distribution of gray values after discretization into intensity level bins was applied.

CT Loclnt peakLocal is the mean intensity level in a $1 \mathrm{~cm}^{3}$ spherical volume, centered on the voxel with the maximum intensity.

CT Wavelet_LLL_GLCM inverseVar is the inverse variance of the gray-level cooccurrence matrix (GLCM) which was calculated after applying a wavelet transform to the original image using low-pass $(\mathrm{L})$ fitering in the $x, y$ and $z$-direction (LLL).

\section{Model 1.2}

CT LoG sigma $25 \mathrm{~mm}$ 3D pos IH maxGradl is the maximum histogram gradient intensity level (maxGradl), which is the discretized intensity level that corresponds to the maximum histogram gradient, of the LoG filtered CT image (as explained before). CT LoG sigma $55 \mathrm{~mm} \mathrm{3D} \mathrm{HH}$ p10 is the $10^{\text {th }}$ percentile of the discretized intensity levels of the LoG filtered CT image.

CT Wavelet $\mathrm{HHH}$ Stats median is the median of the intensity levels of the wavelet filtered $C T$ image using high-pass $(\mathrm{H})$ filtering in the $\mathrm{x}, \mathrm{y}$ and $\mathrm{z}$-direction $(\mathrm{HH})$.

CBCT-Slope Wavelet HHL GLCM maxCorr is the CBCT-slope feature of the maximum correlation coefficient of the GLCM, calculated on the HHL wavelet filtered image.

CBCT-Slope Wavelet_HLH_GLCM infoCorr2 is CBCT-slope feature of the informational measure of correlation 2 of the GLCM, calculated on the $\mathrm{HLH}$ wavelet filtered image. CBCT-Slope Wavelet HLL GLCM inverseVar is the CBCT-slope feature of the inverse variance of the GLCM, calculated on the HLL wavelet filtered image.

\section{Models 3.1 and 3.2}

$\underline{\mathrm{WHO}} 2$ is the dummy variable of a $\mathrm{WHO}$ performance score of 2 (reference $\mathrm{WHO}=$ $0)$.

Concomitant is the dummy variable for concurrent chemotherapy.

Stage II and Stage IIla are dummy variables for overall stage II or stage IIla (reference overall stage I).

Histology (other) and Histology (squamous cell) are the dummy variables for morphology, using adenocarcinoma as reference. 


\section{Supplementary Material 4F - Validation measures of the prognostic index}

\section{Dataset 1}

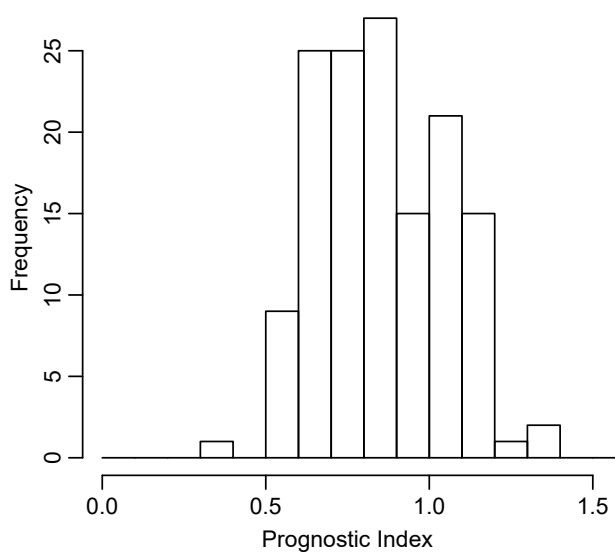

Dataset 3

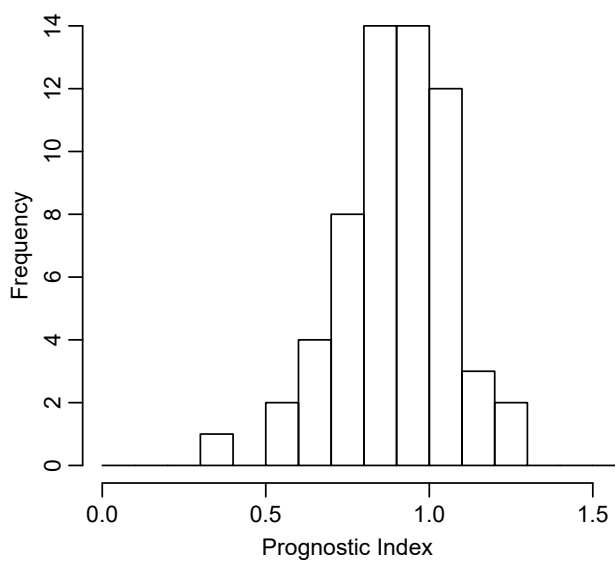

Dataset 2

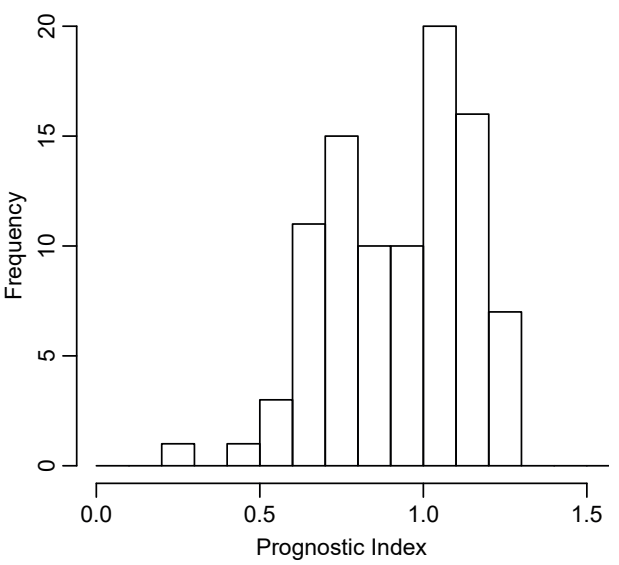

Dataset 4

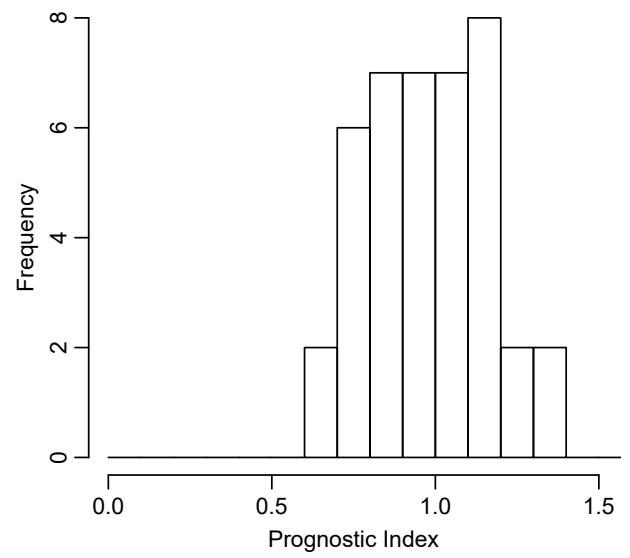

Figure S4.2. Histogram of the prognostic indices for the radiomics model to predict overall survival (model 1.1), shown for all datasets.

Table S4.2. Model validation results for all validation datasets, for the radiomics model to predict overall survival (model 1.1).

Calibration slope on the PI Joint test on all coefficients, (SE, p-value LR test)
Harrell's c-index

(95\% Cl, p-value)

$0.59(0.54-0.65, p=0.0012)$

$0.54(0.43-0.65, p=0.46)$

$0.57(0.47-0.66, p=0.16)$ 


\section{Supplementary Material 4G - Kaplan-Meier curves locoregional recurrence}
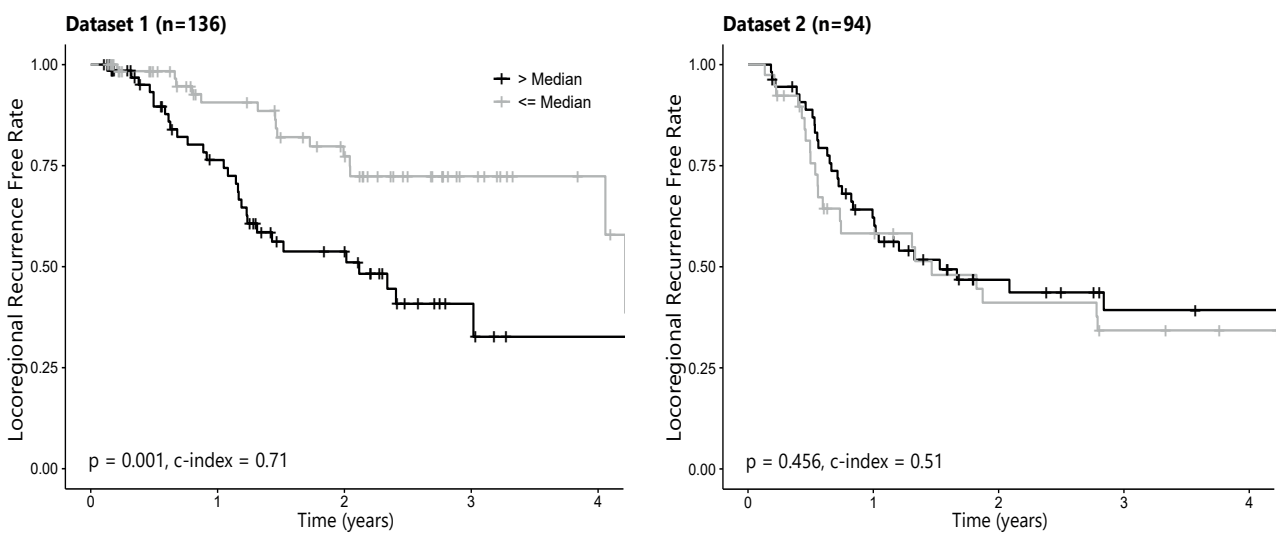

Number at risk

- $68 \quad 39$
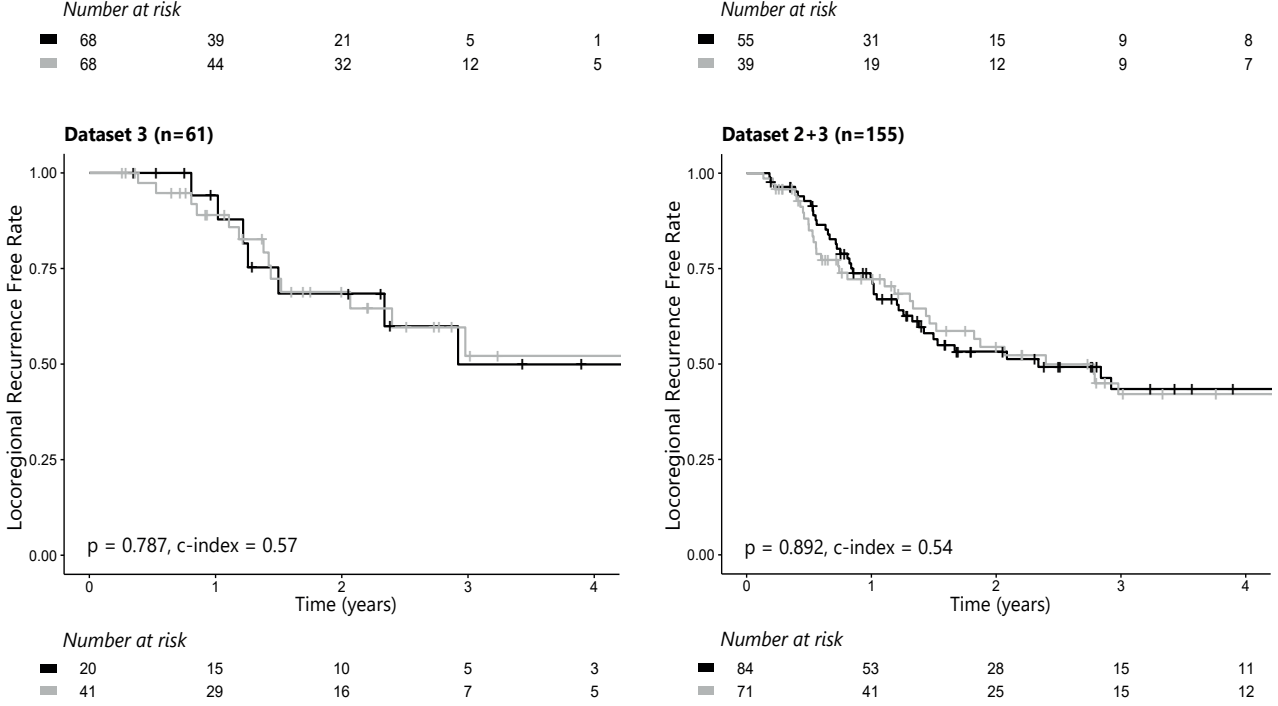

Figure S4.3. Kaplan-Meiers of model 1.2 containing three CT features to predict locoregional recurrence. 


\section{Supplementary Material 4H - TRIPOD Checklist}

\begin{tabular}{|c|c|c|c|c|c|}
\hline \multicolumn{6}{|c|}{ B. TRIPOD ITEMS } \\
\hline & & & $\begin{array}{c}\text { [D] } \\
\text { Development }\end{array}$ & $\begin{array}{c}{[\mathbf{V}]} \\
\text { External validation }\end{array}$ & $\begin{array}{l}{[\mathrm{D}+\mathrm{V}]} \\
\text { Development and } \\
\text { external validation } \\
\text { (of the same model) }\end{array}$ \\
\hline \multicolumn{6}{|c|}{ Title and abstract } \\
\hline \multicolumn{6}{|c|}{$\begin{array}{l}\text { It is suggested to score items } 1 \text { and } 2 \text { (Title and Abstract) after scoring items } 3 \text { to 22, as only after reading the whole publication it can } \\
\text { be judged whether the reporting in the title and abstract is complete. }\end{array}$} \\
\hline \multirow{5}{*}{ Title } & & $\begin{array}{l}\text { Identify the study as developing and/or } \\
\text { validating a multivariable prediction model, } \\
\text { the target population, and the outcome to be } \\
\text { predicted. }\end{array}$ & 1 & 1 & 1 \\
\hline & i & $\begin{array}{l}\text { The words developing/development, } \\
\text { validation/validating, incremental/added value (or } \\
\text { synonyms) are reported in the title }\end{array}$ & 1 & 1 & 1 \\
\hline & ii & $\begin{array}{l}\text { The words prediction, risk prediction, prediction } \\
\text { model, risk models, prognostic models, prognostic } \\
\text { indices, risk scores (or synonyms) are reported in the } \\
\text { title }\end{array}$ & 1 & 1 & 1 \\
\hline & iii & The target population is reported in the title & 1 & 1 & 1 \\
\hline & iv & The outcome to be predicted is reported in the title & 1 & 1 & 1 \\
\hline \multirow{10}{*}{ Abstract } & 2 & $\begin{array}{l}\text { Provide a summary of objectives, study design, } \\
\text { setting, participants, sample size, predictors, } \\
\text { outcome, statistical analysis, results, and } \\
\text { conclusions. }\end{array}$ & 0 & $\mathbf{0}$ & $\mathbf{0}$ \\
\hline & $i$ & The objectives are reported in the abstract & 1 & 1 & 1 \\
\hline & ii & $\begin{array}{l}\text { Sources of data are reported in the abstract } \\
\text { E.g. Prospective cohort, registry data, RCT data. }\end{array}$ & 1 & 1 & 1 \\
\hline & iii & $\begin{array}{l}\text { The setting is reported in the abstract } \\
\text { E.g. Primary care, secondary care, general } \\
\text { population, adult care, or paediatric care. The } \\
\text { setting should be reported for both the development } \\
\text { and validation datasets, if applicable. }\end{array}$ & 1 & 1 & 1 \\
\hline & iv & $\begin{array}{l}\text { A general definition of the study participants is } \\
\text { reported in the abstract } \\
\text { E.g. patients with suspicion of certain disease, } \\
\text { patients with a specific disease, or general eligibility } \\
\text { criteria. }\end{array}$ & 1 & 1 & 1 \\
\hline & $\mathrm{v}$ & The overall sample size is reported in the abstract & 1 & 1 & 1 \\
\hline & vi & $\begin{array}{l}\text { The number of events (or \% outcome together with } \\
\text { overall sample size) is reported in the abstract } \\
\text { If a continuous outcome was studied, score Not } \\
\text { applicable. }\end{array}$ & NA & NA & NA \\
\hline & vii & $\begin{array}{l}\text { Predictors included in the final model are reported in } \\
\text { the abstract. For validation studies of well-known } \\
\text { models, at least the name/acronym of the validated } \\
\text { model is reported } \\
\text { Broad descriptions are sufficient, e.g. 'all information } \\
\text { from patient history and physical examination'. } \\
\text { Check in the main text whether all predictors of the } \\
\text { final model are indeed reported in the abstract. }\end{array}$ & 1 & 1 & 1 \\
\hline & viii & The outcome is reported in the abstract & 1 & 1 & 1 \\
\hline & ix & $\begin{array}{l}\text { Statistical methods are described in the abstract } \\
\text { For model development, at least the type of } \\
\text { statistical model should be reported. For validation } \\
\text { studies a quote like "model's discrimination and } \\
\text { calibration was assessed" is considered adequate. If } \\
\text { done, methods of updating should be reported. }\end{array}$ & 1 & 1 & 1 \\
\hline
\end{tabular}




\begin{tabular}{|c|c|c|c|c|c|}
\hline & $\mathrm{x}$ & $\begin{array}{l}\text { Results for model discrimination are reported in the } \\
\text { abstract } \\
\text { This should be reported separately for development } \\
\text { and validation if a study includes both development } \\
\text { and validation. }\end{array}$ & 1 & 1 & 1 \\
\hline & xi & $\begin{array}{l}\text { Results for model calibration are reported in the } \\
\text { abstract } \\
\text { This should be reported separately for development } \\
\text { and validation if a study includes both development } \\
\text { and validation. }\end{array}$ & 0 & 0 & 0 \\
\hline & xii & $\begin{array}{l}\text { Conclusions are reported in the abstract } \\
\text { In publications addressing both model development } \\
\text { and validation, there is no need for separate } \\
\text { conclusions for both; one conclusion is sufficient. }\end{array}$ & 1 & 1 & 1 \\
\hline \multirow{5}{*}{$\begin{array}{l}\text { Background and } \\
\text { objectives }\end{array}$} & $3 a$ & $\begin{array}{l}\text { Explain the medical context (including whether } \\
\text { diagnostic or prognostic) and rationale for } \\
\text { developing or validating the multivariable } \\
\text { prediction model, including references to } \\
\text { existing models. }\end{array}$ & 1 & 1 & 1 \\
\hline & $\mathrm{i}$ & The background and rationale are presented & 1 & 1 & 1 \\
\hline & ii & $\begin{array}{l}\text { Reference to existing models is included (or stated } \\
\text { that there are no existing models) }\end{array}$ & 1 & 1 & 1 \\
\hline & 3b & $\begin{array}{l}\text { Specify the objectives, including whether the } \\
\text { study describes the development or validation } \\
\text { of the model or both. }\end{array}$ & 1 & 1 & 1 \\
\hline & $\mathrm{i}$ & $\begin{array}{l}\text { It is stated whether the study describes development } \\
\text { and/or validation and/or incremental (added) value }\end{array}$ & 1 & 1 & 1 \\
\hline \multicolumn{6}{|l|}{ Methods } \\
\hline \multirow{10}{*}{ Source of data } & $4 a$ & $\begin{array}{l}\text { Describe the study design or source of data } \\
\text { (e.g., randomized trial, cohort, or registry } \\
\text { data), separately for the development and } \\
\text { validation data sets, if applicable. }\end{array}$ & 1 & 1 & 1 \\
\hline & i & $\begin{array}{l}\text { The study design/source of data is described } \\
\text { E.g. Prospectively designed, existing cohort, existing } \\
R C T \text {, registry/medical records, case control, case } \\
\text { series. } \\
\text { This needs to be explicitly reported; reference to this } \\
\text { information in another article alone is insufficient. }\end{array}$ & 1 & 1 & 1 \\
\hline & $4 b$ & $\begin{array}{l}\text { Specify the key study dates, including start of } \\
\text { accrual; end of accrual; and, if applicable, end } \\
\text { of follow-up. }\end{array}$ & 1 & 1 & 1 \\
\hline & $\mathrm{i}$ & The starting date of accrual is reported & 1 & 1 & 1 \\
\hline & ii & The end date of accrual is reported & 1 & 1 & 1 \\
\hline & iii & $\begin{array}{l}\text { The length of follow-up and prediction horizon/time } \\
\text { frame are reported, if applicable } \\
\text { E.g. "Patients were followed from baseline for } 10 \\
\text { years" and "10-year prediction of..."; notably for } \\
\text { prognostic studies with long term follow-up. } \\
\text { If this is not applicable for an article (i.e. diagnostic } \\
\text { study or no follow-up), then score Not applicable. }\end{array}$ & 1 & 1 & 1 \\
\hline & $5 a$ & $\begin{array}{l}\text { Specify key elements of the study setting (e.g., } \\
\text { primary care, secondary care, general } \\
\text { population) including number and location of } \\
\text { centres. }\end{array}$ & 1 & 1 & 1 \\
\hline & $i$ & $\begin{array}{l}\text { The study setting is reported (e.g. primary care, } \\
\text { secondary care, general population) } \\
\text { E.g.: 'surgery for endometrial cancer patients' is } \\
\text { considered to be enough information about the study } \\
\text { setting. }\end{array}$ & 1 & 1 & 1 \\
\hline & ii & $\begin{array}{l}\text { The number of centres involved is reported } \\
\text { If the number is not reported explicitly, but can be } \\
\text { concluded from the name of the centre/centres, or if } \\
\text { clearly a single centre study, score Yes. }\end{array}$ & 1 & 1 & 1 \\
\hline & & $\begin{array}{l}\text { The geographical location (at least country) of centres } \\
\text { involved is reported }\end{array}$ & & & \\
\hline
\end{tabular}




\begin{tabular}{|c|c|c|c|c|c|}
\hline \multirow[t]{5}{*}{ Participants } & iii & $\begin{array}{l}\text { If no geographical location is specified, but the } \\
\text { location can be concluded from the name of the } \\
\text { centre(s), score Yes. }\end{array}$ & 1 & 1 & 1 \\
\hline & $5 b$ & Describe eligibility criteria for participants. & 1 & 1 & 1 \\
\hline & $\mathrm{i}$ & $\begin{array}{l}\text { In-/exclusion criteria are stated } \\
\text { These should explicitly be stated. Reasons for } \\
\text { exclusion only described in a patient flow is not } \\
\text { sufficient. }\end{array}$ & 1 & 1 & 1 \\
\hline & $5 c$ & Give details of treatments received, if relevant. & 1 & 1 & 1 \\
\hline & $\mathrm{i}$ & $\begin{array}{l}\text { Details of any treatments received are described } \\
\text { This item is notably for prognostic modelling studies } \\
\text { and is about treatment at baseline or during follow- } \\
\text { up. The 'if relevant' judgment of treatment requires } \\
\text { clinical knowledge and interpretation. } \\
\text { If you are certain that treatment was not relevant, } \\
\text { e.g. in some diagnostic model studies, score Not } \\
\text { applicable. }\end{array}$ & 1 & 1 & 1 \\
\hline \multirow{6}{*}{ Outcome } & $6 a$ & $\begin{array}{l}\text { Clearly define the outcome that is predicted by } \\
\text { the prediction model, including how and when } \\
\text { assessed. }\end{array}$ & 0 & 0 & 0 \\
\hline & $\mathrm{i}$ & $\begin{array}{l}\text { The outcome definition is clearly presented } \\
\text { This should be reported separately for development } \\
\text { and validation if a publication includes both. }\end{array}$ & 1 & 1 & 1 \\
\hline & ii & $\begin{array}{l}\text { It is described how outcome was assessed (including } \\
\text { all elements of any composite, for example CVD [e.g. } \\
\text { MI. HF, stroke]). }\end{array}$ & 0 & 0 & 0 \\
\hline & iii & $\begin{array}{l}\text { It is described when the outcome was assessed (time } \\
\text { point(s) since T0) }\end{array}$ & 1 & 1 & 1 \\
\hline & 6b & $\begin{array}{l}\text { Report any actions to blind assessment of the } \\
\text { outcome to be predicted. }\end{array}$ & 1 & 1 & 1 \\
\hline & $\mathrm{i}$ & $\begin{array}{l}\text { Actions to blind assessment of outcome to be } \\
\text { predicted are reported } \\
\text { If it is clearly a non-issue (e.g. all-cause mortality or } \\
\text { an outcome not requiring interpretation), score Yes. } \\
\text { In all other instances, an explicit mention is } \\
\text { expected. }\end{array}$ & 1 & 1 & 1 \\
\hline \multirow{9}{*}{ Predictors } & $7 a$ & $\begin{array}{l}\text { Clearly define all predictors used in developing } \\
\text { or validating the multivariable prediction } \\
\text { model, including how and when they were } \\
\text { measured. }\end{array}$ & 1 & 1 & 1 \\
\hline & $\mathrm{i}$ & $\begin{array}{l}\text { All predictors are reported } \\
\text { For development, "all predictors" refers to all } \\
\text { predictors that potentially could have been included } \\
\text { in the 'final' model (including those considered in any } \\
\text { univariable analyses). } \\
\text { For validation, "all predictors" means the predictors in } \\
\text { the model being evaluated. }\end{array}$ & 1 & 1 & 1 \\
\hline & ii & Predictor definitions are clearly presented & 1 & 1 & 1 \\
\hline & iii & $\begin{array}{l}\text { It is clearly described how the predictors were } \\
\text { measured }\end{array}$ & 1 & 1 & 1 \\
\hline & iv & $\begin{array}{l}\text { It is clearly described when the predictors were } \\
\text { measured }\end{array}$ & 1 & 1 & 1 \\
\hline & $7 \mathbf{b}$ & $\begin{array}{l}\text { Report any actions to blind assessment of } \\
\text { predictors for the outcome and other } \\
\text { predictors. }\end{array}$ & 1 & 1 & 1 \\
\hline & & $\begin{array}{l}\text { It is clearly described whether predictor assessments } \\
\text { were blinded for outcome }\end{array}$ & & & \\
\hline & $\mathrm{i}$ & $\begin{array}{l}\text { For predictors for which it is clearly a non-issue (e.g. } \\
\text { automatic blood pressure measurement, age, sex) } \\
\text { and for instances where the predictors were clearly } \\
\text { assessed before outcome assessment, score Yes. For } \\
\text { all other predictors an explicit mention is expected. }\end{array}$ & 1 & 1 & 1 \\
\hline & ii & $\begin{array}{l}\text { It is clearly described whether predictor assessments } \\
\text { were blinded for the other predictors }\end{array}$ & 1 & 1 & 1 \\
\hline \multirow[b]{2}{*}{ Sample size } & & Explain how the study size was arrived at. & 0 & 0 & 0 \\
\hline & $\mathrm{i}$ & $\begin{array}{l}\text { It is explained how the study size was arrived at } \\
\text { Is there any mention of sample size, e.g. whether } \\
\text { this was done on statistical grounds or } \\
\text { practical/logistical grounds (e.g. an existing study } \\
\text { cohort or data set of a RCT was used)? }\end{array}$ & 0 & 0 & 0 \\
\hline
\end{tabular}




\begin{tabular}{|c|c|c|c|c|c|}
\hline \multirow{5}{*}{ Missing data } & 9 & $\begin{array}{l}\text { Describe how missing data were handled (e.g., } \\
\text { complete-case analysis, single imputation, } \\
\text { multiple imputation) with details of any } \\
\text { imputation method. }\end{array}$ & 1 & 1 & 1 \\
\hline & $i$ & $\begin{array}{l}\text { The method for handling missing data (predictors and } \\
\text { outcome) is mentioned } \\
\text { E.g. Complete case (explicit mention that individuals } \\
\text { with missing values have been excluded), single } \\
\text { imputation, multiple imputation, mean/median } \\
\text { imputation. } \\
\text { If there is no missing data, there should be an } \\
\text { explicit mention that there is no missing data for all } \\
\text { predictors and outcome. If so, score Yes. } \\
\text { If it is unclear whether there is missing data (from } \\
\text { e.g. the reported methods or results), score No. } \\
\text { If it is clear there is missing data, but the method for } \\
\text { handling missing data is unclear, score No. }\end{array}$ & 1 & 1 & 1 \\
\hline & ii & $\begin{array}{l}\text { If missing data were imputed, details of the software } \\
\text { used are given } \\
\text { When under gi explicit mentioning of no missing } \\
\text { data, complete case analysis or no imputation } \\
\text { applied, score Not applicable. }\end{array}$ & 1 & 1 & 1 \\
\hline & iii & $\begin{array}{l}\text { If missing data were imputed, a description of which } \\
\text { variables were included in the imputation procedure is } \\
\text { given. } \\
\text { When under gi explicit mentioning of no missing } \\
\text { data, complete case analysis or no imputation } \\
\text { applied, score Not applicable. }\end{array}$ & 1 & 1 & 1 \\
\hline & iv & $\begin{array}{l}\text { If multiple imputation was used, the number of } \\
\text { imputations is reported } \\
\text { When under gi explicit mentioning of no missing } \\
\text { data, complete case analysis or no imputation } \\
\text { applied, score Not applicable. }\end{array}$ & 1 & 1 & 1 \\
\hline & $10 a$ & $\begin{array}{l}\text { Describe how predictors were handled in the } \\
\text { analyses. }\end{array}$ & 1 & NA & 1 \\
\hline & $\mathrm{i}$ & $\begin{array}{l}\text { For continuous predictors it is described whether they } \\
\text { were modelled as linear, nonlinear (type of } \\
\text { transformation specified) or categorized } \\
\text { A general statement is sufficient, no need to describe } \\
\text { this for each predictor separately. } \\
\text { If no continuous predictors were reported, score Not } \\
\text { applicable. }\end{array}$ & 1 & NA & 1 \\
\hline & ii & $\begin{array}{l}\text { For categorical or categorized predictors, the cut- } \\
\text { points were reported } \\
\text { If no categorical or categorized predictors were } \\
\text { reported, score Not applicable. }\end{array}$ & 1 & NA & 1 \\
\hline & iii & $\begin{array}{l}\text { For categorized predictors the method to choose the } \\
\text { cut-points was clearly described } \\
\text { If no categorized predictors, score Not applicable. }\end{array}$ & 1 & NA & 1 \\
\hline & $10 \mathrm{~b}$ & $\begin{array}{l}\text { Specify type of model, all model-building } \\
\text { procedures (including any predictor selection), } \\
\text { and method for internal validation. }\end{array}$ & 0 & NA & 0 \\
\hline & $\mathrm{i}$ & $\begin{array}{l}\text { The type of statistical model is reported } \\
\text { E.g. Logistic, Cox, other regression model (e.g. } \\
\text { Weibull, ordinal), other statistical modelling (e.g. } \\
\text { neural network) }\end{array}$ & 1 & NA & 1 \\
\hline & ii & $\begin{array}{l}\text { The approach used for predictor selection before } \\
\text { modelling is described } \\
\text { 'Before modelling'means before any univariable or } \\
\text { multivariable analysis of predictor-outcome } \\
\text { associations. } \\
\text { If no predictor selection before modelling is done, } \\
\text { score Not applicable. } \\
\text { If it is unclear whether predictor selection before } \\
\text { modelling is done, score No. } \\
\text { If it is clear there was predictor selection before } \\
\text { modelling but the method was not described, score } \\
\text { No. }\end{array}$ & 1 & NA & 1 \\
\hline
\end{tabular}




\begin{tabular}{|c|c|c|c|c|c|}
\hline \multirow{12}{*}{$\begin{array}{l}\text { Statistical analysis } \\
\text { methods }\end{array}$} & iii & $\begin{array}{l}\text { The approach used for predictor selection during } \\
\text { modelling is described } \\
\text { E.g. Univariable analysis, stepwise selection, } \\
\text { bootstrap, Lasso. } \\
\text { 'During modelling' includes both univariable or } \\
\text { multivariable analysis of predictor-outcome } \\
\text { associations. } \\
\text { If no predictor selection during modelling is done (so- } \\
\text { called full model approach), score Not applicable. } \\
\text { If it is unclear whether predictor selection during } \\
\text { modelling is done, score No. } \\
\text { If it is clear there was predictor selection during } \\
\text { modelling but the method was not described, score } \\
\text { No. }\end{array}$ & 1 & NA & 1 \\
\hline & iv & $\begin{array}{l}\text { Testing of interaction terms is described } \\
\text { If it is explicitly mentioned that interaction terms } \\
\text { were not addressed in the prediction model, score } \\
\text { Yes. } \\
\text { If interaction terms were included in the prediction } \\
\text { model, but the testing is not described, score No. }\end{array}$ & 1 & NA & 1 \\
\hline & $v$ & $\begin{array}{l}\text { Testing of the proportionality of hazards in survival } \\
\text { models is described } \\
\text { If no proportional hazard model is used, score Not } \\
\text { applicable. }\end{array}$ & 0 & NA & 0 \\
\hline & vi & $\begin{array}{l}\text { Internal validation is reported } \\
\text { E.g. Bootstrapping, cross validation, split sample. } \\
\text { If the use of internal validation is clearly a non-issue } \\
\text { (e.g. in case of very large data sets), score Yes. For } \\
\text { all other situations an explicit mention is expected. }\end{array}$ & 0 & NA & 0 \\
\hline & $10 c$ & $\begin{array}{l}\text { For validation, describe how the predictions } \\
\text { were calculated. }\end{array}$ & NA & 1 & 1 \\
\hline & i. & $\begin{array}{l}\text { It is described how predictions for individuals (in the } \\
\text { validation set) were obtained from the model being } \\
\text { validated } \\
\text { E.g. Using the original reported model coefficients } \\
\text { with or without the intercept, and/or using updated } \\
\text { or refitted model coefficients, or using a nomogram, } \\
\text { spreadsheet or web calculator. }\end{array}$ & NA & 1 & 1 \\
\hline & $10 \mathrm{~d}$ & $\begin{array}{l}\text { Specify all measures used to assess model } \\
\text { performance and, if relevant, to compare multiple } \\
\text { models. } \\
\text { These should be described in the methods section of } \\
\text { the paper (item } 16 \text { addresses the reporting of the } \\
\text { results for model performance). }\end{array}$ & 1 & 1 & 1 \\
\hline & i & $\begin{array}{l}\text { Measures for model discrimination are described } \\
\text { E.g. C-index / area under the ROC curve. }\end{array}$ & 1 & 1 & 1 \\
\hline & $\mathrm{ii}$ & $\begin{array}{l}\text { Measures for model calibration are described } \\
\text { E.g. calibration plot, calibration slope or intercept, } \\
\text { calibration table, Hosmer Lemeshow test, O/E ratio. }\end{array}$ & 1 & 1 & 1 \\
\hline & $\mathrm{iii}$ & $\begin{array}{l}\text { Other performance measures are described } \\
\text { E.g. } R^{2} \text {, Brier score, predictive values, sensitivity, } \\
\text { specificity, AUC difference, decision curve analysis, } \\
\text { net reclassification improvement, integrated } \\
\text { discrimination improvement, AIC. }\end{array}$ & 1 & 1 & 1 \\
\hline & $10 \mathrm{e}$ & $\begin{array}{l}\text { Describe any model updating (e.g., } \\
\text { recalibration) arising from the validation, if } \\
\text { done. }\end{array}$ & NA & NA & NA \\
\hline & $\mathbf{i}$ & $\begin{array}{l}\text { A description of model-updating is given } \\
\text { E.g. Intercept recalibration, regression coefficient } \\
\text { recalibration, refitting the whole model, adding a new } \\
\text { predictor } \\
\text { If updating was done, it should be clear which } \\
\text { updating method was applied to score Yes. }\end{array}$ & NA & NA & NA \\
\hline
\end{tabular}




\begin{tabular}{|c|c|c|c|c|c|}
\hline & & $\begin{array}{l}\text { If it is not explicitly mentioned that updating was } \\
\text { applied in the study, score this item as 'Not } \\
\text { applicable'. }\end{array}$ & & & \\
\hline \multirow[b]{2}{*}{ Risk groups } & 11 & $\begin{array}{l}\text { Provide details on how risk groups were } \\
\text { created, if done. }\end{array}$ & 1 & 1 & 1 \\
\hline & i & $\begin{array}{l}\text { If risk groups were created, risk group boundaries } \\
\text { (risk thresholds) are specified } \\
\text { Score this item separately for development and } \\
\text { validation if a study includes both development and } \\
\text { validation. } \\
\text { If risk groups were not created, score this item as } \\
\text { not applicable. }\end{array}$ & 1 & 1 & 1 \\
\hline \multirow[b]{2}{*}{$\begin{array}{l}\text { Development vs. } \\
\text { validation }\end{array}$} & 12 & $\begin{array}{l}\text { For validation, identify any differences from } \\
\text { the development data in setting, eligibility } \\
\text { criteria, outcome and predictors. }\end{array}$ & NA & NA & NA \\
\hline & i & $\begin{array}{l}\text { Differences or similarities in definitions with the } \\
\text { development study are described } \\
\text { Mentioning of any differences in all four (setting, } \\
\text { eligibility criteria, predictors and outcome) is required } \\
\text { to score Yes. } \\
\text { If it is explicitly mentioned that there were no } \\
\text { differences in setting, eligibility criteria, predictors } \\
\text { and outcomes, score Yes. } \\
\text { For incremental value reports, in case additional } \\
\text { predictors are not added to a previously developed } \\
\text { prediction model but rather added to conventional } \\
\text { predictors in a newly fitted model, score Not } \\
\text { applicable. }\end{array}$ & NA & NA & NA \\
\hline \multicolumn{6}{|l|}{ Results } \\
\hline \multirow{11}{*}{ Participants } & $13 a$ & $\begin{array}{l}\text { Describe the flow of participants through the } \\
\text { study, including the number of participants } \\
\text { with and without the outcome and, if } \\
\text { applicable, a summary of the follow-up time. A } \\
\text { diagram may be helpful. }\end{array}$ & 1 & 1 & 1 \\
\hline & $\mathrm{i}$ & The flow of participants is reported & 1 & 1 & 1 \\
\hline & $\mathrm{ii}$ & $\begin{array}{l}\text { The number of participants with and without the } \\
\text { outcome are reported } \\
\text { If outcomes are continuous, score Not applicable. }\end{array}$ & 1 & 1 & 1 \\
\hline & iii & $\begin{array}{l}\text { A summary of follow-up time is presented } \\
\text { This notably applies to prognosis studies and } \\
\text { diagnostic studies with follow-up as diagnostic } \\
\text { outcome. } \\
\text { If this is not applicable for an article (i.e. diagnostic } \\
\text { study or no follow-up), then score Not applicable. }\end{array}$ & 1 & 1 & 1 \\
\hline & $13 b$ & $\begin{array}{l}\text { Describe the characteristics of the participants } \\
\text { (basic demographics, clinical features, } \\
\text { available predictors), including the number of } \\
\text { participants with missing data for predictors } \\
\text { and outcome. }\end{array}$ & 1 & 1 & 1 \\
\hline & $\mathrm{i}$ & Basic demographics are reported & 1 & 1 & 1 \\
\hline & $\mathrm{ii}$ & $\begin{array}{l}\text { Summary information is provided for all predictors } \\
\text { included in the final developed/validated model }\end{array}$ & 1 & 1 & 1 \\
\hline & iii & $\begin{array}{l}\text { The number of participants with missing data for } \\
\text { predictors is reported }\end{array}$ & 1 & 1 & 1 \\
\hline & iv & $\begin{array}{l}\text { The number of participants with missing data for the } \\
\text { outcome is reported }\end{array}$ & 1 & 1 & 1 \\
\hline & $13 c$ & $\begin{array}{l}\text { For validation, show a comparison with the } \\
\text { development data of the distribution of } \\
\text { important variables (demographics, predictors } \\
\text { and outcome). }\end{array}$ & NA & 1 & 0 \\
\hline & & $\begin{array}{l}\text { Demographic characteristics (at least age and gender) } \\
\text { of the validation study participants are reported along } \\
\text { with those of the original development study }\end{array}$ & & & \\
\hline
\end{tabular}




\begin{tabular}{|c|c|c|c|c|c|}
\hline & $\mathrm{i}$ & $\begin{array}{l}\text { For incremental value reports, in case additional } \\
\text { predictors are not added to a previously developed } \\
\text { prediction model but rather added to conventional } \\
\text { predictors in a newly fitted model, score Not } \\
\text { applicable. }\end{array}$ & NA & 1 & 0 \\
\hline & ii & $\begin{array}{l}\text { Distributions of predictors in the model of the } \\
\text { validation study participants are reported along with } \\
\text { those of the original development study } \\
\text { For incremental value reports, in case additional } \\
\text { predictors are not added to a previously developed } \\
\text { prediction model but rather added to conventional } \\
\text { predictors in a newly fitted model, score Not } \\
\text { applicable. }\end{array}$ & NA & 1 & 1 \\
\hline & $\mathrm{iii}$ & $\begin{array}{l}\text { Outcomes of the validation study participants are } \\
\text { reported along with those of the original development } \\
\text { study } \\
\text { For incremental value reports, in case additional } \\
\text { predictors are not added to a previously developed } \\
\text { prediction model but rather added to conventional } \\
\text { predictors in a newly fitted model, score Not } \\
\text { applicable. }\end{array}$ & NA & 1 & 1 \\
\hline \multirow{5}{*}{$\begin{array}{c}\text { Model } \\
\text { development }\end{array}$} & $14 a$ & $\begin{array}{l}\text { Specify the number of participants and } \\
\text { outcome events in each analysis. }\end{array}$ & 1 & NA & 1 \\
\hline & $\mathrm{i}$ & $\begin{array}{l}\text { The number of participants in each analysis (e.g. in } \\
\text { the analysis of each model if more than one model is } \\
\text { developed) is specified }\end{array}$ & 1 & NA & 1 \\
\hline & ii & $\begin{array}{l}\text { The number of outcome events in each analysis is } \\
\text { specified (e.g. in the analysis of each model if more } \\
\text { than one model is developed) } \\
\text { If outcomes are continuous, score Not applicable. }\end{array}$ & NA & NA & NA \\
\hline & $14 \mathrm{~b}$ & $\begin{array}{l}\text { If done, report the unadjusted association } \\
\text { between each candidate predictor and } \\
\text { outcome. }\end{array}$ & NA & NA & NA \\
\hline & $\mathrm{i}$ & $\begin{array}{l}\text { The unadjusted associations between each predictor } \\
\text { and outcome are reported } \\
\text { If any univariable analysis is mentioned in the } \\
\text { methods but not in the results, score No. } \\
\text { If nothing on univariable analysis (in methods or } \\
\text { results) is reported, score this item as Not applicable. }\end{array}$ & NA & NA & NA \\
\hline \multirow{5}{*}{ Model specification } & $15 a$ & $\begin{array}{l}\text { Present the full prediction model to allow } \\
\text { predictions for individuals (i.e., all regression } \\
\text { coefficients, and model intercept or baseline } \\
\text { survival at a given time point). }\end{array}$ & 0 & NA & 0 \\
\hline & $\mathrm{i}$ & $\begin{array}{l}\text { The regression coefficient (or a derivative such as } \\
\text { hazard ratio, odds ratio, risk ratio) for each predictor } \\
\text { in the model is reported }\end{array}$ & 1 & NA & 1 \\
\hline & ii & $\begin{array}{l}\text { The intercept or the cumulative baseline hazard (or } \\
\text { baseline survival) for at least one time point is } \\
\text { reported }\end{array}$ & 0 & NA & 0 \\
\hline & $15 b$ & Explain how to use the prediction model. & NA & NA & NA \\
\hline & $\mathrm{i}$ & $\begin{array}{l}\text { An explanation (e.g. a simplified scoring rule, chart, } \\
\text { nomogram of the model, reference to online } \\
\text { calculator, or worked example) is provided to explain } \\
\text { how to use the model for individualised predictions. }\end{array}$ & NA & NA & NA \\
\hline \multirow{5}{*}{ Model performance } & 16 & $\begin{array}{l}\text { Report performance measures (with } \\
\text { confidence intervals) for the prediction model. }\end{array}$ & 1 & 1 & 1 \\
\hline & i & $\begin{array}{l}\text { A discrimination measure is presented } \\
\text { E.g. C-index / area under the ROC curve. }\end{array}$ & 1 & 1 & 1 \\
\hline & ii & $\begin{array}{l}\text { The confidence interval (or standard error) of the } \\
\text { discrimination measure is presented }\end{array}$ & 1 & 1 & 1 \\
\hline & $\mathrm{iii}$ & $\begin{array}{l}\text { Measures for model calibration are described } \\
\text { E.g. calibration plot, calibration slope or intercept, } \\
\text { calibration table, Hosmer Lemeshow test, O/E ratio. }\end{array}$ & 1 & 1 & 1 \\
\hline & & Other model performance measures are presented & & & \\
\hline
\end{tabular}




\begin{tabular}{|c|c|c|c|c|c|}
\hline & iv & $\begin{array}{l}\text { E.g. } R^{2}, \text { Brier score, predictive values, sensitivity, } \\
\text { specificity, AUC difference, decision curve analysis, } \\
\text { net reclassification improvement, integrated } \\
\text { discrimination improvement, AIC. }\end{array}$ & 1 & 1 & 1 \\
\hline \multirow{6}{*}{ Model updating } & 17 & $\begin{array}{l}\text { If done, report the results from any model } \\
\text { updating (i.e., model specification, model } \\
\text { performance, recalibration). } \\
\text { If updating was not done, score this TRIPOD item as } \\
\text { 'Not applicable'. }\end{array}$ & NA & 1 & 1 \\
\hline & $\mathrm{i}$ & $\begin{array}{l}\text { The updated regression coefficients for each predictor } \\
\text { in the model are reported } \\
\text { If model updating was described as 'not needed', } \\
\text { score Yes. }\end{array}$ & NA & 1 & 1 \\
\hline & ii & $\begin{array}{l}\text { The updated intercept or cumulative baseline hazard } \\
\text { or baseline survival (for at least one time point) is } \\
\text { reported } \\
\text { If model updating was described as 'not needed', } \\
\text { score Yes. }\end{array}$ & NA & 1 & 1 \\
\hline & iii & The discrimination of the updated model is reported & NA & 1 & 1 \\
\hline & iv & $\begin{array}{l}\text { The confidence interval (or standard error) of the } \\
\text { discrimination measure of the updated model is } \\
\text { reported }\end{array}$ & NA & 1 & 1 \\
\hline & v & The calibration of the updated model is reported & NA & 1 & 1 \\
\hline \multicolumn{6}{|l|}{ Discussion } \\
\hline \multirow{2}{*}{ Limitations } & 18 & $\begin{array}{l}\text { Discuss any limitations of the study (such as } \\
\text { nonrepresentative sample, few events per } \\
\text { predictor, missing data). }\end{array}$ & 1 & 1 & 1 \\
\hline & $\mathrm{i}$ & $\begin{array}{l}\text { Limitations of the study are discussed } \\
\text { Stating any limitation is sufficient. }\end{array}$ & 1 & 1 & 1 \\
\hline \multirow{4}{*}{ Interpretation } & 19a & $\begin{array}{l}\text { For validation, discuss the results with } \\
\text { reference to performance in the development } \\
\text { data, and any other validation data. }\end{array}$ & NA & 1 & 1 \\
\hline & $\mathrm{i}$ & $\begin{array}{l}\text { Comparison of results to reported performance in } \\
\text { development studies and/or other validation studies is } \\
\text { given }\end{array}$ & NA & 1 & 1 \\
\hline & $19 b$ & $\begin{array}{l}\text { Give an overall interpretation of the results } \\
\text { considering objectives, limitations, results } \\
\text { from similar studies and other relevant } \\
\text { evidence. }\end{array}$ & 1 & 1 & 1 \\
\hline & $\mathrm{i}$ & An overall interpretation of the results is given & 1 & 1 & 1 \\
\hline \multirow{3}{*}{ Implications } & 20 & $\begin{array}{l}\text { Discuss the potential clinical use of the model } \\
\text { and implications for future research. }\end{array}$ & 1 & 1 & 1 \\
\hline & $\mathrm{i}$ & $\begin{array}{l}\text { The potential clinical use is discussed } \\
\text { E.g. an explicit description of the context in which the } \\
\text { prediction model is to be used (e.g. to identify high } \\
\text { risk groups to help direct treatment, or to triage } \\
\text { patients for referral to subsequent care). }\end{array}$ & 1 & 1 & 1 \\
\hline & ii & $\begin{array}{l}\text { Implications for future research are discussed } \\
\text { E.g. a description of what the next stage of } \\
\text { investigation of the prediction model should be, such } \\
\text { as "We suggest further external validation". }\end{array}$ & 1 & 1 & 1 \\
\hline Total Adherence & 21 & $\begin{array}{l}\text { Calculates the total Adherence to the TRIPOD } \\
\text { statement }\end{array}$ & 0.81 & 0.89 & 0.8 \\
\hline
\end{tabular}




\section{References}

[1] van Timmeren JE, Leijenaar RTH, van Elmpt W, Reymen B, Oberije C, Monshouwer R, et al. Survival prediction of non-small cell lung cancer patients using radiomics analyses of conebeam CT images. Radiotherapy and oncology : journal of the European Society for Therapeutic Radiology and Oncology. 2017;123:363-9.

[2] Feldkamp LA, Davis LC, Kress JW. Practical cone-beam algorithm. J Opt Soc Am A. 1984;1:612-9.

[3] de Jong EEC, Deist TM, Van Elmpt W, Jochems A, Rizzo S, Petrella F, et al. Can quantitative radiomic features describe qualitative semantic features in non-small cell lung cancer patients? Scientific reports. 2018; Submitted.

[4] Larue RTHM, van Timmeren JE, de Jong EEC, Feliciani G, Leijenaar RTH, Schreurs WMJ, et al. Influence of gray level discretization on radiomic feature stability for different CT scanners, tube currents and slice thicknesses: a comprehensive phantom study. Acta oncologica (Stockholm, Sweden). 2017;56:1544-53.

[5] van Timmeren JE, Leijenaar RTH, van Elmpt W, Reymen B, Lambin P. Feature selection methodology for longitudinal cone-beam CT radiomics. Acta Oncologica. 2017:1-7.

[6] Yip SSF, Liu Y, Parmar C, Li Q, Liu S, Qu F, et al. Associations between radiologist-defined semantic and automatically computed radiomic features in non-small cell lung cancer. Scientific reports. 2017;7:3519. [7] Collins GS, Reitsma JB, Altman DG, Moons KG. Transparent Reporting of a multivariable prediction model for Individual Prognosis or Diagnosis (TRIPOD): the TRIPOD statement. Ann Intern Med. 2015;162:55-63. [8] Moons KG, Altman DG, Reitsma JB, loannidis JP, Macaskill P, Steyerberg EW, et al. Transparent Reporting of a multivariable prediction model for Individual Prognosis or Diagnosis (TRIPOD): explanation and elaboration. Ann Intern Med. 2015;162:W1-73. 



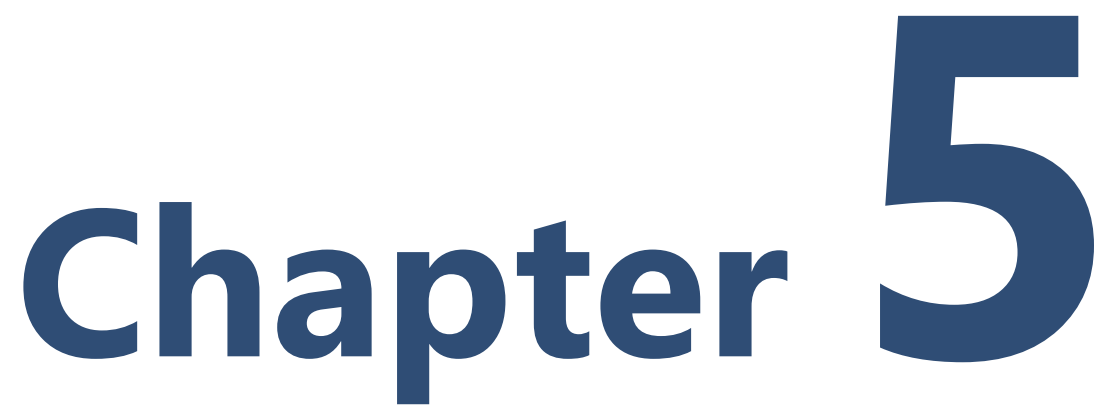

\section{Tumor regression during radiotherapy for non-small cell lung cancer patients using cone-beam computed tomography images}

Janna E. van Timmeren, Wouter van Elmpt, Dirk de Ruysscher, Bart Reymen, Olfred Hansen, Carsten Brink 


\section{ABSTRACT}

\section{Purpose}

Previous literature reported contradicting results regarding the relationship between tumor volume changes during radiotherapy treatment for NSCLC patients and locoregional recurrence free rate or overall survival. The aim of this study is to validate the results from a previous study by using a different volume extraction procedure and evaluating an external validation dataset.

\section{Methods}

For two datasets of 94 and 141 NSCLC patients, gross tumor volumes were determined manually to investigate the relationship between tumor volume regression and locoregional control using Kaplan-Meier curves. For both datasets different subgroups of patients based on histology and chemotherapy regimens were also investigated. For the first dataset $(n=94)$, automatically determined tumor volumes were available from a previous published study to further compare their correlation with updated clinical data.

\section{Results}

A total of 24 out of 94 patients were classified into separate groups when splitting the dataset based on median tumor regression calculated by the two volume extraction methods. Non-adenocarcinoma patients receiving concurrent chemotherapy with large tumor regression show reduced locoregional recurrence free rate in both datasets ( $p<0.050$ in Dataset 2 ). For Dataset 2 , the opposite behavior is observed for patients not receiving any chemotherapy, which was significant for overall survival $(p=0.010)$ but non-significant for locoregional recurrence free rate $(p=0.13)$.

\section{Conclusions}

The tumor regression pattern during radiotherapy is not only influenced by irradiation but largely depends on the delivered chemotherapy schedule, so it follows that the relationship between patient outcome and the degree of tumor regression is also largely determined by the chemotherapy schedule. This analysis shows that the relationship between tumor regression and outcome is complex, and indicates factors that could explain previously reported contradicting findings, which help to guide future studies to fully understand the relationship between tumor regression and outcome. 


\section{Introduction}

Tumor volume is a known prognostic factor for non-small cell lung cancer (NSCLC) patients [1-3]. Nevertheless, the relationship between changes in tumor volume during the course of (chemo)radiotherapy treatment remains unclear. For doseescalation purposes, toxicity estimates, and adaptive radiotherapy strategies, it is important to understand and monitor tumor behavior over the course of treatment. A recent systematic review reports that the majority of retrospective studies found a significant correlation between gross tumor volume (GTV) prior to radiotherapy and overall survival (OS) [1]. However, the results for GTV changes during treatment were contradicting. This literature review describes a total of nine studies that investigated the relationship between GTV changes during treatment and OS. Most studies have used computed tomography (CT) or ${ }^{18} \mathrm{~F}-\mathrm{FDG}$ positron emission tomography imaging to evaluate the changes [4].

Cone-beam computed tomography (CBCT) images are generally acquired daily or weekly prior to radiotherapy for treatment set-up purposes. Therefore, large amounts of images are available, which could additionally be used to investigate tumor volume changes during treatment [5]. Four studies have recently investigated the relationship between tumor volume change and patient outcome for NSCLC patients using $\mathrm{CBCT}$ images acquired during radiotherapy treatment and patient outcome. A study on 38 patients [6] showed improved overall survival for patients with a larger tumor volume reduction. Furthermore, a study on 52 patients [7] also showed that patients with a larger tumor reduction had significantly higher overall survival. On the other hand, a study on 50 NSCLC patients [8] showed worse overall survival for patients with larger tumor shrinkage. Comparable findings were found in a larger study [9]: a significantly reduced locoregional recurrence free rate (LRFR) for patients with large tumor regression during radiotherapy treatment, and worse OS for non-adenocarcinoma patients.

In the study performed by Brink et al. [9], tumor regression was estimated using an automated workflow including a deformable registration of $C B C T$ images on the corresponding planning $\mathrm{CT}$ image followed by the calculation of Jacobian determinants, from which tumor volume regression during treatment could be derived. The data was made available and the aim of the current research was to validate the finding of Brink et al. [9], namely that patients with larger tumor regression show decreased OS and LRFR. Validation was performed by investigating 1) a different method to evaluate the GTV changes during radiotherapy treatment and 2) the GTV changes in NSCLC patients comprising a validation dataset from a different institute, which may lead to a possible explanation for the contradicting findings reported regarding the relation between tumor regression and patient outcome. 


\section{Material and Methods}

\section{Patients}

Dataset 1 is a published $(n=94)$ [10] and is a subset of the original dataset of Brink et al. [9] from Odense University Hospital, Odense. The validation dataset, Dataset 2, consists of 141 stage I-IV NSCLC patients from Maastro Clinic, Maastricht, the Netherlands. The study was approved by Maastro Clinic's Institutional Review Board. Data collection was approved by each institutional ethics committee.

All patients in both datasets were treated radically with (chemo)radiation. Patients with a prior history of lung cancer, simultaneous treatment of brain metastases, stereotactic body radiation treatment (SBRT) and patients who received less than 45 Gy of radiotherapy dose were excluded from the analysis. Moreover, only patients with CBCT images acquired regularly during the course of radiotherapy treatment were included.

Patient and treatment characteristics of Dataset 1 and Dataset 2 were compared using Wilcoxon rank-sum test for continuous variables and Fisher's exact test for categorical variables. P-values below 0.05 were considered significant.

\section{Tumor segmentation}

For all patients included in this study, CBCT images of the first two fractions of radiotherapy treatment were gathered upon availability, as well as bi-monthly (Dataset 1) or weekly (Dataset 2) CBCT images were gathered upon availability. For all patients, the treatment planning $\mathrm{CT}$ images were non-rigidly registered to the corresponding CBCT images. The gross tumor volume (GTV), which was delineated on the treatment planning $\mathrm{CT}$, was used to evaluate tumor volume (regression), containing only primary tumor and not including lymph nodes. One exception was made in Dataset 1, where the entire disease site was contained in lymph node station five, and therefore the combined volume was evaluated for this patient. The deformation field was applied to the treatment planning CT delineations of the GTV to transfer the segmentations onto each CBCT image, using the open-source software 'Reggui' (http://openreggui.org). Afterwards, all CBCT delineations were manually verified and adjusted if needed. Furthermore, an experienced radiation oncologist verified final tumor segmentations for both datasets independently. We did not exclude patients for whom the tumor was positioned partly in the mediastinum, although these tumors might be more difficult to evaluate on CBCT. Possibly, tumor regression was underestimated for these patients.

\section{Volume extraction}

Tumor volume was extracted from all investigated CBCT images in both datasets using the GTV segmentation method as described above (i.e. the 'manual method'). For Dataset 1, the tumor volumes were previously derived from all available CBCT images using an automated procedure as described in [9] (i.e. the 'automatic method'). All available data points were used to perform an exponential fit through the data in order to estimate the tumor volume at day50 of treatment for Dataset 1 [9] and day40 for Dataset 2, which approximately corresponded to the end of the 
radiation treatment.

\section{Comparison of volume extraction methods}

The differences between the manual and automatic method were investigated for all time points at which a manual delineation of the GTV on the CBCT image was available in Dataset 1. Differences between absolute tumor volumes were compared using Bland-Altman plots [11]. Moreover, a linear regression between the two methods of absolute volume extraction was performed and the goodness of fit was evaluated by means of the determination coefficient $R^{2}$. The development of the tumor volume during treatment was evaluated for individual patients. The fitted volumes at the end of radiotherapy were compared using a scatter plot.

\section{Patient outcomes}

Two endpoints were investigated: overall survival (OS) and locoregional recurrence free rate (LRFR). For OS, patients still alive at the end of follow-up were considered right censored. For LRFR, patients without recurrence at death or last follow-up were considered right censored. Information on locoregional recurrence was available for all patients in Dataset 1 and for 137/141 patients in Dataset 2. For Dataset 2, followup CT scans (and/or chest X-rays) were made three months after radiotherapy and then yearly, complemented with a PET/CT when indicated. Also, at clinical suspicion of recurrence, a CT scan and/or X-ray was made, meaning that follow-up scans are performed more frequently in clinical practice. Nonetheless, the exact date of locoregional recurrence is often unknown, resulting in uncertainties in this outcome measure. For Dataset 1, follow-up was performed as described in [9]. In short, patients received a chest radiograph every three months for a period of 2 years. A CT scan was performed only in cases recurrent disease was suspected.

\section{Volume regression relation to outcome}

The relationship between relative volume estimated at the end of treatment and patient outcome was examined. Kaplan-Meier curves were calculated for the two cohorts of patients, stratified according to the median tumor regression at the end of radiotherapy. Log-rank tests were used to test for a significant split of the KaplanMeier curves.

Kaplan-Meier curves were calculated for both datasets, and for Dataset 1 also the two volume extraction methods were also compared. The previous study of Brink et al. [9] showed a significant difference between patients with small and large tumor regression during treatment in relation to LRFR and OS. The effect was most pronounced for non-adenocarcinoma patients, therefore the plots for these patients were recalculated to compare the automatic and manual volume extraction method. Note that all patient outcomes were updated for the current analysis.

The availability of two datasets with different patient characteristics than the previous study is an advantage of this study, since it allows finding an explanation for contradicting previous results. Furthermore, to be able to evaluate the relation between tumor regression and outcome in more homogeneous patient groups, subgroups were created based on histology and chemotherapy regimens. Emphasis 
was placed on non-adenocarcinoma versus adenocarcinoma patients, whichwere also separately evaluated in the previous study of Brink et al. [9]. Moreover, chemotherapy is expected to influence tumor volume changes during radiotherapy - we were able to distinguish a group of patients that did not receive any chemotherapy and a group that received concurrent chemoradiotherapy. Most patients with concurrent chemoradiotherapy already received 1 or 2 cycles prior to the start of radiotherapy, but all of these patients finished chemotherapy during or after radiotherapy. The group of patients who received induction chemotherapy was not evaluated separately in this study. The interval between completion of induction chemotherapy and commencement of radiotherapy was shown to influence tumor growth [12], so the number of patients that received induction chemotherapy in each dataset potentially influences the sign of the relationship between tumor regression and patient outcome. Although the evaluated subgroups are small, they could give an indication of important factors that influence the relationship between tumor regression and outcome.

\section{Absolute tumor volume}

Since baseline tumor volume is a well-investigated prognostic factor [3], some additional analyses were performed for completeness of the current study. The relationship between absolute tumor volume at different time points during treatment and patient outcome was investigated using Kaplan-Meier curves. Moreover, the prognostic value of tumor volume and the influence on the relationship between tumor regression and OS was evaluated.

All analyses were performed in R version 3.4.3, using packages $r m s$, survival, stats and ggplot2.

\section{Results}

\section{Patients}

Patient characteristics of both datasets are shown in Table 1. The World Health Organization (WHO) performance status and the $\mathrm{N}$-stage was significantly different between both datasets ( $p=0.004$ and $p<0.001$, respectively). The radiotherapy schemes differed between Dataset 1 and 2, but also within Dataset 2. For Dataset 1, the interval between the start of chemotherapy and the start of radiotherapy is significantly longer than in Dataset 2: the patients in Dataset 1 often started earlier with the concurrent chemotherapy to prevent patients awaiting treatment while radiotherapy planning is being performed. The distribution and range of baseline tumor volumes was similar $(p=0.23)$.

Eleven patients in Dataset 1 and 43 patients in Dataset 2 did not receive any chemotherapy prior to or during radiotherapy. The median [range] GTV for those patients was $74.9 \mathrm{~cm}^{3}$ [2.4-225] and $28.9 \mathrm{~cm}^{3}$ [0.6-309] for Dataset 1 and 2, respectively. This was not significantly different $(p=0.26)$. Also the distributions of overall stage and WHO performance status were not significantly different these subgroups: $p=0.32$ and $p=0.31$, respectively. In total, 11 patients have overall stage I/ 
Table 5.1. Patient characteristics of both datasets 1 and 2 with corresponding $p$-values to test for differences between the datasets.

\begin{tabular}{|c|c|c|c|}
\hline \multirow{3}{*}{$\begin{array}{l}\text { Gender } \\
\text { Male }\end{array}$} & \multirow[t]{2}{*}{ Dataset $1(n=94)$} & \multirow[t]{2}{*}{ Dataset $2(n=141)$} & \multirow{2}{*}{$p=0.060$} \\
\hline & & & \\
\hline & $\begin{array}{l}45(47.9 \%) \\
49(521 \%)\end{array}$ & $86(61.0 \%)$ & \\
\hline \multicolumn{3}{|l|}{ Age ${ }^{\text {remale }}$} & \multirow{2}{*}{$p=0.10$} \\
\hline $\begin{array}{l}\text { Mean } \pm \text { sd } \\
\text { Median [range] }\end{array}$ & $\begin{array}{l}67.0 \pm 8.5 \\
68[42-83]\end{array}$ & $\begin{array}{l}68.7 \pm 9.5 \\
70[45-86]\end{array}$ & \\
\hline & \multirow[t]{2}{*}{$p=0.38$} \\
\hline $\begin{array}{l}\text { Mean } \pm \mathrm{sd} \\
\text { Median [range] }\end{array}$ & $\begin{array}{l}74.2 \pm 22.9^{*} \\
76[33-135]\end{array}$ & $\begin{array}{l}76.4 \pm 23.7 a \\
78[26-130]\end{array}$ & \\
\hline \multirow{2}{*}{$\begin{array}{l}\text { WHO Performance status } \\
0 \\
1 \\
2 \\
3\end{array}$} & & & \multirow[t]{2}{*}{$p=0.0039$} \\
\hline & $\begin{array}{l}27(28.7 \%) \\
53(56.3 \%) \\
14(14.9 \%) \\
0(0 \%)\end{array}$ & $\begin{array}{l}16(11.3 \%) \\
96(68.1 \%) \\
24(17.0 \%) \\
4(2.8 \%)\end{array}$ & \\
\hline \multirow{5}{*}{$\begin{array}{l}\text { Smoking status } \\
\text { Never } \\
\text { Quit }>10 \mathrm{yr} \\
\text { Quit 1yr-10yr } \\
\text { Current / quit < 1yr } \\
\text { Unknown }\end{array}$} & & & \multirow[t]{5}{*}{$p=0.14$} \\
\hline & $1(1.1 \%)$ & $1(0.71 \%)$ & \\
\hline & $15(16.0 \%)$ & $37(26.2)$ & \\
\hline & $27(28.7 \%)$ & $\begin{array}{l}32(22.7 \%) \\
64(454 \%)\end{array}$ & \\
\hline & $0(0 \%)$ & $\begin{array}{l}64(45.4 \%) \\
7(5.0 \%)\end{array}$ & \\
\hline & \multirow[t]{5}{*}{$p=0.38$} \\
\hline 1 & $13(13.8 \%)$ & 26 (18.4\%) & \\
\hline 2 & $40(42.6 \%)$ & $45(31.9 \%)$ & \\
\hline $\begin{array}{l}3 \\
4\end{array}$ & $14(14.9 \%)$ & $25(17.7 \%)$ & \\
\hline & \\
\hline 0 & 19 (20.2\%) & 38 (27.0\%) & \multirow{4}{*}{$p<0.001$} \\
\hline 1 & $2(2.1 \%)$ & $15(10.6 \%)$ & \\
\hline 2 & $64(68.1 \%)$ & $52(36.9 \%)$ & \\
\hline$\stackrel{3}{3}$ & $9(9.6 \%)$ & 36 (25.5\%) & \\
\hline \multicolumn{3}{|l|}{ Histology } & \multirow[t]{5}{*}{$p<0.001$} \\
\hline Adenocarcinoma & $34(36.2 \%)$ & $37(26.2 \%)$ & \\
\hline $\begin{array}{l}\text { Squamous cell carcinoma } \\
\text { Large cell carcinoma }\end{array}$ & $\begin{array}{l}42(44.7 \%) \\
5(5.3 \%)\end{array}$ & $\begin{array}{l}60(42.6 \%) \\
5(3.5 \%)\end{array}$ & \\
\hline Undifferentiated & $6(6.4 \%)$ & $0(0 \%)$ & \\
\hline NOS & 7 (7.4\%) & $39(27.7 \%)$ & \\
\hline \multicolumn{3}{|l|}{ Chemotherapy } & \multirow[t]{3}{*}{$p<0.001$} \\
\hline No chemotherapy & $11(11.7 \%)$ & $43(30.5 \%)$ & \\
\hline $\begin{array}{l}\text { Neo-adjuvant } \\
\text { Concurrent }+/ \text { - neo-adjuvant }\end{array}$ & $\begin{array}{l}20(21.3 \%) \\
63(67.0 \%)\end{array}$ & $\begin{array}{l}8(5.7 \%) \\
90(63.8 \%)\end{array}$ & \\
\hline & \multirow{2}{*}{$p<0.001$} \\
\hline $\begin{array}{l}\text { Mean } \pm \text { sd } \\
\text { Median [range] }\end{array}$ & $53 \pm 15$ & $17 \pm 11$ & \\
\hline \multicolumn{3}{|l|}{$\begin{array}{l}\text { Medlan [range] } \\
\text { Received radiotherapy dose (Gy) }\end{array}$} & \multirow{2}{*}{$p<0.001$} \\
\hline $\begin{array}{l}\text { Mean } \pm \mathrm{sd} \\
\text { Median [range] }\end{array}$ & $\begin{array}{l}64.3 \pm 2.7 \\
66[60-66]\end{array}$ & $\begin{array}{l}66.4 \pm 5.6 \\
69[45-75.6]\end{array}$ & \\
\hline Planned radiotherapy scheme & & & $p<0.001$ \\
\hline & 94 (100\%) & $0(0 \%)$ & \\
\hline $30 \times 1.5$ Gy (twice daily) + 9-12×2 Gy (daily) & $0(0 \%)$ & $71(50.4 \%)$ & \\
\hline $\begin{array}{l}23-24 \times 2.75 \text { Gy (daily) } \\
38-42 \times 1.8 \text { Gy (daily) }\end{array}$ & $\begin{array}{l}0(0 \%) \\
0(0 \%)\end{array}$ & $\begin{array}{l}28(19.9 \%) \\
26(18.4 \%)\end{array}$ & \\
\hline Other & $0(0 \%)$ & $16(11.3 \%)$ & \\
\hline Interval CT - RT (days) & & & $p<0.001$ \\
\hline Mean \pm sd & $10.9 \pm 2.4$ & $7.2 \pm 1.6$ & \\
\hline Median [range] & $11[5-21]$ & $7[3-16]$ & \\
\hline $\begin{array}{l}\text { Gross tumor volume }\left(\mathrm{cm}^{3}\right) \\
\text { Mean } \pm \mathrm{sd}\end{array}$ & & & $p=0.23$ \\
\hline $\begin{array}{l}\text { Mean } \pm \text { sd } \\
\text { Median [range] }\end{array}$ & $70.3 \pm 74.8$ & $62.7 \pm 70.5$ & \\
\hline $\begin{array}{l}\text { Median [range] } \\
\text { RT = Radiotherapy, CT = Computed Tomography. }\end{array}$ & $38.4[2.1-399.2]$ & $38.3[0.61-341.4]$ & \\
\hline
\end{tabular}


II and 83 patients have overall stage III in Dataset 1. In Dataset 2, 27 have overall stage I/II, 99 have overall stage III and 15 patients have overall stage IV $(p=0.32)$.

Figure 5.1 shows the comparison between OS and LRFR for both datasets.
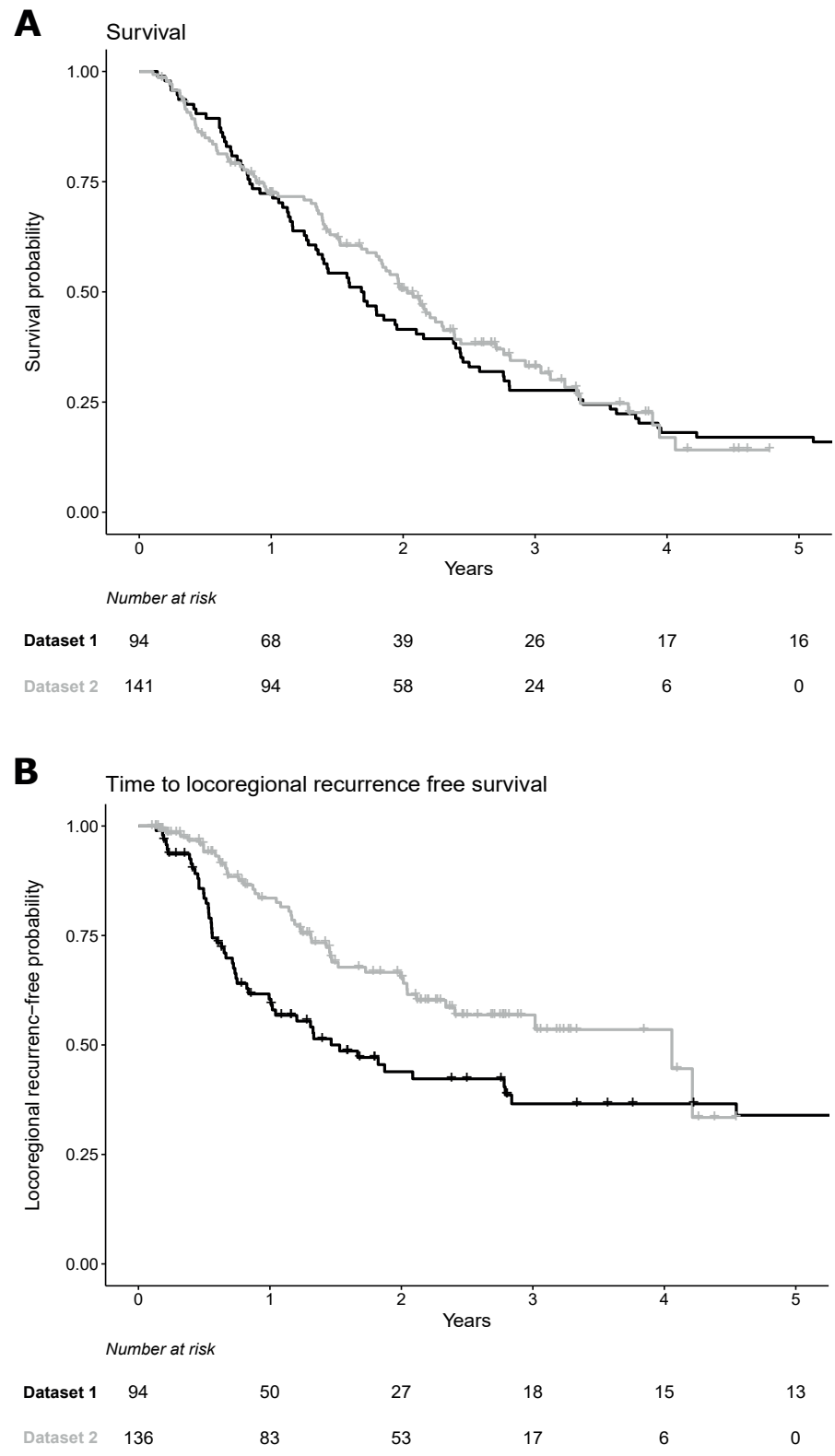

Figure 5.1. Kaplan-Meier curves to compare datasets. (A) OS and (B) LRFR for Dataset 1 (black) and Dataset 2 (gray) and corresponding indication of number of patients at risk per year. 
The median survival was 1.7 years in Dataset 1 and 2.0 years in Dataset 2 . The median time to locoregional recurrence was 1.5 years in Dataset 1 and 4.1 years in Dataset 2 . Due to the large amount of censored data for locoregional recurrence which results in uncertainties, Figure 5.1B was with the sole inclusion of patients with at least two years of follow-up (Figure S5.1).

\section{Images}

Figure S5.2 shows a histogram shown that is representing the number of CBCT images used during treatment to perform the GTV segmentations using the manual method. In total, 454 CBCT images were included in Dataset 1 and 823 CBCT images in Dataset 2, with a median [range] of 5 [4-5] and 6 [5-7] CBCT images per patient for Dataset 1 and 2, respectively.

\section{Comparison of volume extraction methods}

For each time point, the majority of automatically determined tumor volumes in Dataset 1 was estimated to be larger than manual delineations, as indicated in the Bland-Altman plots (Figure S5.3 of Supplementary Material 5C). Moreover, the difference between both methods visually increases with time during treatment. The $R^{2}$ values of the correlation between tumor volumes acquired using the manual and the automatic method at the start of treatment and at time point 2, 3, 4 and 5, were $0.98,0.98,0.98,0.95$ and 0.93 , respectively (plots not shown).

For individual patients, evaluation of tumor volume over the course of treatment was visualized for both volume extraction methods. Six examples are shown in Figure S5.4 that represents cases for which there is a high or low correspondence between the manual and automatic method.

Figure 5.2 shows the Kaplan-Meier plots for LRFR (left) and OS (right) for all non-adenocarcinoma patients of Dataset 1 for the purpose of comparing the manual and automatic method. There is a clear split in Kaplan-Meier curves for both methodologies, which was statistically significant for the manual method $(p=0.029)$ and non-significant for the automatic method $(p=0.057)$. Figure $5.2 \mathrm{C}$ shows the relative volume fitted at day50. The medians for both methods are indicated, which have also been used to split the Kaplan-Meier curves. The gray dots in Figure 5.2C represent the patients that were classified differently, being 24/94 (26\%) patients and $18 / 60$ non-adenocarcinoma patients (30\%).

\section{Absolute tumor volume}

There is a significant split in the Kaplan-Meier curves for OS for absolute tumor volume higher or lower than the median at the start of treatment $(p=0.040)$, at week $2(p=0.018)$ and at week $5(p=0.044)$ for Dataset 2 (Figure S5.5). A scatterplot with the GTV acquired from the first CBCT image plotted against relative tumor volume at the end of treatment shows a weak correlation (Figure S5.6). After combining all data, there was a significant split in OS between patients with small or large tumors for both large regression and small regression $(p=0.018$ and $p=0.030)$, whereas the split was no longer significant after selecting only non-adenocarcinoma patients who received concurrent chemotherapy (Figure S5.7). 
A

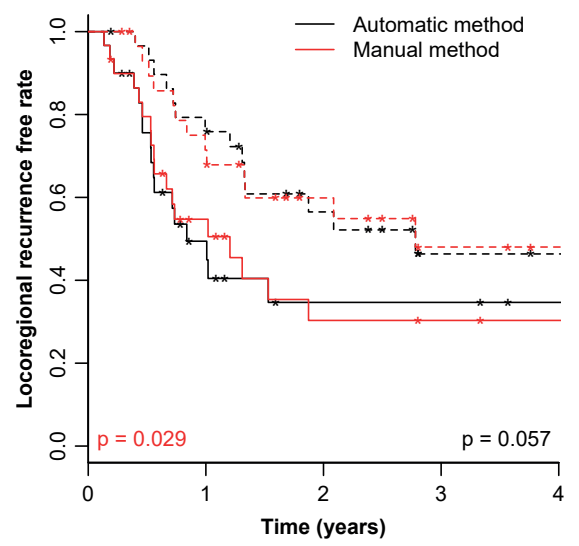

C

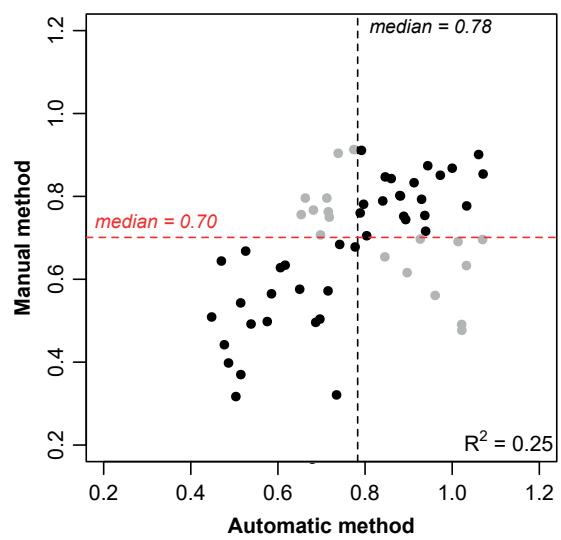

B

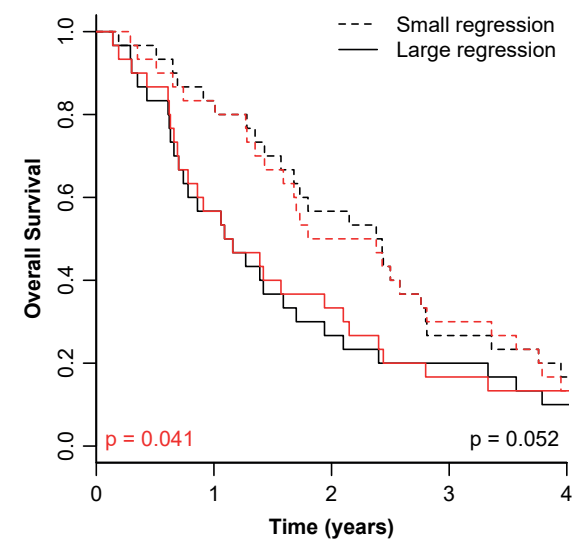

Figure 5.2. Comparison of volume extraction methods. Kaplan-Meier curves for the non-adenocarcinoma patients $(n=60)$ of Dataset 1 , that indicate a difference in LRFR (A) and OS (B) for patients with a tumor regression at the end of treatment larger or smaller than the median, indicated for the automatic (black) and manual (red) method. (C) Relative tumor volume fitted at day50 for the automatic versus manual method. Patients classified differently by both methods are represented by the gray dots.

\section{Volume regression relation to outcome}

Figure 5.3 shows Kaplan-Meier curves describing the relationship between OS and tumor regression at the end of treatment for the patients in Dataset 2. Figure 5.4 shows the relationship with LRFR. When considering all patients, no split was seen for either OS or LRFR. Nevertheless, for the group of patients who did not receive any chemotherapy $(n=43)$, patients with large tumor regression have significantly better survival than patients with relatively small tumor regression (Figure 5.4C). The split in survival curves was not caused by the distribution of overall tumor stage, T-stage or $\mathrm{N}$-stage (Supplementary Material 5E) for OS. Non-adenocarcinoma patients receiving concurrent chemotherapy ( $n=60,50$ also had chemotherapy prior to radiotherapy, 5 had only chemotherapy during radiotherapy, and for 5 no knowledge was available 
of chemotherapy prior to radiotherapy) with large tumor regression showed significantly lower LRFR. 58/60 patients in this group have overall stage III or IV.

A

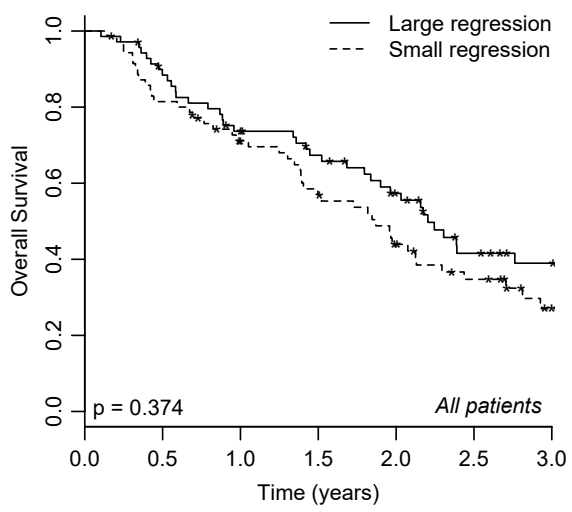

C

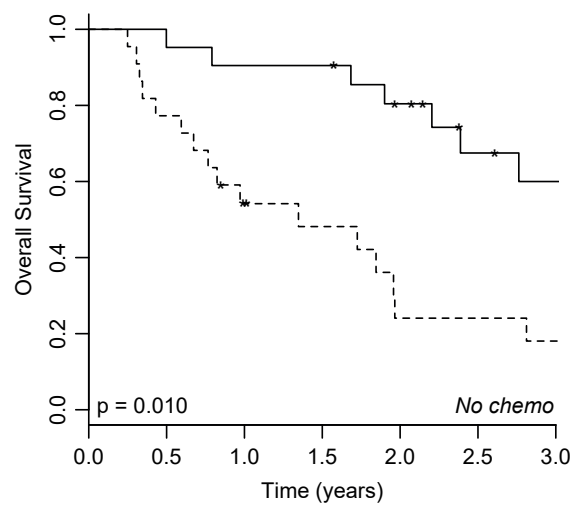

B

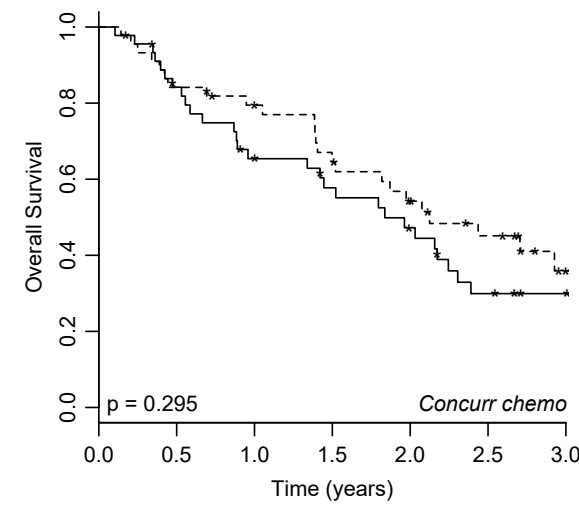

D

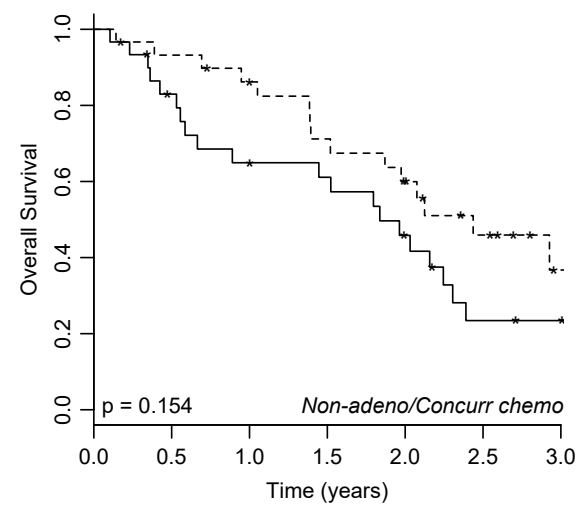

Figure 5.3. Kaplan-Meier curves for overall survival. Data from Dataset 2, split based on the median relative tumor regression at the end of treatment. (A) All patients $(n=141)$, (B) patients that received concurrent chemotherapy $(n=90),(C)$ patients that did not receive chemotherapy $(n=43)$ and (D) non-adenocarcinoma patients that received concurrent chemotherapy $(n=60)$.

In Dataset 1, a significant split was also found for the non-adenocarcinoma patients that received concurrent chemotherapy: patients with large tumor regression at the end of treatment show lower LRFR (Supplementary Material 5F). 34/36 patients in this subgroup have overall stage IIIA or IIIB. This split was not significant for OS.

\section{Discussion}

The aim of this study was to validate the finding of Brink et al. [9] that large tumor regression is related to worse OS and lower LRFR for NSCLC patients. The dataset evaluated in the previous study, as well as a second dataset from a different institute, were evaluated using a manual volume extraction method to investigate the relationship between tumor regression and OS and LRFR. Moreover, automatically 
A

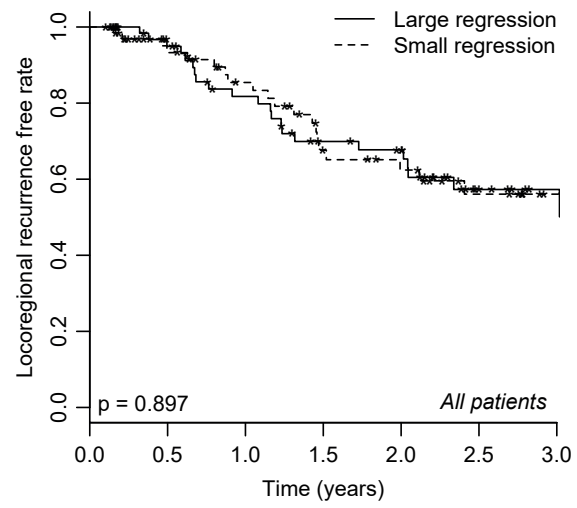

C

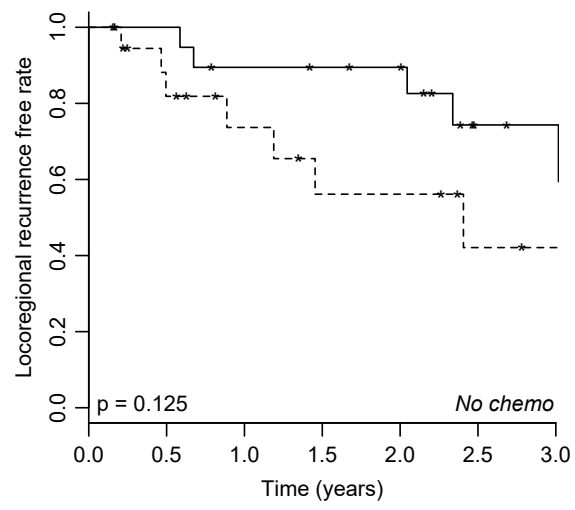

B

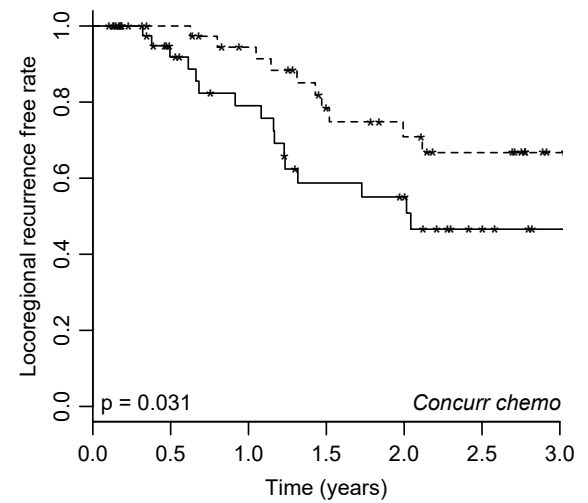

D

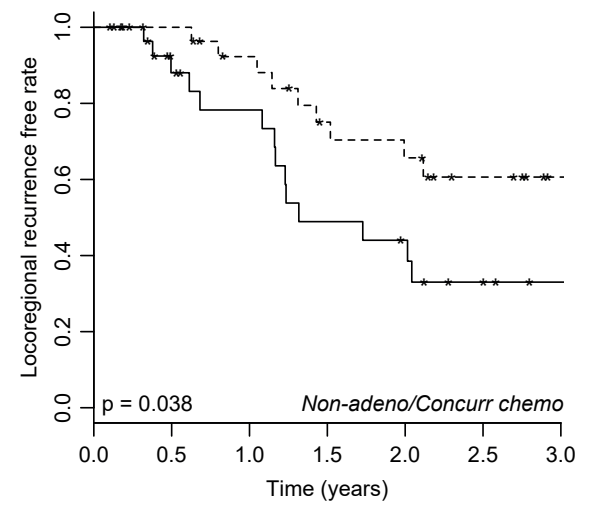

Figure 5.4. Kaplan-Meier curves for locoregional recurrence free rate. Data from Dataset 2, split based on the median relative tumor regression at the end of treatment. (A) All patients $(n=141)$, (B) patients that received concurrent chemotherapy $(n=90),(C)$ patients that did not receive chemotherapy $(n=43)$ and (D) non-adenocarcinoma patients that received concurrent chemotherapy $(n=60)$.

generated delineations on CBCT images of the previous study were available to validate the manual volume extraction method.

The observation of Brink et al. [9] was validated using a different volume extraction method (manually adjusted GTV delineations). Both the manual and the automatic method show that non-adenocarcinoma patients of Dataset 1 with large regression at the end of treatment have reduced LRFR compared to patients with smaller regression, with $p$-values of $p=0.057$ and $p=0.029$ for automatic and manual method, respectively.

Despite the discrepancy in absolute volumes between the two methods, 70/94 patients were assigned to the same tumor regression group. The differences between the volumes estimated at the end of treatment can be explained by several factors. First of all, the number of data points available to perform the fit was much 
lower for the manual method. Therefore, the accuracy of the fit can be largely influenced by one outlier from the manual method. Secondly, the automatic method estimated the volumes in general to be larger than the manual method, and this effect seems to increase with larger tumors. Potentially large regressions are not captured accurately using an automatic deformable registration method. Advantages of the automatic method is that this method is not user-dependent, as it is commonly known that there are large inter-observer variabilities in tumor segmentations [13, 14]. Moreover, the method is much less labor-intensive. The lack of a ground truth for tumor segmentations makes it difficult to specify which method is best, which in turn likely depends on the specific aim for measuring volume changes. In general, there are uncertainties associated with performing tumor segmentations on CBCT due to limited image quality. Improvements of CBCT quality and the use of $4 D C B C T$ instead of 3D could result in more accurate and robust tumor segmentations. Moreover, this would make it possible to include delineations of lymph nodes, which were not performed in the current study. The regression of lymph nodes is potentially a better indicator of treatment response.

For the overall population in Dataset 2, no splitting related to the amount of tumor regression could be found, which could be related to differences within and between datasets as shown in the Results (e.g. WHO performance status, radiotherapy schedule, chemotherapy schedule and histology). A prospective study would enable the selection of a more homogeneous patient population in order to further investigate the influence of these factors. Nevertheless, the current study was able to show that patients receiving chemotherapy prior to and during radiotherapy treatment with large tumor regression have worse OS and lower LRFR in both datasets, despite the fact that the datasets are largely heterogeneous. On the other hand, patients that did not receive any chemotherapy show the inverse relationship between tumor regression and patient outcome.

Chemotherapy type and regimen have not always been taken into account in great detail in previous published analyses. Chemotherapy regimens and their specific timing with respect to the radiation treatment could be the main explanation for the contradicting results of previous studies relating patient outcome to tumor regression during radiotherapy. Most published studies were performed on small cohorts and differences existed in the chemotherapy regimens. In the study of Elsayad et al. [8] patients received different regimens: $16 \%$ did not receive chemotherapy, $60 \%$ received concurrent chemotherapy, $10 \%$ received sequential chemotherapy and $14 \%$ received both concurrent and sequential chemotherapy. The patients in the study of Jabbour et al. [6] started with chemotherapy simultaneously with radiotherapy, whereas the patients in the current study started with chemotherapy already prior to the start of radiotherapy. In the study of Wald et al. [7], all patients but two did not receive any chemotherapy prior to the start of radiotherapy. The results of the current study show the impact of chemotherapy on the tumor volume behavior during treatment: the tumor regression pattern during chemoradiotherapy is the result of irradiation, but largely depends on the delivered chemotherapy schedule. In this study, we have not stratified for different radiotherapy dose schedules. The current 
literature shows that the overall survival of patients receiving 60Gy or 66Gy is very similar [15]. A possible influence of radiotherapy regime, e.g. hypo-fractionated radiotherapy [16], on the relationship between patient outcome and the degree of tumor regression could be of interest, but was outside the scope of the currrent paper.

Besides the influence of chemotherapy, the relationship between GTV changes during treatment and patient outcome is more pronounced for nonadenocarcinoma patients. The impact of histology has been shown before [17] and is another factor that should be taken into account in future analyses. Lastly, tumor volume at the start of treatment has a large influence on these results as well, as it was shown to be related to OS [3]. In the current analysis this parameter was also confirmed to have an influence on both survival and locoregional recurrence (Figure S5.7). Nevertheless, potential other factors are suggested in literature that could influence OS and progression free survival, such as the urokinase plasminogen activator (UPA) system [18], which was not investigated in the current work and is a limitation of this study.

To be able to monitor tumor volume regression during treatment and perform actions accordingly, it is important to fully understand the consequences of a certain behavior seen during radiotherapy. As shown in the current study, the relationship between patient outcome and tumor regression could not be generalized for an entire NSCLC patient population. Preferably, a prospective multi-centric study should be performed in which follow-up protocols are strictly controlled. This would make it possible to more accurately derive the exact relationship between tumor volume regression during treatment and patient outcome and also to define subgroups of patients that would benefit from an adjusted treatment.

Brink et al. [9] showed that patients with large tumor regression had worse overall survival and lower locoregional recurrence free rate. These findings could be confirmed using a different tumor extraction method. Moreover, a similar observation was seen in a validation dataset for a subgroup of non-adenocarcinoma patients receiving concurrent chemoradiotherapy despite the heterogeneities within and between both datasets, confirming the counterintuitive relationship between tumor regression during radiotherapy and patient outcome. An explanation for this behavior is currently unknown, but it is possible that tumor regression is correlated with tumor aggressiveness, which in turn depends on the underlying biological characteristics of the tumor (e.g. histology). This study also shows that this relationship is largely dependent on the administration of chemotherapy prior to or during radiotherapy, histology and tumor volume, hereby indicating factors that help future studies to better understand the complex relation. Larger datasets are needed to further investigate these indications and to identify more specific patient groups for which the tumor behaves similarly during treatment.

\section{Acknowledgments}

$\mathrm{CB}$ and $\mathrm{OH}$ acknowledge support from AgeCare (Academy of Geriatric Cancer Research), an international research collaboration based at Odense University Hospital, Denmark. 


\section{References}

[1] Kasmann L, Niyazi M, Blanck O, Baues C, Baumann R, Dobiasch S, et al. Predictive and prognostic value of tumor volume and its changes during radical radiotherapy of stage III nonsmall cell lung cancer : A systematic review. Strahlenther Onkol. 2018;194:79-90.

[2] Oberije C, De Ruysscher D, Houben R, van de Heuvel M, Uyterlinde W, Deasy JO, et al. A Validated Prediction Model for Overall Survival From Stage III Non-Small Cell Lung Cancer: Toward Survival Prediction for Individual Patients. Int J Radiat Oncol Biol Phys. 2015;92:935-44. [3] Ball DL, Fisher RJ, Burmeister BH, Poulsen MG, Graham PH, Penniment MG, et al. The complex relationship between lung tumor volume and survival in patients with non-small cell lung cancer treated by definitive radiotherapy: a prospective, observational prognostic factor study of the Trans-Tasman Radiation Oncology Group (TROG 99.05). Radiother Oncol. 2013;106:305-11.

[4] Roengvoraphoj O, Wijaya C, Eze C, Li M, Dantes M, Taugner J, et al. Analysis of primary tumor metabolic volume during chemoradiotherapy in locally advanced non-small cell lung cancer. Strahlenther Onkol. 2018;194:107-15.

[5] Elsayad K, Kriz J, Reinartz G, Scobioala S, Ernst I, Haverkamp U, et al. Cone-beam CT-guided radiotherapy in the management of lung cancer: Diagnostic and therapeutic value. Strahlenther Onkol. 2016;192:83-91.

[6] Jabbour SK, Kim S, Haider SA, Xu X, Wu A, Surakanti S, et al. Reduction in Tumor Volume by Cone Beam Computed Tomography Predicts Overall Survival in Non-Small Cell Lung Cancer Treated With Chemoradiation Therapy. Int J Radiat Oncol Biol Phys. 2015;92:627-33.

[7] Wald P, Mo X, Barney C, Gunderson D, Haglund AK, Bazan J, et al. Prognostic Value of Primary Tumor Volume Changes on kV-CBCT during Definitive Chemoradiotherapy for Stage III Non-Small Cell Lung Cancer. J Thorac Oncol. 2017;12:1779-87.

[8] Elsayad K, Samhouri L, Scobioala S, Haverkamp U, Eich HT. Is tumor volume reduction during radiotherapy prognostic relevant in patients with stage III non-small cell lung cancer? J Cancer Res Clin Oncol. 2018.

[9] Brink C, Bernchou U, Bertelsen A, Hansen O, Schytte T, Bentzen SM. Locoregional control of non-small cell lung cancer in relation to automated early assessment of tumor regression on cone beam computed tomography. Int J Radiat Oncol Biol Phys. 2014;89:916-23.

[10] van Timmeren JE, Leijenaar RTH, van Elmpt W, Reymen B, Oberije C, Monshouwer R, et al. Survival prediction of non-small cell lung cancer patients using radiomics analyses of conebeam CT images. Radiother Oncol. 2017;123:363-9.

[11] Bland JM, Altman DG. Statistical Methods for Assessing agreement between two methods of clinical measurement. Lancet. 1986;1:307-10.

[12] El Sharouni SY, Kal HB, Battermann JJ. Accelerated regrowth of non-small-cell lung tumours after induction chemotherapy. Br J Cancer. 2003;89:2184.

[13] Steenbakkers RJ, Duppen JC, Fitton I, Deurloo KE, Zijp L, Uitterhoeve AL, et al. Observer variation in target volume delineation of lung cancer related to radiation oncologist-computer interaction: a 'Big Brother' evaluation. Radiother Oncol. 2005;77:182-90.

[14] van Baardwijk A, Bosmans G, Boersma L, Buijsen J, Wanders S, Hochstenbag M, et al. PETCT-based auto-contouring in non-small-cell lung cancer correlates with pathology and reduces interobserver variability in the delineation of the primary tumor and involved nodal volumes. Int J Radiat Oncol Biol Phys. 2007;68:771-8.

[15] Hansen O, Knap MM, Khalil A, Nyhus CH, McCulloch T, Holm B, et al. A randomized phase II trial of concurrent chemoradiation with two doses of radiotherapy, 60Gy and 66Gy, concomitant with a fixed dose of oral vinorelbine in locally advanced NSCLC. Radiother Oncol. 2017;123:276-81.

[16] Franceschini D, De Rose F, Cozzi L, Navarria P, Clerici E, Franzese C, et al. Radical hypofractionated radiotherapy with volumetric modulated arc therapy in lung cancer : A retrospective study of elderly patients with stage III disease. Strahlenther Onkol. 2017;193:38591.

[17] Kanzaki H, Kataoka M, Nishikawa A, Uwatsu K, Nagasaki K, Nishijima N, et al. Impact of early tumor reduction on outcome differs by histological subtype in stage III non-small-cell 
lung cancer treated with definitive radiotherapy. Int J Clin Oncol. 2016;21:853-61.

[18] Ostheimer C, Evers C, Bache M, Reese T, Vordermark D. Prognostic implications of the codetection of the urokinase plasminogen activator system and osteopontin in patients with non-small-cell lung cancer undergoing radiotherapy and correlation with gross tumor volume. Strahlenther Onkol. 2018;194:539-51. 


\section{Supplementary Material}

Supplementary Material 5A - Locoregional recurrence for both datasets

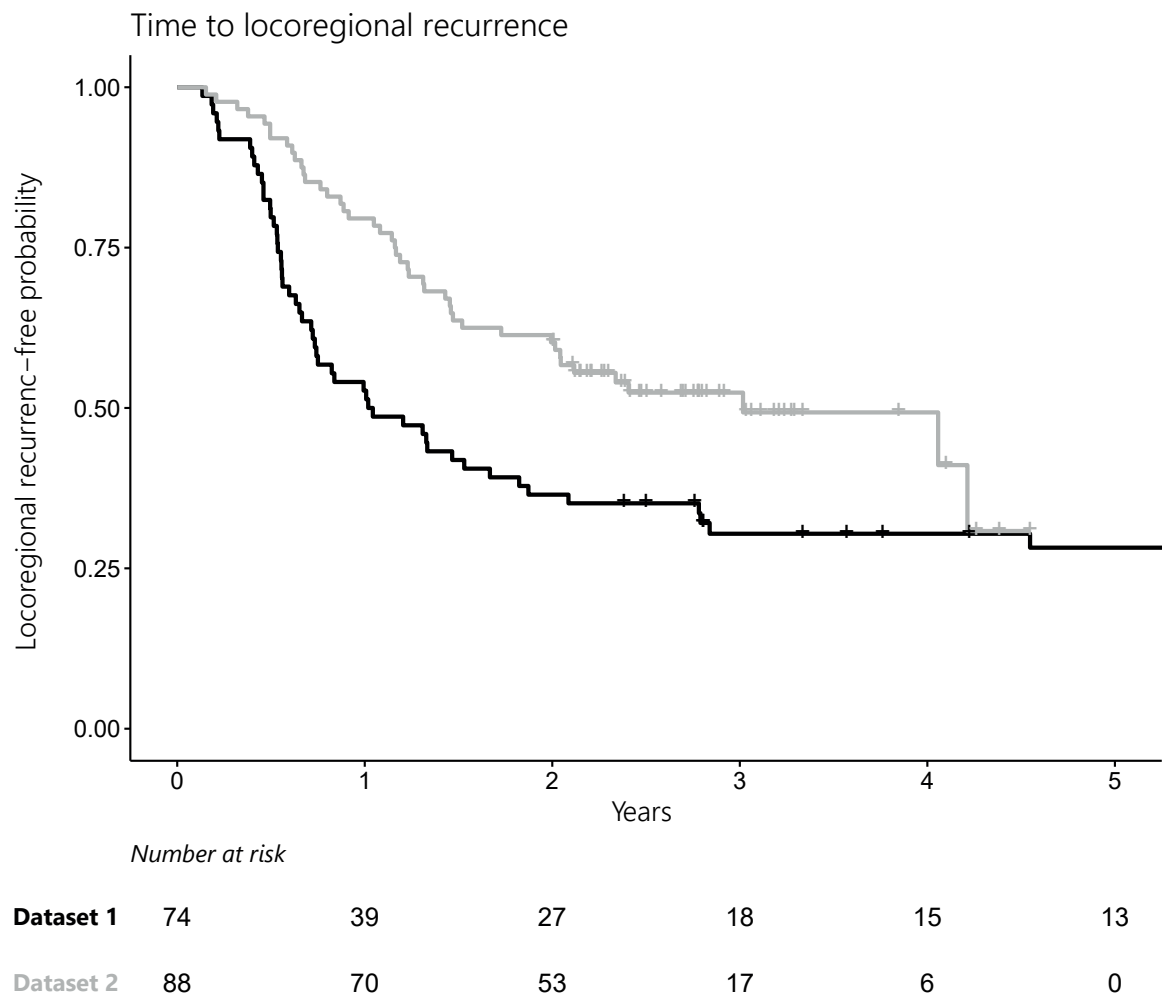

Figure S5.1. Kaplan-Meier curves of locoregional recurrence free rate for both Dataset 1 (black) and Dataset 2 (gray). 


\section{Supplementary Material 5B - Histogram of CBCT images}
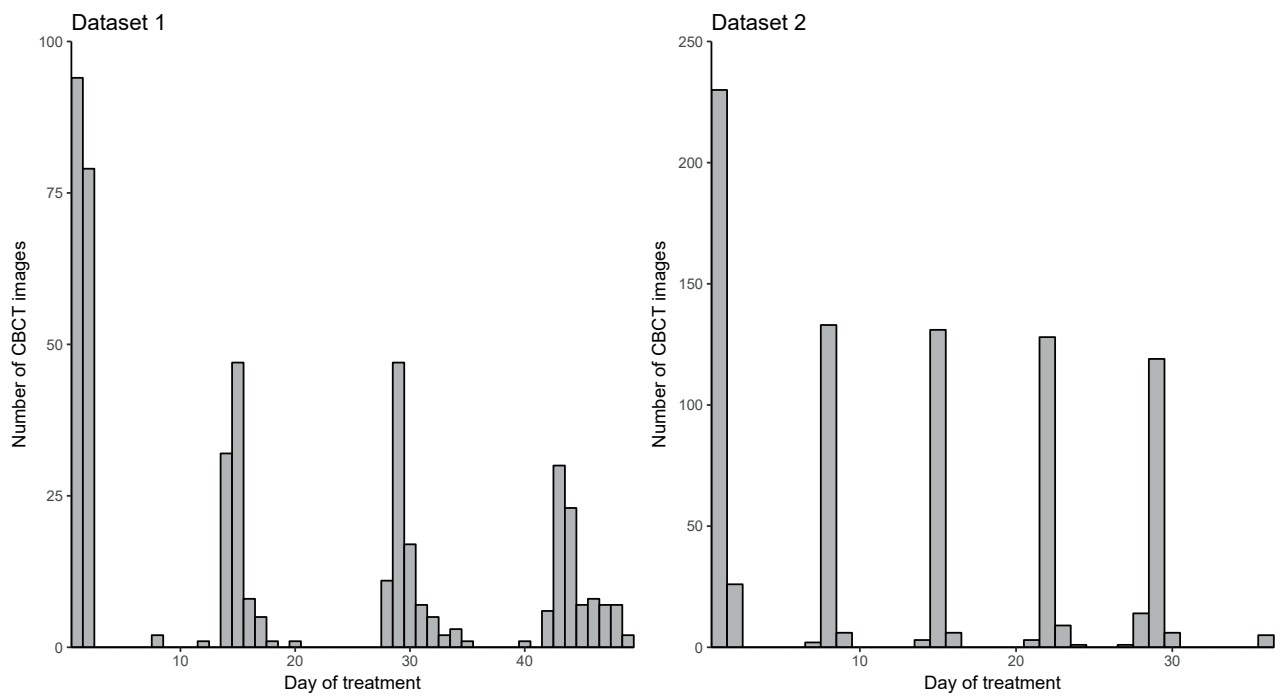

Figure S5.2. Histogram of the number of CBCT images investigated for Dataset 1 and Dataset 2 at each day of treatment. Day 1 corresponds to the CBCT image acquired at the first radiotherapy treatment fraction. 
Supplementary Material 5C - Comparison manual and automatic method
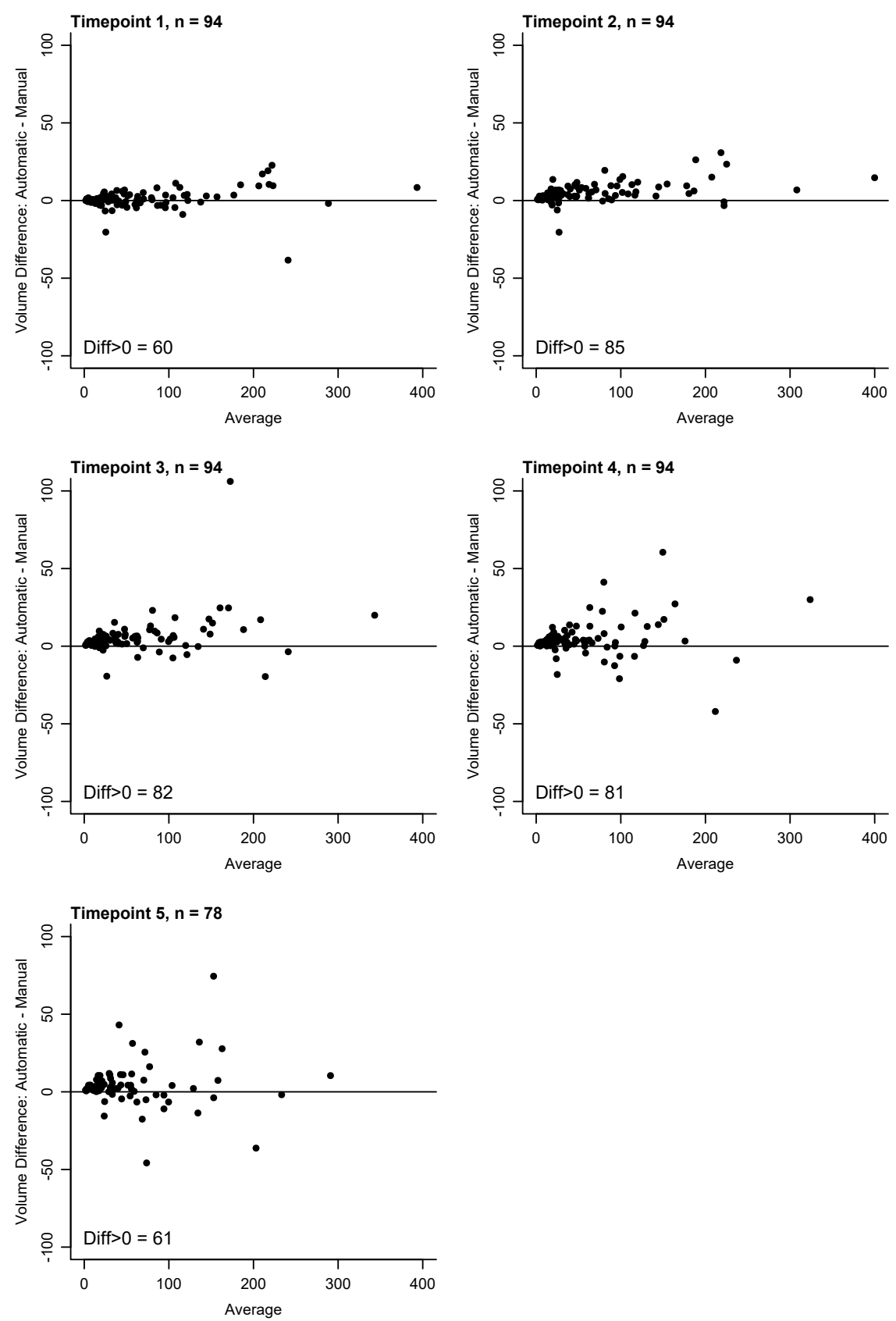

Figure S5.3. Difference in CBCT extracted tumor volumes between the automatic and the manual method, measured at five different time points. 

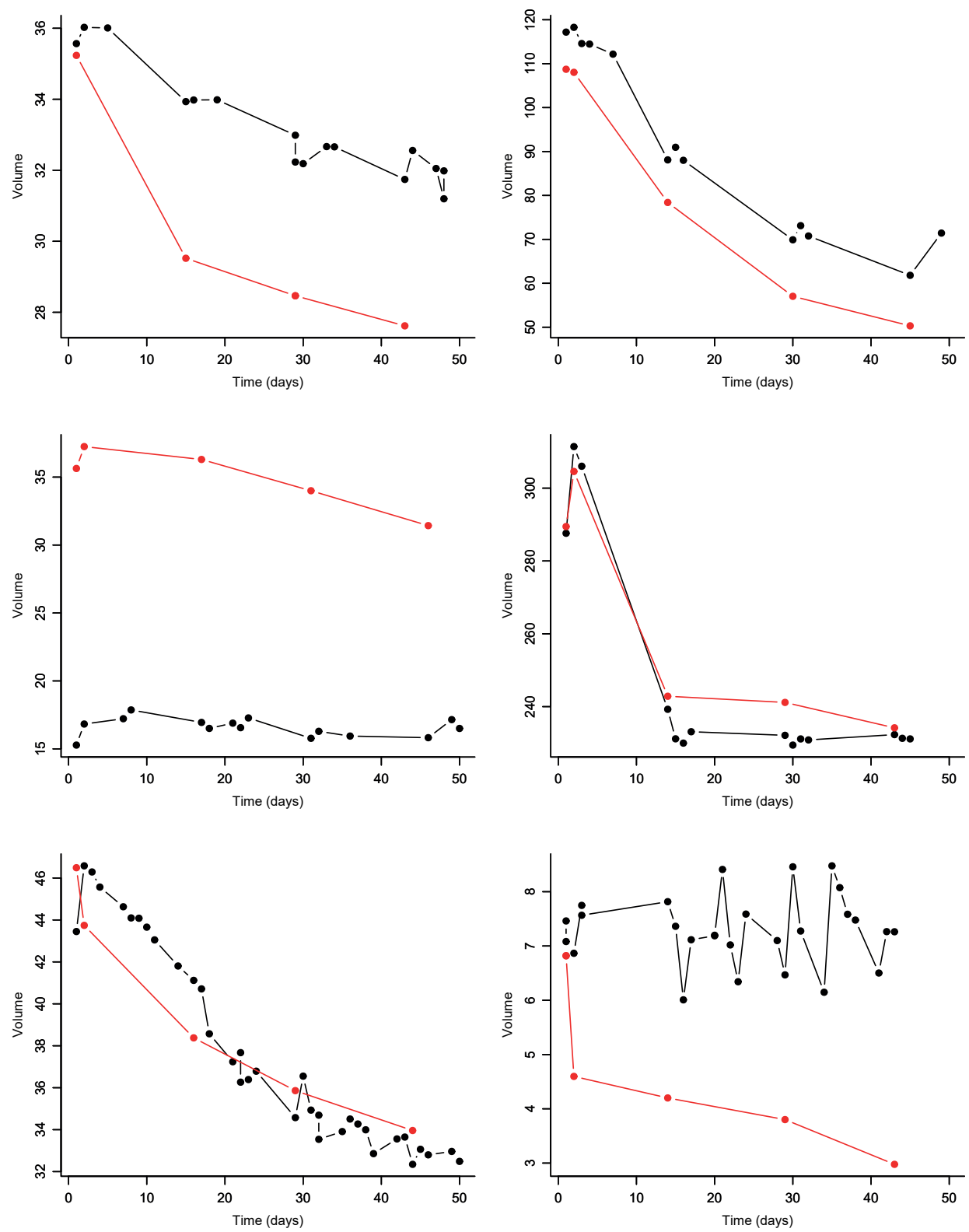

Figure S5.4. Six examples of tumor volume development during treatment for all available data points for the automatic (black) and manual (red) method. 


\section{Supplementary Material 5D - Absolute tumor volume}
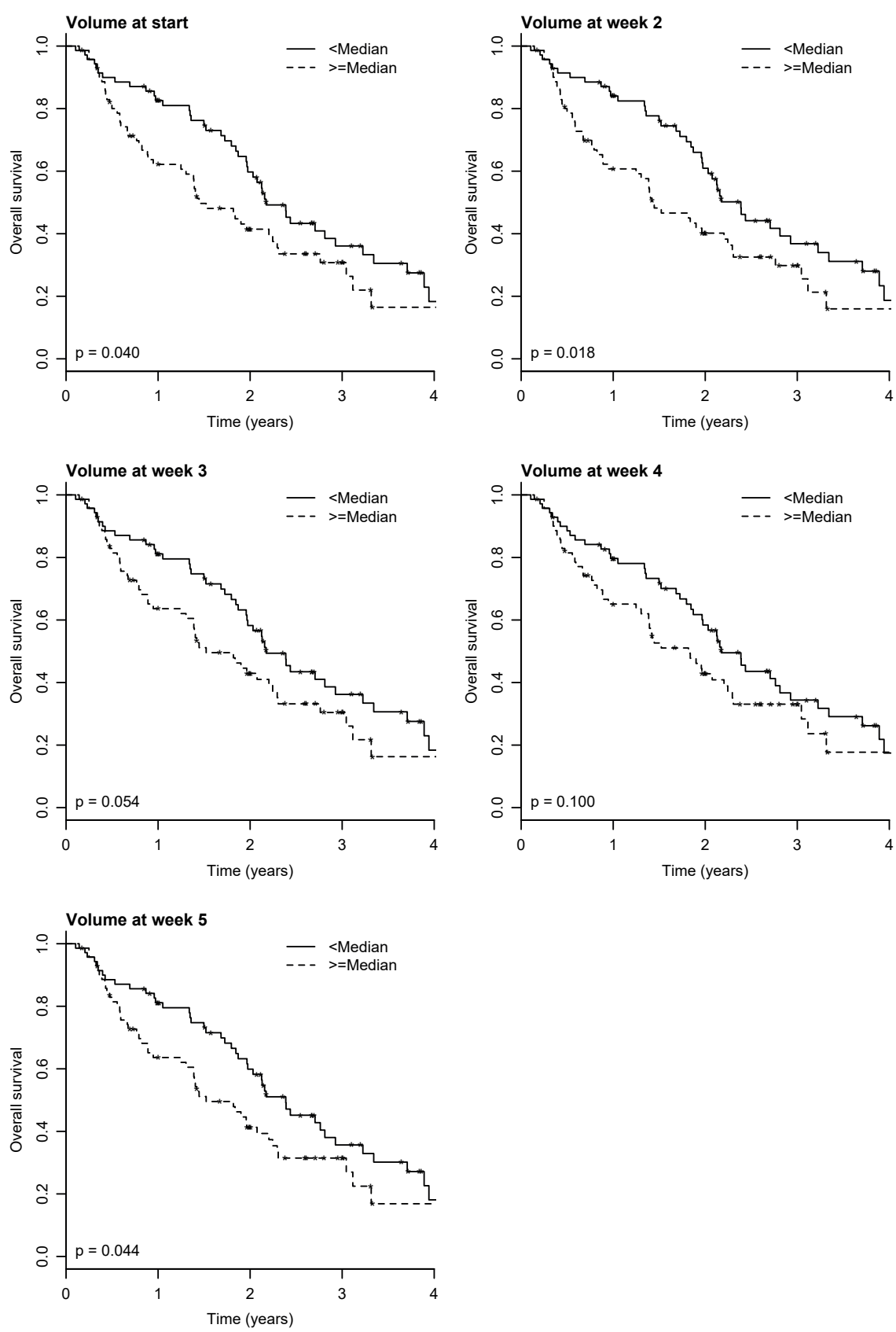

Figure S5.5. Kaplan-Meier curves showing differences in absolute tumor volume extracted from weekly CBCT images of Dataset 2 for overall survival. 

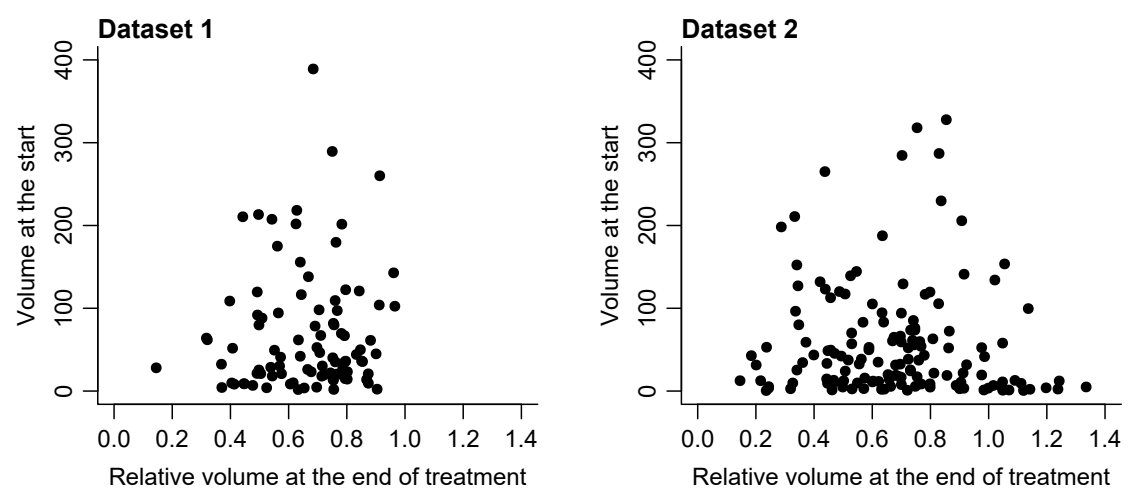

Figure S5.6. Relationship between tumor volume at the start of treatment and the relative volume at the end of treatment, estimated using the manual method for both datasets.

A

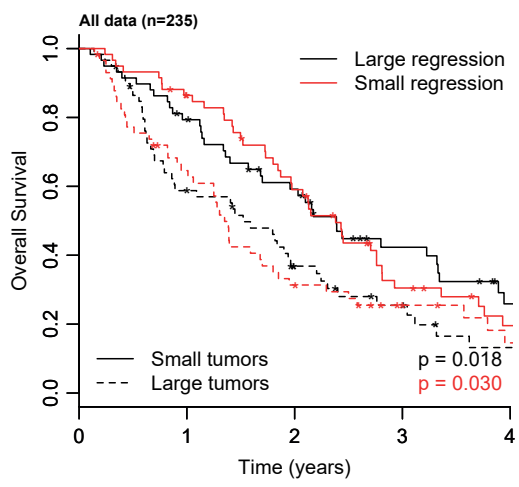

B

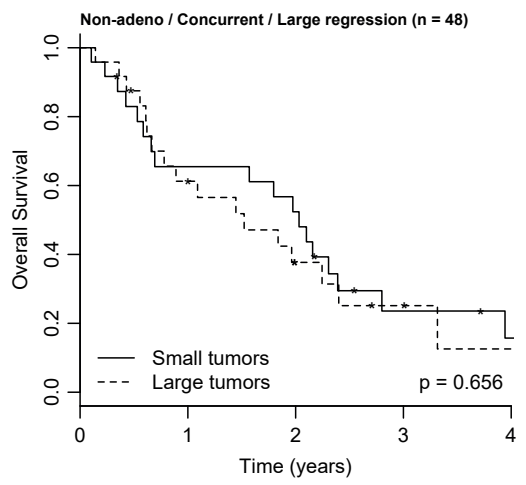

C

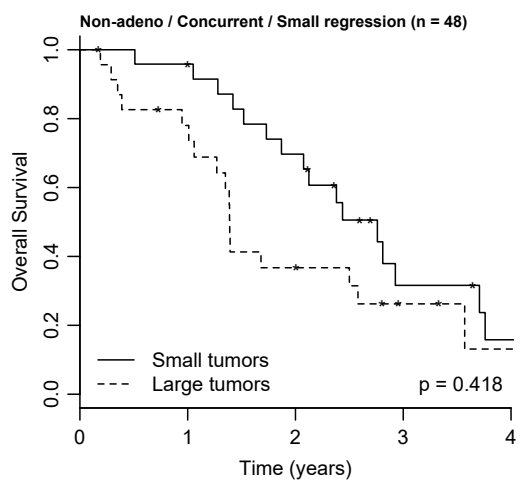

Figure S5.7. Kaplan-Meier curves showing the combined relationship of tumor volume and tumor regression with overall survival, split by median tumor volume and median volume regression. (A) All patients of Dataset 1 and Dataset 2, (B) all non-adenocarcinoma patients of Dataset 1 and 2 receiving concurrent chemotherapy with large (>median) tumor regression, (C) all non-adenocarcinoma patients of Dataset 1 and 2 receiving concurrent chemotherapy with small (>median) tumor regression. 


\section{Supplementary Material 5E - Kaplan-Meier curves for patients without chemotherapy}

A

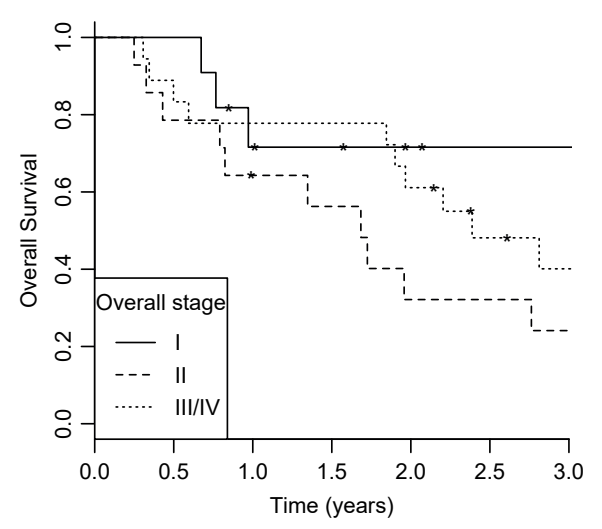

C

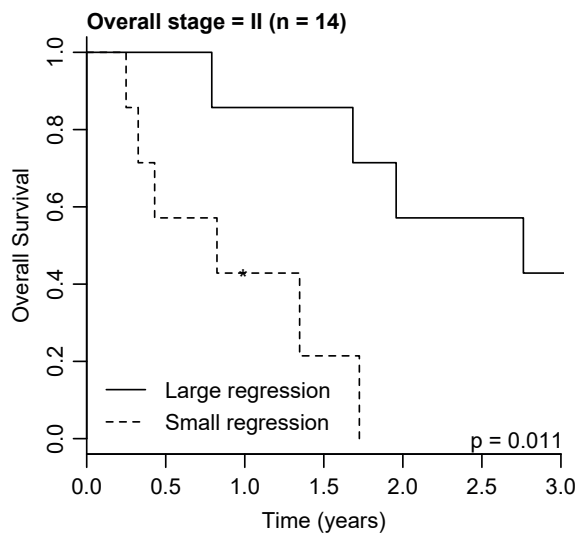

B

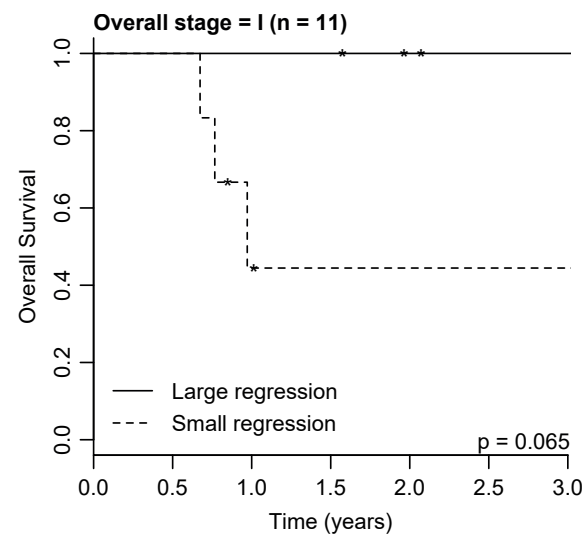

D

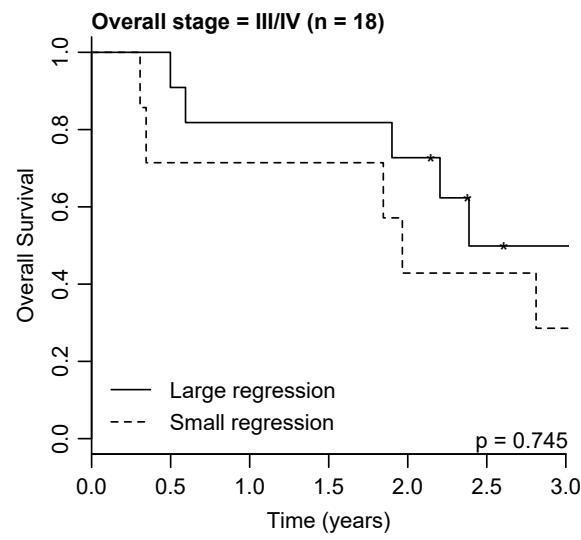

Figure S5.8. Kaplan-Meier curves for overall survival for the patients of Dataset 2 that received radiotherapy without chemotherapy $(n=43)$. (A) Different groups of overall stage, (B) 11 patients with overall stage $I$, split on the median tumor regression, (C) 14 patients with overall stage II, split on the median tumor regression and (D) 18 patients with overall stage III or IV, split on the median tumor regression. 
A

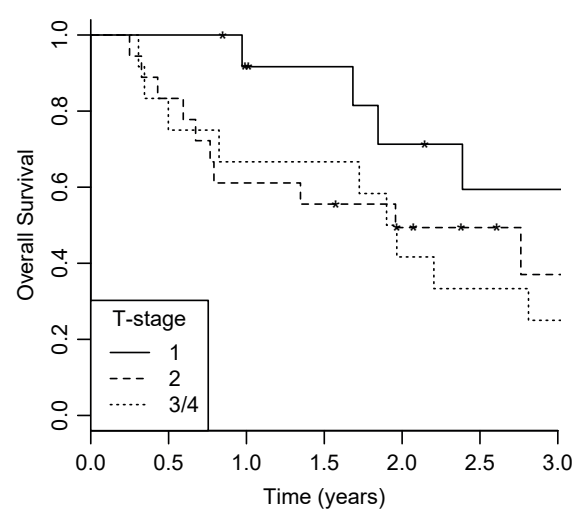

C

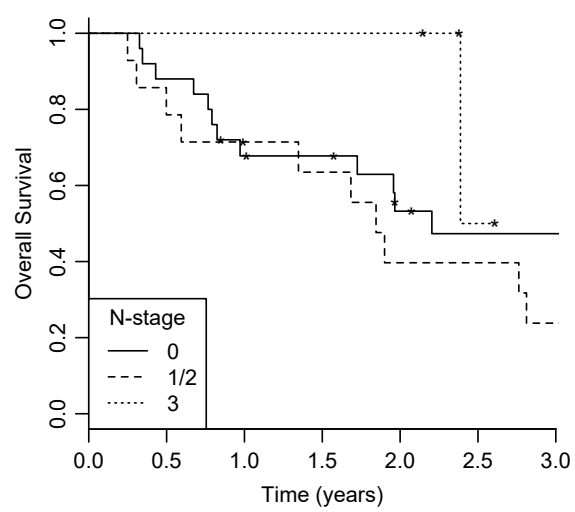

B

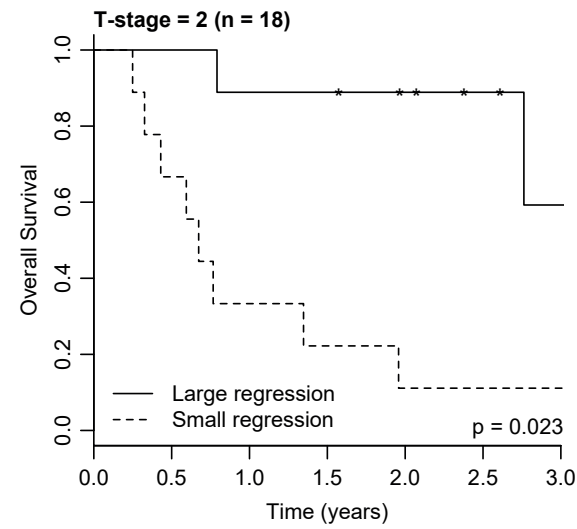

D

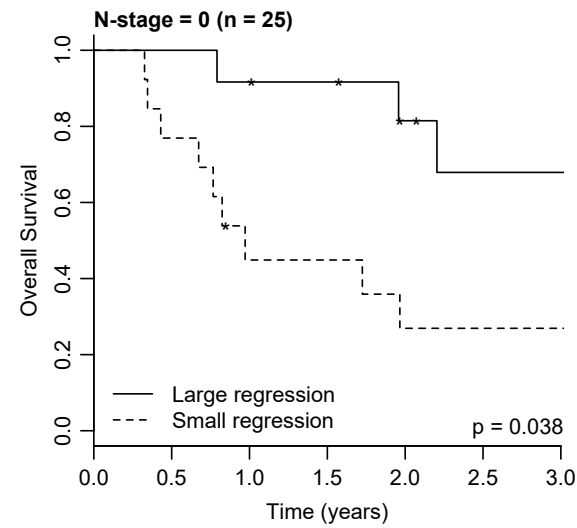

Figure S5.9. Kaplan-Meier curves for overall survival for the patients of Dataset 2 that received radiotherapy without chemotherapy $(n=43)$. (A) Different groups of T-stage, (B) 18 patients with T-stage of 2, split on the median tumor regression, (C) different groups of $\mathrm{N}$-stage and (D) 25 patients with $\mathrm{N}$-stage of 0 , split on the median tumor regression. 
Supplementary Material 5F - Kaplan-Meier curves Dataset 1: relationship between tumor regression and overall survival or locoregional recurrence

A

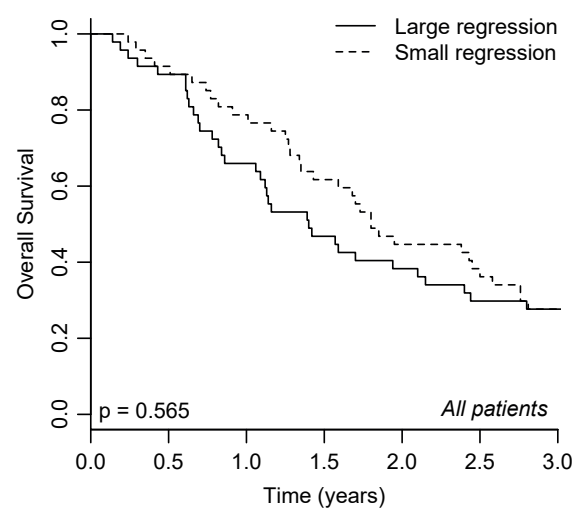

C

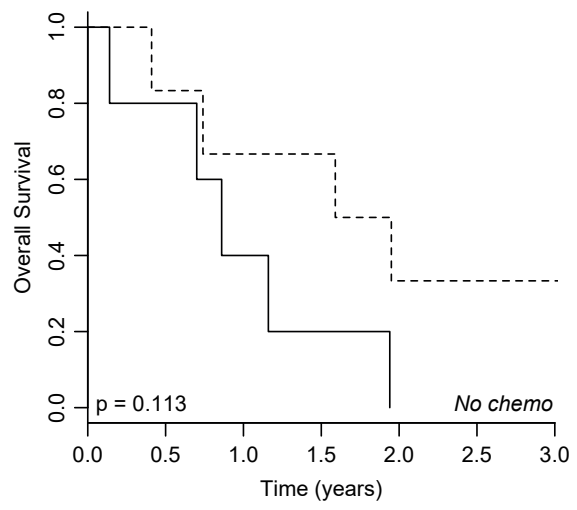

B

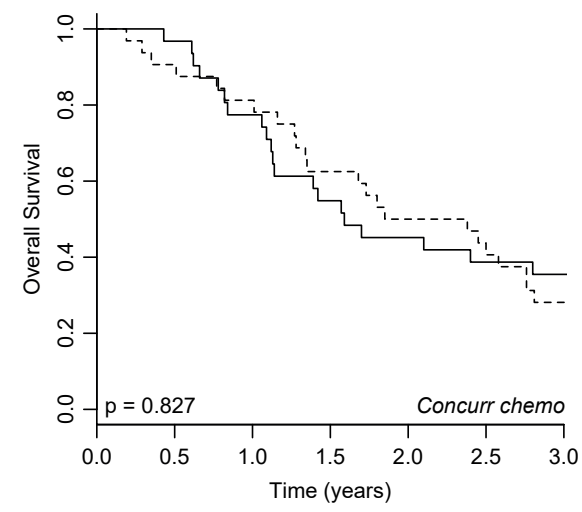

D

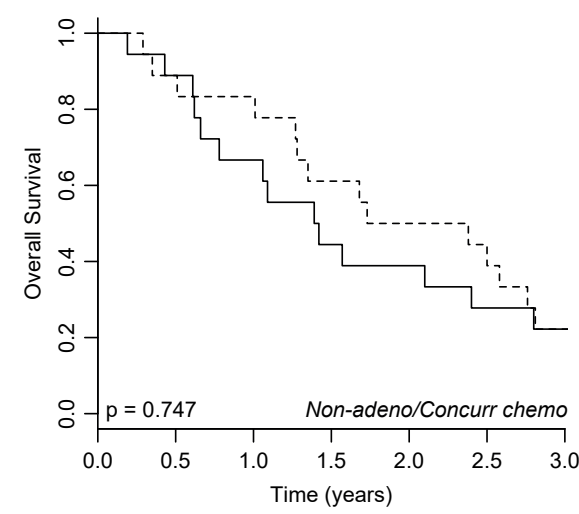

Figure S5.10. Kaplan-Meier curves for overall survival for the patients in Dataset 1, split based on the median relative tumor regression at the end of treatment. (A) All patients $(n=94),(B)$ patients that received concurrent chemotherapy $(n=63),(C)$ patients that did not receive chemotherapy $(n=11)$ and $(D)$ non-adenocarcinoma patients that received concurrent chemotherapy $(n=36)$. 
A

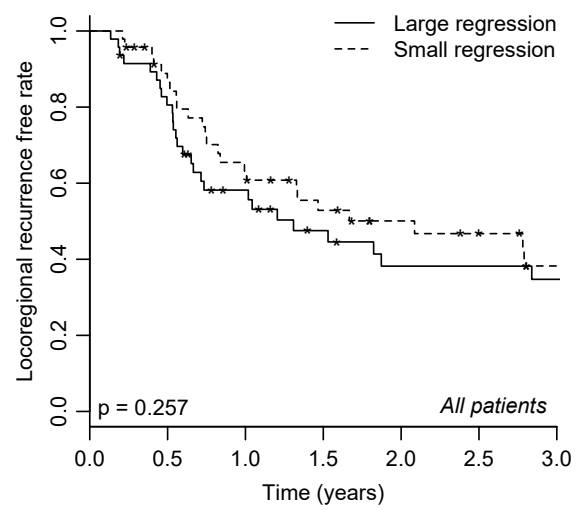

C

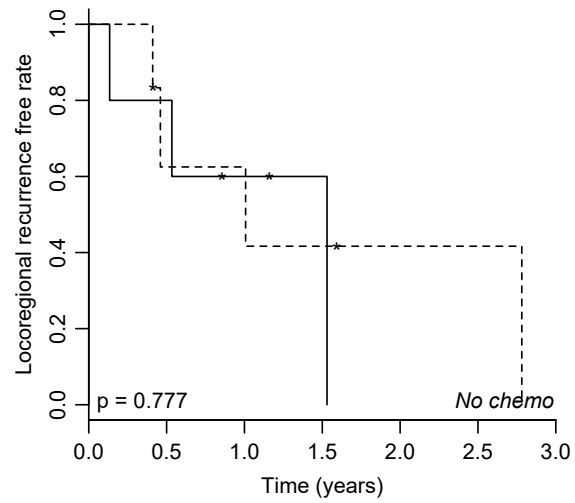

B

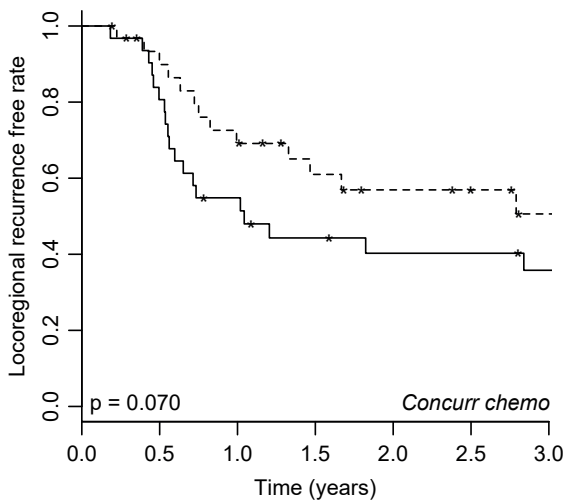

D

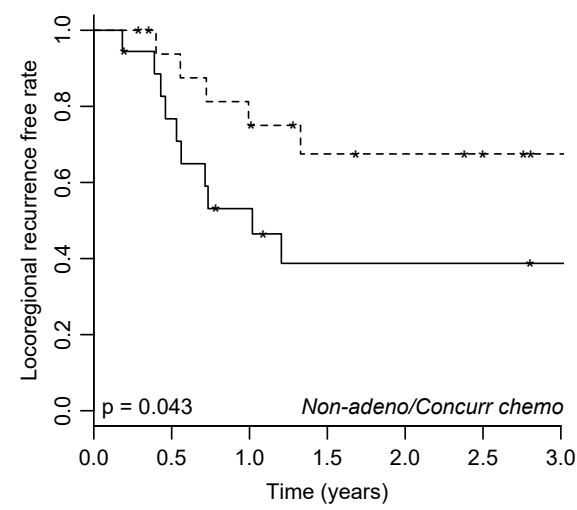

Figure S5.11. Kaplan-Meier curves for locoregional recurrence for the patients in Dataset 1 , split based on the median relative tumor regression at the end of treatment. (A) All patients $(n=94)$, (B) patients that received concurrent chemotherapy $(n=63),(C)$ patients that did not receive chemotherapy $(n=11)$ and (D) non-adenocarcinoma patients that received concurrent chemotherapy $(n=36)$. 


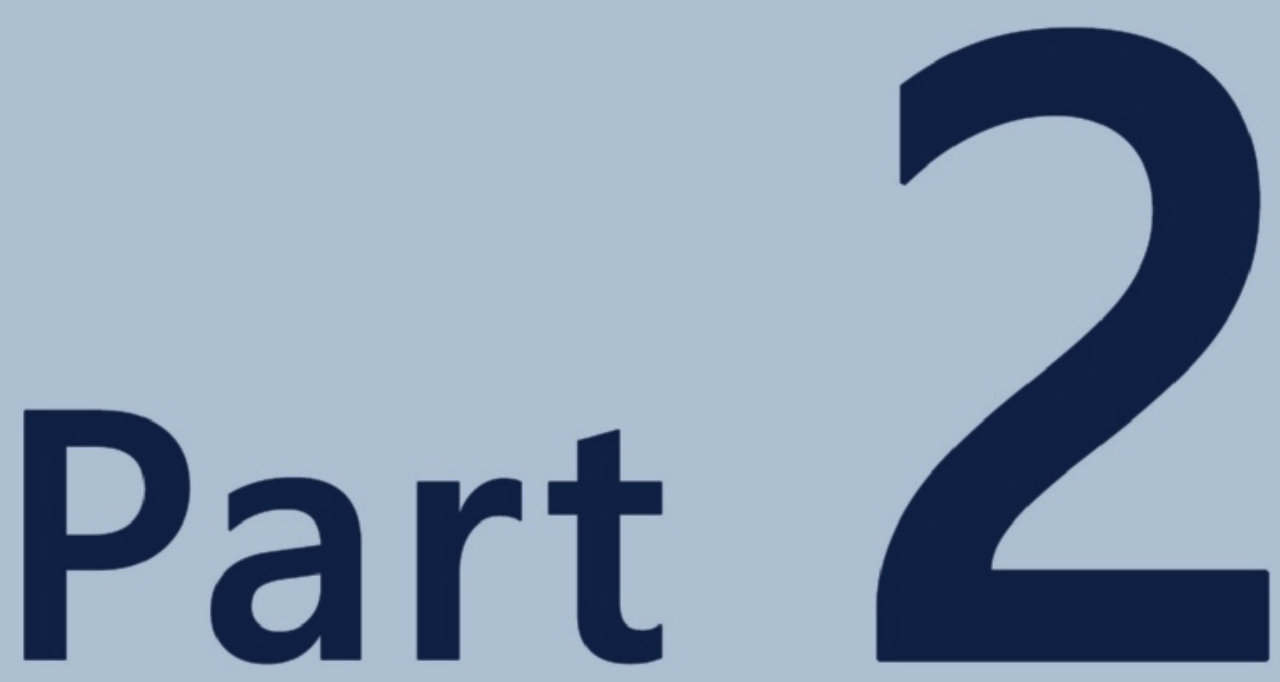



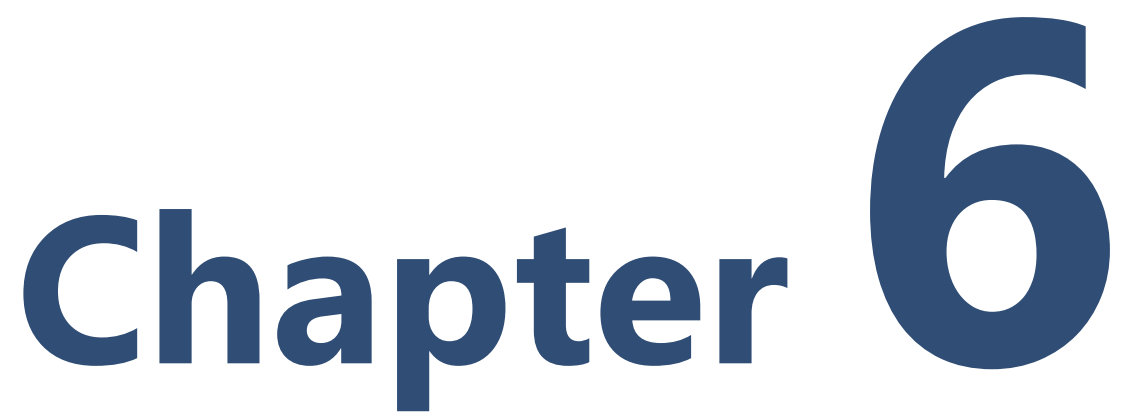

\section{Test-retest data for radiomics feature stability analysis: generalizable or study-specific?}

Authors

Janna E. van Timmeren, Ralph T.H. Leijenaar, Wouter van Elmpt, Jiazhou Wang,

Zhen Zhang, André Dekker*, Philippe Lambin*

Adapted from

Tomography. 2016 Dec; 2(4):361-365.

DOI: http://dx.doi.org/10.18383/j.tom.2016.00208

* These authors contributed equally to this work 


\begin{abstract}
Radiomics is an objective method to extract quantitative information from medical images. However, in radiomics, standardization, overfitting and generalization are major challenges to be overcome. Test-retest experiments can be used to select robust radiomic features that have minimal variation. Currently, it is unknown whether they should be identified for each disease (disease specific) or are only imaging device-specific (computed tomography [CT]-specific). Here, we performed a testretest analysis on CT scans of 40 cancer patients with rectal cancer in a clinical setting. Correlation between radiomic features was assessed using the concordance correlation coefficient (CCC). In total, only 9/542 features have a CCC $>0.85$. Furthermore, results were compared with the test-retest results on CT scans of 27 patient with lung cancer with a 15 -minute interval. Results show that $446 / 542$ features have a higher CCC for the test-retest analysis of the dataset of patients with lung cancer than for the patients with rectal cancer. The importance of controlling factors like scanners, imaging protocol, reconstruction methods and time points in a radiomics analysis is shown. Moreover, the results imply that test-retest analyses should be performed before each radiomics study. More research is required to independently evaluate the effect of each factor.
\end{abstract}




\section{Introduction}

Radiomics is the procedure of extracting features from medical images made in clinical practice, e.g. computed tomography (CT), positron emission tomography or magnetic resonance imaging [1-7]. It is the imaging features' quantitative properties that make radiomics an objective method to quantify the phenotype of the tumor. With radiomics, numerous features are extracted from each image. Therefore, one of the main pitfalls of radiomics is the risk of overfitting. This can be solved, in part, by following a strict policy of feature reduction, for example, with test-retest and multiple delineation studies $[8,9]$, to select only those robust features that provide repeatable and reproducible measurements. These studies are usually performed on imaging datasets following a fixed acquisition and reconstruction protocol and using the same methodology for feature extraction.

However, imaging features have been shown to be influenced by multiple factors, including the type of scanner, imaging settings, reconstruction parameters, delineation of the tumor, and the mathematics of extracting features. Several studies assessed the influence of different scanners and reconstruction settings [10-15]. In addition, the effect of the discretization, for example, standardized uptake value in positron emission tomography radiomics, is a necessary step before feature extraction that requires investigation [16]. Thus, it is evident that standardization is required to generalize the use of radiomics. Thus, a key and unanswered question is whether results from test-retest studies can be generalized or if these also depend on one or more of the same factors that affect imaging features.

Previously, the repeatability of radiomic features in CT imaging was tested in a "coffee-break" test-retest dataset of patients with lung cancer in which 2 scans where made on the same scanner with a 15-minute interval (RIDER dataset [17]) [4]. In such a test one assumes that the tumor has not changed and, therefore, that the radiomic feature values should be repeatable. However, it is unknown if robust features in one tumor site are the same as those in another tumor site. Moreover, it is unknown if radiomic features are also robust in a more clinical test-retest setting, in which the time between scans is in the order of days and different scanners and/ or scanner settings are used. Because of data inhomogeneity in the clinical test-retest setting, we hypothesized that test-retest results of the coffee-break scenario are not generalizable to the clinical scenario. Moreover, we expected that different features may be robust in one tumor site compared with those at another tumor site. To test this hypothesis, we examined the robustness of radiomic features obtained from CT scans of patients with rectal cancer in a clinical scenario [18] and compared this to the robustness of radiomic features for a CT lung cancer dataset in a coffee-break scenario. Moreover, the role of features' correlation with volume on the robustness of radiomic features was assessed to investigate this potential surrogate for feature robustness. 


\section{Methodology}

A clinical dataset of 40 patients with rectal cancer were included in this study. For each patient two $C T$ scans were made prior to treatment acquired from a Brilliance CT Big Bore (Philips Healthcare). The RIDER dataset of lung cancer patients was included in the study as second test-retest dataset, described elsewhere $[4,17,19$ 21]. To avoid discrepancies between methods of analysis, results of the previous study were not reused but obtained again. In total 27 patients of the RIDER dataset were included in the final analysis (data of 5 patients could not be retrieved or had to be excluded because of technical problems). CT parameters are summarized in Table 6.1.

We investigated the test-retest stability on both datasets of a total of 542 radiomic features, divided into the following 4 groups: (1) Tumor intensity $(n=15),(2)$ shape $(n=11)$, (3) texture $(n=44)$ and (4) wavelet $(n=472)$. Mathematical descriptions of all features are published elsewhere [4].

Table 6.1. CT scan parameters for both datasets.

\begin{tabular}{lll} 
& Rectum dataset & RIDER dataset \\
\hline Manufacturer & Philips Healthcare & GE Healthcare \\
Acquisition type & Helical & Helical \\
Tube voltage & $120 \mathrm{kVp}$ & $120 \mathrm{kVp}$ \\
Tube current & 250 or $350 \mathrm{mAs}$ & range $165-549 \mathrm{mAs}$ \\
Slice Thickness & $5 \mathrm{~mm}$ & $1.25 \mathrm{~mm}$ \\
Pixel spacing & range $0.98-1.25 \mathrm{~mm}$ & range $0.51-0.91 \mathrm{~mm}$ \\
Pixels & $512 \times 512$ & $512 \times 512$
\end{tabular}

\section{Statistical Analysis}

The concordance correlation coefficient (CCC) was used to examine agreement between radiomic features derived from the test-retest scans [22]. CCCs were calculated for the RIDER dataset and the rectum dataset and each feature group was compared. For all features in the rectum dataset, we also assessed the correlation with volume using a simple linear regression and $R^{2}$ as correlation parameter. Statistical analysis was performed using the package psych in R (version 3.2.3).

\section{Results}

For each feature group, CCC values between the RIDER dataset and the clinical dataset were compared. Results are shown in Figure 6.1. This analysis was also performed after resampling all data into images with an isotropic voxel size of $3 \mathrm{~mm}$ before feature extraction. These results are shown in Supplemental Data. In total, for $446 / 542$ features (82.3\%) the data points are on the left side of the diagonal, meaning that they have a higher CCC in the RIDER dataset than in the clinical dataset. This is $36 / 44$ features (81.8\%) for the 'Texture' group, 11/11 features (100\%) for 'Shape' group, 10/15 features (66.7\%) for 'Tumor Intensity' group, and 389/472 features (82.4\%) for 'Wavelet' group. 
A

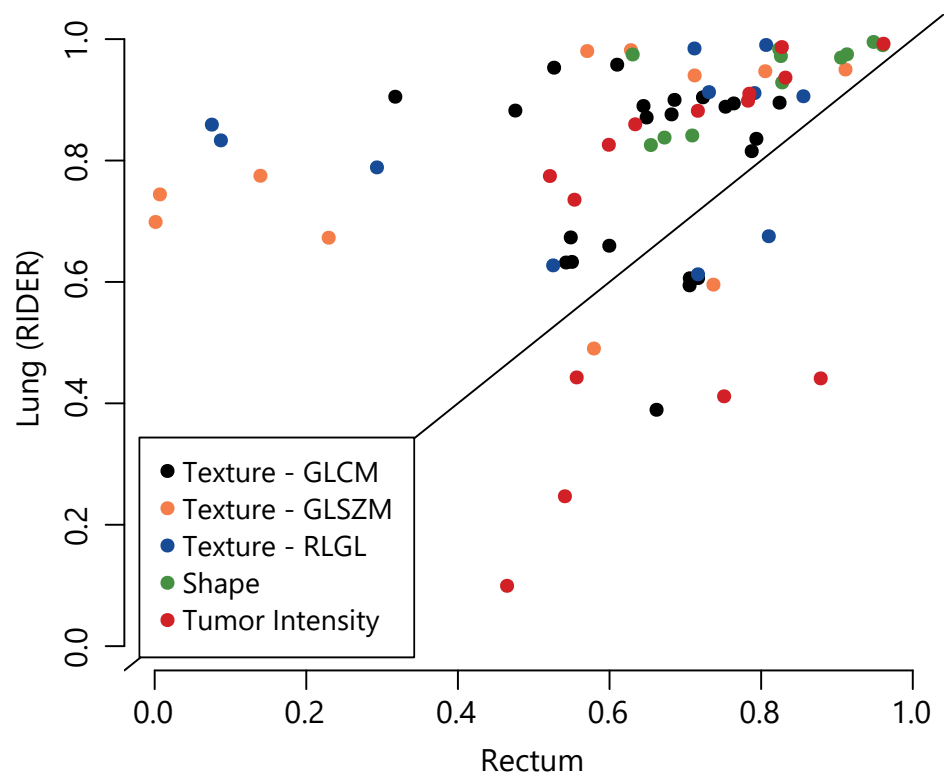

B

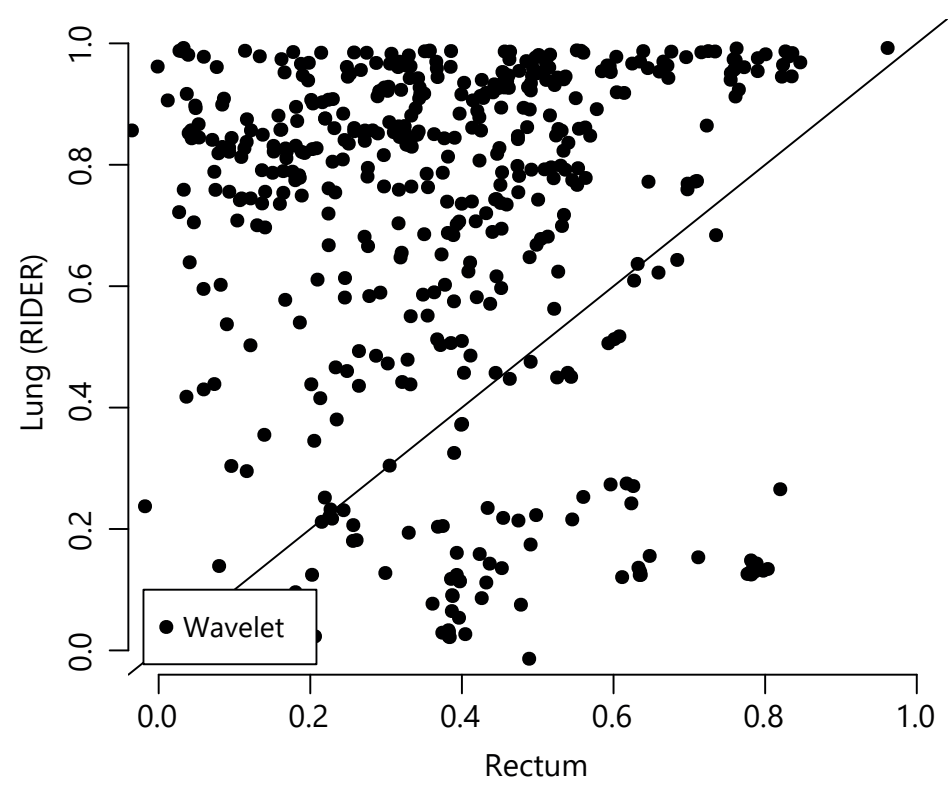

Figure 6.1. Comparison between stability of radiomic features derived from the lung cancer data set (RIDER) and the rectal cancer data set, with feature groups "Texture," "Shape," and "Tumor Intensity" (A) and "Wavelet" (B). Gray-level co-occurrence matrix (GLCM), gray-level size zone matrix (GLSZM), and run-length graylevel (RLGL). 
Shape features were the most reproducible ( $100 \%$ CCC $>0.6$ for both sets), followed by the gray-level co-occurrence matrix features of the 'Texture' group (91\% and $68 \%$ $C C C>0.6$ for the RIDER set and the clinical set, respectively). Wavelet features seemed to be least reproducible in the clinical setting. When using a cut-off CCC of 0.85 , only 9 features were reproducible in the clinical scenario-derived rectal cancer test-retest set, whereas 234 features were reproducible in the coffee-break lung cancer testretest set; 8 of these feature overlapped.

Thirty-six of the 100 most stable features found in the RIDER dataset (range $C C C=0.951-0.995)$ were also present in the 100 most stable features of the clinical dataset, which is unlikely to be coincidental $(p<0.0001)$. The overlapping features included $7 / 11$ 'Shape' features (63.6\%), 1/15 'Tumor intensity' features (6.7\%), 2/44 'Texture' features (4.6\%) and 26/472 'Wavelet' features (5.5\%). The 100 most stable features of both datasets are displayed in Table S6.1.

For all features of the clinical rectum dataset, we assessed the correlation with volume using the coefficient of determination $\left(R^{2}\right)$ of a simple linear regression. Features extracted from the test scan were used for this analysis. Results are shown in Figure 6.2. The $y$-axis represents the robustness of all features, and the $x$-axis represents the $R^{2}$ of the correlation with volume.

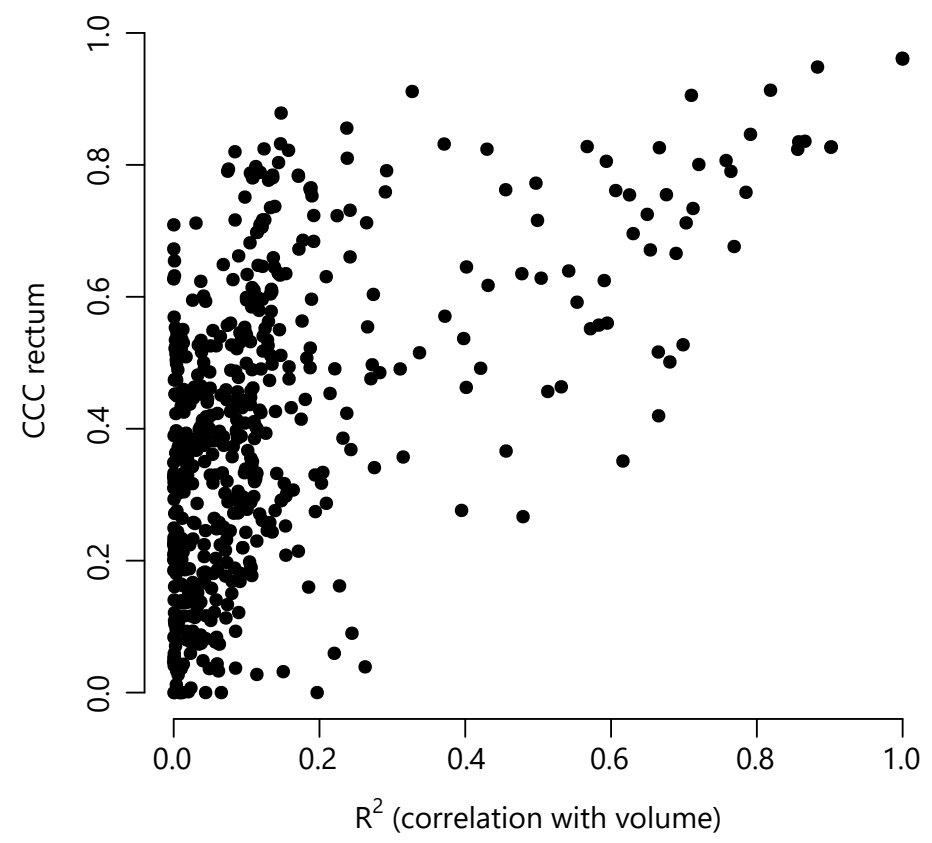

Figure 6.2. Robustness of radiomic features in the rectum data set ( $y$-axis) versus the features' correlation with volume ( $x$-axis).

In total, 4 features had an $\mathrm{R}^{2}>0.9$, meaning a high correlation with volume. These were 'Tumor intensity - Total energy' $\left(R^{2}=1.0\right)$, 'Wavelet - LLL Tumor intensity Total energy' $\left(R^{2}=1.0\right)$, 'Tumor intensity - Energy' $\left(R^{2}=0.90\right)$ and 'Wavelet - LLL Tumor 
intensity Energy' $\left(R^{2}=0.90\right)$. Features were generally more robust with an increase in correlation with volume.

\section{Discussion}

In comparison to the RIDER dataset with the "coffee-break" scenario, features were much less stable in a more clinical scenario of repeated imaging. However, the results show that a subset of stable features derived from the RIDER dataset is also stable in the clinical scenario. There is particularly high correspondence between the 'Shape' features, whereas correspondence between 'Wavelet' features is low. The low correspondence between the clinical dataset and the RIDER dataset can be because of many factors, including the disease site, time between scans and the CT settings. Slice thickness is one of the factors that differs considerably between the CT scans of patients with rectal cancer $(5 \mathrm{~mm})$ and the lung cancer patients $(1.25 \mathrm{~mm})$. However, resampling the data into isotropic voxels of $3 \mathrm{~mm}$ prior to feature extraction did not substantially change the results. Slightly more features were robust after resampling, but the difference in stability between both datasets is similar. The stability of radiomic features may be disease-specific and a function of the time between scans, but we are not able to eliminate the effect of protocol differences between both datasets. If we could show that numerous features are robust in a clinical scenario and that these features correspond to the robust features found by a 'real' test-retest analysis (i.e. with a very short time interval), this could have implied that one extensive test-retest study could provide a set of stable radiomic features that could be used in any further radiomics analysis. However, as this appeared to be not the case, we emphasize, in this study, the importance of a proper test-retest study in each scenario with tight control of influencing parameters and further investigation of the influence of factors like hardware, scan acquisition and reconstruction settings, tumor delineation, and the mathematics of extracting features.

Volume was one of the robust features in the clinical test-retest analysis $(C C C=0.96)$. Out of the 100 most stable features in the rectum dataset, 4 other features highly correlated with volume. Moreover, when the correlation with volume was increasing, features were also more robust. This could partly explain the stability of these features. In total, only a small portion of radiomic features, 9/542, was robust in the clinical test-retest analysis (CCC $>0.85$ ), which suggested that differences between the 2 CT scans influence the stability of the radiomic features. Various causes may explain this. For example, for a subset of 17 patients, a different tube current was used for the test and retest scan-250 mAs and $350 \mathrm{mAs}$ or vice versa-leading to different noise properties in both scans. Another factor that could have reduced the robustness of radiomic features is the voxel resolution due to different reconstruction diameters, which was not constant over all CTs. Moreover, the number of days between the test and retest scan ranged from 5 to 19 (median 8), which is not comparable to the 15-minute interval in the RIDER dataset. It cannot be excluded that in this time period, the tumor changes sub-clinically and that this change is detected by radiomics. Therefore, if this dataset is used for test-retest analysis as shown in this study, it would mean we discard features that are actually informative. If such rapid changes occur in an untreated tumor, future protocols should closely 
control the timing of the $\mathrm{CT}$ (e.g. the time between $\mathrm{CT}$ and treatment) so that the scan is taken at the time when it is most informative. When prognostic information is derived from image features in a radiomics study, one should be aware of changes in a tumor and it is advisable to avoid using features that are not robust in a test-retest study, in which the time interval between scans is large. Future studies, where the predictive performance for the outcome of interest is investigated, of features taken at different time points before treatment are necessary to address these considerations.

Several factors could have reduced the robustness of radiomic features. In this study, the radiomics methodology is controlled, but the hardware, scan acquisition and reconstruction settings, disease site, and scan time interval are different. This study shows that test-retest results are not generalizable, and there is a dependency on one or a combination of these factors. Ideally, one should alter only one of these factors at a time for testing the influence. However, the number of patients in a testretest analysis is usually low. Although in this study we had numerous patients for a test-retest study $(n=40)$, the dataset is very small to be able to analyze subsets in order to test these effects. Phantom measurements could play an important role in accurately assessing the potential influence of differences in scanners, reconstruction methods and imaging settings on radiomic features and may allow for a calibration of the feature values. To conclude, we emphasize that it is important to tightly control all aforementioned factors in a radiomics study. Nevertheless, to minimize the risk of using unstable and unreproducible features in a radiomics analysis, it is advisable to perform treatment site-specific and time-, scanner- and imaging protocol-controlled test-retest analyses.

\section{Acknowledgments}

Authors acknowledge financial support from ERC advanced grant (ERC-ADG-2015, nº 694812 - Hypoximmuno) and the QulC-ConCePT project, which is partly funded by EFPI A companies and the Innovative Medicine Initiative Joint Undertaking (IMI JU) under Grant Agreement No. 115151. This research is also supported by the Dutch technology Foundation STW (grant $n^{\circ}$ 10696 DuCAT \& $n^{\circ}$ P14-19 Radiomics STRaTegy), which is the applied science division of NWO, and the Technology Programme of the Ministry of Economic Affairs. Authors also acknowledge financial support from the EU 7th framework program (ARTFORCE - $n^{\circ} 257144$, REQUITE - $n^{\circ}$ 601826), SME Phase 2 (EU proposal 673780 - RAIL), the European Program H2020-PHC-2015 (BD2Decide - PHC30-689715), Kankeronderzoekfonds Limburg from the Health Foundation Limburg, Alpe d'HuZes-KWF (DESIGN) and the Dutch Cancer Society. 


\section{References}

[1] Lambin P, Rios-Velazquez E, Leijenaar R, Carvalho S, van Stiphout RG, Granton P, et al. Radiomics: extracting more information from medical images using advanced feature analysis. Eur J Cancer. 2012;48:441-6.

[2] Kumar V, Gu Y, Basu S, Berglund A, Eschrich SA, Schabath MB, et al. Radiomics: the process and the challenges. Magn Reson Imaging. 2012;30:1234-48.

[3] Lambin P, van Stiphout RG, Starmans MH, Rios-Velazquez E, Nalbantov G, Aerts HJ, et al. Predicting outcomes in radiation oncology--multifactorial decision support systems. Nat Rev Clin Oncol. 2013;10:27-40.

[4] Aerts HJ, Velazquez ER, Leijenaar RT, Parmar C, Grossmann P, Carvalho S, et al. Decoding tumour phenotype by noninvasive imaging using a quantitative radiomics approach. Nat Commun. 2014;5:4006.

[5] Leijenaar RT, Carvalho S, Hoebers FJ, Aerts HJ, van Elmpt WJ, Huang SH, et al. External validation of a prognostic CT-based radiomic signature in oropharyngeal squamous cell carcinoma. Acta Oncol. 2015;54:1423-9.

[6] Wu W, Parmar C, Grossmann P, Quackenbush J, Lambin P, Bussink J, et al. Exploratory Study to Identify Radiomics Classifiers for Lung Cancer Histology. Frontiers in Oncology. 2016;6:71.

[7] Parmar C, Leijenaar RT, Grossmann P, Rios Velazquez E, Bussink J, Rietveld D, et al. Radiomic feature clusters and prognostic signatures specific for Lung and Head \& Neck cancer. Sci Rep. 2015;5:11044.

[8] Leijenaar RT, Carvalho S, Velazquez ER, van Elmpt WJ, Parmar C, Hoekstra OS, et al. Stability of FDG-PET Radiomics features: an integrated analysis of test-retest and inter-observer variability. Acta Oncol. 2013;52:1391-7.

[9] Parmar C, Rios Velazquez E, Leijenaar R, Jermoumi M, Carvalho S, Mak RH, et al. Robust Radiomics feature quantification using semiautomatic volumetric segmentation. PLoS One. 2014;9:e102107.

[10] Yan J, Chu-Shern JL, Loi HY, Khor LK, Sinha AK, Quek ST, et al. Impact of Image Reconstruction Settings on Texture Features in 18F-FDG PET. J Nucl Med. 2015;56:1667-73.

[11] Galavis PE, Hollensen C, Jallow N, Paliwal B, Jeraj R. Variability of textural features in FDG PET images due to different acquisition modes and reconstruction parameters. Acta Oncol. 2010;49:1012-6.

[12] van Velden FH, Kramer GM, Frings V, Nissen IA, Mulder ER, de Langen AJ, et al. Repeatability of Radiomic Features in Non-Small-Cell Lung Cancer [F]FDG-PET/CT Studies: Impact of Reconstruction and Delineation. Mol Imaging Biol. 2016.

[13] Fried DV, Tucker SL, Zhou S, Liao Z, Mawlawi O, Ibbott G, et al. Prognostic value and reproducibility of pretreatment $C T$ texture features in stage III non-small cell lung cancer. Int J Radiat Oncol Biol Phys. 2014;90:834-42.

[14] Balagurunathan Y, Gu Y, Wang H, Kumar V, Grove O, Hawkins S, et al. Reproducibility and Prognosis of Quantitative Features Extracted from CT Images. Transl Oncol. 2014;7:72-87.

[15] Balagurunathan Y, Kumar V, Gu Y, Kim J, Wang H, Liu Y, et al. Test-retest reproducibility analysis of lung CT image features. J Digit Imaging. 2014;27:805-23.

[16] Leijenaar RT, Nalbantov G, Carvalho S, van Elmpt WJ, Troost EG, Boellaard R, et al. The effect of SUV discretization in quantitative FDG-PET Radiomics: the need for standardized methodology in tumor texture analysis. Sci Rep. 2015;5:11075.

[17] Armato SG, 3rd, Meyer CR, McNitt-Gray MF, McLennan G, Reeves AP, Croft BY, et al. The Reference Image Database to Evaluate Response to therapy in lung cancer (RIDER) project: a resource for the development of change-analysis software. Clin Pharmacol Ther. 2008;84:44856.

[18] Huang YQ, Liang CH, He L, Tian J, Liang CS, Chen X, et al. Development and Validation of a Radiomics Nomogram for Preoperative Prediction of Lymph Node Metastasis in Colorectal Cancer. J Clin Oncol. 2016;34:2157-64.

[19] Zhao B, James LP, Moskowitz CS, Guo P, Ginsberg MS, Lefkowitz RA, et al. Evaluating Variability in Tumor Measurements from Same-day Repeat CT Scans of Patients with NonSmall Cell Lung Cancer. Radiology. 2009;252:263-72. 
[20] McNitt-Gray MF, Bidaut LM, Armato SG, Meyer CR, Gavrielides MA, Fenimore C, et al. Computed Tomography Assessment of Response to Therapy: Tumor Volume Change Measurement, Truth Data, and Error. Transl Oncol. 2009;2:216-22.

[21] Meyer CR, Armato SG, Fenimore CP, McLennan G, Bidautn LM, Barboriak DP, et al. Quantitative Imaging to Assess Tumor Response to Therapy: Common Themes of Measurement, Truth Data, and Error Sources. Transl Oncol. 2009;2:198-210.

[22] Lawrence IKL. A Concordance Correlation Coefficient to Evaluate Reproducibility. Biometrics. 1989;45:255-68. 


\section{Supplementary Material}

\section{Supplementary Material 6A - CCC after resampling}

Prior to feature extraction, all data were resampled into images with a voxel size of $3 \times 3 \times 3 \mathrm{~mm}^{3}$. Results are shown in Figure S6.1. In total, for 530 out of 542 (97.8\%) of the features the data points are on the left side of the diagonal, meaning that they have a higher CCC in the RIDER dataset than in the clinical dataset.

When using the cut-off of $C C C>0.85,17$ features where reproducible in the clinical scenario derived rectal cancer test-retest set while 264 features where reproducible in the coffee-break lung cancer test-retest set. 16 of these feature overlapped. Slightly more features were robust after resampling for both the rectal cancer dataset (17 vs. 9) and the lung cancer dataset (264 vs. 234). 


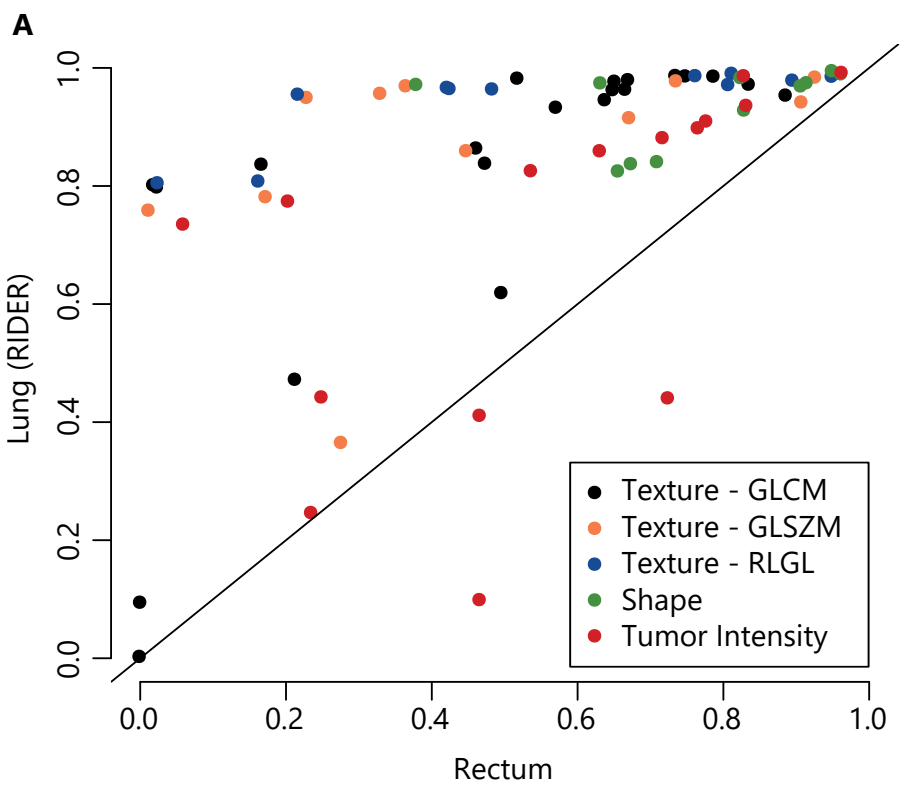

B

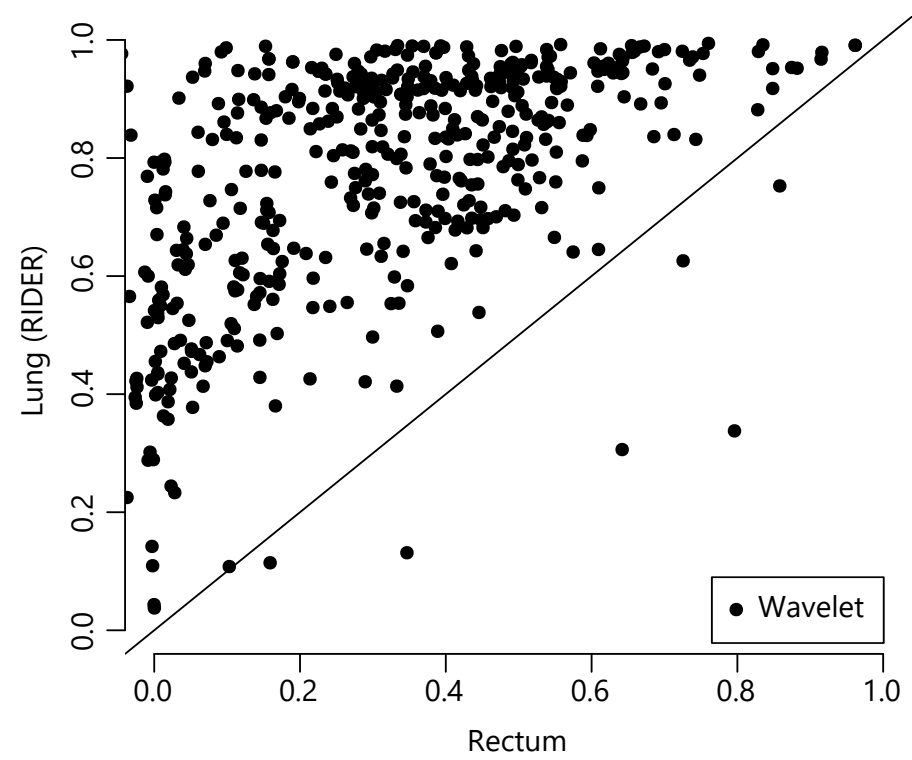

Figure S6.1. Comparison between stability of radiomic features derived from the lung cancer dataset (RIDER) and the rectal cancer dataset, with in panel (A) feature groups 'Texture', 'Shape' and 'Tumor Intensity' and in (B) 'Wavelet'. Images were resampled into isotropic voxels of $3 \mathrm{~mm}$ prior to feature extraction. GLCM: graylevel co-occurrence matrix, GLSZM: gray-level size zone matrix and RLGL: run-length gray-level. 


\section{Supplementary Material 6B - Hunderd most stable features}

Table S6.1. 100 most robust features for both datasets: rectal cancer (left two columns) and lung cancer (right two columns). Common features are indicated bold. Note that feature group 'Tumor intensity' is shortened to 'Stats'.

\begin{tabular}{|c|c|c|c|}
\hline \multicolumn{2}{|l|}{ Rectal cancer } & \multicolumn{2}{|l|}{ Lung cancer } \\
\hline Feature name & $\mathrm{CCC}$ & Feature name & $\mathrm{CCC}$ \\
\hline Wavelet_LLL_stats_totalenergy & 0.962 & Shape_compactness & 0.995 \\
\hline Stats_totalenergy & 0.961 & Stats_totalenergy & 0.992 \\
\hline Shape_volume & 0.960 & Wavelet_HLH_glcm_infoCorr1 & 0.992 \\
\hline Shape_compactness & 0.948 & Wavelet_LLL_stats_totalenergy & 0.992 \\
\hline Shape_surface & 0.913 & Wavelet_LHH_glszm_sizeZoneVariability & 0.992 \\
\hline GLSZM_highIntensityLargeAreaEmp & 0.911 & RLGL_grayLevelNonuniformity & 0.990 \\
\hline Shape_maxDiameter2D3 & 0.905 & Shape_volume & 0.990 \\
\hline Stats_kurtosis & 0.879 & Wavelet_LLH_rlgl_runLengthNonuniformity & 0.988 \\
\hline RLGL_longRunEmphasis & 0.856 & Wavelet_HLL_glcm_infoCorr1 & 0.988 \\
\hline Wavelet_HHH_rlgl_grayLeveINonuniformity & 0.846 & Wavelet_HHH_glcm_infoCorr1 & 0.988 \\
\hline Wavelet_LHL_rlgI_grayLeveINonuniformity & 0.836 & Wavelet_LHL_glcm_infoCorr1 & 0.988 \\
\hline Wavelet_LLH_rilgl_grayLevelNonuniformity & 0.835 & Wavelet_HLH_rlgl_runLengthNonuniformity & 0.988 \\
\hline Stats_median & 0.832 & Wavelet_LLL_glszm_intensityVariability & 0.987 \\
\hline $\begin{array}{l}\text { Wavelet_LLL_glszm_highlntensity } \\
\text { LargeAreaEmp }\end{array}$ & 0.832 & Wavelet_LHH_rlgl_runLengthNonuniformity & 0.987 \\
\hline Shape_maxDiameter2D2 & 0.828 & Wavelet_HLH_glszm_intensityVariability & 0.987 \\
\hline Stats_energy & 0.827 & Wavelet_HLL_rlgl_runLengthNonuniformity & 0.987 \\
\hline Wavelet_LLL_stats_energy & 0.827 & Stats_energy & 0.987 \\
\hline Shape_maxDiameter3D & 0.826 & Wavelet_HHL_rlgl_runLengthNonuniformity & 0.987 \\
\hline GLCM_inverseVar & 0.824 & Wavelet_LLL_stats_ener & 0.987 \\
\hline Shape_surfVolRatio & 0.824 & Wavelet_LHL_glszm_intensityVariability & 0.987 \\
\hline Wavelet_HLL_rlgl_grayLevelNonuniformity & 0.824 & Wavelet_HHL_glszm_intensityVariability & 0.987 \\
\hline Wavelet_LLL_stats_median & 0.822 & Wavelet_HHH_rlgl_runLengthNc & 0.987 \\
\hline Wavelet_LHH_glcm_clusProm & 0.820 & Wavelet_LLH_glszm_intensityVaria & 0.986 \\
\hline RLGL_longRunHighGrayLevEmpha & 0.810 & Wavelet_LHH_glszm_inte & 0.986 \\
\hline RLGL_grayLeveINonuniformity & 0.807 & Wavelet_HHH_glcm_infoCor & 0.986 \\
\hline GLSZM_largeAreaEmphasis & 0.805 & Wavelet_LHL_rlgl_runLengthNonuniformity & 0.985 \\
\hline Wavelet_HLH_glcm_sumVar & 0.803 & let_HLH_glcm_infoC & 0.985 \\
\hline Wavelet_LLL_rlgl_grayLevelNonunifo & 0.801 & RLGL_runLengthNo & 0.985 \\
\hline Wavelet_HHL_glszm_highIntensityEmphasis & 0.797 & Wavelet_! & 0.985 \\
\hline ergy & 0.794 & Wavelet_LLL_rlgl_runLeng & 0.985 \\
\hline RLGL_runPercentage & 0.791 & Wavelet_LHL_glcm_infoCorr2 & 0.985 \\
\hline Wavelet_HLL_stats_median & 0.790 & IL_rlgl_grayLevelNonuniformity & 0.984 \\
\hline Wavelet_HLH_rlgl_grayLevelNonuniformity & 0.790 & Shape_surfVolRatio & 0.984 \\
\hline Wavelet_HHL_glcm_sumVar & 0.789 & Wavelet_HLL_glszm_intensityVa & 0.983 \\
\hline maxProb & 0.788 & LevelNonuniformity & 0.982 \\
\hline Wavelet_HHL_rlgl_high & 0.787 & GLSZM_intensityVariability & 0.982 \\
\hline Wavelet_HLH_glcm_autocorr & 0.784 & Wavelet_LHL_glszm_highlntensityLa & 0.981 \\
\hline Stats_rms & 0.784 & Wav & 0.981 \\
\hline Stats_mean & 0.783 & Wavelet_HHH_glszm_intensityVariability & 0.981 \\
\hline Wavelet_HLH_glcm_sumSquares & 0.782 & GLSZM_sizeZoneVariability & 0.980 \\
\hline Wavelet_HHL_glcm_autocorr & 0.781 & Wavelet_LHH_glcm_infoCorr1 & 0.980 \\
\hline Wavelet_HLH_rlgl_highGrayLevelRunEmphasis & 0.781 & Wavelet_HLL_glcm_infoCorr2 & 0.980 \\
\hline Wavelet_HHL_glcm_sumSquares & 0.780 & Wavelet_LHH_glcm_infoCorr2 & 0.979 \\
\hline Wavelet_HLH_glszm_highlntensityEmphasis & 0.777 & $\begin{array}{l}\text { Wavelet_LLL_glszm_highlntensityLarge } \\
\text { AreaEmp }\end{array}$ & 0.979 \\
\hline $\begin{array}{l}\text { Wavelet_HHL_glszm_highlntensity } \\
\text { LargeAreaEmp }\end{array}$ & 0.772 & Wavelet_LLH_glcm_infoCorr2 & 0.978 \\
\hline Wavelet_LLL_stats_rms & 0.765 & Wavelet_LLL_glszm_zonePercentage & 0.978 \\
\hline GLCM_homōgeneity1 & 0.764 & Wavelet_LHH_stats_energy & 0.977 \\
\hline Wavelet_LHH_glszm_sizeZoneVariability & 0.762 & Wavelet_LLH_stats_energy & 0.977 \\
\hline
\end{tabular}




Wavelet_HHH_stats_energy
Wavelet_LLL_stats_mean
Wavelet_LHL_stats_median
Wavelet_LHH_rlgl_grayLevelNonuniformity
Wavelet_HHH_stats_totalenergy
Wavelet_HLL_glszm_highlntensity
LargeAreaEmp
GLCM_homogeneity2
Stats_skewness
GLSZM_highlntensityEmphasis
Wavelet_LLL_glszm_highlntensityEmphasis
Wavelet_HHH_rlgl_runLengthNonuni-
formity
RLGL_shortRunEmphasis

0.761 Wavelet_HLL_stats_median $\quad 0.976$

0.761 Shape_surface $\quad \mathbf{0 . 9 7 5}$

0.759 Shape_maxDiameter2D1 0.975

$\mathbf{0 . 7 5 8}$ Wavelet_HLL_stats_mean 0.974

$\mathbf{0 . 7 5 5}$ Wavelet_HHL_glszm_sizeZoneVariability 0.974

0.754 Wavelet_LHH_rlgl_grayLevelNonuniformity $\mathbf{0 . 9 7 4}$

0.753 Shape_maxDiameter3D $\mathbf{0 . 9 7 2}$

0.751 Wavelet_HHH_stats_energy $\quad \mathbf{0 . 9 7 2}$

0.737 Wavelet_HLH_stats_energy 0.971

0.735 Wavelet_HHL_rlgl_grayLevelNonuniformity $\mathbf{0 . 9 7 0}$

0.734 Wavelet_HHH_glszm_sizeZoneVariability $\quad 0.970$

0.731 Wavelet_HLH_glszm_sizeZoneVariability 0.970

$\mathbf{0 . 7 2 5}$ Wavelet_HHL_stats_energy 0.970

0.723 Shape_maxDiameter2D3 $\mathbf{0 . 9 7 0}$

0.723 Wavelet_HHH_rlgl_grayLevelNonuniformity $\mathbf{0 . 9 6 9}$

0.717 Wavelet_HHL_stats_totalenergy 0.968

0.717 Wavelet_LHL_glszm_sizeZoneVariability 0.968

0.717 Wavelet_LHL_glszm_zonePercentage 0.968

$\mathbf{0 . 7 1 6}$ Wavelet_LHL_glszm_largeAreaEmphasis $\quad 0.967$

0.712 Wavelet_LLH_glcm_infoCorr1 0.967

$\mathbf{0 . 7 1 2}$ Wavelet_LLL_glszm_sizeZoneVariability 0.966

0.712 Wavelet_HLL_rlgl_grayLevelNonuniformity $\mathbf{0 . 9 6 5}$

0.710 Wavelet_LLL_glszm_largeAreaEmphasis 0.964

0.709 Wavelet_LHH_stats_totalenergy $\quad \mathbf{0 . 9 6 4}$

0.708 Wavelet_HLL_stats_totalenergy 0.963

0.706 Wavelet_HLL_glszm_sizeZoneVariability 0.962

0.706 Wavelet_LLL_glcm_inverseVar 0.962

0.698 Wavelet_HHL_glcm_infoCorr1 0.962

0.697 Wavelet_HHL_glcm_invDiffnorm 0.961

$\mathbf{0 . 6 9 6}$ Wavelet_HLL_glcm_invDiffnorm 0.961

0.686 Wavelet_LHL_stats_totalenergy 0.961

0.684 Wavelet_HLL_stats_energy 0.961

0.682 Wavelet_HHL_glszm_highIntensity $\quad \mathbf{0 . 9 6 1}$ LargeAreaEmp

$\mathbf{0 . 6 7 6}$ Wavelet_LLH_stats_totalenergy 0.960

0.673 Wavelet_LLL_glcm_infoCorr1 0.959

0.672 Wavelet_LHL_glcm_invDiffnorm 0.959

$\mathbf{0 . 6 7 1}$ Wavelet_LHL_glcm_invDiffmomnor 0.958

$\mathbf{0 . 6 6 6}$ GLCM_infoCorr1 0.958

0.662 Wavelet_LHL_stats_median $\quad \mathbf{0 . 9 5 7}$

$\mathbf{0 . 6 6 1}$ Wavelet_LLH_glszm_sizeZoneVariability 0.956

0.659 Wavelet_LLL_glcm_infoCorr2 0.955

0.655 Wavelet_HLH_rlgl_grayLeveINonuniformity $\mathbf{0 . 9 5 4}$

0.649 Wavelet_LLL_glcm_homogeneity2 0.954

0.648 Wavelet_LLL_glcm_homogeneity1 0.954

0.646 Wavelet_LLH_stats_median $\quad \mathbf{0 . 9 5 4}$

$\mathbf{0 . 6 4 5}$ Wavelet_LHL_stats_mean 0.953

0.645 GLCM_infoCorr2 0.953

$\mathbf{0 . 6 3 9}$ Wavelet_LHL_stats_md 0.953

0.636 Wavelet_LLL_rlgl_shortRunEmphasis 0.953

0.635 Wavelet_LHL_stats_energy 0.952

$\mathbf{0 . 6 3 5}$ Wavelet_LLL_stats_uniformity 0.952

0.635 Wavelet_HHH_stats_totalenergy $\quad \mathbf{0 . 9 5 1}$ 




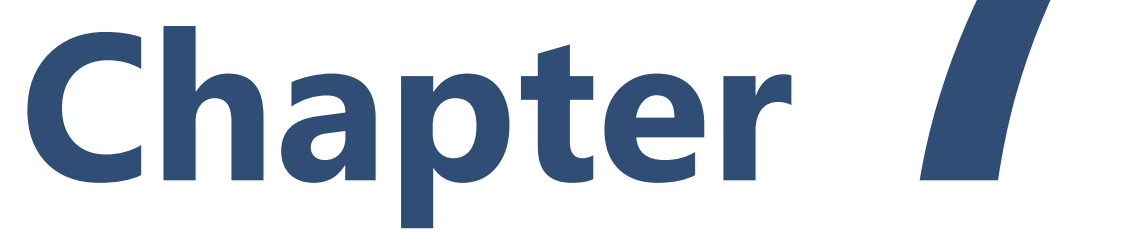

\section{Influence of gray-level discretization on radiomic feature stability for different $\mathrm{CT}$ scanners, tube currents and slice thicknesses: a comprehensive phantom study}

Authors

Ruben T. H. M. Larue*, Janna E. van Timmeren*, Evelyn E. C. de Jong*, Giacomo Feliciani, Ralph T. H. Leijenaar, Wendy M. J. Schreurs, Meindert N. Sosef, Frank H. P. J. Raat, Frans H. R. van der Zande, Marco Das, Wouter van Elmpt, Philippe Lambin 


\section{ABSTRACT}

\section{Background}

Radiomic analyses of CT images provide prognostic information that can potentially be used for personalized treatment. However, heterogeneity of acquisition- and reconstruction protocols influences robustness of radiomic analyses. The aim of this study was to investigate the influence of different CT-scanners, slice thicknesses, exposures and gray-level discretization on radiomic feature values and their stability.

\section{Material and methods}

A texture phantom with ten different inserts was scanned on nine different CTscanners with varying tube currents. Scans were reconstructed with $1.5 \mathrm{~mm}$ or $3 \mathrm{~mm}$ slice thickness. Image pre-processing comprised gray-level discretization in ten different bin widths ranging from 5 to $50 \mathrm{HU}$ and different resampling methods (i.e. linear, cubic and nearest neighbor interpolation to $1 \times 1 \times 3 \mathrm{~mm}^{3}$ voxels) were investigated. Subsequently, 114 textural radiomic features were extracted from a 2.1 $\mathrm{cm}^{3}$ sphere in the center of each insert. The influence of slice thickness, exposure and bin width on feature values was investigated. Feature stability was assessed by calculating the concordance correlation coefficient (CCC) in a test-retest setting and for different combinations of scanners, tube currents and slice thicknesses.

\section{Results}

Bin width influenced feature values, but this only had a marginal effect on the total number of stable features (CCC $>0.85)$ when comparing different scanners, slice thicknesses or exposures. Most radiomic features were affected by slice thickness, but this effect could be reduced by resampling the CT-images before feature extraction. Statistics feature 'energy' was the most dependent on slice thickness. No clear correlation between feature values and exposures was observed.

\section{Conclusions}

CT-scanner, slice thickness and bin width affected radiomic feature values, whereas no effect of exposure was observed. Optimization of gray-level discretization to potentially improve prognostic value can be performed without compromising feature stability. Resampling images prior to feature extraction decreases the variability of radiomic features. 


\section{Introduction}

Extraction of quantitative imaging features, also called radiomics [1], has become an additional source of information for the development of prognostic and predictive models [2-7]. The total number of features that can be calculated is almost unlimited, especially if filter-based features (e.g. Laplacian of Gaussian or wavelet) are also taken into consideration. To incorporate such large numbers of features in prognostic and predictive models, multiple independent and multi-centric datasets are needed for training and validation. However, recent literature shows that there are some challenges to overcome [8].

Several studies already showed that radiomic feature values are influenced by image acquisition and reconstruction settings, like slice thickness and exposure $[2$, 9-12]. For instance, Mackin et al. [13] scanned a phantom with ten unique inserts using different acquisition parameters on computed tomography (CT) scanners of four manufacturers. They demonstrated that the variability in textural features calculated on the phantom scans can be in the same order of magnitude as the variability seen in non-small cell lung cancer patients (NSCLC). Using the same phantom, Shafiq-ul-Hassan et al. [12] investigated voxel-size dependency of radiomic features and found that this dependency can be minimized by resampling to a nominal voxel size or by normalizing the voxel size. Next to that, they found that gray-level dependency also can be reduced by normalization. Since multi-centric data usually are acquired with different CT-scanners using institutional scan protocols, the lack of statistical power in validation datasets might, therefore, be attributed to the different acquisition settings.

In the current study, we investigated the variability in radiomic feature values for scans with different slice thicknesses, different exposures and from different CTscanners and performed a test-retest analysis using the same texture phantom as Mackin et al. and Shafiq-ul-Hassan et al. [12, 13]. In addition to these former studies, we used a more extensive radiomic features dataset, different scanner types and focussed more on the influence of gray-level discretization and resampling of voxel size on interchangeability of radiomic features. Furthermore, we compared the variability of radiomic features values derived from the phantom with those derived from two independent non-small cell lung cancer (NSCLC) patient datasets, to investigate the comparability of the phantom inserts to clinical CT-scans. 


\section{Material and methods}

\section{Image acquisition}

The Credence Cartridge Radiomics phantom (CCR), previously described by Mackin et al. [13], consists of an acrylic case with ten different inserts with different textures and was scanned on nine different CT-scanners. We focussed on the shredded rubber insert, because the CT-properties of this insert are most similar to tumors [13].

An overview of all scanners and settings is provided in Table 7.1. The scans were reconstructed using two different slice thicknesses per scanner. For the ' $\mathrm{H}$ ' scanner, the ' $\mathrm{B}$ ' scanner and the ' $\mathrm{O}$ ' scanner the increment as well as the slice thickness was $1.5 \mathrm{~mm}$ or $3 \mathrm{~mm}$. For the other scanners the increment was $1 \mathrm{~mm}$ for scans with a slice thickness of $1.5 \mathrm{~mm}$ (scanner ' $\mathrm{S}$ ' had a slice thickness of $2 \mathrm{~mm}$ instead of 1.5) and $2 \mathrm{~mm}$ for the scans with a slice thickness of $3 \mathrm{~mm}$. The reconstructed field of view was $500 \mathrm{~mm}$ for all scans.

All scans were performed with a tube voltage of $120 \mathrm{kVp}$ and a pitch of 1.0. The aim was to scan the phantom with the same range of CT-dose index (CTDI) settings [2.17, 3.26, 4.32, 5,43, 10, 20 mGy], but due to hardware (e.g. the risk of overheating) and software limitations (i.e. not all exposures could be set) not all CTDIs could be obtained for all scanners. Philips scans were reconstructed using the B kernel and Siemens scans using the B31f or B31s kernel.

For test-retest purposes, two subsequent CT-scans were acquired on the ' $\mathrm{B}$ ' and the ' $\mathrm{O}$ ' scanner. The phantom was kept in place on the table without changing anything in between, meaning that no other parameters than scanner output fluctuations could have influenced the images.

Table 7.1. Overview scanners and per scanner exposure time, convolution kernel, tube current, focal spot size and collimation.

\begin{tabular}{|c|c|c|c|c|c|c|c|c|}
\hline Hospital & Vendor & Model & Coding & $\begin{array}{l}\text { Exposure } \\
\text { time (ms) }\end{array}$ & $\begin{array}{l}\text { Convolution } \\
\text { kernel }\end{array}$ & $\begin{array}{l}\text { Tube Current } \\
(\mathrm{mA})\end{array}$ & $\begin{array}{l}\text { Focal spot } \\
\text { size }(\mathrm{mm})\end{array}$ & $\begin{array}{l}\text { Collimation } \\
\left(\#{ }^{*} \mathrm{~mm}\right)\end{array}$ \\
\hline \multirow[t]{2}{*}{1} & Siemens & $\begin{array}{l}\text { SOMATOM } \\
\text { Definition Flash }\end{array}$ & $\mathrm{FI}$ & 500 & B31f & $\begin{array}{l}128,160 \\
296,592\end{array}$ & 1.2 & $64 * 0.6$ \\
\hline & Philips & Brilliance 64 & $P$ & 561 & B & $\begin{array}{l}61,91,119 \\
148,274\end{array}$ & 1.0 & $64 * 0.6$ \\
\hline \multirow[t]{3}{*}{2} & Siemens & Biograph 40 & B & 500 & B31f & $40,60,80$ & 1.2 & 24 * 1.2 \\
\hline & Siemens & $\begin{array}{l}\text { SOMATOM } \\
\text { Confidence RT } \\
\text { Pro }\end{array}$ & C & 1000 & B31s & $\begin{array}{l}26,39,52 \\
66,121\end{array}$ & 1.2 & $32 * 0.6$ \\
\hline & Siemens & $\begin{array}{l}\text { SOMATOM } \\
\text { Sensation Open }\end{array}$ & $\mathrm{O}$ & 500 & B31f & $60,80,100$ & 1.2 & 24 * 1.2 \\
\hline 3 & Siemens & $\begin{array}{l}\text { SOMATOM } \\
\text { Definition Flash }\end{array}$ & L & 500 & B31f & $\begin{array}{l}64,96,128, \\
162,296 \\
594\end{array}$ & 1.2 & 64 * 0.6 \\
\hline 4 & Siemens & $\begin{array}{l}\text { SOMATOM } \\
\text { Definition AS+ }\end{array}$ & V & 500 & B31f & $\begin{array}{l}64,96,128, \\
162,298, \\
594\end{array}$ & 1.2 & $64 * 0.6$ \\
\hline 5 & Siemens & Biograph 16 & $\mathrm{H}$ & 500 & B31f & $\begin{array}{l}40,60,84 \\
112,140 \\
216,274\end{array}$ & 0.7 & $16 * 0.75$ \\
\hline 6 & Philips & Gemini TF 16 & S & 534 & B & $\begin{array}{l}58,88,114 \\
143,266 \\
497\end{array}$ & 1.0 & 16 * 1.5 \\
\hline
\end{tabular}




\section{Feature extraction}

A spherical region of interest (ROI) with a volume of $2.1 \mathrm{~cm}^{3}$ was contoured using Mirada RTx (Mirada RTx 1.6, Mirada Medical, Oxford, UK) in the center of every insert. Moreover, for test-retest purposes, the ROI was shifted to the right and downwards. Supplementary Material 7A shows an example CT image of the phantom in which the ROls are indicated in the rubber insert.

One hundred and fourteen radiomic features were extracted for the ROI in every insert using software developed in-house. The histogram of voxel intensity values within the ROI was described by nineteen first order statistics (Stats) features. Textural features were divided in five neighborhood gray-tone difference (NGTDM) features, sixteen neighboring gray-level dependence matrix (NGLDM) features, sixteen gray-level size zone matrix (GLSZM) features, sixteen gray-level run length matrix (GLRLM) features, sixteen gray-level distance zone matrix (GLDZM) features and 26 gray-level co-occurrence matrix (GLCM) features. The definitions of the radiomic features are previously described in van Timmeren et al. [14].

The following pre-processing steps were applied prior to radiomic feature extracting: gray-level discretization and voxel resampling. All features were calculated for ten bin widths ranging from 5 to 50 Hounsfield Units (HU), with a step size of 5 $\mathrm{HU}$. Radiomic features were also calculated with and without applying resampling into voxel sizes of $1 \times 1 \times 3 \mathrm{~mm}^{3}$ using cubic, linear and nearest neighbor interpolation, which was only done for a bin width of 25 .

\section{Analyses}

The variation in feature values due to slice thickness and exposure, calculated per feature for the rubber insert using a bin width of $25 \mathrm{HU}$, was defined as the maximal difference between scans with either the same exposure (variation due to unequal slice thicknesses) or the same slice thickness (variation due to unequal exposures). The ratios between the variations were calculated to investigate which features are most dependent on either slice thickness or exposure. Features were ranked per scanner based on their ratios and afterwards the rankings were summed, with a higher total rank indicating larger dependency on slice thickness, and a lower total rank indicating a larger dependency on exposure.

We compared the radiomic feature values which were calculated using the three different resampling methods. Moreover, for five different scanners we compared the distribution of $\mathrm{HU}$ values in the rubber insert. For all scans we used in this analysis, the phantom was scanned using a CTDI of $5.43 \mathrm{mGy}$ and reconstructed with a slice thickness of $1.5 \mathrm{~mm}$. We also investigated the HU distributions after resampling into voxel sizes of $1 \times 1 \times 3 \mathrm{~mm}^{3}$ using linear interpolation.

To investigate the influence of gray-level discretization on the feature values, each feature was plotted against the bin width. Moreover, the pairwise concordance correlation coefficient (CCC) [15] was used to determine the agreement in feature values over all inserts when comparing (1) a slice thickness of $1.5 \mathrm{~mm}$ with $3 \mathrm{~mm}$, (2) an exposure of $60 \mathrm{~mA}$ with $80 \mathrm{~mA}$, or (3) scanner ' $\mathrm{B}$ ' with 'O' using the same scan protocol. The CCC ranges from -1 (perfect negative agreement) to 1 (perfect positive agreement). A minimum CCC of 0.85 , which was used in previous studies $[16,17]$, was 
used to identify features that were independent of the different scanners or settings that were compared. The total number of stable features per bin width was then used as a measure to investigate the influence of bin width on feature stability.

Moreover, we performed a test-retest analysis to be able to compare results within a controlled setting. For the three sets of scans that were used to perform the CCC analyses described above: 1) B-60mA-3mm and B-60mA-1.5mm, 2) B-60mA-3mm and $B-80 m A-3 m m$ and 3) B-60mA-3mm and O-60mA-3mm, we also calculated the CCC for the test-retest setting of both scans. The minimal CCC of these two test-retest scans was then used to determine the number of stable features $(C C C>0.85)$ per bin width. Moreover, we investigated the variability of radiomic features caused by shifted ROIs (see 'Feature extraction'), by calculating the CCC between the radiomic features extracted from the scan with the ROI in the center and from the same scan with the shifted ROI.

Finally, to test if the variability in tumor feature values is comparable to the variability in feature values measured in the phantom, the results from the ten phantom inserts were compared to two patient datasets. Patient dataset 1 consisted of 157 NSCLC patients for which the CT-scans were acquired in different hospitals in which the phantom was scanned as well. Patient dataset 2 consisted of 168 NSCLC patients for which all CT-scans were performed in a single hospital which is one of the hospitals in which the phantom was scanned. All patient scans had a slice thickness ranging from $1 \mathrm{~mm}$ to $3 \mathrm{~mm}$. To reduce variability between scans, these were resampled to voxels of $1 \times 1 \times 3 \mathrm{~mm}^{3}$ prior to analysis using cubic interpolation, as this corresponds to the voxel size of most the clinical images.

\section{Results}

The feature 'First order statistics (Stats) - Energy' had the highest rank and thus showed the highest dependency on slice thickness and 'GLRLM - Run Length Nonuniformity (RLN)' the second highest dependency (Supplementary Material 7B). The feature 'GLSZM - Small Area Emphasis (SAE)' was least dependent on slice thickness and was ranked last. In further analyses in this study, we focussed on the features 'GLRLM - RLN' and 'GLSZM - SAE', since 'Energy' is independent of graylevel discretization by definition. Figure 7.1 shows that the feature values for 'GLRLM - RLN' for $1.5 \mathrm{~mm}$ and $3 \mathrm{~mm}$ were more similar after resampling, which was not the case for the 'GLSZM - SAE' feature values. The test-retest analysis demonstrated that the feature 'GLRLM - RLN' is a stable feature (CCC $>0.85$ for all bin widths), whereas the feature 'GLSZM - SAE' is unstable (Supplementary Material 7B).

For the feature 'GLRLM - RLN' the cubic, linear and nearest neighbor resampling method resulted in very similar feature ranges after resampling (Figure 7.2). For ' $G L S Z M$ - SAE', however, the nearest neighbor method resulted in a very wide range. When comparing the three resampling methods for all features tested $(n=114)$, for $34(30 \%)$ features cubic interpolation resulted in the narrowest feature range, for 55 (48\%) features this applied to linear interpolation, and the remaining 25 (22\%) features had the narrowest feature range after nearest neighbor interpolation. Nearest neighborhood interpolation had the widest range for the majority (61\%) of the features. For the feature that showed the highest dependency on slice thickness, 
'Stats - Energy', feature values over all scans ranged from $6.3 \times 10^{8}$ to $1.9 \times 10^{9}$ (median $\left.1.2 \times 10^{9}\right)$, which reduced to a range from $5.5 \times 10^{8}$ to $6.9 \times 10^{8}$ (median $6.1 \times 10^{8}$ ) after resampling using cubic interpolation.
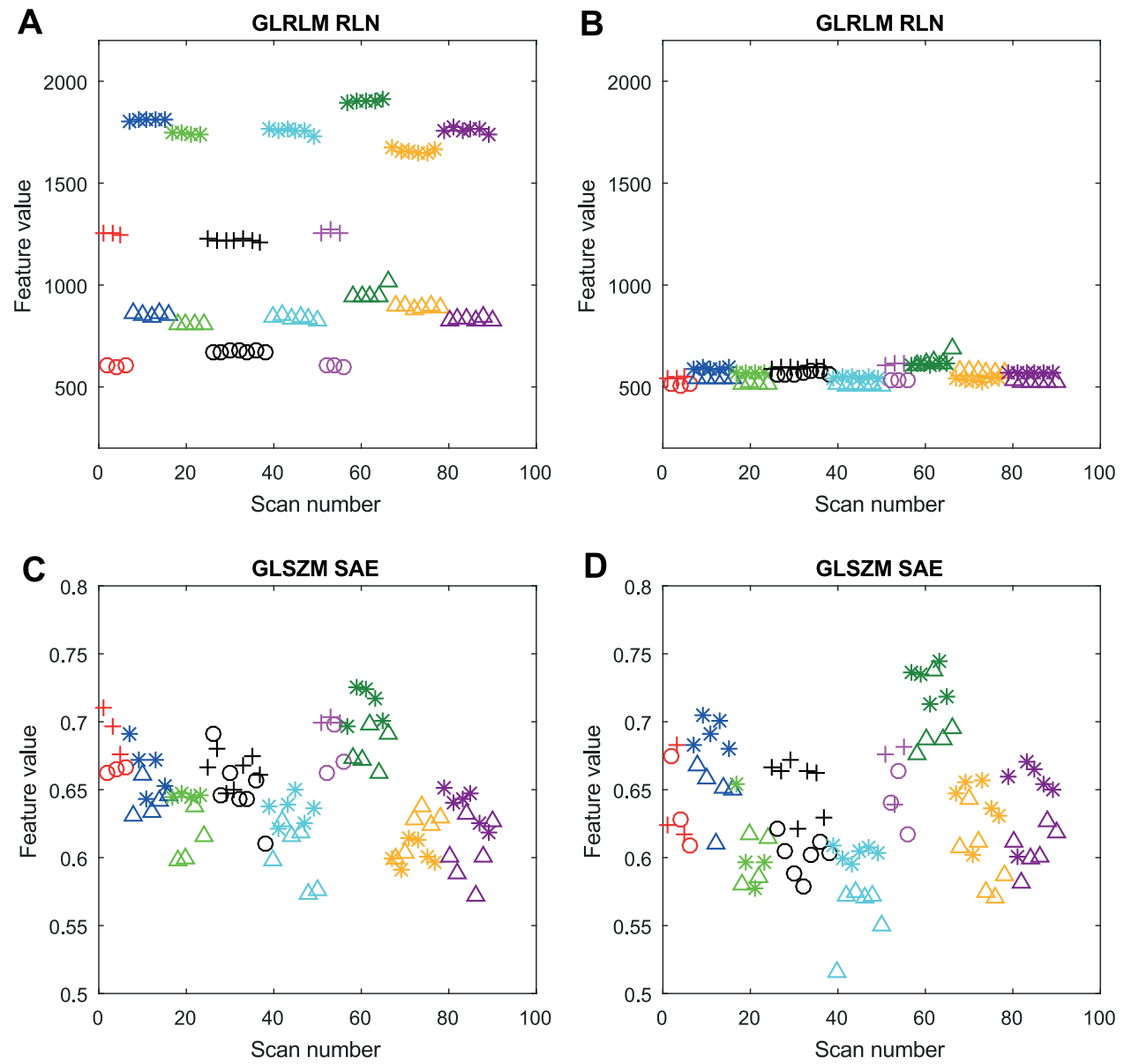

\begin{tabular}{|llll|}
\hline$* 1 \mathrm{~mm}$ & $\bullet \mathrm{B}$ & $\bullet \mathrm{H}$ & $\bullet \mathrm{P}$ \\
$\triangle 2 \mathrm{~mm}$ & $\bullet \mathrm{C}$ & $\bullet \mathrm{L}$ & $\bullet \mathrm{S}$ \\
$+1.5 \mathrm{~mm}$ & $\bullet \mathrm{FI}$ & $\bullet \mathrm{O}$ & $\bullet \mathrm{V}$ \\
$\mathrm{O} 3 \mathrm{~mm}$ & & &
\end{tabular}

Figure 7.1. Scatterplots of feature values for all scanners. Per scanner, the data is sorted from high to low exposure. Left: original data; Right: after resampling; Top: 'GLRLM - RLN', highest slice thickness dependency; Bottom: 'GLSZM - SAE', lowest slice thickness dependency.

Figure 7.3 shows that the scans of the five different scanners had a similar median $\mathrm{HU}$ value: between 931 and $939 \mathrm{HU}$. When comparing the range in $\mathrm{HU}$ of those five scanners, scanner ' $P$ ' had a much wider range than the other four scanners (160-1564 compared to 437-1291). As shown in Figure 7.3 for the feature 'GLCM cluster 
prominence', this also affected radiomic feature values extracted from these images. The same histograms were made after resampling, which show a similar shape as before resampling (shown in Supplementary Material 7C).

A

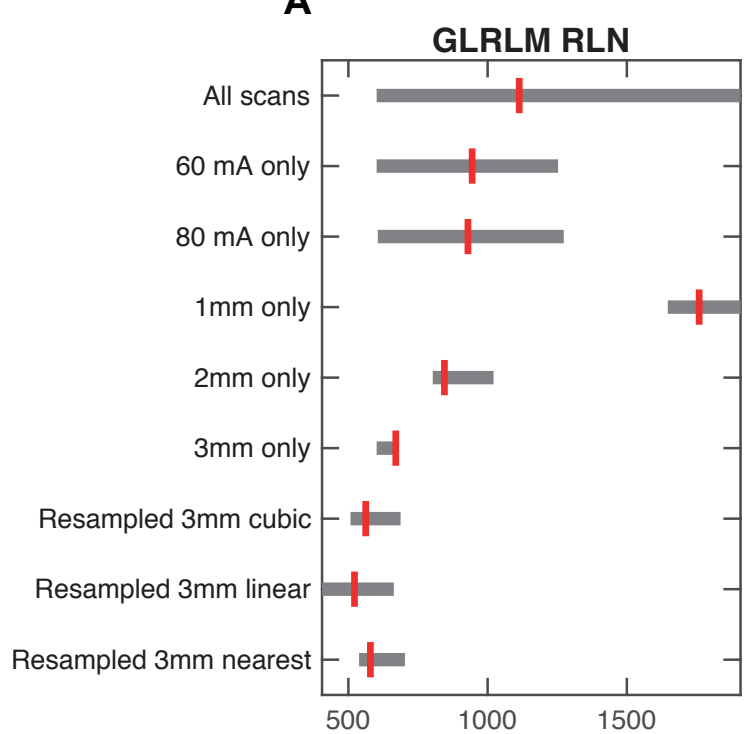

B

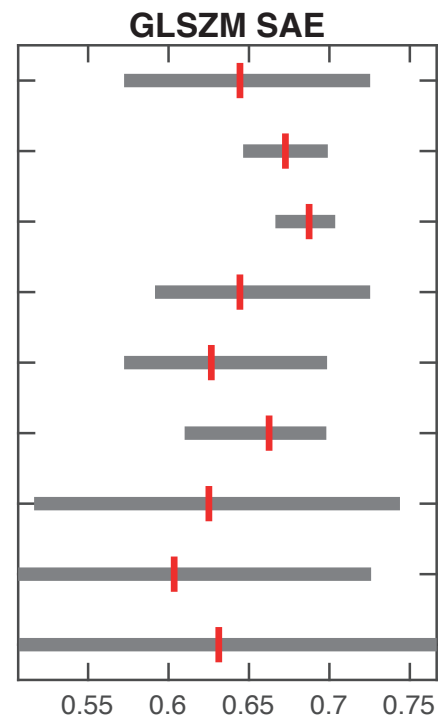

Figure 7.2. Bar plots of the spread of feature values of (A) 'GLRLM - RLN' and (B) 'GLSZM - SAE'. Bars range from the minimum to the maximum observed value, and the vertical lines indicate the median. From top to bottom these bars are shown for all scans $(n=90)$, all scans with an exposure of $60 \mathrm{~mA}(n=6)$, all scans with an exposure of $80 \mathrm{~mA}(n=4)$, all scans with an increment of $1 \mathrm{~mm}(n=34), 2 \mathrm{~mm}(\mathrm{n}=34)$ and $3 \mathrm{~mm}$ $(n=13)$ and all scans after resampling the images into $1 \times 1 \times 3 \mathrm{~mm}^{3}$ using cubic $(n=90)$, linear $(n=90)$ and nearest neighbor $(n=90)$ interpolation.

Figure 7.4 shows that every normalized feature had another bin width dependency. Some features (e.g. 'Energy' and 'Skewness') were not dependent on bin width per definition. In terms of feature stability, when comparing $1.5 \mathrm{~mm}$ scans with $3 \mathrm{~mm}$ scans using an exposure of $60 \mathrm{~mA}$, the total number of stable textural features hardly changed over the different bin widths (Figure 7.5). The median number of stable features was 49 (range 47-53). All CCC numbers for this comparison are shown in Supplementary Material $D$, where the features that were not stable in either the testretest of 'B-60mA-1.5mm' or 'B-60mA-3mm' are also indicated. Also, when comparing an exposure of $60 \mathrm{~mA}$ with $80 \mathrm{~mA}$ on the same scanner, or scanner ' $\mathrm{B}$ ' with ' $\mathrm{O}$ ', no trend indicating a dependency on bin width could be observed. In this case, the median number of stable features was 66.5 (55-70) and 67.5 (56-72), respectively. Note that the total number of stable features was much lower when comparing different slice thicknesses.

The images on the left side of Figure 7.5 show that approximately the same number of features were stable for the test-retest compared to the scans with different exposure or acquired from different scanners. On the other side, more features were stable in the test-retest setting than when slice thicknesses were 

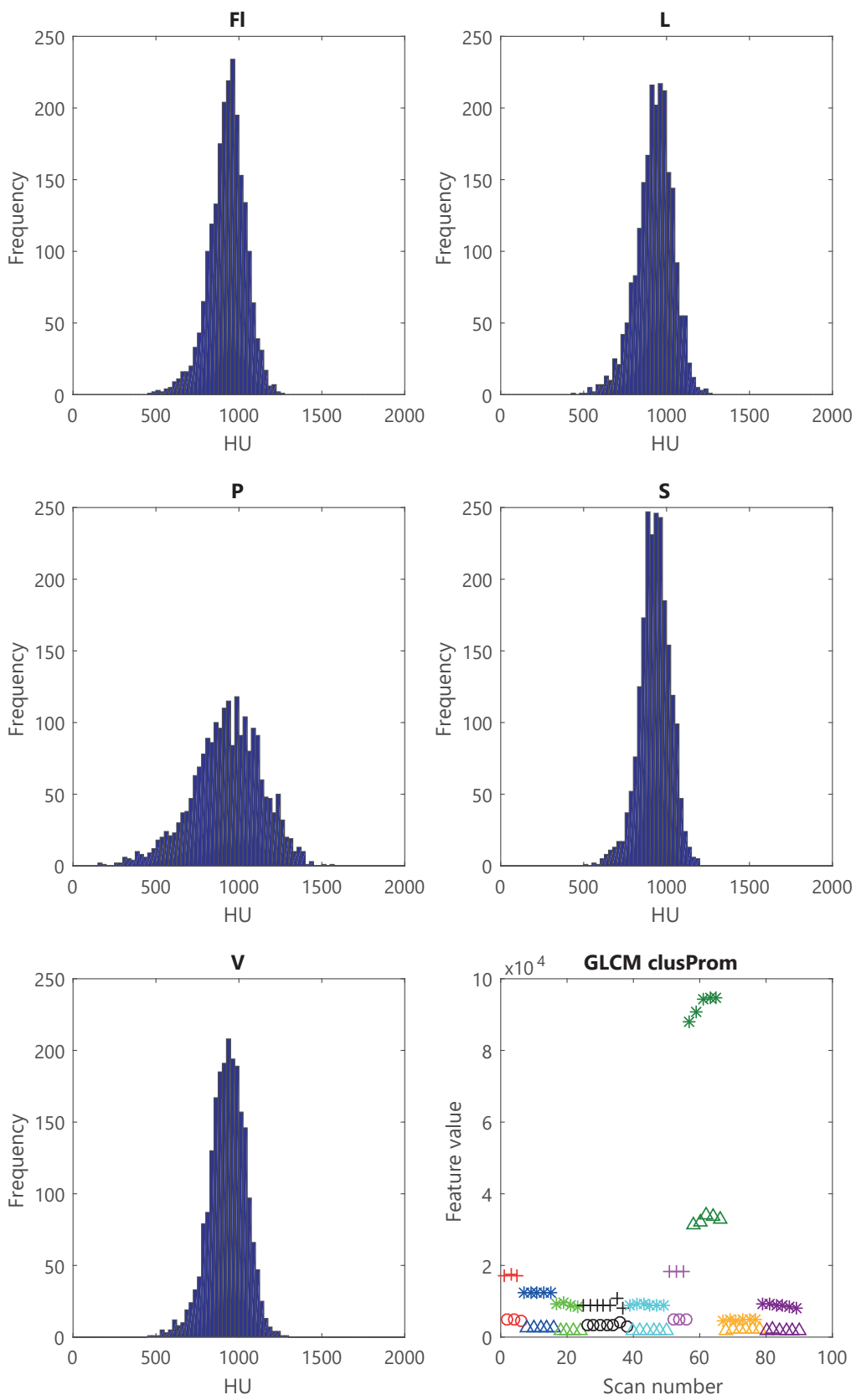

Figure 7.3. HU distributions of the rubber insert scanned with a fixed CDTI of $5.43 \mathrm{mGy}$ on five different Siemens and Philips scanners. From left to right and top to bottom [scanner - tube current]: 'Fl-160mA', 'L-161mA', 'P-148mA', 'S-143mA', and 'V-162mA'. The scatterplot in the bottom right displays the observed values of the feature 'GLCM cluster prominence' over all scanners. The outlier in dark-green is scanner ' $P$ '. 
different. The test-retest analysis of scan 'B-60mA-3mm' (bin width 25) shows that 5 out of 114 had a CCC below 0.85 . When shifting the ROI downwards (ROI '6s324' in Supplementary Material A), there were 8 features with CCC $<0.85$, with $100 \%$ overlap compared to the test-retest. When shifting the delineation to the right ( $\mathrm{ROI}$ ' $6 \mathrm{~s} 234$ '), 14 features were unstable, again with a $100 \%$ overlap.

The range of feature values for all inserts was compared to the range in two independent datasets of NCSLC patients. As shown in Figure 7.6, the 'GLRLM-RLN' feature range observed in the phantom is very limited and substantially lower in comparison with the range observed in the clinical datasets, whereas the 'GLSZM$\mathrm{SAE}^{\prime}$ ranged in the same order of magnitude. The same plots for all features are available in Supplementary Material 7E.

\section{Discussion}

The aim of this study was to investigate the impact of different CT-scanners, slice thicknesses, exposures, gray-level discretization and resampling of voxel sizes on feature values and on their stability using a texture phantom. Moreover, we performed a test-retest analysis. In short, we could show that CT-scanner, slice thickness and gray-level discretization (i.e. bin width) influence feature values. A clear effect of exposure on feature values was not observed. Moreover, the stability of radiomic features is hardly influenced by bin width, i.e. for each bin width the variability across different settings is similar.

Scatterplots of feature values showed that the distribution of feature values is different for each CT-scanner. Since scans were acquired with the same range of CTDI values, this implies that the variability of feature values is affected by different scanners used in that particular study. The feature 'GLSZM - SAE', which has the lowest rank in terms of slice thickness dependency, appeared to be unstable in a testretest analysis. In contrast the feature 'GLRLM - RLN' had a CCC above 0.85 for all test-retest scans, whereas the CCC was low in the comparison between $1.5 \mathrm{~mm}$ and $3 \mathrm{~mm}$ slice thickness (Supplementary Material 7D).

A comparable effect was observed in the HU histograms of five different scans that were acquired with the same CTDI of $5.43 \mathrm{mGy}$. The ' $\mathrm{Fl}$ ', ' $\mathrm{L}$ ', ' $\mathrm{V}$ ', and 'S' scanners had comparable $\mathrm{HU}$ distributions within the rubber insert. However, even though the same radiation dose output of the $\mathrm{CT}$-scanner and the same acquisition protocol was used, scanner ' $P$ ' resulted in a CT-image with a much wider range of $\mathrm{HU}$. The histograms show the same pattern after resampling of the images was applied. The reason for the discrepancy of scanner ' $P$ ' is unknown, but it might be the result of an incorrect calibration of the scanner. So even with similar acquisition protocols, different scanner types within one dataset can influence radiomic feature values. Therefore, we recommend to take this into account when performing radiomic studies with multiple heterogeneous datasets, i.e. to perform a study specific testretest analysis [16] to eliminate features that are not robust across different scanners.

We also showed that a large proportion of features is influenced by the slice thickness used for reconstruction. However, we could show that the variability in feature values decreased after resampling was performed. This is in line with Shafiqul-Hassan et al. [12], who showed that resampling reduced the feature variability 

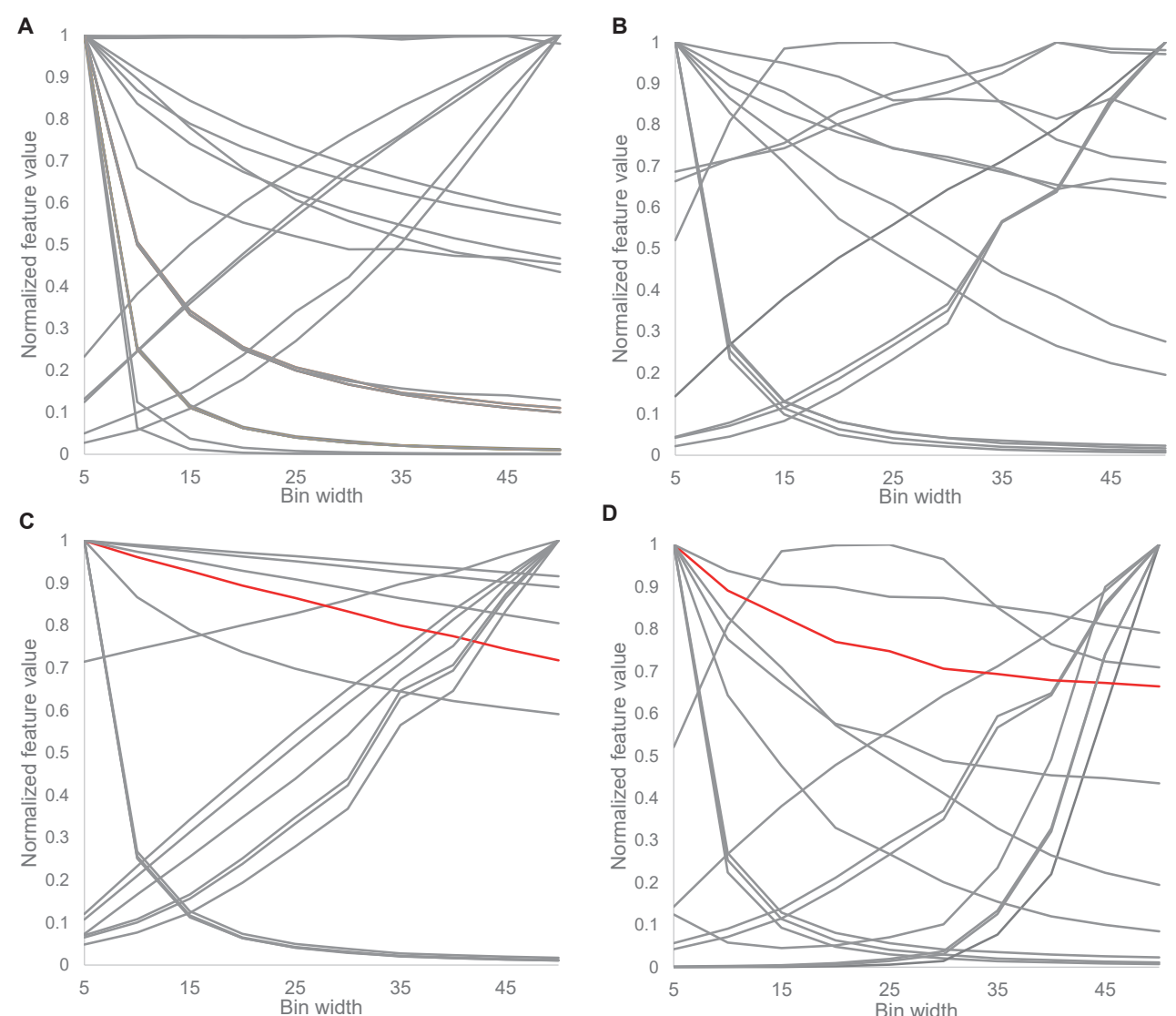

D
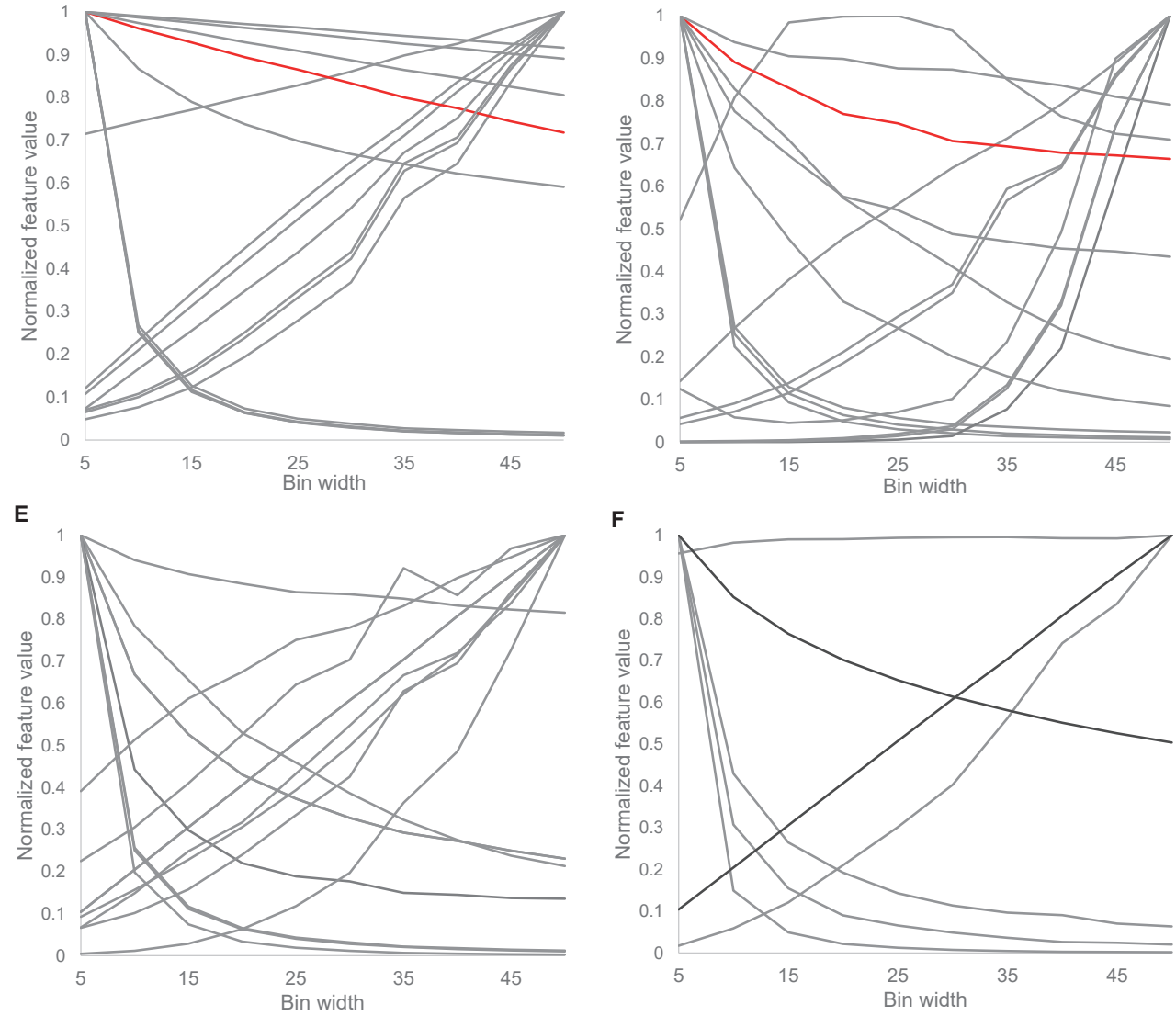

Figure 7.4. Normalized feature values plotted against bin width. (A) GLCM features $(n=26)$, (B) GLDZM features $(n=16),(C)$ GLRLM features $(n=16),(D)$ GLSZM features $(n=16)$, (E) NGDLM features $(n=16),(F)$ NGTDM $(n=5)$ and first order statistics features $(n=2$, dotted lines). The red lines indicate GLRLM RLN and GLSZM SAE. 


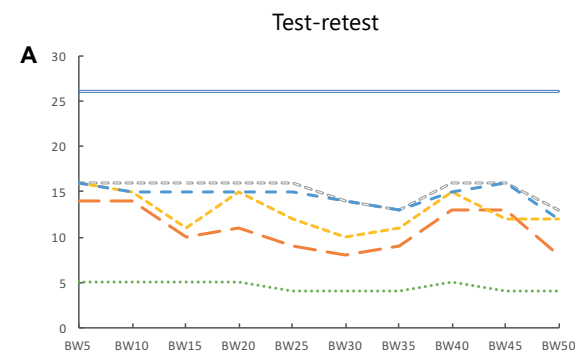

Test-retest

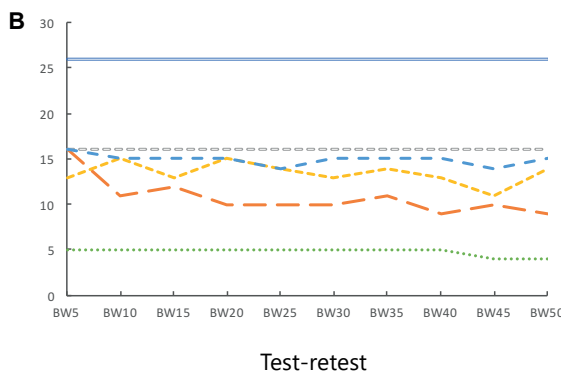

C

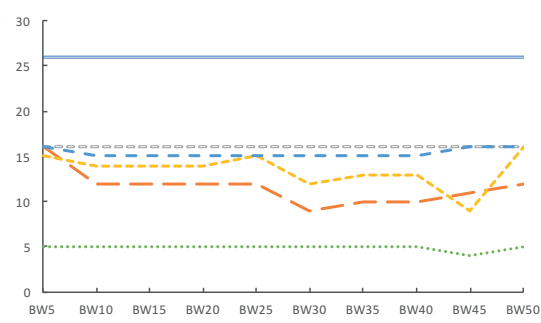

$1.5 \mathrm{~mm}$ vs. $3.0 \mathrm{~mm}$

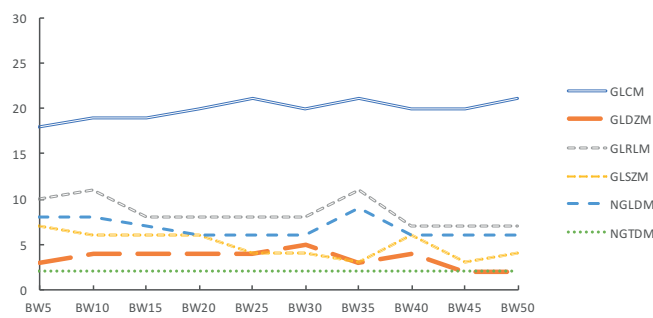

$60 \mathrm{~mA}$ vs. $80 \mathrm{~mA}$

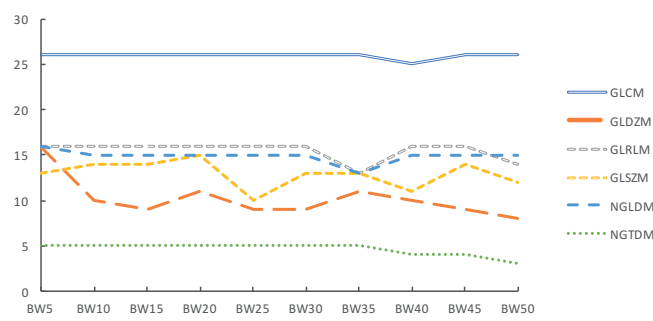

Scanner ' $B$ ' vs. 'O'

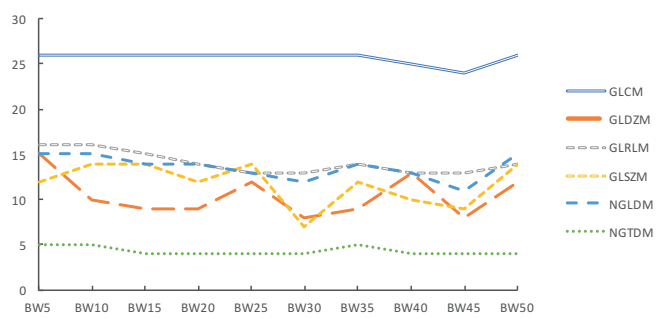

Figure 7.5. The total number of features with a CCC $>0.85$ for each bin width when comparing (A) a slice thickness of $1.5 \mathrm{~mm}$ with $3 \mathrm{~mm}$ (exposure $60 \mathrm{~mA}$, scanner ' $\mathrm{B}$ '), (B) an exposure of $60 \mathrm{~mA}$ with $80 \mathrm{~mA}$ (slice thickness $3 \mathrm{~mm}$, scanner 'B'), or (C) scanner ' $\mathrm{B}$ ' with ' $\mathrm{O}$ ' using the same scan protocol (exposure $60 \mathrm{~mA}$ and slice thickness $3 \mathrm{~mm}$ ). Test-retest figures are based on the minimal observed CCC in both test-retest sets that are compared.

from $\%$ COV $>70 \%$ to $\%$ COV $<30 \%$. Therefore, we recommend to always perform resampling prior to any radiomic analysis. Despite the fact that resampling can greatly improve the robustness of radiomic features [12], we recommend to keep the voxel size as consistent as possible, since the variability in feature values is even lower when the voxel size is equal for all images included in the study. This should also be taken into account when comparing training and validation datasets that might be reconstructed into different voxel sizes, which could have increased the discrepancy between datasets in terms of distribution of radiomic feature values although resampling was applied. In our study the data was resampled to a voxel size of $1 \times 1 \times 3 \mathrm{~mm}^{3}$ using a cubic, linear and nearest neighbor interpolation, while in the study of Shafiq-ul-Hassan et al. [12] the data was resampled to a voxel size of $1 \times 1 \times 2 \mathrm{~mm}^{3}$ using a linear interpolation. Our results demonstrated that linear interpolation resulted in the narrowest feature value range for $48 \%$ of the features 
and cubic interpolation for $30 \%$ of the features, whereas nearest neighbor interpolation had the widest range for $61 \%$ of the features. Therefore, cubic or linear interpolation are preferred over nearest neighbor interpolation when resampling to $1 \times 1 \times 3 \mathrm{~mm}^{3}$ voxels.

Furthermore, we investigated the influence of exposure on the feature values, as tube current modulated CT-scans become more common. We only had data from a few scans per CT-scanner, which made it difficult to investigate the potential influence of exposure on radiomic feature values. For none of the features, we could see a clear relationship between feature value and the exposure. Mackin et al. [13] had quite a large range of exposure values for the different scanners, although the effect of exposure on feature values was not investigated, this might have influenced the scanner comparison results. In the future, the influence of exposure should be explored on a larger dataset.

A

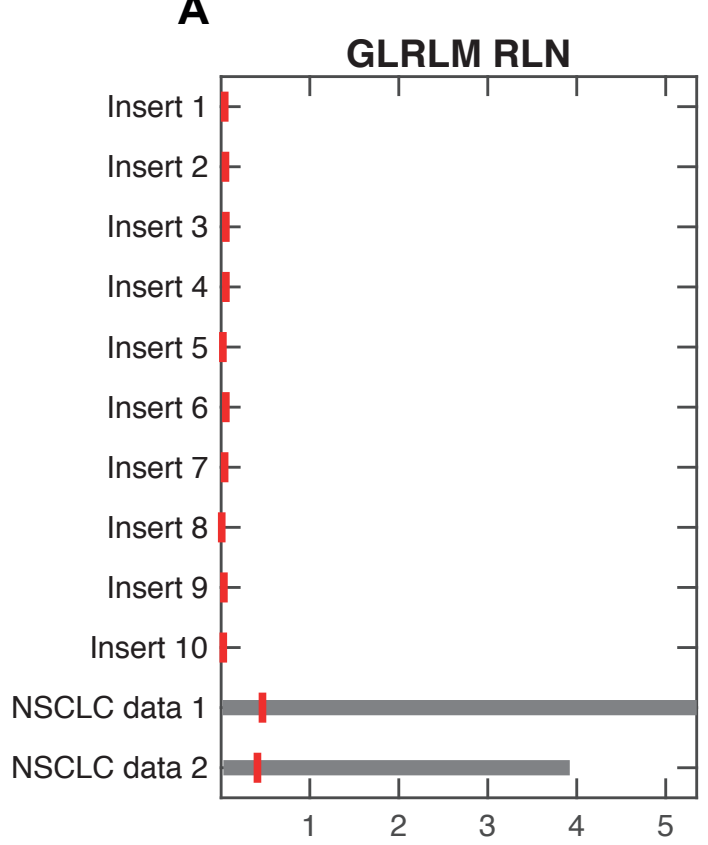

B

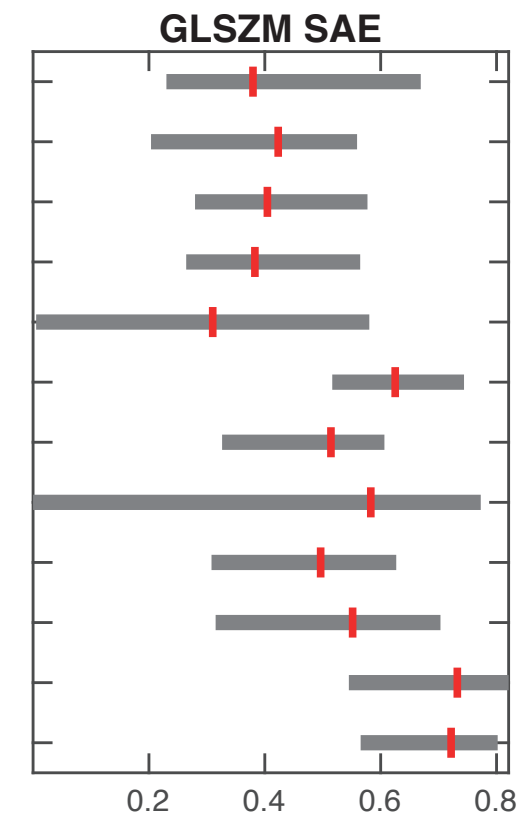

Figure 7.6. Bar plots of the spread of feature values over all phantom inserts and two independent NSCLC datasets for (A) 'GLRLM - RLN' and (B) 'GLSZM - SAE'. The bars indicate the minimum, median and maximum feature values. Scans were resampled to $1 \times 1 \times 3 \mathrm{~mm}^{3}$ voxels using cubic interpolation and a bin width of $25 \mathrm{HU}$ was used.

Finally, we investigated the influence of the pre-processing step of 'graylevel discretization' on feature values and on their stability [18]. The main goal was to find an optimal bin width that could result in the highest reproducibility of radiomic features. Almost all features change in value when choosing another bin width for gray-level discretization. For a subset of features, a very small or very large bin width resulted in very different feature values across scans, whereas feature values are 
more similar with a bin size in the order of 25 (Figure 7.4). Although the feature values change for different bin widths, we were not able to show that the stability of radiomic features is greatly influenced by the choice of bin width (Figure 7.5 and Supplementary Material 7D). Our finding is the contrary of what Shafiq-ul-Hassan et al. described [12]. They found that only seven out of 51 features were reproducible independent of the gray-level discretization. Next to that, they found that seventeen out of 44 features showed a trend with varying number of gray-levels, which could be a linear, quadratic or cubic-type relation. Normalization of feature values by the number of gray-levels reduced the variation in feature values. However, these results imply that the choice of bin width could alter the prognostic value of a certain radiomic signature. The influence of gray-level discretization on the prognostic value of radiomic features has not yet been investigated and needs further research. Therefore, we cannot indicate a certain bin width as being most optimal, but we strongly recommend to be consistent and always clearly report which pre-processing steps have been used to improve the reproducibility and validation of radiomic studies [9].

The test-retest analysis we have performed shows that, even when not changing anything in between two subsequently acquired CT scans, some radiomic features were not robust. When shifting the ROI within the same insert, even more features failed to reach a CCC above 0.85 . The only reason for the instability of features in the test-retest setting could be the variability of scanner output, which is always present and cannot be avoided. Therefore, these features would be too unstable even when imaging protocols are completely standardized. We acknowledge that it is difficult to define a CCC threshold for eliminating the features, but we think that it is reasonable to exclude the non-stable radiomic features with $\mathrm{CCC}<0.85$ in future radiomic studies.

One of the limitations of this study is the ignorance of convolution kernel used during reconstruction. We considered the Philips ' $\mathrm{B}$ ' kernel and the Siemens 'B31s' and 'B31f' as interchangeable, but this was not investigated. We also did not investigate the influence of iterative kernels or others commonly used in clinical practice, which might also affect the variability of radiomic features.

In this study, we investigated the influence of factors which we expected to have a major effect on radiomics. Nonetheless, other parameters influence image quality and therefore could have influenced the results. For example, scanners from different manufacturers have different possibilities for focal spot size and collimation width due to differences in technical design. These parameters do influence the image appearance: a smaller collimation width results in increased noise and a larger focal spot size results in decreased image quality (i.e. reduced sharpness). In the original design of the study we did not control for these parameters and the values were only traced back after the study was performed. In future studies, the influence of these parameters on radiomic features should be evaluated, and we recommend to report as much parameters as possible.

Furthermore, the potential influence of change in volume of the spherical region of interest was not investigated. Although all images were registered to the same reference scan which was used for delineations, this always leads to slightly 
different volumes. Whereas the spherical volume should consistently be $2.1 \mathrm{~cm}^{3}$, we noticed deviations in the order of $0.1 \mathrm{~cm}^{3}$. This could have influenced the results for radiomic features that are correlated to volume.

Another limitation of current and former studies $[12,13]$ is the phantom itself. The squared shape makes the phantom prone to scatter artefacts around the edges. A new cylindrical version of the CCR phantom is therefore being produced and should be used in future CT-scanner variability studies.

In our study we compared the distribution of radiomic features values compared to two clinical NSCLC patient datasets. One possible explanation for the differences between the two patient datasets is that patient dataset 1 was acquired from multiple hospitals and dataset 2 from a single hospital, which might have resulted in a wider distribution of feature values for dataset 1 . Furthermore, the distribution of feature values shows that not all inserts are very representative for clinical NSCLC datasets. Mackin et al. [13] already showed that the mean and standard deviation in $\mathrm{HU}$ of the CCR phantom is different than for patient data. The distribution of HUs in the rubber insert was most comparable to the distribution observed in the NSCLS datasets. However, radiomic features derived from any of the phantom inserts typically had a very limited range when compared to the clinical datasets, as well as a different median value. Inserts with more representative textures are, therefore, warranted for future phantom studies.

In conclusion, this study shows that feature values are influenced by CT-scanner, slice thickness and bin width, whereas the influence of exposure could not be shown. Moreover, the influence of bin width on feature stability was not clear, meaning that we could not indicate an optimal bin width. The test-retest analysis shows that some radiomic features are not robust in a strictly controlled setting: we recommend to exclude those in future radiomic studies. Moreover, we strongly recommend to always perform the pre-processing steps 'resampling' and 'gray-level discretization' for each radiomic study and to clearly report the settings that have been used to improve consistency and reproducibility of radiomic analyses.

\section{Acknowledgments}

The authors would like to acknowledge Dr. D. Mackin, Dr. L. Court and colleagues from The University of Texas, MD Anderson Cancer Center for the opportunity to use their CT-phantom in this study.

\section{Funding}

The authors acknowledge financial support from ERC Advanced Grant (ERC-ADG-2015, no. 694812-Hypoximmuno). This research is also supported by the Dutch Technology Foundation STW (grant no. 10696 DuCAT \& no. P14-19 Radiomics STRaTegy), which is the applied science division of NWO, and the Technology Programme of the Ministry of Economic Affairs. The authors also acknowledge financial support from the EU 7th framework program (ARTFORCEno. 257144, REQUITE-no. 601826), SME Phase 2 (EU proposal 673780-RAIL), EUROSTARS (DART), the European Program H2020-2015-17 (BD2Decide-PHC30-689715 and ImmunoSABRno. 733008), Kankeronderzoekfonds Limburg from the Health Foundation Limburg and the Dutch Cancer Society. 


\section{References}

[1] Lambin P, Rios-Velazquez E, Leijenaar R, Carvalho S, van Stiphout RG, Granton P, et al. Radiomics: extracting more information from medical images using advanced feature analysis. Eur J Cancer. 2012;48:441-6.

[2] Zhang Y, Oikonomou A, Wong A, Haider MA, Khalvati F. Radiomics-based Prognosis Analysis for Non-Small Cell Lung Cancer. Sci Rep. 2017;7:46349.

[3] Leijenaar RT, Carvalho S, Hoebers FJ, Aerts HJ, van Elmpt WJ, Huang SH, et al. External validation of a prognostic CT-based radiomic signature in oropharyngeal squamous cell carcinoma. Acta Oncol. 2015;54:1423-9.

[4] Leijenaar RT, Carvalho S, Velazquez ER, van Elmpt WJ, Parmar C, Hoekstra OS, et al. Stability of FDG-PET Radiomics features: an integrated analysis of test-retest and inter-observer variability. Acta Oncol. 2013;52:1391-7.

[5] Aerts HJ, Velazquez ER, Leijenaar RT, Parmar C, Grossmann P, Carvalho S, et al. Decoding tumour phenotype by noninvasive imaging using a quantitative radiomics approach. Nat Commun. 2014;5:4006.

[6] Coroller TP, Grossmann P, Hou Y, Rios Velazquez E, Leijenaar RT, Hermann G, et al. CTbased radiomic signature predicts distant metastasis in lung adenocarcinoma. Radiother Oncol. 2015;114:345-50.

[7] Scrivener M, de Jong EEC, van Timmeren JE, Pieters T, Ghaye B, Geets X. Radiomics applied to lung cancer: a review. Transl Cancer Res. 2016;5:398-409.

[8] Larue RT, Defraene G, De Ruysscher D, Lambin P, van Elmpt W. Quantitative radiomics studies for tissue characterization: a review of technology and methodological procedures. $\mathrm{Br}$ J Radiol. 2017;90:20160665.

[9] Lambin P, Leijenaar RTH, Deist TM, Peerlings J, de Jong EEC, van Timmeren J, et al. Radiomics: the bridge between medical imaging and personalized medicine. Nat Rev Clin Oncol. 2017; 14:749-62.

[10] Balagurunathan Y, Gu Y, Wang H, Kumar V, Grove O, Hawkins S, et al. Reproducibility and Prognosis of Quantitative Features Extracted from CT Images. Transl Oncol. 2014;7:72-87.

[11] Lu L, Ehmke RC, Schwartz LH, Zhao B. Assessing Agreement between Radiomic Features Computed for Multiple CT Imaging Settings. PLoS One. 2016;11:e0166550.

[12] Shafiq-Ul-Hassan M, Zhang GG, Latifi K, Ullah G, Hunt DC, Balagurunathan Y, et al. Intrinsic dependencies of CT radiomic features on voxel size and number of gray levels. Med Phys. 2017;44:1050-62.

[13] Mackin D, Fave X, Zhang L, Fried D, Yang J, Taylor B, et al. Measuring Computed Tomography Scanner Variability of Radiomics Features. Invest Radiol. 2015;50:757-65.

[14] van Timmeren JE, Leijenaar RTH, van Elmpt W, Reymen B, Oberije C, Monshouwer R, et al. Survival prediction of non-small cell lung cancer patients using radiomics analyses of conebeam CT images. Radiother Oncol. 2017;123:363-9.

[15] Lin LI. A concordance correlation coefficient to evaluate reproducibility. Biometrics. 1989;45:255-68.

[16] van Timmeren JE, Leijenaar RTH, Van Elmpt W, Wang J, Zhang Z, Dekker A, et al. TestRetest Data for Radiomics Feature Stability Analysis: Generalizable or Study-Specific? Tomography. 2016;2:361-5.

[17] Zhao B, Tan Y, Tsai WY, Qi J, Xie C, Lu L, et al. Reproducibility of radiomics for deciphering tumor phenotype with imaging. Sci Rep. 2016;6:23428.

[18] Leijenaar RT, Nalbantov G, Carvalho S, van Elmpt WJ, Troost EG, Boellaard R, et al. The effect of SUV discretization in quantitative FDG-PET Radiomics: the need for standardized methodology in tumor texture analysis. Sci Rep. 2015;5:11075. 


\section{Supplementary Material}

Supplementary Material 7A - CT image of the phantom

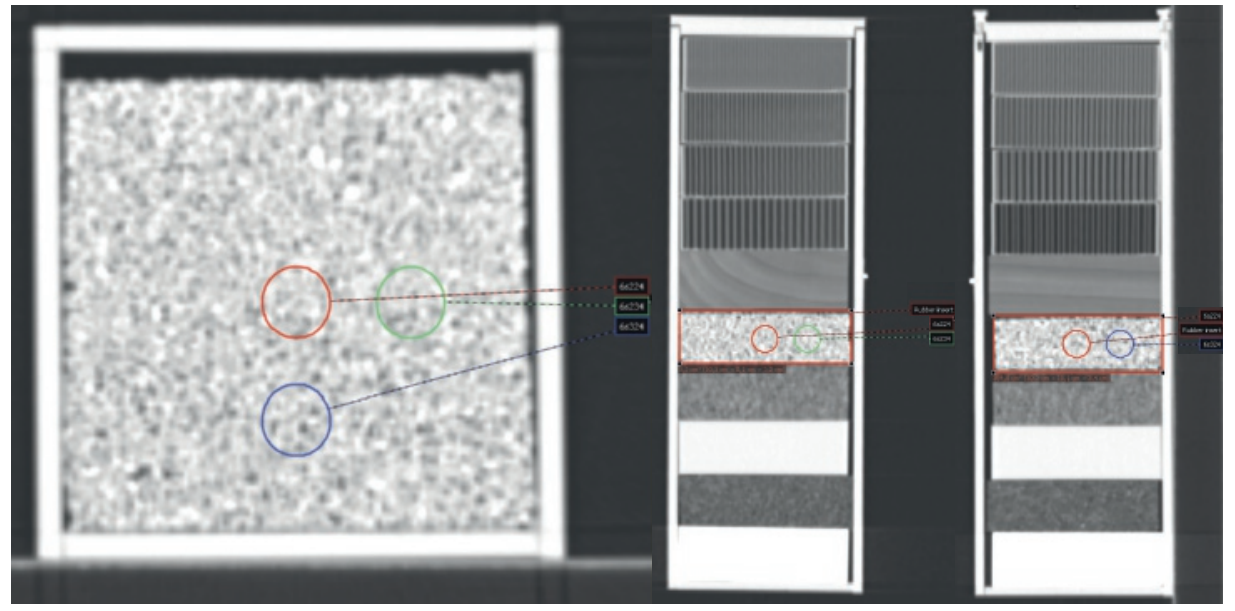

Figure S7.1. Sample CT-image of the phantom including the three ROls used in this study. 


\section{Supplementary Material 7B - Feature ranking indicating slice thickness and exposure dependency}

Table S7.1. Feature ranking based on the ratio between variations due to slice thickness and due to exposure. This ranking is the sum ranking over all scanners.

\begin{tabular}{|c|c|c|c|}
\hline 1. & Stats_energy & 58. & Stats_min \\
\hline 2. & GLRLM_RLN & 59. & GLCM_average \\
\hline 3. & GLCM_sumEntro & 60. & GLCM_sumAvg \\
\hline 4. & GLRLM_GLN & 61. & GLRLM_SRHGE \\
\hline 5. & GLCM_sumVar & 62. & NGLDM_HGSDE \\
\hline 6. & GLCM_clusTend & 63. & NGLDM_LGLDE \\
\hline 7. & GLCM_entrop2 & 64. & GLRLM_HGRE \\
\hline 8. & NGTDM_coarseness & 65. & GLDZM_LISDE \\
\hline 9. & Stats_entropy & 66. & NGLDM_HGE \\
\hline 10. & Stats_std & 67. & Stats_totalenergy \\
\hline 11. & NGLDM_GLV & 68. & Stats_rms \\
\hline 12. & GLRLM_GLV & 69. & GLDZM_HIE \\
\hline 13. & Stats md & 70. & GLRLM_LRLGE \\
\hline 14. & Stats var & 71. & GLSZM_HIE \\
\hline 15. & NGLDM_GLN & 72. & GLCM_autocorr \\
\hline 16. & GLRLM GLNN & 73. & NGLDM LGE \\
\hline 17. & GLRLM_RE & 74. & GLRLM_LRHGE \\
\hline 18. & NGLDM_DN & 75. & GLRLM_LGRE \\
\hline 19. & GLCM_energy & 76. & GLRLM_SRLGE \\
\hline 20. & NGLDM_GLNN & 77. & GLDZM_LIE \\
\hline 21. & GLCM_clusProm & 78. & GLSZM_HISAE \\
\hline 22. & GLCM_sumSquares & 79. & GLSZM_LIE \\
\hline 23. & Stats_uniformity & 80. & GLRLM_SRE \\
\hline 24. & GLDZMM_DZE & 81. & GLSZM_LISAE \\
\hline 25. & GLCM_diffEntro & 82. & GLRLM_RLNN \\
\hline 26. & GLCM_diffAvrg & 83. & GLRLM_RP \\
\hline 27. & Stats_rmd & 84. & GLSZM_ZE \\
\hline 28. & GLCM_dissimilar & 85. & GLDZM_ZP \\
\hline 29. & GLCM_contrast & 86. & GLRLM_LRE \\
\hline 30. & GLDZM_LDE & 87. & GLSZM_ZP \\
\hline 31. & GLCM_diffVar & 88. & NGLDM_LDE \\
\hline 32. & NGLDM̄M_SM & 89. & NGLDM_SDE \\
\hline 33. & Stats_p90 & 90. & NGLDM_DNN \\
\hline 34. & GLCM_homogeneity1 & 91. & GLSZM_L̄ILAE \\
\hline 35. & GLDZM_IN & 92. & GLDZM_HISDE \\
\hline 36. & GLCM_homomogeneity2 & 93. & NGLDM_LGSDE \\
\hline 37. & Stats_p10 & 94. & GLRLM_RLV \\
\hline 38. & GLSZM_IN & 95. & GLCM_infoCorr2 \\
\hline 39. & GLDZM_SDE & 96. & GLCM_infoCorr1 \\
\hline 40. & GLSZM_SZN & 97. & NGLDM_HGLDE \\
\hline 41. & GLDZM_DZN & 98. & NGTDM_contrast \\
\hline 42. & GLCM_clusShade & 99. & GLCM_invDiffmomnor \\
\hline 43. & Stats igr & 100. & GLCM invDiffnorm \\
\hline 44. & GLDZ̄M DZNN & 101. & Stats median \\
\hline 45. & GLDZM DZV & 102. & GLSZM LAE \\
\hline 46. & GLCM_inverseVar & 103. & GLDZM_LILDE \\
\hline 47. & GLDZM_IV & 104. & NGLDM_DV \\
\hline 48. & GLDZM_INN & 105. & GLCM_maxCorr \\
\hline 49. & GLSZM_IV & 106. & GLSZM_SZV \\
\hline 50. & NGTDM_complexity & 107. & Stats_mean \\
\hline 51. & GLDZM_HILDE & 108. & NGTDM_busyness \\
\hline 52. & GLSZM_INN & 109. & NGTDM_strength \\
\hline 53. & Stats_range & 110. & Stats_skewness \\
\hline 54. & GLCM_maxProb & 111. & Stats_kurtosis \\
\hline 55. & GLCM_correl1 & 112. & GLSZM_HILAE \\
\hline 56. & NGLDM_DE & 113. & GLSZM_SZ \\
\hline 57. & Stats_max & & \\
\hline
\end{tabular}




\section{Supplementary Material 7C - HU distributions of the rubber insert}
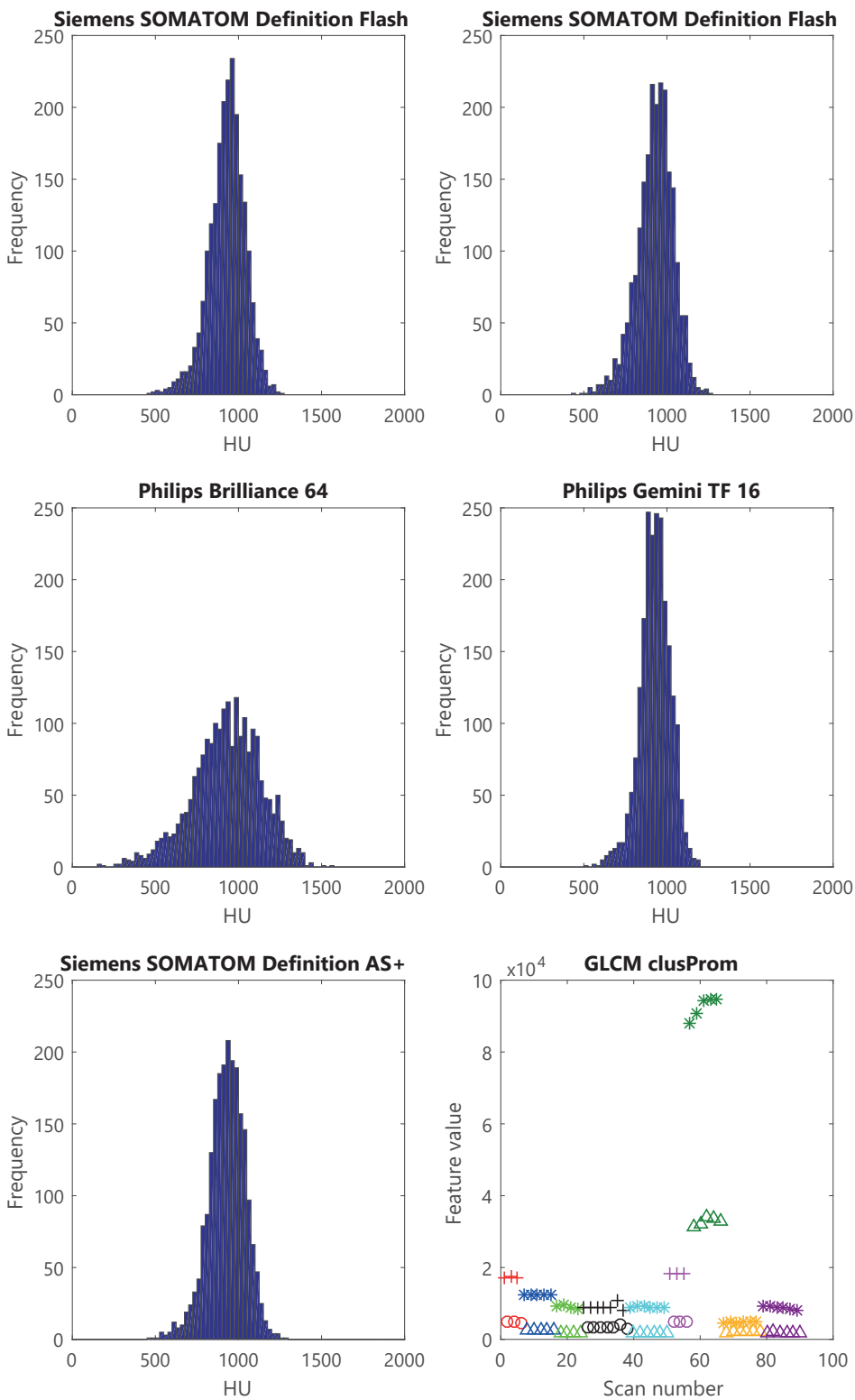

Figure S7.2. HU distributions of the rubber insert scanned with a fixed CDTI of $5.43 \mathrm{mGy}$ on five different scanners, after resampling to voxels of $1 \times 1 \times 3 \mathrm{~mm}^{3}$ using linear interpolation. From left to right and top to bottom [scanner - tube current]: 'Fl-160mA', 'L-161mA', 'P-148mA', 'S-143mA', and 'V-162mA'. The scatterplot in the bottom right displays the observed values of the feature 'GLCM cluster prominence' over all scanners. The outlier in darkgreen is scanner ' $P$ '. 


\section{Supplementary Material 7D - Feature stability for different slice thicknesses}

Table S7.2. Feature stability comparing a slice thickness of $1.5 \mathrm{~mm}$ with $3 \mathrm{~mm}$ (scanner 'B', exposure $60 \mathrm{~mA}$ ). Values with CCC $<0.85$ are indicated in red, and values CCC $>0.85$ in green. The cells marked in bold indicate features with a CCC $<0.85$ in at least one of the testrtest scans [scanner - tube current - slice thickness]: 'B-60mA-3mm' or 'B-60mA-1.5mm'.

\begin{tabular}{|c|c|c|c|c|c|c|c|c|c|c|}
\hline \multirow{2}{*}{ Radiomic feature } & \multicolumn{10}{|c|}{ CCC $1.5 \mathrm{~mm}$ vs. $3.0 \mathrm{~mm}$} \\
\hline & BW 5 & BW 10 & BW 15 & BW 20 & BW 25 & BW 30 & BW 35 & BW 40 & BW 45 & BW 50 \\
\hline GLCM_autocorr & 0,66 & 0,65 & 0,65 & 0,63 & 0,68 & 0,65 & 0,67 & 0,69 & 0,63 & 0,70 \\
\hline GLCM_average & 0,83 & 0,83 & 0,82 & 0,81 & 0,83 & 0,81 & 0,83 & 0,85 & 0,81 & 0,81 \\
\hline GLCM_clusProm & 0,66 & 0,65 & 0,65 & 0,64 & 0,64 & 0,64 & 0,62 & 0,62 & 0,63 & 0,62 \\
\hline GLCM_clusShade & 0,69 & 0,69 & 0,70 & 0,69 & 0,68 & 0,71 & 0,67 & 0,70 & 0,74 & 0,70 \\
\hline GLCM_clusTend & 0,90 & 0,90 & 0,90 & 0,90 & 0,90 & 0,90 & 0,89 & 0,89 & 0,89 & 0,90 \\
\hline GLCM_contrast & 0,93 & 0,93 & 0,93 & 0,93 & 0,93 & 0,93 & 0,93 & 0,92 & 0,93 & 0,93 \\
\hline GLCM_correl1 & 0,91 & 0,90 & 0,90 & 0,90 & 0,89 & 0,91 & 0,89 & 0,89 & 0,90 & 0,90 \\
\hline GLCM_diffAvrg & 0,96 & 0,96 & 0,96 & 0,96 & 0,96 & 0,96 & 0,96 & 0,96 & 0,96 & 0,97 \\
\hline GLCM_diffEntro & 0,96 & 0,96 & 0,96 & 0,97 & 0,97 & 0,97 & 0,97 & 0,97 & 0,97 & 0,96 \\
\hline GLCM_diffVar & 0,92 & 0,92 & 0,92 & 0,92 & 0,92 & 0,92 & 0,92 & 0,92 & 0,92 & 0,93 \\
\hline GLCM_dissimilar & 0,96 & 0,96 & 0,96 & 0,96 & 0,96 & 0,96 & 0,96 & 0,96 & 0,96 & 0,97 \\
\hline GLCM_energy & 0,74 & 0,79 & 0,81 & 0,85 & 0,88 & 0,83 & 0,95 & 0,96 & 0,93 & 0,91 \\
\hline GLCM_entrop2 & 0,75 & 0,89 & 0,92 & 0,93 & 0,94 & 0,94 & 0,95 & 0,95 & 0,95 & 0,95 \\
\hline GLCM_homogeneity1 & 0,95 & 0,96 & 0,96 & 0,96 & 0,97 & 0,97 & 0,97 & 0,97 & 0,97 & 0,97 \\
\hline GLCM_homogeneity2 & 0,93 & 0,95 & 0,96 & 0,96 & 0,97 & 0,97 & 0,97 & 0,97 & 0,97 & 0,97 \\
\hline GLCM_infoCorr1 & 0,83 & 0,85 & 0,92 & 0,96 & 0,97 & 0,99 & 0,98 & 0,97 & 0,99 & 0,99 \\
\hline GLCM_infoCorr2 & 0,99 & 0,98 & 0,98 & 0,98 & 0,98 & 0,98 & ,98 & 0,96 & ,97 &, 96 \\
\hline GLCM_invDiffmomnor & 0,96 & 0,96 & & & 96 & 0,96 & & & & 96 \\
\hline GLCM_invDiffnorm & 0,94 & 0,94 & 0,94 & 0,95 & 0,95 & 0,97 & 0,94 & 0,88 & 0,91 & 0,96 \\
\hline GLCM_inverseVar & 0,93 & 0,95 & 0,95 & 0,95 & 0,95 & 0,93 & 0,98 & 0,98 & 0,97 & 0,88 \\
\hline GLCM_maxCorr & 0,88 & 0,95 & 0,95 & 0,95 & 0,92 & 0,95 & 0,92 & 0,89 & 0,90 & 0,92 \\
\hline GLCM_maxProb & 0,91 & 0,83 & 0,82 & 0,90 & 0,91 & 0,90 & 0,93 & 0,96 & 0,94 & 0,95 \\
\hline GLCM_sumAvg & 0,83 & 0,83 & 0,82 & 81 & 0,83 & 0,81 & 83 & 0,85 &, 81 & 0,81 \\
\hline GLCM_sumEntro & & & & & & 0,94 & 94 & 0,94 & & 0,93 \\
\hline GLCM_sumSquares & 0,91 & 0,91 & 0,91 & 0,91 & 0,91 & 0,91 & 0,91 & 0,90 & 0,91 & 0,91 \\
\hline GLCM_sumVar & 0,90 & 0,90 & 0,90 & 0,90 & 0,90 & 0,90 & 0,89 & 0,89 & 0,89 & 0,90 \\
\hline GLDZM_DZE & 0,63 & 0,68 & 0,68 & 0,67 & 0,69 & 0,73 & 0,69 & 0,60 & 0,70 & 0,64 \\
\hline GLDZM_DZN & 0,64 & 0,86 & 0,93 & 0,94 & 0,95 & 0,93 & 0,94 & 0,93 & 0,95 & 0,95 \\
\hline GLDZM_DZNN & 0,07 & 0,09 & $\underline{0,06}$ & $\underline{0,04}$ & $\underline{0,10}$ & $\underline{0,19}$ & $\underline{0,04}$ & $\underline{0,10}$ & 0,14 & $\underline{0,39}$ \\
\hline GLDZM_DZV & $\underline{0.02}$ & $\underline{0,02}$ & $\underline{0,00}$ & $\underline{0,02}$ & $\underline{0,02}$ & $\underline{0.04}$ & $\underline{0.04}$ & $\underline{0,06}$ & $\underline{0,05}$ & $\underline{0,14}$ \\
\hline GLDZM_HIE & 0,66 & 0,66 & 0,66 & 0,64 & 0,68 & 0,65 & 0,67 & 0,68 & 0,60 & 0,68 \\
\hline GLDZM_HILDE & 0,35 & 0,33 & 0,35 & 0,33 & 0,36 & 0,34 & 0,36 & 0,38 & 0,32 & 0,38 \\
\hline GLDZM_HISDE & 0,84 & 0,85 & 0,82 & 0,81 & 0,84 & 0,80 & 0,81 & 0,82 & 0,75 & 0,81 \\
\hline GLDZM_IN & 0,32 & 0,22 & 0,27 & 0,45 & 0,46 & $\underline{0,45}$ & 0,31 & 0,56 & 0,57 & $\underline{0,53}$ \\
\hline GLDZM_INN & 0,88 & 0,88 & 0,89 & 0,90 & 0,89 & 0,97 & 0,90 & 0,89 & 0,98 & 0,93 \\
\hline GLDZM_IV & 0,90 & 0,90 & 0,89 & 0,87 & 0,86 & 0,87 & 0,88 & 0,84 & 0,82 & 0,83 \\
\hline GLDZM_LDE & 0,04 & 0,04 & $\underline{0,03}$ & $\underline{0,03}$ & $\underline{0,05}$ & $\underline{0,07}$ & $\underline{0,00}$ & 0,03 & $\underline{0,06}$ & $\underline{0,17}$ \\
\hline GLDZM_LIE & 0,64 & 0,30 & $\underline{0.16}$ & 0,19 & $\underline{0,14}$ & $\underline{0,44}$ & $\underline{0.47}$ & 0,88 & 0,80 & $\underline{0.06}$ \\
\hline GLDZM_LILDE & $\underline{0,69}$ & $\underline{0,46}$ & $\underline{0,23}$ & $\underline{0,43}$ & $\underline{0,27}$ & $\underline{0,85}$ & $\underline{0,73}$ & $\underline{0,45}$ & $\underline{0,17}$ & $\underline{0,34}$ \\
\hline GLDZM_LISDE & 0,62 & 0,27 & 0,14 & 0,13 & $\underline{0,11}$ & $\underline{0,34}$ & $\underline{0,28}$ & 0,49 & 0,83 & $\underline{0,02}$ \\
\hline GLDZM_SDE & 0,10 & 0,10 & $\underline{0.08}$ & $\underline{0,06}$ & $\underline{0.11}$ & $\underline{0,17}$ & $-0,01$ & 0,04 & 0,08 & $\underline{0,31}$ \\
\hline GLDZM_ZP & 0,97 & 0,96 & 0,93 & 0,92 & 0,89 & 0,87 & 0,85 & 0,86 & 0,81 & 0,81 \\
\hline GLRLM_GLN & 0,72 & 0,69 & 0,65 & 0,60 & 0,55 & 0,47 & 0,48 & 0,44 & 0,39 & 0,28 \\
\hline
\end{tabular}




\begin{tabular}{|c|c|c|c|c|c|c|c|c|c|c|}
\hline \multirow{2}{*}{ Radiomic feature } & \multicolumn{10}{|c|}{ CCC $1.5 \mathrm{~mm}$ vs. $3.0 \mathrm{~mm}$} \\
\hline & BW 5 & BW 10 & BW 15 & BW 20 & BW 25 & BW 30 & BW 35 & BW 40 & BW 45 & BW 50 \\
\hline GLRLM_GLNN & 0,89 & 0,90 & 0,91 & 0,92 & 0,93 & 0,93 & 0,95 & 0,96 & 0,95 & 0,85 \\
\hline GLRLM_GLV & 0,91 & 0,91 & 0,91 & 0,91 & 0,91 & 0,90 & 0,90 & 0,90 & 0,90 & 0,90 \\
\hline GLRLM_HGRE & 0,66 & 0,66 & 0,66 & 0,64 & 0,69 & 0,66 & 0,67 & 0,69 & 0,63 & 0,70 \\
\hline GLRLM_LGRE & 0,99 & 0,92 & 0,75 & 0,61 & 0,56 & $\underline{0,30}$ & $\underline{0,86}$ & 0,47 & 0,43 & $\underline{0,57}$ \\
\hline GLRLM_LRE & 0,91 & 0,91 & 0,91 & 0,91 & 0,95 & 0,86 & 0,95 & 0,95 & 0,93 & 0,92 \\
\hline GLRLM_LRHGE & 0,66 & 0,66 & 0,65 & 0,62 & 0,67 & 0,62 & 0,64 & 0,66 & 0,57 & 0,59 \\
\hline GLRLM_LRLGE & 0,98 & 0,93 & 0,83 & 0,69 & 0,59 & 0,36 & $\underline{0.85}$ & 0,55 & 0,51 & $\underline{0,55}$ \\
\hline GLRLM_RE & 0,93 & 0,93 & 0,92 & 0,91 & 0,89 & 0,88 & 0,85 & 0,81 & 0,78 & 0,53 \\
\hline GLRLM_RLN & 0,02 & 0,07 & 0,12 & 0,18 & 0,22 & 0,28 & 0,28 & 0,31 & 0,35 & 0,43 \\
\hline GLRLM_RLNN & 0,96 & 0,96 & 0,97 & 0,97 & 0,97 & 0,97 & 0,98 & 0,98 & 0,98 & 0,98 \\
\hline GLRLM_RLV & 0,83 & 0,87 & 0,90 & 0,90 & 0,95 & 0,86 & 0,94 & 0,95 & 0,93 & 0,98 \\
\hline GLRLM_RP & 0,95 & 0,96 & 0,97 & 0,97 & 0,98 & 0,97 & 0,98 & 0,97 & 0,98 & 0,98 \\
\hline GLRLM_SRE & 0,95 & 0,95 & 0,96 & 0,96 & 0,97 & 0,96 & 0,98 & 0,98 & 0,98 & 0,93 \\
\hline GLRLM_SRHGE & 0,66 & 0,65 & 0,66 & 0,64 & 0,69 & 0,66 & 0,67 & 0,69 & 0,63 & 0,71 \\
\hline GLRLM_SRLGE & 0,99 & 0,89 & 0,69 & 0,55 & 0,51 & $\underline{0,25}$ & $\underline{0,85}$ & 0,44 & 0,44 & $\underline{0,54}$ \\
\hline GLSZM_HIE & 0,66 & 0,66 & 0,66 & 0,64 & 0,68 & 0,65 & 0,67 & 0,68 & 0,60 & 0,68 \\
\hline GLSZM_HILAE & 0,84 & 0,67 & 0,66 & 0,68 & 0,40 & 0,52 & $\underline{0,49}$ & 0,89 & 0,51 & 0,51 \\
\hline GLSZM_HISAE & 0,66 & 0,64 & 0,66 & 0,62 & 0,66 & 0,66 & 0,71 & 0,67 & 0,57 & 0,72 \\
\hline GLSZM_IN & 0,32 & 0,22 & 0,27 & 0,45 & 0,46 & $\underline{0,45}$ & 0,31 & 0,56 & 0,57 & $\underline{0,53}$ \\
\hline GLSZM_INN & 0,88 & 0,88 & 0,89 & 0,90 & 0,89 & 0,97 & 0,90 & 0,89 & 0,98 & 0,93 \\
\hline GLSZM_IV & 0,90 & 0,90 & 0,89 & 0,87 & 0,86 & 0,87 & 0,88 & 0,84 & 0,82 & 0,83 \\
\hline GLSZM_LAE & 0,95 & 0,96 & 0,90 & 0,88 & 0,77 & 0,82 & 0,32 & 0,58 & 0,96 & 0,80 \\
\hline GLSZM_LIE & 0,64 & 0,30 & $\underline{0,16}$ & 0,19 & $\underline{0,14}$ & 0,44 & $\underline{0,47}$ & 0,88 & 0,80 & $\underline{0,06}$ \\
\hline GLSZM_LILAE & 0,73 & 0,46 & 0,37 & 0,66 & $\underline{0,50}$ & $\underline{0,51}$ & $\underline{0,15}$ & 0,18 & 0,41 & $\underline{0,78}$ \\
\hline GLSZM_LISAE & 0,32 & $\underline{0.21}$ & $\underline{0,11}$ & $\underline{0,18}$ & $\underline{0,17}$ & $\underline{0.28}$ & $\underline{0,65}$ & $-0,22$ & $\underline{0,05}$ & $\underline{0,10}$ \\
\hline GLSZM_SAE & 0,93 & 0,85 & $\underline{0,30}$ & 0,66 & 0,67 & $\underline{0,81}$ & $\underline{0,13}$ & $-0,18$ & $\underline{0,36}$ & 0,95 \\
\hline GLSZM_SZN & 0,42 & 0,52 & 0,61 & 0,58 & 0,56 & 0,61 & 0,65 & 0,55 & 0,53 & 0,67 \\
\hline GLSZM_SZNN & 0,94 & 0,86 & $\underline{0,48}$ & 0,73 & $\underline{0,65}$ & $\underline{0,83}$ & 0,45 & 0,89 & $\underline{0,66}$ & 0,93 \\
\hline GLSZM_SZV & 0,95 & 0,96 & 0,89 & 0,86 & 0,76 & 0,81 & 0,66 & 0,20 & $\underline{0,74}$ & 0,77 \\
\hline GLSZM_ZE & 0,77 & 0,84 & 0,85 & 0,86 & 0,93 & 0,90 & 0,96 & 0,92 & 0,95 & 0,93 \\
\hline GLSZM_ZP & 0,97 & 0,96 & 0,93 & 0,92 & 0,89 & 0,87 & 0,85 & 0,86 & 0,81 & 0,81 \\
\hline NGLDM_DE & 0,73 & 0,79 & 0,78 & 0,79 & 0,79 & 0,83 & 0,86 & 0,82 & 0,75 & 0,83 \\
\hline NGLDM_DN & 0,32 & 0,34 & 0,36 & 0,37 & 0,37 & 0,36 & 0,30 & 0,31 & 0,34 & 0,32 \\
\hline NGLDM_DNN & 0,94 & 0,93 & 0,95 & 0,95 & 0,95 & 0,94 & 0,95 & 0,94 & 0,96 & 0,96 \\
\hline NGLDM_DV & 0,96 & 0,94 & 0,91 & 0,95 & 0,96 & 0,95 & 0,95 & 0,96 & 0,96 & 0,95 \\
\hline NGLDM_GLN & 0,73 & 0,74 & 0,74 & 0,71 & 0,68 & 0,72 & 0,57 & 0,53 & 0,57 & 0,66 \\
\hline NGLDM_GLNN & 0,89 & 0,90 & 0,91 & 0,93 & 0,94 & 0,92 & 0,96 & 0,96 & 0,95 & 0,95 \\
\hline NGLDM_GLV & 0,91 & 0,91 & 0,91 & 0,91 & 0,91 & 0,91 & 0,91 & 0,91 & 0,91 & 0,91 \\
\hline NGLDM_HGE & 0,66 & 0,66 & 0,65 & 0,64 & 0,69 & 0,66 & 0,67 & 0,69 & 0,63 & 0,70 \\
\hline NGLDM_HGLDE & 0,66 & 0,66 & 0,59 & 0,56 & 0,57 & $\underline{0,55}$ & $\underline{0,55}$ & 0,76 & 0,62 & 0,53 \\
\hline NGLDM_HGSDE & 0,66 & 0,62 & 0,64 & 0,59 & 0,62 & 0,59 & 0,63 & 0,60 & 0,53 & 0,69 \\
\hline NGLDM_LDE & 0,94 & 0,94 & 0,95 & 0,97 & 0,98 & 0,95 & 0,97 & 0,95 & 0,97 & 0,97 \\
\hline NGLDM_LGE & 0,99 & 0,95 & 0,85 & 0,74 & 0,69 & 0,46 & $\underline{0,90}$ & 0,51 & 0,43 & $\underline{0,73}$ \\
\hline
\end{tabular}




\begin{tabular}{|c|c|c|c|c|c|c|c|c|c|c|}
\hline \multirow{2}{*}{ Radiomic feature } & \multicolumn{10}{|c|}{ CCC $1.5 \mathrm{~mm}$ vs. $3.0 \mathrm{~mm}$} \\
\hline & BW 5 & BW 10 & BW 15 & BW 20 & BW 25 & BW 30 & BW 35 & BW 40 & BW 45 & BW 50 \\
\hline NGLDM_LGLDE & 0,99 & 0,97 & 0,92 & 0,77 & 0,63 & 0,40 & 0,88 & 0,56 & 0,46 & 0,66 \\
\hline NGLDM_LGSDE & 0,73 & $\underline{0,43}$ & $\underline{0.23}$ & $\underline{0.21}$ & $\underline{0,34}$ & $\underline{0,49}$ & $\underline{0.69}$ & $\underline{0,76}$ & 0,74 & 0,26 \\
\hline NGLDM_SDE & 0,96 & 0,94 & 0,92 & 0,93 & 0,91 & 0,91 & 0,92 & 0,93 & 0,91 & 0,94 \\
\hline NGLDM_SM & 0,44 & 0,40 & 0,41 & 0,34 & 0,35 & 0,31 & 0,26 & 0,23 & 0,27 & 0,45 \\
\hline NGTDM_busyness & 0,77 & 0,75 & 0,51 & 0,63 & $\underline{0,72}$ & $\underline{0,45}$ & $\underline{0,73}$ & 0,27 & $\underline{0,00}$ & 0,44 \\
\hline NGTDM_coarseness & 0,35 & 0,30 & 0,28 & 0,28 & 0,23 & 0,25 & 0,23 & 0,23 & 0,25 & 0,30 \\
\hline NGTDM_complexity & 0,69 & 0,63 & 0,63 & 0,60 & 0,61 & 0,58 & 0,56 & 0,56 & 0,49 & 0,59 \\
\hline NGTDM_contrast & 1,00 & 1,00 & 1,00 & 0,99 & 1,00 & 1,00 & 0,99 & 0,96 & 0,98 & 0,99 \\
\hline NGTDM_strength & 0,90 & 0,93 & 0,94 & 0,95 & 0,96 & 0,94 & 0,95 & 0,95 & 0,90 & 0,95 \\
\hline Stats_energy & 0,70 & 0,70 & 0,70 & 0,70 & 0,70 & 0,70 & 0,70 & 0,70 & 0,70 & 0,70 \\
\hline Stats_entropy & 0,94 & 0,95 & 0,95 & 0,95 & 0,95 & 0,95 & 0,96 & 0,96 & 0,96 & 0,95 \\
\hline Stats_iqr & 0,98 & 0,98 & 0,98 & 0,98 & 0,98 & 0,98 & 0,98 & 0,98 & 0,98 & 0,98 \\
\hline Stats_kurtosis & 0,95 & 0,95 & 0,95 & 0,95 & 0,95 & 0,95 & 0,95 & 0,95 & 0,95 & 0,95 \\
\hline Stats_max & 0,99 & 0,99 & 0,99 & 0,99 & 0,99 & 0,99 & 0,99 & 0,99 & 0,99 & 0,99 \\
\hline Stats_md & 0,96 & 0,96 & 0,96 & 0,96 & 0,96 & 0,96 & 0,96 & 0,96 & 0,96 & 0,96 \\
\hline Stats_mean & 1,00 & 1,00 & 1,00 & 1,00 & 1,00 & 1,00 & 1,00 & 1,00 & 1,00 & 1,00 \\
\hline Stats_median & 1,00 & 1,00 & 1,00 & 1,00 & 1,00 & 1,00 & 1,00 & 1,00 & 1,00 & 1,00 \\
\hline Stats_min & 0,99 & 0,99 & 0,99 & 0,99 & 0,99 & 0,99 & 0,99 & 0,99 & 0,99 & 0,99 \\
\hline Stats_p10 & 1,00 & 1,00 & 1,00 & 1,00 & 1,00 & 1,00 & 1,00 & 1,00 & 1,00 & 1,00 \\
\hline Stats_p90 & 1,00 & 1,00 & 1,00 & 1,00 & 1,00 & 1,00 & 1,00 & 1,00 & 1,00 & 1,00 \\
\hline Stats_range & 0,82 & 0,82 & 0,82 & 0,82 & 0,82 & 0,82 & 0,82 & 0,82 & 0,82 & 0,82 \\
\hline Stats_rmd & 0,98 & 0,98 & 0,98 & 0,98 & 0,98 & 0,98 & 0,98 & 0,98 & 0,98 & 0,98 \\
\hline Stats_rms & 1,00 & 1,00 & 1,00 & 1,00 & 1,00 & 1,00 & 1,00 & 1,00 & 1,00 & 1,00 \\
\hline Stats_skewness & 0,98 & 0,98 & 0,98 & 0,98 & 0,98 & 0,98 & 0,98 & 0,98 & 0,98 & 0,98 \\
\hline Stats_std & 0,95 & 0,95 & 0,95 & 0,95 & 0,95 & 0,95 & 0,95 & 0,95 & 0,95 & 0,95 \\
\hline Stats_totalenergy & 1,00 & 1,00 & 1,00 & 1,00 & 1,00 & 1,00 & 1,00 & 1,00 & 1,00 & 1,00 \\
\hline Stats_uniformity & 0,89 & 0,90 & 0,91 & 0,93 & 0,94 & 0,92 & 0,96 & 0,96 & 0,95 & 0,95 \\
\hline Stats_var & 0,92 & 0,92 & 0,92 & 0,92 & 0,92 & 0,92 & 0,92 & 0,92 & 0,92 & 0,92 \\
\hline
\end{tabular}


Supplementary Material 7E - Feature range phantom and patients
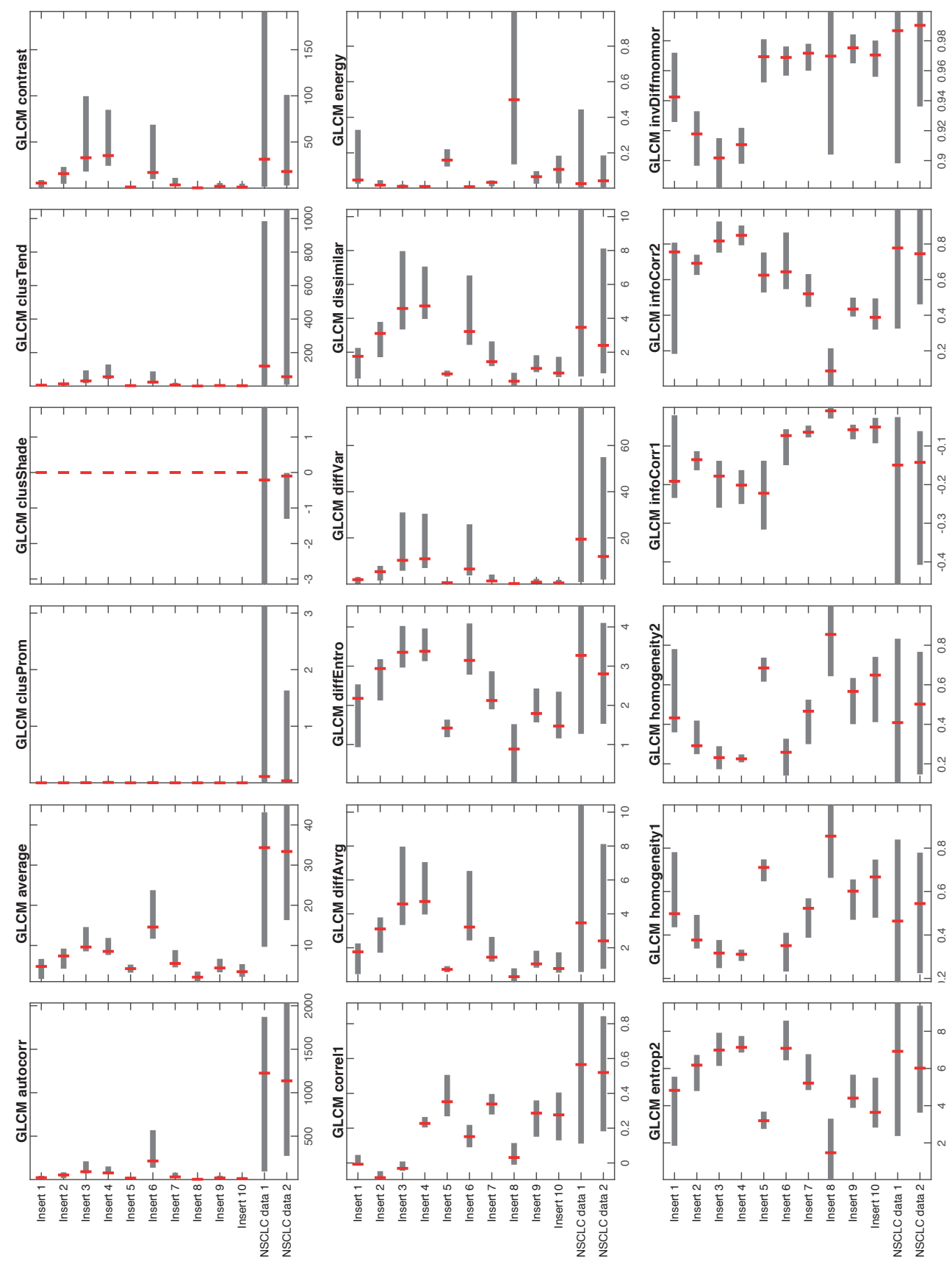

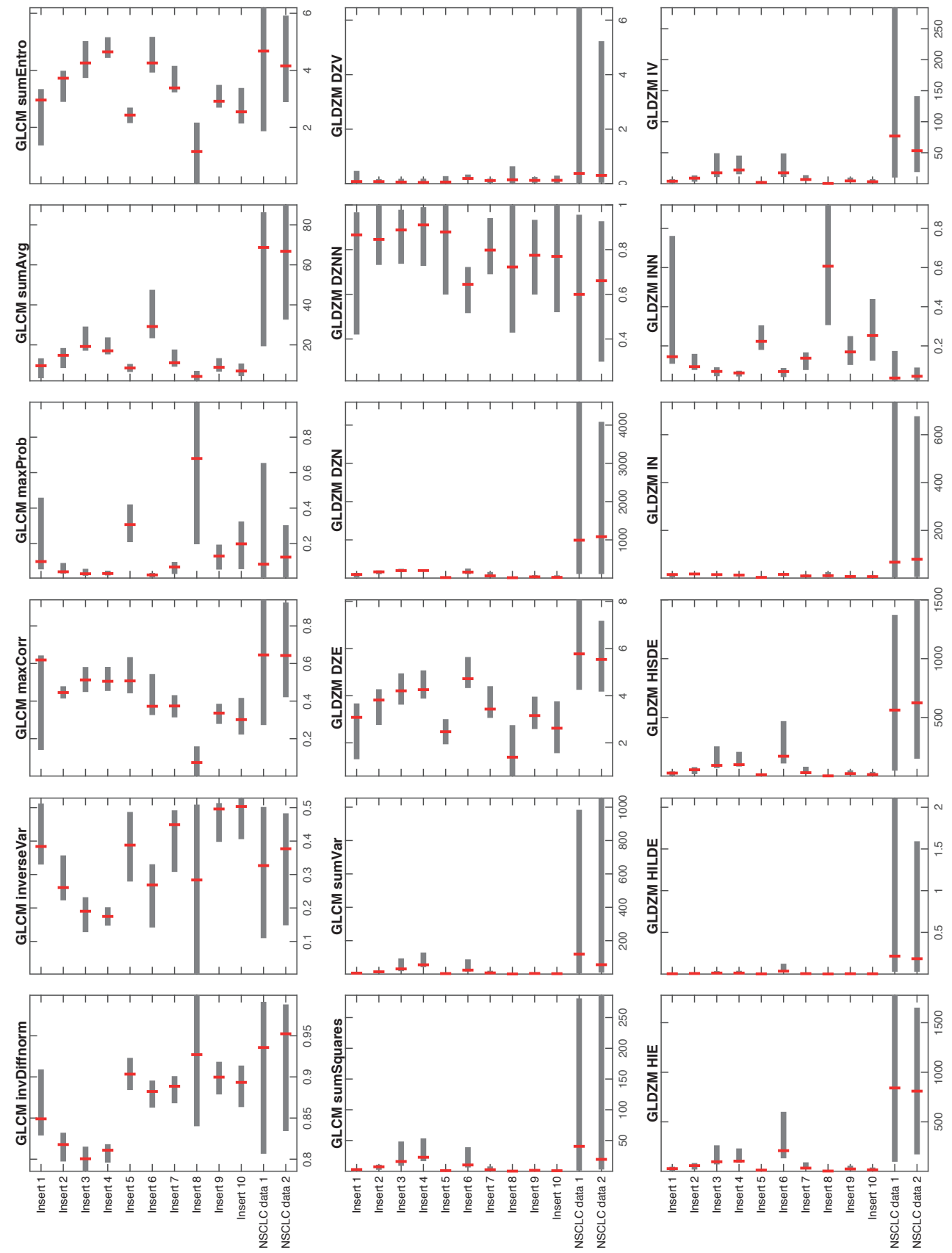

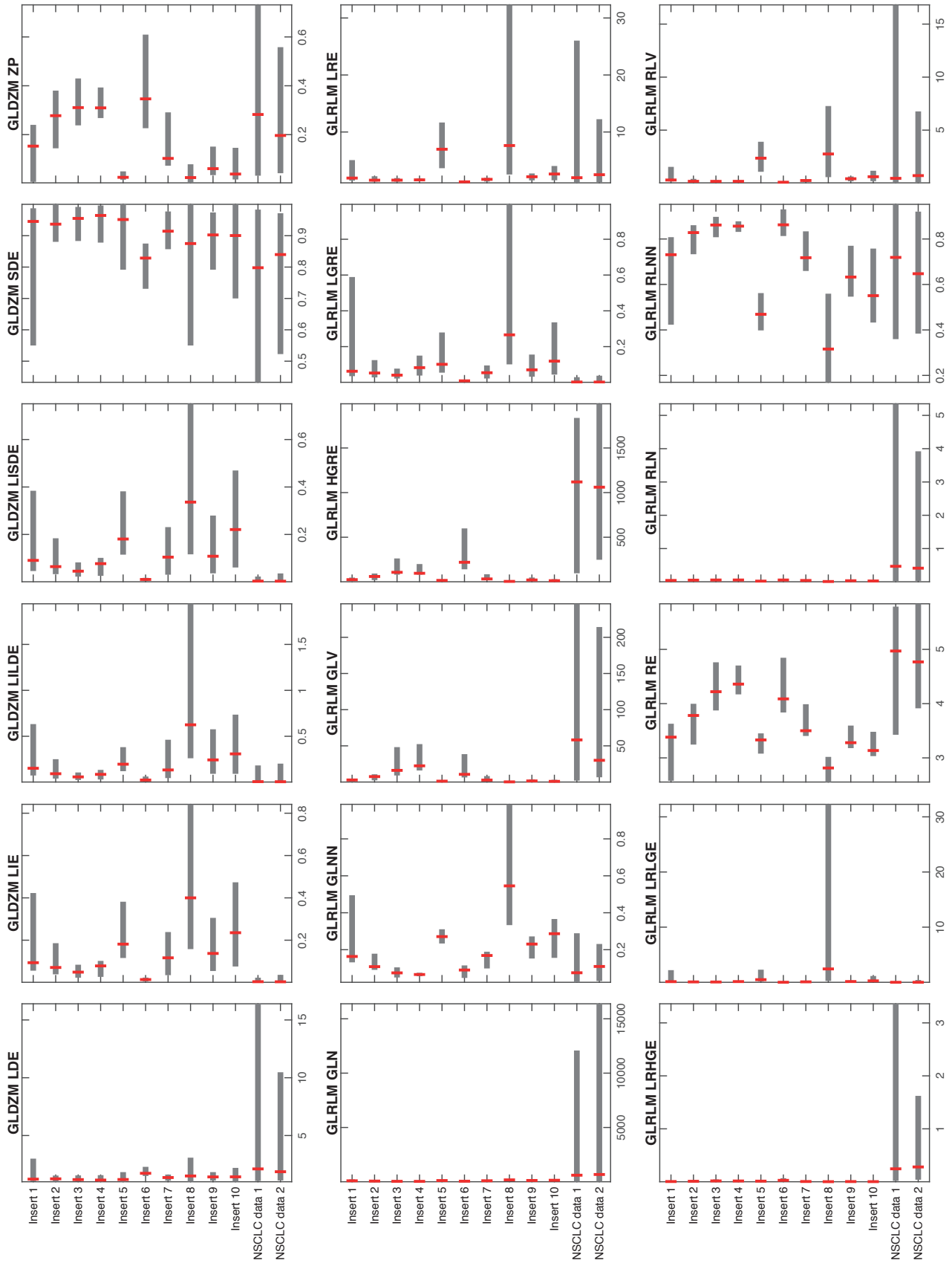

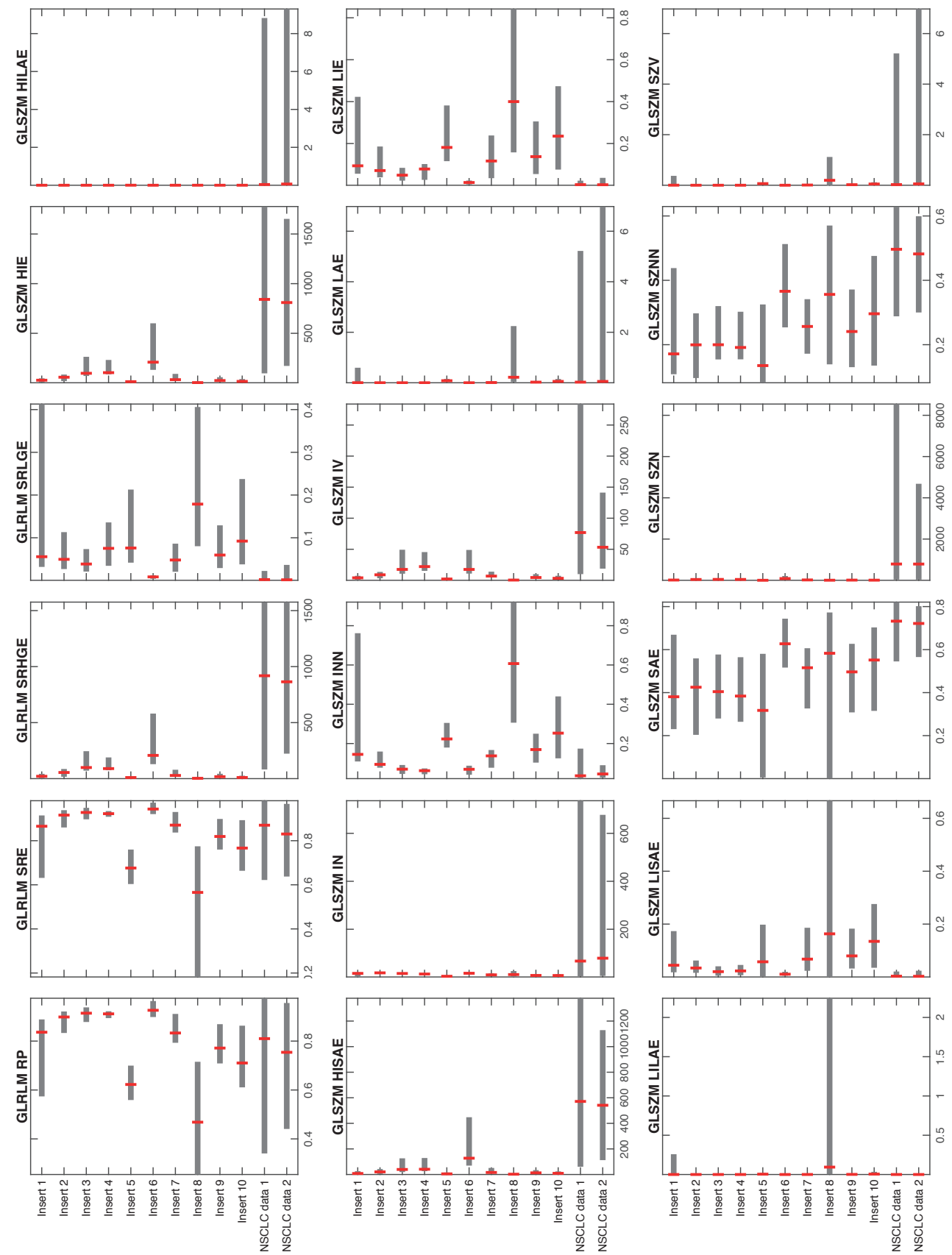

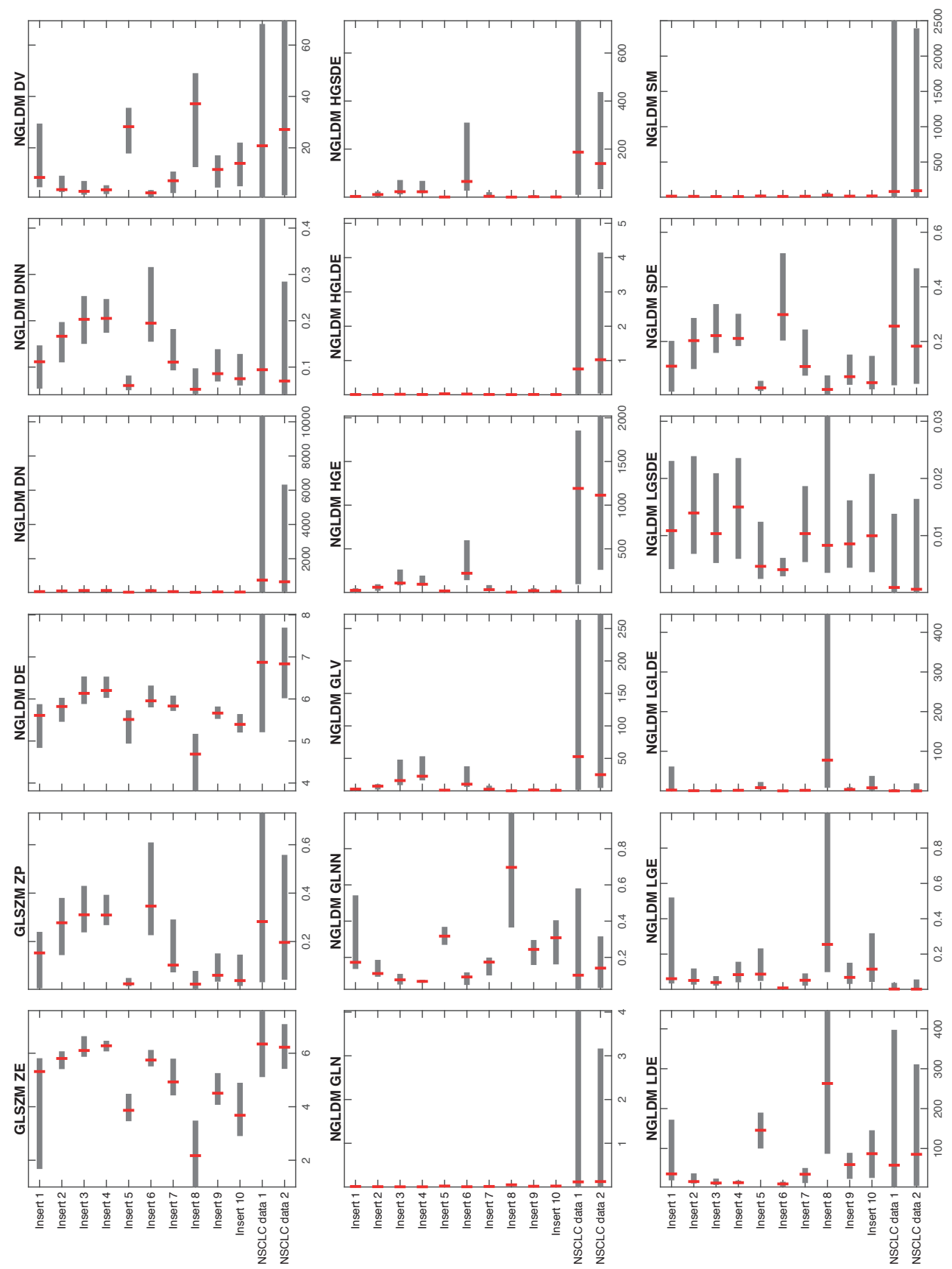

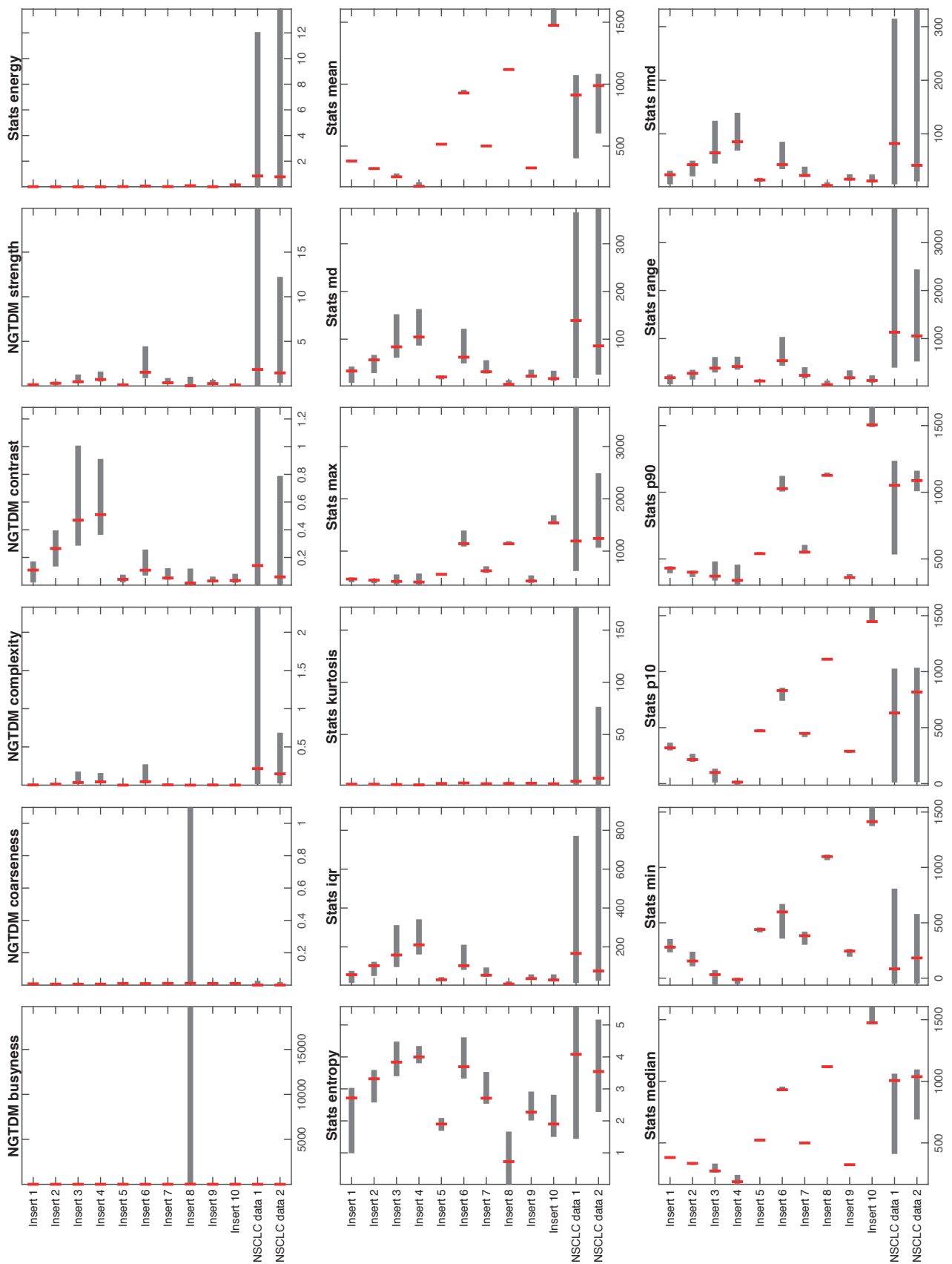

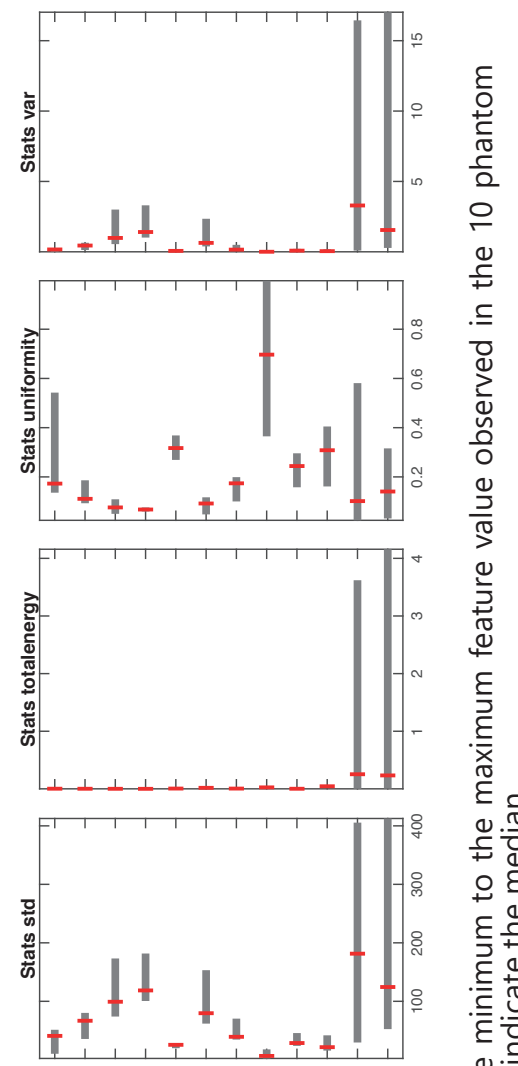

है

†

으

ह®

.

.

든

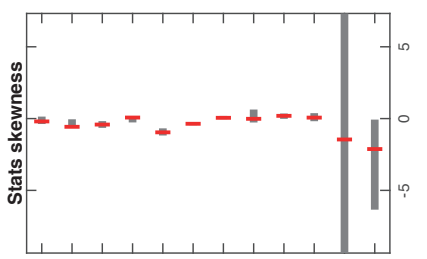

$\varepsilon \cong$

은 망

ㅁำ

잉

可尝

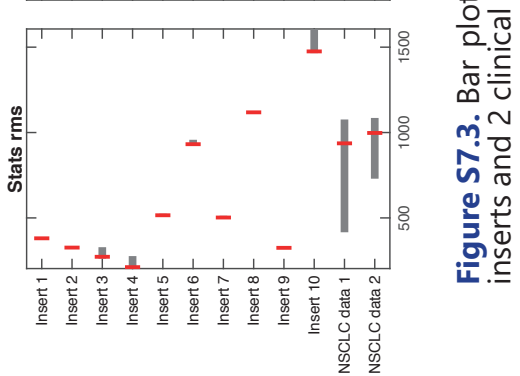




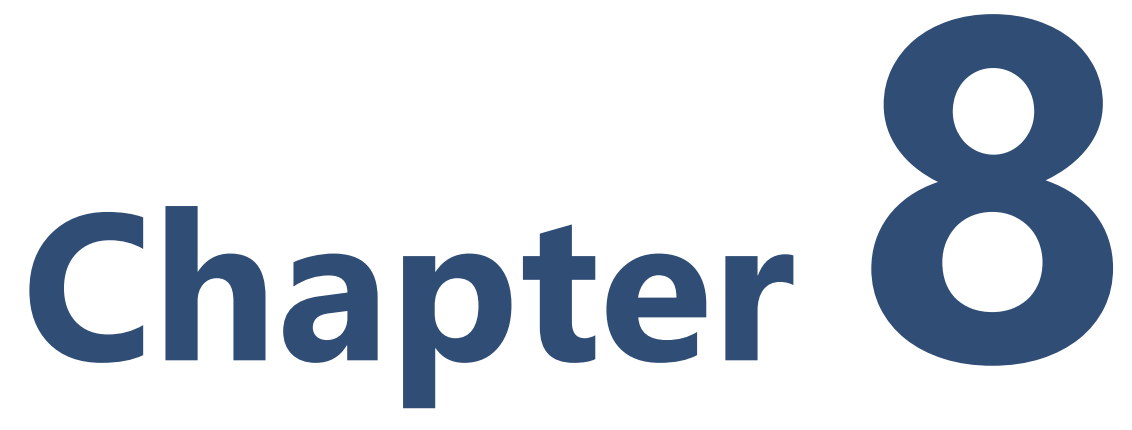

\section{Challenges and caveats of a multi-center retrospective radiomics study: an example of early treatment response assessment for NSCLC patients using FDG-PET/CT radiomics}

Authors

Janna E. van Timmeren*, Sara Carvalho*, Ralph T.H. Leijenaar, Esther G.C. Troost, Wouter van Elmpt, Dirk de Ruysscher, Jean-Pierre Muratet, Fabrice Denis, Tanja Schimek-Jasch, Ursula Nestle, Arthur Jochems, Henry C. Woodruff, Cary Oberije, Philippe Lambin 


\begin{abstract}
Background

Prognostic models based on individual patient characteristics can improve treatment decisions and outcome in the future. In many (radiomic) studies, small size and heterogeneity of datasets is a challenge that often limits performance and potential clinical applicability of these models. The current study is an example of a retrospective multi-centric study with challenges and caveats. To highlight common issues and emphasize potential pitfalls, we aimed for an extensive analysis of these multi-center pre-treatment datasets, with an additional ${ }^{18} \mathrm{~F}$-fluorodeoxyglucose (FDG) positron emission tomography/computed tomography (PET/CT) scan acquired during treatment.
\end{abstract}

\title{
Material and methods
}

The dataset consisted of 138 stage II-IV non-small cell lung cancer (NSCLC) patients from four different cohorts acquired from three different institutes. The differences between the cohorts were compared in terms of clinical characteristics and using the so-called 'cohort differences model' approach. Moreover, the potential prognostic performances for overall survival of radiomic features extracted from CT or FDG-PET, or relative or absolute differences between the scans at the two time points, were assessed using the LASSO regression method. Furthermore, the performances of five different classifiers were evaluated for all image sets.

\section{Results}

The individual cohorts substantially differed in terms of patient characteristics. Moreover, the cohort differences model indicated statistically significant differences between the cohorts. Neither LASSO nor any of the tested classifiers resulted in a clinical relevant prognostic model that could be validated on the available datasets.

\section{Conclusions}

The results imply that the study might have been influenced by a limited sample size, heterogeneous patient characteristics, and inconsistent imaging parameters. No prognostic performance of FDG-PET or CT based radiomics models can be reported. This study highlights the necessity of extensive evaluations of cohorts and of validation datasets, especially in retrospective multi-centric datasets. 


\section{Introduction}

Prognostic models based on individual patient derived factors are essential to better estimate patient's outcome prior to or during treatment. These models help to improve individualized treatment decisions (personalized medicine) that may lead to better patient outcomes [1, 2]. Medical images are an example of an information source, which could be used to derive important patient-specific prognostic information. Large amounts of quantitative features can be calculated from medical images acquired during a patient's course of treatment, e.g. positron emission tomography (PET) or computed tomography (CT). This principle of extracting imaging features is called 'radiomics', which is a rapidly evolving field of interest [3-5]. Multiple studies have shown radiomics' potential to derive prognostic information for patient outcomes. Despite the promising results, radiomics faces multiple challenges [6]. An important challenge is the collection and acquisition of (large amounts of) suitable imaging data, which is difficult due to evolving technology, lack of standardization protocols and differences in cohorts and protocols between institutes.

Imaging data collected from a single institution often results in a more homogeneous dataset, e.g. the images are acquired using the same settings (with the institute's acquisition and reconstruction protocols) on the same scanner. Also, with regard to clinical characteristics the patient population is usually more homogeneous. However, a prognostic model developed based on these data might fail when applied to validation data from an external institute, due to the lack of transferability of the model (i.e. the model is specific for a population) [7, 8]. Therefore, one might argue for using more heterogeneous datasets to train the model to broaden the clinical applicability. In practice, it appears difficult to find these datasets and to validate such a model. Besides the variability in imaging and/or clinical characteristics within a dataset, the dimensionality of the data is often an issue, e.g. having over 1000 radiomic features with often only a limited number of patients. $A$ review published in 2015 has shown that many published (radiomics) studies contain models based on a limited number of patients without validation on an independent dataset, resulting in high probabilities of false positive results [9].

In this study, PET and CT images were collected from non-small cell lung cancer (NSCLC) patients who underwent an ${ }^{18} \mathrm{~F}$-fluorodeoxyglucose (FDG) positron emission tomography/computed tomography (PET/CT) scan prior to and during (chemo)radiotherapy to be able to evaluate radiomics' potential to assess early response. Previous studies have explored (early) response assessment using information derived from FDG-PET/CT scans [10-15]. Since the PET/CT scans obtained during treatment are generally not acquired in clinical practice, it is difficult to gather large datasets of this type. Therefore, the data in the current study were collected from three different institutes where small sub-cohorts were available and is therefore a typical example of a multi-centric study with retrospectively collected data.

Retrospective studies, although collected from centers with high quality care, often suffer from heterogeneities within and between datasets, which is unavoidable in clinical practice. Therefore, the current study aims to evaluate the multicenter patient data in terms of cohort characteristics and prognostic performance, intending to highlight common issues and emphasizing pitfalls for future (radiomic) studies. 


\section{Materials and Methods}

\section{Study cohort}

The entire study cohort consists of 138 stage II-IV NSCLC patients, all treated with curatively intended (chemo)radiotherapy. The study population was divided into four different datasets from three different institutes. Datasets 1 and 2 originated from MAASTRO Clinic, Maastricht, the Netherlands. Dataset 3 is from Clinique Victor Hugo, Le Mans, France, and Dataset 4 was obtained from University Medical Center Freiburg, Freiburg, Germany. Dataset 1 was prospectively collected with approval of the Institutional Review Board of the Department of Radiation Oncology of Maastricht University Medical Center (Maastro Clinic) (clinicaltrials.gov NTC00522639). The original study that collected the data in Dataset 2 was approved by the Institutional Board of the Department of Radiation Oncology of Maastricht University Medical Center (Maastro Clinic), as stated in [12]. Dataset 3 was collected in a previous study [16] and approved by the appropriate Institutional Review Board. Dataset 4 was prospectively collected with approval of the Ethics Committee of the Albert-Ludwig University Freiburg, Germany Freiburg (clinicaltrials.gov NCT00697333).

All patients underwent an ${ }^{18} \mathrm{~F}-\mathrm{FDG}-\mathrm{PET} / \mathrm{CT}$ scan before radiotherapy for treatment planning purposes and an additional scan approximately two weeks after the start of treatment. The scanning parameters are summarized in Table 8.1. Detailed cohort descriptions are provided in Supplementary Material 8A. For all patients, overall survival (OS), calculated from the start of radiotherapy, was collected to serve as a clinical endpoint in this study. A patient still alive at the time of study analysis was considered right-censored. Median follow-up times were calculated using the Kaplan-Meier estimator with reversed events, considering death as censored data.

\section{Tumor segmentation and image analysis}

The primary gross tumor volume (GTV) was delineated by experienced radiation oncologists on pre- and during-radiotherapy fused FDG-PET/CT images using the following WL settings, W:1700 L:-300 (lung) and for W:600 L:40 (mediastinum). These target delineations were not evaluated by other specialists and the reproducibility of these segmentations was not investigated in this study, as this segmentation process was just part of normal clinical practice. FDG-PET/CT images were converted into standard uptake value (SUV) prior to analysis [17]. A total of 1295 radiomic features were extracted from CT images and 1400 radiomic features were extracted from PET images. Prior to radiomics feature extraction, all images were resampled into voxel dimensions $1 \times 1 \times 3 \mathrm{~mm}^{3}$ for $\mathrm{CT}$ and $4 \times 4 \times 3 \mathrm{~mm}^{3}$ for PET, which corresponded to the average voxel dimensions of all images rounded to the nearest integer. Resampling decreases the variability of radiomic features [18] and was performed using cubic interpolation. For one PET scan the resampling resulted in a segment splitting off from the original volume resulting in two separate volumes. As radiomic features have a different interpretation when extracted from multiple volumes, this patient was excluded from the analyses to avoid inconsistencies. 
Table 8.1. Scanning parameters for all scans included in the study.

\begin{tabular}{|c|c|c|c|c|c|}
\hline \multicolumn{2}{|c|}{ Parameters } & $\begin{array}{l}\text { Dataset } 1 \\
(n=100)\end{array}$ & $\begin{array}{l}\text { Dataset } 2 \\
(n=62)\end{array}$ & $\begin{array}{l}\text { Dataset } 3 \\
(n=54)\end{array}$ & $\begin{array}{l}\text { Dataset } 4 \\
(n=60)\end{array}$ \\
\hline \multicolumn{2}{|c|}{ Manufacturer } & Siemens & Siemens & Philips Healthcare & Philips Healthcare \\
\hline \multirow[t]{5}{*}{ CT } & Tube voltage & $120 \mathrm{kVp}$ & $120 \mathrm{kVp}$ & $\begin{array}{l}120 \mathrm{kVp}(\mathrm{n}=39) \\
140 \mathrm{kVp}(\mathrm{n}=15)\end{array}$ & $120 \mathrm{kVp}$ \\
\hline & Tube current & $\begin{array}{l}120 \mathrm{~mA}(n=3) \\
160 \mathrm{~mA}(n=15) \\
173 \mathrm{~mA}(n=1) \\
240 \mathrm{~mA}(n=72)\end{array}$ & $\begin{array}{l}80 \mathrm{~mA}(\mathrm{n}=13) \\
336 \mathrm{~mA}(\mathrm{n}=49)\end{array}$ & $\begin{array}{l}\text { Median [range] } \\
183 \text { mA [115-277] }\end{array}$ & $\begin{array}{l}\text { Median [range] } \\
183 \mathrm{~mA}[35-337]\end{array}$ \\
\hline & Convolution kernel & $\begin{array}{l}\text { B19f }(n=97) \\
\text { B30f }(n=2) \\
\text { B41f }(n=1)\end{array}$ & $\begin{array}{l}\text { B19f }(n=37) \\
\text { B30f }(n=12) \\
\text { B41f }(n=13)\end{array}$ & B & B \\
\hline & Slice thickness & $3 \mathrm{~mm}$ & $3 \mathrm{~mm}$ & $5 \mathrm{~mm}$ & $\begin{array}{l}1 \mathrm{~mm}(n=16) \\
2 \mathrm{~mm}(n=16) \\
3 \mathrm{~mm}(n=4) \\
4 \mathrm{~mm}(n=24)\end{array}$ \\
\hline & Pixel spacing & $0.98 \mathrm{~mm}$ & $0.98 \mathrm{~mm}$ & $\begin{array}{l}1.2 \mathrm{~mm}(\mathrm{n}=44) \\
1.4 \mathrm{~mm}(\mathrm{n}=10)\end{array}$ & $\begin{array}{l}\text { Median [range] } \\
1.2 \mathrm{~mm}[0.7-1.4]\end{array}$ \\
\hline \multirow[t]{5}{*}{ PET } & $\begin{array}{l}\text { Reconstruction } \\
\text { algorithm }\end{array}$ & OSEM2D 4i8s & OSEM2D 4i8s & BLOB-OS-TF & BLOB-OS-TF \\
\hline & Slice thickness & $3 \mathrm{~mm}$ & $3 \mathrm{~mm}$ & $4 \mathrm{~mm}$ & $\begin{array}{l}2 \mathrm{~mm}(n=38) \\
4 \mathrm{~mm}(n=22)\end{array}$ \\
\hline & Pixel spacing & $4.07 \mathrm{~mm}$ & $4.07 \mathrm{~mm}$ & $4 \mathrm{~mm}$ & $\begin{array}{l}2 \mathrm{~mm}(\mathrm{n}=38) \\
4 \mathrm{~mm}(\mathrm{n}=22)\end{array}$ \\
\hline & Injected FDG dose & $\begin{array}{l}\text { Median [range] } \\
180 \mathrm{MBq} \\
{[113-354]}\end{array}$ & $\begin{array}{l}\text { Median [range] } \\
302 \mathrm{MBq} \\
{[175-482]}\end{array}$ & $\begin{array}{l}\text { Median [range] } \\
290 \mathrm{MBq} \\
{[139-474]}\end{array}$ & $\begin{array}{l}\text { Median [range] } \\
340 \mathrm{MBq} \\
{[198-434]}\end{array}$ \\
\hline & $\begin{array}{l}\text { Planned interval FDG } \\
\text { injection - image } \\
\text { acquisition }\end{array}$ & 60 minutes & 60 minutes & 60 minutes & 60 minutes \\
\hline
\end{tabular}

The image analysis included the investigation of different feature groups: 1) Morphological 'Shape', 2) Fractal, 3) Local Intensity 'Loclnt', 4) Statistical features 'Stats', 5) Intensity-volume histogram 'IVH', 6) Textural features, including gray-level co-occurrence matrix ' $G L C M$ ', gray-level run length matrix ' $G L R L M$ ', gray-level size zone matrix 'GLSZM', neighborhood gray-tone difference matrix 'NGTDM', gray-level distance zone matrix 'GLDZM', neighborhood gray-level dependence matrix 'NGLDM', 7) Laplacian of Gaussian filter 'LoG' (prior to group 4), and 8) Wavelet filter (prior to group 6). The feature descriptions of the feature groups (fractal, local intensity and intensity histograms) can be found in Supplementary Material $8 \mathrm{C}$. Other feature descriptions can be found elsewhere [19]. The IVH features were extracted from PET images only and these allow to retrieve the metabolic tumor volume (MTV), which is defined as the volume of voxels with an intensity above $\mathrm{x} \%$ of SUVmax within the lesion (from 10 to 90\% (MTV10\% - MTV90\%)) [20] and total lesion glycolysis (TLG), by multiplying MTV by the corresponding mean SUV within the segmented volume: TLG10\% - TLG90\% [20, 21].

Image analysis was performed in Matlab R2014a (The Mathworks, Natick, MA) using in-house developed software used for feature extraction. The absolute variation (abs) and percentage (rel) variation between subsequent scans were also 
derived, defined as:

\author{
Abs $=$ During_treatment- Pre_treatment (1) \\ Rel $=($ During_treatment- Pre_treatment)/Pre_treatment (2)
}

In total, eight sets of radiomic features were derived from each dataset, being CTscan1 (pre-treatment), CT-scan2 (during treatment), PET-scan1, PET-scan2, CT-abs, CT-rel, PET-abs and PET-rel.

\title{
Cohort comparison
}

To compare the four cohorts, two different approaches were applied. First of all, clinical characteristics were compared to test univariate cohort differences using a Wilcoxon rank test for continuous variables or a chi-square test for categorical variables. P-values below 0.05 were considered significant. Note that the p-values of these analyses were not corrected for multiple testing.

Secondly, the cohort differences (CD) model approach described in detail by van Soest et al. $[7,8]$ was used to assess the multivariate cohort differences by predicting to which cohort a patient belongs. It provides as summarizing measure of the level of generalizability of the model, ranging from reproducibility to transferability. Two CD-models were created. The first CD-model included two-year survival (binary variable) as an independent variable, as well as radiomic features of a model developed in the current study (see next section 'Model development'). The second CD-model also included the clinical variables 'Gender' and 'Overall Stage' as independent variables to investigate whether potential differences between the cohorts can be explained by those clinical parameters. As two-year survival was used as input variable, four patients of Dataset 2 were not included in this analysis due to shorter follow-up times. For the CD-model, stage was converted into dummy variables, using three categories II, IIla or IIIb/IV. Stage II was used as the reference category.

The binary dependent variable of the CD-models was cohort A or B. A simple logistic regression was used to train the regression beta coefficients and predict to which cohort the data belongs. This procedure was applied to all possible combinations of two datasets. The performance of the CD-models for each combination of two datasets was evaluated by their Receiver Operating Curve (ROC). High CD Area under the Curve (AUC) values would indicate a large difference between the distributions in the cohorts and imply that the model tests transferability rather than reproducibility $[7,8]$.

\section{Model development}

Each of the four datasets was used to train a model, which was subsequently validated on the three remaining datasets. Moreover, a model was developed by combining all data into one large dataset $(n=138)$ which was split randomly into training $(75 \%$, $n=103)$ and validation $(25 \%, n=35)$, as proposed in [22].

For model development, a least absolute shrinkage and selection operator 
(LASSO) method was applied [23]. A 10-fold cross validation procedure was repeated 200 times to optimize the penalty coefficient lambda, i.e. to find the smallest error, and to stabilize the method. Inputs for LASSO were the total of extracted radiomic features from either CT-scan1, CT-scan2, PET-scan1, PET-scan2, CT-abs, CT-rel, PETabs or PET-rel. The performance of the penalized Cox model was evaluated using Harrell's concordance index (c-index), for which 1 indicates perfect discrimination and a value of 0.5 no discrimination (no greater than chance expectation) [24]. Moreover, prognostic index $(\mathrm{PI})$ values, defined as $\Sigma_{i} \beta_{i} x_{i}$, were calculated for all four datasets. These were analyzed to give insight in discriminative ability of the model, as proposed by Royston and Altman [25].

\section{Classification comparison}

For the combined dataset, five different classifiers were investigated according to the methodology described by Deist, Dankers et al. [26]. These include glmnet (penalized generalized linear models), rf (random forest), svm (support vector machine), LogitBoost (boosting) and rpart (regression trees). The neural network classifier was not investigated, as these required too long computation times for a large number of input parameters and extensive tuning. The classifiers deal with binary outcomes, therefore 'two-year survival' was used as endpoint. Since four patients in Dataset 4 did not reach the minimum of two years of follow-up, these patients were excluded for this specific analysis. The maximum number of repetitions was increased to 47 (limited by available calculation time on the computer) for this study and default tuning was turned off. All other settings were kept to the standards as described in [26].

All statistical methods were performed in R (version 3.4.3), using the packages survcomp, survival and glmnet. For the classification comparison, additional packages were used as described in [26]. P-values below 0.05 were considered significant.

\section{Univariable analysis}

The performance of commonly assessed PET metrics as potential prognostic factors was also investigated in an attempt to validate previous findings [10-16]. Also, radiomic features can be hard to interpret, whereas these PET metrics are wellknown. A univariable Cox proportional hazard regression was computed for the percentage variation of most commonly assessed PET metrics: volume, maximum SUV (maximum image intensity value), mean SUV, peak SUV (maximum average SUV in a $1 \mathrm{~cm}^{3}$ spherical volume), MTV50\% (volume above $50 \%$ of intensity) and TLG50\% (TLG for the volume above $50 \%$ of intensity). This analysis was performed on the combined dataset of 138 patients.

\section{Results}

Characteristics of the cohorts are summarized in Table 8.2, which shows that all clinical parameters were significantly different between one or more datasets, except age (indicated with the bold numbers). All patients from Datasets 1, 3 and 4 received concurrent chemoradiotherapy and no other treatment between the first PET scan 
and the start of radiotherapy (RT). In Dataset 2, 55\% of patients received sequential chemoradiotherapy and one patient did not receive any chemotherapy. Because of the limited sample size available to us for this study, we have decided to not exclude outliers (e.g. PET scans separated by long time intervals) in an effort to make the dataset more homogeneous.

Median [range] survival was $1.6[0.1-4.5], 2.3$ [0.2 - 7.0], 1.8 [0.3 - 5.9] and $3.1[0.1-5.2]$ years for Dataset $1,2,3$ and 4 , respectively (Figure 8.1 ).

Table 8.2. Patient characteristics for all four datasets used in this study. The bold, dashed numbers $1,2,3$ or 4 indicate the datasets from which the variable was significantly different.

\begin{tabular}{|c|c|c|c|c|}
\hline & $\begin{array}{l}\text { Dataset } 1 \\
(n=50)\end{array}$ & $\begin{array}{l}\text { Dataset } 2 \\
(n=31)\end{array}$ & $\begin{array}{l}\text { Dataset } 3 \\
(n=27)\end{array}$ & $\begin{array}{l}\text { Dataset } 4 \\
(n=30)\end{array}$ \\
\hline Age [years] & - & - & - & - \\
\hline Range (median) & $35-86(63)$ & $46-82(64)$ & $41-76(62)$ & $47-83(64)$ \\
\hline Mean \pm SD & $62 \pm 11$ & $65 \pm 9$ & $61 \pm 8$ & $65 \pm 9$ \\
\hline Gender & 3 & 3 & $1,2,4$ & 3 \\
\hline Male & 25 (50\%) & 22 (71\%) & 25 (93\%) & $19(63 \%)$ \\
\hline Female & 25 (50\%) & 9 (29\%) & $2(7 \%)$ & $11(37 \%)$ \\
\hline Stage & $2,3,4$ & 1 & 1 & 1 \\
\hline II & - & $2(6 \%)$ & $4(15 \%)$ & $1(3 \%)$ \\
\hline IIla & 17 (34\%) & $14(45 \%)$ & $16 *(59 \%)$ & $16(53 \%)$ \\
\hline IIIb & 27 (54\%) & $15(48 \%)$ & 7 (26\%) & $13(43 \%)$ \\
\hline IV & $6(12 \%)$ & - & - & - \\
\hline Histology & 3,4 & 3,4 & 1,2 & 1,2 \\
\hline Adenocarcinoma & $18(36 \%)$ & $6(19 \%)$ & $11(41 \%)$ & $9(30 \%)$ \\
\hline Squamous cell carcinoma & $14(28 \%)$ & $9(29 \%)$ & $14(52 \%)$ & $18(60 \%)$ \\
\hline NSCLC Otherwise Specified & $18(36 \%)$ & $16(52 \%)$ & $2(7 \%)$ & $3(10 \%)$ \\
\hline Radiotherapy [dose] & 2,3 & $1,3,4$ & 1,2 & 2 \\
\hline Range (median) & $45-69(69)$ & $46-70(61)$ & $66-70(66)$ & $30-74(66)$ \\
\hline Mean \pm SD & $64 \pm 6$ & $61 \pm 7$ & $68 \pm 2$ & $66 \pm 8$ \\
\hline Chemotherapy & 2 & $1,3,4$ & 2 & 2 \\
\hline Concurrent & 50 (100\%) & $13(42 \%)$ & 27 (100\%) & 30 (100\%) \\
\hline Sequential & - & 17 (55\%) & - & - \\
\hline No & - & $1(3 \%)$ & - & - \\
\hline Interval pre-PET - First RT [days] & 3,4 & 3,4 & $1,2,4$ & $1,2,3$ \\
\hline Range (median) & $4-16(7)$ & $2-13(7)$ & $5-93(33)$ & $2-37(16)$ \\
\hline Mean \pm SD & $7 \pm 2$ & $8 \pm 2$ & $38 \pm 21$ & $17 \pm 7$ \\
\hline Interval First RT - during-PET [days] & 2,3 & $1,3,4$ & $1,2,4$ & 2,3 \\
\hline Range (median) & $5-20(15)$ & $6-19(8)$ & $15-32(21) b$ & $14-24(15)$ \\
\hline Mean \pm SD & $15 \pm 2$ & $9 \pm 3$ & $21 \pm 4$ & $16 \pm 3$ \\
\hline Interval between PET scans [days] & $2,3,4$ & $1,3,4$ & $1,2,4$ & $1,2,3$ \\
\hline Range (median) & $19-27(22)$ & $10-24(16)$ & $21-110(52)$ & $22-59(33)$ \\
\hline Mean \pm SD & $22 \pm 2$ & $17 \pm 3$ & $59 \pm 21$ & $34 \pm 8$ \\
\hline
\end{tabular}

* Includes one TXN2MO patient, for which the merged structure between node and tumor was analyzed.

+ Two patients' first PET scans were acquired more than 30 days after start of radiotherapy. 
Median follow-up was 3.8 [2.5 - 4.5], 6.9 [6.6 - 7.0], 4.0 [2.6 - 5.9] and 3.4 [1.3 - 5.2] years for Dataset 1, 2, 3 and 4, respectively.

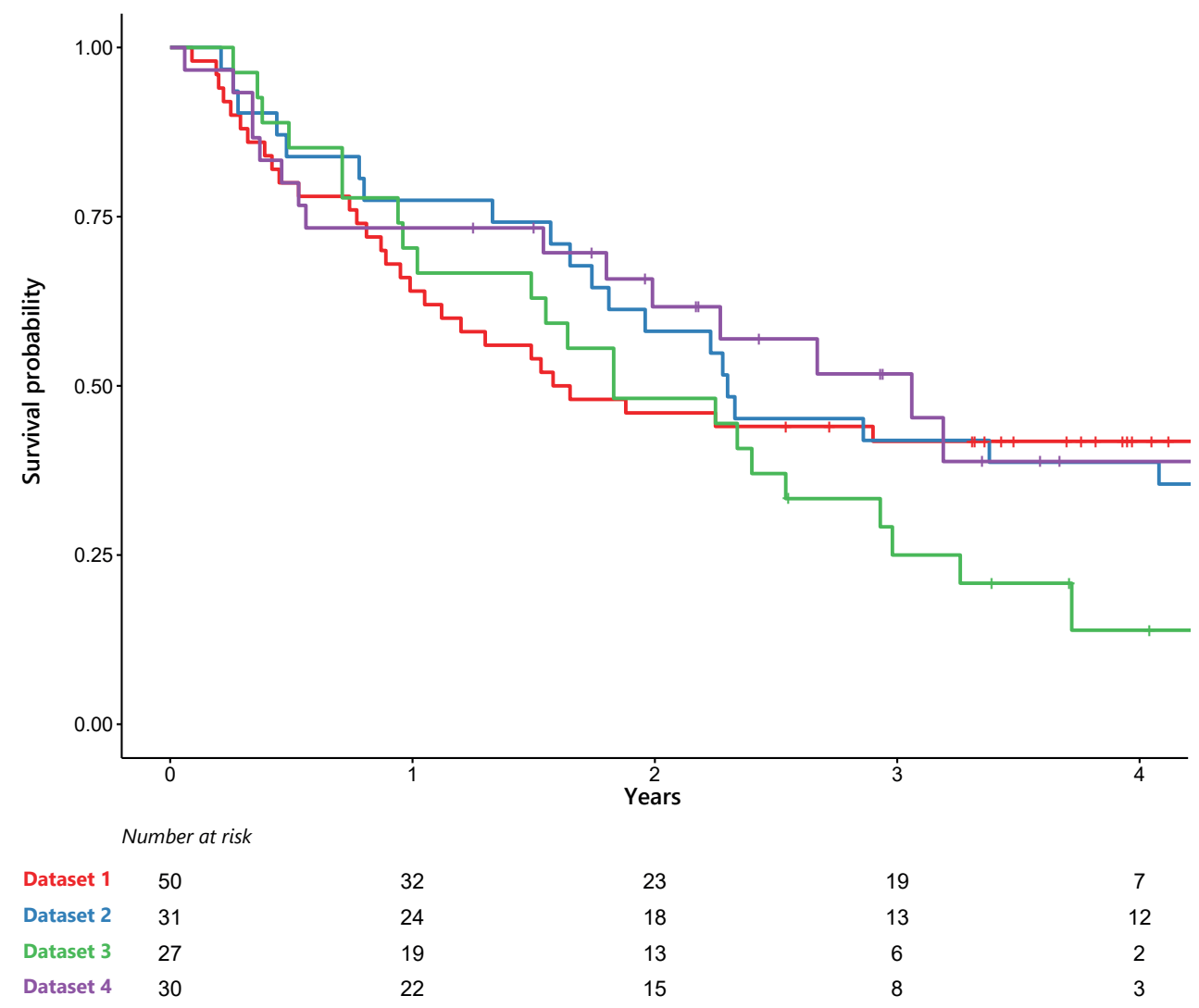

Figure 8.1. Kaplan-Meier curves for overall survival of all datasets.

Figure 8.2 shows the ROCs of the CD-model for each combination of two datasets. AUC values ranged from 0.66 (Dataset 3 versus 4) to 0.89 (Dataset 2 versus 4). None of the confidence intervals included 0.5 , meaning that all AUC values were significantly different from 0.5 (not shown). The ROC curves of the CD-model, which included gender and overall stage, resulted in slightly higher AUC values, ranging from 0.77 to 0.92 (Figure S8.1).

To assess prognostic performance, models were developed using all four different datasets once as training and the remainders as validation. The first models were trained on the image sets of the largest dataset, Dataset $1(n=50)$. Using LASSO, prognostic predictors were identified for the image sets CT-scan1, CT-rel and PETabs. For the other image sets, no prognostic models were identified. Since Dataset 1 was the largest dataset and CT scans prior to treatment are currently most commonly used to assess prognostic performance, the model belonging to CT-scan 1 of Dataset 1 was chosen to be used to construct the CD-model (Figure 8.2). This model, built 
using LASSO, consisted of 3 variables: 1) Wavelet LLH (Low Low High) Fractal sd (standard deviation), 2) Wavelet LLH GLDZM IV (Inverse Variance) and 3) Wavelet LLH GLSZM IV. The corresponding beta coefficients of the Cox model were 1.38, 5.0*10-17 and $2.9^{*} 10^{-32}$, respectively. Figure 8.3 shows the ranges of the prognostic index (PI) for all four datasets for this model.

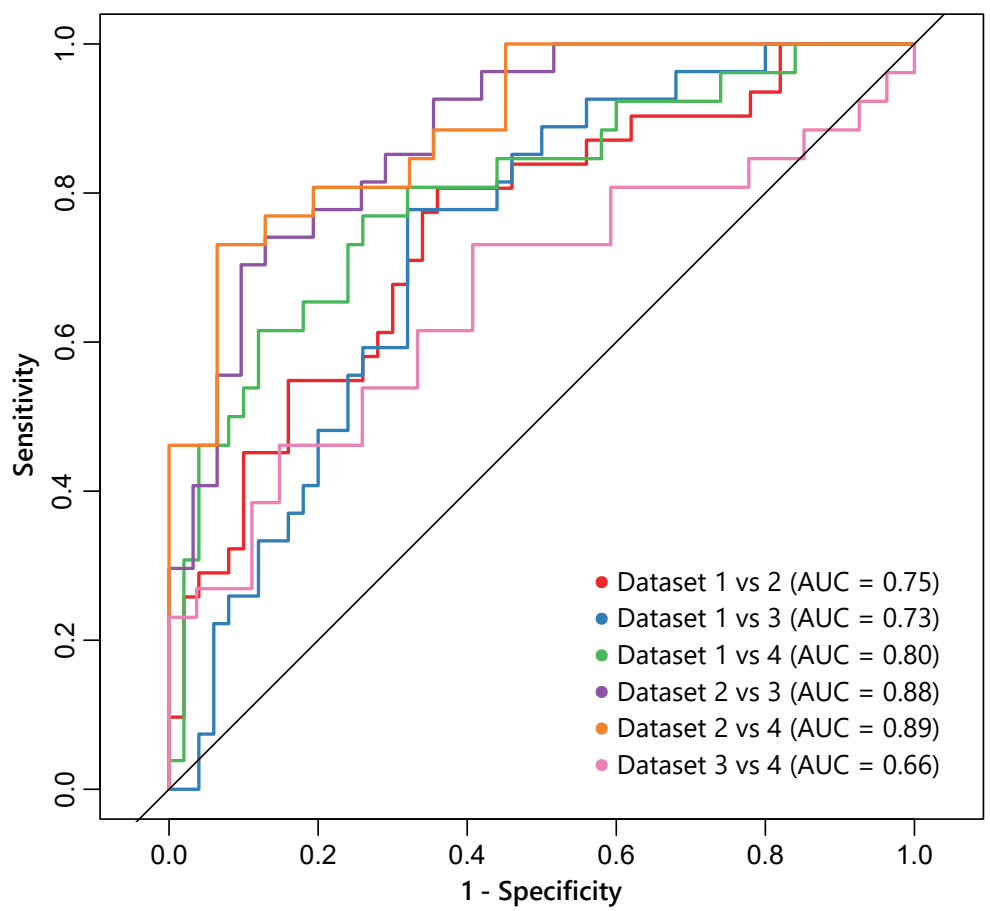

Figure 8.2. Receiver Operator Curves (ROC) for the cohort difference (CD) model for each combination of datasets, including three radiomic features and 'two-year survival' as independent variables.

Table 8.3 shows the results for Harrell's concordance index for the imaging features extracted from all image sets and the calculated differences. The performances of models trained on Dataset 2, 3 or 4 are shown in Supplementary Material 8D. Furthermore, all data was combined into one large cohort and randomly split into training $(n=103)$ and validation $(n=35)$. For CT-rel and CT-abs, models were selected with c-indices significantly different from 0.5 : $0.68[95 \% \mathrm{Cl} 0.61-0.74]$ and $0.68[95 \%$ $\mathrm{Cl} 0.61-0.74]$, respectively. However, none of these models could be validated on the validation subgroup, reaching c-indices of $0.54[95 \% \mathrm{Cl} 0.42-0.67]$ and 0.50 $[95 \% \mathrm{Cl} 0.37-0.62]$. For the other image sets, subsets of regressors were identified which resulted in predictions (i.e. relative risks) equal to 1 for all patients, meaning that the model did not contain any prognostic information.

Besides LASSO, different classifiers were investigated to further examine the prognostic value of the radiomic features for 2-year survival. The mean AUC values are shown in the heatmap of Figure 8.4. 
Table 8.4 shows the results of the univariable analysis of the prognostic value of percentage variation of common PET imaging descriptors on the combined cohort of 138 patients. The results for hazard ratio (HR), corresponding confidence interval $(\mathrm{Cl})$ and the c-index were shown.

Dataset 1

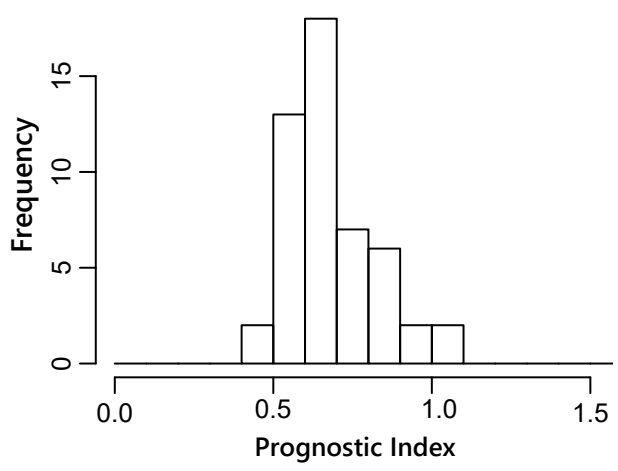

Dataset 3

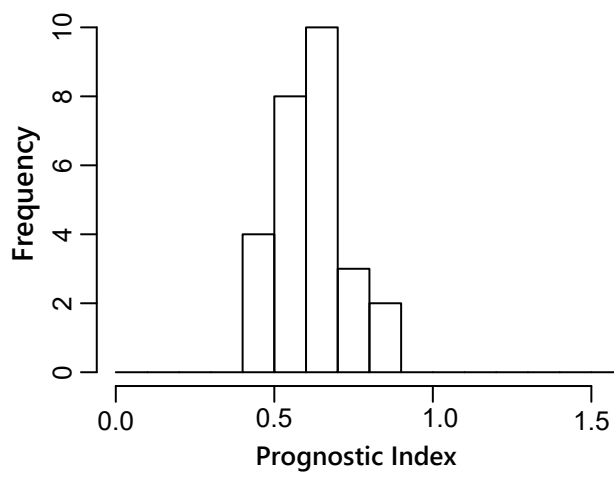

Dataset 2

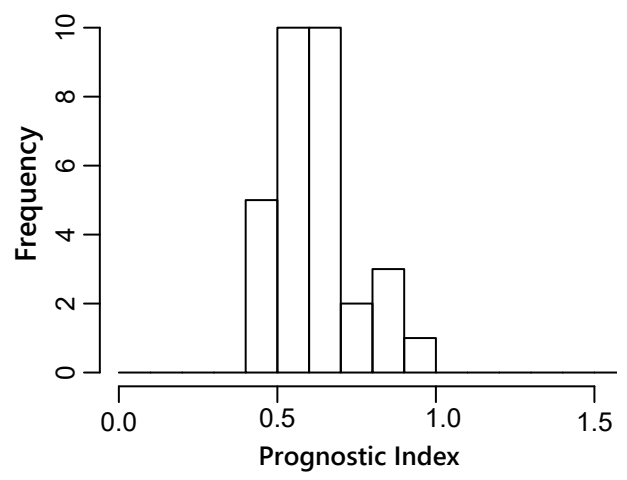

Dataset 4

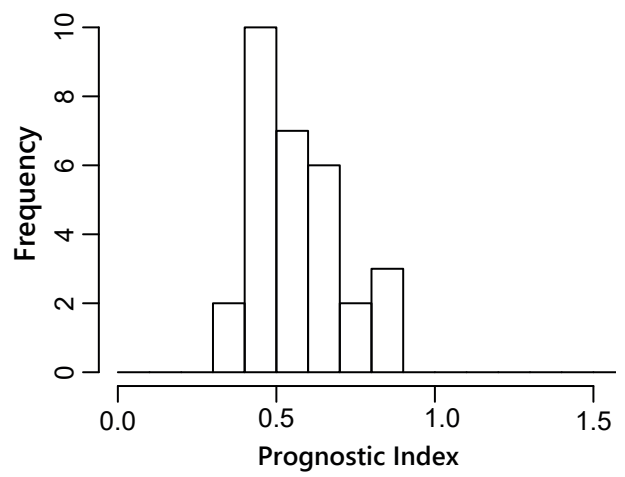

Figure 8.3. Prognostic index $(\mathrm{PI})$ ranges for all datasets based on the model developed on CT-scan1, using Dataset 1 as training dataset.

\section{Discussion}

This study aimed to show potential issues using a typical problematic example of retrospective multi-centric study, by investigating different methodologies and approaches for comparing cohort characteristics and assessing prognostic performance in a radiomic study. The analysis was based on a combined evaluation of four sub-cohorts, which resulted in the inclusion of 138 patients. The acquisition of a larger dataset is challenging for this research question, as FDG-PET/CT scans during treatment are not standard in clinical routine. As far as we are aware, the current dataset is one of the largest available. Nevertheless, the amount of data is probably less than required in order to build a generalized feature model for repeated 
Table 8.3. Values of Harrell's concordance index with $95 \%$ confidence intervals, in the case Dataset $1(n=50)$ was used as training $(T)$ to develop a model using LASSO. Validation (V) results are shown for Dataset 2,3 and 4. Significant values are indicated in gray. A hyphen indicates that either all coefficients were forced to zero, or all predictions were equal to one, meaning that no linear combination of any subset of regressors was useful in predicting the outcomes.

\begin{tabular}{|c|c|c|c|c|c|c|c|c|}
\hline & CT-scan 1 & CT-scan2 & PT-scan1 & PT-scan2 & CT-rel & CT-abs & PT-rel & PT-abs \\
\hline T-Dataset 1 & 0.68 & - & - & - & 0.67 & & - & 0.64 \\
\hline T-Lower bound & 0.59 & - & - & - & 0.57 & & - & 0.56 \\
\hline T-Upper bound & 0.76 & - & - & - & 0.76 & & - & 0.73 \\
\hline V-Dataset 2 & 0.55 & - & - & - & 0.58 & & - & 0.56 \\
\hline V-Lower bound & 0.40 & - & - & - & 0.48 & & - & 0.43 \\
\hline V-Upper bound & 0.69 & - & - & - & 0.69 & & - & 0.70 \\
\hline V-Dataset 3 & 0.41 & - & - & - & 0.53 & & - & 0.56 \\
\hline V-Lower bound & 0.27 & - & - & - & 0.39 & & - & 0.44 \\
\hline V-Upper bound & 0.54 & - & - & - & 0.68 & & - & 0.68 \\
\hline V-Dataset 4 & 0.49 & - & - & - & 0.62 & & - & 0.52 \\
\hline V-Lower bound & 0.37 & - & - & - & 0.48 & & - & 0.36 \\
\hline V-Upper bound & 0.62 & - & - & - & 0.75 & & - & 0.68 \\
\hline
\end{tabular}

PET/CT studies. Although the size of this dataset may not be sufficient from a statistical point of view, its results may provide clinical relevant insights to be obtained in order to improve future studies with limited sample sizes. Therefore, we think it is important to publish these results in an attempt to guide reflections and considerations with respect to analyses and conclusions in future (radiomic) studies. In this study, both the characteristics of the study cohort and the prognostic performance of imaging features were extensively explored.

Table 8.4. Cox regression on the percentage variation of the PET imaging descriptors most commonly used, reporting the univariable hazard ratio (HR), 95\% confidence interval $(\mathrm{CI})$ of the HR and corresponding $\mathrm{p}$-value. Univariable performance is reported in terms of the concordance-index (c-index). Absolute values of Scan 1 and 2, and percentage variation between PET acquisitions of the analyzed metrics are also presented (mean \pm standard deviation).

\begin{tabular}{llllllll} 
& Scan 1 & Scan 2 & $\begin{array}{l}\text { Percentage } \\
\text { difference }\end{array}$ & HR & 95\% Cl & -value & c-index \\
\hline Volume $\left[\mathrm{cm}^{3}\right]$ & $85 \pm 113$ & $68 \pm 94$ & $-20 \pm 31$ & 1.11 & $0.55-2.25$ & 0.77 & 0.50 \\
SUV max & $12 \pm 6.2$ & $9.0 \pm 4.1$ & $-20 \pm 39$ & 1.16 & $0.73-1.83$ & 0.53 & 0.52 \\
SUV mean & $5.3 \pm 2.5$ & $4.1 \pm 1.8$ & $-12 \pm 71$ & 1.09 & $0.87-1.36$ & 0.45 & 0.55 \\
SUV peak & $8.8 \pm 4.3$ & $6.4 \pm 2.8$ & $-18 \pm 51$ & 1.14 & $0.83-1.56$ & 0.43 & 0.54 \\
MTV 50\% & $26 \pm 39$ & $20 \pm 25$ & $-9.5 \pm 46$ & 1.72 & $1.09-2.71$ & 0.02 & 0.54 \\
TLG 50\% & $266 \pm 499$ & $132 \pm 212$ & $-25 \pm 61$ & 1.19 & $0.92-1.54$ & 0.18 & 0.56
\end{tabular}

First of all, comparison of the cohort characteristics of all datasets showed that all variables were significantly different between two or more datasets, except for age. The overall stage, as well as the received radiotherapy dose of the patients in Dataset 1 , was significantly different compared to the other three datasets. In addition, the timing of chemotherapy was different, since $55 \%$ of the patients from 


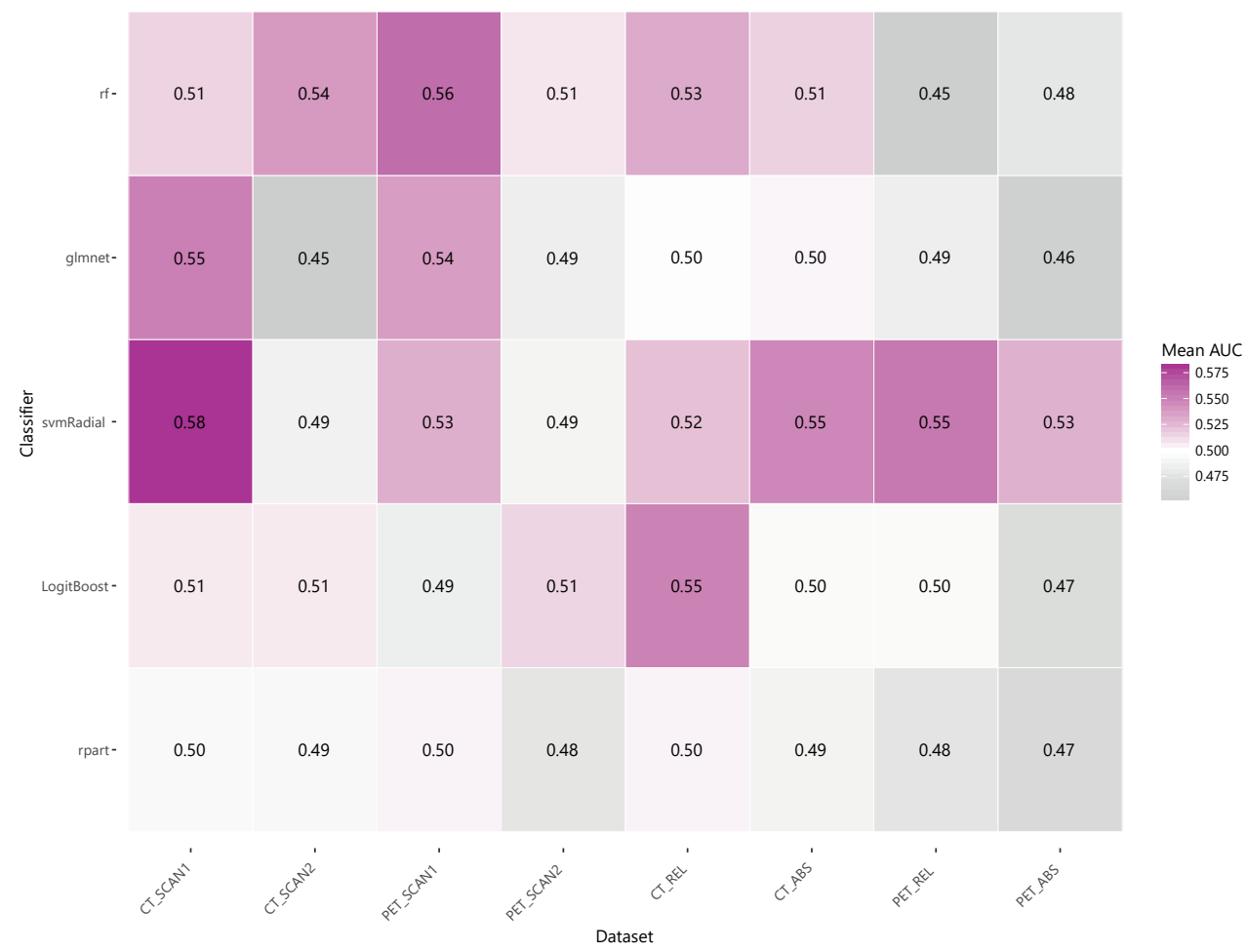

Figure 8.4. Heatmap with the mean AUC values found for each classifier and each image set, predicting two-year survival. The entire cohort with at least two-years of follow-up $(n=134)$ was used in this investigation.

Dataset 2 received sequential chemotherapy, whereas all other patients received concurrent chemotherapy. Furthermore, the intervals between the two FDG-PET/CT scans was quite different between the datasets, since this was a retrospective study and the initial purposes of the studies were different. The variations in treatment types as well as differing outcomes between the cohorts are a probable source of noise that dilutes any signal present within the radiomic features. Furthermore, heterogeneities introduced by differences in timing of the imaging sessions, e.g. between PET scans, are a well-known confounding factor in retrospective imaging studies. Besides the large variabilities of clinical variables between the datasets, the CD-model showed high AUC values $(0.65-0.88)$. The high AUC values of the CDmodel indicate high ability to predict to which dataset the data belongs. In other words, high AUC values mean that the (distribution of) the input variables of the cohort are very different. This may be caused by differences in radiomic features since the survival is very similar for all datasets (Figure 8.1). Moreover, this shows that the model tests the transferability rather than the reproducibility of the model, i.e. the model is specific for a population. While the exclusion of patients with characteristics considered to be outliers would have resulted in a more homogeneous dataset, we decided against this approach as it would have resulted in too small a 
dataset to draw any conclusions from.

Secondly, the prognostic performance of the radiomic features extracted from CT, PET, delta-CT or delta-PET, was investigated. The models were selected using a LASSO procedure. High values of Harrell's concordance index (around 0.7 and even reaching 0.86 ) indicate good prognostic performance of the models trained on Dataset 2. Nevertheless, the results could not be validated on independent datasets, with the only significant c-index being 0.64 achieved on Dataset 4 for PETscan1. This c-index was the only value being significantly different from 0.5 . When one of the other datasets was used as training, no model achieved a c-index significantly different from 0.5 on any of the remaining validation datasets. In terms of discriminative power of the model, the spread of prognostic indices shown for the model trained on CT-scan 1 of Dataset 1 does not show relevant outliers or differences between the datasets. In summary, the model performance results show the importance of validation: one should be careful with presenting results that are developed on (small) datasets without validation, as they are likely to be overoptimistic. In case only one (large) dataset is available, a cross-validation procedure could be applied to reduce the risk of overfitting. A recent paper summarizes the need for validation to assess the clinical usefulness of prognostic models [27].

Potentially, LASSO (e.g. penalized regression) was not the optimal method to select a prognostic model. Therefore, multiple other classifiers [28] were investigated using the methodology described by Deist et al. [26]. Since the sub-cohorts were small, all data were combined into one large cohort to investigate the ability of five different classifiers to predict 2-year survival. None of the classification methods was able to produce a clinical significant result, with the highest mean AUC being 0.58 for the radiomic features extracted from $\mathrm{CT}$ images prior to treatment. This result was achieved by the support vector machine (svm), which overall obtained the highest AUC results. Combining datasets into one large cohort did also not result in significant c-indices, potentially influenced by the heterogeneity within the combined dataset, which makes it rather difficult to develop a prognostic model.

The performance of the percentage variation of commonly used PET metrics was also investigated. Only MTV50\% achieved a significant Hazard Ratio, but the corresponding c-index of the Cox model was not significantly different from 0.5 . This performance was also performed on the PET features extracted from either scan 1 or 2 instead of the percentage variation. None of the metrics was significantly related to overall survival (results not shown). This contradicts previously published results, which have shown the importance of these metrics in a prognostic setting [29, 30].

The results do not show conclusive results for the possibility to perform early treatment response assessment using an additional ${ }^{18} \mathrm{~F}-\mathrm{FDG}-\mathrm{PET} / \mathrm{CT}$ scan acquired at intermediate time point during treatment with a radiomics analysis. However, the potential prognostic value of differences between two time points was investigated before and showed promising, but also conflicting results [10, 12, 14, 16, 31]. Cremonesi et al. [32] reports that the differences in PET parameters are a main limitation for 21 recent studies in early response assessment for NSCLC patients. In general, radiomic features are highly affected by different acquisition and reconstruction settings [33-39]. This is one of the factors that could (partly) explain 
the results in the current study. In this study, there were large differences between datasets, but even within a dataset the acquisition and reconstruction settings were not identical, as shown in Table 1. The inconsistency of settings within a dataset is an issue which is difficult to overcome, especially in retrospective studies. Initiatives to provide protocols and guidelines will hopefully improve standardization in the future [40-42]. Moreover, a recent study proposed a 'post-reconstruction harmonization method' to reduce the variability in radiomic features extracted from PET images from different institutes [43]. Besides the inconsistency in acquisition and reconstruction settings within a dataset, other parts of the radiomics workflow including segmentation [44], pre-processing $[45,46]$ and feature extraction, remain problematic for multi-center studies and the transferability and reproducibility of developed models. A recent review proposed a harmonization for the radiomics methodology [47]. Moreover, the image biomarker standardization initiative (ISBI) attempts to standardize radiomics in terms of feature definitions and processing [48]. Nevertheless, the lack of standardization remains one the main limitations for radiomic studies. Several factors can improve the quality of future radiomic studies and reduce the risk of false positive results, as summarized in the recently proposed Radiomics Quality Score (RQS) [3].

The descriptive character of this study is intended to serve as a tool for highlighting common issues in radiomics literature and to emphasize pitfalls for future studies. Moreover, we emphasize the urgent need to publish negative results to avoid publication bias. With the current study, we would like to highlight the importance of proper validation of the results, but also the consideration of the feasibility of performing a (radiomic) study. We would recommend to perform statistically rigorous sample size calculations upfront and to perform an extensive cohort investigation to decrease the risk of false positive findings. It is essential to reduce this risk, as the main goal is to improve individual treatment decisions for better patient outcome, which can only be achieved when proper statistically sound investigations are reported.

\section{Conclusion}

In an attempt to inform future radiomic studies, we illustrate possible problems that can be encountered in retrospective multi-centric study by evaluating cohort characteristics and clinical characteristics. The models presented do not support any correlation between radiomic features acquired from FDG-PET/CT scans and overall survival. Further investigations indicate that the radiomic analysis was influenced by the limited sample size and heterogeneous imaging and clinical characteristics.

\section{Acknowledgments}

The authors would like to thank Frank Dankers and Timo Deist for their help and support using the classifier comparison methodology. The authors would also like to thank Johan van Soest for his support using the cohort differences model. 


\section{References}

[1] Lambin P, Zindler J, Vanneste BG, De Voorde LV, Eekers D, Compter I, et al. Decision support systems for personalized and participative radiation oncology. Adv Drug Deliv Rev. 2016.

[2] Lambin P, van Stiphout RG, Starmans MH, Rios-Velazquez E, Nalbantov G, Aerts HJ, et al. Predicting outcomes in radiation oncology--multifactorial decision support systems. Nat Rev Clin Oncol. 2013;10:27-40.

[3] Lambin P, Leijenaar RTH, Deist TM, Peerlings J, de Jong EEC, van Timmeren J, et al. Radiomics: the bridge between medical imaging and personalized medicine. Nat Rev Clin Oncol. 2017;14:749-62.

[4] Lambin P, Rios-Velazquez E, Leijenaar R, Carvalho S, van Stiphout RG, Granton P, et al. Radiomics: extracting more information from medical images using advanced feature analysis. Eur J Cancer. 2012;48:441-6.

[5] Alic L, Niessen WJ, Veenland JF. Quantification of heterogeneity as a biomarker in tumor imaging: a systematic review. PLoS One. 2014;9:e110300.

[6] Kumar V, Gu Y, Basu S, Berglund A, Eschrich SA, Schabath MB, et al. Radiomics: the process and the challenges. Magn Reson Imaging. 2012;30:1234-48.

[7] Debray TP, Vergouwe Y, Koffijberg H, Nieboer D, Steyerberg EW, Moons KG. A new framework to enhance the interpretation of external validation studies of clinical prediction models. J Clin Epidemiol. 2015;68:279-89.

[8] van Soest J, Meldolesi E, van Stiphout R, Gatta R, Damiani A, Valentini V, et al. Prospective validation of pathologic complete response models in rectal cancer: Transferability and reproducibility. Med Phys. 2017;44:4961-7.

[9] Chalkidou A, O'Doherty MJ, Marsden PK. False Discovery Rates in PET and CT Studies with Texture Features: A Systematic Review. PLoS One. 2015;10:e0124165.

[10] Usmanij EA, de Geus-Oei LF, Troost EG, Peters-Bax L, van der Heijden EH, Kaanders JH, et al. 18F-FDG PET early response evaluation of locally advanced non-small cell lung cancer treated with concomitant chemoradiotherapy. J Nucl Med. 2013;54:1528-34.

[11] Massaccesi M, Calcagni ML, Spitilli MG, Cocciolillo F, Pelligro F, Bonomo L, et al. (1)(8) F-FDG PET-CT during chemo-radiotherapy in patients with non-small cell lung cancer: the early metabolic response correlates with the delivered radiation dose. Radiat Oncol. 2012;7:106. [12] van Elmpt W, Ollers M, Dingemans AM, Lambin P, De Ruysscher D. Response assessment using 18F-FDG PET early in the course of radiotherapy correlates with survival in advancedstage non-small cell lung cancer. J Nucl Med. 2012;53:1514-20.

[13] Huang W, Zhou T, Ma L, Sun H, Gong H, Wang J, et al. Standard uptake value and metabolic tumor volume of (1)(8)F-FDG PET/CT predict short-term outcome early in the course of chemoradiotherapy in advanced non-small cell lung cancer. Eur J Nucl Med Mol Imaging. 2011;38:1628-35.

[14] Banna GL, Anile G, Russo G, Vigneri P, Castaing M, Nicolosi M, et al. Predictive and Prognostic Value of Early Disease Progression by PET Evaluation in Advanced Non-Small Cell Lung Cancer. Oncology. 2017;92:39-47.

[15] Hofheinz F, Hoff J, Steffen IG, Lougovski A, Ego K, Amthauer H, et al. Comparative evaluation of SUV, tumor-to-blood standard uptake ratio (SUR), and dual time point measurements for assessment of the metabolic uptake rate in FDG PET. EJNMMI Res. 2016;6:53. [16] Yossi S, Krhili S, Muratet JP, Septans AL, Campion L, Denis F. Early assessment of metabolic response by $18 \mathrm{~F}-\mathrm{FDG}$ PET during concomitant radiochemotherapy of non-small cell lung carcinoma is associated with survival: a retrospective single-center study. Clin Nucl Med. 2015;40:e215-21.

[17] Kinahan PE, Fletcher JW. Positron emission tomography-computed tomography standardized uptake values in clinical practice and assessing response to therapy. Semin Ultrasound CT MR. 2010;31:496-505.

[18] Larue RTHM, van Timmeren JE, de Jong EEC, Feliciani G, Leijenaar RTH, Schreurs WMJ, et al. Influence of gray level discretization on radiomic feature stability for different CT scanners, tube currents and slice thicknesses: a comprehensive phantom study. Acta Oncol. 2017;56:154453. 
[19] van Timmeren JE, Leijenaar RTH, van Elmpt W, Reymen B, Oberije C, Monshouwer R, et al. Survival prediction of non-small cell lung cancer patients using radiomics analyses of conebeam CT images. Radiother Oncol. 2017;123:363-9.

[20] Van de Wiele C, Kruse V, Smeets P, Sathekge M, Maes A. Predictive and prognostic value of metabolic tumour volume and total lesion glycolysis in solid tumours. Eur J Nucl Med Mol Imaging. 2013;40:290-301.

[21] Visser EP, Philippens ME, Kienhorst L, Kaanders JH, Corstens FH, de Geus-Oei LF, et al. Comparison of tumor volumes derived from glucose metabolic rate maps and SUV maps in dynamic 18F-FDG PET. J Nucl Med. 2008;49:892-8.

[22] Hastie T, Tibshirani R, Friedman J. The Elements of Statistical Learning. 2 ed: SpringerVerlag New York; 2008.

[23] Tibshirani R. Regression shrinkage and selection via the lasso. J Royal Stat Soc. 1996:26788.

[24] Harrell FE, Jr., Califf RM, Pryor DB, Lee KL, Rosati RA. Evaluating the yield of medical tests. Jama. 1982;247:2543-6.

[25] Royston P, Altman DG. External validation of a Cox prognostic model: principles and methods. BMC Med Res Methodol. 2013;13:33.

[26] Deist TM, Dankers F, Valdes G, Wijsman R, Hsu IC, Oberije C, et al. Machine learning algorithms for outcome prediction in (chemo)radiotherapy: An empirical comparison of classifiers. Med Phys. 2018.

[27] Zwanenburg A, Löck S. Why validation of prognostic models matters? Radiother Oncol.

[28] Kourou K, Exarchos TP, Exarchos KP, Karamouzis MV, Fotiadis DI. Machine learning applications in cancer prognosis and prediction. Comput Struct Biotechnol J. 2015;13:8-17.

[29] Paesmans M, Garcia C, Wong CY, Patz EF, Jr., Komaki R, Eschmann S, et al. Primary tumour standardised uptake value is prognostic in nonsmall cell lung cancer: a multivariate pooled analysis of individual data. European Respiratory Journal. 2015;46:1751-61.

[30] Fried DV, Mawlawi O, Zhang L, Fave X, Zhou S, Ibbott G, et al. Stage III Non-Small Cell Lung Cancer: Prognostic Value of FDG PET Quantitative Imaging Features Combined with Clinical Prognostic Factors. Radiology. 2016;278:214-22.

[31] Fave X, Zhang L, Yang J, Mackin D, Balter P, Gomez D, et al. Delta-radiomics features for the prediction of patient outcomes in non-small cell lung cancer. Sci Rep. 2017;7:588.

[32] Cremonesi M, Gilardi L, Ferrari ME, Piperno G, Travaini LL, Timmerman R, et al. Role of interim (18)F-FDG-PET/CT for the early prediction of clinical outcomes of Non-Small Cell Lung Cancer (NSCLC) during radiotherapy or chemo-radiotherapy. A systematic review. Eur J Nucl Med Mol Imaging. 2017;44:1915-27.

[33] Galavis PE, Hollensen C, Jallow N, Paliwal B, Jeraj R. Variability of textural features in FDG PET images due to different acquisition modes and reconstruction parameters. Acta Oncol. 2010;49:1012-6.

[34] van Velden FH, Kramer GM, Frings V, Nissen IA, Mulder ER, de Langen AJ, et al. Repeatability of Radiomic Features in Non-Small-Cell Lung Cancer [F]FDG-PET/CT Studies: Impact of Reconstruction and Delineation. Mol Imaging Biol. 2016.

[35] Yan J, Chu-Shern JL, Loi HY, Khor LK, Sinha AK, Quek ST, et al. Impact of Image Reconstruction Settings on Texture Features in 18F-FDG PET. J Nucl Med. 2015;56:1667-73.

[36] Nyflot MJ, Yang F, Byrd D, Bowen SR, Sandison GA, Kinahan PE. Quantitative radiomics: impact of stochastic effects on textural feature analysis implies the need for standards. J Med Imaging (Bellingham). 2015;2.

[37] Mackin D, Ger R, Dodge C, Fave X, Chi P-C, Zhang L, et al. Effect of tube current on computed tomography radiomic features. Sci Rep. 2018;8:2354.

[38] Mackin D, Fave X, Zhang L, Fried D, Yang J, Taylor B, et al. Measuring Computed Tomography Scanner Variability of Radiomics Features. Invest Radiol. 2015;50:757-65.

[39] Bailly C, Bodet-Milin C, Couespel S, Necib H, Kraeber-Bodere F, Ansquer C, et al. Revisiting the Robustness of PET-Based Textural Features in the Context of Multi-Centric Trials. PLoS One. 2016;11:e0159984.

[40] Boellaard R, O'Doherty MJ, Weber WA, Mottaghy FM, Lonsdale MN, Stroobants SG, et al. 
FDG PET and PET/CT: EANM procedure guidelines for tumour PET imaging: version 1.0. Eur J Nucl Med Mol Imaging. 2010;37:181-200.

[41] Boellaard R, Delgado-Bolton R, Oyen WJ, Giammarile F, Tatsch K, Eschner W, et al. FDG PET/CT: EANM procedure guidelines for tumour imaging: version 2.0. Eur J Nucl Med Mol Imaging. 2015;42:328-54.

[42] de Jong EEC, van Elmpt W, Hoekstra OS, Groen HJM, Smit EF, Boellaard R, et al. Quality assessment of positron emission tomography scans: recommendations for future multicentre trials. Acta Oncol. 2017;56:1459-64.

[43] Orlhac F, Boughdad S, Philippe C, Stalla-Bourdillon H, Nioche C, Champion L, et al. A postreconstruction harmonization method for multicenter radiomic studies in PET. J Nucl Med. 2018.

[44] Parmar C, Rios Velazquez E, Leijenaar R, Jermoumi M, Carvalho S, Mak RH, et al. Robust Radiomics feature quantification using semiautomatic volumetric segmentation. PLoS One. 2014;9:e102107.

[45] Leijenaar RT, Nalbantov G, Carvalho S, van Elmpt WJ, Troost EG, Boellaard R, et al. The effect of SUV discretization in quantitative FDG-PET Radiomics: the need for standardized methodology in tumor texture analysis. Sci Rep. 2015;5:11075.

[46] Mackin D, Fave X, Zhang L, Yang J, Jones AK, Ng CS, et al. Harmonizing the pixel size in retrospective computed tomography radiomics studies. PLoS One. 2017;12:e0178524.

[47] Sollini M, Cozzi L, Antunovic L, Chiti A, Kirienko M. PET Radiomics in NSCLC: state of the art and a proposal for harmonization of methodology. Sci Rep. 2017;7:358.

[48] Zwanenburg A, Leger S, Vallieres M, Lock S. Image biomarker standardisation intitiative feature definitions. CoRR. 2016;abs/1612.07003. 


\section{Supplementary Material}

\section{Supplementary Material 8A - Dataset descriptions}

\section{Dataset 1}

The first dataset consists of 50 stage IIla-IV NSCLC patients treated between September 2010 and March 2012. Data was prospectively collected (NTC00522639). All patients have given written informed consent. Patients with prior history of lung cancer or patients undergoing surgery were excluded. All patients were treated with curatively intended (chemo)radiotherapy. A larger cohort of patients from the same clinical trial were studied in [1], but use in the current study was limited due to availability of during treatment FDG-PET/CT images.

Before scanning, patients fasted for a minimum of 6 hours. The total administered dose of FDG was calculated as (bodyweight (Kg) $\times 4+20)$ MBq. FDG$\mathrm{PET} / \mathrm{CT}$ images were acquired 60 minutes post injection on a Siemens Biograph 40 Truepoint PET/CT (Siemens AG, Munich, Germany). An Ordered Subset Expectation Maximisation 2D 4 iterations 8 subsets (OSEM2D 4i8s) algorithm with a $5 \mathrm{~mm}$ postreconstruction Gaussian smoothing filter was used for PET image reconstruction. Voxel size was $4.07 \times 4.07 \times 3.0 \mathrm{~mm}^{3}$. All PET scans were corrected for attenuation using the mid-ventilation phase of the $4 D C T$ or a 3DCT thorax, case the 4DCT was not of sufficient image quality due to irregular breathing of the patient. Model-based methods were applied for scatter correction, and all scans were corrected for random events and decay. All CT images were acquired using a peak tube voltage of $120 \mathrm{kVp}$ and a tube current of $120 \mathrm{~mA}, 160 \mathrm{~mA}, 173 \mathrm{~mA}$ or $240 \mathrm{~mA}$. All images were reconstructed to a voxel size of $0.977 \times 0.977 \times 3.0 \mathrm{~mm}^{3}$ using the kernel B19f in $97 \%$ of the cases.

\section{Dataset 2}

The second dataset comprised 32 stage IIb-IIIb NSCLC patients, originating from a dataset described previously by van Elmpt et al. [2]. Patients were treated between July 2008 and December 2008. The same procedure for PET imaging and the same imaging protocol and settings were used to acquire the PET/CT images as described for dataset 1. The tube currents used were $80 \mathrm{~mA}$ and $336 \mathrm{~mA}$ and the convolution kernels were B19f, B30f and B40f.

\section{Dataset 3}

Dataset 3 consists of 27 stage II-IIIb NSCLC patients. Characteristics of this dataset has been described before [3]. Patients were treated between June 2008 and December 2012. FDG-PET/CT images were acquired 60 minutes post injection on a Philips Gemini TF 64 PET/CT System (Philips Healthcare, Cleveland, OH). The second PET/CT scan was acquired after 30 Gy was given. PET images were reconstructed to isotropic voxels of $4.0 \times 4.0 \times 4.0 \mathrm{~mm}^{3}$ using the default algorithm BLOB Ordered Subset Time of Flight (BLOB-OS-TF). CT images were acquired using a peak tube voltage of 120 or $140 \mathrm{kVp}$ and variable tube currents ranging between 115 and 277 
$\mathrm{mA}$. The images were reconstructed to a pixel size of $1.17 \times 1.17 \mathrm{~mm}^{2}$ or $1.37 \times 1.37$ $\mathrm{mm}^{2}$ and a slice thickness of $5.0 \mathrm{~mm}$ using kernel $\mathrm{B}$.

\section{Dataset 4}

The fourth dataset consists of 30 stage II-IIIb NSCLC patients, recruited in a subproject of a multicenter PET-PLAN trial (drks.de DRKS00002178). Patients were treated between August 2010 and July 2015. Inclusion and exclusion criteria were according to the main study 'PET-PLAN'. All patients received concurrent chemotherapy, either Cisplatin or Carboplatin on day 1 and 5 of week 1 and 5 of radiotherapy and Vinorelbine (Navelbine) on day 1 of week 1, 2, 3, 5, 6 and eventually week 7 . The second PET/CT was made after between 16 and 30 Gy was given (median $\pm \mathrm{sd}=22$ \pm 3 ). PET/CT images were acquired on a Philips Gemini TF 64 PET/CT System or the Philips Gemini TF Big Bore PET/CT (Philips Healthcare, Cleveland, OH). PET images were reconstructed using the default algorithm (BLOB-OS-TF). All images were attenuation corrected and corrected for random events, scatter and decay. Except for one scan $\left(5.35 \times 5.35 \times 3.0 \mathrm{~mm}^{3}\right)$, all PET images were reconstructed into a voxel size of $4.07 \times 4.07 \times 3.0 \mathrm{~mm}^{3}$. CT images were acquired using a peak tube voltage of $120 \mathrm{kVp}$ and a tube current ranging between $35 \mathrm{~mA}$ and $337 \mathrm{~mA}$. CT pixels sizes range between $0.684 \times 0.684 \mathrm{~mm}^{2}$ and $1.37 \times 1.37 \mathrm{~mm}^{2}$, and slice thickness between $1 \mathrm{~mm}$ and $5 \mathrm{~mm}$. Kernel B was used. 


\section{Supplementary Material 8B - ROCs Cohort difference model}

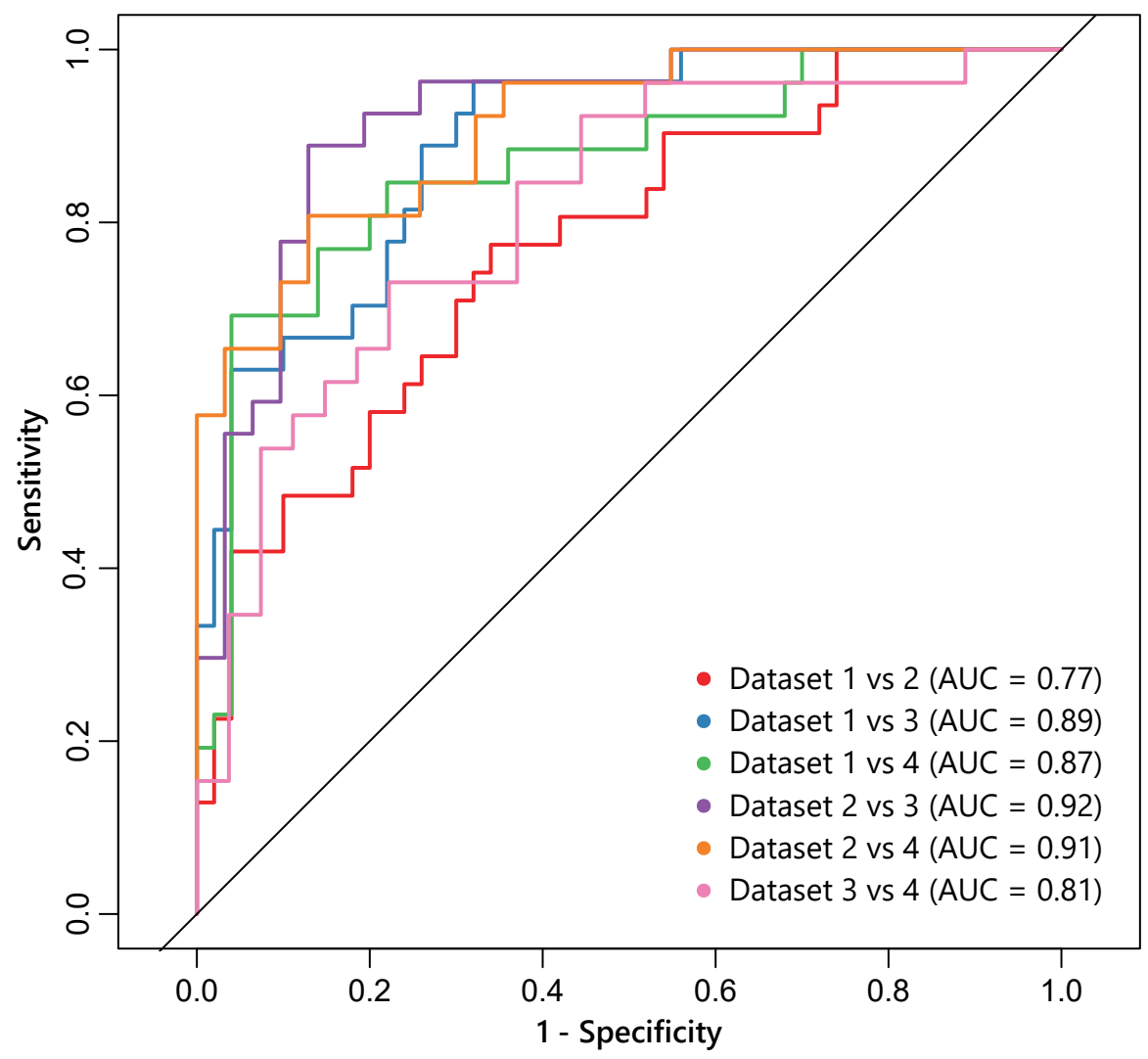

Figure S8.1. Receiver Operator Curves (ROC) for the cohort difference (CD) model for each combination of datasets, including three radiomic features, 'two-year survival', 'gender' and 'stage' as independent variables. 


\section{Supplementary Material $8 \mathrm{C}$ - Feature descriptions}

\section{Fractal Dimension features}

The fractal dimension (FD) of the image is computed as described by Al-Kadi and Watson [4]. Given the FD processed image $I$, with $N$ elements:

\section{Average:}

$$
\text { average }=\frac{1}{\mathrm{~N}} \sum \mathrm{I}
$$

\section{Lacunarity}

\section{Standard deviation:}

$$
\text { lacunarity }=\frac{\frac{1}{\mathrm{~N}} \sum \mathrm{I}^{2}}{\frac{1}{\mathrm{~N}^{2}} \sum \mathrm{I}}-1
$$

$$
\text { standard deviation }=\left(\frac{1}{\mathrm{~N}-1} \sum(\mathrm{I}-\overline{\mathrm{I}})^{2}\right)^{1 / 2}
$$

Where $\bar{I}$ is the mean of $I$.

\section{Local Intensity features}

Local Intensity (Loclnt) features are defined based on local intensity values around a center voxel [5].

\section{Local intensity peak}

Mean intensity level in a $1 \mathrm{~cm}^{3}$ spherical volume, centered on the voxel with the maximum intensity level in the volume of interest. In case multiple voxels contain the maximum intensity level, the highest mean intensity level of all spherical volumes is used.

\section{Global Intensity peak}

Similar to local intensity peak, but in this case the mean intensity level in a $1 \mathrm{~cm}^{3}$ spherical volume is calculated for every voxel in the volume of interest. The highest mean intensity level of all spherical volumes is selected as the global intensity peak feature. 


\section{Intensity histogram features}

Intensity histogram features describe the distribution of gray values within the volume, after discretization into intensity level bins was applied. Let:

$$
X_{d}=\left\{X_{d, 1}, X_{d, 2}, \ldots, X_{d, N_{v}}\right\} 10
$$

be the set of discretized intensity values of the $N_{v}$ voxels in the volume of interest,

$H=\left\{n_{1}, n_{2}, \ldots\right\}$ be the histogram with frequency count $n_{i}$ of each discretized intensity level $i$ in $X_{d}$,

$N_{g}$ be the number of discretized intensity values (bins) in the volume of interest,

$p_{i}$ be the occurrence probability for each bin $i$ of the histogram $N_{g}$, where $p_{i}=n_{i} / N_{v}$.

\section{Coefficient of variance (cov)}

$$
\operatorname{cov}=\frac{\text { standard deviation }}{\text { mean }}
$$

\section{Energy}

$$
\text { energy }=\sum_{j=1}^{N_{\mathrm{v}}} \mathrm{X}_{\mathrm{d}}(\mathrm{j})^{2}
$$

Energy is also known as the sum of squares.

\section{Entropy}

$$
\text { entropy }=-\sum_{\mathrm{i}=1}^{\mathrm{N}_{\mathrm{g}}} \mathrm{P}(\mathrm{i}) \log _{2} \mathrm{P}(\mathrm{i})
$$

\section{Interquartile range (iqr)}

$$
\mathrm{IQR}=\mathrm{P}_{75}-\mathrm{P}_{25}
$$

where $P_{25}$ and $P_{75}$ are the $25^{\text {th }}$ and $75^{\text {th }}$ percentile of $X_{d}$, respectively.

\section{Kurtosis}

where $\bar{X}_{d}$ is the mean of $X_{d}$.

$$
\text { kurtosis }=\frac{\frac{1}{\mathrm{~N}_{\mathrm{v}}} \sum_{\mathrm{j}=1}^{\mathrm{N}_{\mathrm{v}}}\left(\mathrm{X}_{\mathrm{d}}(\mathrm{j})-\overline{\mathrm{X}}_{\mathrm{d}}\right)^{4}}{\left(\frac{1}{\mathrm{~N}_{\mathrm{v}}} \sum_{\mathrm{j}=1}^{\mathrm{N}_{\mathrm{v}}}\left(\mathrm{X}_{\mathrm{d}}(\mathrm{j})-\overline{\mathrm{X}}_{\mathrm{d}}\right)^{2}\right)^{2}}
$$

\section{Maximum}

The maximum discretized intensity value of $X_{d}$.

$$
\text { maximum }=\max \left(X_{d}\right)
$$

\section{Maximum histogram gradient (maxgrad)}

$$
\operatorname{maxgrad}=\max \left(\mathrm{H}^{\prime}\right)
$$

Where $H^{\prime}$ is the histogram gradient, defined as:

$$
\mathrm{H}^{\prime}=\left\{\mathrm{H}(2)-\mathrm{H}(1), \ldots, \frac{\mathrm{H}(\mathrm{i}+1)-\mathrm{H}(\mathrm{i}-1)}{2}, \ldots, \mathrm{H}\left(\mathrm{N}_{\mathrm{g}}\right)-\mathrm{H}\left(\mathrm{N}_{\mathrm{g}}-1\right)\right\}
$$

\section{Maximum histogram gradient intensity level (maxgradi)}

The discretized intensity level $i$ corresponding to the maximum histogram gradient. 


\section{Mean}

The mean discretized intensity value of $X_{d}$.

$$
\text { mean }=\frac{1}{N_{v}} \sum_{j=1}^{N_{v}} X_{d}(j)
$$

15. Mean absolute deviation (meand)

The mean of the absolute deviations of all discretized intensity levels around the mean of $X_{d}$.

$$
\text { meand }=\frac{1}{N_{v}} \sum_{j=1}^{N_{v}}\left|X_{d}(j)-\overline{X_{d}}\right|
$$

where $\bar{X}_{d}$ is the mean of $X_{d}$.

\section{Median}

The sample median of $X_{d}$ or the $50^{\text {th }}$ percentile of $X_{d}$.

\section{Median absolute deviation (mediand)}

The dispersion from the median of $X_{d}$.

where $M$ is the median of $X_{d}$.

$$
\text { mediand }=\frac{1}{N_{v}} \sum_{j=1}^{N_{v}}\left|X_{d}(j)-M\right|
$$

\section{Minimum}

The minimum discretized intensity value of $X_{d}$.

$$
\text { minimum }=\min \left(X_{d}\right)
$$

\section{Minimum histogram gradient (mingrad)}

$$
\text { mingrad }=\min \left(\mathrm{H}^{\prime}\right)
$$

Where $H^{\prime}$ is the histogram gradient, defined as:

$$
H^{\prime}=\left\{H(2)-H(1), \ldots, \frac{H(i+1)-H(i-1)}{2}, \ldots, H\left(N_{g}\right)-H\left(N_{g}-1\right)\right\}
$$

\section{Minimum histogram gradient intensity level (mingradi)}

The discretized intensity level $i$ corresponding to the minimum histogram gradient.

\section{Mode}

The mode of $X_{d}$ is the most frequently occurring discretized image level present. In case multiple bins have the highest count $n_{i}$, the mode is the smallest of those values.

\section{Uniformity}

\section{Range}

$$
\text { uniformity }=\sum_{\mathrm{i}=1}^{\mathrm{N}_{\mathrm{g}}} \mathrm{P}(\mathrm{i})^{2}
$$

The range of bins in the histogram, i.e. the width of the histogram.

$$
\text { range }=\max \left(X_{d}\right)-\min \left(X_{d}\right)
$$


24. Root mean square (RMS)

$$
\text { RMS }=\sqrt{\frac{\sum_{j=1}^{N_{\mathrm{v}}} \mathrm{X}_{\mathrm{d}}(\mathrm{j})^{2}}{\mathrm{~N}_{\mathrm{v}}}}
$$

\section{Robust mean absolute deviation (rmeand)}

Similar to mean absolute deviation, but in this case only considering the set of intensity levels in the range between the $10^{\text {th }}$ and $90^{\text {th }}$ percentile of $X_{d}$.

$$
\text { rmeand }=\frac{1}{N_{10-90}} \sum_{j=1}^{N_{10-90}}\left|X_{d, 10-90}(j)-\bar{X}_{d, 10-90}\right|
$$

where $X_{10-90}$ represents the set of $N_{10-90}$ voxels in $X_{d}$ whose discretized intensity levels fall within the range of the $10^{\text {th }}$ till the $90^{\text {th }}$ percentile of $X_{d}$.

\section{Skewness}

$$
\text { skewness }=\frac{\frac{1}{N_{v}} \sum_{j=1}^{N_{v}}\left(X_{d}(j)-\bar{X}_{d}\right)^{3}}{\left(\sqrt{\frac{1}{N_{v}} \sum_{j=1}^{N_{v}}\left(X_{d}(j)-\bar{X}_{d}\right)^{2}}\right)^{3}}
$$

\section{Standard deviation}

$$
\text { standard deviation }=\left(\frac{1}{\mathrm{~N}_{\mathrm{v}}-1} \sum_{\mathrm{j}=1}^{\mathrm{N}_{\mathrm{v}}}\left(\mathrm{X}_{\mathrm{d}}(\mathrm{j})-\overline{\mathrm{X}}_{\mathrm{d}}\right)^{2}\right)^{1 / 2}
$$

\section{Variance}

The variance of $X_{d}$.

$$
\text { variance }=\frac{1}{N_{v}-1} \sum_{j=1}^{N_{v}}\left(X_{d}(j)-\overline{X_{d}}\right)^{2}
$$

where $\overline{X_{d}}$ is the mean of $X_{d}$.

\section{9. $10^{\text {th }}$ percentile}

The $10^{\text {th }}$ percentile of $X_{d}$.

30. $90^{\text {th }}$ percentile

The $90^{\text {th }}$ percentile of $X_{d}$.

\section{Quartile coefficient of dispersion (qcod)}

The quartile coefficient of dispersion is a robust alternative to the coefficient of variance.

$$
q \operatorname{cod}=\frac{\mathrm{P}_{75}-\mathrm{P}_{25}}{\mathrm{P}_{75}+\mathrm{P}_{25}}
$$

where $P_{25}$ and $P_{75}$ are the $25^{\text {th }}$ and $75^{\text {th }}$ percentile of $X_{d}$, respectively. 


\section{Supplementary Material 8D - Model performance}

Values of Harrell's concordance index with $95 \%$ confidence intervals for models trained on Dataset 2, 3 or 4 and validated on the remaining datasets. Values significant different from 0.5 are indicated in gray. A hyphen indicates that either all coefficients were forced to zero, or all predictions were equal to one, meaning that no linear combination of any subset of regressors was useful in predicting the outcomes. For Dataset 4, no subset of prognostic regressors was identified using the LASSO procedure for any of the images sets.

Table S8.1. Values of Harrell's concordance index with $95 \%$ confidence intervals, in the case Dataset $2(n=31)$ was used as training (T) to develop a model using LASSO. Validation (V) results are shown for Dataset 1,3 and 4. Significant values are indicated in gray. A hyphen indicates that either all coefficients were forced to zero, or all predictions were equal to one, meaning that no linear combination of any subset of regressors was useful in predicting the outcomes.

\begin{tabular}{lclllllll} 
& CT-scan1 & CT-scan2 & PET-scan1 & PET-scan2 & CT-rel & CT-abs & PET-rel & PET-abs \\
\hline T-Dataset 2 & 0.76 & - & 0.84 & 0.71 & - & - & 0.84 & 0.86 \\
T-Lower bound & 0.66 & - & 0.75 & 0.64 & - & - & 0.79 & 0.82 \\
T-Upper bound & 0.87 & - & 0.93 & 0.78 & - & - & 0.89 & 0.91 \\
V-Dataset 1 & 0.44 & - & 0.47 & 0.55 & - & - & 0.51 & 0.51 \\
V-Lower bound & 0.34 & - & 0.37 & 0.45 & - & - & 0.41 & 0.42 \\
V-Upper bound & 0.53 & - & 0.57 & 0.66 & - & - & 0.62 & 0.61 \\
V-Dataset 3 & 0.53 & - & 0.51 & 0.40 & - & - & 0.54 & 0.59 \\
V-Lower bound & 0.41 & - & 0.36 & 0.28 & - & - & 0.40 & 0.47 \\
V-Upper bound & 0.64 & - & 0.65 & 0.52 & - & - & 0.69 & 0.71 \\
V-Dataset 4 & 0.53 & - & 0.64 & 0.47 & - & - & 0.31 & 0.34 \\
V-Lower bound & 0.44 & - & 0.52 & 0.32 & - & - & 0.21 & 0.21 \\
V-Upper bound & 0.62 & - & 0.76 & 0.61 & - & - & 0.41 & 0.46
\end{tabular}


Table S8.2. Values of Harrell's concordance index with $95 \%$ confidence intervals, in the case Dataset $3(n=27)$ was used as training $(T)$ to develop a model using LASSO. Validation (V) results are shown for Dataset 1,2 and 4. Significant values are indicated in gray. A hyphen indicates that either all coefficients were forced to zero, or all predictions were equal to one, meaning that no linear combination of any subset of regressors was useful in predicting the outcomes.

\begin{tabular}{lllllllll} 
& CT-scan1 & CT-scan2 & PT-scan1 & PT-scan2 & CT-rel & CT-abs & PT-rel & PT-abs \\
\hline T-Dataset 3 & - & - & - & - & 0.71 & 0.74 & - & - \\
T-Lower bound & - & - & - & - & 0.59 & 0.64 & - & - \\
T-Upper bound & - & - & - & - & 0.84 & 0.83 & - & - \\
V-Dataset 1 & - & - & - & - & 0.59 & 0.54 & - & - \\
V-Lower bound & - & - & - & - & 0.50 & 0.42 & - & - \\
V-Upper bound & - & - & - & - & 0.68 & 0.65 & - & - \\
V-Dataset 2 & - & - & - & - & 0.60 & 0.55 & - & - \\
V-Lower bound & - & - & - & - & 0.48 & 0.44 & - & - \\
V-Upper bound & - & - & - & - & 0.72 & 0.67 & - & - \\
V-Dataset 4 & - & - & - & - & 0.62 & 0.52 & - & - \\
V-Lower bound & - & - & - & - & 0.51 & 0.37 & - & - \\
V-Upper bound & - & - & - & - & 0.74 & 0.68 & - & -
\end{tabular}

Table S8.3. Values of Harrell's concordance index with $95 \%$ confidence intervals, in the case Dataset $4(n=30)$ was used as training $(T)$ to develop a model using LASSO. Validation (V) results are shown for Dataset 1,2 and 3. A hyphen indicates that either all coefficients were forced to zero, or all predictions were equal to one, meaning that no linear combination of any subset of regressors was useful in predicting the outcomes. No prognostic models could be identified using LASSO for any of the available image sets.

\begin{tabular}{|c|c|c|c|c|c|c|c|c|}
\hline & CT-scan1 & CT-scan2 & PT-scan1 & PT-scan2 & CT-rel & CT-abs & PT-rel & PT-abs \\
\hline T-Dataset 4 & - & - & - & - & - & - & - & - \\
\hline T-Lower bound & - & - & - & - & - & - & - & - \\
\hline T-Upper bound & - & - & - & - & - & - & - & - \\
\hline V-Dataset 1 & - & - & - & - & - & - & - & - \\
\hline V-Lower bound & - & - & - & - & - & - & - & - \\
\hline V-Upper bound & - & - & - & - & - & - & - & - \\
\hline V-Dataset 2 & - & - & - & - & - & - & - & - \\
\hline V-Lower bound & - & - & - & - & - & - & - & - \\
\hline V-Upper bound & - & - & - & - & - & - & - & - \\
\hline V-Dataset 3 & - & - & - & - & - & - & - & - \\
\hline V-Lower bound & - & - & - & - & - & - & - & - \\
\hline V-Upper bound & - & - & - & - & - & - & - & - \\
\hline
\end{tabular}




\section{References}

[1] Carvalho S, Leijenaar RTH, Troost EGC, van Timmeren JE, Oberije C, van Elmpt W, et al. 18F-fluorodeoxyglucose positron-emission tomography (FDG-PET)-Radiomics of metastatic lymph nodes and primary tumor in non-small cell lung cancer (NSCLC) - A prospective externally validated study. PLoS One. 2018;13:e0192859.

[2] van Elmpt W, Ollers M, Dingemans AM, Lambin P, De Ruysscher D. Response assessment using 18F-FDG PET early in the course of radiotherapy correlates with survival in advancedstage non-small cell lung cancer. J Nucl Med. 2012;53:1514-20.

[3] Yossi S, Krhili S, Muratet JP, Septans AL, Campion L, Denis F. Early assessment of metabolic response by 18F-FDG PET during concomitant radiochemotherapy of non-small cell lung carcinoma is associated with survival: a retrospective single-center study. Clin Nucl Med. 2015;40:e215-21.

[4] Al-Kadi OS, ast, Watson D. Texture Analysis of Aggressive and Nonaggressive Lung Tumor CE CT Images. IEEE Trans Biomed Eng. 2008;55:1822-30.

[5] Wahl RL, Jacene H, Kasamon Y, Lodge MA. From RECIST to PERCIST: Evolving Considerations for PET response criteria in solid tumors. J Nucl Med. 2009;50 Suppl 1:122s-50s. 


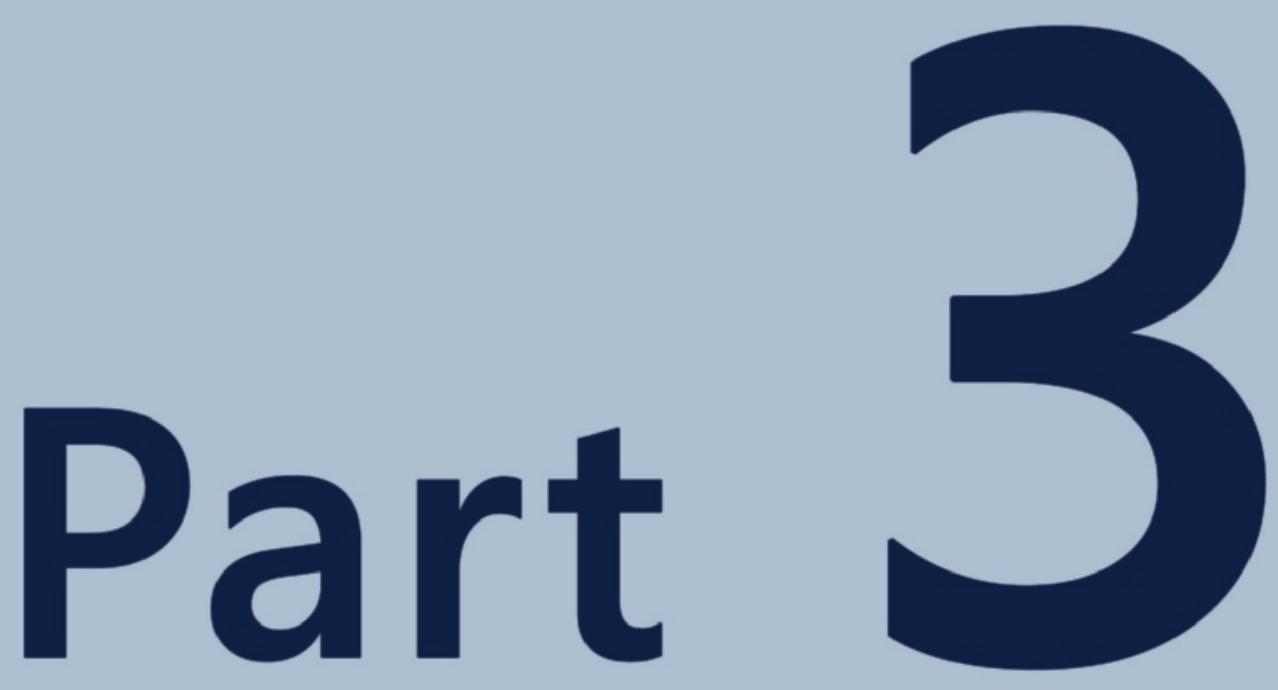



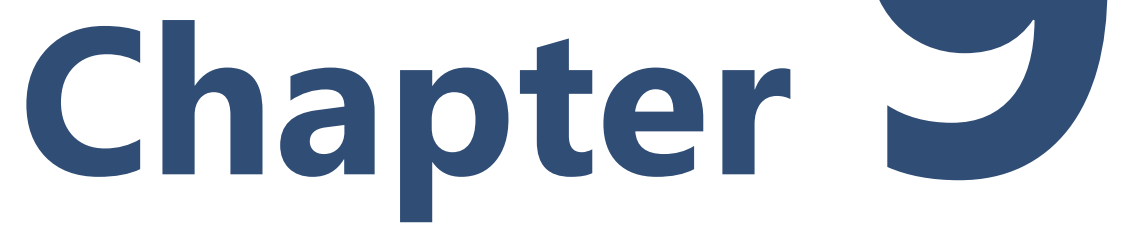

\section{Complementary value of deep learning and radiomics for prognosis and histology classification in non-small cell lung cancer}

Authors

Janna E. van Timmeren, Sergey Primakov, Manon Beuque, Wouter van Elmpt, Ralph T.H. Leijenaar, Bart Reymen, Carsten Brink, Olfred Hansen, Leen Paelinck, Evelien Bogaert, Carlos De Wagter, Elamin Elhaseen, Yolande Lievens, René Monshouwer, Johan Bussink, Abdalla Ibrahim, Philippe Lambin*, Arthur Jochems*

Adapted from In preparation 


\section{ABSTRACT}

\section{Background}

Deep learning has gained renewed interest for applications in quantitative medical imaging because of its improved performance in image classification contests. The aim of this study was to compare the performance of deep learning and radiomics for two-year survival prediction of non-small cell lung cancer (NSCLC) patients, and classification of the histological subtypes adenocarcinoma (AD) and squamous cell carcinoma (SCC).

\section{Material and methods}

A total of 760 CT scans and clinical parameters of stage I-IV NSCLC patients were available for two-year survival analysis and 500 patients for histology, originating from five different centers. Datasets were split into training, test and validation. Regularized generalized linear models (glmnet) were used to develop radiomic models, using radiomic features extracted from CT scan of the primary tumor (GTV). A deep learning network was built based on the Xception model. Model performance of the test set and validation set were described using the AUC. Model performance of deep learning and radiomics for two-year survival prediction were compared to a model containing clinical parameters: age, gender, TNM-stage and tumor volume.

\section{Results}

Radiomics and deep learning achieved equal performance for two-year survival prediction on the validation dataset $(A \cup C=0.62)$, which was similar to the performance of the clinical model (AUC = 0.63). On the other hand, deep learning outperformed radiomics for histological subtype classification on the validation dataset (AUC $=0.66$ and $A \cup C=0.55$, respectively).

\section{Conclusions}

This study shows the potential of deep learning for medical imaging classification. Future steps will include the collection of larger and more homogeneous datasets in terms of imaging settings and patient characteristics. Moreover, attempts will be made to improve the deep learning pre-processing and deep learning network. Furthermore, the complementary value of 'deep features' and radiomics needs further investigation. 


\section{Introduction}

The number of estimated cancer deaths in 2018 is 9.6 million, among which $18.4 \%$ are caused by lung cancer [1]. The 5-year survival rate is low: approximately $12.6 \%$ in Europe [2]. Quantitative image analysis of medical images has been proposed to contribute to prediction models, which enables improving personalized medicine [3]. Due to better informed treatment decisions, patient outcome could potentially be improved. One of the proposed methods is radiomics, which is defined as the extraction of large amounts of quantitative image features from medical images [47]. A region of interest $(\mathrm{ROI})$ should be defined prior to the extraction of radiomic features. ROI segmentation is usually performed manually and is thus prone to interand intra-observer variabilities [8-10]. Recently, deep learning has gained renewed interest for image classification, mainly caused by its high performance in, for example, the ImageNet contest [11]. It has been applied for several tasks, including segmentation, detection and classification [12]. Deep learning for medical image classification to derive prognostic information for NSCLC patients has not yet been studied extensively. A recent study investigated the performance of deep learning for 1,194 NSCLC patients for two-year survival and showed improved performance compared to a random forest model using radiomic features [13]. An earlier study from 2016 combined radiomic features with deep neural network features and achieved an AUC of 0.935 to classify lung adenocarcinoma patients into short survivors and long survivors [14], but this was trained on 81 NSCLC patients and no validation dataset was available.

Due to the effect of second-line and third-line treatments, other prognostic factors and because death is not necessarily caused by the cancer, the maximum achievable prediction for two-year survival based on only pre-treatment characteristics is never perfect. In the current study, we have also investigated the performance of quantitative image analysis for the classification between the two main histological subtypes of NSCLC: adenocarcinoma (AD) and squamous cell carcinoma (SCC). To summarize, the aim of the current study was to compare and investigate the power of radiomics and deep learning for two distinct applications: prediction of two-year survival and classification of histological subtypes (adenocarcinoma versus squamous cell carcinoma) for NSCLC patients. 


\section{Methods}

\section{Patients}

This study includes stage I-IV non-small cell lung cancer (NSCLC) patients that all received (chemo)radiotherapy with curative intent. Patients with available information about either two-year survival or histology (AD/SCC) were included in the study. $A$ total of 760 patients were available for two-year survival analysis and 500 patients for histology. The data originates from four different centers, consisting of five different datasets. An overview of the datasets and the numbers is presented in Table 9.1. Reasons for excluding data are explained below. Dataset 1, 3, 4 and 5 were combined into one training dataset and dataset 2 was used as test set.

Table 9.1. Overview of the data used in this study.

\begin{tabular}{llllll} 
Center & Treated between & $\begin{array}{l}\text { Total } \\
\text { available }\end{array}$ & $\begin{array}{l}\text { Total } \\
\text { included }\end{array}$ & $\begin{array}{l}\text { Two-year } \\
\text { survival }\end{array}$ & Histology \\
\hline Maastro Clinic* & $2004-2010$ & 422 & 352 & 351 & 173 \\
Maastro Clinic & $2012-2015$ & 256 & 242 & 214 & 166 \\
Odense University Hospital & $2007-2011$ & 94 & 89 & 89 & 72 \\
Radboud University Hospital & $2009-2011$ & 60 & 58 & 58 & 41 \\
Ghent University Hospital & $2010-2016$ & 61 & 53 & 48 & 48 \\
\hline TOTAL & & 893 & 794 & 760 & 500
\end{tabular}

* This dataset is a subset of the 'Lung 7' dataset of the publication of Aerts et al. [7], which is publically available (https://wiki.cancerimagingarchive.net/display/Public/NSCLC-Radiomics).

\section{Image of interest}

This study investigates computed tomography (CT) images, acquired for each patient at radiotherapy treatment planning and retrospectively collected. Therefore, there was quite a variation in image acquisition and reconstruction parameters within and between all datasets. All settings are summarized in Table S9.1. Nineteen patients in dataset 1 and ten patients in dataset 2 were excluded because of irregular slice spacing or missing slices in the scan.

\section{Region of interest}

The region of interest (ROI) in this study was the primary tumor. The gross tumor volume (GTV) was segmented in the clinical workflow for treatment planning and this radiotherapy structure set (RTSTRUCT) was used in this study for both radiomics and deep learning. For six patients in dataset 1, the RTSTRUCT-file was missing, therefore those patients were excluded. Also the cases for which the GTV consisted of multiple volumes were removed from the analysis in this study. Radiomic features have a different interpretation when calculated from multiple volumes, and choosing one volume might result in an unfair comparison between radiomics and deep learning. This was the case for 45 patients in dataset 1, four patients in dataset 2, five patients in dataset 3, two patients in datasets 4 and eighth patients in dataset 5. 


\section{Radiomic feature extraction}

A total of 2812 radiomic features were extracted from the images using RadiomiX (OncoRadiomics SA, Liège, Belgium), consisting of 1) Morphological, 2) Fractal, 3) Local Intensity, 4) Statistical features, 5) Intensity-volume histogram, 6) Textural features, 7) Laplacian of Gaussian filter and 8) Wavelet filter. Mathematical descriptions of all features are described in [15]. Gray-level discretization was performed using a bin width of $25 \mathrm{HU}$ prior to calculation of all features except those in group 4. Moreover, all images were resampled to isotropic voxel size of $1 \times 1 \times 3 \mathrm{~mm}^{3}$ using cubic interpolation, as this improves the stability of radiomics $[16,17]$ and allows for more meaningful feature comparisons between scans with different voxel sizes.

\section{Radiomics feature selection}

To reduce the risk of overfitting and to improve radiomic model robustness, several methods were applied to reduce feature dimensionality. First of all, features that contain missing values were removed. Subsequently, features with one unique value or with a number of unique values that is much lower than the number of samples, were removed using the function nearZerovar of the package caret in $\mathrm{R}$ (version 3.4.3). Furthermore, unstable radiomic features were removed by means of a testretest analysis and a multiple observer analysis. Two publically available datasets were used for this purpose, including the RIDER dataset [18] and the Maastro Clinic inter-observer reproducibility test series [7, 9]. For the RIDER dataset, 27 patients could be included, the other patients could not be retrieved due to technical problems, e.g. missing RTSTRUCT, scan/slices or link between the two, in either the test or the retest scan. All images were resampled to an isotropic voxel size of $0.65 \times 0.65 \times 1 \mathrm{~mm}^{3}$ (average dimensions of images) using cubic interpolation. The multiple delineations dataset consists of 18 patients, but three of them had multiple volumes separately delineated by all five observers. Therefore, in total 21 volumes were delineated by five different observers and used to acquire feature stability. All images in this dataset had the same voxel size, so no resampling was applied prior to radiomic feature extraction.

Stability was assessed by calculating the concordance correlation coefficient (CCC) for test-retest [19] and the overall CCC (OCCC) for multiple delineations [20] using the functions epi.ccc and epi.occc of the package epiR in $\mathrm{R}$ (version 3.4.3). Features that were stable for both the test-retest analysis and the multiple observer analysis, using a threshold value of 0.85 , were included in further analysis.

\section{Radiomics analysis}

In order to assess the performance of radiomic features for prediction of two-year survival or adenocarcinoma (AD) versus squamous cell carcinoma (SCC), the machine learning classification comparison of Deist, Dankers et al. [21] was used to select the most appropriate classifier, as this study concludes that "an informed pre-selection of classifiers based on existing datasets can improve discrimination over random selection". The classifier comparison procedure compares 5 different machine learning classifiers, being glmnet (penalized generalized linear models), rf (random forest), svm (support vector machine), LogitBoost (boosting) and rpart (regression 
trees). The maximum number of cross-validation repetitions was set to 50 . Default tuning was turned off and all other settings were kept to standard as described in the original publication [21]. Classifier performance was assessed using the area under the receiver operating characteristic (AUC). The classifier with the highest average performance for the training dataset of 546 patients (two-year survival) or 334 patients (histology) was chosen as the most appropriate machine learning classifier. Subsequently, the selected classifier was optimized using the training dataset. The final model was validated on dataset 2 ( $n=214$ and $n=166$, respectively).

\section{Deep learning preprocessing}

Images and corresponding segmentation (RTSTRUCT) were preprocessed into suitable dimensions for using the Xception model developed by Chollet in 2016 [22]. The input of the Xception model is $n \times n \times m$, where $n$ are the weight and height (299 in the case of Xception) and $m$ are the number of channels (RGB: 3 channels).

For preprocessing, pixel values below -1000 were set to -1000 , histogram equalization was performed and window/level settings of [1500:-600] were applied. For two-year survival prediction, the tumor segmentation was used to select the slice of interest, which was set to the slice that contains the most tumor pixels. For histology, all slices that contain tumor, except for the first and last slice, were included.

Next, a lung segmentation algorithm was applied in order to remove the background. This image was set as the R-channel (Figure 9.1). For the G-channel the tumor was extracted from the image using the available RTSTRUCT and the B-channel is a bounding box that has the size of the smallest box around the tumor plus a 20 pixel extension on each side. All images were zoomed to match the desired size in one dimension to make sure original image proportions remain unchanged. Finally, image padding with -1000 was applied to achieve the desired size of $299 \times 299$ pixels. Final images were normalized between 0 and 1 prior to model training.

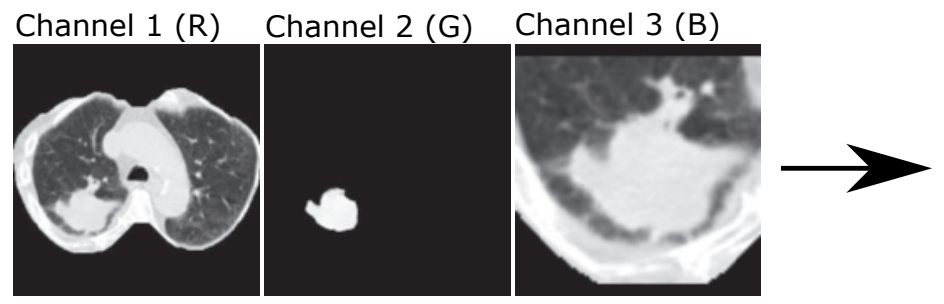

Figure 9.1. Overview of image slices used as input for the deep learning network.

\section{Deep learning network}

The architecture of the Xception model was used, where Xception is a model developed based on the Inception architecture and called "Extreme Inception" (Xception) [22]. One dense layer with 1024 units and ReLu (Rectified Linear Units) activation function followed by a dense layer with 1 output unit and sigmoid activation function represented the classification part of the network. The model was compiled using the Adam optimizer. The entire model was trained using randomized 
initialization of the weights.

The training set was used to train the model and split into training/test (0.75/0.25). The model was initially trained using a batch size of 10 and 100 epochs. Learning rate annealing was applied to gradually decrease the learning rate while training using $1 \times 10^{-4}$ as starting value. Afterwards, model weights were fine-tuned using data augmentation with random and varying rotations, shifts, zoom and flips using a rotation limit of 5 degrees, width and height shifts of maximum 0.05 , zooming limited to 0.01 and allowing horizontal flip. Augmentation data generator with a batch size of 10, 20 steps per epoch and 100 epochs was used to fine-tune the weights of the model. Model weights that achieved the highest accuracy on the test dataset were saved and final model performance was evaluated on the validation set (dataset 2, $\mathrm{n}=214$ or $\mathrm{n}=166$ ).

\section{Clinical model}

To compare performance to established prognostic factors for NSCLC, we also assessed the prognostic value of TNM-stage, tumor volume, age and gender to predict two-year survival, as these parameters were available for all patients. TNMstage was converted to dummy variables for T-stage and dummy variables for $\mathrm{N}$ stage. T-stage was divided into $\mathrm{T} 1, \mathrm{~T} 2$ and $\mathrm{T} 3 / 4$, and $\mathrm{N}$-stage was divided into N0, $\mathrm{N} 1 / 2$ and N3. T1 and N0 were used as reference categories. Recursive feature elimination using 10 -fold cross-validation with the linear discriminant function was performed on the training set was to select prognostic features, using the rfe function of the R-package caret.

\section{Results}

Patient characteristics are summarized in Table S9.2.

\section{Radiomics - two-year survival}

Feature numbers were reduced to 2012 after removing features with missing values or low/zero variance. Afterwards, 468 of them remained after excluding unstable features. These 468 features were used as input for the classifier comparison. The machine learning classifier 'glmnet' achieved the highest average AUC over all iterations for the training dataset. Therefore, this model was optimized for the training dataset and finally applied to the validation dataset. A 10-fold crossvalidation was repeated 200 times on the training data to find the most optimal lambda value, i.e. the lambda value with the minimum mean-cross validation error.

A model of two features was developed for 2-year survival prediction: "shape - compactness" and "shape - major axis length". These features are correlated to tumor volume: Pearson's correlation coefficients are 0.95 and 0.79 , respectively. The model achieved an AUC of 0.63 for training and 0.62 for validation. ROC curves are shown in Figure 9.2.

Radiomics - histology

A total of 466 stable features remained after the feature selection procedure. For histology classification, i.e. adenocarcinoma (AD) vs. squamous cell carcinoma (SCC), 
also the machine learning classifier 'glmnet' achieved the highest performance for the training set. Subsequently, a model of six features was developed, including four wavelet-filtered features, one LoG-filtered feature and one neighborhood gray-level different matrix feature. The model and its coefficients are shown in Supplementary Material 9C. The model achieved an AUC of 0.63 on training and 0.55 on validation.

A

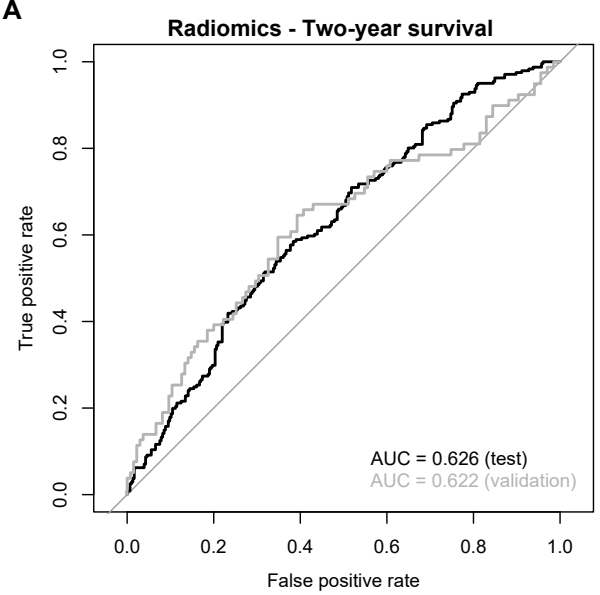

C

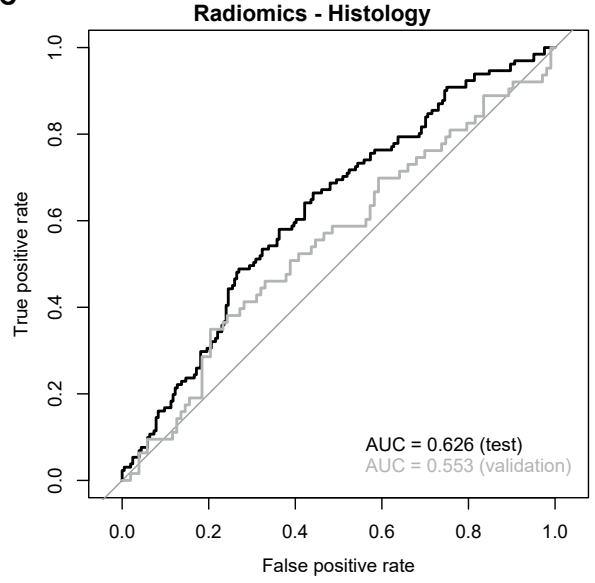

B

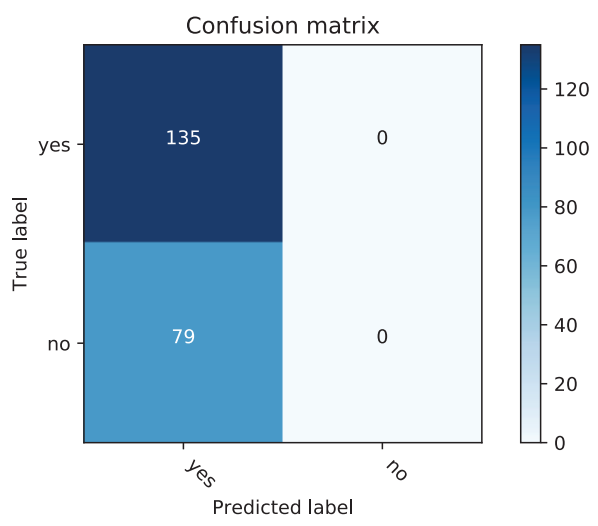

D

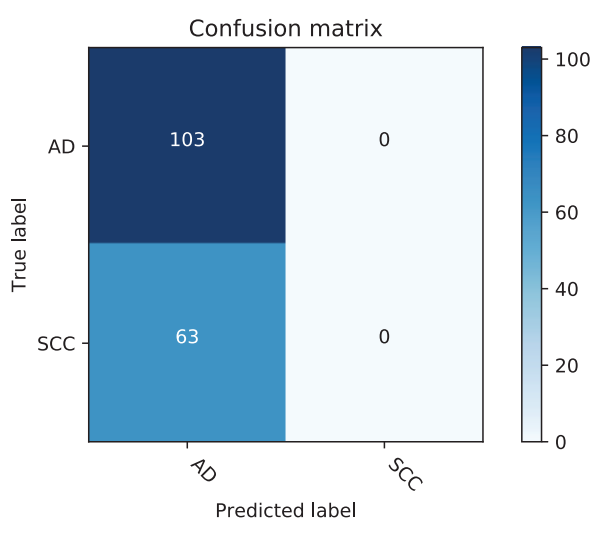

Figure 9.2. Receiver Operating Characteristic (ROC) curves of the performance of radiomics for prediction of two-year survival $(A)$ or histology $(B)$, with corresponding AUC values.

\section{Deep learning - two-year survival}

The training set of 546 patients with one slice per patients was used to train the deep learning network, using $0.75 / 0.25$ split for training/test. The remaining patients of Dataset $2(n=214)$ were used to validate to model. The network performed with an AUC of 0.61 [95\% Cl $0.51-0.71$ ] on the test-set and 0.62 [95\% $\mathrm{Cl} 0.53-0.70$ ] on the validation set. The ROC-curve is shown in Figure 9.3. 


\section{Deep learning - histology}

For histology, each slice of the training set that contains tumor was included to have larger dataset to train the model. This resulted in a total of 5540 images available for training, originating from 334 patients. The final model was validated on the remaining 166 patients of Dataset 2: only the slice that contains most tumor was included here. The model achieved a performance of 0.74 [95\% $\mathrm{Cl} 0.72-0.77]$ on the test-set and a performance of $0.66[95 \% \mathrm{Cl} 0.57-0.75]$ on the validation set of 166 patients.

A

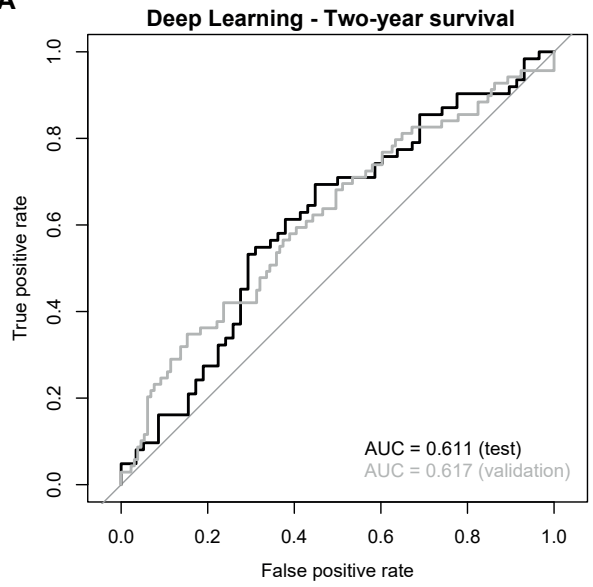

C

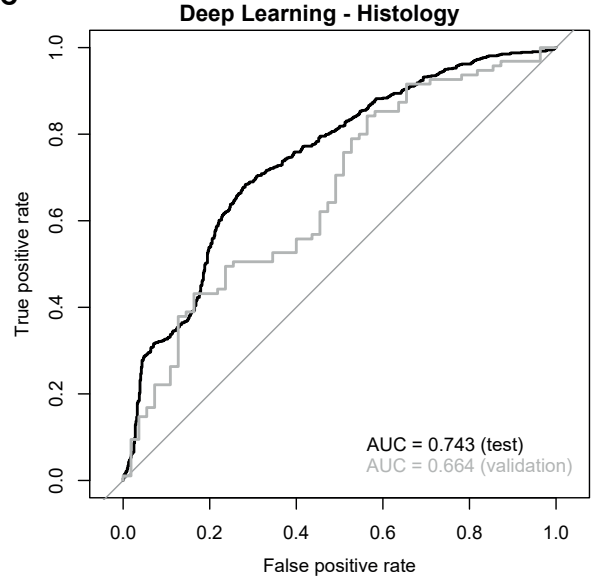

B

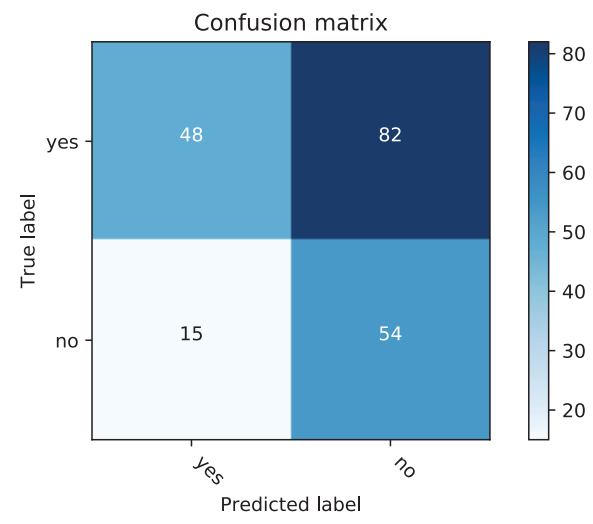

D

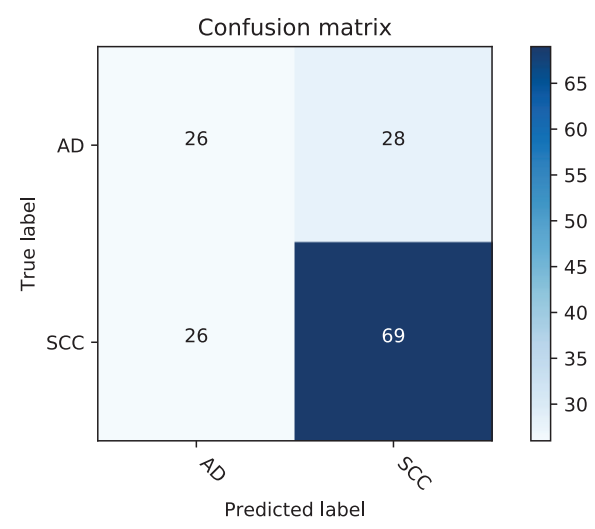

Figure 9.3. Receiver Operating Characteristic (ROC) curves of the performance of deep learning for prediction of two-year survival (A) or histology (B), with corresponding AUC values.

\section{Clinical model}

Seven features were imputed into the recursive feature elimination method, being T-stage 2, T-stage 3/4, N-stage 1/2, N-stage 3, age, gender and tumor volume. Information about age was missing for Only one feature was selected: tumor volume. This achieved an accuracy of $0.63[95 \% \mathrm{Cl} 0.54-0.70]$ on the validation dataset. 


\section{Discussion}

This study aimed to investigate the complementary value of radiomics and deep learning for two-year survival prediction and classification between adenocarcinoma/ squamous cell carcinoma for NSCLC patients.

The prognostic value of radiomics for two-year survival reached a performance of 0.63 for test $(n=546$ : dataset $1,3,4$ and 5$)$ and 0.62 for validation $(n=214$ : dataset 2). On the other hand, the performance for deep learning was 0.61 and 0.62 , respectively. This shows that radiomics and deep learning have equal performance on the validation dataset for two-year survival estimation. The recursive feature elimination on clinical features only included tumor volume in the final model, which achieved a performance of 0.60 in test and 0.63 in validation. The features in the radiomics model were highly correlated to volume $(\rho=0.95$ and $\rho=0.79)$. This shows that both the deep learning and the radiomics model had no additional value compared to a model with just tumor volume.

For histology, AD versus SCC, less data was available $(n=335$ and $n=166$ for training and test, respectively), but here all slices that contain tumor, except for the first and last slice, were included for model training. The performance of the radiomics model was 0.63 for training and 0.55 for validation, whereas deep learning achieved a performance of 0.74 for training and 0.66 for validation, which shows that deep learning had improved performance over radiomics. The validation results are comparable to results found in literature: an AUC of 0.655 was found for AD versus SCC using support vector machine algorithms on radiomic features [23], but this model was only trained on 181 NSCLC patients and no independent validation was available. In the current study, a similar performance was achieved on the training dataset using radiomics, but could only be achieved on the validation dataset using deep learning. Potentially, the amount of data available for training boosted the deep learning model performance compared to the two-year survival prediction performance. For two-year survival we did not use all tumor slices but instead only the slice that contains the largest tumor area, because we assumed that the size of the tumor is an important parameter that should be taken into account for two-year survival prediction.

Radiomics and deep learning use different kinds of information. Radiomics uses a region of interest, the gross tumor volume in this study, to calculate three-dimensional features. On the other hand, the current deep learning network learns from twodimensional images. However, the use of different inputs for each RGB-channel results in a more extensive information source than just the use of one CT-scan slice. In the first channel the entire lungs were used, whereas the second channel contains only the tumor but this includes information about location. Furthermore, several studies have shown that the boundaries around the tumor also contain prognostic information, which is used in the third channel. In this study, deep learning was applied to 2D-slices, but it would also be possible to train the network on the entire 3D image. The information available for radiomics and deep learning is different and it is not yet known what the best approach is. Potentially, both approaches should be combined to extract the most information out of the images, as was shown by Paul 
et al. in 2016 [14], which will be investigated in the future.

The lack of interpretability of radiomics and deep learning could be an issue for clinical implementation. The underlying biological processes of radiomic features is not yet known, but more research is being performed relating genes and frozen sections to feature values. Moreover, the features itself are calculated by a welldefined mathematical formula and especially the simpler features that describe the shape of a tumor or the coarseness of the ROI are understandable. On the other hand, the process from input images to a prediction is more a black box in deep learning, which could be a disadvantage for understanding a good or bad model performance. Visualization techniques, for instance saliency maps that show the importance of each pixel in the image for the final prediction, will help to raise understanding of the process within a deep learning network.

Both radiomics and the deep learning workflow used in this study require segmentation of the tumor. This could be made easier in the future by using deep learning to segment the tumor prior to the prediction step. This will not only increase speed of the process, it will also ensure more robust segmentations (i.e. no inter- or intra-observer variabilities). This will also make it easier to increase cohort sizes with images for which segmentation was not performed or not available, e.g. open source data without target volume delineations.

The amount of data available is an important factor for the success-rate of radiomics and deep learning. A previous study trained and tuned the network on a total of 464 patients and tested the model on 211 patients. This study achieved and AUC of 0.70 for overall survival. For the current study, less patients were available for training and validation, which could have hampered the performance. The cohort size will be increased in the future to evaluate the performance of deep learning and radiomics for larger datasets.

Another limitation of this study is the heterogeneity in the dataset in terms of image acquisition- and reconstruction settings. The dataset might not be large enough to capture all variabilities in the dataset to ensure generalizability of the model, which could have influenced the performance on the validation datasets. A larger dataset would allow to capture more variations in imaging parameters to improve validation performance.

\section{Conclusion}

In this study, no superiority of deep learning or radiomics could be found for the prediction of two-year survival and both models performance similarly compared to a model containing just tumor volume. On the other hand, deep learning performed better for histological subtype classification, radiomics predicted the same class for each patient. This work shows the potential of deep learning for classification in medical imaging. Potentially, the combination of information sources has improved prognostic value, so future work will include combining radiomic features with deep learning features. Moreover, larger datasets will be collected, and an attempt will be made to improve the deep learning pre-processing and deep learning network. 


\section{Acknowledgments}

Authors acknowledge financial support from ERC advanced grant (ERC-ADG-2015, $\mathrm{n}^{\circ} 694812$ - Hypoximmuno), ERC-2018-PoC ( $\left.n^{\circ} 81320-C L-I O\right)$. This research is also supported by the Dutch technology Foundation STW (grant $n^{\circ}$ P14-19 Radiomics STRaTegy), which is the applied science division of NWO, and the Technology Programme of the Ministry of Economic Affairs. Authors also acknowledge financial support from SME Phase 2 (RAIL - $n^{\circ} 673780$ ), EUROSTARS (DART, DECIDE, COMPACT), the European Program H2020-2015-17 (BD2Decide - PHC30689715, ImmunoSABR - $n^{\circ} 733008$, PREDICT - ITN - $n^{\circ}$ 766276), TRANSCAN Joint Transnational Call 2016 (JTC2016 "CLEARLY"- n UM 2017-8295), Interreg V-A Euregio Meuse-Rhine ("Euradiomics"), Kankeronderzoekfonds Limburg (KOFL) from the Health Foundation Limburg and the Dutch Cancer Society.

\section{References}

[1] Bray F, Ferlay J, Soerjomataram I, Siegel RL, Torre LA, Jemal A. Global cancer statistics 2018: GLOBOCAN estimates of incidence and mortality worldwide for 36 cancers in 185 countries. CA Cancer J Clin. 2018.

[2] Francisci S, Minicozzi P, Pierannunzio D, Ardanaz E, Eberle A, Grimsrud TK, et al. Survival patterns in lung and pleural cancer in Europe 1999-2007: Results from the EUROCARE-5 study. Eur J Cancer. 2015;51:2242-53.

[3] Lambin P, van Stiphout RG, Starmans MH, Rios-Velazquez E, Nalbantov G, Aerts HJ, et al. Predicting outcomes in radiation oncology--multifactorial decision support systems. Nat Rev Clin Oncol. 2013;10:27-40.

[4] Gillies RJ, Kinahan PE, Hricak H. Radiomics: Images Are More than Pictures, They Are Data. Radiology. 2016;278:563-77.

[5] Lambin P, Rios-Velazquez E, Leijenaar R, Carvalho S, van Stiphout RG, Granton P, et al. Radiomics: extracting more information from medical images using advanced feature analysis. Eur J Cancer. 2012;48:441-6.

[6] Lambin P, Leijenaar RTH, Deist TM, Peerlings J, de Jong EEC, van Timmeren J, et al. Radiomics: the bridge between medical imaging and personalized medicine. Nat Rev Clin Oncol. 2017;14:749-62.

[7] Aerts HJ, Velazquez ER, Leijenaar RT, Parmar C, Grossmann P, Carvalho S, et al. Decoding tumour phenotype by noninvasive imaging using a quantitative radiomics approach. Nat Commun. 2014;5:4006.

[8] van Baardwijk A, Bosmans G, Boersma L, Buijsen J, Wanders S, Hochstenbag M, et al. PETCT-based auto-contouring in non-small-cell lung cancer correlates with pathology and reduces interobserver variability in the delineation of the primary tumor and involved nodal volumes. Int J Radiat Oncol Biol Phys. 2007;68:771-8.

[9] Leijenaar RT, Carvalho S, Velazquez ER, van Elmpt WJ, Parmar C, Hoekstra OS, et al. Stability of FDG-PET Radiomics features: an integrated analysis of test-retest and inter-observer variability. Acta Oncol. 2013;52:1391-7.

[10] Parmar C, Rios Velazquez E, Leijenaar R, Jermoumi M, Carvalho S, Mak RH, et al. Robust Radiomics feature quantification using semiautomatic volumetric segmentation. PLoS One. 2014;9:e102107.

[11] Krizhevsky A, Sutskever I, Hinton GE. ImageNet classification with deep convolutional neural networks. Proceedings of the 25th International Conference on Neural Information Processing Systems - Volume 1. Lake Tahoe, Nevada: Curran Associates Inc.; 2012. p. 1097-105. [12] Litjens G, Kooi T, Bejnordi BE, Setio AAA, Ciompi F, Ghafoorian M, et al. A survey on deep learning in medical image analysis. Med Image Anal. 2017;42:60-88.

[13] Hosny A, Parmar C, Coroller TP, Grossmann P, Zeleznik R, Kumar A, et al. Deep learning for lung cancer prognostication: A retrospective multi-cohort radiomics study. PLoS Med. 2018;15:e1002711.

[14] Paul R, Hawkins SH, Balagurunathan Y, Schabath MB, Gillies RJ, Hall LO, et al. Deep Feature Transfer Learning in Combination with Traditional Features Predicts Survival Among Patients 
with Lung Adenocarcinoma. Tomography. 2016;2:388-95.

[15] de Jong EEC, Deist TM, Van Elmpt W, Jochems A, Rizzo S, Petrella F, et al. Can quantitative radiomic features describe qualitative semantic features in non-small cell lung cancer patients? Sci Rep. 2018; Submitted.

[16] Shafiq-Ul-Hassan M, Zhang GG, Latifi K, Ullah G, Hunt DC, Balagurunathan Y, et al. Intrinsic dependencies of CT radiomic features on voxel size and number of gray levels. Med Phys. 2017;44:1050-62.

[17] Larue RTHM, van Timmeren JE, de Jong EEC, Feliciani G, Leijenaar RTH, Schreurs WMJ, et al. Influence of gray level discretization on radiomic feature stability for different CT scanners, tube currents and slice thicknesses: a comprehensive phantom study. Acta Oncol. 2017;56:154453.

[18] Armato SG, 3rd, Meyer CR, McNitt-Gray MF, McLennan G, Reeves AP, Croft BY, et al. The Reference Image Database to Evaluate Response to therapy in lung cancer (RIDER) project: a resource for the development of change-analysis software. Clin Pharmacol Ther. 2008;84:44856.

[19] Lawrence IKL. A Concordance Correlation Coefficient to Evaluate Reproducibility. Biometrics. 1989;45:255-68.

[20] Barnhart HX, Haber M, Song J. Overall concordance correlation coefficient for evaluating agreement among multiple observers. Biometrics. 2002;58:1020-7.

[21] Deist TM, Dankers F, Valdes G, Wijsman R, Hsu IC, Oberije C, et al. Machine learning algorithms for outcome prediction in (chemo)radiotherapy: An empirical comparison of classifiers. Med Phys. 2018.

[22] F. C. Xception: Deep Learning with Depthwise Separable Convolutions. CoRR. 2016; abs/1610.02357.

[23] E L, Lu L, Li L, Yang H, Schwartz LH, Zhao B. Radiomics for Classification of Lung Cancer Histological Subtypes Based on Nonenhanced Computed Tomography. Acad Radiol. 2018. 


\section{Supplementary Material}

\section{Supplementary Material 9A - Imaging parameters}

Table S9.1. Image parameters for the entire cohort.

\begin{tabular}{|c|c|c|}
\hline \multicolumn{2}{|c|}{ Parameters } & Entire cohort $(n=824)$ \\
\hline \multicolumn{2}{|c|}{ Manufacturer } & Siemens $(n=501)$ \\
\hline \multirow[t]{9}{*}{ CT } & Tube voltage [kVp] & $\begin{array}{l}\text { Mean } \pm \text { SD: } 122 \pm 5.97 \\
\text { Median [range]: } 120 \text { [120 - 140] } \\
\text { Unknown }(n=143)\end{array}$ \\
\hline & Tube current [mA] & $\begin{array}{l}\text { Mean } \pm \text { SD: } 181 \pm 97.9 \\
\text { Median [range]: } 188 \text { [40-642] } \\
\text { Unknown }(n=143)\end{array}$ \\
\hline & Convolution kernel & $\begin{array}{l}\text { B10f }(n=1) \\
\text { B18f }(n=21) \\
\text { B19f }(n=183) \\
\text { B19s }(n=13) \\
\text { B30f }(n=206) \\
\text { B30s }(n=7) \\
\text { B31f }(n=24) \\
\text { B40f }(n=16) \\
\text { B40s }(n=11) \\
\text { B41f }(n=21) \\
\text { B60s }(n=2) \\
\text { I30f } \mid 3(n=2) \\
\text { B }(n=68) \\
\text { FC13 }(n=25) \\
\text { Unknown }(n=179)\end{array}$ \\
\hline & Exposure Time [ms] & $\begin{array}{l}\text { Mean } \pm \text { SD: } 487 \pm 485 \\
\text { Median [range]: } 361 \text { [361 - 8333] } \\
\text { Unknown }(n=209)\end{array}$ \\
\hline & Exposure [mAs] & $\begin{array}{l}\text { Mean } \pm \text { SD: } 578 \pm 454 \\
\text { Median [range]: } 400[30-1700] \\
\text { Unknown }(n=143)\end{array}$ \\
\hline & $\begin{array}{l}\text { Reconstruction diame- } \\
\text { ter }[\mathrm{mm}]\end{array}$ & $\begin{array}{l}\text { Mean } \pm \text { SD: } 509 \pm 35.6 \\
\text { Median [range]: } 500[436-880] \\
\text { Unknown }(n=143)\end{array}$ \\
\hline & Patient position & HFS $(n=824)$ \\
\hline & Slice thickness (mm) & $\begin{array}{l}\text { Mean } \pm \text { SD: } 3.02 \pm 0.49 \\
\text { Median [range]: } 3[2-5]\end{array}$ \\
\hline & Pixel spacing (mm) & $\begin{array}{l}\text { Mean } \pm \text { SD: } 0.99 \pm 0.06 \\
\text { Median [range]: } 0.98[0.72-1.72]\end{array}$ \\
\hline
\end{tabular}

* Due to anonymization, this information was removed from the DICOM header for a subset of CT images in the dataset. 


\section{Supplementary Material 9B - Patient characteristics}

Table S9.2. Patient characteristics for all datasets.

\begin{tabular}{|c|c|c|c|c|c|}
\hline & $\begin{array}{l}\text { Dataset } 1 \\
(n=352)\end{array}$ & $\begin{array}{l}\text { Dataset } 2 \\
(n=242)\end{array}$ & $\begin{array}{l}\text { Dataset } 3 \\
(n=89)\end{array}$ & $\begin{array}{l}\text { Dataset } 4 \\
(n=58)\end{array}$ & $\begin{array}{l}\text { Dataset } 5 \\
(n=53)\end{array}$ \\
\hline \multicolumn{6}{|l|}{ Age } \\
\hline Range (median) & $33-91(68)$ & $38-89(70.5)$ & $42-83(67)$ & $41-85(63.5)$ & $47-83(65)$ \\
\hline Mean \pm SD & $67.8 \pm 10.1$ & $69.2 \pm 9.5$ & $66.5 \pm 8.6$ & $63.8 \pm 10.9$ & $64.5 \pm 8.7$ \\
\hline \multicolumn{6}{|l|}{ Gender } \\
\hline Male & 242 (68.8\%) & 158 (65.3\%) & 42 (47.2\%) & 39 (67.2\%) & 41 (77.5\%) \\
\hline Female & $110(31.3 \%)$ & 84 (34.7\%) & 47 (52.8\%) & 19 (32.8\%) & $12(22.6 \%)$ \\
\hline T-stage & & & * & & \\
\hline 1 & 82 (23.3\%) & 45 (18.6\%) & $12(13.4 \%)$ & 7 (12.1\%) & 11 (20.8\%) \\
\hline 2 & $136(38.6 \%)$ & 75 (31.0\%) & 38 (42.7\%) & 18 (31.0\%) & 17 (32.8\%) \\
\hline 3 & $41(11.6 \%)$ & 41 (16.9\%) & 13 (14.6\%) & 22 (27.9\%) & 10 (18.9\%) \\
\hline 4 & 93 (26.4\%) & 75 (31.0\%) & 25 (28.1\%) & $10(17.2 \%)$ & 15 (28.3\%) \\
\hline \multicolumn{6}{|l|}{$\mathrm{N}$-stage } \\
\hline 0 & 147 (41.8\%) & 62 (25.6\%) & 17 (19.1\%) & 12 (20.7\%) & 5 (9.4\%) \\
\hline 1 & $20(5.7 \%)$ & $18(7.3 \%)$ & $2(2.2 \%)$ & $6(10.3 \%)$ & $3(5.7 \%)$ \\
\hline 2 & 114 (32.4\%) & 88 (36.4\%) & 62 (69.7\%) & 32 (55.2\%) & $34(64.2 \%)$ \\
\hline 3 & 68 (19.3\%) & 67 (27.7\%) & $8(9.0 \%)$ & 7 (12.1\%) & 11 (20.8\%) \\
\hline \multicolumn{6}{|l|}{ Stage } \\
\hline I & 83 (23.6\%) & $23(9.5 \%)$ & $4(4.5 \%)$ & $4(6.9 \%)$ & $0(0 \%)$ \\
\hline II & 36 (10.2\%) & $29(12.0)$ & $6(6.7 \%)$ & $8(13.8 \%)$ & 2 (3.8\%) \\
\hline Illa & $89(25.3 \%)$ & 71 (29.3\%) & 48 (53.9\%) & 33 (56.9\%) & 33 (62.3\%) \\
\hline IIIb & 144 (40.9\%) & 92 (38.0\%) & 31 (34.8\%) & $12(20.7 \%)$ & 18 (34.0\%) \\
\hline IV & $0(0 \%)$ & $26(10.7 \%)+$ & $0(0 \%)$ & $0(0 \%)$ & $0(0 \%)$ \\
\hline \multicolumn{6}{|l|}{ Histology } \\
\hline Adenocarcinoma & $46(13.1 \%)$ & 64 (26.4\%) & 35 (39.3\%) & 20 (34.5\%) & 30 (56.6\%) \\
\hline Squamous cell carcinoma & $127(36.1 \%)$ & $104(43.0 \%)$ & $37(41.6 \%)$ & $21(36.2 \%)$ & $18(34.0 \%)$ \\
\hline Large cell carcinoma & $92(26.2 \%)$ & $10(4.1 \%)$ & $4(4.5 \%)$ & $5(8.6 \%)$ & $3(5.7 \%)$ \\
\hline Other & $0(0 \%)$ & $1(0.4 \%)$ & $6(6.7 \%)$ & $2(3.4 \%)$ & 1 (1.9\%) \\
\hline Not otherwise specified & 87 (24.7\%) & $63(26.0 \%)$ & 7 (7.9\%) & $10(17.2 \%)$ & $1(1.9 \%)$ \\
\hline \multicolumn{6}{|l|}{$\mathrm{GTV}\left(\mathrm{cm}^{3}\right)$} \\
\hline Range (median) & $0.53-660(35)$ & $0.60-812(40)$ & 2.2-397 (41) & $1.0-482(80)$ & $1.5-427(32)$ \\
\hline Mean \pm SD & $67.3 \pm 90.6$ & $74.9 \pm 100$ & $69.4 \pm 72.0$ & $108 \pm 108$ & $71.0 \pm 97.8$ \\
\hline \multicolumn{6}{|l|}{ Overall survival (year) } \\
\hline Median [range] & $1.7[0.1-4.8]$ & $1.7[0.1-5.5]$ & $1.7[0.1-9.8]$ & $1.7[0.1-6.5]$ & $\begin{array}{l}2.8[0.4- \\
7.7]\end{array}$ \\
\hline
\end{tabular}

Events at time of analysis
Survival
308
169
69
47
28

* One patient in this dataset was classified as TON2, since all disease was in lymph node station 5 and no primary tumor could be distinguished. The entire volume was used as ROI.

+ This group of patients is treated with curative intent and has similar prognosis and the stage III patients. 


\section{Supplementary Material 9C - Models}

Table S9.3. Model coefficients and feature explanations for the radiomics model to predict two-year survival.

\begin{tabular}{lll} 
Feature name & Coefficient & Feature explanation \\
\hline Shape_compactness & -0.00162 & $\begin{array}{l}\text { Compactness is a measure of how much the volume resem- } \\
\text { bles a sphere }\end{array}$ \\
Shape_majoraxislength & -0.0288 & $\begin{array}{l}\text { The major axis length is the largest eigenvalue of a princi- } \\
\text { pal component analysis (PCA) on the } x, y \text { and } z \text { coordinates } \\
\text { of all voxels within the volume, which is a measure of the } \\
\text { extent of the volume along its three principle axes. }\end{array}$
\end{tabular}

Table S9.4. Model coefficients and feature explanations for the radiomics model to classify histological subtype (adenocarcinoma versus squamous cell carcinoma).

\begin{tabular}{|c|c|c|}
\hline Feature name & Coefficient & Feature explanation \\
\hline LoG_sigma_4_5_mm_3D_pos_IH_p10 & 0.0158 & $\begin{array}{l}\text { The } 10^{\text {th }} \text { percentile of the Intensity Histogram } \\
\text { (IH) calculated from the positive (pos) part of } \\
\text { the filtered image. The image is filtered with } \\
\text { a Laplacian of Gaussian (LoG) filter with a } \\
\text { Gaussian standard deviation (radius) } \sigma \text { of } 4.5 \\
\text { mm. The IH is the distribution of gray values } \\
\text { after discretization into intensity level bins } \\
\text { was applied. }\end{array}$ \\
\hline NGLDM_LGLDE & -0.0165 & $\begin{array}{l}\text { This is a feature calculated on the neighbor- } \\
\text { ing gray-level dependence matrix (NGLDM) } \\
\text { called 'low gray-level large dependence } \\
\text { emphasis' (LGLDE) that capture coarseness } \\
\text { of the overall ROI in the image. This feature } \\
\text { emphasizes the part of the NGLDM (upper } \\
\text { right quadrant) that locate high dependence } \\
\text { counts and low gray levels. }\end{array}$ \\
\hline Wavelet_HLH_Stats_energy & $-3.13 * 10^{-10}$ & $\begin{array}{l}\text { First order statistics (Stats) feature that rep- } \\
\text { resents the energy of the image, calculated } \\
\text { from the distribution of gray values of the } \\
\text { original images. This feature was calculated } \\
\text { after applying a wavelet transform to the } \\
\text { original image using high-pass (H) filtering } \\
\text { in the } x \text {, low-pass filtering in the y and high- } \\
\text { pass filtering in the z-direction (HLH) }\end{array}$ \\
\hline Wavelet_HLL_Fractal_lacunarity & -4.70 & $\begin{array}{l}\text { Fractal dimension (measuring the geomet- } \\
\text { rical complexity of images) lacunarity, which } \\
\text { describes the heterogeneity and the gaps in } \\
\text { an image. This feature was calculated after } \\
\text { applying a wavelet transform to the original } \\
\text { image using } H \text {, } L \text { and } L \text { filtering in the } x-, y- \\
\text { and } z \text {-direction, respectively (HLL). }\end{array}$ \\
\hline Wavelet_LLL_GLCM_invDiffMomNor & -4.84 & $\begin{array}{l}\text { This is a gray-level co-occurrence matrix } \\
\text { (GLCM) feature: inverse difference moment } \\
\text { normalized (invDiffMomNor), which is nor- } \\
\text { malized feature that represents homogeneity. } \\
\text { This feature was calculated after applying a } \\
\text { wavelet transform to the original image using } \\
\text { L filtering in the } x-, y \text { - and z-direction (LLL). }\end{array}$ \\
\hline Wavelet_LLL_GLCM_inverseVar & -0.710 & $\begin{array}{l}\text { The inverse variance (inverseVar) of the } \\
\text { GLCM, calculated after applying a wavelet } \\
\text { transform to the original image using low- } \\
\text { pass filtering in the } x-, y \text { - and } z \text {-direction } \\
\text { (LLL). }\end{array}$ \\
\hline
\end{tabular}


Table S9.5. Model coefficients and feature explanations for the clinical model to predict twoyear survival.

Feature name

Shape_volume
Coefficient

$-0.00569$

\section{Feature explanation}

Shape - volume is the volume of the region of interest in $\mathrm{cm}^{3}$. 



\section{Chapter Discussion and future perspectives}



Radiomics is an increasing field of interest and is currently being investigated for many different image modalities and disease sites, aiming to support treatment individualization and improve treatment outcome. Numerous proof-of-concept studies show the prognostic or predictive potential of radiomics for multiple disease sites using different treatment modalities, describing phenotypes of a certain region of interest by means of quantitative imaging features. This thesis focused on lung cancer, currently the main cause of death from cancer worldwide, and in particular on non-small cell lung cancer (NSCLC).

Most studies in the field of radiotherapy focus on image features extracted prior treatment for the prediction of patient outcome. Potentially, the change of radiomic features over time contains additional prognostic information, which could make it possible to assess (early) tumor response during treatment, allowing to adjust treatment accordingly on an individual basis. Therefore, the following hypothesis was investigated in this thesis: Quantitative information derived from medical images acquired prior to and during treatment describing the (changing) phenotype of the tumor, provides prognostic information for non-small cell lung cancer patients.

All studies in this work were retrospective studies and thus the heterogeneity and size of the datasets was the most apparent challenge in this thesis, whereas prospective studies have the opportunity to control for many parameters, in order to generate a homogeneous dataset. But, besides cohort size and intra- and interdataset heterogeneity, many more barriers should be overcome in the field of radiomics [1-4]. The following sections discuss the main challenges for each step of the radiomics workflow in relation to the chapters in this thesis, comprising image acquisition, segmentation, feature extraction, feature selection and modeling.

\section{Image acquisition and reconstruction}

\section{Acquisition}

Across different institutes and even within an institution, imaging systems are often from different manufacturers. A recent study showed that less than half of 177 radiomic features was reproducible when extracted from five different $C T$ scanners [5]. Moreover, tube current, energy and scanner type all influence feature values [6]. Hard- and software limitations or discrepancies between manufacturers make standardization difficult. In this thesis, the multi-center studies contained images acquired from different imaging systems, resulting in variations in acquisition parameters and image quality. This also influenced the results of chapter 2-5, because the cone-beam CT images were acquired from three different systems: Varian Truebeam, Elekta XVI and Varian Clinac iX. Ideally, images are all obtained using controlled acquisition settings, but the availability of this type of data is often limited. A prospective study could overcome this issue, whereas a phantom study could help to investigate the influence of image acquisition parameters on radiomic feature values and possibly allow for post-harmonization, which is discussed later in this section. 


\section{Reconstruction}

Besides image acquisition, also multiple image reconstruction settings are generally used within datasets. Some studies have investigated the influence of reconstruction settings on feature stability $[7,8]$ and showed that a selection of features is insensitive to a wide range of settings. Nevertheless, the application of the results of these studies is hampered by the lack of standardized feature naming and implementation convention. Moreover, the continuous development of new features makes it hard to have up-to-date studies. This means that it is becoming more and more important to implement guidelines, as discussed later in this chapter under Feature extraction Radiomics implementation.

The variation between reconstruction settings can be reduced by standardization and consensus, for instance about the slice thickness that should be used based on the tradeoff between the required image quality and the delivered radiation dose [9]. To circumvent the lack of standardization, the collection of raw data, e.g. CT projections, sound attractive, as these images could be reconstructed according to a predefined protocol which would facilitate model validation across different centers. This, however, is not feasible in current clinical practice due to the enormous file size of these projections. On the other hand it would be possible to reconstruct an image twice: one according to the local protocol and one according to published guidelines.

Heterogeneities in datasets caused by different scanners, image acquisition and reconstruction settings are especially challenging for retrospective multi-centric radiomic studies like the one described in chapter 8 , since these factors have impact on radiomic features values and thus hamper the interoperability of radiomic models. A generalizable model could potentially be built in case (much) larger datasets are used. Another option to circumvent the variability in settings is to apply postreconstruction feature harmonization to eliminate the batch-effect. Moreover, phantom studies can be used to investigate the impact of these variabilities on feature values.

\section{Phantom studies}

For the sensitivity of radiomic features for acquisition and reconstruction settings, phantom studies play an important role [10]. In the ideal situation, one would have a phantom with texture inserts that, when imaged, have similar characteristics to patient tissue. Moreover, one should keep all settings consistent when only changing one parameter at a time and then test all possible combinations of settings on each CT scanner type. One combination of acquisition settings, reconstruction settings and imaging system should then be set as a reference. All other settings could then be used to determine a correction factor or calibration curve that could be used to correct radiomic features accordingly. This process is an enormous challenge and should also be kept up-to-date to involve new developments. A large phantom study was recently performed by Ger et al. [11], using a round phantom with 6 inserts containing different materials. A total of 100 CT scanners at 35 clinics were included. The variability of 49 radiomic features was investigated for controlled scanning protocols and local scanning protocols. The results show that the variability decreases 
by $50 \%$ when controlled protocols were used. This implies that standardization and consensus, where possible, are of major importance to reduce feature variability.

A phantom study to assess radiomic feature stability was performed in chapter 7 of this thesis, aiming to investigate the influence of CT-scanners, slice thickness, exposure and gray-level discretization on radiomic feature stability, by scanning a phantom with texture inserts on multiple different scanners using different acquisition and reconstruction settings. Feature values were influenced by CT-scanner and slice thickness. Also, the study showed that resampling images into equal voxel sizes improves the stability of radiomic features, which was also shown by Shafiq-ulHassan et al. [12]. Resampling images prior to feature extraction also results in a more meaningful comparison when two datasets with different voxel sizes are involved.

\section{Post-reconstruction harmonization}

Retrospectively collected imaging data will generally be heterogeneous due to varying imaging protocols and different scanner systems. Therefore, a recent study proposed to apply a post-reconstruction harmonization procedure [13], which is an open source batch-effect correction tool called ComBat [14]. This harmonization of radiomic features is intended for multicenter studies that used different reconstruction methods, or had a different scanner manufacturer. In a study in which all parameters are controlled except one, e.g. scanner type, this method could be a potential solution for harmonization. Nevertheless, for studies in which multiple factors differ substantially, this method might not be suitable, as this method is only able to correct for one variable. Moreover, the post-reconstruction harmonization method does not take into account the interpretability of radiomic features. For instance, feature values can become negative, which does not always result in realistic values (e.g. negative tumor volumes). Since complete image acquisition standardization is practically impossible, the proposal would be to develop more sophisticated techniques to perform feature harmonization that can handle more than one variable.

\section{Big(ger) data}

When large amounts of data could be used, variation in data plays a less important role and a generalizable model could be built that captures all heterogeneities present. For example, extracting all available scans from a PACS (Picture Archiving and Communication System) and apply modeling on these data in an automated fashion could be a solution for variations in image- and reconstruction settings and heterogeneity in patient characteristics. This approach would be faster than performing prospective studies that controls all settings and would also be more widely applicable.

\section{Segmentation}

Segmentation of the ROI is prone to intra- and inter-observer variation [15], which could be reduced by protocols or guidelines for target delineations. In 2017, a study investigated the influence of multiple observers on CT-extracted radiomic feature stability for three different tumor sites, including 11 NSCLC patients [16]. The study 
showed varying stabilities per tumor site, but for NSCLC $90 \%$ of 1404 features were considered stable using a threshold of 0.8 for the intra-class correlation coefficient (ICC). A study with larger patient numbers should be performed to more accurately assess the influence of segmentation on radiomic feature stability. Delineations will be more consistent in case of (semi-)automatic segmentation methods (e.g. atlasbased or using deep learning) $[15,17]$. In 2014, a study showed that semi-automatic segmentation improved radiomic feature stability [17]. Fully automated segmentations save time, are user-independent and consistent: they are preferable over manual segmentations for quantitative image analysis purposes as this eliminates concerns about intra- and inter-observer variations. The quality of the automated segmentations should be judged by experts prior to implementation in clinical practice.

The study of Lustberg et al. [18] compared atlas-based, deep learning and manual delineations of OARs and showed that the automatic methods reduce delineation time. Moreover, a recent study from 2018 showed that OAR segmentations in thoracic cancers using an atlas-based approach of 5000 atlases reach a dice similarity coefficient (DSC) of minimal 0.80 for all organs [19]. Automatic tumor delineation methods would be of great value for longitudinal CBCT radiomics, as the cumbersome delineation process currently limits the inclusion of daily CBCT images for pattern evaluations as described in chapter 5 .

\section{Feature extraction}

\section{Radiomics pre-processing}

Prior to feature extraction, several pre-processing steps are involved, including conversion from segmentation (e.g. polygon data in case the segmentation comes from an RTSTRUCT file) to mask, voxel resampling and gray-level discretization. The latter was also investigated in chapter 7. This phantom study showed that radiomic features values were influenced by bin size. The importance of the pre-processing step of image discretization was already shown in a previous study [20]. Nevertheless, this chapter shows that the stability of radiomic features was not influenced much by the bin size.

Voxel size is often inconsistent within datasets, meaning that images often undergo resampling processes prior to radiomic feature extraction to improve the interoperability between datasets and the stability of radiomic features [9]. This resampling into equal voxel sizes, however, means that information is lost when resampling to larger voxel sizes and interpolation of data when resampling to smaller voxel sizes. Also, there is yet no consensus about the voxel size or the resampling method (e.g. nearest neighbor, linear interpolation, cubic convolution). The influence of resampling on the prognostic value of radiomics has not yet been investigated.

\section{Radiomics implementation}

All over the world, radiomics solutions are being developed. This results in different terminology and implementations of mathematical equations, algorithms and preprocessing options. A study in head-and-neck cancer showed that only $12 \%$ of 649 PET-extracted radiomic features were reproducible over two different radiomics 
software packages [21], which was mainly caused by different implementations of filters (e.g. wavelet).

Several initiatives, including the 'Imaging Biomarker Standardization Initiative' (IBSI) have been taken to provide mathematical descriptions of radiomic features, guidelines for pre-processing images, and checklists of reporting settings and parameters $[22,23]$. This initiative also provides a digital phantom for comparing pre-processing implementations $[3,24]$. The distribution of open-source software or commercial solutions that are conform the guidelines in this initiative, would improve the reproducibility of radiomics in general.

\section{Interpretability}

Feature interpretation has always been a challenge for the implementation of radiomics in clinical practice [25]. One could argue that the biological process underlying the feature value might not be relevant as it does not influence the feature's prognostic value, but it definitely would improve better understanding for radiomics in general. A recent study showed correlation between molecular pathways and radiomic imaging features [26] and another study investigated the relationship between genetic changes and radiomic features [27]. Also the correlation between radiomic features and semantic features could help to gain knowledge about the meaning of radiomic features [28]. To further improve the understanding of radiomic features, it is important to continue the work about correlating radiomics to genomics, histological patterns and pathology.

\section{Feature selection}

Up until now, no consensus has been reached upon the procedure of feature selection. The elimination of unstable or redundant features is essential to prevent the inclusion of non-informative features or features that represent noise. Moreover, feature reduction could help to prevent overfitting, as the number of features is generally much larger than the number of features. However, multiple methods are possible for deciding on the features to be removed. The library of radiomic features contains highly correlated features which raises the question on which of the correlated features to remove [28]. Depending on the solution used, one feature or another will be included in a prognostic model having the same accuracy. Furthermore, feature selection often involves test-retest datasets, for instance the publicly available Reference Image Database to Evaluate Response (RIDER) dataset [29] consisting of 32 NSCLC patients who were scanned twice within a 15-minute interval. Chapter 6 compared two test-retest studies which were performed in different disease sites, since it was unknown whether feature stability is study-specific. The chapter shows that test-retest results are not generalizable and points out the importance of including test-retest analyses in study protocols to reduce the number of unstable and unreproducible radiomic features. Furthermore, this chapter emphasizes that it is important to control factors related to scanner, image protocol and reconstruction settings for a reproducible radiomics study. The observation that test-retest studies are not generalizable was also confirmed by Larue et al. [30], who compared two test- 
retest studies in NSCLC and oesophageal cancer and a stability study using 4D imaging.

Besides test-retest, other methods can be used to perform feature reduction, for example a multi-observer delineation study to assess features' sensitivity to segmentation variations [31]. Unfortunately, these stability datasets often comprise only tens of patients, meaning the correlation coefficient between radiomic features can be influenced by a single outlier easily. The range of feature values in the testretest dataset is also of major importance on the value of this relative measure of correlation, which might not be directly translatable to feature robustness. Furthermore, the decision on stable features is often based on an arbitrarily chosen threshold value, whereas a general applicability requires agreement on this stability threshold.

Thus, although these steps are an important step prior to modeling, the general applicability is currently limited. One could propose a list of unstable features for each disease site which should be used by everyone. Nevertheless, this approach would only be feasible if the radiomics implementation is standardized. Moreover, feature stability might be not be generalizable over different scanners or acquisitionand reconstructing settings.

In a longitudinal setting, a different feature selection procedure should be used as described in this thesis, since robust and reproducible features should be selected, but they are not informative for the longitudinal part of the model if they are not changing throughout the entire course of treatment. This was described in chapter 3 of this thesis, where a Bland-Altman approach was used to determine the absolute feature variability over the first two radiotherapy fractions, to be able to define a 'real' change in feature values that is not noise.

\section{Modeling}

\section{Development and validation}

The process of developing and validating a model and reporting the workflow properly has been a point of discussion in recent studies [32]. The 'Transparent reporting of a multivariable prediction model for individual prognosis or diagnosis' (TRIPOD) statement provides guidelines for developing and validation a model and for reporting in the scientific publication [33, 34]. The TRIPOD statement also contains a checklist that can be consulted when developing or validating a prediction model. This helps to improve the reproducibility of (radiomic) models and the possibility of validating a published model, whereas nowadays mostly not enough information is provided to be able to perform the validation or to reproduce the results.

A systematic review from 2015 showed that the majority of published radiomic studies have high risk of type-I errors [35], caused by a lack of (external) validation and statistical multiple testing corrections. Chapter 8 of this thesis showed the relevance of performing an external validation of a developed model to investigate the applicability of a model and to reduce the risk of false positive results. For this study, data was collected in which patients received during-treatment PET/CT scans, to study the prognostic value of differences between radiomic features in order to 
capture an early treatment response. Because these during-treatment PET/CT scans are not performed in clinical practice, the study cohorts were small. Besides that, the data originated from different centers and were collected in an uncontrolled setting, implying that large inter- and intra-dataset heterogeneities were present. Moreover, despite inclusion criteria to select NSCLC patients, there is generally still large variation within and between datasets, since patients have a different gender, age, general health and tumor stage and are thus receiving a different treatment in terms of type of chemotherapy, radiation dose and fractionation schedule. A model could be developed based on the training dataset, but this model has low transferability [36].

\section{Machine learning}

Several studies have investigated the performance of multiple machine learning approaches to prognostic model building [37-40]. A comparison of classifiers was also applied in chapter 8 . The choice of classification method is an important parameter for model performance, as the study of Deist et al. [37] showed that model's discrimination performance can be improved in case one makes an informed decision about the classifier. An earlier study from 2015 showed that the largest variability in predictive model performance in radiomic studies could be explained by the classifier method [39]. In the last chapter of this thesis, chapter 9, we proposed to use the training data to perform a classifier comparison and subsequently use the best performing classifier for further fine-tuning, also using only the training data. To more accurately choose the best classifier, larger amounts of data are needed.

\section{Clinical outcome}

Prediction models are often used to predict an outcome (far) in future, for example overall survival or locoregional recurrence. Second-line or third-line treatments have major impact on the outcome and are not considered in the variables of prediction models that can be acquired prior to or during a treatment. Moreover, the cause of death can be unrelated to the disease, whereas the prognostic factors were intended to predict disease development. Locoregional recurrence is an outcome measure which is also prone to uncertainties, which potentially influenced the results in chapter 4 and 5 . In clinical routine of for example lung cancer, follow-up imaging is performed three months after treatment and then on a yearly basis. Therefore, the exact date of the recurrence is mostly unknown. Furthermore, it can be very challenging to distinguish between a recurrence or inflammation or scar tissue, meaning that a misclassification is not uncommon. Therefore, the prognostic value of a model tested at time of treatment is limited by definition and the maximum achievable prognostic value is currently unknown. Chapter 9 showed that both radiomics and deep learning were not able to achieve an accuracy above around $65 \%$ for the prediction of two-year survival for NSCLC patients.

\section{Future prospects in radiomics}

Radiomics has the potential to serve as an additional information source for the development of prediction models to improve personalized treatment. Nevertheless, 
the extensive list of barriers shows that radiomics has a challenging position in the field of radiotherapy and outcome prediction. The above mentioned challenges should be overcome before it can significantly contribute to current clinical practice. Moreover, it is important to assess whether radiomic features complement wellestablished prognostic factors. It is likely that radiomics will be incorporated in a prognostic model with other information sources, including biological factors, clinical parameters or tumor related factors. A recent study showed that a combination of information sources leads to improved prognostic performance [26].

Good efforts are currently being made to improve the field in terms of standardization, quality and reproducibility. These include amongst others the IBSI and TRIPOD initiatives. Moreover, the recently proposed radiomics quality score (RQS) can serve as workflow guidelines to improve study quality [3]. Furthermore, guidelines for improved quality and standardization for imaging are performed, for instance the EANM guidelines for FDG PET/CT [41]. Also, a recent publication proposed a radiomics workflow including feature selection and machine learning approaches that decreases risk of a false positive finding [42]. These guidelines and initiatives will improve the general applicability of radiomics in the future.

The rest of this chapter describes some future perspectives in the field of radiomics, including longitudinal radiomics and the combination of radiomics and deep learning.

\section{Longitudinal radiomics - current status}

The new development longitudinal or 'delta radiomics' has been investigated in this thesis using CBCT images. CBCT images are available in clinical practice and therefore are an ideal candidate to extract quantitative longitudinal information. Several studies in literature have investigated the change of image features extracted from CBCT for NSCLC, including the density change of the lung $[42,43]$, which was indicated to be related to a patient's susceptibility to radiation. Another study showed that CBCTextracted radiomic feature changes for head-and-neck cancer can predict xerostomia [44]. These studies showed the potential of using CBCT images for during-treatment monitoring and (early) adaptive radiotherapy. Prior to our investigations, the prognostic value of radiomic features extracted from CBCT for prognosis in NSCLC was not yet explored. Therefore, chapter 2-5 investigated the feasibility of longitudinal $\mathrm{CBCT}$ radiomics for prognosis in NSCLC. The principle of longitudinal radiomics was shown schematically in Figure 10.1.

In chapter 2, the feasibility of extracting radiomic features from $C B C T$ images was evaluated by investigating the interchangeability between CT- and CBCTextracted radiomic features. The study showed that a large number of features are interchangeable between $\mathrm{CT}$ and $\mathrm{CBCT}$, including the features of a previously published prognostic radiomic signature [46]. The prognostic performance of this signature was similar in $\mathrm{CBCT}$ and $\mathrm{CT}$ for three independent datasets. This showed the potential of using CBCT to extract radiomic features for prognosis in NSCLC. Therefore, in chapter 3 , a feature selection methodology was described for a longitudinal approach that selects reproducible features that change over the course of treatment. This chapter showed that radiomic features extracted weekly from 


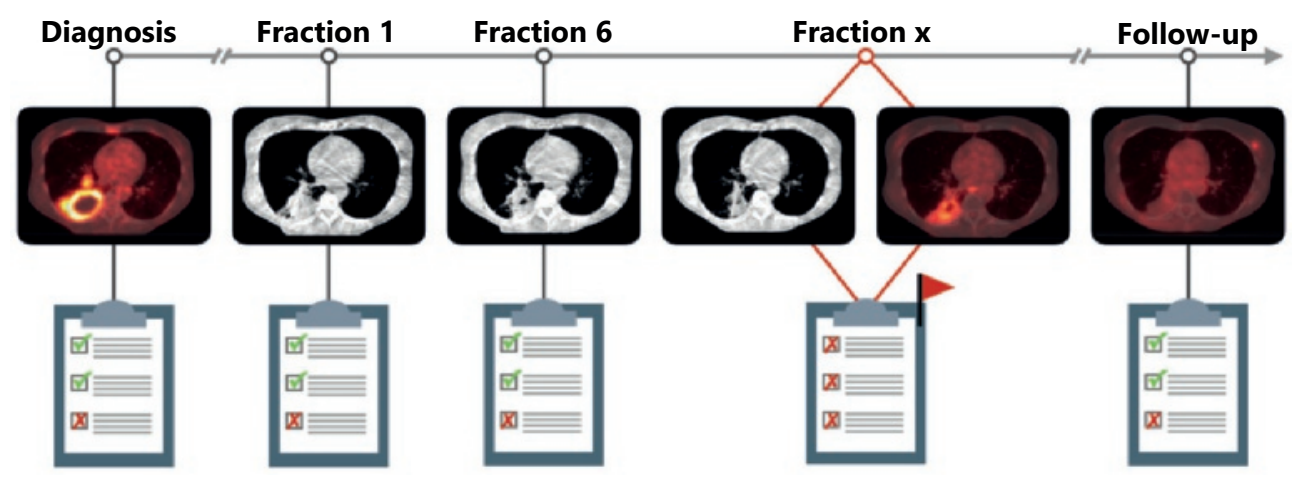

Figure 10.1. Principle of longitudinal radiomics: medical images will be acquired over the course of treatment to monitor tumor changes. Adapted from our review "Radiomics: de toekomst in medische beeldvorming" published in Nederlands Tijdschrift voor Oncologie [45].

$\mathrm{CBCT}$ images during treatment change more than the day-to-day variability. Moreover, the features selected in an early stage during treatment remain important in subsequent weeks, showing the reproducibility of selecting features. In chapter 4, the feature selection method of chapter 3 was applied to larger datasets of four different centers to investigate the prognostic value for overall survival and locoregional recurrence. However, no complementary value of $\mathrm{CBCT}$ to $\mathrm{CT}$ extracted features was observed in this study. On the other hand, also clinical variables and CT extracted features had poor performance in validation datasets for both overall survival and locoregional recurrence. This could probably be caused by limited cohort sizes and inter- and intra-dataset heterogeneities in terms of patient characteristics and acquisition- and reconstruction parameters, as discussed earlier in this chapter. Whereas chapter 4 investigated longitudinal radiomic features, chapter 5 focused on tumor volume changes as the potential prognostic factor of interest for NSCLC patients. Tumor volume prior to treatment is an important prognostic factor, but several studies showed conflicting results about the prognostic value of volume changes during treatment [47-50]. Chapter 5 investigated the relationship between the change of tumor volume during treatment and overall survival and locoregional recurrence. It confirmed the observation of a previous study that NSCLC with large tumor regression show decreased overall survival and locoregional recurrence free survival [51]. Moreover, it showed that the tumor behaves differently for different groups of patients, influenced by initial tumor size, type of chemotherapy and histology. Because of the limited cohort sizes in the study, it was not possible to further investigate the tumor volume changes for specific subgroups, but this could explain the discrepancies between studies in literature. To investigate the tumor volume behavior in each of these subgroups, tumor volume changes should be evaluated in large homogeneous cohorts, e.g. patients that received equal treatments and had similar tumor sizes at the start of treatment, but a different histology classification. 


\section{Longitudinal radiomics - future perspectives}

An improved prognostic performance of longitudinal $\mathrm{CBCT}$ radiomic features extracted from the primary GTV of NSCLC patients compared to baseline (CB)CT features could not be demonstrated. However, all studies in this thesis were affected by limited sample sizes and heterogeneities within and between datasets, which potentially caused poor generalizability and low validation performance. Moreover, the field of longitudinal CBCT radiomics is challenging in many ways. Cone-beam CT images have low image quality, increased noise, lower contrast and they are more susceptible for artifacts (e.g. caused by high density implants) compared to diagnostic CT imaging, all these factors potentially hamper the discovery of a strong prognostic signal. In future, the use of iterative reconstruction methods or artefact reduction techniques could improve image quality [53] and 4D radiomics with motion correction could be helpful for radiomics applied for lung cancer [54]. Therefore, despite the fact that the hypothesis of improved prognostic value for NSCLC patients using longitudinal $C B C T$ radiomics could not be confirmed in this thesis, this does not mean that there is no prognostic information in these images. The potential of CBCT radiomics should be further explored, especially when larger datasets and higher quality images are available.

Longitudinal radiomics has also been investigated for other image modalities and other disease sites. These studies included small cohorts and did not perform external validation. One study investigated delta radiomic features extracted from CT images of 137 NSCLC patients, showing improved performance using CT features extracted at the end of treatment compared to baseline for prediction of locoregional recurrence [55], but not for overall survival. A second study used during-treatment CT scans to extract radiomic features for prediction of overall survival and locoregional tumor control in 78 head-and-neck cancer patients [56]. The results of this study showed that radiomic features extracted at the second week of treatment have improved performance over baseline features. A recent study proposed a new methodology for longitudinal quantitative imaging features to predict 2-year survival [57], using intensity changes at the border of the tumor using both PET and CT images. The study showed promising results for the performance of these longitudinal "pattern" features, but only 30 NSCLC patients were included in this study. Another study used delta radiomic features extracted from nodules images on low-dose CT to predict lung cancer incidence in a lung cancer screening trial, and showed that delta radiomic features improved the incidence prediction [58]. These results should be validated in independent datasets.

The development of MR guided radiotherapy (MRgRT) is interesting for a longitudinal radiomics approach, since these MR images are acquired every fraction and could be a potential candidate to monitor tumor changes over the course of treatment. A recent study by Boldrini et al. [59] investigated the potential prognostic value of delta radiomics using $0.35 \mathrm{~T} \mathrm{MRI} \mathrm{in} \mathrm{an} \mathrm{MRgRT} \mathrm{setting} \mathrm{for} \mathrm{clinical} \mathrm{complete}$ response in rectal cancer. This study showed promising results for radiomic features extracted at the second week of treatment. To conclude, there is still room for improvement in the field of longitudinal radiomics for prognosis. 


\section{Radiomics for diagnosis}

This thesis focusses on the application of radiomics for predicting patient outcome. However, radiomics is also applied to aid in diagnosis. The hypothesis is that radiomics' quantitative features can distinguish a benign and malignant lesion and could therefore contribute to the workflow and help radiologists. A review study from 2017 describes the application of radiomics for lung cancer diagnosis [60]. One of these studies showed that radiomics was able to discriminate between invasive pulmonary adenocarcinomas (IPAs) and preinvasive lesions on CT images with an AUC of 0.98 [61]. Instead of trying to predict an event far in future, radiomics potentially has a more valuable contribution in quantifying the phenotype of a region of interest for cancer diagnosis.

\section{Deep learning}

Deep learning can contribute significantly to the field of radiomics. For instance, automatic segmentations using deep learning approaches could overcome the limitation of this cumbersome process. Especially for longitudinal CBCT radiomics, automatic segmentation methods would improve the applicability. Chapter 9 investigated the complementary value of radiomic and deep learning features to predict two-year survival or histology (adenocarcinoma versus squamous cell carcinoma). The study showed that deep learning and radiomics had similar performance for two-year survival prediction ( 0.60 and 0.62 , respectively), whereas deep learning had improved performance for the classification between adenocarcinoma and squamous cell carcinoma (0.66 compared to 0.55). Data augmentation and normalization are generally applied to prevent over-fitting, as this increases the variabilities in the training dataset that could represent general data heterogeneities. The models developed in this chapter still tend to over-fit on the training data and thus show a large discrepancy between performance in training and validation, but similar performance in validation and test. Larger datasets should be used in the future to develop more generalizable models and to more accurately train the model.

Deep learning for classification has the advantage that the entire image can be used as input in the network and no segmentation is needed. Deep learning has also several disadvantages over radiomics: the process is even more a black box than radiomics. Efforts to visualize the steps in the deep learning network could potentially overcome this. Furthermore, larger datasets are required to achieve a reasonable performance as many more parameters need to be trained in deep learning. A recent study used deep learning algorithms to detect head CT scan abnormalities on more than 20.000 CT scans and achieved AUC values around 0.95 , showing high potential for deep learning in automated diagnosis [62].

Potentially, the combination of deep learning features and radiomic features are complementary, as shown by a few recent studies [63-65]. For example, Lao et al. [65] showed that a model with both deep learning features and radiomic features has improved prognostic value for overall survival in glioblastoma multiforme. 


\section{Conclusion}

This thesis shows that quantitative information derived from medical images acquired prior to treatment describing the phenotype of the tumor, provides prognostic information for non-small cell lung cancer patients. Nevertheless, the complementary value of longitudinal radiomics on currently acquired imaging modalities (mainly CBCT) could not be shown. This indicates that the most advantaged techniques (i.e. radiomics) are not able to extract the prognostic information, or longitudinal CBCT data does not contain prognostic information. Recent literature shows that there is a continuous development of quantitative imaging, including improvements of techniques, standardization and consensus. This indicates a strong belief that techniques such as radiomics have the potential to significantly contribute to the clinical decision making process in the future, which could improve patient outcome since treatment decisions are better informed. But, the puzzle has not yet been solved. 


\section{References}

[1] Hatt M, Tixier F, Pierce L, Kinahan PE, Le Rest CC, Visvikis D. Characterization of PET/CT images using texture analysis: the past, the present... any future? Eur J Nucl Med Mol Imaging. 2017:44:151-65.

[2] Kumar V, Gu Y, Basu S, Berglund A, Eschrich SA, Schabath MB, et al. Radiomics: the process and the challenges. Magn Reson Imaging. 2012;30:1234-48.

[3] Lambin P, Leijenaar RTH, Deist TM, Peerlings J, de Jong EEC, van Timmeren J, et al. Radiomics: the bridge between medical imaging and personalized medicine. Nat Rev Clin Oncol. 2017;14:749-62.

[4] Yip SS, Aerts HJ. Applications and limitations of radiomics. Phys Med Biol. 2016;61:R150-66.

[5] Berenguer R, Pastor-Juan MDR, Canales-Vazquez J, Castro-Garcia M, Villas MV, Mansilla Legorburo F, et al. Radiomics of CT Features May Be Nonreproducible and Redundant: Influence of CT Acquisition Parameters. Radiology. 2018;288:407-15.

[6] Mackin D, Ger R, Dodge C, Fave X, Chi P-C, Zhang L, et al. Effect of tube current on computed tomography radiomic features. Sci Rep. 2018;8:2354.

[7] van Velden FH, Kramer GM, Frings V, Nissen IA, Mulder ER, de Langen AJ, et al. Repeatability of Radiomic Features in Non-Small-Cell Lung Cancer [F]FDG-PET/CT Studies: Impact of Reconstruction and Delineation. Mol Imaging Biol. 2016.

[8] Zhao B, Tan Y, Tsai WY, Qi J, Xie C, Lu L, et al. Reproducibility of radiomics for deciphering tumor phenotype with imaging. Sci Rep. 2016;6:23428.

[9] Mackin D, Fave X, Zhang L, Yang J, Jones AK, Ng CS, et al. Harmonizing the pixel size in retrospective computed tomography radiomics studies. PLoS One. 2017;12:e0178524.

[10] Mackin D, Fave X, Zhang L, Fried D, Yang J, Taylor B, et al. Measuring Computed Tomography Scanner Variability of Radiomics Features. Invest Radiol. 2015;50:757-65.

[11] Ger RB, Zhou S, Chi PM, Lee HJ, Layman RR, Jones AK, et al. Comprehensive Investigation on Controlling for CT Imaging Variabilities in Radiomics Studies. Sci Rep. 2018;8:13047.

[12] Shafiq-Ul-Hassan M, Zhang GG, Latifi K, Ullah G, Hunt DC, Balagurunathan Y, et al. Intrinsic dependencies of CT radiomic features on voxel size and number of gray levels. Med Phys. 2017:44:1050-62.

[13] Orlhac F, Boughdad S, Philippe C, Stalla-Bourdillon H, Nioche C, Champion L, et al. A postreconstruction harmonization method for multicenter radiomic studies in PET. J Nucl Med. 2018.

[14] Johnson WE, Li C, Rabinovic A. Adjusting batch effects in microarray expression data using empirical Bayes methods. Biostatistics. 2007;8:118-27.

[15] van Baardwijk A, Bosmans G, Boersma L, Buijsen J, Wanders S, Hochstenbag M, et al. PETCT-based auto-contouring in non-small-cell lung cancer correlates with pathology and reduces interobserver variability in the delineation of the primary tumor and involved nodal volumes. Int J Radiat Oncol Biol Phys. 2007;68:771-8.

[16] Pavic M, Bogowicz M, Wurms X, Glatz S, Finazzi T, Riesterer O, et al. Influence of interobserver delineation variability on radiomics stability in different tumor sites. Acta Oncol. 2018;57:1070-4.

[17] Parmar C, Rios Velazquez E, Leijenaar R, Jermoumi M, Carvalho S, Mak RH, et al. Robust Radiomics feature quantification using semiautomatic volumetric segmentation. PLoS One. 2014;9:e102107.

[18] Lustberg T, van Soest J, Gooding M, Peressutti D, Aljabar P, van der Stoep J, et al. Clinical evaluation of atlas and deep learning based automatic contouring for lung cancer. Radiother Oncol. 2018;126:312-7.

[19] Schipaanboord B, Boukerroui D, Peressutti D, van Soest J, Lustberg T, Kadir T, et al. Can atlas-based auto-segmentation ever be perfect? Insights from Extreme Value Theory. IEEE Trans Med Imaging. 2018.

[20] Leijenaar RT, Nalbantov G, Carvalho S, van Elmpt WJ, Troost EG, Boellaard R, et al. The effect of SUV discretization in quantitative FDG-PET Radiomics: the need for standardized methodology in tumor texture analysis. Sci Rep. 2015;5:11075.

[21] Bogowicz M, Leijenaar RTH, Tanadini-Lang S, Riesterer O, Pruschy M, Studer G, et al. Post- 
radiochemotherapy PET radiomics in head and neck cancer - The influence of radiomics implementation on the reproducibility of local control tumor models. Radiother Oncol. 2017;125:385-91.

[22] Zwanenburg A, Leger S, Vallieres M, Lock S. Image biomarker standardisation intitiative feature definitions. CoRR. 2016;abs/1612.07003.

[23] Zwanenburg A, Leger S, Vallieres M, Lock S. Image biomarker standardisation initiative. arXiv preprint arXiv:1612.07003.

[24] Lambin P. Radiomics Digital Phantom. CancerData. 2016.

[25] Morin O, Vallieres M, Jochems A, Woodruff HC, Valdes G, Braunstein SE, et al. A Deep Look into the Future of Quantitative Imaging in Oncology: A Statement of Working Principles and Proposal for Change. Int J Radiat Oncol Biol Phys. 2018.

[26] Grossmann P, Stringfield O, El-Hachem N, Bui MM, Rios Velazquez E, Parmar C, et al. Defining the biological basis of radiomic phenotypes in lung cancer. Elife. 2017;6.

[27] Panth KM, Leijenaar RT, Carvalho S, Lieuwes NG, Yaromina A, Dubois L, et al. Is there a causal relationship between genetic changes and radiomics-based image features? An in vivo preclinical experiment with doxycycline inducible GADD34 tumor cells. Radiother Oncol. 2015;116:462-6.

[28] Yip SSF, Liu Y, Parmar C, Li Q, Liu S, Qu F, et al. Associations between radiologist-defined semantic and automatically computed radiomic features in non-small cell lung cancer. Sci Rep. 2017;7:3519.

[29] Armato SG, 3rd, Meyer CR, McNitt-Gray MF, McLennan G, Reeves AP, Croft BY, et al. The Reference Image Database to Evaluate Response to therapy in lung cancer (RIDER) project: a resource for the development of change-analysis software. Clin Pharmacol Ther. 2008;84:44856.

[30] Larue RTHM, Van De Voorde L, van Timmeren JE, Leijenaar RTH, Berbee M, Sosef MN, et al. 4DCT imaging to assess radiomics feature stability: An investigation for thoracic cancers. Radiother Oncol. 2017;125:147-53.

[31] Leijenaar RT, Carvalho S, Velazquez ER, van Elmpt WJ, Parmar C, Hoekstra OS, et al. Stability of FDG-PET Radiomics features: an integrated analysis of test-retest and inter-observer variability. Acta Oncol. 2013;52:1391-7.

[32] Zwanenburg A, Löck S. Why validation of prognostic models matters? Radiother Oncol.

[33] Moons KG, Altman DG, Reitsma JB, loannidis JP, Macaskill P, Steyerberg EW, et al. Transparent Reporting of a multivariable prediction model for Individual Prognosis or Diagnosis (TRIPOD): explanation and elaboration. Ann Intern Med. 2015;162:W1-73.

[34] Collins GS, Reitsma JB, Altman DG, Moons KG. Transparent Reporting of a multivariable prediction model for Individual Prognosis or Diagnosis (TRIPOD): the TRIPOD statement. Ann Intern Med. 2015;162:55-63.

[35] Chalkidou A, O'Doherty MJ, Marsden PK. False Discovery Rates in PET and CT Studies with Texture Features: A Systematic Review. PLoS One. 2015;10:e0124165.

[36] van Soest J, Meldolesi E, van Stiphout R, Gatta R, Damiani A, Valentini V, et al. Prospective validation of pathologic complete response models in rectal cancer: Transferability and reproducibility. Med Phys. 2017;44:4961-7.

[37] Deist TM, Dankers F, Valdes G, Wijsman R, Hsu IC, Oberije C, et al. Machine learning algorithms for outcome prediction in (chemo)radiotherapy: An empirical comparison of classifiers. Med Phys. 2018.

[38] Sun W, Jiang M, Dang J, Chang P, Yin F-F. Effect of machine learning methods on predicting NSCLC overall survival time based on Radiomics analysis. Radiat Oncol. 2018;13:197.

[39] Parmar C, Grossmann P, Bussink J, Lambin P, Aerts HJ. Machine Learning methods for Quantitative Radiomic Biomarkers. Sci Rep. 2015;5:13087.

[40] Leger S, Zwanenburg A, Pilz K, Lohaus F, Linge A, Zophel K, et al. A comparative study of machine learning methods for time-to-event survival data for radiomics risk modelling. Sci Rep. 2017;7:13206.

[41] Boellaard R, Delgado-Bolton R, Oyen WJ, Giammarile F, Tatsch K, Eschner W, et al. FDG PET/CT: EANM procedure guidelines for tumour imaging: version 2.0. Eur J Nucl Med Mol 
Imaging. 2015;42:328-54.

[42] Chatterjee A, Vallières $M$, Dohan A, Levesque IR, Ueno Y, Bist V, et al. An empirical approach for avoiding false discoveries when applying high-dimensional radiomics to small datasets. IEEE Plasma Sci. 2018:1-.

[43] Bertelsen A, Schytte T, Bentzen SM, Hansen O, Nielsen M, Brink C. Radiation dose response of normal lung assessed by Cone Beam CT - a potential tool for biologically adaptive radiation therapy. Radiother Oncol. 2011;100:351-5.

[44] Bernchou U, Hansen O, Schytte T, Bertelsen A, Hope A, Moseley D, et al. Prediction of lung density changes after radiotherapy by cone beam computed tomography response markers and pre-treatment factors for non-small cell lung cancer patients. Radiother Oncol. 2015;117:17-22.

[45] Rosen BS, Hawkins PG, Polan DF, Balter JM, Brock KK, Kamp JD, et al. Early changes in serial CBCT-measured parotid gland biomarkers predict chronic xerostomia after head and neck radiotherapy. Int J Radiat Oncol Biol Phys. 2018.

[46] Leijenaar RTH, De Jong EEC, Larue RT, Van Timmeren JE, Lambin P. Radiomics: de toekomst in medische beeldvorming. Nederlands Tijdschrift voor Oncologie. 2017;14:82-9.

[47] Aerts HJ, Velazquez ER, Leijenaar RT, Parmar C, Grossmann P, Carvalho S, et al. Decoding tumour phenotype by noninvasive imaging using a quantitative radiomics approach. Nat Commun. 2014;5:4006.

[48] Jabbour SK, Kim S, Haider SA, Xu X, Wu A, Surakanti S, et al. Reduction in Tumor Volume by Cone Beam Computed Tomography Predicts Overall Survival in Non-Small Cell Lung Cancer Treated With Chemoradiation Therapy. Int J Radiat Oncol Biol Phys. 2015;92:627-33.

[49] Elsayad K, Samhouri L, Scobioala S, Haverkamp U, Eich HT. Is tumor volume reduction during radiotherapy prognostic relevant in patients with stage III non-small cell lung cancer? J Cancer Res Clin Oncol. 2018.

[50] Kasmann L, Niyazi M, Blanck O, Baues C, Baumann R, Dobiasch S, et al. Predictive and prognostic value of tumor volume and its changes during radical radiotherapy of stage III nonsmall cell lung cancer : A systematic review. Strahlenther Onkol. 2018;194:79-90.

[51] Kanzaki H, Kataoka M, Nishikawa A, Uwatsu K, Nagasaki K, Nishijima N, et al. Impact of early tumor reduction on outcome differs by histological subtype in stage III non-small-cell lung cancer treated with definitive radiotherapy. Int J Clin Oncol. 2016;21:853-61.

[52] Brink C, Bernchou U, Bertelsen A, Hansen O, Schytte T, Bentzen SM. Locoregional control of non-small cell lung cancer in relation to automated early assessment of tumor regression on cone beam computed tomography. Int J Radiat Oncol Biol Phys. 2014;89:916-23.

[53] Zhu L, Xie Y, Wang J, Xing L. Scatter correction for cone-beam CT in radiation therapy. Med Phys. 2009;36:2258-68.

[54] Star-Lack J, Sun $M$, Oelhafen $M$, Berkus T, Pavkovich J, Brehm $M$, et al. A modified McKinnon-Bates (MKB) algorithm for improved 4D cone-beam computed tomography (CBCT) of the lung. Med Phys. 2018.

[55] Fave X, Zhang L, Yang J, Mackin D, Balter P, Gomez D, et al. Delta-radiomics features for the prediction of patient outcomes in non-small cell lung cancer. Sci Rep. 2017;7:588.

[56] Leger S, Zwanenburg A, Pilz K, Zschaeck S, Zophel K, Kotzerke J, et al. CT imaging during treatment improves radiomic models for patients with locally advanced head and neck cancer. Radiother Oncol. 2018.

[57] Buizza G, Toma-Dasu I, Lazzeroni M, Paganelli C, Riboldi M, Chang Y, et al. Early tumor response prediction for lung cancer patients using novel longitudinal pattern features from sequential PET/CT image scans. Phys Med. 2018;54:21-9.

[58] Cherezov D, Hawkins SH, Goldgof DB, Hall LO, Liu Y, Li Q, et al. Delta radiomic features improve prediction for lung cancer incidence: A nested case-control analysis of the National Lung Screening Trial. Cancer Med. 2018.

[59] Boldrini L, Cusumano D, Chiloiro G, Casa C, Masciocchi C, Lenkowicz J, et al. Delta radiomics for rectal cancer response prediction with hybrid $0.35 \mathrm{~T}$ magnetic resonance-guided radiotherapy (MRgRT): a hypothesis-generating study for an innovative personalized medicine approach. Radiol Med. 2018. 
[60] Thawani R, McLane M, Beig N, Ghose S, Prasanna P, Velcheti V, et al. Radiomics and radiogenomics in lung cancer: A review for the clinician. Lung Cancer. 2018;115:34-41.

[61] Chae HD, Park CM, Park SJ, Lee SM, Kim KG, Goo JM. Computerized texture analysis of persistent part-solid ground-glass nodules: differentiation of preinvasive lesions from invasive pulmonary adenocarcinomas. Radiology. 2014;273:285-93.

[62] Chilamkurthy S, Ghosh R, Tanamala S, Biviji M, Campeau NG, Venugopal VK, et al. Deep learning algorithms for detection of critical findings in head CT scans: a retrospective study. Lancet. 2018;392:2388-96.

[63] Paul R, Hawkins SH, Balagurunathan Y, Schabath MB, Gillies RJ, Hall LO, et al. Deep Feature Transfer Learning in Combination with Traditional Features Predicts Survival Among Patients with Lung Adenocarcinoma. Tomography. 2016;2:388-95.

[64] Kooi T, Litjens G, van Ginneken B, Gubern-Merida A, Sanchez Cl, Mann R, et al. Large scale deep learning for computer aided detection of mammographic lesions. Med Image Anal. 2017;35:303-12.

[65] Lao J, Chen Y, Li ZC, Li Q, Zhang J, Liu J, et al. A Deep Learning-Based Radiomics Model for Prediction of Survival in Glioblastoma Multiforme. Sci Rep. 2017;7:10353. 



$$
\text { Appendices }
$$





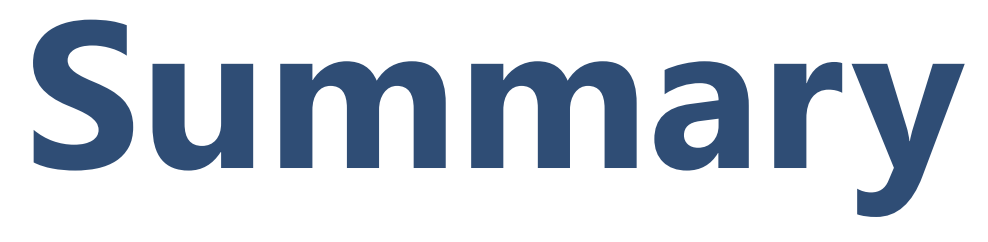

Summary \& Samenvatting 



\section{Summary}

The 5-year survival rate of lung cancer patients is about $20 \%$ and the puzzle of curing this disease is still unsolved. It is known that several individual patient characteristics influence disease development, but fitting all pieces together is challenging. In case a treatment is specifically tailored to an individual based on a combination of multiple information sources like demographics, blood biomarkers, genes and medical images, optimal disease outcome could be achieved since treatment decisions are better informed. This concept is known as "personalized treatment". To this extent, it is essential to select parameters that are able to predict or monitor the development of a disease. This thesis focused on image derived parameters for prognosis (e.g. overall survival and locoregional recurrence) in non-small cell lung cancer (NSCLC) patients, the largest subgroup of all lung cancer patients, treated with radiotherapy. Besides regular image modalities like computed tomography (CT) and positron emission tomography (PET), the potential of monitoring disease development during radiation treatment was evaluated using cone-beam $C T$ images $(C B C T)$. The latter are generally acquired in clinical practice for patients receiving radiotherapy in order to achieve correct patient positioning, thus lots of images are available per patient and at multiple time points during treatment. Quantitative image features, defined by mathematical algorithms, were derived from these medical images in order to unravel the prognostic potential of this particular information source; this principle is called radiomics. The longitudinal radiomics approach was studied in the first part of the thesis using CBCT images, whereas the second part focused on (technical) challenges in the radiomics field using either $C T$ or PET imaging, and the last part investigated a deep learning approach. The potential of longitudinal CBCT radiomics was confirmed in this thesis by showing that a subset of features is interchangeable between CT and $C B C T$, and showing that the radiomic features do change during the course of treatment. Nevertheless, the prognostic value of longitudinal CBCT radiomics did not outperform that of $\mathrm{CT}$ images acquired prior to radiotherapy. This, however, does not mean that there is no prognostic value, but current study designs were not able to show this. The difficulties in detecting true prognostic indicators are potentially caused by the heterogeneous character of the datasets and/or the inferior image quality of $\mathrm{CBCT}$ images. We explored in this thesis possible explanations and future directions to work towards the goal of achieving better prognosis models. The heterogeneity within and between datasets, as well as cohort size were shown to hamper to development of prognostic models in the second part of the thesis. Moreover, several other factors influence (the robustness of) radiomic feature values, including image acquisition- and reconstruction parameters. Improvements of imaging techniques, and standardization and consensus in settings, radiomics methodology and statistical approaches are of major importance for the success factor of radiomics. The development of deep learning might raise the potential of the medical image information source, as shown in the last part of this thesis. There is still potential for image features derived from medical images to contribute to personalized treatment, although there is still lot to be done and improvements to be made. But, every piece of the puzzle that falls into place is a step towards improved lung cancer management. 



\section{Samenvatting}

De 5-jaarsoverleving van longkankerpatiënten is ongeveer $20 \%$, en de puzzel van de genezing van deze ziekte is nog niet opgelost. Het is alom bekend dat individuele patiënt karakteristieken het ziekteverloop beïnvloeden, maar het is een uitdaging om deze puzzelstukjes in elkaar te passen. Wanneer een behandeling volledig wordt geïndividualiseerd met behulp van informatiebronnen, zoals demografie, bloed biomarkers, genen en medische beelden, kan optimale ziekte-uitkomst bereikt worden, doordat beslissingen tijdens de behandeling op een volledigere informatievoorziening gebaseerd zijn: gepersonaliseerde geneeskunde. Het is daarom essentieel om factoren te identificeren die de ontwikkeling van een ziekte kunnen voorspellen of volgen. Dit proefschrift focust op eigenschappen afgeleid uit medische beelden voor de prognose (overleving en lokaal regionale controle) in patiënten met niet-kleincellig longcarcinoom, de grootste subgroep van alle longkankerpatiënten, die behandeld zijn met radiotherapie. Naast de reguliere beelden, zoals computertomografie (CT) en positron emissie tomografie (PET), werd de potentie van het monitoren van ziekteverloop onderzocht met behulp van conebeam CT (CBCT) beelden. Deze CBCT beelden worden in de klinische praktijk gemaakt tijdens een radiotherapie behandeling om de positie van de patiënt te verifiëren, waardoor er voor elke patiënt een groot aantal beelden beschikbaar zijn voor analyse. Kwantitatieve beeldeigenschappen, gedefinieerd door wiskundige formules, werden berekend uit deze medische beelden om de prognostische waarde van deze informatiebron te onderzoeken: een principe genaamd radiomics. Het principe van longitudinale radiomics werd onderzocht in het eerste deel van dit proefschrift, terwijl het tweede deel focust op (technische) radiomics uitdagingen en het laatste deel de toegevoegde waarde van deep learning onderzocht. De potentie van longitudinale $C B C T$ radiomics werd bevestigd, doordat een deel van de radiomics beeldeigenschappen uitwisselbaar bleek te zijn tussen CT en CBCT, en door te laten zien dat deze beeldeigenschappen veranderen tijdens de behandeling. Desalniettemin was de prognostische waarde van longitudinale $C B C T$ radiomics niet hoger dan die van CT-beelden die voor de behandeling werden gemaakt. Dit betekent echter niet dat longitudinale $C B C T$ radiomics ook daadwerkelijk geen prognostische waarde heeft. In dit proefschrift hebben we gekeken naar mogelijke verklaringen en toekomstige onderzoeksrichtingen om betere prognostische modellen te ontwikkelen. Mogelijk werden de resultaten beïnvloed door de lage beeldkwaliteit van CBCT's, of het feit dat er gebruik werd gemaakt van kleine, heterogene datasets. Het tweede deel van dit proefschrift laat zien dat de heterogeniteit binnen en tussen datasets, en de grootte van de datasets, de ontwikkeling van prognostische modellen hinderen. Bovendien zijn er verschillende factoren die de (robuustheid van) radiomics beeldeigenschappen beïnvloeden, zoals de instellingen bij het verkrijgen en reconstrueren van de medische beelden. Verbetering in beeldvormingstechnieken, net als standaardisatie en consensus in instellingen, radiomics methodologie en statistische technieken, zijn van groot belang voor de succesfactor van radiomics. Zoals het laatste deel van de thesis laat zien, kan ook de ontwikkeling van deep learning bijdragen aan de potentie van medische beelden als prognostische informatiebron. Kortom, er is nog steeds veel potentie voor medische beeldeigenschappen om bij te dragen aan gepersonaliseerde geneeskunde, maar is er nog een lange weg te gaan. Doch elk puzzelstukje dat op z'n plek valt, zal bijdragen aan verbeterde overlevingskansen voor longkankerpatiënten. 

Valorization
addendum 



\section{Personalized medicine}

Cancer is estimated to cause 9.6 million deaths worldwide in 2018. This number is increasing yearly, caused by a growing population and aging. Cancers accounting for a large proportion of cancer deaths include breast cancer, colorectal cancer, stomach and liver cancer, but of all cancer types, lung cancer causes most cancer deaths worldwide. Lung patients currently have a poor 5 -year survival rate of about $20 \%$. A more effective use of healthcare data could help to choose the optimal treatment for each patient: personalized treatment, replacing the one-size-fits-all principle.

In today's society, more and more data are collected, saved and analyzed. These large amounts of data (i.e. 'big data') are no longer restricted to large companies. The trend of big data is also apparent in healthcare. For each individual patient, information such as demographics, medical history, medical images and laboratory results are collected and saved in Electronic Health Records (EHR). These data can be mined to find trends on population basis, which could help to improve general disease management. Moreover, disease prevention is also facilitated by big data in healthcare. Data in the EHRs, as well as patient preferences, help to guide individual treatment decisions using so-called clinical decision support systems (CDSS). To this extend, prediction models are being built in order to translate different sources of information to a predictive value (i.e. effectiveness of one treatment compared to another) or prognostic value (i.e. treatment outcome irrespective of the type of treatment). The hypothesis is that prediction models are able to improve the survival chances of patients because of better informed decisions about the treatment initialization or adaptations, which could also reduce the risk of toxicities. This also implies that the collection of data in healthcare is able to reduce healthcare costs by disease detection, prevention and more effective treatments.

\section{Prognostic value}

Suitable data for prediction models include patient related factors, information about treatment, stage of the disease and imaging data. This thesis focused on prognosis for non-small cell lung cancer (NSCLC), the major subtype of lung cancer. Several prognostic factors have already been identified for NSCLC patients, including TNMstage, histological subtype (e.g. adenocarcinoma, squamous cell carcinoma) and mutation status. Besides that, parameters derived from medical images (e.g. radiomics) are being investigated for their relationship with disease development and patient outcome, which was the main topic of this thesis. In case of a positive correlation, these image-derived features could be incorporated in a CDSS to improve patient outcome by enhanced personalized medicine. Whereas the prognostic value of radiomics was described for lung cancer in this thesis, the methods, approaches and conclusions are easily transferable to other disease sites as well, e.g. head-andneck cancer, esophageal or rectal cancer. Moreover, the patients that were evaluated in this thesis have all received radiation treatment, but the methodologies could also be applied for patients receiving chemotherapy or surgery. So, although the research question of this thesis was relatively specific, the principle of acquiring quantitative features from medical images acquired in clinical practice could contribute to the general management of cancer, as this principle is applicable to many disease sites 
and treatment choices.

\section{Data sharing}

The distribution of prognostic models over the scientific community is important to further develop and validate them, to be able to proceed to a clinical implementation. One of initiatives to distribute prediction models is the website www.predictcancer.org, which is a database of prognostic and predictive models for several disease sites and the corresponding publication. The prognostic model that was validated in chapter 2 of this thesis was also published on this website. There exist also a mobile application of predictcancer, which is intended to serve as a decision support aid. The entire community can publish their models on this website, which contributes to the general use and evaluation of prognostic and predictive models. Currently, the website contains 25 cancer prediction models for a total of seven different cancers, including four for lung cancer. A platform that collects validated prediction models facilitates the integration of a prediction model in commercially available CDSSs, for instance implemented in mobile apps.

Besides sharing prognostic models, publicly available data will help the development and validation of these models. The data of chapter 2 was made publicly available on www.cancerdata.org (http://dx.doi.org/10.17195/ candat.2017.02.1), which is an initiative of Maastro Clinic to share datasets, protocols and guidelines. Furthermore, The Cancer Imaging Archive (TCIA) on www. cancerimagingarchive.net collects medical images and corresponding patient information. This allows for finding external validation datasets more easily, but also to find suitable datasets for test-retest purposes, like the RIDER dataset which was used in chapter 6 and 9 . Also one of the datasets that was used for model development in chapter 9 was acquired from an open-source publication.

\section{Radiomics}

The software that was used in this thesis is a research version of a commercially available software RadiomiX, which is distributed by the company Oncoradiomics (www.oncoradiomics.com). Besides the feature extraction procedure, it is also possible in this software to extract a radiomic signature or apply a certain machine learning approach in one-go. Another software that is used worldwide is PyRadiomics, which is an open source radiomics software developed in Python. Distribution of radiomics software, that are in agreement with standardization guidelines, is essential to spread the worldwide applicability of prognostic models that are being developed that contain radiomic features, as well as the development and validation of new prognostic models. The community is able to contribute to these open source implementations, which allows for knowledge exchange. Companies will benefit from this knowledge in the development of their commercial solutions available for use in clinical practice.

Information about radiomics is also published on www.radiomics.world. This website also contains the radiomics quality score (RQS), which was developed by our group in 2018 and is intended to improve the quality of future radiomic studies in terms of methodology, external validation and protocol descriptions. In chapter 4 of 
this thesis, the RQS was used to improve the quality of the study. The website also provides the links to the digital phantom, which is intended to compare different radiomics implementations and to harmonize them. Standardization initiatives and guidelines for publications are essential to ensure robustness and generalizability of radiomics studies. This type of research will also improve the quality of commercial products, as manufacturers aim to develop state-of-the-arts solutions that satisfy existing quality standards.

\section{Innovation}

The main innovation of this thesis is the use of cone-beam CT images for radiomics and the corresponding longitudinal approach. These studies will help to guide future research for further development of this methodology, which could be of interesting for integration of tumor monitoring during treatment using conventional linacs (e.g. Varian, Elekta). The feature selection methodology described in chapter 3 of this thesis is relevant for other longitudinal studies, not necessarily including CBCT images. A potential future application includes radiomics analyses of longitudinal MRI images acquired from novel MR-linac systems (e.g. Elekta Unity, MRIdian linac, ViewRay), which could help future patients by allowing for early response assessment.

The last part of the thesis and specifically chapter 9, describes the potential of the recent development of deep learning for prognosis in NSCLC. Since the successes of Google contests using deep learning networks for image classification, for instance the ImageNet challenge, there is renewed interest for deep learning in medical image segmentation, analysis and classification. The application of deep learning for segmentation is beneficial for many fields: not only for radiomics which required a defined region of interest, but also for radiotherapy planning, including definition of the target and surrounding organs at risk, which could be integrated in existing treatment planning software (e.g. Eclipse, Raystation). Although the automatic segmentations might still require verification by a radiation oncologist, the use of deep learning would greatly reduce the amount of time required for treatment planning, which is beneficial for both the medical specialists and the patients awaiting treatment. The use of deep learning for classification includes differentiation between benign and malignant lesions, which reduces the amount of unnecessary biopsies. Moreover, as shown in chapter 9, it helps to improve predictions, potentially in combination with radiomics. The potential of deep learning in medicine is huge and it is to be expected that it will play a major role in modern healthcare. 



\section{Curriculum vitae}

Curriculum vitae $\&$ list of publications 

Janna Elizabeth (Janita) van Timmeren was born on the 11th of November 1992 in Groningen, the Netherlands. After finishing secondary education at the Zernike College in Haren in 2010, she studied Medical Natural Sciences at the VU University in Amsterdam. She gained interest in the field of medical physics, which increased during her minor in Medical Imaging. During her bachelor's studies, she performed an internship at the Radiotherapy department of VU Medical Center about electron film dosimetry and she completed the honours program. After

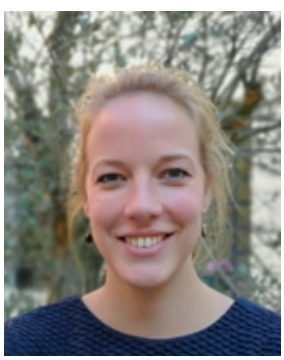
obtaining her bachelor's degree cum laude in 2013, she enrolled in the M.Sc. program of Medical Natural Sciences - Medical Physics. During her master, she performed an internship of 5 months at the department of Radiotherapy of National University Hospital Galway in Ireland, which focused on IMRT treatment plan verification using Octavius 4D dosimetry. She performed the major internship at the department of Nuclear Medicine and Radiology of VU Medical Center in Amsterdam, which focused on the derivation of ejection fraction from ${ }^{15} \mathrm{O}$ water-gated PET images. After her graduation cum laude, she started on the PhD project at Maastricht University in September 2015 to investigate the prognostic value of radiomic features derived from cone-beam CT (CBCT) images for non-small cell lung cancer. During her PhD, a GROW travel grant allowed her to visit the research group of Prof. Dr. Carsten Brink at the Odense University Hospital in Denmark for a period of 3.5 months to collaborate on the CBCT radiomics project.

\section{Grants}

- NRS Young Investigator Travel Grant, ESTRO 37 Congress, Barcelona, Spain

- GROW Travel Grant, Visiting scientist at the Radiotherapy Department of Odense University Hospital, Odense, Denmark

- ARTFORCE-ESTRO Grant, Research Masterclass for Beginners in Research (ESTRO course), 2017, Florence, Italy

- BiGART2017 Travel Grant, Biology-Guided Adaptive Radiotherapy, 2017, Aarhus, Denmark

- NRS Young Investigator Travel Grant, Annual Meeting of the American Society for Radiation Oncology, 2016, Boston, US

- ARTFORCE-ESTRO Grant, Quantitative Methods in Radiation Oncology (ESTRO course), 2015, Brussels, Belgium 



\section{Scientific publications}

- van Timmeren JE, van Elmpt W, Leijenaar RTH, Reymen B, Monshouwer R, Bussink J, Paelinck L, Bogaert E, De Wagter C, Elhaseen E, Lievens Y, Hansen O, Brink C, Lambin P. Longitudinal radiomics of cone-beam CT images for nonsmall cell lung cancer patients: Evaluation of the added prognostic value for overall survival and locoregional recurrence. Radiother Oncol. 2019 Jul; 136:7885. doi:10.1016/j.radonc.2019.03.032

- Walsh $\mathrm{S}$, de Jong EEC, van Timmeren JE, Ibrahim A, Compter I, Peerlings J, Sanduleanu S, Rafaee T, Keek S, Larue RTHM, van Wijk Y, Even AJG, Jochems A, Barakat MS, Leijenaar RTH, Lambin P. Decision Support Systems in Oncology. JCO Clinical Cancer Informatics. 2019 Feb; (3):1-9. doi:10.1200/CCI.18.00001

- van Timmeren JE, van Elmpt W, de Ruysscher D, Reymen R, Hansen O, Brink C. Tumor regression during radiotherapy for non-small cell lung cancer patients using cone-beam computed tomography images. 2018 Dec; Submitted

- *van Timmeren JE, *Carvalho S, Leijenaar RTH, Troots EGC, van Elmpt W, de Ruysscher D, Muratet JP, Denis F, Schimek-Jasch T, Nestle U, Jochems A, Woodruff $\mathrm{HC}$, Oberije C, Lambin P. Challenges and caveats of a multi-center retrospective radiomics study: an example of early treatment response assessment for NSCLC patients using FDG-PET/CT radiomics. 2018 Jun; Submitted

- Sanduleanu S, Woodruff HC, de Jong EEC, van Timmeren JE, Jochems A, Dubois L, Lambin P. Tracking tumor biology with radiomics: A systematic review utilizing a radiomics quality score. Radiother Oncol. 2018 Jun; 127[3]:349-360. doi:10.1016/j.radonc.2018.03.033

- Carvalho S, Leijenaar RTH, Troots EGC, van Timmeren JE, Oberije C, van Elmpt W, de Geus-Oei LF, Bussink J, Lambin P. 18-F-fluorodeoxyglucose positronemission tomography (FDG-PET)-Radiomics of metastatic lymph nodes and primary tumor in non-small cell lung cancer (NSCLC) - A prospective externally validated study. PloS One. 2018 Mar; 13[3]:e0192859. doi:10.1371/journal. pone.0192859

- Lambin P, Leijenaar RTH, Deist TM, Peerlings J, de Jong EEC, van Timmeren J, Sanduleanu S, Larue RTHM, Even AJG, Jochems A, van Wijk Y, Woodruff $H$, van Soest J, Lustberg T, Roelofs E, van Elmpt W, Dekker A, Mottaghy FM, Wildberger JE, Walsh S. Radiomics: the bridge between medical imaging and personalized medicine. Nat Rev Clin Oncol. 2017 Dec; 14[12]:749:762. doi: 10.1038/ nrclinonc. 2017

\section{*equal contribution}


- *Larue RTHM, *van Timmeren JE, *de Jong EEC, Feliciani G, Leijenaar RTH, Schreurs WMJ, Sosef MN, Raat FHMJ, van der Zande FHR, Das M, van Elmpt W, Lambin P. Influence of gray level discretization on radiomic feature stability for different CT scanners, tube currents and slice thicknesses: a comprehensive phantom study. Acta Oncol. 2017 Nov; 56[11]:1544-1553. doi: 10.1080/0284186X.2017.1351624

- van Timmeren JE, Leijenaar RTH, van Elmpt W, Reymen B, Lambin P. Feature selection methodology for longitudinal cone-beam CT radiomics. Acta Oncol. 2017 Nov; 56[11]:1537-1543. doi: 10.1080/0284186X.2017.1350285

- Larue RTHM, van de Voorde L, van Timmeren JE, Leijenaar RTH, Berbée M, Sosef MN, Schreurs WMJ, van Elmpt W, Lambin P. 4DCT imaging to assess radiomics feature stability: An investigation for thoracic cancers. Radiother Oncol. 2017 Oct; 125[1]:147-153. doi: 10.1016/j.radonc.2017.07.023

- van Timmeren JE, Leijenaar RTH, van Elmpt W, Reymen B, Oberije C, Monshouwer R, Bussink J, Brink C, Hansen O, Lambin P. Survival prediction of non-small cell lung cancer patients using radiomics analyses of cone-beam CT images. Radiother Oncol. 2017 Jun; 123[3]:363-369. doi: 10.1016/j.radonc.2017.04.016

- Lambin P, Zindler J, Vanneste BG, De Voorde LV, Eekers D, Compter I, Panth HM, Peerlings J, Larue RT, Deist TM, Jochems A, Lustberg T, van Soest J, de Jong EE, Even AJ, Reymen B, Rekers N, van Gisbergen M, Roelofs E, Carvalho S, Leijenaar $R T$, Zegers CM, Jacobs $M$, van Timmeren J, Brouwers $P$, Lal JA, Dubois $L$, Yaromina A, Van Limbergen EJ, Berbee $M$, van Elmpt W, Oberije C, Ramaekers B, Dekker A, Boersma LJ, Hoebers F, Smits KM, Berlanga AJ, Walsh S. Decision support systems for personalized and participative radiation oncology. Adv Drug Deliv Rev. 2017 Jan; 109:131-153. doi: 10.1016/j.addr.2016.01.006

- Leijenaar RTH, de Jong EEC, Larue RTHM, van Timmeren JE, Lambin P. Radiomics: de toekomst in medische beeldvorming. Nederlands Tijdschrift voor Oncologie. 2017 May; 14[3]: 82-89.

- van Timmeren JE, Leijenaar RTH, van Elmpt W, Wang J, Zhang Z, Dekker A, Lambin P. Test-Retest Data for Radiomics Feature Stability Analysis: Generalizable or Study-Specific? Tomography. 2016 Dec; 2[4]:361-365. doi: 10.18383/j. tom.2016.00208 
- *Driessen RS, *van Timmeren JE, Stuijfzand WJ, Rijnierse MT, Danad I, Raijmakers PG, Beek AM, van Rossum AC, Nijveldt R, Lammertsma AA, Harms HJ, Huisman MC, Knaapen P. Measurement of LV Volumes and Function Using Oxygen-15 Water-Gated PET and Comparison With CMR Imaging. JACC Cardiovasc Imaging. 2016 Dec; 9[12]:1472:1474. doi: 10.1016/j.jcmg.2016.01.014

- *Scrivener M, *de Jong EEC, *van Timmeren JE, Pieters T, Ghaye B, Geets X. Radiomics applied to lung cancer: a review. Translational Cancer Research. 2016;5(4):398-409. doi: 10.21037/tcr.2016.06.18

\section{Presentations}

- van Timmeren JE, Lambin P. Radiomics: transforming standard imaging into mineable data related to biology. Refresher course. Presented at European Congress of Medical Physics, Copenhagen, Denmark, August 2018

- van Timmeren JE, Leijenaar RTH, van Elmpt W, Lambin P. Prognostic value of longitudinal $C B C T$ radiomics for non-small cell lung cancer patients: potential for adaptive radiotherapy. Presented at the 15th Acta Oncologica conference (BiGART), Aarhus, Denmark, June 2017

- van Timmeren JE, Leijenaar RTH, van Elmpt W, Lambin P. Interchangeability of a radiomic signature between conventional and weekly cone-beam $\mathrm{CT}$ allowing response prediction in non-small cell lung cancer. Presented at the ASTRO annual meeting, Boston, USA, September 2016

- van Timmeren JE, Leijenaar RTH, van Elmpt W, Lambin P. Validation of a prognostic radiomic signature on $\mathrm{kV}$ cone-beam $\mathrm{CT}$ images in non-small cell lung cancer patients. Presented at the 18th International Conference on the use of Computers in Radiation Therapy, London, UK, June 2016

- van Timmeren JE, Leijenaar RTH, van Elmpt W, Lambin P. Can we replace high quality simulation $\mathrm{CT}$ by simple $\mathrm{kV}$ cone-beam $\mathrm{CT}$ to extract an externally validated radiomic signature? Presented at International Conference on Translational Research in Radio-Oncology, Geneva, Switzerland, February 2016

*equal contribution 

Dankwoord 

"Bijna af, alleen het dankwoord nog ... ". Niet het minst onbelangrijke hoofdstuk van het proefschrift en zeker niet het minst gelezen. Terwijl ik dit aan het schrijven ben, realiseer ik me hoe ongelofelijk veel ik heb meegemaakt tijdens mijn promotietraject. Ik heb veel mensen leren kennen, vrienden voor het leven gemaakt en binnen en buiten Europa op reis geweest: congressen in Géneve, Londen, Kopenhagen, Aarhus, Boston, Barcelona en Milaan, cursussen in Brussel, Florence, Lyon, Le Bono, Utrecht en Maastricht en niet te vergeten 3 maanden promotiesamenwerking in Odense. Al met al heb ik veel ervaring opgedaan de afgelopen jaren en daar kijk ik met heel veel tevredenheid op terug! Ik ben veel mensen dank verschuldigd die hebben bijdragen aan dit proefschrift, mijn ontwikkeling of de nodige ontspanning naast werk. Ik zal beginnen bij het begin, de wortels van dit proefschrift en dus het allerbelangrijkst voor het tot stand komen van dit proefschrift: mijn promotieteam. Allereerst mijn promotor, prof. dr. Philippe Lambin, bedankt voor uw vertrouwen en alle kansen die ik tijdens mijn promotietraject heb gekregen. De mogelijkheden om congressen en cursussen te bezoeken of bij een ander instituut op bezoek te gaan, net als de belangrijke connecties die ik heb opgedaan dankzij uw uitgebreide netwerk, zijn voor mij erg waardevol geweest. Ik bewonder uw inspiratie en creativiteit, nooit zit u zonder ideeën. Daarnaast ben ik heel veel dank verschuldigd aan mijn eerste copromotor: dr. Wouter van Elmpt. Wouter, je nuchtere kijk op het werk en het bedenken van simpele oplossingen zorgden ervoor dat ik na een overleg altijd weer verder kon. Bedankt dat je, hoe druk ook, altijd weer ergens tijd wist te vinden om te helpen, met het onderzoek, de manuscripten en later ook een Grant aanvraag. Ik waardeer de tijd die je voor de vele overleggen hebt vrijgemaakt en ook je bereidheid om mee te denken over de vervolgstappen na deze promotie. Het was fijn om altijd bij iemand terecht te kunnen voor een goed gesprek of advies. Daarnaast ook dank voor mijn tweede co-promotor dr. Ralph Leijenaar. Ralph, je kennis van radiomics en statistiek hebben mij erg geholpen en je kritische commentaar op een bepaald plan van aanpak hebben de papers beter gemaakt. De wandelingen tijdens de lunch hielpen voor een frisse neus en nieuwe energie om weer achter de computer te kruipen. Ik ben jullie alle drie, Philippe, Wouter en Ralph, dankbaar voor de begeleiding tijdens mijn promotietraject, ik heb onwijs veel van jullie geleerd. We zullen elkaar in de toekomst ongetwijfeld weer tegen komen.

Daarnaast wil ik graag de leden van mijn beoordelingscommissie (prof. dr. F.J.W. Verhaegen, prof. dr. R. Boellaard, prof. dr. A-M. Dingemans, prof. dr. M.E. Kooi en prof. dr. J.J. Sonke) bedanken voor het kritisch lezen en beoordelen van mijn proefschrift.

Next, I would like to give a big thanks to Professor Carsten Brink. We started the collaboration in the beginning of $\mathrm{my} \mathrm{PhD}$ and this resulted in a three month visit to Odense in the third year of my PhD. I have felt very welcome and these months gave me new energy for my projects. Besides the great experience of working on my research abroad, these months also served as an 'active recovery' during my PhD. Carsten, thank you for your time and the fruitful meetings we had and also for the continuous support and collaboration after my visit. Your positivity is really inspiring! Also thanks for Professor Olfred Hansen, for collecting the data, helping with any questions about the data and the continuous collaboration throughout my PhD. I 
would also like to thank my temporary-colleagues in Denmark for the company in the office, lunch in the 'hullet', after-work drinks and the Danish Christmas party. Tak for alt!

Prof. dr. Yolande Lievens, drs. Evelien Bogaert, dr. Leen Paelinck, prof. dr. Carlos De Wagter en dr. Elamin Elhaseen, bedankt voor de samenwerking voor het cone-beam CT radiomics project en dat ik naar Gent kon komen om het project en mijn werk nader toe te lichten.

Dr. René Monshouwer en prof. dr. Johan Bussink, bedankt voor het leveren van data, het beantwoorden van mijn vragen omtrent de data en het lezen van de manuscripten. En Frank, ik ben je hulp met het scannen van het fantoom in Nijmegen nog niet vergeten, bedankt!

Veder wil ik ook graag Hubert bedanken voor je tijd en hulp bij het scannen van het fantoom bij Maastro. De Servicedesk en Data managementteam van Maastro wil ik ook graag bedanken voor het verzamelen van data (in het bijzonder Joeri, bedankt!) en voor alle support omtrent ICT: de deur stond altijd open voor vragen.

André Dekker, bedankt voor de samenwerking omtrent het test-retest project, dit was mijn eerste artikel en ik heb hier meteen veel van kunnen leren. Bedankt ook voor de gesprekken begin vorig jaar en hulp met connecties in Australië. Daarnaast, André, Cheryl en Ellen, bedankt voor jullie werk omtrent de PhD discussies bij Maastro.

Cary Oberije, bedankt voor de fijne samenwerking bij een aantal projecten en daarnaast voor je kennis over statistiek, betrokkenheid en gezelligheid.

Verder wil ik Floor en Rianne graag bedanken, we kunnen altijd bij jullie terecht voor vragen over van alles en nog wat en hebben gedurende mijn $\mathrm{PhD}$ veel geholpen en geregeld, van DTA's tot handtekeningen en van grant-aanvragen tot het inplannen van afspraken. Daarnaast Natascha, het laatste kantoorgenootje van het andere vrouwenkantoor op de D-Lab, met Cary, Floor en Rianne, bedankt voor je betrokkenheid en gezelligheid.

Arthur en Henry, onze postdocs, bedankt voor alles wat jullie voor de groep doen en hebben gedaan. Jullie staan altijd voor de groep klaar en zijn bereid overal mee te helpen, bedankt! Daarnaast, Arthur, bedankt dat je mij op weg hebt geholpen met het deep learning project.

I would also like to thank all my colleagues for the good atmosphere at work, coffee breaks, vlaai, treats from all over the world, lunch, walks, PhD parties, carnival, drinks at Thembies and participation at $\mathrm{PhD}$ lunch meetings. With the danger of forgetting someone here: Abdalla, Adriana, Alberto, Alex, Ana, Aniek, Anshu, Arthur, Brent, Cecile, Damiënne, Daniela, Elizaveta, Esma, Esther, Evelyn, Fadila, Frank, Gabriel, Giacomo, Guangyao, Henry, Inge, Isabel, Iva, Joana, Johan, José, Jurgen, Karen, Leonard, Lotte, Lucas, Manon, Mariana, Mark, Marta, Matilde, Murillo, Relinde, René, Rianne, Ruben, Sanaz, Sara, Séan, Sebastian, Sergey, Shane, Simon, Skadi, Stefan, Timo, Turkey, Wouter, Yvonka, Zhen and Zhenwei: thanks for creating these great memories. Sara, you left Maastro already a while ago, but I will always remember your enthusiasm and sparkling personality. Isabel, thank you for sharing your passion about korfball, I loved our trainings together. Although I haven't played in a while, I 
hope I can still enjoy your games in the national team of Portugal for a long time, you're a great player! Ana, we met each other already in Galway, and I was really happy to see you again in Maastricht. You didn't have the best start, but I have a lot of respect for your perseverance. Gabriel and Loise, thank you for your friendliness and hospitality. Timo, thank you for your great company and your willingness to help everyone anytime with anything. Jurgen, je Belgische accent moest ik even aan wennen, maar je gezellige humor werd altijd zeer gewaardeerd. Gelukkig zijn we uiteindelijk nog langs gekomen in je thuishaven Antwerpen. Ik vond het heel bijzonder en een eer om je paranimf te zijn, bedankt! Enne, volgens mij heb ik nog steeds een paarse aardappel van je tegoed. Aniek, ik waardeer je nuchtere persoonlijkheid en 'yolo' instelling. Je flauwe grappen samen met Ruben zorgden af en toe voor wat welkome afleiding op kantoor. Waar Mark en jij nu ook op de wereld zijn, ik hoop dat jullie nog lang van deze wereldreis mogen genieten! Ik kom graag eens luisteren naar jullie verhalen. Ruben, je organisatie skills hebben bijgedragen aan vele leuke PVM-activiteiten: kookworkshop, Efteling, winterwandeling en uiteraard carnaval, waaraan mijn herinneringen aan jou onlosmakelijk verbonden zijn. Als jij in de buurt was, werd er altijd wel een gezellig feestje gevierd! En of het nu het schrijven van een paper is of het maken van een PhD filmpje, ik weet dat ik kan vertrouwen op je last-minute capaciteiten, en dat maakt ons een goed team. Also thank you for the great weekend in Cambridge, Sara, Nuno, Daniel, Ruben, Jurgen, Evelyn and Karen. Daarnaast wil ik graag mijn sportmaatjes Aniek, Cecile, Ruben, Evelyn en Jurgen bedanken voor het squashen, klimmen en vele groepslesjes bij UM sport: club power, core\&more, super HIIT, bootcamp, enzovoort. Shane, thank you for still being my Tuesday morning 7.15am spinning companion (at least, mostly). Henry, thank you for the weekly climbing sessions, for teaching me and your endless enthusiasm and passion about climbing. Also thanks for your nice company in the office and conversations at work or during climbing, I've really enjoyed those. I hope that we can go climbing outdoors a few times this spring. Iva, my dear office mate, you just started climbing but I hope you're going to love it as much as I do!

Wat betreft sport, een belangrijke periode tijdens het derde jaar van mijn PhD was de Roparun. Mijn nieuwe motivatie voor hardlopen en het enthousiasme van collega's heeft mij uiteindelijk doen besloten in mei 2018 met team 18 Maastro Clinic mee te gaan als Roparunner. De voorbereidingen en de activiteiten vormden een belangrijk onderdeel van de voorpret en opbouw van de spanning. Ik vond het een fantastisch en heel bijzonder weekend om nooit meer te vergeten! Mijn hardloopteammaatjes Robert, José en Harvey, fietsers Britt, Dianne, Helen en Mirthe, en chauffeur Lucas, bedankt voor de super goede sfeer onderweg en het oppeppen van elkaar, ondanks vermoeidheid of blessures. Daarnaast de rest van het team ook enorm bedankt voor deze prestatie en alle (extra...) kilometers, de vele voorbereidingen en acties om geld binnen te halen. Ik ben super trots op iedereen: Angele, Benny, Cathelijn, Cheryl, Claudia, Dianne, Fenne, Gloria, Harvey, Henny, Indra, John, Jolein, José, Lieke, Lucas, Manon, Mirthe, Rafiq, Rik, Rob, Robert, Stefan en Tiny. Jullie hebben het geweldig gedaan.

Verder wil ik in het bijzonder graag Evelyn bedanken, ik was vanaf dag 1 blij met mijn gezellige buurvrouw die me alles over het reilen en zeilen bij Maastro kon 
vertellen. We mochten beiden een presentatie geven bij ASTRO in Boston en hebben toen besloten het bezoek aan dit congres aan te vullen met twee heerlijke weken vakantie in Canada en Amerika, waar we de meest bijzondere walviservaring hadden. Daarnaast deden we samen mee aan de tenniscursus, gingen we klimmen of gewoon gezellig kletsen. Ik heb er een goede vriendin bijgekregen waar ik erg dankbaar voor ben. Na een tijdje kregen we een nieuwe collega: Cecile, je werd al snel een vriendin van ons beiden. Samen werden we Maastrio en konden we al kletsend aardig wat koffiepauzes en avondjes vullen. Cecile, bedankt voor de gezelligheid en onze heerlijke dagen Barcelona tijdens en na de ESTRO afgelopen jaar. Relinde, ik leerde jou goed kennen toen je je PhD bij de universiteit bent begonnen en mijn kantoormaatje werd. Dit was altijd erg gezellig en ik ga je droge grappen tussen het werken door zeker missen! Mijn drie paranimfen: Relinde, Evelyn en Cecile, bedankt voor de gezellige avondjes samen eten en borrelen en steun door de jaren heen. Het was erg fijn om altijd je verhaal kwijt te kunnen. Daarnaast vond ik het echt superleuk dat jullie me in Odense kwamen opzoeken! Sindsdien gaan we regelmatig gezellig samen een weekendje weg: Brugge, Porto of een mini-roadtrip door België, Frankrijk, Luxemburg en Duitsland. In zo'n weekend is er heel veel tijd voor gezelligheid, ontspanning, bijkletsen, sightseeing en lekkere hapjes en drankjes. Als het aan mij ligt houden we deze weekendjes erin!

Naast iedereen die ik dankzij mijn PhD heb leren kennen, zijn er nog heel veel mensen die ik wil bedanken omdat ze hebben bijgedragen aan ontspanning en leuke activiteiten in de weekenden, interesse hebben getoond in mijn werk en me hebben gemotiveerd om door te gaan. Allereerst de vriendinnengroep die ik het langst ken, sommigen zelfs al sinds groep 2, en beter bekend staat als The Gang. Lieve meiden, Ellen, Eva, Gea-marit, Gwendolien, Henrieke, Jolien, Julia, Nadine en Sarie, jullie zijn geweldig. Verjaardagen, weekendjes weg, Sinterkerst, samen koken, borrelen, uiteten of op stap, in Groningen, Amsterdam, Zwolle, Utrecht, Cardiff, Odense, Bad Bentheim, Zeewolde, Enschede en niet te vergeten, Maastricht. Ik vind het heel bijzonder dat we na al die jaren, waarin er veel veranderd is en nog blijft veranderen (met de komst van Gangbaby\#1 Nore), zulke goede vriendinnen zijn gebleven. Ik hoop dat dit altijd zo zal blijven, maar daar heb ik alle vertrouwen in!

Daarnaast wil ik mijn lieve vriendinnetje Regina bedanken voor de gezellige dagjes theedrinken, winkelen en kletsen. Bovendien heeft onze fantastische backpack vakantie in Slovenië in 2017 voor altijd een bijzonder plekje in mijn hart! We zijn ter voorbereiding allebei wat fanatieker gaan hardlopen en hebben we in oktober 2017 samen de halve marathon gelopen in Breda. Deze momenten zijn altijd een zeer belangrijke afleiding geweest tijdens mijn promotie.

Mijn motivatie om te hardlopen is ook aangewakkerd dankzij Gijs. Ik wil jou ook bedanken voor de mooie tijd die we samen hebben gehad. Ik heb hele mooie herinneringen aan de gezellige weekendjes, hardloop- en wandelrondes in de bossen en de weekenden in Odense en Hamburg. Ik zal dit jaar nooit vergeten.

Kirsten en Richard, ik kom net bij jullie vandaan terwijl ik dit aan het schrijven ben, en we hebben weer een ouderwets gezellig weekendje samen gehad. Kirsten, zoals je zei toen ik binnenkwam: "je bent weer thuis". Zo voelt het altijd nog een 
beetje. Ondanks dat we elkaar natuurlijk nu niet meer zo vaak zien, voelt het elke keer weer alsof het gisteren was. Bedankt voor deze bijzondere vriendschap en ik ben heel trots dat ik getuige mag zijn bij jullie aankomende bruiloft.

Ten slotte wil ik nog graag mijn familie bedanken voor de gezelligheid op verjaardagen, een bijzondere bruiloft, feestjes of familiedag: Janet, Jan en Grietje, Erik-Jan en Rianna, Wouter en Manuela, Anne-Marie en Bas, Mattheus en Henriette, Bert-jan, André, Xander, Regina en Frank en nu ook Bert en Vera. Daarnaast bedankt voor jullie interesse rondom mijn promotie. Ook al is het misschien voor jullie niet altijd helemaal duidelijk geweest waar ik al die tijd mee bezig was, ik waardeer het zeker!

Lieve zus en zwager, lieve Lianne en Ricardo, bedankt dat ik altijd welkom bij jullie ben, bij jullie ben ik ook altijd een beetje thuis. Ik kan altijd bij jullie terecht voor een slaapplekje, een middagje puzzelen, spelletjes spelen, gezellig dinertje, high-tea of borreltje en een luisterend oor. Jullie bruiloft afgelopen jaar was erg bijzonder en ik ben heel dankbaar dat ik zowel ceremoniemeester als getuige mocht zijn. Zus, ondanks dat we vroeger veel ruzie hadden, zijn we inmiddels de beste vriendinnen. We hebben de afgelopen jaren helaas niet dicht bij elkaar gewoond: ik studeerde in Amsterdam terwijl jij in Groningen studeerde en toen jij naar de randstad verhuisde begon ik aan mijn promotie in Maastricht. Wie weet gaat dat binnenkort veranderen.. Gelukkig zien we elkaar regelmatig en ik weet bovendien dat je er altijd voor me bent. Lieve zus, bedankt voor alles!

Ik ben ontzettend dankbaar voor al deze lieve mensen om mee heen. Dit is enorm waardevol en ik ben heel blij dat ik altijd bij iemand terecht kan als er iets is. Hiervoor wil ik ook tenslotte heel graag m'n ouders bedanken. Lieve pappa en mamma, bedankt dat jullie er altijd voor ons zijn en achter onze keuzes staan. Ik vind het heel mooi om te zien hoe trots jullie zijn, daar ben ik dan weer trots op. Ik heb me altijd gesteund, begrepen en aangemoedigd gevoeld. Ik kan niet in woorden uitdrukken hoe dankbaar ik jullie ben, maar ik hoop dat jullie dat weten. Bedankt voor alles, ik houd van jullie. 



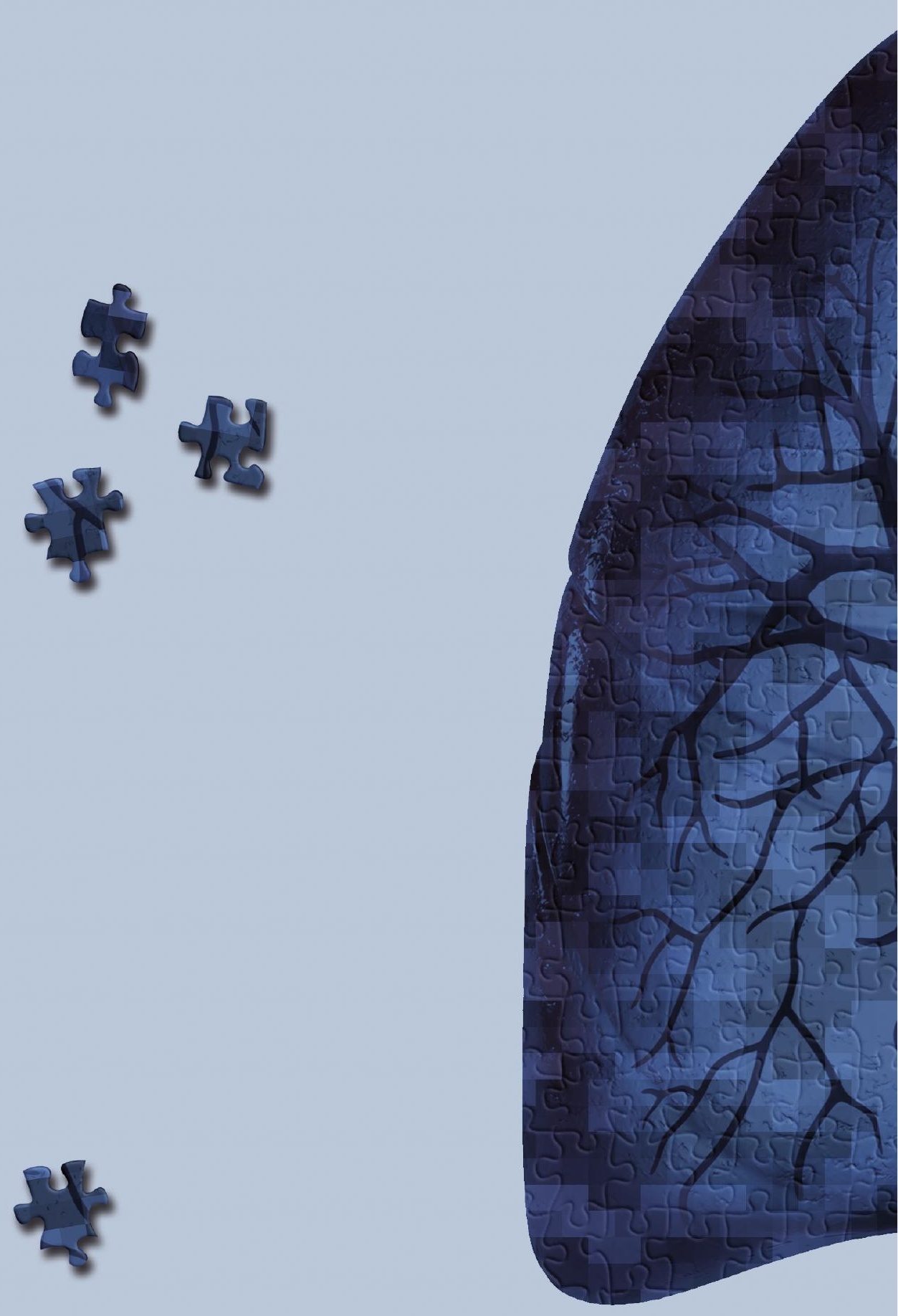